
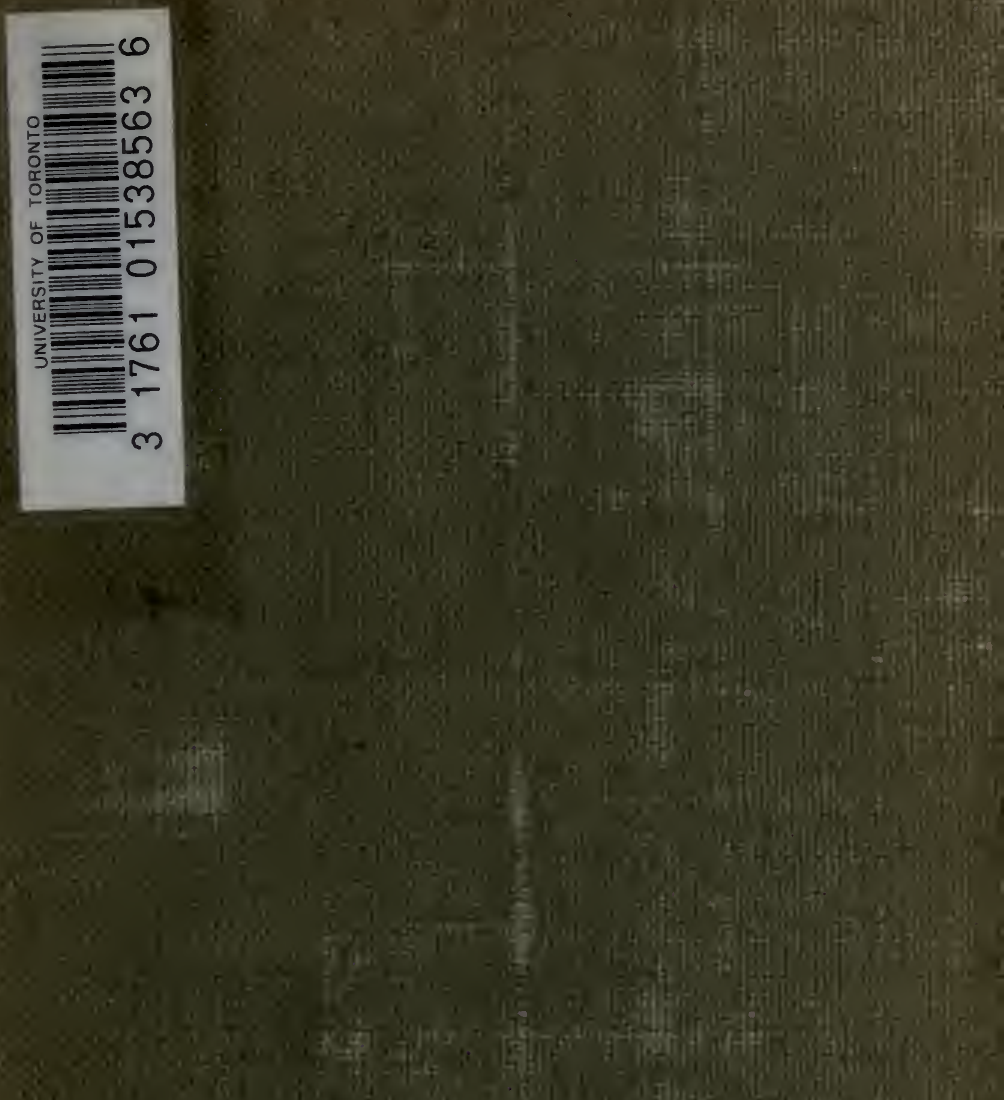

Shy

ous torits

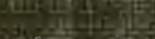

$$
=40,14 \text {. }
$$

\title{
Uitu: of
}

Tinse

LIESARY
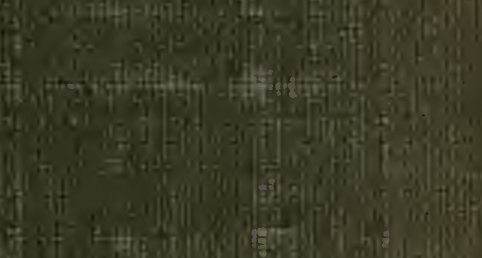
Nof 



\section{LIBRARY \\ FACULTY OF FORESTRY UNIVERSITY OF TORONTO}



Digitized by the Internet Archive in 2007 with funding from Microsoft Corporation 



\title{
ENGYCLOPÉDIE AGRICOLE
}

Publiée sous la direction de G. WERY

\author{
Albert FroN
}

SYLVIGULTURE 


\section{ENCYCLOPÉDIE AGRICOLE}

Publlée par une réunlon d'Ingénleurs agronomes

SOUS LA DIRECTIOX DE

G. WERY

Ingénieur agronone

Sous-Directeur de l'Institut Xational Agronomique

\section{Introduction par le Dr P. REGNARD}

Directeur de l'Institut National Agronomique

Mlembre de la Société Nationale d'Agriculture de France.

:- volumes in-16 de chacun 400 à 500 pages, illustrès de nombreuses figures.

Chaque volume : broches, $5 \mathrm{fr}$.; cartonné, 6 fr.

\section{Agriculture générale...........}

Industries agricoles de fermen. iation (Cidrerie, Brasserie, Mydromels, Distillerir) ........

Engrals..................... Plantes fourrageres...........

Oralnage et Irrlgations.......

Plantes industrielles...........

cortales.

Cultures potagdres ............. Arboriculeure..................

syloleulture.................

viticulture .................. Vinincation (in, linaigre.

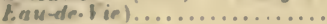

Zoologle agricole

Zootechnie gendrale. Zootechnle spoclale (Races)..... spécinl d'agriculture.

machlnes agricoles............ M. Cotras, ingẻnieur agronome, répetiteur h

Constructions rurales.........

Economie agricole

It ceislation rurale.

Technolocle asticole (Suererie, feevierie, meunerie, buvlange-

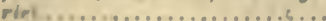

Lalterlo.....................

Aquleulture.................. l'Institut untional ngronomique.

M. DanguY, ingénieur agronome, directeur des études a l'Ficole nationale d'agriculture de Girignon.

M. P. Diffloth, ingénieur agronome, professeur spécial d'agriculture.

II. Bocluaxgen, ingénieur agronome, chef de Laboratoire à l'Institut Pasteur de Lille.

M. Garola, iugénieur agronome, professeur départemental d'ugriculture a Charties.

II. Risikn, directeur honoraire de I'Institut national agronomique, Menubre de la Société Nationale d'Agriculture de France.

M. G. W Wıx, ingénieur agronone, sous-directeur de l'Institut national agromonique.

M. Tnocnr, ingénieur agronome, professeur à l'École nationale des industries agricoles de Douai.

M. Laval..és, ingénieur agronome, ancien chef des travaux de la Station expérimentale agricole de Cappelle.

M. Leon Bussard, ingénieur agronome, chef des travaux de la Station d'essais de semences, a l'lustitut untional agrononique, professeur à l'Ecole nationnle d'horticulture.

Il. Finox, ingénieur agronome, professeur à l'École forestière des Barres (Loiret).

M. Pacutrit, ingénieur agronome, répétiteur à I'Institut national agronomique.

M. P'ACutrer, ingénieur agronone.

II. Georges Gunxacx, ingénieur agronome, répétiteur a l'lustitut nntional agrononique.

II. Joezan, iugénieur ngronome, prof sseur à l'École untionale d'agriculture de Rennes.

M. SAll.Al1b, ingénieur agronome, professeur a I'Eccole nationale des industries agricoles de Douni.

.M. Malur, ingénieur agronome, ancien directeur de l'lecole nationalo d'industrie laitiére de Mamirolle.

It. Desoscs.k, ingéuieur agronome, inspecteur général de In piscitulture. 


\section{ENGYGLOPÉDIE AGRIGOLE}

Publiée par une réunion d'Ingénieurs agronomes SOUS LA DIRECTION DE G. WERY

\section{SYLVICULTURE \\ nin}

\section{Albert FRON}

INGÉNEUR AGROXOME

INSPECTECR ADJOINT DES EATX ET FORETS

Introduction par le D: P. REGNARD

DIRECTEUR DE L'INSTITCT NATIONAL AGRONOMIQUE

MEMBRE DE LA SOCIÉTÉ N $^{\text {le }}$ D'AGRICULTURE DE FRANCE

Avec 55 figures intercalées dans le texte
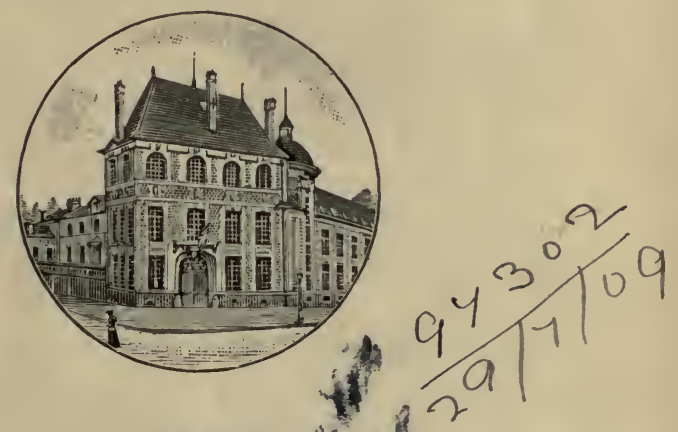

PARIS t

LIBRAIRIE J.-B. BAILWUERE ET FILS 19, rue Hauteleuille, près du Bgutyred

190,3 है

Tous droits $x$ servés. 


$$
\begin{aligned}
& S D \\
& 391 \\
& F 65
\end{aligned}
$$




\section{ENCIYCLOPEDIE AGRICOLE}

\section{INTRODUG'TION}

Si les choses se passaient en toute justice, ce n'est pas moi qui devrais signer cette préface.

L'honneur en reviendrait bien plus naturellement à Iun de mes deux éminents prédécesseurs.

a Eugène Tisserand, que nous devons considérer comme le véritable créateur en France de l'enseignement supérieur de l'Agriculture : n'est-ce pas lui qui, pendant de longues années, a pesé de toute sa valeur scientifique sur nos gouvernements, et obtenu qu'il fùt créé à Paris un Institut agronomique comparable à ceux dont nos voisins se montraient fiers depuis déjà longtemps?

Eugène Risler, lui aussi, aurait dù plutòt que moi présenter au public agricole ses anciens élèves devenus des maitres. Près de douze cents Ingénieurs Agronomes, répandus sur le territoire français, ont été façonnés par lui : il est aujourd'hui notre vénéré doyen, et je me souviens toujours avec une douce reconnaissance du jour où j'ai débuté sous ses ordres et de celui, 
proche encore, oủ il m’a dẻsigné pour être son successeur.

Mais, puisque les éditeurs de cette collection ont voulu que ce fùt le directeur en exercice de l'Institut agronomique qui présentat aux lecteurs la nouvelle Encyclopédie, je vais tàcher de dire brièvement dans quel esprit elle a été conçue.

Des Inge̊nieurs Agronomes, presque tous professeurs d'agriculture, tous anciens élèves de l'Institut national agronomique, se sont donné la mission de résumer, dans une série de volumes, les connaissances pratiques absolument nécessaires aujourd'hui pour la culture rationnelle du sol. Ils ont choisi pour distribuer, rigler et diriger la besogne de chacun, Georges Wery, que j’ai le plaisir et la chance d'avoir pour collaborateur et pour ami.

L'idée directrice de l'œuvre commune a été celle-ci : extraire de notre enseignement supérieur la partie immédiatement utilisable par l'exploitant du domaine rural ef faire connaitre du même coup à celui-ci les données scientifiques définitivement ac(quises sur lesquelles la pratique actuelle est basee.

Ce ne sont donc pas de simples Manuels, des Formulaires irraisonnés que nous offrons aux cultivateurs, ce sont de brefs Traités, dans lesquels les résultats. incontestables sont mis en évidence, à côté des bases scientifiques qui ont permis de les assurer.

Je voudrais qu'on puisse dire qu'ils représentent le réritable esprit de notre Institut, arec cette restriction quils ne doivent ni ne peuvent contenir les discussions, les erreurs de route, les rectifications qui ont fini par établir la vérité telle qu'elle est, toutes choses que l'on diveloppe longuement. daus notre enseigne- 
ment, puisque nous ne devons pas seulement faire des. praticiens, mais former aussi desintelligences élevées, capables de faire avancer la science au laboratoire et sur le domaine.

Je conseille done la lecture de ces petits rolumes à nos anciens élèves, qui y retrouveront la trace de leur première éducation agricole.

Je la conseille aussi à leursjeunes camarades actuels, qui trouveront là, condensées en un court espace, bien des notions qui pourront leur servir dans leurs. études.

J'imagine que les élèves de nos Écoles nationales. d'Agriculture pourront y trouver quelque profit, et que ceux des Écoles pratiques devront aussi les consulter. utilement.

Enfin, c'est au grand public agricole, aux cultivateurs. que je les offre avec confiance. Ils nous diront, après. les avoir parcourus, si, comme on l'a quelquefois prétendu, l'enseignement supérieur agronomique est exclusif de tout esprit pratique. Cette critique, usée, disparaîtra définitivement, je l'espère. Elle n'a d'ailleurs jamais été accueillie par nos rivaux d'Allemagne et d'Angleterre, qui ont si magnifiquement dèveloppé chez eux l'enseignement supérieur de l'Agriculture.

Successivement, nous mettons sous les yeux du lecteur des volumes qui traitent du sol et des façons qu'il doit subir, de sa nature chimique, de la manière de la corriger ou de la compléter, des plantes comestibles. ou industrielles qu'on peut lui faire produire, des animaux qu'il peut nourrir, de ceux qui lui nuisent.

Nous étudions les manipulations et les transforma-tions que subissent, par notre industrie, les produits de la terre : la vinification, la distillerie, la panifica- 
tion, la fabrication des sucres, des beurres, des fromages.

Nous terminons en nous occupant des lois sociales qui régissent la possession et l'exploitation de la propriété rurale.

Nous avons le ferme espoir que les agriculteurs feront un bon accueil à l'œurre que nous leur offrons.

\section{Dr Paul Regiard,}

Membre de la Société nationale

d'Agriculture de France,

Directeur de l'Institut national agronomique. 


\section{P RÉ F A G E}

Répandre au sein des populations agricoles les notions classiques de sylviculture afin de faire comprendre la forèt, de la faire aimer et respecter; donner en même temps les notions pratiques nécessaires au propriétaire qui gère un domaine boisé : tel est le double but de cet ouvrage.

Professeur à l'École forestière des Barres, chargé arec nos collègues de préparer aux fonctions d'agent une élite de préposés forestiers, nous avons entrepris ce travail à la demande d'un grand nombre de nos camarades de l'Agriculture qui n'ont pas, comme nous, suivi les cours spéciaux de l'École forestière de Nancy, et qui ont soit à enseigner comme professeurs d'agriculture, soit à appliquer comme propriétaires forestiers les règles générales de la sylviculture. C'est à eux que nous le dédions.

A tous points de vue, d'ailleurs, l'art forestier est à divulguer auprès du propriétaire foncier.

Sil s'agit d'un domaine forestier, beaucoup trop de propriétaires, en en exceptant toutefois les professionnels, considerent la forèt comme un bien qui se gère tout seul, sans règle, au hasard des caprices; ils considèrent le terrain sur lequel repose la forèt comme un sol indéf:niment fertile, susceptible de donner des produits ligneux et aussi de la litière, sans exiger aucune restitution; 
souvent alor's, après des exploitations trop hâtives, après des fautes culturales répélées, le massif s'interrompt, les homnes essences disparaissent et sont progressivement remplacées par des mort bois, des épines, des ronces, de la bruyère ou des genèts; le sol s'appaurrit el à la forèt se substituent peu à peu des friches incultes et improductives.

S'il s'agil d'un domaine agricole, beaucoup trop de propriétaires dédaignent la forêt, et ne comprennent pas le rôle qu'elle est appelée à jouer pour améliorer les mauvaises terres et pour équilibrer les cultures. Aujourd'hui où la culture intensive s'impose plus que jamais en agriculture, oủ la main-d'œurre de plus en plus rare ne peut se multiplier sur d'immenses surfaces à faible rendement, le propriétaire parait avoir intérèt à concentrer ses efforts sur les terres de honne et de moyenne qualité; il doit rendre les maurais sols à la culture forestière; c'est une conséquence des conditions économiques actuelles et de l'emploi raisonné du fumier et des engrais; c'est aussi une conséquence des défrichements exagérés qui ont été eflectués au cours des siècles précélents, alors que la culture extensive demandait d'immenses surfaces pour assurer la production normale des denrées agricoles.

S'il s'agit enfin des terres définitivement abandonnées, qu'on laisse à tort à l'état de friches ou de pâtures dégradées, beaucoup trop de propriétaires ne paraissent pas se douter, tout au moins dans certaines régions, qu'on peut les restaurer progressivement, et souvent mème les remettre en valeur par la culture forestière et les présbois.

Dans notre carrière forestière, nous avons toujours été en contact arec e propriétaire foncier, possesseur du sol contigu aux forts soumises au régime forestier; mainte fois des propriétaires de terres et de bois, des régisseurs de domaines boisés, des camarades de l'Agrri- 
culture et aussi des instituteurs s'intéressant aux queslions forestières, nous ont demandé des conseils pratiques sur les choses concernant notre profession spéciale. En publiant dans l'Encyclopédie agricole un volume de sylviculture, nous avons voulu compléler et généraliser ces conseils, et nous ne saurions trop remercier MM. Regnard et Wery, qui ont pris linitiative de cette Encyclopédic, d'avoir bien voulu entrer dans nos vues, et de nous avoir fourni l'occasion de réaliser notre projet.

Aujourd'hui l'enseignement forestier, longtemps négligé en dehor's des Écoles spéciales et de nos grandes Écoles d'agriculture, tend à se répandre dans toutes les classes de la société, et à prendre dans nos établissements publics le rang auquel il a droit. Des cours adaptés à leur auditoire spécial se créent un peu partout, dans les Facultés, dans les Lycées et les Collèges, dans les Écoles normales d'instituteurs et les Écoles primaires; un peu partout, des hommes déroués à la sylviculture cherchent à faire comprendre l'utilité des massifs boisés et le rôle que les for'èts et le reboisement sont appelés à jouer de nos jours dans l'économie générale du pays. Des suciétés forestières, grandes ou petites, s'ouvrent à l'iniative privée, et parmi elles nous voulons citer les sociétés scolaires forestières, nées hier en Franche-Comté, et dont les premiers travaux paraissent déjà couronnés de succis sur plusieurs points de notre territoire. Les divers services du Ministère de l'Agriculture et les pouroirs publics encouragent ce mourement naissant qui peut avoir de si heureuses conséquences pour notre pays.

En résumant dans un ourrage classique les principes fondamentaux de la sylviculture, nous avons voulu aider les débutants dans ces cuvres nouvelles, et pour cela nous nous sommes donné comme règle d'étayer nos opinions et nos conseils sur des citations prises dans les 
"uvracres forestiers les plus sérieux, persuadé que le lecteur aura tout intérèt à se reporter aux auteurs que nous signalons ainsi à son attention.

Pour donner plus d'intérèt à nos descriptions de massils boisés, nous avons reproduit une série de rues pholographiques prises par nous au cours de nos tournćes forestières. Nous avons été heureux de pouroir y joindre quelques-unes des photograrures qui illustrent lintéressant ouvrage de notre ancien maître M. Boppe et de notre camarade Jolyet.

Enfin pour suppléer aux excursions en forêt, si nécessaires pour comprendre et faire aimer les bois, il manquait, à notreavis, dans les collections scolaires des rues de massifs hoisés pouvant ètre projetées derant un auditoire. M.M. Radiguet et Massiot, dont les collections de vues de projection sont déjà si variées, ont bien roulu accepter de commencer avec nos clichés des séries forestières destinées à des cours et conférences de sylviculture. Nous tenons à leur en exprimer ici tous nos remerciements.

II nous reste un agréable devoir à remplir: c'est de remercier les agents de l'Administration des eaux el forèts qui ont bien voulu nous aider de leurs conseils ; nous nous faisons un honneur de citer parmi eur les noms de M.M. Marchand, directeur de l'Ecole foreslière des Barres, Broilliard, Sanglé-Ferrière, Barret, Pardé, Delaunoy, Galmiche, et enfin celui de M. Riret, notre premier professeur de sylviculture, lorsque nous étions blive à l'Institut national agronomique. A tous nous leur exprimons nos sentiments de respeclueuse gratilude.

A. Finos.

Nogent-sur-Vernisson, 1er mai 1903. 


\section{SYLVICULTURE}

\section{PREMIERE PARTIE}

\section{LA FORÊT EN GÉNÉRAL ET SES ÉLÉMENTS CONSTITUTIFS}

\section{PREMIÉRE SEGTION}

\section{Vie de l'arbre en général.}

\section{GÉNĖRALITĖS}

Tout végétal dont la durée embrasse un nombre d'années considérahle, qui présente à la base une tige ligneuse, nue et simple, portant à une hauteur plus ou moins grande une couronne formée ou d'un faisceau de feuilles, ou d'un grand nombre de branches subdivisées en rameaux sur lesquels les feuilles sont fixées, est un arbre.

Un arbre, dans nos climats, est un régétal phanérogame (1), c'est-à-dire un être vivant, du règne végétal,

(1) Les phanérogames se subdivisent en gymnospermes (sapin, épicéa, pin, mélèze, arbres résineux) et en angiospermes; les angiospermes se subdivisent en monocotylédones (palmiers, etc., arbres à tige cylindrique appelée stpie) et en dicotylédones (chêne, hêtre, etc., grands arbres feuillus de nos

A. Fron. -Sylviculture. 
pourvu de racines, d'une tige, de feuilles, et qui donne naissance à des organes apparents qu'on désigne sous le nom de fleurs.

La fleur, dont les parties essentielles sont les étamines (organe mâle) et le pistil (organe femelle), donne après fécondation le fruit et la graine. Le régétal arbre, parvenu à l'àge adulte, dissémine ses graines tout autour de lui, dans un rayon plus ou moins grand suivant que la semence est lourde (gland, faîne, etc.), légère (bouleau, elc.), ailée (arbres résineux), ou munie d'organes accessoires facilitant son transport à distance (peuplier, orme, érable, etc.).

De la graine, jlacée dans des conditions favorables à la germination, naît un nouvel individu, semblable à ses parents (1).

\section{I. - GERMINATION}

Lorsqu'on place une graine d'arbre, par exemple un gland (semence du chène), dans un sol meuble, c'est-àdire perméable à l'air, humide et abrité par des feuilles contre la vive lumière du soleil, on voit au bout de quelques jours, si la temperature est suffisamment élevée, ce gland se gonfler, et son enveloppe écailleuse se fendre. L'embryon contenu dans cette graine passe de l'état de vie ralentie à l'état de vie active, et développe d'abord sa radicule en une petite racine qui se dirige de haut en bas dans le sol; puis les deux cotylédons, en s'écartant, laissent sortir la gemmule qui se dirige vers le ciel. Dans le chêne, la germination est hypogée; la tigelle ne s'ac-

forèts). Cette distiuction entraine une très grande différence daus la constitu. tion des organes de la plante, du bois par exemple.

(t) Ia reproduction par voie de semences coniporte une hérédité avec variations possibles; il $n^{\prime} y$ a hérédité sans variations possibles que par les procédés de multiplication, spore, houture, marcotte, greffe, rejet affranchi, drageon, et le nouvel individu ainsi ohtenu est identique a celui dont il est tosu. Cette distinction, très inportante en horticulture, l'est moins dans le domaine de la praticjue on sylviculture. 
croît que faiblement, et les cotylédons restent emprisonnés dans le sol (fig. 1). Pendant cette première période de son existence, le jeune chène, encore atta. ché au gland d'où il est sorti, se nourrit des éléments de réserve contenus dans les cotylédons; bientôt la radicelle s'implante dans le sol, la jeune pousse donne naissance à des feuilles normales qui s'étalent audessus du sol; les cotylédons épuisés, flétris, devenus inutiles, se détachent et le petit chène vit de sa vie propre. Il est, à la vérité, à l'état d'ébauche mais il est complet. Son accroissement ultérieur s'opérera par la multiplication et la différenciation progressive des organes dont il est pourvu à ce moment.

Une faîne (semence de hètre) germe dans des conditions analogues; toutefois la tigelle, en se déve-

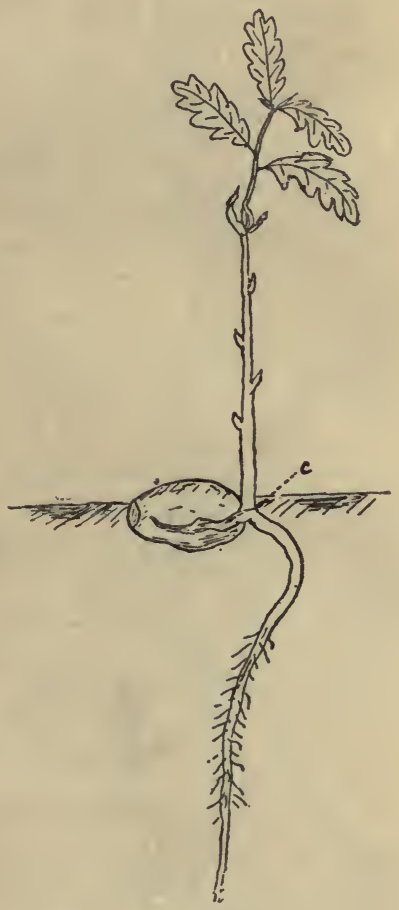

Fig. 1. - Jeune plant de chêne (germination hypogée). $c$, collet de la racine. loppant, porteàl'extérieur du sol les deux cotylédons contenus dans la graine, qui s'épanouissent en feuilles cotylédonaires; on dit dans ce cas que la germination est épigée (fig. 2). Chez le sapin, la germination est épigée et le jeune plant possède cinq à sept feuilles cotylédonaires. 
Toutes les graines qui tombent sur le sol ne germent

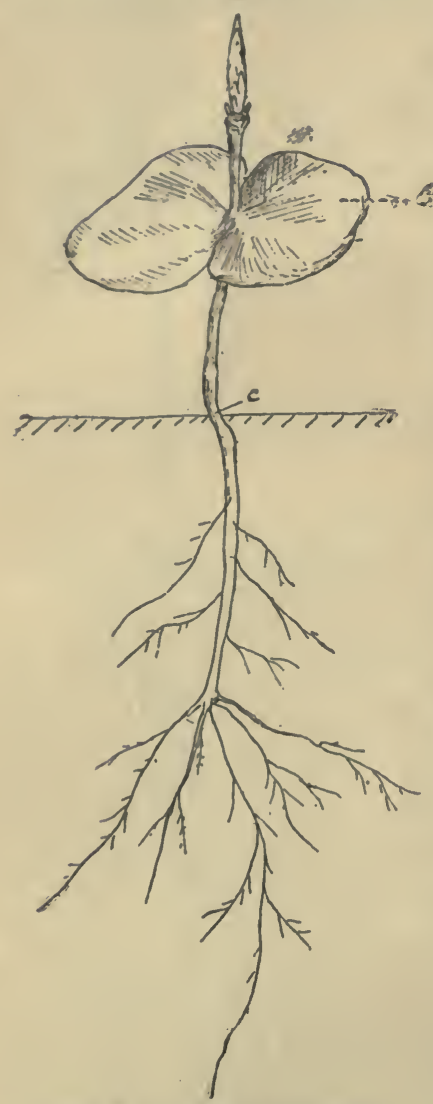

Fig. 2. - Jeune plant do hêtre

(germination épigée).

c, collet de la racine; $C$, feuilles cotylédonaires. pas; beaucoup servent de nourriture aux animaux; il s'en perd un grand nombre, faute d'avoir trouvé, sur le point où elles sont tombées, les conditions nécessaires à leur germination : dans un terrain trop humide, elles pourrissent; elles se dessèchent au contraire, si l'eau fait défaut. Le froid arrète la germination, une chaleur modérée l'accélère, mais la vive lumière lui est défavorable.

De mème, toutes les graines qui ont commencé à germer ne réussissent pas ; pour que la jeune racine puisse pénétrer dans le sol et s'y développer, il faut, non seulement que ce sol soit meuble et humide, mais encore que la graine en germination ne soit pas séparée du sol par un obstacle, tel qu'une couche trop épaisse de feuilles mortes; si le sol est dur, tassé, la radicelle trop tendre ne peut percer la couche 
superficielle; elle se dessèche et la plante meurt. Elle meurt aussi quand le sol est trop aqueux, trop compact et par suite insuffisamment aéré.

Un jeune plant(1), pendant toute cette première phase de la végétation, est constitué par des tissus pleins de liquide et par suite très tendres; il ne présente pas la mème vigueur et la mème force de résiștance contre les excès du froid, de la chaleur et de la sécheresse, que l'arbre fait; un hâle de quelques heures, un coup de soleil suffisent pour le flétrir; aussi, dans les premières années de leur existence, beaucoup de jeunes semis ontils besoin d'abri. En général, la nature a pris soin de leur assurer elle-mème cette protection, d'abord par les feuilles mortes qui courrent le sol, puis par le feuillage des arbres porte-graines; plus les jeunes plants sont sensibles, plus ces abris naturels sont puissants, et on voit presque toujours les arbres à feuillage touffu, à semences lourdes, produire de jeunes plants délicats (c'est-à-dire ayant besoin d'abri pendant leur toute jeunesse), tandis que les arbres à feuillage léger donnent naissance à des plants robustes (c'est-à-dire susceptibles de se passer d'abri dès leur jeune âge, tout au moins dans les circonstances climatériques qui leur sont appropriées).

\section{II. - ENRACINEMENT}

La racine se développe en profondeur dans le sol, de telle manière que l'extrémité de la racine principale aille verticalement vers le centre de la terre. Elle est constituée en principe par un axe conique allongé qui présente une région de poils absorbunts (2) (fig. 3); le sommet de

(1) Jeune arbre issu directement d'une graine.

(2) La région des poils absorbants, située à une distance déterminée du son net de la racine, se déplace dans le sol, a's fur et à mesure de l'allongement de la racine, ce qui permet à l'arbre d'aller progressivement puiser dans un milieu non encore épuisé les aliments nécessaires. Enfin, si on casse la partie 
la racine (extrémité tournée ver's le bas) est recouvert par un tissu de protection, la coiffe. De bonne heure cet axe principal émet latéralement des ramifications de premier ordre, ayant la mème constitution que la racine principale; ces ramifications émettent

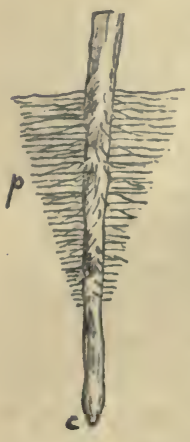

Fig. 3. - Partie terminale d'une racine. elles-mèmes des ramifications de second ordre, qui deviennent de plus en plus grèles, et ainsi de suite; ainsi se constitue l'enracinement de l'arbre, avec une ou plusieurs grosses racines, des radicelles et enfin un enchevêtrement de filaments très grêles qu'on appelle le chevelu.

Dans le genre chène, l'axe principal ou pirot s'enfonce, sauf obstacle, profondément dans le sol, continuant à s'accroìtre aussi verticalement que possible, et il conserve toujours une prédominance marquée sur les racines latérales dont les principales ont une tendance à s'enfoncer plus ou moins verticalement, $c$, coiffe; $p$, ré- surtout si le pivot vient à ètre oblitéré gion pilifère. de bonne heure. L'enracinement est dit pirotant, ou plutôt profond.

Dans le genre épicéa, au contraire, la vitalité se maintient peu active sur le pivot, et se reporte de bonne heure sur les ramifications latérales, qui ont une tendance à s'écarter horizontalement; l'enracinement est dit traçant, ou plutot superficiel.

Dans le genre châtaignier, l'enracinement se compose de quelques racines à peu près de mème force pénétrant obliquement dans le sol à une assez grande profondeur; il est dit obligue.

On trouve d'ailleurs dans les enracinements des arbres forestiers, tous les termes intermédiaires ou mixtes. cesse de functionner, et des racines laterales prennent sa place et ses fonctions. 
II est à remarquer que, dans leur jeune âge, la plupart des essences ont des racines pivotantes, et ce n'est que peu à peu que se caractérise l'enracinement.

L'axe du pivot est dans le prolongement de l'axe de la tige; on appelle collet de la racine la région de partage entre la racine et la tige, région qui se trouve normalement au niveau du sol.

Mycorhizes (1). - Chez un grand nombre d'arbres forestier's, on trouve des racines associées à des filaments délicats qui sont un mycélium de champignon. L'union est si intime et si régulière que la racine constitue avec le mycélium un tout morphologique, défini avec la netteté d'un organe normal. Cette formation qui n'est ni racine ni champignon, mais qui tient à la fois de la racine et du champignon, a reçu de Frank le nom de mycorhize (fig. 4). Par l'intermédiaire de cet organe mixte, l'arbre et le champignon harmonisent leurs fonctions pour le plus grand profit de la communauté; il y a symbiose (lans le sens le plus étroit du mot. En ce qui concerne le champignon, la symbiose est utile à son développement, car l'arbre élabore pour lui des aliments; en ce qui concerne l'arbre forestier, la symbiose est peut-ètre moins nécessaire, car il trouve dans ses organes propres les conditions suffisantes à son alimentation; toutefois l'association lui assure en outre la faculté de se nourrir en saprophyle. Remarquons que le développement des mycorhizes est lié à la présence de l'humus; dans le groupe des Cupulifères (chêne ou hètre par exemple) ces lormations font défaut chez les racines qui ont traversé le manteau d'humus pour se répandre dans la profondeur du sol; mais les racines indépendantes qu'on observe en ces points émettent peu de radicelles, tandis qu'elles produisent d'exubérants systèmes de mycorhizes, dès qu'elles arrivent au contact des couches ou des nids d'humus.

(1) D'après P Vuillemin. “ Les Mycorhizes " (Revue générale des sciences 1890). 
Or, l'engrais naturel des forèts consiste dans les déchets périodiques du corps des arbres; ces déchets sont principalement les racines usées et les feuilles tombées. La perte de celte masse considérable de substances n'est que momentanée; par l'adaptation réciproque des essences forestières et des champignons, chaque arbre réalise l'important problème de réparer ses pertes de la façon la plus prompte et la plus complète sans épuiser le sol. I)es cryptogames quelconques, bactéries ou autres, peuvent transformer les feuilles et les détritus diver's qui forment l'humus, mais, par l'association mycorhizienne, la préférence est accordée, dans les conditions normales, aux champignons des racines; ceux-ci assimilent les substances à leur portée dans l'humus, et ils paient à l'arbre leur place privilégiée en le faisant participer à leur nutrition. Les forestiers savent bien que les arbres souffrent autant de l'enlerement des feuilles dont ils se sont dépouillés, que les plantes des champs profitent peu de l'apport de ces débris. Cela tient précisément à ce que cetle fumure de la forêt, faite pour les arbres, est directement utilisée par les mycorhizes adaptés à ce milieu spécial, tandis qu'elle est moins complètement à la portée d'herbes quelconques, en dépit de l'action réelle des microorganismes extérieurs (1).

Les mycorhizes constituent donc l'organe habituel de l'absorption de l'humus par les plantes supérieures (Vuillemin).

\section{III. - TIGE FEUILLEE, RAMIFICATION}

Le petit chène dont nous avons suivi l'évolution jusqu'au momeṇt où il a commencé à vivre de sa vie propre, est composé de parties bien distincto's, la ti!je, la racine. les fenilles qui s'ćtalent à l'extrémité de la tige, le bour-

(1) Double raison pour laisser sur le sol de la forit la converture morte of les débris végélutux de toule nature. Tout enlivenent de litière est uno opérit. tion mauvaive, trìs préjudiciable à la forèt. 
geon terminal, sommet extrème de la tige et les bourgeons axillaires nés à l'aisselle des feuilles, c'est-à-dire à l'angle d'insertion de celles-ci sur la tige.

La ramification est due à l'épanouissement des bourgeons axillaires qui donnent, en se développant, des rameaux ou branches attachés sur les flancs de l'axe principal. Ces rameaux se courrent, comme la tige, de feuilles et de bourgeons, et ils peuvent fournir de nouvelles ramifications; l'arbre peut ainsi porter des axes de deux, de trois, de quatre ordres, etc., suivant le nombre de générations par lesquelles ces axes ont été créés, ét d'autant plus àgés qu'ils sont plus éloignés du sommet.

La tige et ses ramifications ne s'allongent, d'année en année, que par le développement de nouvelles pousses, issues du bourgeon terminal des pousses de l'année précédente (1); la hauteur totale d'un arbre est dès lors égale à la somme des pousses annuelles de sa tige principale. Chez tous nos arbres, la ramification se produit ainsi; elle est dite latérale (2).

Chez le chêne, le hêtre, etc., les branches ne tardent pas à l'emporter en croissance sur la tige principale, dont l'importance diminue progressivement; à un moment donné le bourgeon terminal de cet axe principal s'étiole, la tige principale ne continue plus à s'accroittre, et la croissance en hauteur de l'arbre est terminée.

De mème la croissance des ramifications de second

(1) Dans ure jeune pousse on distingue la croissance terminale (qui se produit daus le bourgeon terminal) et la croissance intercalaire, qui se produit au-dessous du summet de la tige, et porte généralement sur les entre-nceuds, et' d'une façon nulle ou beaucoup moins accentuée sur les uoxuds. Ces deux croissances provoyuent l'allongement de la tige. Lorsque cet allonyement de la partie la plus jeune de la tige a cessé de se produire, la pousse furméo ne s'allonge plus jamans. Si la tige s'accrolt encore, ce ne peut étre qu'en épaisseur.

(2) La ramification termimale, peu répandue, ne se rencontre guère que chez les cryjtogames, les lycopodiacées, les fougèros et chez certaines mousses; les vigettaux qui offrent ce mode de ranification n'ont que des bourgeons terminaux. 
ordre l'emporte sur la croissance des branches de premier ordre, et ainsi de suite; on dit que le chène a une ramification en cime; autrement dit, la tête de l'arbre se présente sous la forme d'une cime plus ou moins étalée. Dans cette cime, les branches de la partie supérieure, les plus récemment formées, conservent la direction des pousses qui les ont produites; les branches plus anciennement formées fléchissent peu à peu sous leur propre poids, et font avec l'axe principal un angle d'autant plus ouvert qu'elles sont plus âgées.

Ajoutons enfin que chez le chène, les rameaux se développent à l'aisselle des feuilles, en des points quelconques de la pousse (1); la ramification est plus ou moins irrégulière ou diffuse (fig. 5).

Chez le Sapin, l'Épicéa, etc., la croissance de la tige principale conserve toujours une avance sur celle des branches; de mème la croissance des axes de premier ordre conserve une avance sur les ramifications de second ordre, et ainsi de suite, ce qui constitue botaniquement une ramification en grappe, type très distinct de la ramification en cime; l'ensemble de la tige et de ses ramifications prend la forme générale d'une pyramide plus ou moins élancée.

En outre, les bourgeons axillaires de la tíge, se développent seulement à l'extrémité de chaque pousse annuelle, presque immédiatement au-dessous du bourgeon terminal de méme âge qu'eux; leur base est tellement rapprochée qu'elle semble disposée dans ứ mème plan horizontal; la ramification de l'axe príncipal prend l'aspect d'une forme dite verlicillée (2). Mais ce caraclère ne s'observe que sur l'axe principal; sur les branches,

(1) En réalité, les feuilles s'insèrent toujours suivant une spire déterminée par l'indice d'insertion, mais des causes nombreuses viennent modifier cetle régularité et elles présentent.un aspect diffus.

(2) Chaque verticille correspondant à la pousse d'uue année, le nombre des verticilles qu'on peut compter sur l'axe principal dome l'ảge de l'arbre (arbres jeunes). 
rameaux ef ramules, la ramification peut ètre différente. Chez le sapin, les branches sont forles; elles s'étalent

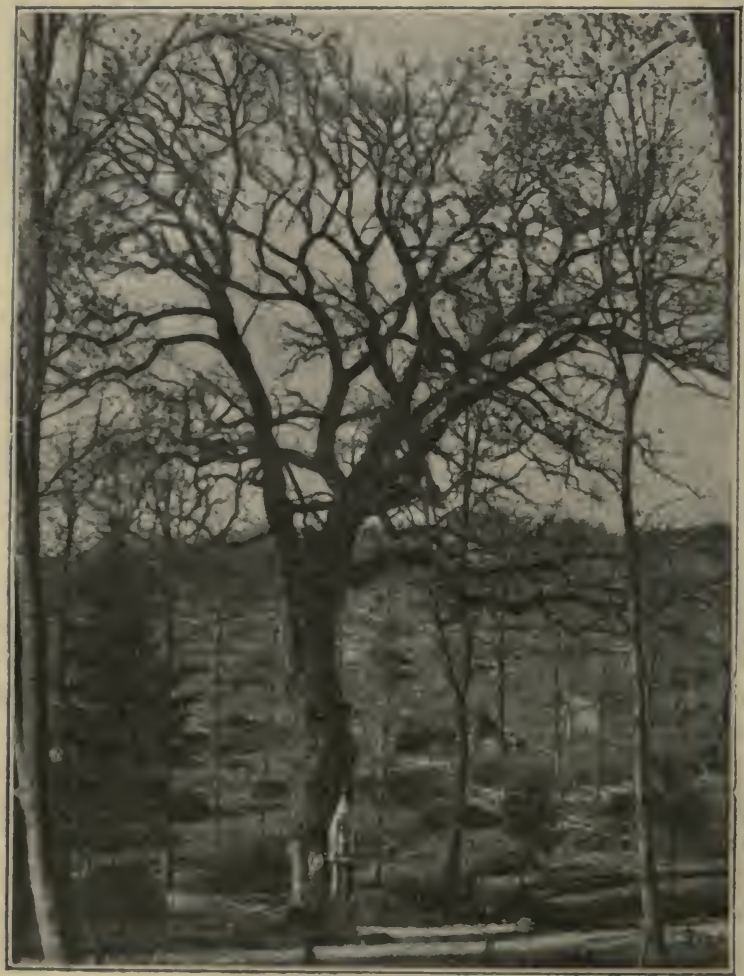

Jig. 5. - Vieux chène de Chaville (seine-et-Oise).

horizontalement, se divisent en rameaux etramules sonsiblement opposés, situés dans un mème plan horizontal (fig. 6).

Chez l'épicéa, les branches sont grêles, sourent 


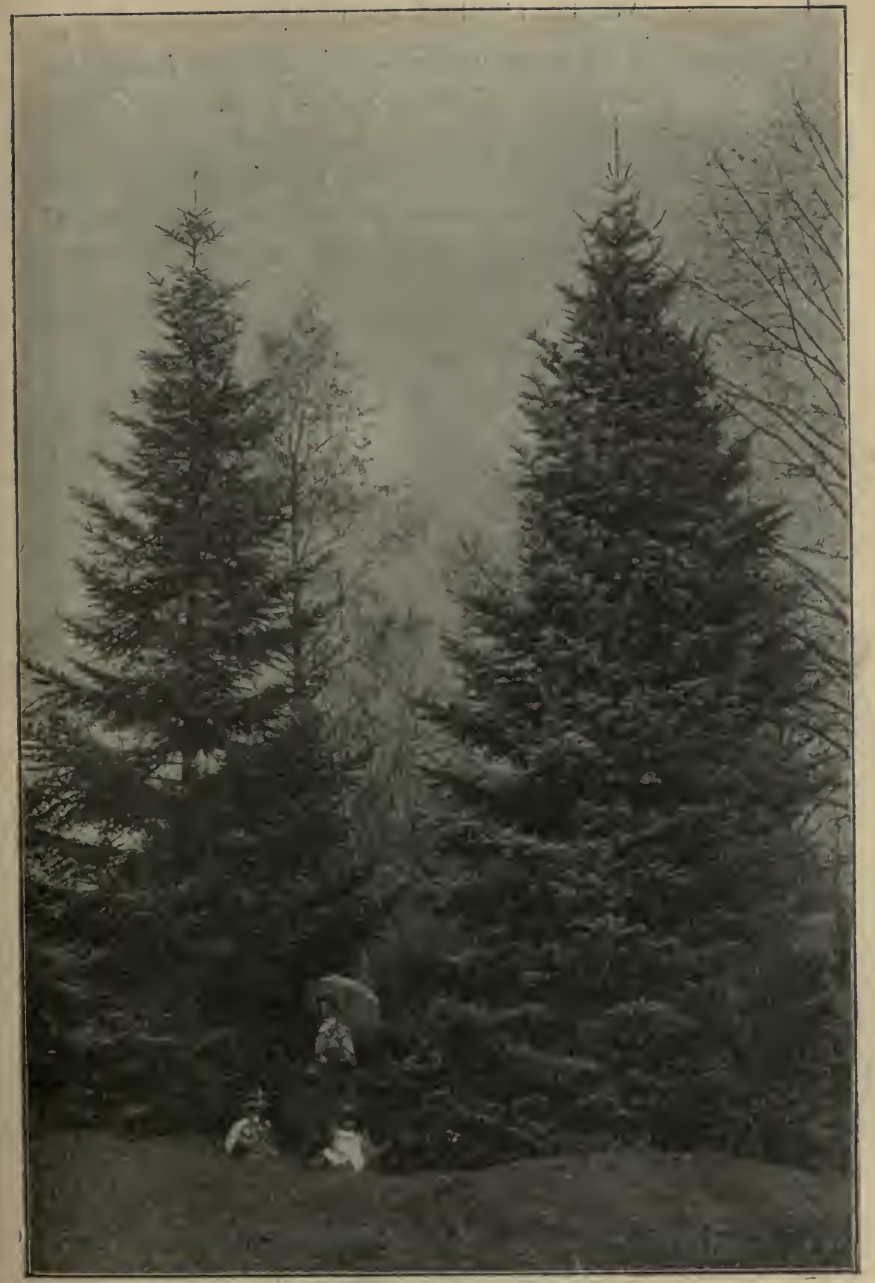

Fig. 6. - Abies Numidica. De Lannoy. Abies Concolor. Lindl. (Arboretum national des Barres.); 
aryuées, plus ou moins retombantes, et ne se redressent que vers l'extrémilé; elles se divisent en rameaux et ramules sensiblement opposés, qui, au lieu de s'étaler dans un mème plan, retombent de chaque côté des branches. Les nombreuses diflérences qui existent donnent à la ramification de chaque essence un aspect caractéristique.

En sylviculture, on distingue : les essences à feuilles caluques, chez lesquelles les feuilles nées au printemps tombent avant l'hiver de la mème année, ou tout au moins avant la pousse du printemps suivant (1) et les essences à feuilles persistuntes chez lesquelles les feuilles restent sur l'arbre pendant plusieurs années, de sorte que celui-ci n'est jamais dépouillé de feuilles pendant l'hiver.

On appelle tronc ou fît la tige principale proprement dite, jusqưà la naissance des plus grosses branches; le restant de l'arbre forme la tête de l'arbre, qu'on désigne aussi sous le nom de cime ou de houppier.

A l'état isolé, le fút de l'arbre, du chène par exemple, s'allonge peu, les branches principales, spécialement les branches basses, prennent un développement considérable; la cime sétale et prend une forme plus ou moins arrondie (fig. 7). Mais un phénomène important vient, suivant les essences, faire varier cetle forme théorique, c'est l'élagage na:urel.

Durant toute la période le croissance en hauteur de l'arbre, les branches basses de plus en plus dominées et privées de nourriture par les ramifications quai se forment et se déreloppent dars la partie supérieure de la címe, cessent rapidement de s'accroiltre, puis dépérissent lentement, se dessèchent et finissent par tomber, laissant sur le tronc de larbre une cicatrice peu importante ct

- qui se ferme d'elle-mème, sans causer le dommage sen-

(1) Si les feuilles persistent a l'élat desséché sur l'arbre pendant l'automne et une partio de l'hiver, on les dit mir'cessantes. 
TIGE FEUILLEE, RAMIFICATION.

sible au fủt. Cet élagage naturel a pour effet d'élever la

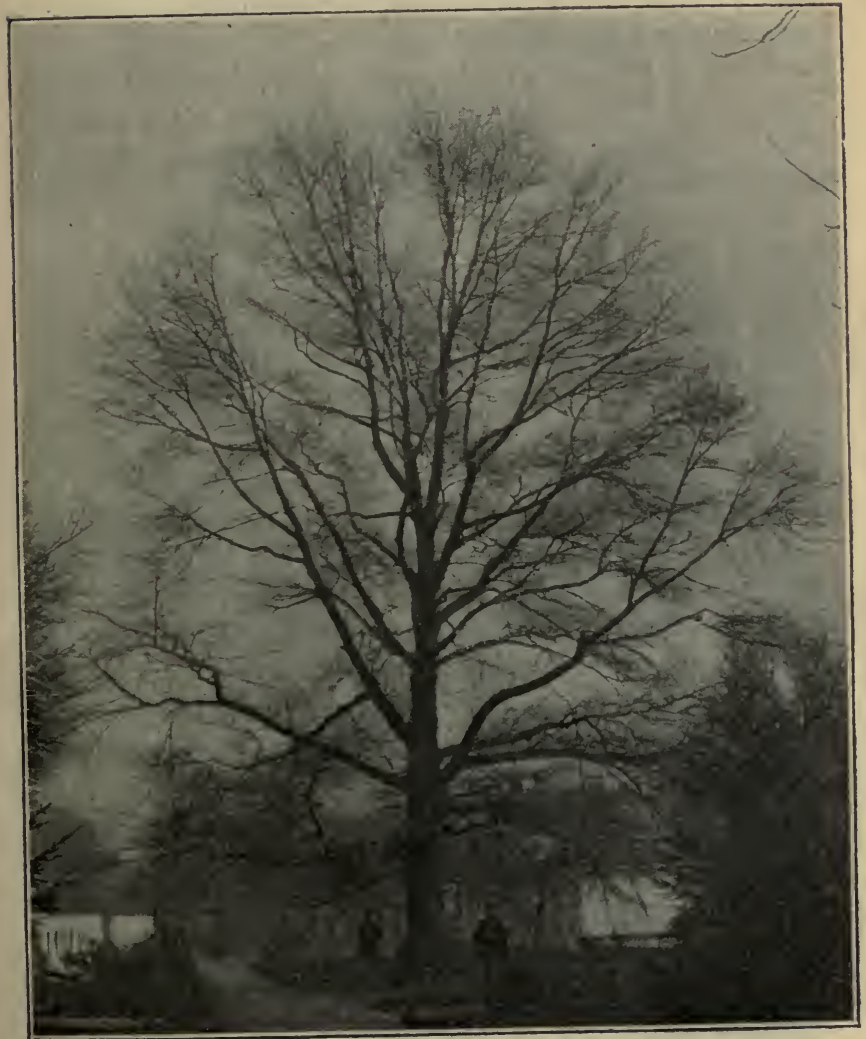

Fig. 7. - Chène isolé (forme spécifique).

Quercus heterophylla. Mich. (Arboretum national des Barres)

cime au-dessus du sol et par suite d'augmenter la hauteur du fût; un moment vient toutefois où la croissance 
en hauteur se ralentit beaucoup, et dès lors les rameaux des régions basses mieux nourris ont leur existence assurée ; ils s'alfirment, s'allongent, grossissent et se constituent en branches principales.

D'une importance relativement faible, lorsque l'arbre croissant à l'état isolé est frappé de tous côtés par la lumière, l'élagage naturel prend une importance capitale dans un massif(1).

L'ensemble du fût et de la cime constitue un facies spécial auquel on donne le nom de port de l'arbre. Le port d'un arbre dépend beaucoup de sa ramification; il varie d'abord avec l'espèce, et dans une même espèce, il varie suivant l'âge du sujet, et aussi suivant les conditions de fertilité du sol, de végétation et de climat (fig. 8). Ce facies de l'arbre isolé est fortement modifié quand l'arbre croît en massif; tous les sujets tendent à y prendre une mème forme, dite forme forestière (fig. 10).

Le port d'un arbre, sa ramification plus ou moins serrée, son feuillage plus ou moins abondant et régulier, permettent de concevoir une notion nouvelle, très importante en sylviculture, celle du couvert d'un arbre. Par couvert il faut entendre l'abri exercé par la masse de feuillage sur l'espace situé au-dessous de lui, abri qui se traduit par une diminution dans l'intensité et par conséquent dans l'action des radiations solaires (chaleur et lumière) et des précipitations atmosphériques sur les plantes qui croissent au-dessous de lui. Le couvert, qui empèche aussi la formation de la rosée, exerce son influence sur la projection horizontale de la tète de l'arbre, c'est-à-dire sur la surfacequi est immédiatement dominée par la cime et les branches.

(1) Il permet aux jeunes sujets de se constituer un fùt élancé sans que l'homme ait à intervenir. Notous ici qu'un élagage artificiel effectué en conpant des branches vivantes ou mortes sur le fint de l'arbie, laisse, quel que soit le diamètre de ces branches, une blessure dont on retrouvera la trace et suuvent lis graves conséquences dans le bois. 


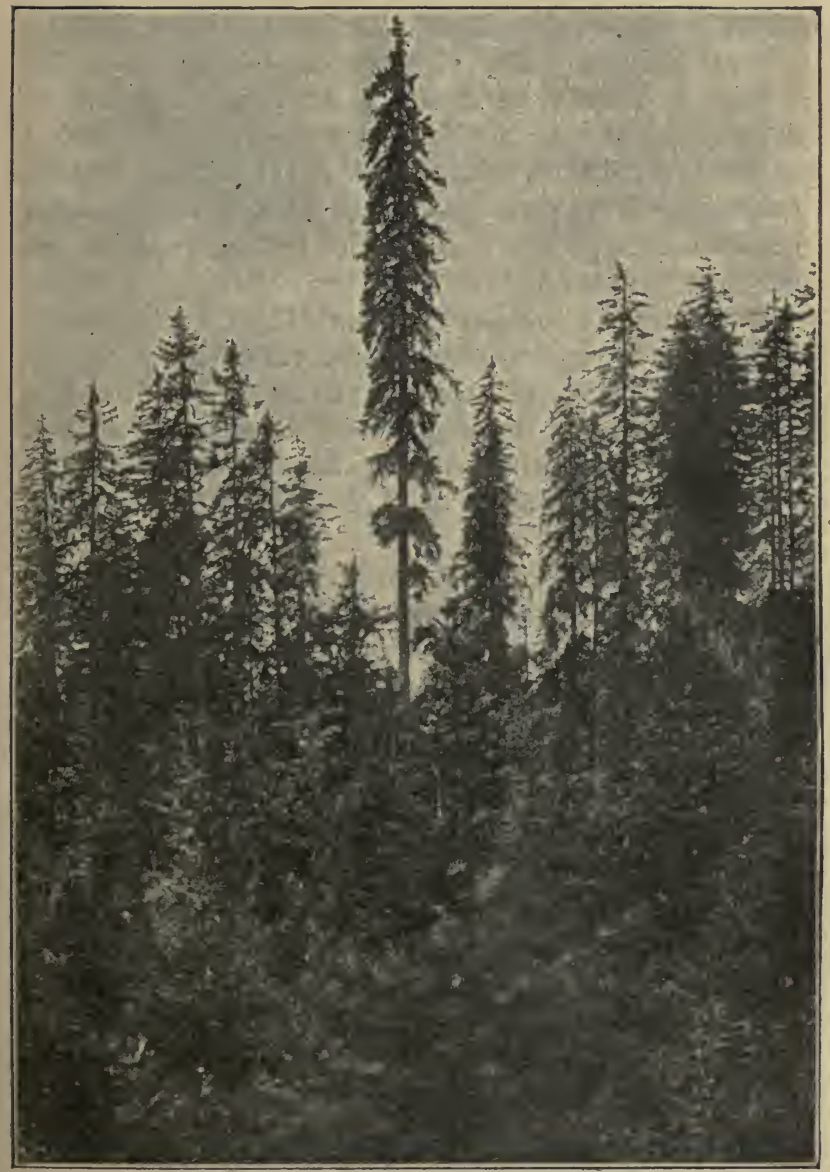

Fig. 8. - Epicéa columnaire, forêt du Risoux (Jura). (Photographie de M. P. Galland).

Extrait de "Les Forêts" par MiI. Boppe et Jolyet. 
Suivant les cas, le couvert est dit épais, léger ou trés léger; le couvert des arlores, ulile en certains cas, est en général nuisible à la végétation sous-jacente; son effet dépend de l'âge et de l'essence des arbres dominés et dominants. Toutes choses égales d'ailleurs, il varie : $1^{\circ}$ avec la fertilité du sol, le climat et l'exposition; $2^{\circ}$ a vec la hauleur de la cime au-dessus du sol, car plus les branches sont près de la terre, plus le mème couvert est écrasant et nuisible.

Le couvert ne doit pas ètre confondu avec l'ombrage, ombre portée par un arbre, frappé obliquement par les rayons solaires; l'ombrage diminue simplement l'ardeur du soleil, empêche la trop grande dessiccation du sol, sans toutefois soustraire les végétaux à l'action utile et nécessaire de la lumière, de la chaleur, de la pluie et de la rosée.

Les essences à couvert épais, ont généralement de jeunes plants à tempérament délicat; dès lors elles sont susceptibles de se maintenir et de continuer à vivre, sinon à s'accroître, pendant un certain temps sous le couvert d'arbres plus âgés; en sylviculture, on leur donne la dénomination d'essences d'ombre.

Par opposition, on appelle essences de lumière celles qui ne peuvent pas persister sous un certain couvert, et que l'absence de lumière fait disparaitre promptement; généralement, les essences de lumière ont le couvert léger, et le jeune plant issu de leurs graines a le tempérament robuste.

Remarquons toutefois que celle aptitude spéciale de vive ou de disparaitre sous l'influence diun couvert prolongé, varie non seulement avec l'essence, mais souvent, pour une mẻme essence avec l'âge, la station el aussi, dans une grande mesure, avec le degré de fertilité dus sol. 


\section{IV. - CROISSANCE EN HAUTEUR. - LONGËVITÉ. DIMENSIONS}

Dans la première partie de son existence, le jeune plant croît en général très lentement; il pousse des branches et des feuilles, mais l'effort de la végétation se porte principalement sur le développement des racines, et la tige s'élève peu. Dans des conditions normales, elle s'élève par exemple de s̀ à 6 centimètres par an pour le sapin et l'épicéa; de 8 à 12 centimètres par an pour le chène, le hêtre, le pin sylvestre, etc. Dès l'âge de six à dix ans, la croissance en hauteur s'accentue, les jeunes plants prennent leur essor, les pousses annuelles mesurent 10 à 20 centimètres, parfois 30 centimètres et plus; en mème temps l'arbre croît en grosseur et cet accroissemen l se traduit à l'extérieur par une augmentation du diamètre, et de la circonférence ou tour de l'arbre.

D’après M. Bouquet de la Grye (1), la vie d'un arbre se compose de trois périodes, dont la durée est variable, suivant les essences et les conditions locales: la première; celle de la jeunesse, se manifeste à l'extérieur par la tendance de la tige à s'élever; pendant cette période, le feuillage est abondant, l'écorce est lisse et saine, les jeunes pousses sont longues et droites. La deuxième période est celle de la maturité; pendant sa durée, l'arbre cesse peu à peu de croître en hauteur, mais son accroissement en grosseur ne subit pas de ralentissement; les branches prennent un grand développement, le feuillage reste vigoureux, l'écorce est encore saine, mais elle devient rugueuse, l'arbre prend une cime plus ou moins arrondie, plus ou moins relevée. A cette période succède celle de v'etour ou de la décrépilude qui se manifeste d'abord par le desséchement lent et progressif des branches du

(1) A. Bouquet de la Grye, Eléments de Sylviculture. - Guide du Forestier, t. I. 
sommel de la cime (1); celles-ci meurent les unes après les autres, et finissent par tomber, en laissant après la tige des chicots qui se décomposent et deviennent spongieux. Dis lors, la décomposition de l'arbre commence au centre et à la base du tronc, et s'étend progressivement; l'arbre continue à virre par ses couches superficielles jusqu'à la fin de cette phase qui se traduit par la mort ou la chute de l'arbre.

En général, les arbres qui ont une croissance très rapide atteignent plus tôt que les arbres à croissance lente le terme de leur développement et mème de leur existence. Les essences forestières présentent à ce point de vue de très grandes différences.

\section{V. - FLORAISON. - FRUCTIFICATION}

L'arbre, pendant le cours de son existence, se met à fleurir et à fructifier; en général, les fleurs de nos arbres forestiers sont petites ou peu apparentes et, à ce point de vue, ces végétaux phanérogames sont dits monoïques, dioïques, hermaphrodites ou polygames (2).

Les fruits de nos arbres forestiers ont des formes très variables suivant les essences; il en est de mème des graines. En sylviculture, on donne le nom de semence Iantôt à des fruits, tantot à de simples graines, et on distingue au point de vue de la dissémination: les semences lourdes (gland, faîne, etc.) qui tombent en raison de leur poids, au pied des arbres porte-graines; les sc-

(1) Il ne faut pas toujours prendre pour un symptóme de décrépilude l'apparition de brauches morles vers le sommet d'arbres sains d'ailleurs; ce flienoméne peut étre produit par des causes accidentelles auxquelles il est parfuis possible do remédier.

(2) Monołque : l'arbre porte sur le même pied, mais sur des rameaux ou à des places diflérentes, des fleurs males et des fleurs femelles (chene, sapin); Diotque : l'arlire n'a que des fleurs malez (pied male) ou des fleurs femelles (pied femelle) (saule); Ilermaphrodite : larbre porle des fleurs munies chacune d'étumines et de pistil (tilleul, érable); P'olỵgame : l'arlore porte à la fois des fleurs màles, des fleurs femelles, el des fleurs hermaplirodites (frêne). 
mences légères ou graines légères, généralement ailées ou munies d'organes spéciaux qui facilitent leur transport à de grandes distances au moment de la dissémination.

Pour la formation de la graine, nous savons qu'il est nécessaire que l'arbre ait accumulé dans ses tissus une certaine réserve d'éléments nutritifs; la qualité de cette réserve dépend de l'âge du régétal, de la station, de la lumière, de l'essence, du climat, de l'année, des conditions de végétation, etc.; l'âge auquel commence une fructification normale et abondante est dès lors assez indéterminé pour nos arbres forestiers. Toutefois, on peut dire que l'âge de la plus forte production de graine est, en général, celui qui suit la période du plus grand accroissement en hauteur, celui où la cime commence à se développer et où la production du bois de tige se ralentit; cette période se prolonge souvent jusqu'à un âge avancé.

D’après M. Gayer (1), les graines contenant beaucoup d'éléments minéraux, et une grande accumulation et assimilation de principes nutritifs étant nécessaires à leur formation abondante, il est logique qu'en général les terrains frais et riches donnent une fructification plus abondante et des graines plus fertiles que les terrains maigres; mais en outre, la somme d'énergie vitale (chaleur et lumière) nécessaire à la maturation des graines et des fruits est plus considérable que celle qui est requise pour la formation du bois ; de là l'importance de la station au point de vue du climat, et, en particulier, de la latitude et de l'altitude; de là l'importance d'une assimilation abondante, et par suite d'un feuillage développé, bien exposé à l'accès de la lumière. Il est un fail reconnu depuis longtemps, c'est que seuls les arbres dont la cime est bien développée et baignee de lumière ont une abondante fructification, tandis que ceux qui en sont privés pour une raison quelconque, ne portent point de fruits.

(1) Dr Karl Gayer, Traité de Sylviculture. - (Traduit par E. Visart de Bocarmé, Munich, 1901). 
Selon l'essence, le climat et les conditions de végétation, un arbre parrenu à l'âge adulte, fructifie d'une façon abondante, soit régulièrement toutes les années, soit irrégulièrement, et dans ce dernier cas, les années à graines ne se reproduisent que périodiquement à des intervalles plus ou moins éloignés.

\section{VI. - CROISSANCE EN DIAMĖTRE. - BOIS}

Un jeune bourgeon terminal présente dans la région en voie de croissance une structure très simple, très peu différenciée. Une jeune pousse, qui n'a pas encore terminé sont accroissement en longueur, présente sous l'épiderme une ou plusieurs assises corticales, entourant un cylindre central de structure primaire. Un axe, dont la croissance en longueur est terminée, continue à s'accroître en diamètre ; cet accroissement est dû au fonctionnement de deux assises de cellules génératrices, l'une interne, le cambium, située entre le liber primaire et le bois primaire, formant un anneau complet dans le cylindre central; l'autre externe, située à des profondeurs variables dans le parenchyme cortical. L'accroissement en diamètre se produit d'une façon inégale suivant les circonstances, mais il dure pendant toute l'existence de l'arbre.

C'est à la multiplication des cellules dans la zone génératrice interne, multiplication qui ne s'effectue dans nos climats que pendant la durée de la saison de régétation, qu'est due la formation lente et progressire du bois secondaire ou bois proprement dit. Cette multiplication se fait, chez les essences indigènes du moins, de l'intérieur vers l'extérieur dans le sens du rayon sur' une section transversale du füt, de telle sorte que la masse de bois fabriqué est formée d'une série de couches annuelles, dont les plus anciennes sont au centre de l'arbre et les plus jeunes vers le cambium.

Il est, en général, facile, sur la tranche d'une tige 
exploitée, de distinguer les différentes couches ligneuses. Tantôt, en effet, d'après MII. Boppe et Jolyet (1), le bois fabriqué au début de la saison de végétation, dit bois de printemps, est franchement distinct du bois d'été qui se forme plus tard; et comme dans ce cas la caratéristique du bois de printemps est d'être constitué par des éléments à parois minces et à grosses cavités intérieures, il apparaît toujours sous l'aspect d'un tissu tendre et blanchâtre, par opposition au tissu plus dur et plus coloré du bois d'été (chêne, sapin, etc.). Tantôt, au contraire, bois de printemps et bois d'été se ressemblent; mais alors les derniers éléments de celui-ci, ceux qui bordent la couche vers l'extérieur, sont très minces et souvent colorés en brun, ce qui rend encore les formes annuelles distinctes l'une de l'autre, avec plus de difficulté toutefois (hêtre, bouleau, charme, fruitiers).

Dans le premier cas, le bois est dit non homogène; il est beaucoup plus homogène dans le second cas. La plupart de nos essences feuillues et toutes nos essences résineuses ont un bois non homogène, dans lequel la zone de printemps est moins dense et de qualité inférieure à celle d'été.

La couche annuelle, produit de l'activité du végétal pendant une saison.de végétation, a une épaisseur qui varie avec cette activité. Dans les mèmes lieux et pour une mème essence, si la croissance est rapide, la couche annuelle est plus épaisse; si l'inverse se produit, la couche annuelle est moins épaisse, et dès lors l'activité de la régétation est susceptible d’influer sur la qualité du bois. Chez les essences feuillues à bois homogène ou sensiblement homogène, les qualités du bois paraissent indépendantes de l'épaisseur de la couche annuelle; chez le chène, et généralement chez tous les arbres feuillus à bois non homogène, l'épaisseur de la formation de prin-

(1) Boppe et Jolyet, Les Foréts, 1900. 
temps reste sensiblement la mème; une croissance rapide, les conditions de végétation très favorables tendent à accroitre dans chaque couche annuelle la proportion de bois d'été, et comme la différence entre la qualité du bois de printemps et celle du bois d'été est prononcée, le bois tend à devenir plus dur, plus résistant, plus nerveux, en un mot mieux lignifié.

Mais pour que cette loi soit exacte, il ne faut comparer que des arbres soumis aux mêmes conditions de climat; le climat et l'exposition ont, en effet, une influence analogue sur la proportion de bois d'été et de printemps dans la couche annuelle. C'est ainsi que le chène, mème à croissance rapide, a un bois moins dense, moins dur s'il provient des régions septentrionales de son aire d'habitation.

Chez le sapin, au contraire, et généralement chez tous les arbres résineux, c'est l'épaisseur de la formation d'été qui semble rester sensiblement constante; une végétation active tend à augmenter la proportion de bois de printemps; par suite la densité et les qualités du bois sont inversement proportionnelles à la rapidité et à la vigueur de la végétation, et par conséquent à l'épaisseur des couches annuelles (1).

Aubier. Bois parfait. - Chez la plupart de nos grandes essences on distingue ordinairement, dans le bois d'un arbre, deux régions, l'aubier et le bois parfait.

L'aubier est le bois jeune, périphérique, que recouvre immédiatement l'écorce ; sa couleur est légèrement jaunâtre ou blanchâtre, ce qui permet de le distinguer du bois parfait plus coloré; gorgé de sève ou, en hiver, de réserves nutritives, il est par suite sujet à la pour-

(1) Commo conséquence, cliez la plupart des arbres fevillus, le bois des branches, qui pousse plus lentement, est inférieur au bois des axes pri.acipaux; il est plus poreux, plus léger, moins dense, par suite inférieur comme combustible. Chez les arbres résineux le bois des branches, parce qu'il pousse plus lentement est plus dense et de beaucoup supérieur comme combustible h celui du tronc. 
riture et à la vermoulure quand il est mis en œurre.

Le bois parfait est formé par les couches les plus internes, c'est-à-dire les plus anciennement formées du bois, couches qui, en vieillissant, se sont modifiées physiquement et chimiquement; leur couleur devient plus foncée, leur densité, leur dureté augmentent; les cellules qu'elles renferment se vident de leur protoplasme, perdent une partie de leur eau, épaississent leurs membranes qui s'incrustent de substances nouvelles, très riches en carbone et en hydrogène; leurs tissus cessent de laisser circuler la sève, et le bois parfait ainsi constitué d'éléments morts n'est plus susceptible de se modifier ultérieurement, sinon par altération, puis par décomposition et destruction des tissus. Ce bois est plus dur, plus dense que l'aubier, peu sujet aux fermentations; il est de meilleure qualité et moins exposé aux ravages des insectes (1).

Chez certaines essences, l'aubier est de très mauvaise qualité et doit ètre rejeté ; chez les autres, il est moins mauvais, et il s'emploie concurremment a vec le bois parfait dont il diffère peu.

En général, les bois parfaits, les meilleurs au point de vue de la dureté et de la résistance à la décomposition, sont accompagnés du plus mauvais aubier (chênes et pins), tandis que ceux de qualité inférieure offrent un aubier passable (peupliers, sapins, épicéas).

Enfin, certaines essences ont beaucoup d'aubier (pins); d'autres passablement (chênes); il en est qui en présentent fort peu (châtaignier, mélèze); autrement dit, l'àge auquel l'aubier passe à l'état de bois parfait

(1) Les arbres très âgés présentent souvent dans le cœur du bois parfait une coloration plus foncée, variable avec les essences et qui, sur une section transversale de la tige, apparaft en tache à contours irréguliers, dont le périmètre ne suit pas les limites des couches annuelles; c'est un commencement d'altéralion, et la désorganisation lente des tissus finit par constituer dans l'axe de la tige et tout d'abord à la base du tronc, des régions creuses oủ le bois se dissocie peu à peu.

A. Fron. - Sylviculture, 
est variable chez les diverses essences; de plus de quarante ans chez le frène et chez certains arbres où le bois demeure indéfiniment en apparence à l'état initial, il est de quinze à vingt ans en moyenne chez le chêne, et seulement de quatre à cinq ans chez le châtaignier, le robinier. Toutefois ces chiffres ne sont que des moyennes, car le climat, la nature du sol, le mode de culture et l'âge des arbres influent sur l'épaisseur de l'aubier. Il résulte des fails constatés que, toutes choses égales d'ailleurs, la proportion qui existe entre l'aubier et le bois parfait diminue avec l'âge, fait intéressant à noter pour les arbres à bois précieux, qu'il y a à ce point de vue intérèt à conserver sur pied aussi longtemps que possible.

En sylviculture, on classe les principales essences, par rapport à leur bois, sous les dénominations de :

Bois dur's : Chène ; châtaignier ; hêtre ; charme ; frêne ; érable ; orme ; micocoulier ; fruitiers.

Bois demi-durs : bouleau; aune.

Bois tendres (1) : tilleul ; peuplier; saule.

Bois résineux : sapin ; épicéa ; mélèze ; pins.

\section{VII. - BOURGEONS}

A l'extrémité des pousses des arbres, et généralement à la base des feuilles, on observe des corps de forme assez variable, d'où doivent sortir les pousses nouvelles et les fleurs; ces corps sont les bourgeons, dits bourgeons nol'maux (2).

(1) On désigne dans lo langage habituel les bois tendres sous le nom de bois blancs, ee qui est un terme assez impropre, d'après M. Fliche, certains bois lourds, le charme par exemple, étant parfaitement blancs, tandis que des bois légers peuvent offrir des colorations assez vives.

(2) Remarquons avec M. Fliche que les bourgeons a fleurs sont plus r'nflés que les lourgeons à feuilles, que les premiers se forment à l'aisselle d'une feuille l'année qui précède leur développement; qu'on peut ainsi préciser une anuée a l'avance une fructification abondante; enfin, que ce n'est pas une, mais deux années favorables qui sont nécessaires pour assurer une fructification abondante. 
Ces bourgeons sont soit terminaux, et alors ils concourent à l'allongement de l'axe qu'ils terminent, soit axilluires et alors ils concourent à la formation de la ramification de l'arbre. Les bourgeons axillaires occupent sur l'axe qui les porte une place déterminée par l'arrangement des feuilles sur la tige et sur les rameaux, arrangement soumis à des lois dont la rigueur rappelle celle des lois mathématiques; suivant les cas, on les dit alternes, opposés ou verticillès (1).

Le bourgeon normal, formé ou plutot simplement ébauché à l'aisselle de la feuille d'une année, ne se dé veloppe en principe que l'année qui suit celle de sa formation; toutefois il arrive que pour des causes accidentelles, certains bourgeons se développent dans li saison mẻme où ils se sont formés, ce qui leur a valu la dénomination de prompts bourgeons. Par opposition, on appelle bourgeons dormunts ceux qui, une fois formés, passent au moins un hiver sans se développer.

Tous les bourgeons dormants ne se développent pas forcément l'année qui suit leur formation; un certain nombre peuvent avorter complètement (2); beaucoup d'entre eux, privés de nourriture par leurs voisins plus robustes ou privés de la lumière nécessaire à leur développement, peuvent être susceptibles de rester à l'état de

(1) Alternes : on n'observe qu'une feuille ou un bourgeon à un niveau donné, et cette disposition présente un grand nombre de variations, suivant l'indice d'insertion dont les plus communs sont $1 / 2$ (disposilion distique), 1/3, $2 / 5,3 / 8$, etc.

Opposés : les feuilles ou bourgeons s'attachent sur l'axe dans un même plan horizontal, aux deux extrémités d'un même diamètre; les diamètres passant par les points d'insertion de deux paires voisines sont perpendiculaires l'un à l'autre.

Verticillés : si au lieu de deux feuilles ou bourgeons au mème niveau, il en existe trois, quatre, cinq ou davantage; dans tous les cas, les points d'insertion d'un même verticille sont toujours également espacés et alternent (en projection) avec ceux du verticille inférieur ou supérieur.

(2) Chez les conifères ou arbres résineux, l'avortement des bourgeons axillaires se produit avec une périodicité régulière sur l'axe principal, si bien que quelques bourgeons situés à l'aisselle des feuilles de l'extrémité de la tige se montrent seuls fertiles. 
vie latente; ils suivent lentement la croissance de la tige à laquelle ils appartiennent, sans se développer à l'extérieur et constituent ce qu'on appelle des bourgeons proventifs.

Les essences feuillues offrent à cet égard de très grandes différences; suivant l'essence, le nombre des bourgeons proventifs est très variable et leur vitalité, à cet état de vie ralentie, se conserve plus ou moins longtemps. Tant que dure cette vitalité, le bourgeon proventif est susceptible de se réveiller et de reprendre un développement normal, si les causes qui l'ont arrêté dans sa croissance viennent à cesser. Ainsi un fût de chêne, mis en pleine lumière après avoir été longlemps abrité, se couvre de branches gourmandes, dont la formation est due au brusque réveil des bourgeons proventifs, et c'est pour la même cause que certains arbres feuillus, le chêne par exemple, réparent facilement les accidents survenus dans leur cime, el sont susceptibles de remplacer par des rameaux jeunes et vigoureux, les quelques branches dont les vents, le givre, la gelée, etc., ont pu déterminer la mort.

Indépendamment de ces bourgeons normaux, dont la position est définie sur le régétal par une rìgle commune à l'espèce, il peut s'en produire d'autres dits bourgeons adrentifs qui n'ont pas de situation déterminée sur le végétal; on les voit apparaître sur les organes les plus divers, déjà même arancés en âge : racines, tiges, branches, etc., peuvent émettre des bourgeons adrentifs et lescauses les plus diverses favorisent le développement de ces bourgeons spéciaux; une des plus déterminantes est une blessure voulue ou accidentelle assez profonde ponr alleindre la couche génératrice ou cambium; les rollules de ce tissu tendent à fermer la plaie en formant un bourrelet cicatriciel ; le bourgeon adventif naît d'un hourgeonnement de ce tissu cicatriciel auquel il aplartient; à la différence du bourgeon proventif, il n'est pas, 
au moment de son développement, intimement lié comme ce dernier à l'axe mème à la surface duquel il apparaît.

\section{VIII. - REJETS DE SOUCHE. - DRAGEONS}

Certains arbres possèdent la faculté d'émettre des rejets de souche et des drageons. Ces rejets et drageons ont une grande importance forestière; c'est sur leur production qu'est basé le traitement en taillis.

Rejets de souche. - On appelle rejets de souche les jeunes pousses feuillées qui se forment sur la section d'abatage d'un arbre. Pour exploiter l'arbre rez terre, on a dû faire à la cognée une section nette du tronc; la zone génératrice ou cambium, alimentée par les réserves nutritives contenues dans la souche, forme circulairement sur le pourtour de la section un bourrelet cicatriciel; chez les arbres susceptibles de rejeter de souche, on voit se produire sur ce bourrelet un plus ou moins grand nombre de bourgeons adventifs; ces bourgeons entrent immédiatement en évolution, et donnent naissance à une première série de rejets de souche, insérés sur la section mêrne de la souche, entre l'écorce et le bois. Ces rejets sont relativement grêles et assez fragiles, en raison de leur faible adhérence à la souche ellemême.

En outre, la partie de la tige comprise au-dessous de la section développe sous l'influence de l'afflux de nourriture et de lumière qui se produit alors, un certain nombre de bourgeons proventifs; ces bourgeons donnent naissance à de noureaux rejets, généralement plus nombreux et plus vigoureux que les précédents, et surtout mieux assis, en raison de leur origine antérieure qui les relie intimement avec le corps de la souche. Ces rejets d'origine proventive sont insérés latéralement sur le pourtour de la souche (fig. 9).

La faculté que possède un arbre de rejeter de souche, 
varie d'abord avec l'essence; elle varie aussi avec l'àge du sujet exploité; disons plus exactement que les bourgeons proventifs dont nous avons besoin pour obtenir de bons rejets de souche, ont une vitalité qui ne dure

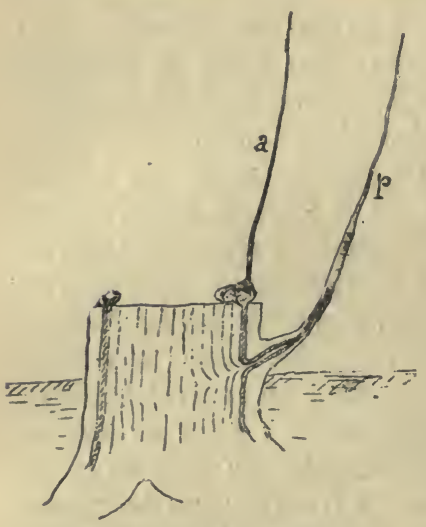

Fig. 9. - Rejets de souche.

$a$, d'origine adventive; $p$, d'origine proventive.

pas indéfiniment; cette vitalité, longue chez le chêne, puisqu'elle persiste parfois au delà de cent ans, ne dure en moyenne chez toutes les essences susceptibles de rejeter de souche, qu'environ une quarantaine d'années; passé cet âge, la vitalité du bourgeon proventif disparaît ou tend à disparaître, et celui-ci n'est plus susceptible de donner une pousse feuillée (1).

Le rejet proventif, inséré lutéralement autour de la souche, se trouve de bonne heure en contact avec le sol; il est dis lor's dans d'excellentes condition pour se constituer rapidement un enracinement propre; très fréquemment, il s'isole ainsi de la souche pour vivre d'une vie indépendante (2). Nourri d'abord abondamment par les réserves accumulées dans la souche, il présente une

(1) Le chine rejette bien de souche; lo hètre rejette mal de souche; les diverses essences feuillues présentent sous ce rapport de grandes différences; nos essences résineuses ne rejettent pas de souche, tout au moins en principe, car on doit faire certaines rest $ı$ ictions, notamment en ce qui concerne certains jounes pins.

(2) Si les sujets nouveaux parviennent à prendre racine, ils deviennent indépendants de la kouche; ce mode de propagation est une véritable multiplication par division de la plante mère au inème litre que la bouture et la. uturcotte. 
végétation vigoureuse, supérieure à celle d'un semis de même âge; mais issu d'un bourgeon resté longtemps en souffrance, il ne conserve pas cette vigueur; dès lors, le rejet se développe moins bien; il possède, toutes choses égales d'ailleurs, une longévité moins grande que l'arbre de franc pied.

Quant au rejet adventif, il s'affranchit plus difficilement; inséré sur une souche qui ne tarde pas à périr et à se décomposer, il est dans des conditions moins favorables, et se ressent toute sa vie de cette tare originelle.

En sylviculture, on donne le nom de cépée aux divers rejets issus d'une mème souche.

Yotons ici que sur certains terrains, on voit apparaître de véritables rejets de souche à la base d'arbres (de chènes, notamment) trop âgés pour le sol qui les porte ; la maturité de ces arbres est arrivée, et elle se traduit en mème temps par l'apparition de branches mortes dans la cime. La nature donne dans ce cas au propriétaire des arbres une leçon de sylviculture.

Drageons. - On donne le nom de drageons aux produits des bourgeons adventifs qui naissent sur les racines et donnent naissance à des pousses feuillées s'élevant au-dessus du sol. Le développement de ces bourgeons est dû à une mutilation soit de la tige, soit de la racine; il se produil quelquefois quand les arbres sont trop vieux, observation à rapprocher de la même remarque faite au sujet des rejets de souche.

Les jeunes pousses issues de ces bourgeons prennent rapidement un enracinement propre à leur base, puis ils s’isolent du pied mère et constituent des pieds indépendants.

Les essences forestières présentent à ce point de vue de très grandes différences; certaines essences susceptibles d'émettre un grand nombre de drageons sont dites drageonnantes. 


\section{IX. - COMPOSITION ET ALIMENTATION DE L'ARBRE}

L'analyse chimique des divers arbres y fait retrouver constamment les éléments suivants : carbone, oxygène, hydrogène, azote; soufre, phosphore, silicium, chlore, potassium, sodium, calcium, magnésium, manganèse, fer, etc.

Les quatre premiers sont des éléments organiques; les composés ternaires (formés de carbone, d'oxygène et d'hydrogène) tels que la cellulose, la cutine, la lignine, l'amidon, les sucres; ou quaternaires (formés de carbone, hydrogène, oxygène et azote) matières albuminoïdes par exemple, qui résultent de leur combinaison, ne se rencontrent que dans les corps organisés. Ces principes organiques constituent essentiellement la trame du végétal, et des divers composés qui se forment en lui sous l'influence de la vie; soumis à la combustion, ils disparaissent à l'état de gaz ou de vapeurs et c'est à ce titre qu'on les appelle des corps volatils. 90 à $9 \breve{s}$ p. 100 de la substance des arbres sont fournis par eux.

Les autres éléments sont dits inorganiques ou minéraux; ils se retrouvent dans les cendres après la combustion et existent en proportion variable, mais toujours très faible dans les arbres; on les appelle principes fixes.

Tous ces corps se combinent entre eux de façons très diverses, pour former des composés dont la nature varie avec les difrérentes essences, et dans chaque arbre avec les différents organes qui le constituent.

Analyse du bois. - La composition chimique élémentaire du bois est, à peu près la même pour toutes lés 
essences; d'après les analyses de Payen et de Chevandier on peut la représenter par :

Carbone................

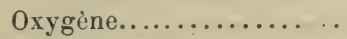

Hydrogène.............

Azote..................

Cendres................

50 à 53 p. 100
40 à $43 \quad-$
6,08 à $6,28-$
0,78 à 1,41 -
0,8 à $1,8-$

En moyenne l'azote n'atteint pas 1 p. 100 ; quant aux cendres, leur proportion varie entre 0,9 et 1,3 p. 100 .

Si l'on veut tenir compte de l'eau que renferme le bois, on arriverait au résultat ci-après donné par II. Jìne :

Eau 14,20

Carbone........ 42,08

Hydrogène.... $\quad 5,10$

Oxygène....... 37,18

Azote........ 0,57

Cendres........ 0,87

Pour du sapin ayant séjourné à l'air et à couvert pendant plus d'un an, ayant par conséquent déjà perdu une partie de son eau qui, lorsque le bois est fraîchement abattu, atteint de 3 à 40 p. 100.

Analyse des cendres. - $1^{\circ}$ Proportion. - La proportion des cendres varie selon la partie de l'arbre que l'on envisage. D'après les expériences de M. Chevandier, on aurait les proportions suivantes p. 100 de matière en :

Bois du tronc........ 0,296 Éंerce du tronc...... 1,129

Petites branches..... 0,304 Écorce des moyennes

Racines............. 0,223 racines.......... 1,643

Feuilles ............. 7.118 Chevelu des racines... 5,007

Écorce des pet.branches. 3,454

Dans les graines, la proportion est encore plus forte que dans les feuilles.

$2^{\circ}$ Composition. - La composition des cendres varie beaucoup arec les essences; elles renferment surtout les éléments : chaux, soude, silice, magnésie, potasse, alumine, acide carbonique, acide phosphorique. 
Le tableau suivant, donnant l'analyse des cendres de quelques essences principales, fait ressortir les diffẻrences d'une espèce à l'autre.

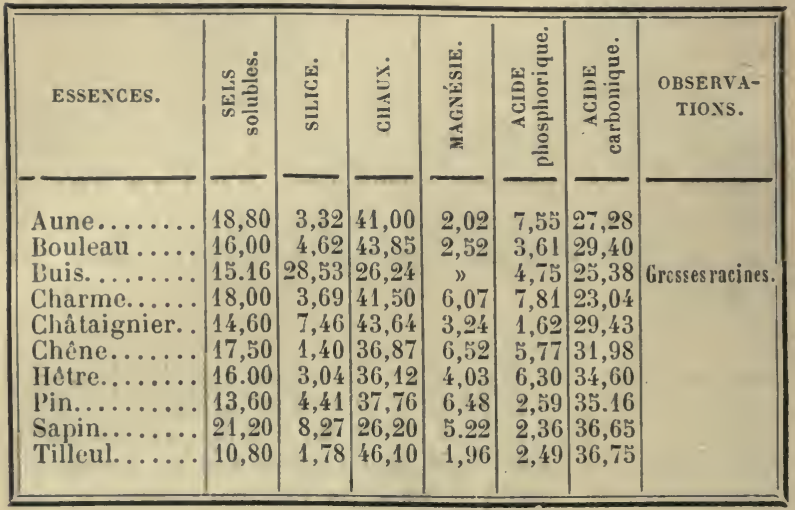

Alimentation de l'arbre. - L'arbre vivant, qu'il soit issu de l'embryon contenu dans la graine ou du développement d'un simple bourgeon, demande tous ces principes aux milieux extérieurs sous forme d'aliments; il puise ses aliments dans l'atmosphère et dans le sol, sous l'action de deux fonctions primordiales qui caractérisent un végétal supérieur: la respiration et la fonction chlorophyllienne.

Respiration. - Dans tout ètre vivant, végétal ou animal, le protoplasme (1) est le siège exclusif de la vie, et cette dernière s'y manifeste toujours par les mèmes phénomènes essentiels; partout le protoplasme absorbe de l'oxygène, rejelte de l'acide carbonique, et cet échange qu'on appelle respiration est le résultat d'une oxydation, d'une combustion lente de la matière organique. Cette oxydation s'accompagne de la mise en liberté d'une cer-

(1) Matière granuleuse alhuminolide, qui est la substunce essenticllement vivante de la cellule, végétale ou animale. 
taine quantité de force vive, susceptible d'ètre transformée en un travail interne (1).

La respiration de l'arbre se traduit par l'absorption de l'oxygène de l'air, et le rejet dans l'atmosphère de l'acide carbonique.

Cet échange gazeux avec l'atmosphère s'effectue aussi bien le jour que la nuit et l'activité seule du protoplasme contenu dans les cellules vivantes y suffit.

Fonction chlorophyllienne. - Gràce à la vitalité du protoplasme et sous l'influence de certaines radiations solaires, il se développe dans l'intérieur des tissus de la feuille et des jeunes pousses, un composé spécial, la chlorophylle; c'est la présence des grains de chlorophylle dans les cellules qui donne leur couleur aux organes verts de l'arbre.

Le grain de chlorophylle (2), sous l'influence de la lumière du jour, absorbe certaines radiations solaires, et accumule ainsi une force ou calorique qu'il est susceptible de dépenser en phénomènes chimiques très importants qui se traduisent par :

$1^{\circ}$ L'assimilation chlorophyllienne. - L'acide carbonique de l'atmosphère interne des organes verts, en contact du grain de chlorophylle et sous l'action des radiations solaires est décomposé en oxygène qui est mis en liberté au moins partiellement, et en carbone. Ce carbone à l'état naissant, se fixe aux éléments de l'eau, peut-ètre aussi à l'hydrogène et à l'oxygène d'autres composés, pour former des hydrates de carbone, tout d'abord du glycose; pour se transformer ensuite en

(1) Chez les animaux, une partie de cette force vive se transforme en calorique pour entretenir la chaleur animale du corps vivant.

(2) M. Jean Friedel a obtenu l'assimilation chlorophyllienne, en dehors de l'organisme, sans l'intervention du protoplasme vivant, par l'action d'une diastase qui utilise l'énergie des rayons solaires (comptes rendus de l'Ac. des Sciences, 6 mai 1901). D'après ses expériences, l'agent principal de l'assimilation chlorophyllienne dans la plante verte serait un ferment soluble (enzyme) et le pigment chlorophyllien ne fonctionnerait que comme un sensibilisateur chimique. (Revue générale de botanique, T. XV.) 
d'autres composés ternaires, en amidon par exemple qui est le produit presque immédiat et le plus apparent de l'assimilation du carbone par les feuilles, pour se combiner à l'azote des nitrates ou à d'autres éléments, l'action de la chlorophylle et de la lumière n'est plus nécessaire, l'activité du protoplasme suffit.

Quant à l'acide carbonique décomposé, il est remplacé par une quantité équivalente d'acide carbonique que l'organe vert prend à l'atmosphère en vertu des lois générales d'équilibre et de diffusion des gaz, de même qu'en vertu des mêmes lois, l'oxygène mis en liberté se dégage à l'extérieur.

Ainsi l'arbre, sous l'action de la chlorophylle et de la lumière, prend à l'air extérieur de l'acide carbonique et rejette dans l'atmosphère de l'oxygène. Ce phénomène d'assimilation ne cesse que pendant la nuit, car la chlorophylle ne se développe et n'agit que sous l'influence des radiations lumineuses (lumière et certaine quantité de chaleur).

$2^{\circ}$ La chlorovaporisation. - De même, et grâce au fonctionnement de la chlorophylle, l'arbre transpire pendant le jour une très grande quantité d'eau qui se perd dans l'atmosphère à l'état de vapeur d'eau.

Pour être remplacée dans le végétal au fur et à mesure de sa disparition, cette eau est puisée par les racines dans le sol. Quelques chiffres sont utiles pour donner une idée du besoin d'eau qu'ont les plantes, et spécialement les végétaux forestiers.

D’après Haberland, la quantité d'eau absorbée dans le sol pendant une période annuelle de végétation par les racines d'une futaie de hêtre âgée de cent quinze ans, serait représentée par une lame d'eau épaisse de $0^{\mathrm{m}}, 450(1)$.

(1) A celte eau transpirée, s'ajoute la perte d'eau par simple évaporation qui s'effectue à l'air chez les plantes vivantes aussi bien que chez les plantes mortes 
Cette épaisseur serait seulement :

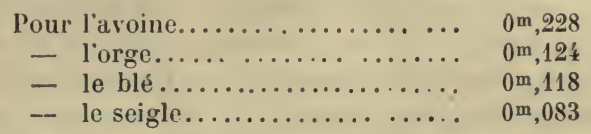

Enfin, d'après Lawes et Gilbert, la formation d'un kilogramme de matière sèche nécessiterait l'absorption de 230 à $3 \ddot{30}$ kilogrammes d'eau.

Cette énorme masse d'eau absorbée chemine par voic d'osmose et de diffusion au travers des cellules, fibres ou canaux qui lui sont ménagés à cet effet dans les tissus du bois, et se trouve appelée ainsi, en vertu des lois d'équilibre, vers les cellules des tissus verts, afin de les maintenir turgescentes.

Dès lors, l'alimentation de l'arbre feuillé est assurće; il élabore des hydrates de carbone dans ses tissus verts, en décomposant l'acide carbonique de l'air; il absorbe directement l'oxygène de l'air'; il est capable, à l'aide de l'eau qui circule dans ses tissus, d'aller chercher par voie d'osmose dans les parties du sol où fonctionnent ses poils radicaux et ses mycorhizes, tous les éléments organiques : carbone, hydrogène, oxygène et azote, ainsi que tous les éléments minéraux qui lui sont nécessaires et qui s'y trouvent, en solution ou autrement. L'oxygène lui est apporté par l'air, par l eau, et par les composés oxygénés puisés dans le sol; l'hydrogène provient de l'eau et de l'ammoniaque fournies par le sol et l'atmosphère; l'azole est fourni par le sol, sous forme de sels minéraux; dans une certaine mesure, il est fourni par les matières nitreuses, en dissolution dans les eaux mé-

et les corps inertes, d'après les lois physiques, lor'squ'elles renferment plus d'eau que l'air ambiant.

Pour se rendre compte de la quantité énorme d'eau transpirée par le régétal, il suffit de se rappeler qu'en France, pays très bien arrosé par les pluies, la quantité d'eau qui tombe annuellement sur l'ensemble du territoire peut étre représentée par une lame d'eau d'une épaisseur movenne de 80 centimètres environ. 
téorigues; mais, pour l'arbre, la véritable source de l'azote parait ètre dans l'humus; cet humus, produit par la décomposition des matières végétales et de tous les détritus d'origine organique renferme de lui-mème une certaine quantité d'azote (1); mais en outre, d'aptès les recherches de $\mathbf{M}$. Henry, les feuilles mortes ont la faculté de fixer directement l'azote de l'air, par l'intermédiaire d'infiniment petits, encore mal déterminés; elles jouent ainsi, pour les sols forestiers, le rôle des légumineuses en culture agricole, et enrichissent le sol en azote; cet azote peut être fourni à l'arbre par l'intermédiaire de ses mycorhizes.

Quant aux éléments minéraux, c'est également le sol qui doit en pourvoir la plante, mais les mycorhizes semblent jouer ici un rôle important, en assimilant les substances à leur portée dans l'humus, et en restituant ainsi rapidement au végétal ce qu'il a perdu momentanément, par la chute d'organes, relativement riches en mèmes éléments minéraux.

Ainsi s'établit chez les végétaux forestiers une rotation, une circulation continue d'éléments nécessaires à la vie, qui dans une certaine mesure permet d'expliquer :

$1^{\circ}$ Pourquoi les végétaux forestiers sont moins exigeants que les végétaux agricoles sur la composition chimique des sols;

2 'Pourquoi les végétaux forestiers peuvent se passer des engrais qu'on emploie en agriculture, et ont mème la réputation, justement méritée, d'entretenir la fertilité d'un sol el mème de l'améliorer; ceci, à la condition expresse, que la culture soit bien laite, et qu'elle ne prive

(1) Quand on suit la décomposition des matières végétales contenues dans un courant d'air, on les voit dégager, sous l'influence des êtres vivants qui pullulent daus ces débris, des quantités notables d'acide carbonique, tandis que les dégagements d'ammoniaque sont rares ou nuls, l'azote étant utilisé par les etres vivants. Ces dibris perdant constamment du carbone, tandis que l'azote demeure, on congoit que l'humus soit plus riche en azote que les débris végétaux dunt il provient. 
le sol d'aucun des débris végétaux qui constituent sous un arbre ou sous une forèt, la couverture morte ou litière et par suite l'humus.

Si l'on ajoute à ces considérations que la récolte du produit bois enlève au sol beaucoup moins d'éléments que la récolte agricole, on comprendra pourquoi la culture forestière, ou culture d'arbres, est moins épuisante que la culture agricole et peut se maintenir indéfiniment et dans de bonnes conditions sur le mème sol, sans l'intervention de l'homme.

Phénomènes d'osmose et de diffusion. - Quoi qu'il en soit, le végétal trouve dans le sol des aliments assimilables et c'est à l'état de dissolution dans l'eau, par un phénomène d'osmose et de diffusion, c'est-à-dire par un échange de liquides de densités différentes, à travers les membranes végétales, que s'opère le passage de ces éléments entre le sol, les poils absorbants (racines ou mycorhizes) et les cellules végétales successives.

On comprend aisément, qu'en vertu des lois de l'équilibre osmotique, des échanges aient lieu dans les diverses parties du végétal; celui-ci élabore, élimine, consomme et transforme dans ses tissus verts, et la perte d'eau et de substances utilisées ou transformées provoque de proche en proche une rupture de l'équilibre existant et par suite un appel d'eau et des substances azotées et minérales qu'elle tient en solution, au travers des cellules spéciales du bois adaptées à ce rôle; c'est ce qu'on a longtemps appelé courant de sève ascendante.

D'autre part, tous les organes en voie de croissance, depuis le chevelu des racines, jusqu au bourgeon terminal de la tige ou des branches supérieures, l'assise cambiale, les cellules vivantes des tissus où s'accumulent des matériaux de réserve, choisissent dans ce liquide les corps qui leur conviennent et donnent naissance à des produits variés résultant du travail spécial auquel ils se livrent.

Cette rupture d'équilibre provoque un appel des élé- 
ments utiles de proche en proche, au travers des rellules spéciales du liber, a daptées à ce rôle; c'est ce qu'on a longtemps appelé à tort courant descendant de sève élaborée.

C'est à la réunion des aliments hydrocarbonés produits par les feuilles et des aliments azotés fournis par les racines qu'est due la multiplication cellulaire. Dans la translation de ces substances, il n'y a pas à considérer de direction ascendante ou descendante, pas plus que de circulation. Pendant la période de grande croissance, les aliments sont attirés presque uniquement par les tissus en évolution ; ils se rendent, pour s'y accumuler, aux tissus de réserve, quand cette activité diminue; et cela quelle que soit la situation des organes par rapport à l'axe du végétal.

Le végétal, par le fait de son travail, opère ainsi une véritable sélection; il est susceptible de demander au milieu ambiant les éléments dont il a besoin, au fur et à mesure qu'il les consomme, et les échanges, d'où résulte la nutrition de la plante, se poursuivent sans arrèt pendant la durée de la végétation. A la fin de la jériode de végétation, toule vie apparente cesse, dans nos climats tout au moins, pour reprendre à nouveau au printemps suivant.

Remarquons enfin que dès le début de cette nouvelle période de végétation, l'organisme est mis en mouvement par l'action de diastases spéciales qui, sous l'influence de l'eau et d'une température convenable, transforment les éléments de réserve en principes assimilables; ces principes entrent en dissolution dans l'eau. Immédiatement utilisables, ils servent au premier travail du végétal, à l'épanouissement des premiers organes verts et par suite, suivant l'expression courante, au départ de lı sèce. 


\section{DEUXIEME SECTION \\ Forêt et peuplements.}

\section{GÉNÉRALITÉS.}

Un simple assemblage d'arbres qui naissent, grandissent et meurent dans un sol supposé indéfiniment iertile constitue une for'èt.

Telles étaient les forèts de l'ancienne Gaule qui couvraient la presque totalité du territoire.

Aujourd'hui, la manière d'envisager la forèt est différente; elle est présentée ainsi par M. Boppe : comme les productions de la terre, plus encore que toutes les autres, la production forestière est soumise à des lois qu'on ne peut méconnaitre; en fait, si la forèt se développe sous la seule action des forces qui se meuvent à la surface du globe, son état de végétation est fonclion des

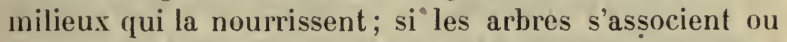
s'excluent, c'est pour obéir à des exigences physiologiques. Aussi de semblables associations ne peuvent ètre considérées comme une réunion fortuite de sujets indépendants les uns des autres, car dès que les arbres sont ainsi groupés en massif, on les voit perdre peu à peu leur individualilé, pour concourir à la formation de cet ètre nouveau, unique qu'on appelle la forêt. Celle-ci, avec des conditions d'existence et des propriétés spéciales, avec des aptitudes et des besoins qui lui sont particuliers, fonctionne à la façon d'un organisme complexe, dans lequel les végétaux, l'atmosphère et le sol entrent comme facteurs. 
En sylvieulture on entend : par massif forestier, ou massif boise (1) un ensemble de bois et forèts, autrement dit une superficie plus ou moins étendue de terrain occupée par des arbres, sans aucune distinction d'essences, d'âge, d'origine et de groupement; par peuplement, la réunion d'un grand nombre d'arbres, en un toul limité, de même nature et indépendant, qui fait l'ohjet d'un traitement ou d'une exploitation forestière. La réunion d'un certain nombre de peuplements forme une forèt.

Si une partie du peuplement se distingue de l'ensemble, par l'essence, l'âge ou la croissance les sujets, mars se trouve en rapport plus ou moins intime avec lui, elle forme ce qu'on appelle un bouquet ou un groupe (le groupe a moins d'étendue que le bouquet).

\section{I. - FORMATION EN MASSIF}

Pour qu'un espace de terrain soit dit boisé, il faut qu'il soit occupé par des arbres, autant que possible à l'exclusion de toule aulre végétation; lintervalle des arbres peut itre tris variable.

Si ce terrain, occupé par un certain nombre d'arbres est entièrement couvert par leurs cimes, le peuplenent est dit formé en massif; il importe peu pour cela que les cimes se superposent ou non en plusieurs étages.

Celte formation en massif comporte une infinité de degrés intermédiaires entre l'état parfait où les cimes se superposent, et empiètent largement les unes sur les autres, et l'état opposé où le couvert n'existe que partiellement; c'est ce degré qu'on exprime en pratique, en se servant des termes:

Massif serré, bon massif, si les cimes de tous les arbres so fouchent et sont entrelacées;

Massif suffisant, ou simplement massif, si les cimes se

(1) Dans le langage courant, on appelle bois une petite étendue de terrain boisé, et forêt une grande etendue de terrain boisé. 
touchent, sans s'entrelacer, sans être agitées par le vent; Massif interrompu, si les cimes ne se touchent que par certains points;

Massif clairiér's, incomplet, insuffisant; si le peuplement renferme de nombreuses clairieres;

Massif clair, entrecoupé, si le peuplement est parsemé le virles.

On désigne : par clairière, toute portion de forêt à peu - près dégarnie de bois, où les arbres sont rares et disséminés et où, dans l'intervalle des arbres, le sol est encore recouvert d'essences inférieures ; par vide, toute portion de forèt complitement dégarnie d'arbres, tout au plus couverte de morts-bois, c'est-à-dire d'arbustes ou d'arbrisseaux. Enfin, si les clairières ou vides occupent de très grandes étendues, le terrain prend la dénomination de terres vaines ou vagues.

Remarquons que l'espace occupé par chaque arbre dans l'atmosphìre, correspond à un espace semblable, occupé par les racines dans le sol; ces deux causes réunies agissent sur la végétation.

Le degré de perféction d'un massif dépend de trois causes principales : la fertilité de la station; le tempérament et le couvert de l'essence; l'àge du peuplement.

La fertilité d'une station influe en général sur la qualité du massif; dans une bonne station, où les sujets vigoureux tendent à prendre une cime forte et bien fournie, c'est moins le nombre des sujets que le développement individuel des cimes qui concourt à la perfection du massif.

Le tempérament et le couvert d'une essence sont en rapport intime avec le massif qu'elle forme; les essences d'ombre à feuillage épais donnent des peuplements mieux formés en massif que les essences de lumière à feuillage clair.

L'âge du peuplement agit sur l'état de massif en ce sens que, dans la jeunesse et l'àge adulte, le massif est 
généralement plus parfait que dans la vieillesse et dans la vieillesse extrème, époques auxquelles il se fait du desserrement et des clairières.

\section{I1. - INFLuence dE L'ÉtAT dE MASSIF SUR LES INDIVIDUS}

Forme forestière. - L'arbre qui croit en massif, enserré de toutes parts par les sujets voisins, est soumis à des canditions spéciales de milieu, qui modifient d'une façon sensible sa manì̀re d'exister; privé latéralement de lumière par le feuillage des arbres voisins, il cherche toujours à s'élever au-dessus du massif; lous les sujels se poussent en hauteur, il y a lutte pour la lumière, c'est-à-dire pour la vie; les dernières branches seules restent assez éclairées pour demeurer vivantes, et la tige se dégarnit de plus en plus; d'ailleurs ces branches ellesmèmes ne peuvent s'allonger sans se heurter à celles des arbres voisins. Aussi la forme spécifique de chaque sujet disparait-elle ou à peu près pour faire place à une forme forestière uniforme. L'arbre tend à prendre un fût démesurément long surmonlé par une cime grèle (fig. 10).

Nous examinerons successivement l'influence qu'exerce l'étal te massif sur l'accroissement en hauteur, l'accroissement en diamètre, puis en volume.

$1^{\circ}$ Accroissement en hauteur. - Nous distinguons trois cas :

A. L'arbre est susceptible de dépasser en hauteur le massif qui l'entoure. - Cet étal se produit soil que ce massif soit constilué par des végétaux de moindre importance forestiòre, incapables d'avoir la mème régélation, et par suite incapables d'atteindre la mème hauleur; soit que ce massif soit constitué par des végétaux du mème genre, recépés périorliquement avant qu’ils n’aient alteint la hauteur qu'ils sont susceptibles d'acquérir. 
Dans ce cas, le massif par le couvert latéral qu'il exerce, augmente l'effet de l'élagage naturel; l'arbre allonge ses jeunes pousses terminales pour aller chercher la lumière au-dessus du massif, pendant que les

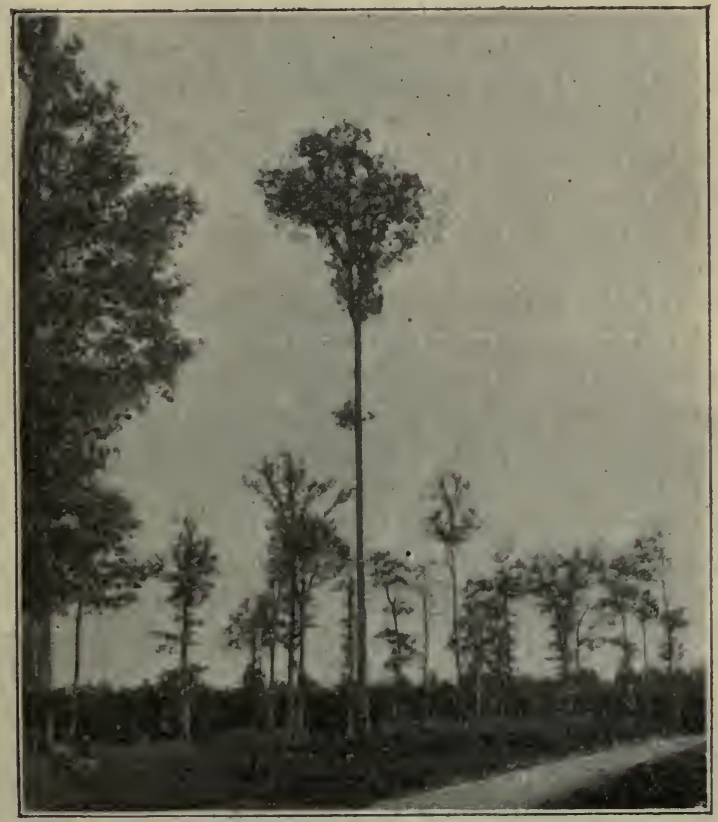

Fig. 10. - Chène de futaie (forme forestière). ropèt domaniale de Bercé (Sarthe).

branches, privées de lumière par le massif contigu, disparaissent peu à peu dans toute la région enserrée par les sujets environnants.

Le fùt de l'arbre est donc plus long que dans le cas de l'arbre isolé, et la cime s'étale au-dessus du massif, tendant à y prendre sa forme naturelle. 
Celle forme forestière ne diffère de la forme spécifique de l'arbre que par une cimé plus éleveée, mais normalement étalée et par un fùt plus allongé. Ce cas se présente pour toutes les réserves de taillis sous futaie (fig. 11).

Pendant la croissance de l'arbre, spécialement chez. certaines essences (le chène, par exemple), un grand nombre de bourgeons ont pu rester à l'état proventif dans toute la partie de la tige privée de lumière par le massif. Lorsque, pour une cause quelconque, le massif vient à disparaitre, un certain nombre de ces bourgeons, frappés par la lumière, se développent et donnent naissance, le long du fût de l'arbre, à des branches gourmandes.

B. L'arbre fait partie intégrante d'un massif serré(1). - Cet état se produit si l'arbre est destiné à croître avec le massif qui le suit en hauteur.

Dans ce cas, la lutte pour la vie est intense; chaque arbre allonge ses pousses terminales pour aller chercher la lumière au-dessus du massif et tous les sujets se poussent en hauteur, pendant que leurs branches latérales, serrées par les sujets voisins, tendent à disparaitre et que la tige se dégarnit de plus en plus. Les derniires branches seules sont assez éclairées pour demeurer vivantes, mais elles ne peuvent s'étaler librement; elles se bornent à occuper la place qui leur est strictement mesurée; la cime reste grèle, resserrée par celles des sujets voisins qui l'arrètent dans son développement, et est portée à l'extrémité d'un fùt démesurément long (fig. 10 .

Cet élat persiste tant que les circonslances spéciales qui l'ont provoriué ne viennent pas à changer. Deux modifications peuvent survenir :

a. Cette cime étriquée troure tout à coup, par la disparition voulue ou accidentelle d'un ou de plusieur's sujets voisins, une nouvelle place au soleil; les branches

(1) Dans un massif trop serré, les arbres se développent mal, Innt en hau. teur qu'en diamètre. 
INFLUENGE DE L'ETAT DE MASSIF.

demeurées_vivantes vont aussitôt chercher à s'étendre, à

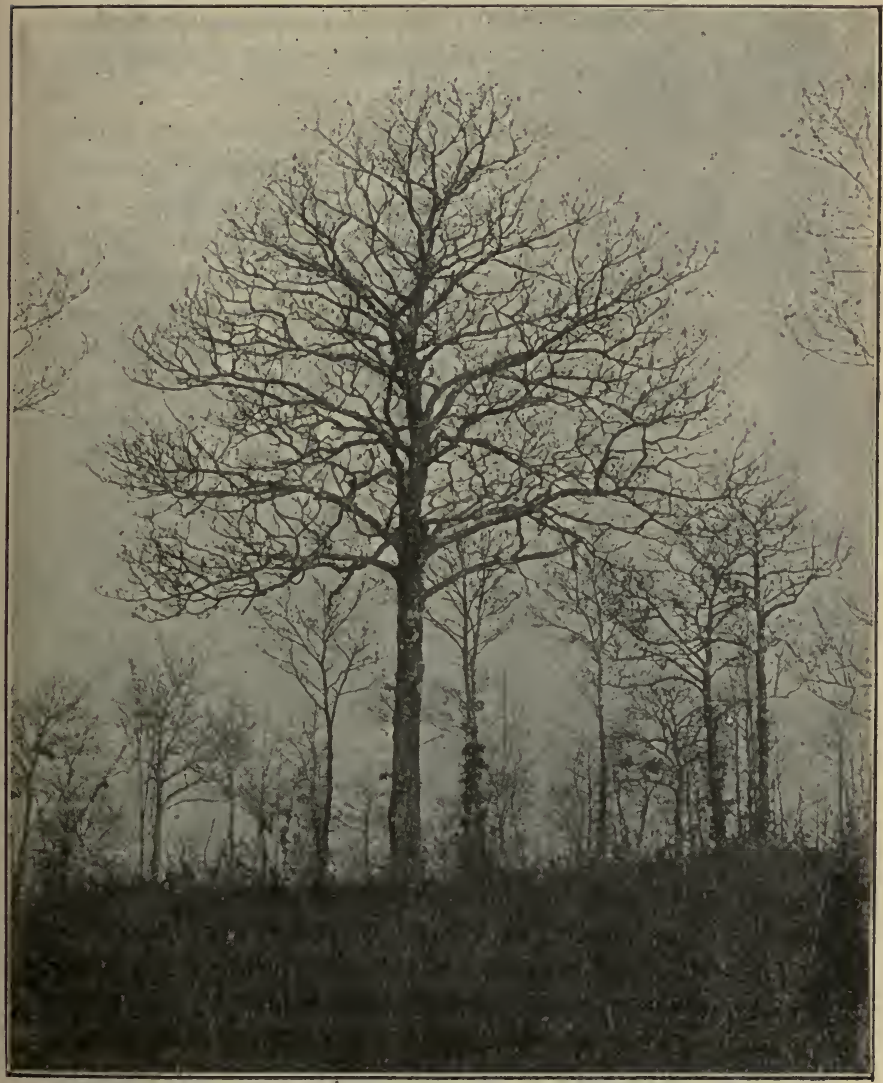

Fig. 11. - Cinene de taillis sous futaie (forme forestière), forèt de Chargeylès-Port (Haute-Saòne).

"Les Forêts " par M.M. Boppe et Jolyet.

sétaler librement du côté du jour, jusqu'ì ce qu'elles 
rencontrent un obstacle qui les arrète. Nous verrons plus tard que dans un massif, l'homme sait créer ainsi artificiellement des places libres à côté des cimes qu'il veut développer pour favoriser la croissance des sujets d'avenir; c'est le principe des éclaircies.

b. L'arbre se trouve subitement isolé de tous les sujets qui l'entourent; on voit alors sa cime dépérir; son fût est souvent envahi par des branches gourmandes qui hâtent la mort en cime, et l'arbre est destiné à mourir, s'il n'est pas capable (soit en raison de son essence ou de son âge, soit en raison de conditions favorables de végétation) de développer en dessous de la première cime, un certain nombre de bourgeons proventifs, et de se créer une nouvelle tète. L'arbre présente alors une cime plus basse de forme plus normale, et porte tout à fait au sommet de l'arbre, des branches mortes.

Ajoutons qu'un arbre subitement isolé, est très exposé ì ètre renversé par les vents, si on n'a pas eu soin de le préparer progressivement à cet état d'isolement.

C. L'arbr'e ayant cr'û à l'état isolé, se trouve peu à peu enserré au milieu d'un jeune massif qui s'élève autour de lui.

$\mathrm{Au}$ fur et à mesure que ses branches basses sont atteintes par le couvert qui se crée autour d'elles, elles dípérissent et meurent; les pousses terminales recommencent à croître, et l'arbre cherche à se constituer une nouvellecime, au-dessus du massif naissant qui l'envahil, ou dans la place quelui laissent encore les sujets voisins.

Il est à remarquer que dans ce cas la mort des grosses branches latérales est la cause de tares nombreuses qui se propagent daus le bois el abrègent l'existence de l'arbre.

$2^{\circ}$ Accroissement en diamètre. - Puur un mème arbre, et dans des conditions de sol, de climat et d'exposition idenliques, l'épaisseur des accroissements en diamètre varie beaucoup sur toute la hauteur de l'arbre, ce qui tend à donner au lül une forme géonétrique se 
rapprochant tantôt du cylindre, tantôt du cône, tantôt de volumes plus compliqués. MM. Boppe et Jolyet rapportent les faits suivants exposés par M. le docteur Nordlinger.

a. Dans un jeune arbre garni de ses branches depuis le sol, conséquemment isolé, les accroissements s'amincissent régulièrement de la base au sommet; la forme de la tige est conique.

b. Quand les branches inférieures sèchent naturellement, les accroissements présentent leur plus grande épaisseur dans le voisinage et au-dessous des premières branches vives; ils s'amincissent de là jusqu'au pied; la tige se rapproche du paraboloïde.

c. A l'état de massif, les couches deviennent de plus en plus larges par le haut, souvent deux ou trois fois plus larges qu'au pied; elles donnent à la tige une forme cylindrique.

d. L'arbre en massif qu'on isole s'accroît dans l'ordre inverse, c'est-à-dire que les grossissements supérieur's s'amincissent, tandis que les inférieurs s'élargissent; la tige a des tendances à revenir à la forme conique qui est la plus générale chez les arbres crûs isolément.

$3^{\circ}$ Accroissement en volume. - L'accroissement en volume d'un arbre est la résultante de son accroissement en hauteur et de son accroissement en diamètre.

Nous empruntons à M. le professeur Huffel (1) les conclusions suivantes :

a. Pour un arbre isolé, très vigoureux, dont rien ne vient contrarier le libre développement dans toutes les directions, aussi bien dans le sol que dans l'atmosphère, les accroissements annuels de volume (quantité de bois qu'un arbre ajoute chaque année à son volume) suivent une marche ascendante depuis la naissance, et cette marche ascendante persiste parfois jusqu'à la maturité de l'arbre, c'est-à-dire jusqu'à l'époque où on ne peut

(1) G. Huffel " Les arbres et les peuplements forestiers, " 
plus différer sa rérolte sans le voir perdre de sa qualité pour la consommation.

Le plus sourent néanmoins cette marche ascendante s'arrète de bonne heure, à partir par exemple du moment où l'accroissement en hauteur se ralentit d'une façon notable; les accroissements annuels de volume deviennent presque constants, de sorte que ce volume varie alors à peu près proportionnellement au temps. Quelquefois même, des arbres qui ont eu dans la jeunesse une croissance rapide, la ralentissent à un âge plus ou moins a vancé.

b. Pour un arbre réservé dans les tuillis sous futaie, dont la cime reste toujours à l'état libre au-dessus du taillis, mais dont les fûts sont tantôt exposés à la lumière et à la chaleur, tantôt englobés dans ur massif, il paraît probable que la coupe du taillis produit une augmentation dans l'accroissement des arbres de réserve (1);

c. Pour un arbre enserré dans un massif. Le plus ou moins d'espace dont dispose l'arbre influe sur la marche de l'accroissement; l'état de massif serré entrave d'ume façon générale le développement de l'arbre, et par contre (fait tris important, sur lequel repose toute la pratique des éclaircies), si l'on vient à dégager un arbre dont la vécrétation a été contrariée, on le voit bientòt reprendre un accroissement beaucoup plus considérable.

II. Huffel conclut de ce qui précède que l'élat de massif exerce une influence prépondérante sur les conditions de la croissance; le développement relatif de la tige el des branches des arbres, el la proportion entre la hauteur et le diamitre du trone en dépendent principalement.

Chez l'arbre ayant crû à l'état isolé, le bois des hran-

(1) On peut admettre qu'il se présente à ce moment dams le sol, pour les racines des arbres de réserve, une question de moindre concurrence, au moins piudant quelques années. 
ches a beaucoup plus de développement que celui du tronc, parfois mème il le dépasse notablement en volume; rette proportion varie d'ailleurs avec les essences, suivant leur port; chez beaucoup d'essences, elle varie d'une façon notable avec le sol, car un sol qui n'est pas profond, meuble et frais, nuit au développement en hauteur et par suite en longueur du fût, spécialement chez l'arbre isolé. La supériorité de la production d'un arbre isolé ne peut se traduire que par une augmentation de diamètre du tronc, et du développement des branches.

Chez l'arbre ayant crû à l'état de massif, le fût est très développé en longueur et la cime est resserrée, étriquée, confinée à la partie supérieure de l'arbre. Le bois des branches a beaucoup moins de développement que celui du trone, et lui est toujours notablement inférieur en volume.

\section{III. - INFLUENCE DE L'ÉTAT DE MASSIF SUR LA FERTILITÉ DE LA STATION}

$1^{\circ}$ Fertilité de la station. - Toute culture, quelle soit agricole ou forestière, est dirigée dans le but d'exporter au dehors de la station, au moment de la récolte, un produit utile, plus ou moins riche en matériaux enlevés au sol.

Pour maintenir la fertilité de la station, on doit tenir compte de la loi de restitution qui s'impose à tous les genres de production, car un sol auquel on enlève sans resse des récoltes sans rien lui restituer s'épuise fatılement.

Cette loi rend indispensables en culture agricole, le labour et l'apport de fumier ou d'engrais; en culture forestière, cette loi est aussi rigoureuse, mais les conditions économiques de la sylviculture actuelle ne permettent pas à l'homme de restituer au sol sous forme de fumure artificielle des éléments fertilisants.

L'homme exige de la forèt qu'elle donne sur le mème 
sol des récoltes successives, et la forèt, si on veut bien la laisser faire et ne pas la contrarier, est capable, par ses seules forces naturelles, d'entretenir le sol dans un état de fertilité satisfaisant et mème de l'améliorer. Ce phénomène tout spécíal tient, d'une part, à la nature de la récolte, d'autre part, à la périodicité de cette récolte, et enfin à l'action réparatrice de la forèt elle-mème sur la fertilité de la station.

Nous arons à examiner successivement ces trois points:

a) Nature de la récolte. - L'exploitation forestière produit des arbres, mais la récolte ne consiste pas à enlever le végétal dans son intégralité; les racines restent dans le sol, sauf parfois les parties les plus âgées des axes principaux (extraction de souches); le fût ou tronc, ainsi que les branches principales et secondaires, constituent la matière ligneuse demandée par la consommalion, mais ce produit ne représente qu'une faible portion de la production tolale (1); les jeunes branches, branchelles ou brindilles, les feuilles et les semences, ne présentent aucun intérèt au point de vue de la récolte, tout au moins en lant que produit ligneux (2), et sont, soit annuellement, soit périodiquement, soit enfin à l'époque mème de l'exploitation, restituées au sol.

L'analyse chimique a démontré (p. 32) que le bois constitué de tout échantillon ayant dépassé les dimensions de branchettes ct de brindilles renferme en quantités tris faibles les matériaux rares et précieux comme l'azole, l'acide phosphorique et la polasse; il en est de mème pour les autres principes minéraux, el les parties cons-

(1) Les feuilles qui tombent aunuellement par hectire sous un courert constant, représentent un poils sec qui, d'après M. Grandeau, est seusibleusut superieur au poids sec du bois qui se forme ou est récolté sur cette surface peulaut le nène teups.

(2) Si on récolte les feuilles et les briudilles comme fuurrage, les fruits ou semences pour les douner au bétail, la litièro des forèts pour l'utiliser ailleurs, on ne fait plus de la culturo forestière, mais véritablement une culture agrıcole sounise coume olle à la loi impérieuse de la restifution. 
titutives de l'arbre peurent être classées dans l'ordre suivant quant à leur richesse absolue en éléments incombustibles : les feuilles et les aiguilles sont de beaucoup les plus riches du végétal; à poids égal elles renferment trois fois plus de matières minérales que le bois; puis viennent l'écorce, les jeunes branches, les branches plus grosses, et enfin le fût proprement dit. Le bois parfait est, de toutes les parties de l'arbre, le plus pauvre en principes incombustibles.

Dans la racine, les parties les plus jeunes sont les plus riches; enfin le végétal accumule dans la graine d'abondantes réserves alimentaires qui la rendent riche en principes utiles, notamment en azote.

La récolte forestière, si elle est strictement limitée au bois constitué, n'exporte donc que les parties les plus pauvres en matériaux utiles; elle est, par ce fait, beaucoup moins épuisante que la récolte agricole.

b) Périodicité de la récolte. - Pour atteindre les dimensions qui permettent de l'utiliser et par suite de la récolter, la matière ligneuse demande un nombre d'années plus ou moins grand. Il s'en suit que les récoltes se font périodiquement sur le mème point, toujours à des intervalles assez éloignés pour permettre qu'une longue jachère sépare les exploitations successives.

c) Action réparatrice de la forêt elle-même sur la fertilité de la station. - L'instrument essentiel dont se sert la forêt pour exercer cette action, est la couvertnre morte, c'est-à-dire la couche superficielle qui recouvre immédiatement le sol minéral.

Couverture morte. - La couverture morte joue dans lá vie de la forèt un rôle capital. Il ne faut pas être bien attentif pour remarquer en se promenant sous bois que le sol est formé d'une véritable litière, ou paillis de détritus de tous genres tels que : feuilles, aiguilles, brindilles et branches mortes, lambeaux d'écorce, fruits et graines, déjections et débris d'animaux, etc. Sous un 
peuplement en massif serré, aucune végétation herbacée ne vient interrompre cette couverture morte, sur laquelle on éprouve, en marchant, une sensation toute spéciale d'élasticité.

Cette couverture morte, due à la présence et à l'action du peuplement, suffit pour entretenir la fertililé de la station et pour l'améliorer.

A la surface du sol on trouve tous les déchets de la végétation qui se fragmentent d'une façon incessante, se pourrissent et se transforment au fur et à mesure qüils sont recouverts par de nouveaux débris; envahis par les champignons et les ferments divers qui agissent dans ce milieu favorable, ces débris se transforment peu à peu en une matière pulvérulente, de couleur foncée, souvent complitement noire qui dégage une odeur de moisissure spéciale et qu'on appelle humus ou terreau. L'humus forme la partie la plus profonde de cette couverture morte, et au-dessous se trouve le sol minéral.

Les conditions nécessaires à la formation de cet humus sont la chaleur, l'humidité et un air calme; ces conditions se trouvent réalisées sous un massif dont le couvert est complet et constant (1).

Róle physique de la couverture morte et de l'humus. - La couverture morte joue par sa constitution et sa place à la surface du sol, un rôle physique des plus importants.

D'après M. le professeur Ebermayer : $1^{\circ}$ elle offre de nombreux espaces capillaires, des sortes de canaux qui la rendent comparable à une éponge et lui permettent de retenir une très grande quantité d'eau par imbibition,

(1) Dans des conditions normales, la transformation des feuilles en hunus seffectue plus ou moins rapidement suivant la nature des feuilles et débris végétaux d'une part, et d'autre part, suivant la nature du sol. Il faut en uoyenne deux d̀ cinq ans pour les feuilles, huit ans environ pour les aiguilles de résineux. Sur les sols siliceux, il faut plus de temps que sur les autres. Les feuilles du bouleau, du tremble se modifient plus rapidenient que celles du chène et celles-ci plus rapidement que celles du hètre. 
quantité qui parfois peut atteindre deux fois et demi son poids.

2o Elle protège le sol contre l'accès direct de l'air, et le met partiellement à l'abri des mouvements de l'atmosphère, empèchant ainsi une trop active évaporation.

$3^{\circ}$ Enfin l'air renfermé dans ces canaux agit, comme dans le cas de la neige, en rendant la couverture peu conductrice de la chaleur, et diminue ainsi tanlot le rayonnement du sol, tantôt la quantité de chaleur qu'il absorberait s'il était nu; la couverture empêche donc la couche superficielle du sol de s'échauffer ou de se refroidir trop rapidement.

Il est évident en outre qu'elle s'oppose au tassement du sol en brisant le choc des gouttes qui viendraient le haltre pendant les grandes pluies; qu'elle prérient le ruissellement et le ravinement des terres; qu'elle facilite enfin la pénétration dés eaux almosphériques dans les couches profondes.

A ces propriétés physiques, s'ajoute l'action physique prépondérante de l'humus, matière essentiellement hygroscopique, susceptible de fixer 100 p. 100 de son poids d'eau, qu'il prend au sol ou à l'atmosphère ; aussi filtrant qu'hygroscopique, il reste en général frais sans devenir mouilleux; de consistance moyenne, il donne de la ténacité aux terres légères, de la perméabilité aux terres compactes, et se montre en toutes choses le pondérateur merveilleux des qualités physiques du sol. Bien plus, le terreau forestier attire et fait vivre une multitude d'ètres organisés qui le perforent de leurs galeries, le parcourent et le remuent sans cesse, et mélangent peu à peu le sol minéral et le terreau; il semble que par cet ameublissement naturel du sol foreslier, la nature ait voulu remplacer elle-même le travail de la pioche ou de la charrue.

Le végétal complète ce travail par l'action des racines 
qui pénètrent dans le sol à de grandes profondeurs, qui se glissent dans les fissures des roches sous-jacentes; par pression ou par contact, ces racines les disloquent ou les attaquent lentement avec des dissolvants spéciaux, et contribuent ainsi d'une manière directe à donner au sol, sinon l'ameublissement, du moins la perméabilité.

Avec le temps, l'ensemble de ces actions physiques contribue à transformer peu à peu le terrain en celle lerre i bois spéciale au sol boisé.

Róle chimique de la couverture morte et de l'humus. La couverture morte et l'humus jouent un rôle chimique qui n'est pas moins important; grâce à eux, le sol forestier s'enrichit par restitution, par mise en valeur d'éléments nouveaux dans le sol, el enfin par fixation directe de l'azote de I'air.

a) Restitution. - La récolte du produit bois n'exporte en dehors qu'une faible partie des éléments puisés par l'arbre dans le sol; la majeure parlie de ces matériaux précieux persiste à l'état de débris végétaux dans la couverture morte. L'analyse les y a recherchés el II. le professeur Henry (1) a trouvé dans la couverture morte de taillis sous futaie âgés de vingtans, aux environs de Naucy, les poids suirants à l'hectare :

\begin{tabular}{|c|c|c|}
\hline 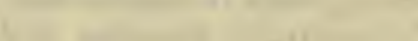 & Sol calcaire. & Sul argileux. \\
\hline Acide phosphorique........... & $\begin{array}{l}\text { Kil. } \\
23\end{array}$ & $\begin{array}{l}\text { Kil. } \\
20\end{array}$ \\
\hline 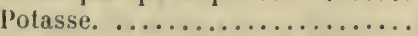 & 16 & 33 \\
\hline
\end{tabular}

Ces éléments constituent un premier gain pour le sol forestier.

b) Mise en valeur d'élèments nouveaux dans le sol. Le sol minéral renferme une réserve d'éléments tels que silicates de potasse, de chaux, de magnésie; etc., non

(1) E. Henry. "Poids et Composition de la couverture morte des forèts." (Comples rendus Ac. des Sciences 1896). 
immédiatement assimilables, c'est ce que M. Henry appelle la réserve du sol. Cette réserve se mélange peu à peu au terreau, comme nous l'avons vu précédemment; elle se trouve dans un milieu meuble, humide, riche en acide carbonique produit par les microorganismes de toute nature qui transforment la couverture morte en terreau; l'acide carbonique dissout les sels nutritifs et les transforme en éléments assimilables.

Ces éléments constituent un nouveau gain pour le sol forestier.

c) Fixalion directe de l'azole de l'air. - M. le professeur Henry a démontré que par l'intermédiaire de microorganismes encore mal déterminés, analogues à ceux des nodosités radiculaires des légumineuses, les feuilles mortes ont la faculté de fixer directement l'azote de l'air.

Avec le temps, lensemble de ces actions contribue à enrichir d'une fuçon notable le sol forıstier, et à accroîlre, ou toul au moins, à maintenir la fertilite de la station.

$2^{\circ}$ Influence du massif. - ll résulte de ce qui précède qu'un couvert prolongé et ininterrompu exerce une influence salutaire sur la fertitité du sol.

Si ce couvert vient à disparaitre (exploitation à blane étoc) le sol, exposé à l'action des vents et à l'ardeur du soleil, perd beaucoup d'humidité; la couverture morte se dessìche et est bientôt entraînée par le vent; l'humus fait alors défaut et son rôle hienfaisant n'existe plus: pendant les sécheresses de l'été et dans les années sèches, le terrain perd son eau, non seulement dans les couches superficielles mais jusque dans les couches profondes et le sol se contracte et se durcit; les éléments minéraux restent inertes parce qu'ils n'ont plus à leur portée l'atmosphère saturée d'acide carlonique nécessaire pour les dissoudre, et les éléments solubles qui ne sont pas immédiatement en contact avec des organes d'absorption, n'étant plus retenus par la matière humique, sont en- 
trainés dans les profondeurs du sol par les eaux pluviales; le grain du sol en azote n'existe plus. Le sol arrivé à cet état a perdu sa fertilité, et par suite son aptitude à produire des végétaux. S’il est pauvre en éléments nutritifs et s'il n'est pas entretenu par d'autres sources d'humidité, il peut ètre totalement ruiné.

$\mathrm{Si}$, au contraire, le mème terrain est occupé par une forèt formée en massif, et si le couvert est constitué de telle sorte que le vent et le soleil ne puissent pas y pénétrer, les causes de desséchement du sol sont en grande partie écartées; la couche d'air humide qui repose à sa surface n'est plus mise en mouvement, et unie à la couche de litière qui se décompose lentement, elle constitue un manteau protecteur qui retient l'humidité.

Ainsi une forêt bien traitée se ferme aux influences extérieures, et pourvoit elle-mème à la conservation des qualités du sol (Gayer).

$3^{\circ}$ Couverture vivante du sol. - Sous un peuplement clairiéré, des taches de végétation herbacée viennent interrompre la couverture morte et le sol est plus ou moins recouver't d'un tapis végélal ou couverture vivante, composé de plantes de petite taille, ligneuses ou herbacées, qui verdissent à la surface du sol, sans jamais s'élever au point d'ètre confondues aver. le sous bois.

Un bon état de massif, dans lequel le courert est ininterrompu, entrave la formation à la surface du sol de ce tapis végétal; par contre, la présence d'une couverture vivante à la surface du sol, est loujours l'indice d'une dégradation dans l'état du peuplement.

Cette couverture vivante, indépendamment de lobstacle qu'elle met à l'installation et à la croissance des jeunes semis, présente l'inconvénient d'absorber à son profit les réserves alimentaires du terrain; néanmoins elle protige dans une certaine mesure le sol, empèche le tassement, et par les détritus végétaux et débris morts 
qu'elle abandonne, elle fournit au terrain des éléments organiques dont les grands arbres peuvent profiter; à ce point de vue, un tapis végétal est encore préférable à un état de dénudation complet du sol.

\section{IV. - ACCROISSEMENT TOTAL DES PEUPLEMENTS}

Dans les peuplements, l'entretien continu du massif exerce une grande influence sur l'activité du sol; il en résulte qu'à ce premier point de vue l'état de massif favorise directement la croissance, et par suite la production totale de bois.

Nais par contre, dans les peuplements formés en massif, chaque arbre dispose de peu d'espace pour chercher sa nourriture et ne reçoit pas la lumière de toutes parts; il en résulte que le développement individuel de chaque sujet considéré isolément sera, au bout d'un certain temps, moins accentué que si le terrain était planté d'arbres largement espacés, ce qui ne veut pas dire toutefois que le volume total du produit sera plus considérable dans le second cas que dans le premier, car, en général, le nombre des sujets compense et au delà leur infériorité individuelle.

Entre ces deux limites, il existe un certain état de massif, permettant au terrain d'ètre garni du plus grand nombre possible d'arbres, ayant chacun leur maximum de développement.

Ce n'est donc, ni l'espacement exagéré, ni le massif serré qui donnera le plus fort volume de bois sur un espace donné, mais une mesure moyenne, caractérisant l'état de bon massif, dans lequel chaque arbre dispose continuellement de l'espace nécessaire pour se développer rapidement.

Remarquons que cette considération suppose toujours la conservation constante des qualités du sol et que, dans un massif moyen, la forte production est due 
simultanément à l'effet du couvert continu sur la fertilité du sol, et à l'utilisation de celle-ci par le nombre d'arbres.

\section{V. - PHASES SUCCESSIVES DE LA VIE. DES PEUPLEMENTS}

Modifications intérieures. - Quelle que soit l'or'i-

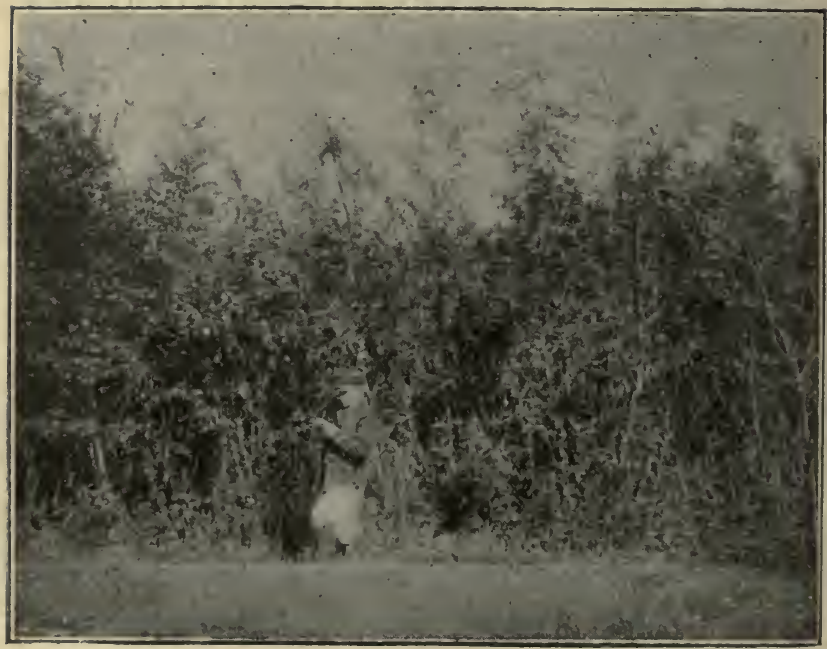

Fig. 12. - Peuplement de chène à létat de fourré, forêt domaniale de Bercé (Sarthe).

gine des éléments qui constituent un peuplement en voie de croissance, les arbres qui avancent en âge demandent un espace de plus en plus grand pour y développer leurs cimes et leurs racines; la lutle pour la vie qui s'établit dés le début de la croissance dans un peuplement formé en massif, tend à y créer une séparation en plusicurs parties distinctes. Il est en effet nécessaire qu'un grand nombre 
PllaSES SUCCEsSIVES DE LA VIE deS PEUPLEMENTS. 61

des sujets existant au commencement disparaissent peu à peu, pour céder la place à ceux qui restent. Les arbres les plus forts soit par leur énergie vitale individuelle dans la station, soit en raison de la qualité de la semence dont ils proviennent ou des conditions dans lesquelles ils se sont développés, prennent peu à peu et très lentement l'avantage dans cette lutte pour l'exis-

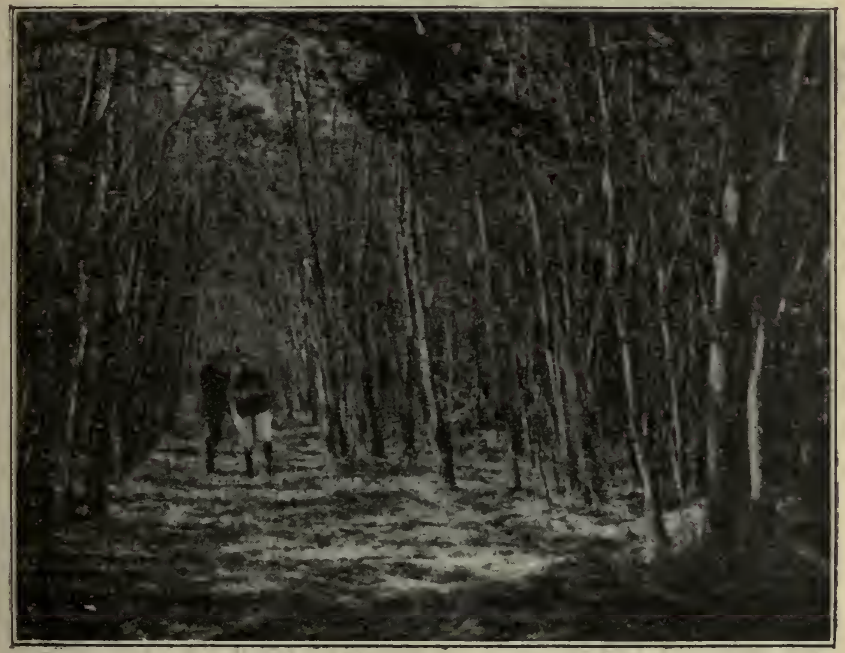

Fig. 13. - Peuplıment de chène à l'état de gaulis, forèt domaniale de Bercé (Sarthe).

tence qui dure aussi longtemps que la vie du peuplement; les autres sont dès lors destinés à dépérir et à être éliminés progressivement.

Dans tout peuplement abandonné à lui-mème, on trouve toujours pendant toute la phase active de son exis.ence et tant que l'ininterruption du couvert continue à exister, un ensemble de tiges d'élite qui dominent les

A. Irox. - Sylviculture. 
autres, et se développent avec énergie, et un ensemble de tiges à toutes les phuses du processus de dépérissement; le peuplement est divisé en deux parties distinctes ; le peuplement principal et le peuplement accessoire.

Modifications extérieures. -- Au fur et à mesure de l'accroissement des sujets qui constituent le peuplement, ce dernier change d'aspect, et il est d'usage de caractériser ces modifications extérieures par le degré de son développement. On distingue;

$1^{\circ}$ La période de fourré qui caractérise l'état de jeunesse du peuplement jusqu'au commencement de l'élimination des produits accessoires. Le fourré, dit M. Broilliard, n'est composé que de tout jeunes brins, dont les branches persistent jusqu'au sol et ordinairement mème s'entrelacent; il est assez difficile de pénétrer dans l'intérieur d'un fourré.

Les fourrés naturels offrent généralement des tiges de hauteurs très inégales, pressées et formant un ensemble confus de jeunes sujets de bonnes essences avec des espèces secondaires et des morts bois, dont la présence hàte la formation du fourré et lui donne la densité désirable (fig. 12, p. 60).

$2^{\circ}$ La période de perchis suit la précédente; elle dure jusqu'à ce que les tiges aient 20 centimètres de diamètre à hauteur d'homme. Cette période se subdivise en celle de bas perchis ou gaulis et celle de haut perchis.

D'après M. Broilliard le gaulis (fig. 13, p. 61) est formé de baguettes ou gaules flexibles, ayant perdu leur branches basses. L'appareil de feuillage est complet sinon maximum, car dès lor's il disparaìl chaque année à peu près autant de feuilles et de bourgeons au-dessous des cimes, qu'il s'en forme de nouveaux à la partic supérieure. Le sol s'améliore rapidement par l'effet du couvert bas et complet, et des détritus végétaux qui s'accumulent. Ise nombre des petites cimes qui luttent entre elles en s'éle- 
PHASES SUCGESSIVES DE LA VIE DES PEUPLEMENTS. 63

vant pour prendre place au soleil, diminue d'année en année, pour ainsi dire à vue d'œil.

Si les tiges ont plus de 10 centimètres de diamètre, le peuplement est principalement constitué par des perches dont les cimes suffisent à former un étage su-

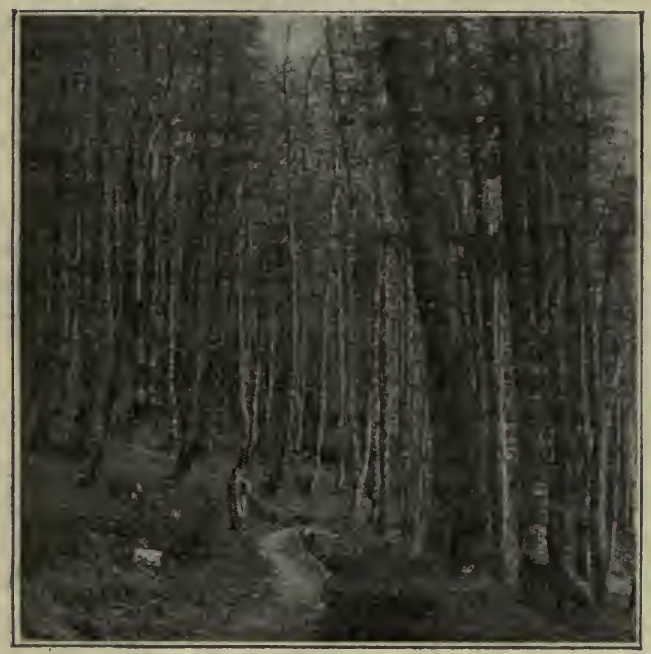

Fig. 14. - Haut perchis de hètre dans le Morvan, forèt domaniale du Breuil (Nièrre).

périeur complet, bien qu'il se trouve encore entre elles, et au-dessous d'elles, beaucoup de gaules formant un peuplement accessoire sans avenir. Cet état correspond à la période de perchis proprement dite, dans laquelle la production annuelle en bois arrive à son maximum; l'élagage naturel des branches basses s'opère encore avec. rapidité, et le nombre des tiges diminue de mème (fig. 14).

Lorsque les tiges atteignent une moyenne de 20 centimìtres de diamètre à hauteur d'homme, le peuplement 
principal est constitué par des fûts ayant déjà acquis une grande hauteur. Les cimes dont les branches principales sont déjà fortes occupent chacune une place assez large; les plus faibles résistent longtemps avant de périr sous l'étreinte de leurs voisines. L'allongement du fút se ralentit, et le massif de feuillage commence à se desserrer.

Cet état correspond à la période dite de haut perchis.

$3^{\circ}$ La période de futaie commence à ce moment, lorsque le peuplement principal a en moyenne plus de 20 centimètres de diamètre de tiges. On peut la subdiviser : en basse futaie tant que les tiges ont de 20 à 3:) centimètres de diamètre à hauteur d'homme; moyenne

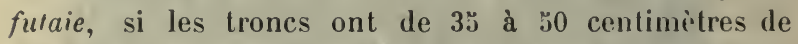
diamètre à hauteur d'homme; haule futaie si les arbres ont plus de 50 centimètres de diamètre à cette même lıauteur (fig. 10̈).

D'après M. Broilliard, dans cetle période de futaie, les cimes élevées ont de fortes branches qui persisteront à peu près indéfiniment ou ne disparaîtront à la longue qu'en laissant au tronc des tares amenant la dégradation lente des arbres les plus faibles. Les trouées qui viennent à se produire se comblent dès lor's difficilement, et au-dessous d'elles, des semis se montrent en permanence sur le sol. La production du nassif est un peu plus faible que dans les perchis.

Chacun de ces états persiste un temps plus long que celui qui l'a précédé; le bas perchis plus longtemps que le gaulis, mais moins longtemps que le haut perchis, et celui-ci moins encore que la futaie. Celle-ei prend le nom de vieille futrie quand les arbres, devenus gros, approchent de la maturité.

\section{VI. - CONCLUSIONS GÉNÉRALES}

Nous ne sommes plus aujourd'hui au temps où les forêts, non encore exploitées, croissaient sansl'intervention 
de l'homme. Une protection méthodique est devenue nécessaire à la forèt pour qu'elle puisse subsister et que son existence reste assurée malgré l'esprit de destruction qui se manifeste un peu partout, et la menace permanente qu'est pour elle le progrès de

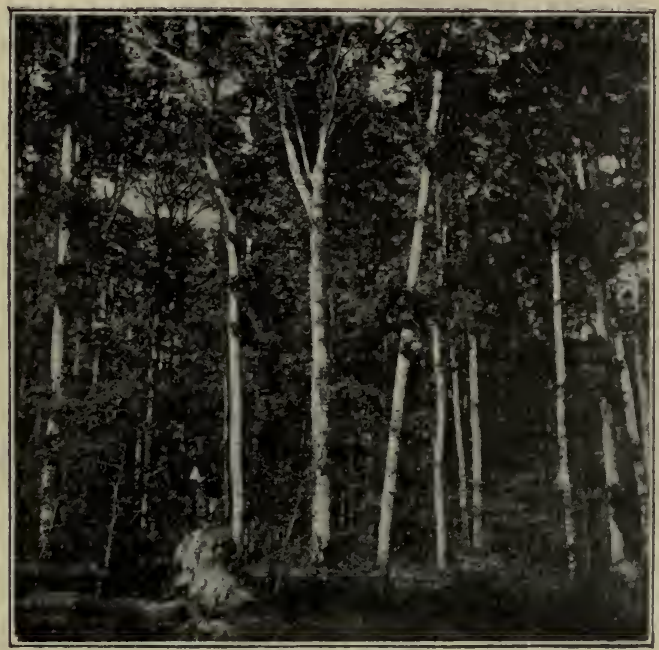

Fig. 1ว̆. - Peuplement de chêne à l'état dè futaie, (forêl domaniale de Bercé (Sarthe).

la civilisation et des besoins. C'est pourquoi la forèt est devenue, comme le champ du laboureur, un objet de culture.

Forces productrices. - Contrairement à ce qui se passe en agriculture, les moyens de la sylviculture sont essentiellement naturels; ramenés à leurs causes premières, ils se trouvent toujours et uniquement, en fin de compte, dans ces divers éléments naturels qui constituent la force productrice des stations. 
M. Gayer pose nettement le principe suivant: La cullure forestiere doit avoir pour principe fonilamental d'entourer de soins assidus et permanents les agents naturels de production des stations et de les maintenir sans interruption en pleine activité et aptes à remplir le rôle qu'elle en altend.

Toutes les opérations forestières doivent respecter cette loi suprème; nous dirons mème plus: tout résultat à obtenir, par quelque mesure culturale que ce soit, méme les profits en apparence justifiés, on doit y renoncer si on ne les obtient qu'au détriment de l'entretien de la station (1).

Line des causes générales d'appauvrissement de la fertilité des stations dans beaucoup de forèts, sur laquelle insiste l'auteur que nous venons de citer, est la diminution progressive de l'humidité du sol, et par conséquent la diminution de son activité à tous les points de rue. II. Gayer constate que c'est là la cause des nombreux obstacles que rencontre souvent la culture forestière, et il en conclut que le soin le plus assidu doit être donné à la conservation de cette humidité; les arbres ont besoin de beaucoup d'eau, et l'eau, véhicule de la vie or'ganique, est la principale condition de la fertilité du sol $(\mathcal{Z})$.

Audouble point de vue du maintien de la fertilité et de la quantité d'eau disponible, le principal moyen de conserver d'une facon permanente les forces productrices d'une station consiste dans l'entretien continu d'une régétation forestière formée en massif et en rapport avec les qualités du sol.

(1) Nous sommes loin ici de la méthode adoptée par un grand uombre de propriètaires particuliers, que des raisons d'ordre économique souvent mal entendues, conduisent peu à peu, mais sùrement, à la ruine de leur propriété boisće, et par suite au rendement nul, par l'emploi abusif d'une culture essentiellement. appauvissante, c'est-à-dire par la négligeuce absolue des conditions de ferilité de la station.

(z) Nous avons vu trop souvent des propriétaires ouvrir systcimatiquement en forét des fossés d'assainissement. Sauf dans le cas d'emplacements essen. ticllement mouilleux, ce travail est inutile ou mème nauvais; ces fossés d'asséchement vont à l'encontre des besoins de la végétation; heureusement qu'en général, ces fossés ouverts par des ouvriers inexpérịmentés n’onł pạs la pente voulue et qu'alors ils ne font pas to ual qu'ils qermient faire. 
Deux pratiques désastreuses viennent troubler et pour ainsi dire détruire l'effet améliorant d'un couvert ininterrompu et prolongé; ce sont : l'enlèvement des feuilles mortes ou de la litière et l'écobuage.

Enlèvement des feuilles mortes. - Dans certaines contrées où la culture manque de paille pour faire de la litière, on a recours aux feuilles mortes de la forèt. Nous avons suffisamment montré dans ce qui précède l'utilité de la couverture morte pour qu'il soit inutile d'insister sur les eflets désastreux de cette pratique, spécialement lorsque cet enlèvement de litière se répète toutes les années.

Dans le même ordre d'idées, on doit respecter les arbusles qui végètent en sous bois, les mousses et toute la végétation herbacée qui peuvent se trouver à la surface du sol, et dont les débris augmentent l'épaisseur de la couverture morte; tout enlèvement de cette couverture virante sous forme de nettoiements mal entendus, peut ètre considéré comme nuisible au mème titre que l'exportation des feuilles mortes, et se traduit par une diminution sensible dans la fertilité de la station.

Écobuage. - L'écobuage consiste à faire suivre claque récolte de bois ou chaque exploitation d'une récolte de céréales, sans addition d'engrais, après avoir brulé au préalable sur le parterre de la dite coupe les herbes et lous les rémanents de l'exploitation, ramilles, brindilles, etc.

Dans les Ardennes, cette pratique prend le nom de sarlage et est appliquée à des taillis exploités périodiquement tous les quinze ou vingt ans.

Nalgré certains a vantages, notamment en sol schisteux, froids et pauvres, un tel procédé de culture est fâcheux el tend d'ailleurs à disparaitre. D'abord la récolte agricole prive la végétation forestière d'une partie notable de la richesse minérale du sol; ensuite on brûle sans profit les principes organiques de la couverture morte; enfin, en dehors des dangers d'incendie, la dénudation et l'ameu- 
blissement des terres sur les pentes rapides sont dangereux, car on les expose sans protection à l'action mécanique des pluies.

L'écobuage, en rompant l'équilibre qui doit exister dans un sol forestier, est une opération beaucoup plus funeste qu’utile, et généralement toujours appauvrissante pour la fertilité de la station.

\section{INFLUENCE DES FORÊTS SUR LE RÉGIME GĖNÉRAL DES EAUX (1)}

Il résulte de très nombreuses observations faites à la fois en Russie, en Allemagne et en France que les forèts agissent de la manière suivante sur le régime général des eaux :

a) Elles provoquent les chutes de pluie et de neige;

b) Elles diminuent considérablement ou mème suppriment le ruissellement sur les terrains en pente (2); dans les cas où elles ne suppriment pas ce ruissellement, elles en allongent considérablement la durée et le rendent inoffensif ;

c) Elles dessèchent très puissamment le sol, et peuvent abaisser au-dessous d'elles de 10 mètres le niveau de la couche des eaux souterraines;

d) Elles maintiennent l'humidité de la surface du soì, mais sur une couche mince de $0^{\mathrm{m}}, 15$ à $0^{\mathrm{m}}, 20$ d'épaisseur (influence de l'humus);

e) Dans un sol boisé les eaux s'infiltrent moins que dans un sol nu;

(1) D'après le rapport de M. IJenry au Congres international de Sylviculture de 1900 à Paris.

(2) La quantité d'eau qui s'écoule inmédiatement par ruissellement varie selon la raidour des pentes et l'abondance des pluies violentes; dans le

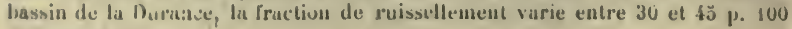
de l'eau tominie. 
INFLUENCE DES FORÊTS SUR LE RÉGIME DES EAUX. 69

f) Un sol boisé est dans son ensemble moins humide qu'un sol nu;

g) Les couches d'air qui couvrent une forèt sont jusqu'à 1500 mètres de hauteur plus humides que celles qui couvrent les cultures agricoles.

Ces principes pris isolément sont invariables, mais les circonstances locales influent sur leur importance relative.

Comme. conclusion en ce qui concerne l'action de la forét sur le régime des sources, on paraît pouvoir dire aujourd'hui que cetle action en plaine est sûrement différente de ce qu'elle est dans les régions montagneuses. 11 est incontestable que les forêts de montagne favorisent, en général, la production des sources; les exemples abondent de sources qui ont tari après des coupes ou des déboisements, et qui ont reparu avec la forèt. Quant aux forêts de plaine, leur action lirecte sur les sources parait moins nette; tantôt elles les font disparaître en desséchant le sol, tantôt elles peuvent en provoquer l'apparition en appelant les pluies, notamment s'il s'agit d'une région déboisée.

II. Henry compare les forèts à une pompe aspirante et foulante puisant l'eau dans les nuages pour faire vivre les végétaux, la leur rendant ensuite grâce à la chlorovaporisation. Ainsi la forèt, comme créatrice des pluies, exerce une action bienfaisante sur nos campagnes, et ce rôle est digne de lui acquérir de nouveaux titres à notre reconnaissance. 


\section{TROISIEMME SECTION}

\section{Principales essences forestières.}

\section{GËNÉRALITĖS}

Les essences forestières de nos massifs boisés, se divisent en deux groupes: les essences feuillues et les essences résineuses. Parmi les premières, il en est qui peuvent à elles seules former des massifs purs, ce sont les essences sociules, d'autres au contraire ne se trouvent dans un bois que par bouquets ou sujets isolés, sans arriver à former à elles seules un peuplement ou mème une partie notable de peuplement; ce sont les essences disséminécs. Quant aux essences résineuses, elles sont toutes sociales.

En sylviculture, on dit qu'une essence est dominante, quand, dans la région, elle entre à ellé seule en proportion notable dans la constitution des peuplements; dans le cas contraire, elle est dite subordonnée.

On dit qu'une essence est à tempèrament roluste quant, dans les circonstances climatériques qui lui sont appropriées, les jeunes plants decelte essence peuvent ètreélevés sans abri, dès l'époque de leur levée ; toutefois un léger abri peut ètre utile à plusieurs d'entre eux pendant la première année, et quelquefois la seconde année de leur existence, surtout contre les chaleurs de l'été, quand les circonstances de sol ou d'exposition sont peu favorables. 
L'essence est dite à tempérament assez robuste, si dans la plupart des cas, ses jeunes plants demandent à ètre abrités, au moins pendant quelques années. Enfin une essence est à tempérument délicat, lorsqu'elle demande à ètre protégée dans sa période de jeunesse pendant plusieurs années (sapin); les jeunes plants de cette essence supportent le couvert, mème quand il ne leur est plus utile; leur végétation est sans doute peu active, mais quand on vient à les découvrir peu à peu ils renaissent insensiblement et ne tardent pas à s'élancer; le sapin est surtout remarquable à cet égard. Le sapin et les essences susceptibles de vivre ou tout au moins de persister sous le couvert, sont dites essences d'ombre.

Au contraire, pour la plupart des essences à tempérament robuste, un couvert prolongé entrave pour longtemps et parfois mème pour toujours la végétation du semis; beaucoup mène ne tardent pas à dépérir (chène rouvre, chène pédonculé, pin sylvestre); ces essences, qui ont absolument besoin de lumière non seulement pour vivre mais pour pouvoir subsister, sont dites essences de lumiere.

Ajoutons toutefois que les conditions de fertilité du sol peuvent modifier le tempérament d'une essence.

\section{I. - ChÊnE ROUVRE ET CHÈNE PÉdONCULÉ (1)}

Le chène rouvre et le chène pédonculé sont très souvent réunis sous la simple dénomination de chêne.

Qualités. - Usages. - Arbre de première grandeur; bois dur, de construction, de travail, de fente (merrain, échalas, etc.); bon combustible; écorces à tan.

(1) Nons avons emprunté une partie des renseignements qui concernent les essences à l'Atlas de statistique forestière de l'administration des Eaux el Forèts, à l'Agenda du forestier et à MM. Malhieu, Muel, Lorenta et Tassy. 
Importance. - Essence la plus importante entre toutes par ses qualités et par la surface qu'elle occupe; couvrant 29 p. 100 du sol des forèts soumises au régime forestier, soit 7ä4144 hectares et par analogie 2663740 hectares pour la totalité des forèts de France.

Distribution. - Véritable essence nationale, couvrant toute la france du nord au sud, de l'est à l'ouest, sans en excepter un seul département; ne variant que par le degré d'abondance ou de rareté.

Dominant sur presque toute l'étendue du payss, le chêne n'est subordonné, rare ou très rare que dans dans l'extrème midi, sur le littoral méditerranéen, dans la région calcaire du pin d'Alep et sur le littoral te rOcéan, dans une partie de la région sablonneuse du pin maritime. Il décroìt en importance avec l'altitude dans les Vosges, les Alpes, le Plateau central et les Pyrénées; manque mème entièrement dans les régions élevées des trois premières de ces contrées montagneuses.

Mélangés sur beaucoup de points, le chène pédonculé et le chêne rourre s'isolent sur beaucoup d'autres; le premier devient dominant et mème se rencontre à peu près seul dans les grandes plaines, basses et humides; il forme ces haies plantées d'arbres de haute tige qui hordent les herbages du centre et de l'ouest; le second apparait dès que le sol se relève et s'assainit; il produit alors les belles futaies de l'Allier, du Cher, du Loir-etCher, d'Indre-et-Loire, de la Sarthe, de l'orne, ete. S'accommodant mieux de la chaleur et de la sécheresse que son congénère, le rouvre s'avance plus que lui vers le sud-est et préfère alors l'exposition nord; on le rencontre dans les montagnes de l'Ardèche, du Gard, etc., situées au-dessous de la latitude de Valence oì il devient très pubescent, et se trouve le plus souvent désigné sous le nom de chène blanc; il s'élève aussi plus haut dans la 
montagne, en raison de ses moindres exigences en humidité ; il se plaît sur les versants des montagnes, les plateaux, s'élève jusqu'à la région du sapin et se rencontre jusqu'à l'altitude de 1000 mètres.

Départements non occupés. - Le chène ne fait absolưment défaut dans aucun département; il manque partiellement dans quelques-uns d'entre eux; Vosges, Doubs, Savoie, Isère, Hautes-Alpes, Ardèche et Corse.

Le rouvre se rencontre dans tous les départements; le pédonculé n'existe pas dans un certain nombre appartenant, sauf un seul, au sud-est de la France : Corse, Var, Bouches-du-Rhône, Pyrénées-Orientales, Aude, Loż̀re, Ardèche, Drôme, Haules-Alpes et Basses-Alpes.

Altitude. - Limite inférieure au bord de la mer. Le chène pédonculé recherche les plaines et le fond des vallées; on le trouve cependant dans la région des coteaux, mais mélangé au rouvre; le chène rouvre recherche les régions accidentées, les collines, les plateaux, les contreforts des montagnes. Ces deux chènes atteignent leur maximum d'expansion dans la zône tempérée, où ils caractérisent par leur abondance une région se confondant sourent avec celle de la vigne et à laquelle succède la région froide du sapin.

La limite supérieure varie comme il suit avec les altitudes et l'espèce.

\begin{tabular}{|c|c|c|c|c|c|c|}
\hline & Vosges. & JuRA. & $\begin{array}{c}\text { Atrís } \\
\text { DEL.'Is } \\
-\end{array}$ & $\begin{array}{l}\text { Platead } \\
\text { cextral. } \\
\text { - }\end{array}$ & Prákenes. & Consr. \\
\hline & $\begin{array}{l}\text { m. } \\
800\end{array}$ & $\begin{array}{l}\mathrm{m.} \\
850\end{array}$ & $\begin{array}{c}\text { m. } \\
1200\end{array}$ & $\begin{array}{l}\text { m. } \\
900\end{array}$ & $\begin{array}{c}\text { m. } \\
1200\end{array}$ & m. \\
\hline & 1000 & 900 & 1500 & 1150 & $1 \breve{00}$ & 1500 \\
\hline
\end{tabular}

Croissance et longévité. - Les chènes rouvre et pédonculé croissent lentement, mais pendant une longue période d'années, jusqu'à l'àge de cent quatre-vingts à deux cents ans; le pédonculé a toutefois une croissance un peu moins lente que le rouvre; ils peuvent maintenir un massif jusque vers trois cents ans et mème plus.

A. Frox. - Sylviculture. 
La tige présente un fût droit, cylindrique, pouvant atteindre une hauteur de 20 mètres sans branches. Ces deux essences acquièrent 2.) à 30 mètres de hauteur et parfois 33 mètres, sur 4 à 6 mètres de circonférence et mème plus. Dans des conditions spéciales, elles peuvent vivre jusqu'à cinq et six siècles.

Sol. - Ces deux chènes paraissent assez indifférents quant à la nature minéralogique du sol; on les trouve dans les sols calcaires, argileux, siliceux.

Le pédonculé aime les terrains frais, sinon humides, profonds, substantiels, argileux, pourvu qu'ils ne soient pas trop compacts (sols argilo-siliceux ou sols argilocalcaires). On le trouve avec une belle croissance sur le bord des eaux, dans les plis de terrains et dans toutes les places où une dépression un peu marquée du sol, en permettant à l'humidité de s'y amasser, assure à ses racines la fraìcheur nécessaire à leur croissance.

Le rouvre, au contraire, ne réussit pas sur les sols trop argileux ou trop humides; il aime les terrains meubles, graveleux (calcaires ou siliceux), frais, sablonneux, pourvu qu'il y ait un peu d'argile pour y maintenir une certaine fraicheur. Il réussit quelquefois sur des versants assez arides; il craint moins la chaleur et le froid que le chène pédonculé.

Le chêne pédonculé n'a pas d'exposition préférée; le rouvre aime les expositions firaiches.

Gouvert. - Le couvert du chène rouvre est léger, celui du chène pédonculé est incomplet et plus léger.

Caractères généraux. - Germination hypogrée; tempérament robuste les jeunes chènes réclament l'action de la lumière directe dès leur jeune âge); enracinement pivotant; feuilles alternes, caduques; floraison monoïque, à la fin d'avril ou au commencement de inai; semences lourdes (gland).

Le chène (rouvre ou pédonculé) fructifie d'une façon régulière et abondante vers soixante ou cent ans suivant 
qu'il est isolé ou en massif; les glandées abondantes sont plus ou moins nombreuses selon les climats; elles peuvent arriver à deux ans d'intervalle dans les climats doux et à dix ans dans les climats moins favorables; les rejets fructifient dès vingt ans; le gland tombe dès qu'il est mûr; la dissémination a lieu l'année mème de la floraison. Il 'rejette très bien de souche, surtout le rouvre.

Allures forestieres. - Essences sociales; ces deux chènes se prêtent au traitement en taillis (taillis à écorce) et en futaie; ils donnent de très belles réserves dans le taillis composé; en futaie, ils deviennent les géants de la plaine. Les massifs ont hesoin d'ètre desserrés de bonne heure. 11 ne convient pas de les élever à l'état pur, mais en mélange avec le hêtre ou le charme (souvent, le chêne pédonculé avec le charme et le chêne rouvre avec le hêtre); quand on n'a pas de mélange, il est désirable d'a voir des sous-bois.

\section{II. - HÊTRE}

Qualités. - Usages. - Arbre de première grandeur, bois dur à aubier pas distinct; bois de travail et de feu.

Importance. - Tient par la surface qu'il occupe dans les forêts, le deuxième rang parmi les essences forestières les plus importantes; couvre 19 p. 100 du sol des forêts soumises au régime forestier, c'est-à-dire 494339 hectares, ce qui représente par analogie, pour toutes les forêts réunies, une surface de 1743209 hectares.

Répartition. - Végétalion surtout belle sur la montágne de hauteur moyenne; arbre des zones tempérées et froides, répandu à peu près dans toute la France, si ce n'est en trois zones principales : dans les hautes régions des Alpes; sur tout le litloral méditerranéen, de Nice à Perpignan; sur le littoral océanique du sud de Bayonne aux Sables-d'Olonne; il fait également défaut sur la pointe méridionale de la Corse, par laquelle passe la limite 
équatoriale de l'aire d'habitation de l'essence en Europe.

Sur la vaste étendue qu'il occupe, le hêtre est dominant dans les régions de collines et dans les régions montagneuses de moyenne élévation, telles que les Vosges, les collines jurassiques de Lorraine et de Franche Comté, les collines de l'Oise et du Bocage, le Jura, une grande partie du plateau central, des Alpes, des Pyrénées ; il est subordonné, rare et très rare dans les grandes plaines de la Flandre, de la Champagne, de la Brie, de la Beauce, dans la Bresse et le long de la vallée du Rhône.

Départements non occupés. - Dans les Alpes : partie de la Savoie et des Hautes-Alpes. Sur le littoral de la Méditerranée : partie des Alpes-Maritimes, du Var, du Vaucluse, de la Drôme, du Gard, de l'Hérault, de l'Aude, des Pyrénées-Orientales et totalité des Bouches-duRhône. - En Corse : partie méridionale. - Sur le littoral océanique du sud : totalité des Landes, du Lot-etGaronne, de la Fironde, de la Dordogne, partie de la Charente-Inférieure et de la Vendée.

Altitude. - Descend au niveau de la mer dans le nord, à $3 \ddot{30} 0$ mètres dans les Alpes du sud; commence à 70 mètres au-dessus de la mer dans les Pyrénées, à 700 mètres seulement en Corse. Les altitudes supérieures sont : dans les Vosges, 1250 mètres; dans le plateau central, 1540 mètres; dans le Jura, 1550 mètres; dans les Alpes méridionales, 2000 mètres; dans les Pyrénées, 2100 mètres; en Corse, 1800 mètres.

Sol. -- Indifférent à la composition minérale du sol, le hêtre se contente de toute espèce de terrain, pourvu que ce terrain soit divisé; il n'exige qu'une profondeur médiocre, à la condition toutefois que l'épaisse couche de feuilles mortesqu'il produit chaque année se maintienne à la surface; il prospère sur les terrains profonds et frais.

Croissance et longévité. - Le hêtre pousse lentement pendant les six à dix premières années, puis sa végélation s'accélère et devient rapide jusqu'à l'âge de 
cent vingt ans environ; il s'élève à 30 et 40 mètres, prend jusqu'à 1 mètre et $1^{\mathrm{m}}, 0 \mathrm{0} 0$ de diamètre à la base (de 3 à 5 mètres de circonférence) et prospère exceptionnellement en sol convenable pendant trois cents ans.

Renseignements généraux. - Couvert très épais; germination épigée; tempérament délicat; essence d'ombre; enracinement surtout traçant, peu profond; feuilles alternes, caduques; floraison unisexuée monoïque, en avril-mai; semence lourde (faìne). Le hètre fructifie entre soixante et quatre-vingts ans en massif, entre quarante et cinquante ans s'il est isolé; les fainées sont abondantes tous les cinq ou six ans. Malgré son enracinement superficiel, le hètre ne drageonne pas; il rejette mal de souche, sauf dans certaines régions (Pyrénées).

Allures forestières. - Essence sociale; le hètre ne forme pas de beaux taillis; l'état de futaie est celui qui lui convient le mieux; en fulaie, il se régénère très bien, et peut constituer des peuplements purs. En mélange, il est souvent envahissant. En mélange avec le chène et le sapin il donne de belles futaies.

\section{III. - CHARME}

Qualités. - Usages. - Arbrede seconde grandeur; bois dur, fournissant un combustible de première qualité, accessoirement du bois de travail ; tige cannelée plus ou moins profondément.

Importance. - Tient le troisième rang parmi les essences indigènes par la surface qu'il occupe; recouvre 16 p. 100 du sol des forêts soumises au régime forestier, soit 297113 hectares; par analogie couvre 1102237 hectares du sol des forèts de toutes calégories.

Distribution. - Caractéristique de la zone tempérée; occupe les plaines et collines du nord et de l'est, soit toute la partie de la france située au nord d'une ligne 
lirée de Grenoble à l'embouchure de la Gironde, sans néanmoins s'élever dans les hautes Vosges, ni sur les plateaux supéricurs du Jura ou sur les parties culminantes du plateau central; se montre accidentellement au sud de la ligne indiquée plus haut, sur une bande dirigée nord-sud, qui passe par '1ulle, Cahors, Montauban, Toulouse, pour rejoindre quelques stations éparses de l'espèce, sur les premier's contreforts des Pyrénées.

Dominant vers le nord-est où se remarque le maximum d'expansion, décroît assez régulièrement en abondance vers le sud-ouest, où il n'est plus que subordonné, rare ou très rare, où mème il fait absolument défaut.

Départements non occupés. - Manque entièrement dans 14 départements : Corse, Alpes-Maritimes, Var, Basses-Alpes, Hautes-Alpes, Vaucluse, Bouches-duRhône, Gard, Ardèche, Lozère, Aude, Pyrénées-Orientales, Landes et Gironde.

Manque dans partie de 19 départements : Haute-Saroie, Savoie, Jura, Drôme, Haule-Loire, Puy-le-Dôme, Cantal, Aveyron, Hérault, Tarn, Ariège, Haute-Garonne, Hautes-Pyrénées, Basses-Pyrénées, Charente-Inférieure, Vendée, Jura, Doubs et Vosges.

Altitude. - Arbre des régions tempérées, mais supporte les climats rigoureux; se trouve dans les plaines, sur les coteaux et les montagnes de moyenne élération ; arrive dans le nord au niveau de la mer, mais ne s'élève pas daus la région froide au-delà de 760 mètres dans les Vosges, de 900 mètres dans le Jura, le plateau central et les Pyrénées; parvient à 1400 mètres dans les Alpes du sud.

Sol. - Indilrérent à la composition minéralogique du terrain; les sols argilo-sablonneux, perméables, meubles et frais ou mème humides sont ceux qu’il préfère; il se développe encore bien dans les terres argileuses el calcaires qui ne sont pas trop compacles; mais il redoute les terrains marécageux ou les sables brûlants. 
Groissance et longévité. - Le charme croit lentement, sa croissance est toujours plus lente que celle des chènes rourre et pédonculé, et du hêtre; dans les quarante premières années, cette croissance égale celle du hètre, mais après cette époque, il se laisse dépasser; il ne vit guère au delà de cent cinquante ans, ne s'élève qu'exceptionnellement au-dessus de 20 à 24 mètres sur $1^{\mathrm{t}}, 50$ à 2 mètres de circonférence, quoique celle-ci puisse atteindre 4 mètres. Exploité en taillis, sa végétation est assez active, ses cépées bien fournies; la croissance des rejets de charme est beaucoup plus rapide que celle des brins de semence et se maintient assez bien pendant les trente premières années; à vingt ans, ses produits sont deux fois plus abondants que ceux du hêtre.

Caractères généraux. - Couvert assez épais, moins épais que celui du hêtre; germination épigée; teınpérament assez robuste; enracinement à court pivot avec racines latérales; feuilles alternes caduques; floraison monoïque, au commencement de mai ; semence à cupule foliacée facilitant la dissémination. Le charme fructifie de bonne heure, dès l'âge de vingt ans; les années de semence sont très fréquentes et la graine très abondante, mais entre ces années fécondes, il y a absence complète de graines. La dissémination se fait de suite ou plus généralement à l'automne et au printemps suivant. Malgré leur forme presque traçante, les racines de charme ne drageonnent presque pas, mais la souche rejette abondamment; les bourgeons proventifs ont une vitalité persistante (quatre-vingts ans) et sont nombreux; les bourgeons adventifs sont rares.

Allures forestieres. - Essence sociale; le charme vit bien en massif serré; il ne saurait constituer des futaies pures, mais il est précieux comme essence accessoire; il se laisse dominer. C'est une essence très propre au régime des taillis, mais très envahissante. 


\section{IV. - SAPIN PECTINÉ}

Qualités. - Usages. - Arbre de première grandeur; bois résineux (sans canaux résinifères); bois deconstruction et de travail (sciage) de premier ordre; maurais chauffage; charbon peu estimé.

Importance. - D'après la surface occupée, tient le quatrième rang parmi les essences principales, le premier parmi celles qui sont résineuses; recouvre 7 p. 100 de la surface des forêts soumises au régime forestier, soit 112117 hectares, et par analogie 642 971 hectares de l'ensemble des forêts de toutes catégories.

Distribution. - En relation exacte arec l'orographie du payss, et n'en occupant, à l'état spontané, que les régions véritablement montagneuses : Vosges, Jura, Plateau central, Alpes, Pyrénées el Corse. Il caractérise une région bien définie, comprise entre celle de la vigne et du chêne pédonculé, d'une part, et celle de l'épicéa, d'autre part. Une atmosphère humide (moins humide que pour l'épicéa) convient bien au sapin.

En France, il ne se rencontre qu'à l'est et au sud d'une ligne brisée qui, partant d'Épinal, passe successivement à Bourg, Clermont, Aurillac, Carcassonne et aboutit, en suivant les Pyrénées, à Bayonne. A l'ouest de sa limite occidentale on le troure par taches isolées, notamment aux environs de Bagnoles, dans l'Orne.

Départements occupés. - Englobe dans ses six stations principales, soit partiellement, soit totalement, 33 départements ainsi répartis :

Groupe Vosgien. - 4 départements : petite partie de Meurthe-et-Moselle; moitié des Vosges; très petites portions de la Haute-Saône et du territoire de Belfort.

Groupe $d u J u r^{\circ}$. -3 départements : la plus grande partie du Doubs; environ moitié du Jura et de l'Ain.

Groupe des Alpes. - 9 départements : totalité de la Hauts-Savoie, de la Saroie el des Hautes-Alpes; partie 
de l'Isère, de la Dròme, des Basses-Alpes, des AlpesMaritimes, du Var et du Vaucluse.

Groupe du Plateau Central. -9 départements : Loire, Haute-Loire, Cantal et Puy-de-Dôme en entier; Rhône et Ardèche presque en entier; Allier, Aveyron et Lozère en partie seulement.

Groupe des Pyrénées. - 7 départements : totalité de l'Ariège et des Pyrénées-Orientales, partie de l'Aude, de la Haute-Garonne, des Hautes-Pyrénées, des BassesPyrénées et du Tarn.

Groupe de la Corse. - Tout le département.

Stations isulées. - En Normandie (Orne).

Altitude. - Caractérise le commencement de la région froide; succède à celle du charme, à celle où prospère le chène, il présente les altitudes inférieures et supérieures qui suivent :

$\begin{array}{cccccc}\text { Vosges. } & \text { Jura. } & \text { Plateau centr. } & \text { Alpes. } & \text { Pyránís. } & \text { Cons: } \\ \text { m. } & \text { m. } & \text { m. } & \text { m. } & \text { m. } & \text { m. } \\ 250-1200 & 400-1400 & 500-1500 & 600-2000 & 700-2100 & 800-2100\end{array}$

Sol. - Indifférent à la composition minéralogique du sol; aime les sols profonds, frai:; et divisés, fertiles; prospère sur les plateaux calcaires Ju Jura, où il fait pénétrer ses racines dans les moindres fissures des rochers. Craint les sols marécageux, les sables maigres.

Croissance. - Longévité. - Le sapin pectiné pousse très lentement, jusqu'à dix ou quinze ans ; après dix ans, il forme des verticilles réguliers, et sa végétation est assez rapide; il s'élance, s'accroît assez rapidement d'une façon soutenue; la cime devient pyramidale et il conserve bien cette forme jusqu'à soixante-dix, quatre-vingts ans; il parvient généralement à $3 \breve{3}$ ou 40 mètres de hauteur, sur 4 à 6 mètres de circonférence; il vit deux à trois cents ans et parfois davanlage.

Caractères généraux. - Couvert épais; germination épigée; tempérament délical; essence d'ombre; enraci- 
nement profond et puissant; feuilles persistantes; floraison unisexuée monoïque, fin avril ou commencement de mai; fructification au commencement d'octobre de la mème année el dissémination dans le courant du mème mois. La fructification est assez régulière et assez abondante à partir de soixante, soixante-dix ans; presque annuelle; semence assez légère, graine ailée.

Allures forestières. - Le sapin ne se traite qu'en futaie, à l'état pur ou en mélange. C'est essentiellement l'arbre des peuplements irréguliers et du jardinage. En dehors de ses stations naturelles, il ne donne rien de bon.

\section{V. - PIN SYLVESTRE}

Qualités. - Usages. - Arbre de première grandeur; bois résineux, riche en canaux résinifères; bois de construction, de travail et de feu; n'est point soumis au gemmage.

Importance. - Tient par son importance superficielle le cinquième rang parmi les principales essences forestières, le second parmi celles qui sont résineuses. Occupe, spontané ou introduit, 4,5 p. 100 de la surface des forèts soumises au régime forestier, soit 118392 hectares, ce

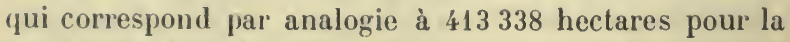
totalité du domaine forestier.

Distribution. - Essence des plaines du nord de l'Europe, n'existant en France à l'état spontané que dans les régions montagneuses de moyenne élévation, où elle a reçu une grande extension artificiellement; partout propagée comme principale espice de boisement, si ce I'est au sud-ouest dans la région du pin maritime, et sur le littoral de la Méditerranée, dans la partie de la région du pin d'Alep.

Ne parait réellement spontané que dans les Vosges, les Alpes, le plateau central et sur quelques points des 
Pyrénées; manque absolument dans la partie calcaire et montagneuse du Jura.

Départements occupés - A l'état spontané, se rencontre sur partie ou totalité de 19 départements, savoir :

Pour les Vosges : partie du département de ce nom ;

Pour le plateau central: Rhône, Loire, Puy-de-Dôme, Cantal, Haute-Loire, Ardèche, Lozère et Hérault ;

Pour les Alpes: Haute-Savoie, Savoie, lsère, Drôme, Hautes-Alpes, Basses-Alpes, Alpes-Maritimes;

Pour les Pyrénées: Pyrénées-Orientales, Aude et Hautes-Pyrénées.

Propagé par la culture (semis ou plantation), sans qu'aucune loi préside à sa distribution, dans la plupart des autres départements au nombre de 59 ; ne manque absolument que dans 10, appartenant tous au groupe du sud-ouest: Deux-Sèvres, Charente-Inférieure, Gironde, Dordogne, Lot, Lot-et-Garonne, Landes, Basses-Pyrénées, Gers, Tarn-et-Garonne.

Altitude. -- Dans les stations où il est spontané, présente les altitudes inférieures et supérieures qui suivent :

$\begin{array}{cccc}\begin{array}{c}\text { Vosges. } \\ \text { m. }\end{array} & \begin{array}{c}\text { Plateau central. } \\ \text { m. }\end{array} & \begin{array}{c}\text { Alpes. } \\ \text { m. }\end{array} & \begin{array}{c}\text { Pyrénérs. } \\ \text { m. }\end{array} \\ 289-1100 & 200-1550 & 300-1700 & 300-2000\end{array}$

Limites inférieures moins certaines que les supérieures, à raison de l'extension donnée à l'espèce par la culture, et de la difficulté de reconnaitre si elle est spontanée ou introduite.

Sol. - Sans refuser de croître sur les sols calcaires, préfère de beaucoup ceux qui ne le sont pas. En plaine, les sols sablonneux, mélangés d'argile, profonds et frais, sont ceux qui lui conviennent le mieux. Le pin sylvestre réussit même dans les sables purs, aux expositions chaudes, là où aucune essence ne se maintiendrait; pourvu que le sous-sol contienne une humidité souter- 
raine qui, par les grandes sécheresses, pourra remonter par capillarité pour maintenir la végétation.

Croissance. - Longévité. - Le pin sylvestre a une croissance rapide dès la deuxième ou troisième année de son existence; elle varie d'ailleurs notablement avec les conditions de sol, de climat et d'altitude. Il s'élève à 30 ou 35 mètres de hauteur, mais il est loin d'atteindre la grosseur du sapin et de l'épicéa, bien qu'il soit capable de mesurer jusqu'à 3 et 4 mètres de tour; il vit deux à trois sièrles.

Caractères généraux. - Couvert léger ; toutefois la durée de persistance des feuilles varie avec les climats et avec l'âge; pendant les vingt-cinq à trente premières années, elles persistent quatre ans, et le couvert en est moins léger et mème assez épais; c'est pour cela que le pin sylvestre a été employé pour refaire les terrains ruinés; mais si un jeune perchis de pin sylvestre est très bon pour améliorer le sol, un massif âgé laisse le sol se détériorer; germination épigée; tempérament robuste : il languit et succombe en peu de temps sous un couvert même léger; essence de lumière; enracinement généralement pivotant el développé ; feuilles persistantes; floraison unisexuée monoïque, mai-juin ; fructification septembre-octobre de la seconde année après la floraison; dissémination au printemps suivant, vingtdeux mois après la floraison; la fructification du pin sylvestre est précoce, vers quinze ans si l'arbre est isolé; vers cinquante ans s'il est en massif; elle est abondante tous les trois, cinq ans; semence légère, graine ailée.

Allures forestières. - Dans les limites de son aire d'habitation, le pin sylvestre est traité en futaies, aux révolutions de cent quarante, cent quatre-vingts à deux cents ans. En plaine ou hors de sa station naturelle, il constitue des plantations qu'on exploite vers trentecinqy à soixante ans et qu’on régénìre artificiellement. Essence précieuse pour reboiser les terrains nus. 


\section{VI. - CHÊNE YEUSE OU CHÊNE VERT}

Qualités. - Usages. - Arbre de troisième dimension, à bois très dur, très lourd, extrèmement compact, fort sujet à se gercer et à se tourmenter, peu convenable pour le travail, mais combustible de première qualité; excellentes écorces pour le tannage.

Importance. - Tient, parmi les essences forestières principales, le sixième rang par la surface qu il recouvre; occupe 4 p. 100 de l'étendue des forèts soumises au régime forestier, soit 10933 hectares et par analogie 367412 hectares de l'ensemble de toutes les forêts du pays.

Distribution. - Essence des lieux arides et découverts de la France méridionale, tout particulièrement méditerranéenne; abonde sur tout le littoral, de Menton à Céret; s'avance dans les Alpes, par les grandes vallées, jusqu'à Digne, Sisteron, Nyons, Montélimar' remonte le Rhône jusqu'au delà de Valence; garnit les contreforts des Cévennes, pénètre vers le sud dans le plateau central par la région calcaire des Causses, jusqu'à Florac, Rodez, Saint-Affrique, Castres et vers l'ouest se prolonge jusqu'à Toulouse et Saint-Gaudens.

Reparaît sur le littoral de l'Océan sous fornie de bande, qui de la Teste de Buch, dans la Gironde, se dirige presque sans discontinuité jusqu'à Saint-Brieuc, sur la Manche, et atteint son maximum d'expansion dans la Charente-Inférieure, où elle s'élargit vers l'est jusqu'au delà d'Angoulême. Généralement rare ou très rare, cette essence est loin d'avoir, sur le littoral de l'Océan, la mème importance forestière que sur le littoral méditerranéen.

Départements occupés. - Essence dominante ou subordonnée des forèts de 11 départements qu'elle occupe entièrement, sauf quelques hauts sommets: Corse, Alpes-Maritimes, Var, Bouches-du-Rhône, Vaucluse, Gard, Hérault, Aveyon, Aude, Pyrénées-Orientales et 
Charente-Inférieure. Disséminée, rare ou très rare dans 13 départements : Basses-Alpes, Drôme, Ardèche, Lozère, Tarn, Ariège, Ilaute-Craronne, Gironde, Charente, Vendée, Loire-Inférieure, Morbihan et Côtes-du-Nord.

Altitude. - Des bords de la mer, s'élève dans les Cérennes à 800 mètres dans les environs d'Alais; dans les Alpes, à 1100 mètres au-dessus de Grasse; dans les Pyrénées, à 800 mètres aux environs de Prades, et à 1110 mètres aux environs de Quillan; parvient en Corse à 1150 mètres.

Sol. - Il se contente des sols les plus médiocres, mêmes arides, aux expositions les plus chaudes; manifeste une préférence marquée pour les sols calcaires; de là son abondance sur le littoral méditerranéen, si ce n'est dans les montagnes granitiques des Maures; et sa rareté sur le littoral sablonneux de l'Océan, excepté là où reparaissent les calcaires, dans les Charentes; de là, enfin, son apparition dans la région calcaire des Causses, sur le plateau central.

Croissance et longévité. - Le chène yeuse est tantôt à l'état de buisson, tantôt un bel arbre de 15 à 18 mètres de hauteur el de 2 à 3 mètres de tour (Algérie); en lirance et en Corse, il ne dépasse pas 10 mètres de hauteur; sa croissance est assez rapide dans la jeunesse, puis elle se ralentit; il peut vivre trois siècles et plus.

Curactères généraux. - Couvert épais ; germination hypogée; tempérament très robuste; enracinement pivotant avec racines latérales; feuilles alternes persistantes; floraison unisexuée monoïque en avril-mai; fructification en septembre de la mème année; semence lourde (gland). Le chène yeuse fructifie d'une façon abondante et régulière dès huit, dix ans. Il rejette de souches et drageonne abondamment.

Allures forestieres. - En France, le chène yeuse ne forme que des taillis. Les sous-bois de chène yeuse sont tries beaux sous le pin d'Alep. 


\section{VII. - PIN MARITIME}

Qualités. - Usages. - Arbre de première dimension; bois résineux avec de nombreux canaux résinifères; bois de construction et de travail; soumis au gemmage au sud de l'embouchure de la Gironde; employé à la fixation des dunes.

Importance. - Tient, au point de vue de la surface qu'il recouvre, le septième rang parmi les dix principales essences forestières, le troisième rang parmi celles d'entre elles qui sont résineuses; occupe 3 p. 100 de l'étendue des forêts soumises au régime forestier, soit 93343 hectares, et par analogie 275359 hectares de toute la propriété boisée.

Distribution. - Comme le pin sylvestre, très fréquemment employé au boisement, de sorte qu'il est difficile d'en déterminer nettement l'aire d'habitation; en tout cas, espèce essentiellement littorale à l'état spontané, abondamment répandue dans la région sablonneuse qui borde le golfe de Gascogne, de Bayonne à la Rochelle; se retrouvant sur les versants méditerranéens des Pyrénées, puis dans la région granitique et porphyrique des Maures et de l'Esterel; enfin dans la Corse; propagée abusivement par semis bien au delà de son aire, jusque dans le centre et l'est et mème jusqu'au nord.

Départements occupés. - Occupe à l'état spontané, totalement ou partiellement 13 départements, savoir : la Corse; partie des Alpes-Maritimes et du Var; partie des Pyrénées-Orientales et de l'Aude; partie des BassesPyrénées; les Landes, le Gers, le Lot-et-Garonne, la Gironde, la Dordogne, la Charente et la Charente-Inférieure; propagé artificiellement dans 46 autres départements et tout particulièrement dans ceux de l'ouest; ne faisant entièrement défaut que dans 28.

Altitude. - Croît au hord de la mer, sur les dunes et dans les Landes, pour s'élever spontanément à 700 mè- 
tres dans les Pyrénées-Orientales, à 800 mètres dans les Alpes-Marilimes. Introduit dans le centre et propagé à tort el sans grand succìs dans des régions trop septentrionales et trop éloignées de son aire d'habitation, où il redoute la gelée et les grands froids.

Sol. - Essence calcifuge, très caractéristique des sols exempts de calcaire, tels que les sols granitiques, porphyriques, schisteux, sablonneux, elc. A part les sols calcaires, compacts, marécageux, qui lui sont contraires, il vient sur tous les sols, de préférence sur les sols meubles, profonds et frais.

Croissance et longévité. - Le pin maritime a une végétation très prompte, longtemps soutenue, et peut, à cent ans, atteindre 3 mètres et plus de tour.

Caractères généraux. - Couvert faible; germination épigée; tempérament robuste; enracinement pivotant et traçant ; feuilles persistantes ; floraison monoïque avril-ınai; fructification, automne de la seconde année; dissémination, printemps suivant; graines ailées. Le pin maritime fructifie d'une façon très précoce, vers quinze ans, abondante et presque continue à l'âge moyen.

Allures forestières. - Futaies gemmées sur le liltoral du golfe de Gascogne; ne produisant plus suffisamment de gemme au nord de la Gironde et sur le littoral de la Méditerranée pour ètre résiné.

\section{VIII. - ÉPICÉA COMMUN}

Qualités. - Usages. - Arbre de prenière grandeur; bois résineux, à canaux résinifères, de service, de construction, de travail, de sciage, de fente (merrain).

Importance. - Placé au huitième rang au point de vue de la surface qu'il boise; occupe 2,9 p. 100 du sol des forèts soumises au régime forestier, c'est-à-dire 75346 heclares; par analogie, couvre 275559 hectares dans l'ensemble des forèts de toutes catégories. 
Distribution. - Ne se rencontre à l'état spontané que dans les régions montagneuses où il occupe une zone supérieure à celle du sapin, mais beaucoup plus restreinte; forme sur la frontière orientale, et du nord au sud, une longue bande qui couvre les hautes Vosges centrales, les plateaux et les crêtes élevées du Jura, les régions moyennes des Alpes. Disséminé, rare ou très rare dans le plateau central (Cantal, Haute-Loire, Aveyron, Hérault) et dans les Pyrénées (Pyrénées-0rientales et Ariège) où il a été introduit par la culture; manque en Corse.

Départements occupés. - 11, savoir: parties les plus élevées des Vosges, du Doubs, du Jura, de l'Ain ; totalité de la Haute-Savoie et de la Savoie; partie de l'Isère, de la Drôme, des Hautes-Alpes, des Basses-Alpes et des Alpes-Marilimes.

Altitude. - En mélange avec le sapin dans la zone moyenne de ce dernier; devient plus abondant à mesure que s'accroît l'altitude, et finit, au-dessus de cette zone, par former des peuplements purs. Il présente les altitudes inférieures et supérieures qui suivent :

$\begin{array}{ccc}\begin{array}{c}\text { Vosges. } \\ \text { m. }\end{array} & \begin{array}{c}\text { Jura. } \\ \text { m. }\end{array} & \begin{array}{c}\text { Alpes. } \\ \text { m. }\end{array} \\ 500 \text { à } 1250 & 700 \text { à } 1550 & 900 \text { à } 2400\end{array}$

Il est introduit artificiellement à des altitudes inférieures. Contrairement au sapin, l'épicéa devient buissonnant aux altitudes supérieures à sa zone normale de végétation et disparait gráduellement.

Sol. - Indifférent à la nature minérale du terrain; il croît aussi bien sur les grès et les granits des Vosges que sur les calcaires du Jura et des Alpes; il demande des sols frais et humides; ce qu'il lui faut surtout, c'est une atmosphère humide, des pluies fréquentes, de fortes rosées, une grande fraîcheur à la surface du sol.

Groissance et longévite. - D'une façon générale, dans 
les stations où il est spontané, l'épicéa a une croissance lente; cependant, au début, elle est moins lente que celle du sapin; son développement s'active dès l'âge de cinq ou six ans; sa longévité et ses dimensions sont à peu près semblables à celles du sapin; cependant, à un âge avancé, son diamètre est ordinairement moins fort, mais sa hauteur peut être supérieure.

Caractères généraux. - Couvert épais; germination épigée; tempérament assez robuste, toutefois le jeune plant a besoin d'abri dans son extrême jeunesse, car il craint beaucoup la sécheresse; essence de lumière, il craint davantage le couvert que le sapin; toutefois, en raison de son port élancé, l'épicéa est susceptible de former des massifs très serrés; enracinement faible et traçant; feuilles persistantes; l'épicéa a la faculté de développer en dehors de ses bourgeons normaux, d'autres bourgeons de nature axillaire disposés sans ordre, dont la présence masque la régularité de la ramification, et accentue beaucoup l'intensité du couverl. Cette faculté de développer facilement des bourgeons axillaires, rend l'épicéa propre à la taille (haies); floraison unisexuée monoïque fin mai, commencement de juin ; fructification octobre de la même année; dissémination immédiatement après la fructification, ou plutôt au printemps suivant; graines légères, ailées; l'épicéa fructifie en massif vers cinquante ans, mais? moins régulièrement que le sapin; la fructification n'est abondante que lous les deux à six et mème huit ans.

Allures forestières. - L'épicéa ne peut se traiter qu'en futaie; il aime à ètre en massif serré el se conduit bien en peuplement pur et régulier; il donne de très bons mélanges, notanment avec le sapin et le hètre ; il se montre envahissant daus les pâturages et les coupes de taillis voisins, où les graines sont transportées par le vent. Ciest un des arbres les plus précieux que nous possiodions pour effectuer des travaux de repeuplement et 
de reboisement, en raison de la facilité avec laquelle il s'élève en pépinière et de la sûreté de sa reprise sur un sol découvert.

\section{IX. - MÉLĖZE}

Qualités. - Usages. - Arbre de premier ordre par ses dimensions et ses qualités; dans sa station naturelle, le mélèze est le roi des forèts de montagne, au mème titre que le chène dans les forèts de plaine. Bois de construction et de travail; n'est point soumis au résinage.

Importance. - Parmi les dix essences dominantes principales, tient le neuvième rang d'importance superficielle, occupe 2 p. 100 de l'étendue totale des forêts soumises au régime forestier, soit 42829 hectares; par analogie, recouvre 183706 hectares de l'ensemble des forèts du pays.

Distribution. - N'apparaît à l'état spontané que dans les régions élevées des Alpes; manque dans tous les autres groupes montagneux; introduit en tous lieux par la culture, du département du Nord aux Pyrénées, des Vosges et du Jura à la pointe du Finistère, sans présenter sous cette forme, une importance forestière réelle. A l'élat spontané forme une bande dirigée du nord au sud, de Saint-Gervais (Haute-Savoie) à Nice, laquelle couronne tous les sommets de la région alpestre en s'appuyant constamment sur la partie orientale de la frontière qui sépare la France de l'Italie.

Départements occupés. - Croît spontanément sur 6 départements et n'en occupe que la région orientale la plus élevée sur une partie plus ou moins étendue de leur territoire : Alpes-Maritimes, Basse-Alpes, HautesAlpes, Isère, Savoie et Haute-Savoie.

Altitude. - Forme des massifs à partir de 800 mètres jusqu'à 2300 mètres.

Sol. - Semble ne manifester aucune préférence à cet égard, mais pour atteindre ses dimensions normales il 
lui faut des terrains meubles, légers, profonds, les sols argileux compacts lui sont contraires; il recherche des situations abrilées contre les vents âpres et secs des lıautes régions, et préfère d'autre part les expositions froides (expositions nord et est).

Croissance et Iongévité. - Le mélèze a une végétation remarquablement prompte aux basses altitudes, mais dans les $A l$ pes il croît très lentement: sa croissance est régulière jusqu'à cent cinquante ans ; dans sa station naturelle, le mélèze peut vivre trois à quatre siècles et mesurer 40 à 50 mètres de hauteur sur 3 à 5 mètres de circonférence au pied, tandis que, en dessous de sa siluation naturelle (dans les Vosges, par exemple), ses dimensions et sa longévité sont très restreintes, et de bonne heure, il présente les marques d'une caducité prématurée; son bois change alors et est de qualité inférieure.

Caractères généraux. - Couvert très léger; germination épigée; tempérament robuste, le jeune plant est susceptible de résister aux froids comme aux arleurs du soleil, mais dans les climats doux on fera bien de l'ombrager; essence de lumière; enracinement pivotant et traçant; feuilles caduques ; floraison unisexuée monoïque, en juin (en avril en plaine et dans les stations basses); fructification : automne de l'année de la floraison ; dissémination: printemps suivant; graines légères, ailées.

La fructification du mélèze est précoce, surtout lorsqu'il est cultivé dans les régions tempérées, mais alors les graines en sont vaines, et ce n'est qu'à l'âge moyen qu'elle devient régulière.

Allures forestieres. - Le mélèze est traité en futaie rlaire, soit seul, soit associé au sapin, à l'épicéa ou au pin cembro. Il convient bien pour faire des prés bois (prés hois des Alpes). Dans la limite inférieure de sa station, on le mélange avec le sapin, l'épicéa, le pin sylvestre el on le traite en peuplement irrégulier. 


\section{X. - PIN D'ALEP}

Qualités. - Usages. - Arbre de deuxième grandeur; bois de menue charpente, de travail et de feu; non soumis au gemmage.

Importance. - Placé au dernier rang des dix essences principales de la France, couvre 0,5 p. 100 de la surface des forêts soumises, soit 11980 hectares; par analogie, occupe 43926 hectares dans les forèts réunies.

Distribution. - Aire plus méridionnale encore que celle du chène yeuse, exclusivement confinée au littoral de la Méditerranée, sans dépasser vers l'est le Gard, vers le nord les limites méridionnales des Hautes-Alpes, de la Drôme et de l'Ardèche.

Départements occupés. - Six, savoir: 4 sur toute leur étendue: Var, Bouches-du-Rhòne, Vaucluse et Gard; 2 sur une portion seulement de leur surface: Alpes-Maritimes et Basses-Alpes. Introduit sur une petite échelle dans partie de 3 départements limitrophes : Pyrénées-Orientales, Hérault et Ardèche; manque complètement en Corse.

Altitude. - Borde le littoral au niveau de la mer, s'élève dans les Alpes-Marilimes et auprès des côtes à 900 mètres et mème, par pieds isolés, à 1050 mètres.

Sol. - Préfère les terrains calcaires légers, mais vit également sur ceux qui sont siliceux; abonde dans les Alpes méridionnales calcaires, manque ou devient rare dans les montagnes granitiques des Maures; très précieux par la faculté de croître jusque sur Jes rochers.

Croissance et longévité. - Le pin d'Alep reste jusqu'à l'àge de dix à douze ans branchu et feuillé dès la base; la cime en est alors conique, l'accroissement rapide. Vers vingt ans, il forme un arbre à tige grêle, flexueuse, dont la végétation se ralentit; la cime s'étale largement; à cinquante ans, il atteint en moyenne 12 mètres de haut sur 1 mètre de tour en France et exceptionnellement 20 mètres de hauteur sur $3^{\mathrm{m}}, 50$ de tour. 
Caractères généraux. - Couvert très léger; germination épigée; lempérament très robuste, essence de lumière. Enracinement pirotant et en mème temps largement développé; feuilles persistantes; graine ailée; fructification précoce et abondante, vers dix-huit, vingt ans; dissémination en mai de la troisième année après la floraison.

Allures forestières. - Futaies claires avec sous-étage de chène kermès et de chêne vert ou de chène blanc, souvent traités en taillis; les pins sont alors disséminés au-dessus du taillis.

\section{XI.? - CHÊNE TAUZIN}

i Qualités. - Usages. - Arbre de seconde grandeur ; bois très raide, se gerçant et se tourmentant beaucoup, employé pour menue charpente travail; combustible et écorces de bonne qualité.

Importance. - Plutôt disséminé qu'à l'état de massif.

Distribution. - Essence propre au sud-ouest, caractérisant le versant océanique; du sud au nord, s'étend des Pyrénées à la Loire, et mème un peu au delà jusqu'au Mans; de l'ouest à l'est, s'étend des bords de l'Océan à une ligne tirée d'Orléans à Tulle et à Toulouse; atteint son maximum d'expansion sur le littoral, dans les Landes, la Gironde, le Lot-el-Garonne, la Dordogne et la Charente-Inférieure; décroît en abondance et en continuitésur les bords septentrionaux et occidentaux de son aire, où il devient subordonné et très rare; quelques stations douteuses sont signalées en dehors de cette aire, dans le voisinage de Castres, Saint-Afrrique, Tarascon et Mont-louis. Arbre de plaines, de collines et de montagues peu élevées; souvent associé au chène pédonculé.

Despartements occupés. - L'aile d'habitation embrasse entièrement 13 départements : Gers, Landes, Tarn-etGaronne, Lot-el-Garonne, Lot, Corrèse, Dorlogne, 
Gironde, Charente, Charente-Inférieure, Deux-Sèvres, Vienne et Loire-Inférieure ; partiellement, 7 départements : Basses-Pyrénées, Hautes-Pyrénées, HauteGaronne, Indre-et-Loire, Cher, Sarthe et Vendée. Les stations douteuses appartiennent à l'Aveyron, au Tarn, à l'Ariège et aux Pyrénées-Orientales.

Altitude. - Limite inférieure à quelques mètres audessus du niveau de l'Océan; limite supérieure à 150 mètres dans la Loire-Inférieure, à 200 mètres à Chinon (Indre-et-Loire), 8300 à 900 mètres dans les Pyrénées.

Sol.- Semble préférer les sols siliceux légers et frais, les dunes; il vit sur les sols les plus ingrats, mais il réclame un climat doux, tempéré ; croit plutôt dans les stations du chêne pédonculé que dans celles du chêne rouvre.

Groissance, longévité. - Végétation lente, le fût tortueux n'a pas d'élevation et ce n'est qu'exceptionnellement et dans des circonstances favorables qu'on lui voit prendre une tige droite et assez élancée; il atteint rarement 20 mètres de haut; sa longévité se rapproche de celle des rouvre et pédonculé.

Caractères généraux. - Couvert léger; germination hypogée; tempérament robuste; enracinement pivotant et traçant; feuilles alternes caduques ; floraison unisexuée monoïque mai-juin; fructification : septembre de la mème année. Le chène tauzin possède au plus haut degré la faculté de drageonner; il se reproduit aussi par rejets de souches avec une grande vigueur.

Allures forestières. - 11 forme des taillis très fourrés et de longue durée. Ses racines traçantes et sa faculté de rejeter et de drageonner abondamment le font employer pour le boisement des dunes et des landes.

\section{XII. - CHÊNE OCCIDENTAL}

Qualités. - Usages. - Arbre à maturation bisannuelle, uniquement exploité pour l'écorce qui fournit une partie du liège du commerce; bois de feu. 
Importance. - Très faible par l'étendue occupée ; il a été longtemps confondu avec le chène-liège.

Distribution. - Essence exclusivement océanique, appartenant au littoral du golfe de Gascogne; disséminé dans un espace triangulaire, limité à l'ouest par le littoral, au nord-est par la Garonne ou peu s'en faut, et au sud-est par une ligne allant de Bayonne à Agen; il a été acclimaté jusqu'aux Charentes sur les parties voisines du littoral (Belle-Ile-en-Mer).

Departements occupés. - Quatre, savoir : partie des Basses-Pyrénées, des Landes, de la Gironde et la totalité du Lot-et-Garonne.

Altitude. - S'élève des bords de la mer à 183 mètres seulement.

Sol. - Essence des sols siliceux et argilo-siliceux, calcifuge; se plait dans les terrains de transport siliceux ou argilo-siliceux des Landes, aux expositions chaudes et abritées des vents.

Croissance, longévité. - Croissance assez lente; vers cent ans, ne mesure que $1^{\mathrm{n}}, 50$ à 2 mètres de circonférence; sa longévité peut être très grande.

Caractères généraux. - C'est l'arbre à liège du littoral de l'ouest et il présente les mêmes caractères généraux que le chène-liège.

Allures forestières. - Futaie très claire; disséminé plutot qu'en massif; il s'accommode particulièrement bien du mélange avec le pin maritime sous lequel il trouve protection et souffre peu de son couvert très léger.

\section{XIII. - CHÊNE-LIĖGE}

Qualités. - Usages. - Arbre de deuxième grandeur à maturation annuelle, uniquement exploité pour l'écorce qui fournit le liège du commerce; bois de feu.

Importance. - Très faible par l'étendue occupée. 
Distribution. - Essence exclusivement méditerranéenne; se trouvant en France à la limite septentrionale de son aire d'habitation; répandu dans toute la Corse et sur les bords de la Méditerranée, formant trois stations disjointes : $1^{\circ}$ dans les Maures et l'Esterel, de Grasse à Hyères ; $2^{\circ}$ dans le Gard et l'Hérault ; $3^{\circ}$ dans les PyrénéesOrientales.

Départements occupés. - Six, savoir : la Corse en totalité, les Alpes-Maritimes, le Var, le Gard, l'Hérault, et les Pyrénées-Orientales en partie.

Altitude. - S'élève de l'altitude 0, au bord de la mer à celle de 500 mètres dans les Pyrénées-Orientales, de 780 mètres dans les Maures (Var) et de 700 mètres en Corse.

Sol. - Sols granitiques, porphyriques, feldspathiques, schisteux; il parait redouter les sols. calcaires ; les sols compacts lui sont contraires.

Groissance, longévité. - Croissance assez rapide dans la jeunesse; il prend bientot une forme trapue; en France, il s'élève à 10-12 mètres de haut et peut atteindre 4-ว̆ mètres de tour; l'enlèvement du liège, s'il est bien fait ne lui est pas nuisible; il vit cent cinquante à deux cents ans et plus.

Caractères généraux. - Couvert léger; germination hypogée; tempérament robuste; enracinement pivotant ou traçant suivant le sol ; feuilles alternes, persistantes, mais feuillage grêle et rare; floraison unisexuée monoïque, avril-mai ; fructification et dissémination : commencement d'octobre à la fin de décembre de la mème année. La fructification se produit dès l'âge de quinze ans, mais elle n'est abondante et soutenue qu'à partir de trente ans. Le chêne-liège rejette vigoureusement; il drageonne quelque peu.

Allures forestières. - Le chêne-liège est essentiellement traité pour son écor'ce; on ne l'élève qu'en futaie claire, le plus souvent plantée.

A. Fron. - Sylviculture. 


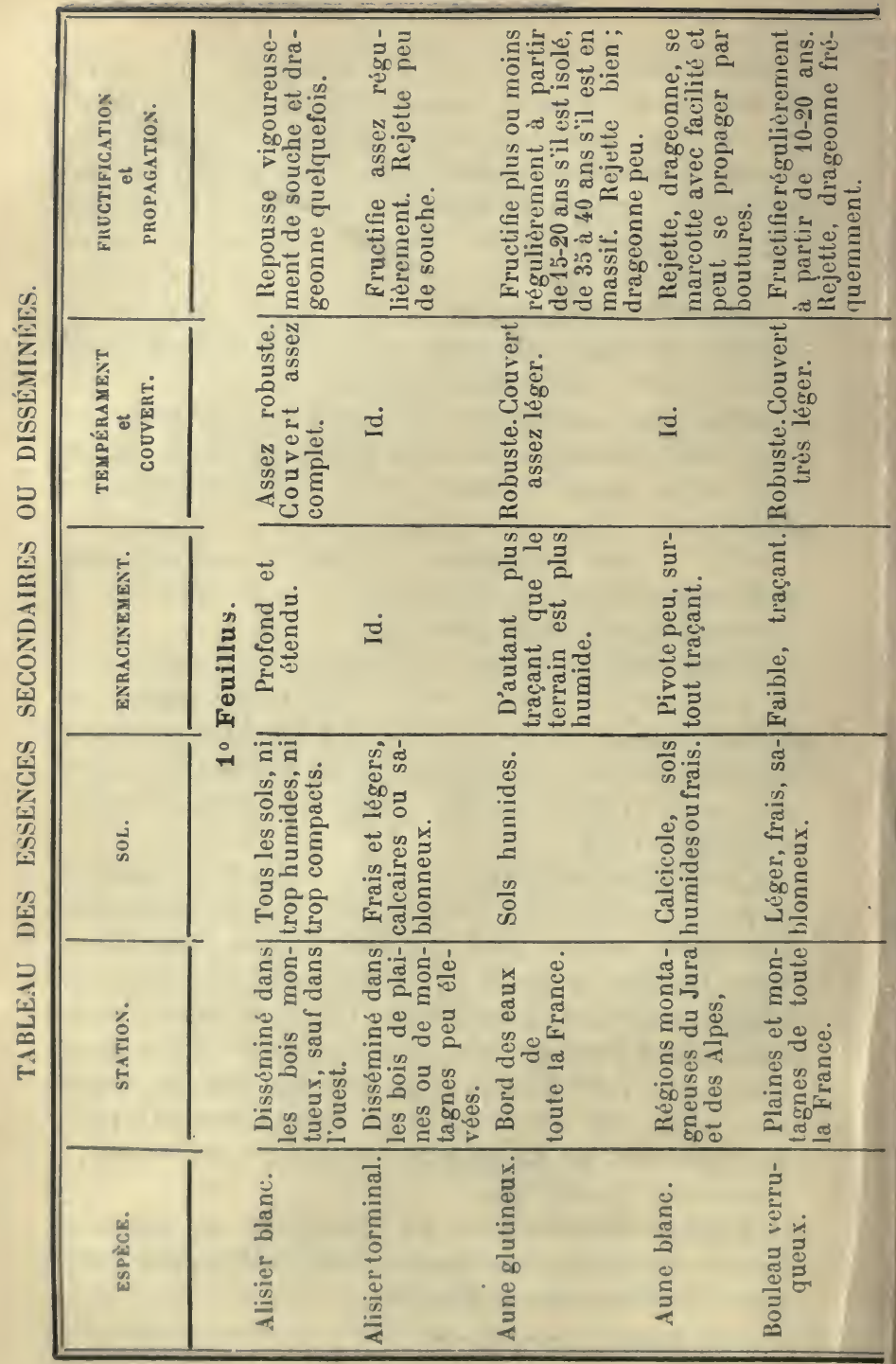




\begin{tabular}{|c|c|c|c|c|c|c|}
\hline 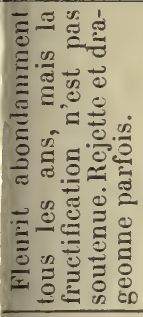 & 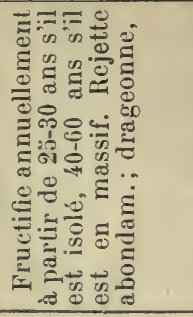 & 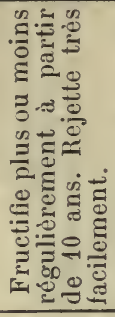 & 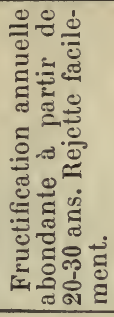 & ฮ่ & 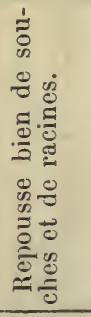 & 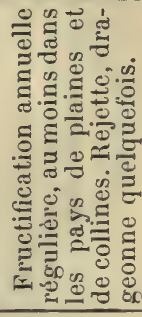 \\
\hline 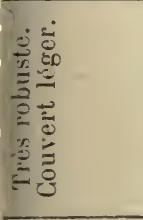 & 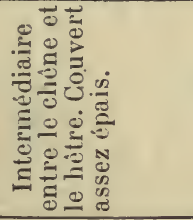 & 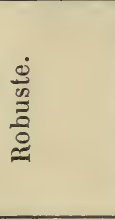 & 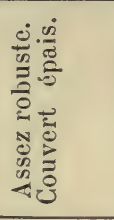 & $\check{\check{D}}$ & 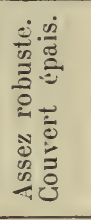 & 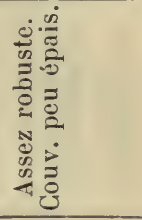 \\
\hline 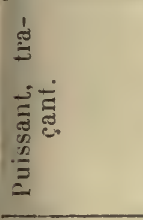 & 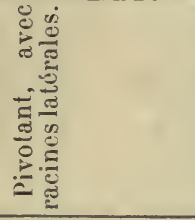 & 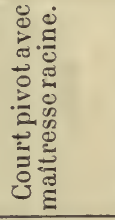 & 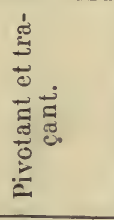 & $\stackrel{\Xi}{ }$ & 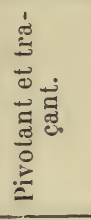 & I \\
\hline 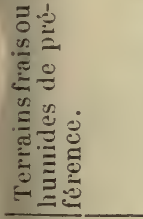 & 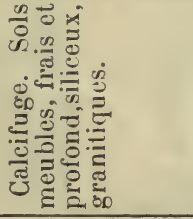 & 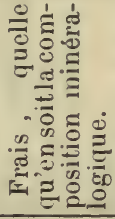 & 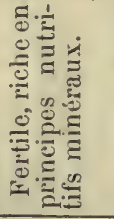 & 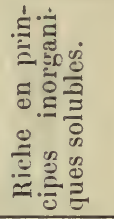 & 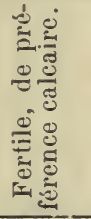 & 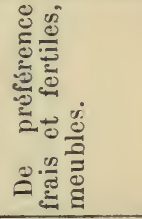 \\
\hline 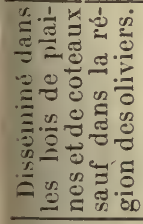 & 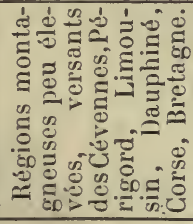 & 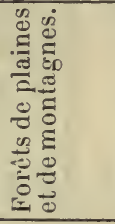 & 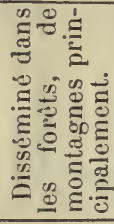 & 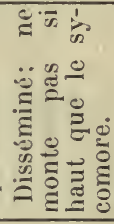 & 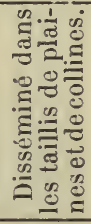 & 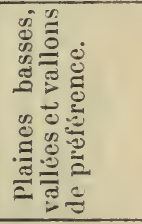 \\
\hline $\begin{array}{l}\stackrel{1}{\Xi} \\
\frac{0}{0} \cdot \frac{0}{\infty} \\
\frac{0}{0}\end{array}$ & 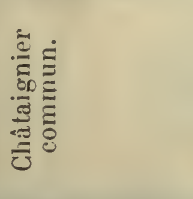 & 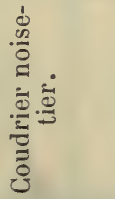 & 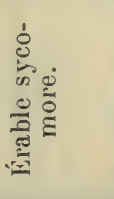 & 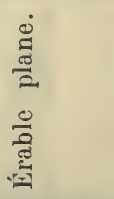 & 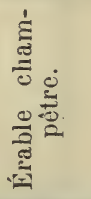 & 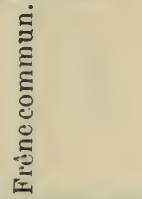 \\
\hline
\end{tabular}




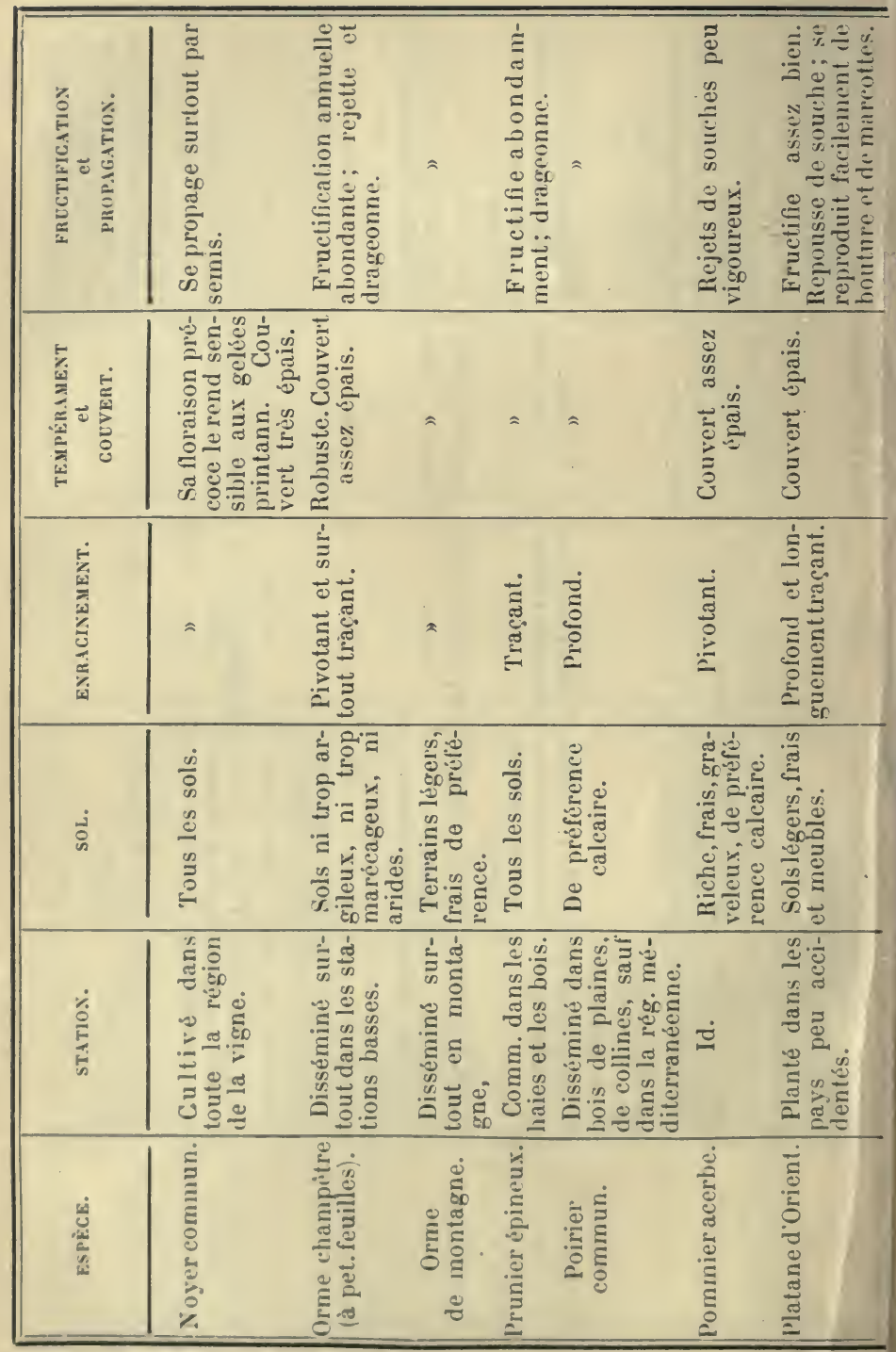




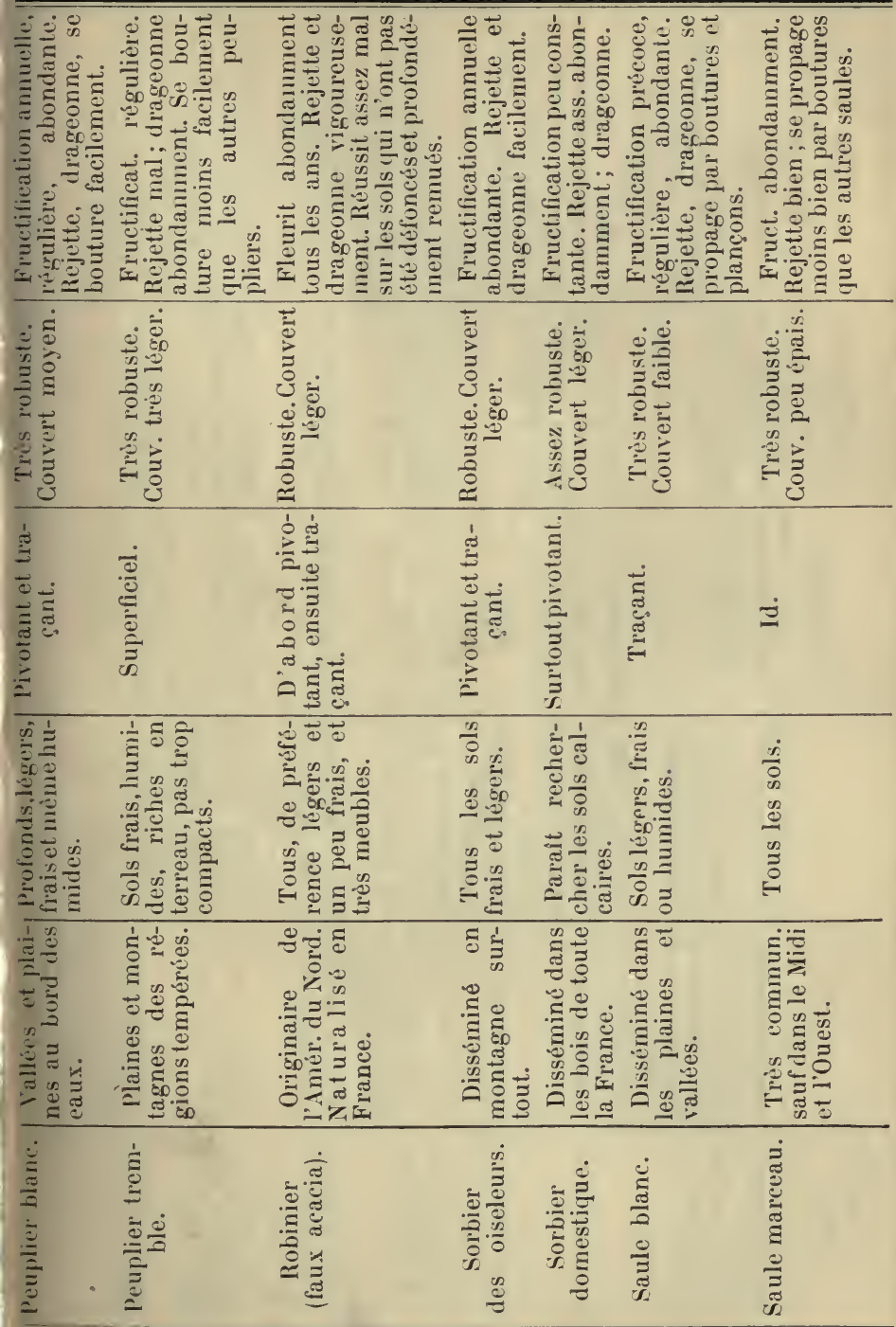




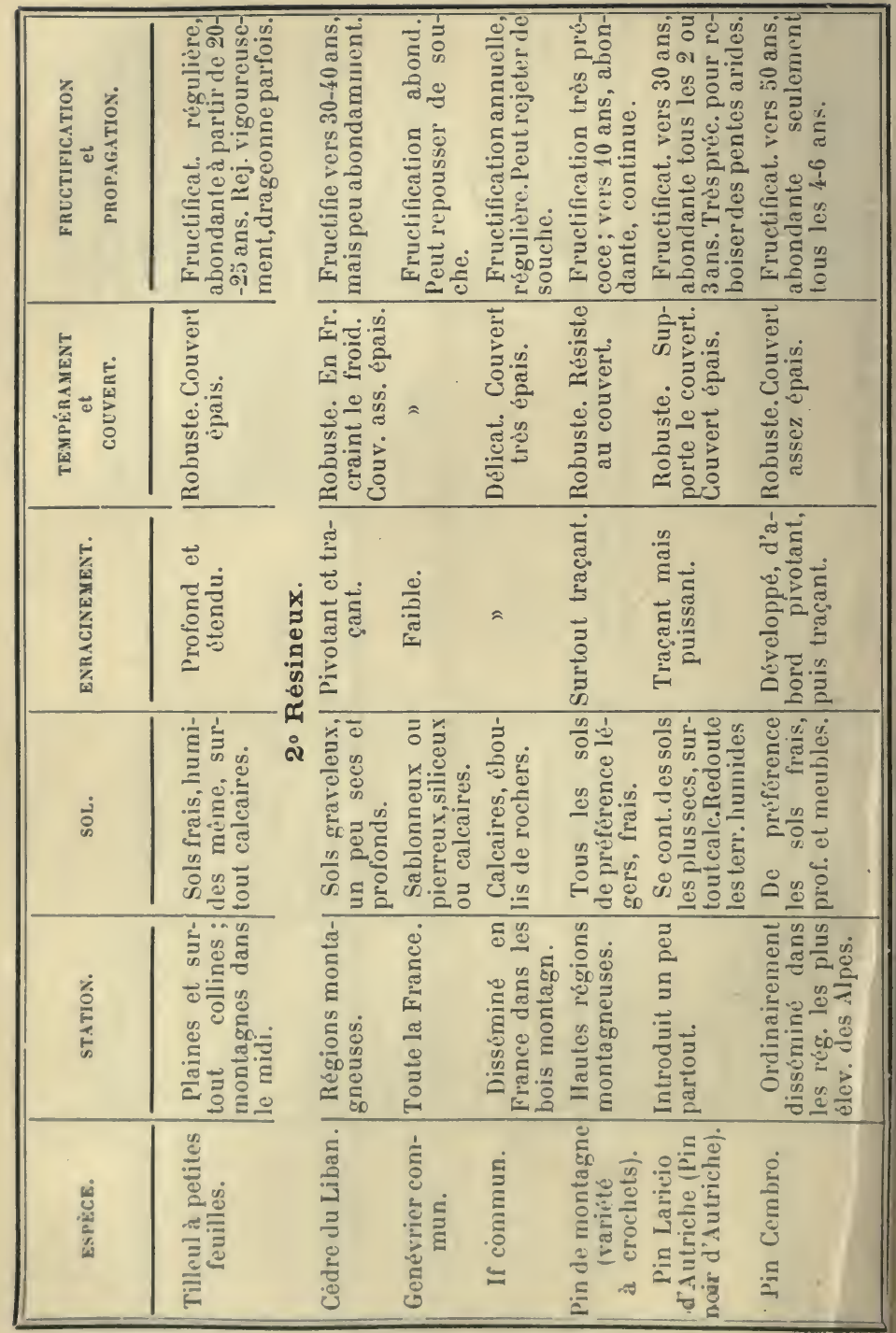




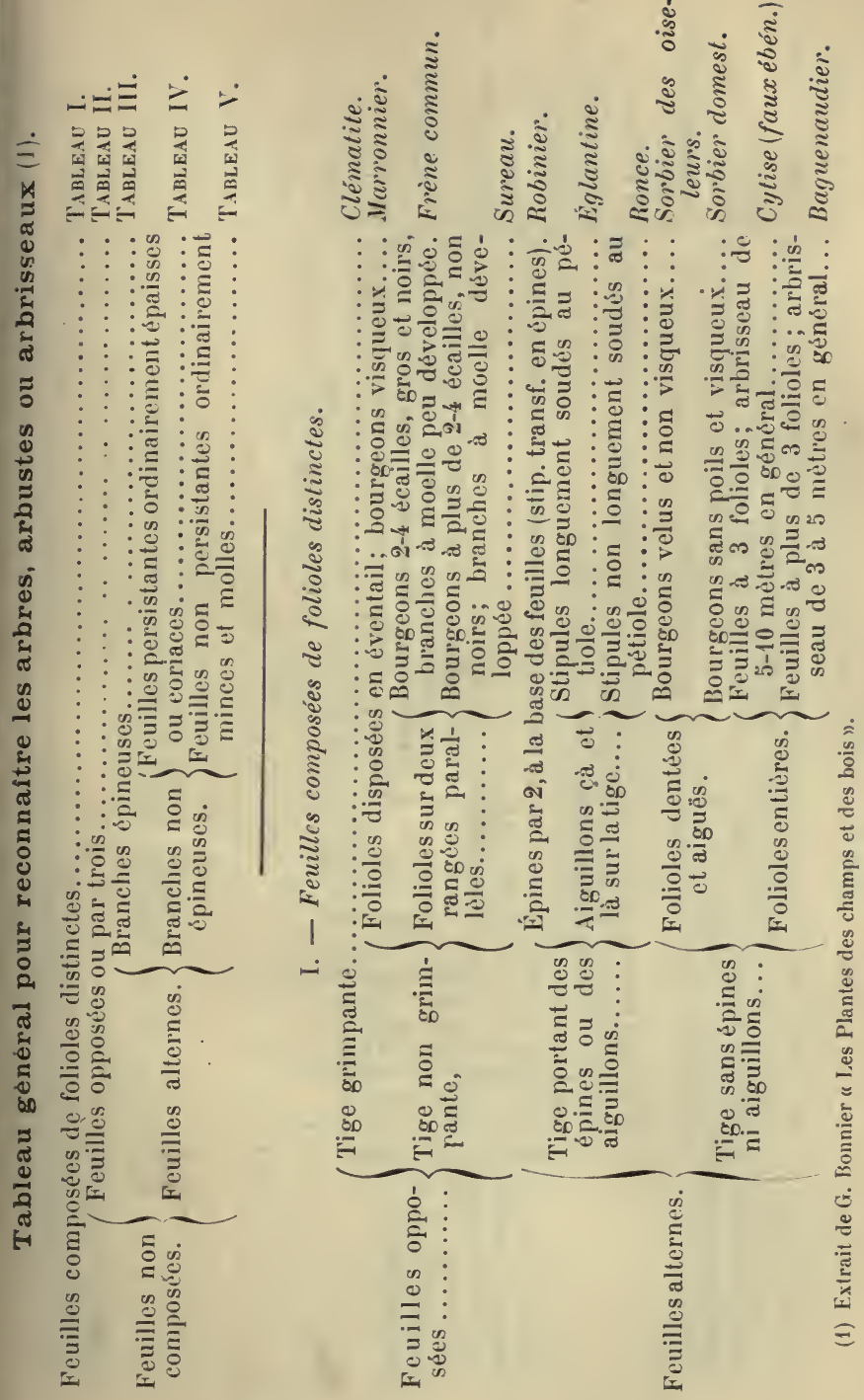




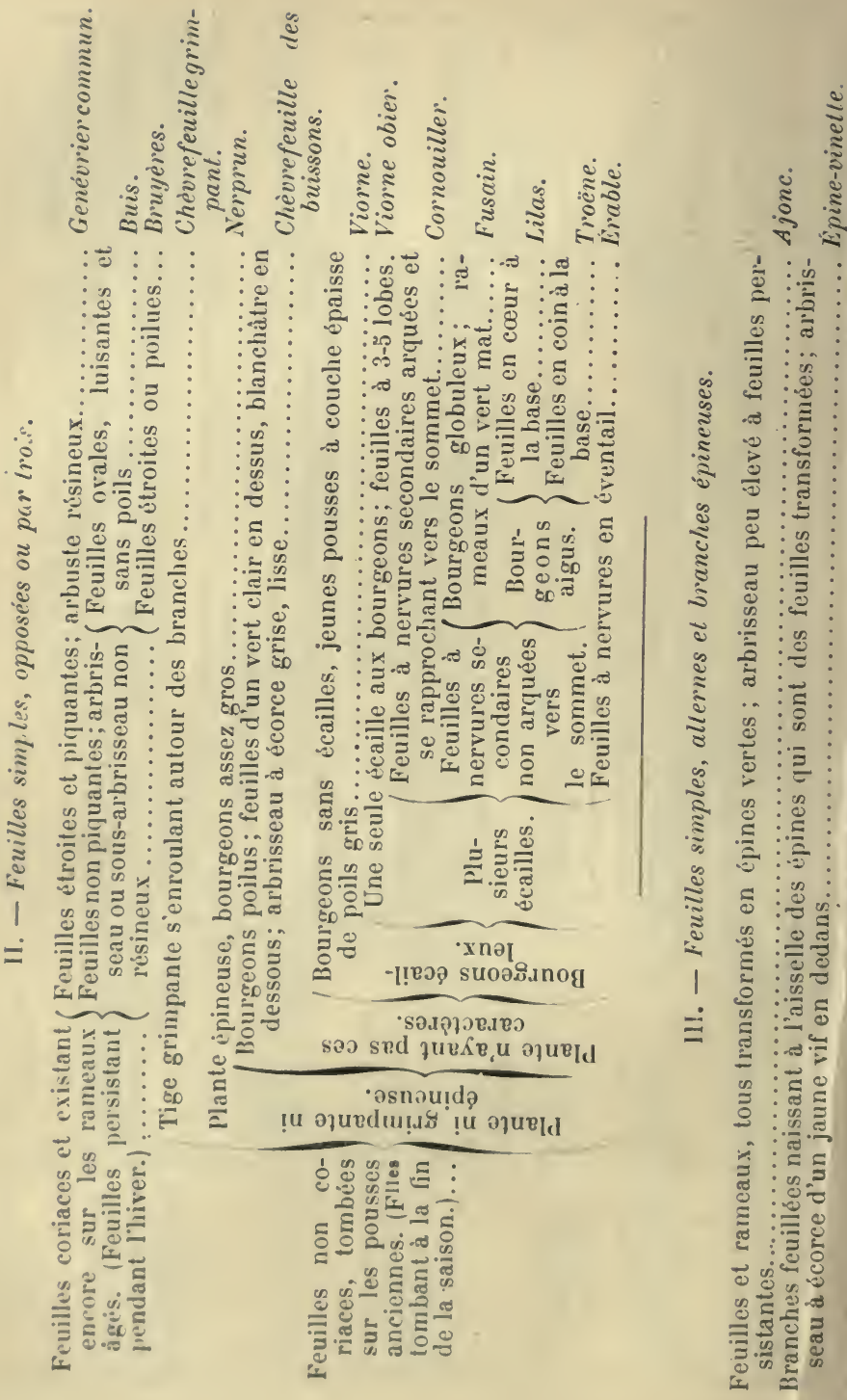




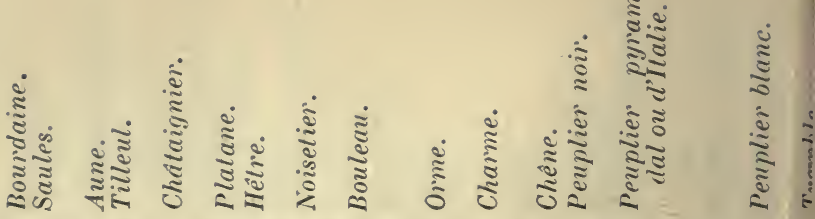

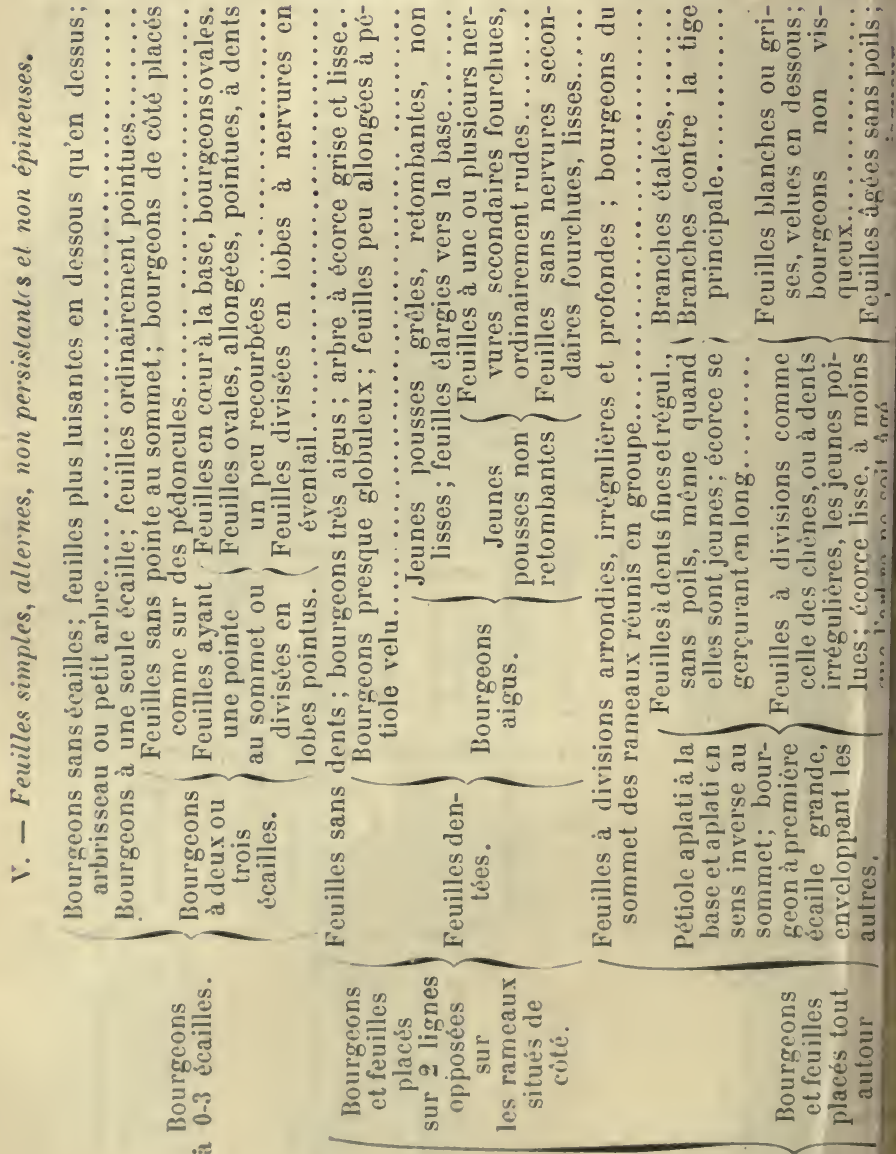




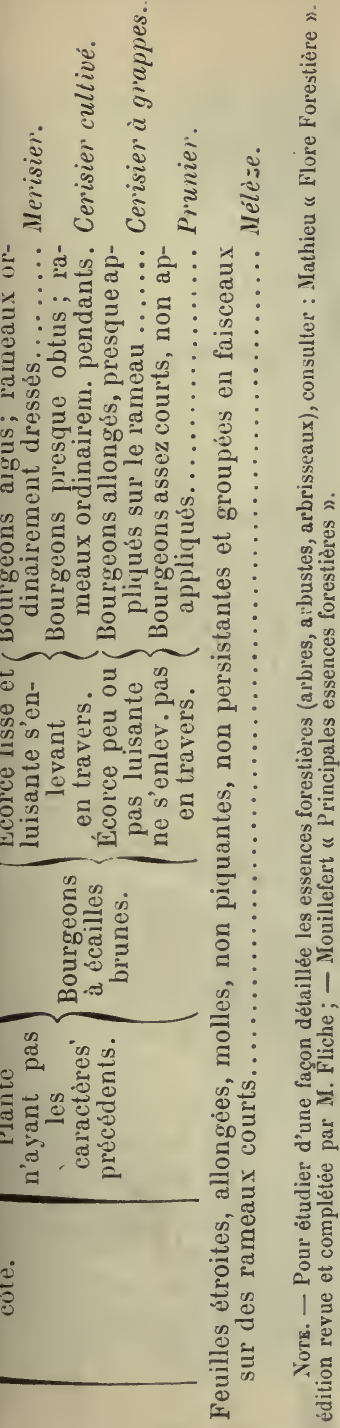




\section{QUATRIEME SECTION}

\section{Diverses formes de peuplements.}

\section{|GĖNÉRALITÉS}

Forét naturelle. - Action déla nature. - A l'époque reculée où les familles humaines vivaient à l'étal isolé, se nourrissant de fruits sauvages et des produits de la chasse et de la pèche, la surface de la France élait presque entièrement couverte de forêts. Peu à peu, les progrès de la civilisation, le développement de l'agriculture et de l'industrie eurent pour conséquence la disparition progressive de cet immense domaine forestier, qu'une longue suite de siècles a réduit à l'état où nous le trourons aujourd'hui.

Mais que l'homme abandonne au hasard les lerrains qu'il a conquis sur la forèt, qu'il veuille bien ne pas contrarier la nature et la laisser agir seule! il ne faudra pas un siècle pour voir se rétablir spontanément de proche en proche toule une végétation ligneuse, là où la forêt n'existe plus.

La nature poursuit lentement son cuvre, à l'aide des moyens qui lui sont propres; en montagne, les pâturages se laissent envahir par des semis d'épicéa provenant de la forèt voisine; en plaiıe, sur la friche ahandonnée, apparaît le coudrier qui, sur les sols ealcaires mème les plus mauvais, est un des premier's arbrisseaux, et un des plus actifs parmi ceux qu'utilise la nature ; avec lui peuvent se montrer l'épine noire, le saule marceau et d'au- 
tres morts-bois qui s'emparent peu à peu du sol (fig. 16). En même temps apparaissent suivant les sols et les stations, tilleuls, érables, merisiers, bouleaux, trembles et toutes autres essences secondaires qui viennent prendre rang au-dessus des morts-bois. Alors se montrent peu à peu les essences feuillues plus précieuses, le chêne d'abord, dont nous voyons tous les jours l'apparition spontanée sous les plantations résineuses quelque peu desserrées, puis le charme, puis tous les bois feuillus spontanés de la station.

Ainsi se constitue peu à peu par voie de semis la forèt naturelle; et si on réfléchit aux innombrables combinaisons des forces créatrices de la nature, on reconnait que les massifs de la forêt vierge doivent nécessairement avoir pour caractère une certaine variété d'aspects, d'après les exigences et la variété des essences.

Dans de tels massifs, les arbres arrivés à l'àge de la fructification fournissent des semences, qui tôt ou tard donnent naissance à des semis; ces semis végètent un certain temps mais, privés de lumière, ils disparaissent successivement sous le couvert, jusqu'à ce que les arbres qui leur ont donné naissance et qui forment l'étage supérieur, disparaissent à leur tour.

Le vieux massif se trouve remplacé sans que le sol ait cessé d'être complètement couvert par une nouvelle génération de plants qui s'élèvent en massif serré. La lutte pour l'espace s'engage entre les sujets voisins, au fur et à mesure qu'il leur faut plus de place, dans la terre pour développer leurs racines et dans l'air pour étendre leurs branches et participer à l'influence de la lumière ; bientot tous ne peuvent plus trouver place dans le massif, leur nombre diminue peu à peu et les brins les plus bfailes, surtout les brins dominés à tempérament robuste succombent dans la lutte, sèchent et disparaissent. Pendant la première jeunesse, cet'état de plus en plus serré n'est réellement préjudiciable qu'aux essences dominées 
par les plus vigoureuses, car le sol est de plus en plus couvert, car les brins se procurent un mutuel appui contre les intempéries, tout en se poussant à s'accroitre en hauteur. Les plus vigoureux qui dominent, forment et allongent leurs fûts en se débarrassant des branches basses. Cette première lutte pousse à l'élongation des fûts.

Mais plus tard, cette lutte pour l'existence entraine un ralentissement marqué dans l'accroissement des bois, car les tiges dominées, quoique privées de lumière, sont d'autant plus lentes à disparaître qu'elles ont plus de développement. Cependant la diminution du nombre de tiges continue suivant la mème loi, jusqu'à ce que ce nouveau massif cède lui-même place à un autre.

A l'état de nature, le sol est complètement couvert et la forèt se développe librement, au hasard, sous l'impulsion des forces naturelles, forces dont l'effet varie suivant les lieux, les époques et le caprice des accidents.

Toutefois, dans les phases successives de son existence, se trouve toujours le principe de conservation, dont l'effet est de maintenir et d'augmenter constamment la fertilité du sol et par suite d'assurer la perpétuité de la forêt.

Forêt cultivée. - Action de l'homme. - La forèt cultivée n'est pas ainsi abandonnée à elle-mème ; destinée à l'exploitation, elle doit être disposée de manière que la récolte puisse se faire régulièrement et l'homme exerce sur toutes les phases de son développement une action, bonne ou mauvaise, qui modifie le mode selon léquel les peuplements prennent naissance et les conditions même de leur croissance.

Sous cette action, les peuplements prennent une composition et une forme déterminée, correspondant au mode de traitement qu'on impose à la forèt.

En sylviculture, nous appelons: forme d'un peuplement, la constitution générale de ce peuplement au point de 
vue de l'origine, de l'àge et des conditions de croissance de ses différentes parties;

Régime, la méthode générale de culture à laquelle la forèt est soumise; le régime est caractérisé par la façon dont on opère la régénération ;

Mode de traitement, le procédé d'éducation et d'exploitation dans chaque régime; il correspond à une forme déterminée de peuplement.

Le nombre des formes de peuplement est très considérable, et nous derons nous contenter de définir les formes principales, qui satisfont dans une mesure suffisante, tant aux lois de la nature qu'aux conditions de l'exploitation.

Nous avons à distinguer trois régimes :

$1^{\circ}$ Régime de la futaie. - Une forèt est soumise au régime de la futaie, lorsque la régénération est effectuée par brins de semence; le peuplement est renouvelé par des sujets issus chacun directement d'une graine. On dit dans ce cas, que le peuplement est une futaie.

$2^{\circ}$ Régime du taillis. - Lne forèt est soumise au régime du taillis, lorsque la régénération a lieu par rejets ou drageons; en fait, le peuplement est plutot rajeuni par l'évolution des rejets et des drageons qui proviennent des souches récemment exploitées. On dit dans ce cas que le peuplement est un taillis.

$3^{\circ}$ Régime du taillis composé. - Une forêt est soumise au régime du taillis composé (ou taillis sous futaie), lorsque la régénération a lieu par rejets, drageons et brins de semence; un tel peuplement participe des deux états précédents et présente un caractère mixte; on dit dans ce cas indistinctement que le peuplement est un taillis composé ou un taillis sous futaie.

A chacun de ces régimes correspondent dirers modes de traitement, et par suite de formes de peuplements que nous allons examiner successivement, tant au point de vue de leur origine, qu'à celui de leur constitution, des 
dangers extérieurs qui les menacent, de leur production et de leur influence sur la fertilité de la station.

Nous avons adopté dans cette étude la division de M. Gayer, auquel nous avons emprunté une partie des renseignements qui suivent.

\section{I. - FUTAIE}

Définitions. - Un peuplement est dit régulier quand les différences d'âge de chacun des éléments qui le constituent ne dépassent généralement pas une dizaine d'années; ces différences d'âge cessent d'ètre appréciables dès la période de perchis, et donnent une futaie adulte dont les sujets ont en apparence le même âge.

Il en est autrement quand la période de régénération dure plus de vingt à trente ans sur la mème surface; les différences d'àge des éléments qui constituent le peuplement ne s'eflacent plus complètement, et restent encore appréciables lorsque la futaie est parvenue à l'état adulte; le peuplement ne présente plus une apparence absolument régulière et il est dit irrégulier.

Nous avons donc à distinguer: Ies peuplements réguliers et les peuplements irréguliers.

\section{1. - Peuplements réguliers.}

Premier type; Futaie régulière obtenue par coupe unique.

$1^{0}$ Or'igine et caractères. - Sous ce premier mode de traitement, la création du peuplement se fait par voie de semis ou de plantation directe sur une surface nue, el exceptionnellement par ensemencement latéral des portegraines voisins. Dans de bonnes conditions, la croissance peut être vigoureuse dès le début; néanmoins, l'état de fourré ne se forme généralement qu'au bout d'un certain nombre d'années qui dépend du mode de repeuplement 
et des essences employées, ainsi que de la fertilité du sol et des influences météoriques.

Souvent, pendant cette période de début, notamment dans les coupes blanches (1) effectuées à côté d'autres peuplements fertiles, la surface du terrain reboisé est envahie spontanément par une foule encombrante de morts-bois à graines ailées ou légères qui proviennent des sujets voisins. Ces semis naturels sont plus ou moins envahissants, suivant les stations, suivant la première croissance des essences plantées; parfois ils forment en peu de temps un couvert qui peut être utile pour le développement ultérieur du jeune peuplement artificiel.

Dès la formation du fourré, l'accroissement en hauteur devient rapide, les branches et le feuillage se joignent, et forment dès lors sur le sol un couvert ininterrompu et très bas, qui s’élève peu à peu pendant la période de gaulis sous l'influence de l'élagage naturel (2). A ce moment commence l'élimination active du peuplement accessoire, et la prépondérance du peuplement principal; c'est la période du perchis, qui dure plus ou moins longtemps suivant l'essence et la station, et qui correspond à la formation complète du massif et à un notable accroissement en hauteur; le couvert ininterrompu s'élève de plus en plus en vieillissant et finit par laisser entre lui et le sol un espace considérable qu'occupent seuls les fûts dégarnis de feuillage, et déjà desserrés. C'est généralement à la fin de cette période de perchis que correspond le maximum de l'énergie du peuplement.

(1) Coupes blanches, coupes à blanc étoc, coupes en bloc, c'est-à-dire surfaces sur lesquelles on exploite en bloc tous les arbres qui garnissent le sol.

(2) Un grand nombre de propriétaires ont l'habitude, détestable à notre avis, de couper rez tronc toules les branches basses dans un peuplement de ce genre, avant qu'clles ne disparaissent naturellement, spécialement dans les plantations de pins. Cetle opération n'est pas avantageuse pécuniairement, la valeur du bois coupé ne payant mème pas les frais de main d'œu vre. Elle est déplorable au point de vue cultural; elle est mauvaise au point de vue de la qualité future du bois. Considérons cette habitude commie un sacrifice important qu'on fait au coup d'wil et a la commodité du chasseur. 
Alors commence l'état de futaie à partir duquel les arbres s'espacent de plus en 'plus; le couvert diminue progressivement d'épaisseur suivant l'essence et les qualités de la station.

$2^{\circ}$ Danger's extérieurs. - Dans un grand nombre de circonstances le peuplement, ainsi créé artificiellement en terrain nu, est exposé à des dangers extérieurs dont l'influence varie suivant la résistance de l'essence et les conditions de la station. Pendant toute la période de jeunesse, il peut y avoir à craindre :

$a$. La gelee, qui peut frapper les jeunes plants, parce qu'ils sont directement exposés à son influence, sans aucun abri; un certain nombre d'essences trop sensibles ne résisteront jamais dans ces conditions.

b. La sécheresse de l'été, due à l'action solaire qui frappe directement le sol sans abri et les jeunes plants.

c. Les variations extrêmes de température qui ne sont pas atténuées en terrain découvert, et sont défavorables à une bonne végétation forestière.

d. L'envahissemenl de la végètation herbacée, fa vorisée par un découvert total, qui épuise inutilement le terrain, tend à étouffer les jeunes plants, et trop souvent attire le bétail que le propriétaire ne prend pas le soin d'écarter; le pâturage toléré dans de jeunes repeuplements est la ruine certaine de l'état boisé.

e. L'invasion des champignons et surtout des insectes, plus dangereuse dans les peuplements réguliers que dans les autres.

$3^{\circ}$ Production. - La fulaie régulière, en raison même de sa constitution, tend à produire des sujets de même dimensíon, qui n'ont disposé chacun pour se développer que du minimum d'espace nécessaire, tant dans le sol pour le développement des racines, que dans l'atmosphère pour le développement des cimes; elle ne tend pas à favoriser le développement de sujets d'élite ayant des dimensions et des qualités spéciales. 
$4^{0}$ Influence sur la fertilité de la station. - Nous distinguerons trois phases:

$1^{\text {re }}$ phase. - Le sol est complètement mis à découvert par l'exploitation à blanc; par suite, il est exposé directement à toutes les intempéries, il s'appauvrit, et cet effet est plus ou moins néfaste pour la génération suivante, selon la qualité initiale de la station.

$2^{\text {e }}$ phase. - Le couvert se rétablit dès l'état de fourré; il commence lentement à exercer son action conservatrice des qualités du sol et à rendre à celui-ci les éléments qu'il a perdus pendant la mise à découvert; mais il n'acquiert réellement cette aptitude qu'à partir de la période de perchis; c'est à ce moment que règne dans la futaie régulière le massif le plus parfait, et cette situation se traduit par un redoublement de l'activité de la végétion; mais on le roit, pour que le sol récupère ses qualités, il faut un bon massif, maintenu aussi longtemps que possible, condition que remplissent bien les essences d'ombre, mais non pas, en général, les essences de lumière.

$3^{e}$ phase. - Le massif tend à s'éclaircir dès la période de futaie, le couvert diminue, et cela d'autant plus que le feuillage s'élève, surmontant un espace libre qu'occupe seulement le fût des arbres. Par suite, plus le peuplement est ancien, plus le vent peut s'introduire dans le voisinage de son sol (surtout dans les situations exposées, sur les lisières, dans les parcelles isolées, etc.).L'air humide du sous-bois est entrainé, l'évaporation s'active, la couche de feuilles et d'humus se dessìche et est emportér par le vent, et en fin de compte le sol se durcit, s'amaigrit et se couvre de mauvaises herbes; aussi, dans la futaie régulière, des périodes de révolution très longues entravent-elles souvent l'action conservatrice des forèts sur les qualités du sol.

Les inconvénients de la méthode par coupe unique, s'accentuent en raison directe del'élendue du peuplement, et c'est surtout dans les grandes coupes qu'ils se font 
sentir; ils s'atténuent quand il s'agit de petits peuplements et de bouquets de bois environnés de peuplements d'un caractère différent.

Deuxième type. - Futaie régulière obtenue par coupes successives, avec réserves d'ensemencement et d'abri.

$1^{\circ}$ Origine et caractères. - Dans une vieille futaie, parvenue à l'époque de l'exploitation, on peut, au lieu d'enlever en bloc tout le matériel sur pied dans une parcelle donnée, le réaliser par fractions en dirigeant les exploitations de telle sorte que le peuplement nouveau résultera: soit de l'ensemencement naturel fourni par des porte- graines, répartis uniformément en plus ou moins grand nombre sur le terrain à repeupler; soit du repeuplement artificiel effectué sous l'abri d'arbres réservés lors de l'exploitation du peuplement antérieur.

Dans ces deux cas, le peuplement en voie de formation ainsi que le sol sur lequel il s'installe sont partiellement couverts et par suite protégés par les arbres réservés. L'enlèvement total de cet abri n'a lieu que quelques années plus tard, quand la reprise du peuplement est assurée.

a. On opère par régénération naturelle; c'est le cas le plus général.

Nous avons vu précédemment que la fructification des arbres dont la cime est isolée est plus abondante que celle des arbres en massif; qu'en outre, les influences les plus favorables à la germination d'une semence, ainsi qu'au développement des jeunes plantules issues de la graine sont dues à l'action des trois éléments : air calme, chaleur et humidité, agissant simultanément; quant à la lumière, son action est peu nécessaire pour la germination et pour le premier développement du tout jeune plant; ce n'est qu'un peu plus tard, quand les racines de ce jeune plant ont percé la terre pour s'y fixer et que ses feuilles commencent à fonctionner, qu'il réclame plus ou moins 
vite, suivant les essences et les stations, l'action de la lumière; pour ètre bienfaisante à ce moment, cette action doit lui être dispensée avec mesure.

Cet ensemble de conditions favorables peut ètre réalisé dans une mesure aussi variable que l'exigent l'essence et la station, par une série de coupes successives.

Les premières exploitations faites dans le vieux peuplement ont pour but d'isoler les cimes des portegraines, et d'obtenir le semis ; les suivantes ont pour but de faire disparaitre l'abri dès qu'il devient inutile au recru.

Un réensemencement naturel complet n'est généralement pas obtenu de suite dès la première année ; on doit alors attendre le produit d'une nouvelle année fertile, ou combler artificiellement les vides par semis ou plantation. Malgré cela, les différences d'âge qui en résultent ne dépassent généralement pas une dizaine d'années.

Les graines, spécialement les semences lourdes, et par suite leurs jeunes plants, ont une tendance à se répartir par bouquets à proximité des porte-graines; l'abondance des semis naturels (1) est généralement telle que les jeunes plants voisins forment massif dès leur première jeunesse et atteignent rapidement la période de fourré; les difrérences d'âge s'accusent de moins en moins au fur et à mesure que les bouquets se rejoignent; dès le début de la période de gaulis, le massif est continu et le couvert presque sans lacunes; l'élimination des tiges accessoires et dominées s'effectue dès lors d'une façon très active, en raison de la densité considérable du massif; la tendance à l'uniformité, aidée des soins de la culture, achève de donner au peuplement les caraclères de la futaie régulière.

b. On opère par semis artificiel sur toute l'étendue du terrain à repeupler et sous l'abri des arbres réservés.

Ce cas rentre immédiatement dans le précédent, si les

(1) Ce ne sout pas toujours les semis les plus serrés qui donnent les meilleurs résullats. 
arbres réservés sont suffisamment nombreux au moment où l'on effectue le semis.

Mais le semis peut être fait sous un couvert insuffisant ou même complètement nul, en raison du petit nombre l'arbres formant abri et de la hauteur de leurs cimes; le jeune peuplement qui provient de ce semis est alors exposé aux mêmes dangers et aux mèmes inconvénients que le repeuplement d'une coupe blanche.

$2^{\circ}$ Danger's extérieur's. - Les conditions sont ici bien plus favorables que dans le cas précédent. L'abri formé par les arbres réservés comme porte-graines atténue les effets des écarts brusques de température et ceux de la gelée sur les jeunes plants; il les préserve de la sécheresse et des variations dangereuses de température en conservant au sol un certain couvert et par suite une certaine humidité ; il s'oppose, jusqu'à un certain point, au développement de la végétation herbacée et à l'envahissement des morts-bois.

$3^{\circ}$ Production. - Les conditions de production sont à peu de choses près identiques à celles des futaies régulières de premier type.

$4^{\circ}$ Influence sur la fertilité de la station. - Dans la futaie traitée par coupes successives, le repeuplement se fait sur une surface toujours protégée par un couvert plus ou moins interrompu; le jeune plant naît et se développe à l'abri des porte-graines et le sol reste toujours partiellement couvert ; lorsque ces réserves d'abri viennent à disparaître, le repeuplement est à l'état de fourré, et sa densité étant considérable, la protection du sol est suffisamment assurée. Les peuplements, ainsi obtenus, sont incontestablement préférables à ceux qui sont créés en terrain dénudé, au point de vue de la conservation des qualités du sol.

Toutefois les conditions dans lesquelles s'opère cette régénération ne suffisent pas toujours à prévenir l'influence pernicieuse de la dénudation du sol. ll arrive 
parfois, qu'après les exploitations qui ont pour but d'isoler les cimes des porte-graines, la fructification se fasse longtemps attendre, ou que le recru soit détruit; le sol alor's est envahi de plus en plus par les mauvaises herbes; l'abri des porte-graines étant en somme incomplet, le vent et le soleil font leur œuvre. Dans ce cas, quelques bouquets de semis préexistant, mème isolés, peuvent devenir fort utiles en maintenant entre eux des couches d'air relativement tranquilles.

\section{2. - Peuplements irréguliers.}

Troisième type. - Futaie traitée par coupes successives ayant un caractère jardinatoire.

$1^{0}$ Origines et caractères. - Si dans un peuplement de futaie, les éléments ont des âges variant entre eux, non plus dans les limites de zéro à dix ans, mais dans les limites de zéro à vingt ou de zéro à quarante ans autrement dil, si la pèriode de régénération d'une surface donnée dure de vingt à quarante ans, les diverses parties de ce peuplement ne se raccordent plus aussi facilement; les différences d'âge restent apparentes, non seulement pendant la période de jeunesse, mais pendant Iongtemps. Le peuplement est composé de groupes et bouquets dont l'ensemble forme un peuplement irrégulier oû le massif des couronnes des arbres ne se tient plus à un niveau uniforme; il offre des ondulations correspondant à l'âge des divers bouquets sans pour cela présenter de lacunes; enfin ce n'est qu'à un âge avancé que le niveau s'établit plus ou moins, en même temps que le couvert se desserre de lui-mème.

Sous un tel massif, parvenu à l'àge d'exploitation, peuvent déjà se trouver des semis, disséminés par taches, partout où le couvert s'est accidentellement interrompu.

Lor's de la régénération, pour maintenir l'état irrégulier, on est conduit à obtenir celte régénération par placeaux, 
en coupant des arbres de place en place, spécialement là où se trouvent déjà des semis; sous les trouées ainsi faites dans le couvert, viennent s'installer de nouveaux semis qui constituent un groupe ou bouquet de semis; dans les exploitations suivantes, on a soin de venir élargir peu à peu ces places. En opérant ainsi, on obtient pendant la période de régénération, un nouveau peuplement qui se compose de nombreux bouquets d'âges diflérents, entre lesquels se trouvent des cordons irréguliers du massif originaire.

A mesure que les jeunes bouquets se développent et qu'il s'en forme de nouveaux dans les parties non encore ensemencées, le peuplement ancien disparaît, au fur et à mesure des exploitations. Supposons la période de régénération fixée à trente ans; les derniers arbres de l'ancien peuplement tombent à la fin de cette période, et lis divers bouquets, âgés de un à trente ans sont à ce moment presque partout formés en massif ; le peuplement ainsi créé, est prèt alors à passer par les périodes du perchis et de la futaie, pour arriver finalement à sa maturité.

A ce moment on recommence une nouvelle période de régénération; l'exploitation se porte sur les bouquets les plus avancés en âge, et les coupes successives se continuent comme nous venons de l'indiquer.

Les tendances de la sylviculture moderne se portent vers des peuplements ainsi constitués.

La durée de la période de régénération influe sur la forme du peuplement; elle a pour conséquence de provoquer des différences d'âge dans le jeune peuplement, différences d'autant plus appréciables que cetle période de régénération est plus longue. Avec une période de régénération courte, inférieure à vingt ans par exemple, on se rapproche du peuplement régulier de deuxième type (futaie régulière obtenue par coupes successives); avec une période de régénération longue, supérieure à 
quarante ans, par exemple, on tend vers la futaie jardinée.

Le concours du repeuplement artificiel n'est pas exclu de cette méthode ; dans certaines circonstances même, il peut être très utile et il intervient alors sous forme de bouquets artificiellement plantés, là où devraient exister des semis naturels. Cette plantation doit être effectuée assez longtemps avant les premières coupes de régénération, en plusieurs opérations plus ou moins espacées; remarquons quon peut agir ainsi artificiellement pour introduire par places, des essences qui font défaut ou ne sont guère représentées dans le peuplement originaire, et que la manière de conduire les coupes au-dessus et autour de chaque bouquet laisse une grande latitude à cet égard.

2 o Dangers extérieur's. - Cetle forme de peuplement tend à se rapprocher beaucoup de la forèt naturelle; les jeunes bouquets de régénération abrités par le peuplement qui les domine ou les entoure, se trouvent assez longtemps protégés contre la gelée et la sécheresse; la même cause agit pour les abriter contre les variations extrêmes de température et, dans une certaine mesure, contre l'invasion de la végétation herbacée. Enfin l'action des vents sur les arbres porte-graines est moins à redouter en raison de leur isolement progressif et de l'abri que leur donnent les cordons du vieux peuplement conservé au moins pendant un certain temps autour des bouquets.

$3^{\circ}$ Production totale. - Les ondulations qu'offre le niveau des cimes de ce peuplement irrégulier permettent aux sujets'les plus vigoureux de prendre peu à peu le dessus pendant la période du perchis et de la futaie; dans les bouquets les plus anciens, formés en massifs quasil'éguliers, les sujets d'élite s'espacent avec l'âge, en mème temps que le feuillage des cimes s'élève; le couvert diminue; il permet l'installation des premiers semis 
et par suite le commencement des coupes de régénération. Plus on approche de la fin de la période de régénération, dit M. Gayer, plus les sujets aptes à prendre un grand développement s'espacent et sont mis en lumière parmi les bouquets de repeupleument. Cette transition graduelle de l'état de massif à l'état dégagé permet aux arbres de s'adapter facilement aux nourelles conditions d'existence qui leur sont faites; l'augmentation de l'afflux de lumière et la bonne conservation de l'humidité du sol font que le peuplement conserve sa vitalité jusqu'à un âge avancé.

Le traitement par coupes jardinatoires permet de favoriser le développement de sujets d'élite, et de mettre à profit leur valeur individuelle.

$4^{\circ}$ Influence sur la tertilité de la station. - L'action conservatrice des qualités du sol est très nette, et il n'est pas besoin d'iusister; elle est due simultanément aux causes qui agissent dans les futaies du second type, et à ce fait qu'on n'opère la régénération que sur de petites surfaces, autour desquelles persistent les restes du vieux peuplement.

Quatrieme type. - Futaie jardinée.

$1^{\circ}$ Caractère et origine. - Le jardinage est une méthode de traitement en futaie qui consiste en principe à enlever çà et là chaque année et par places suffisamment éloignées les unes des autres, des arbres à réaliser au fur et à mesure qu'ils arrivent à une dimension déterminée; et accessoirement, à faire tomber par points isolés ceux qui sont viciés, ou d'une végétation mauvaise.

Cette manière de procéder provoque ainsi indéfiniment ét sans règle la régénération naturelle du peuplement par points ou par places disséminées sur toute la surface de la forêt.

En théorie, le peuplement doit être constitué de telle manière que tous les degrés d'âge, depuis le jeune brin de l'année jusqu'à l'arbre exploitable y soient représentés, 
soit isolés, soit surtout à l'état groupé et cela d'une manière continue (fig. 17).

La futaie jardinée est, de toutes les formes, celle qui comprend les differences d'âge les plus élevées.

La futaie jardinée, ainsi comprise, se rapproche beaucoup de la forèt naturelle dont elle présente les principaux caractères; lorsqu'un gros arbre vient à tomber, la trouée produite permet à l'air et à la lumière de pénétrer jusqu'à la place qu'il occupait sur le sol en quantité suffisante pour y constituer des conditions favorables à l'installation d'un semis; si des semences aptes à germer ou de jeunes semis s'y trouvent, l'arbre tombé est bientôt remplacé par de jeunes plants qui garnissent la trouée; mais, s'il l'on n'a fait tomber qu'un arbre, fût-il gros, la trouée se referme assez vite et les jeunes plants qui occupent la place de l'arbre enlevése trouvent gènés dans leur croissance; s'il s'agit d'essences de lumière, ils disparaissent rapidement; s'il s'agit d'essences d'ombre, ils se maintiennent, presque sans se développer, attendant pour repartir la disparition d'arbres voisins; leur végétation repart alor's franchement jusqu'à ce qu'ils soient de nouveau recouverts par le peuplementsupérieur dont les branches viennent à nouveau fermer la trouée, et ainsi de suite; pendant un certain temps, tout au moins, ils vivent par à coups, ainsi que d'autres semis, venus plus tard dans les mèmes conditions qu'eux.

Si, au lieu de considérer une petite trouée, nous supposons qu'on agisse par grandes trouées, en prenant plus d'un arbre en chaque point, le couvert se referme plus difficilement; les essences de lumière nées en bouquets dans la trouée, peuvent subsister jusqu'à ce que l'enlèvement d'un bouquet voisin vienne les sauver délinitivement. Les groupes et bouquets croissent à l'état de massif; les liges se dépouillent et se développent en hauteur, et les sujets particulièroment bien doués, qui se dégagent pour former des arbres de fortes dimensions, 
conservent la faculté de développer leur couronne

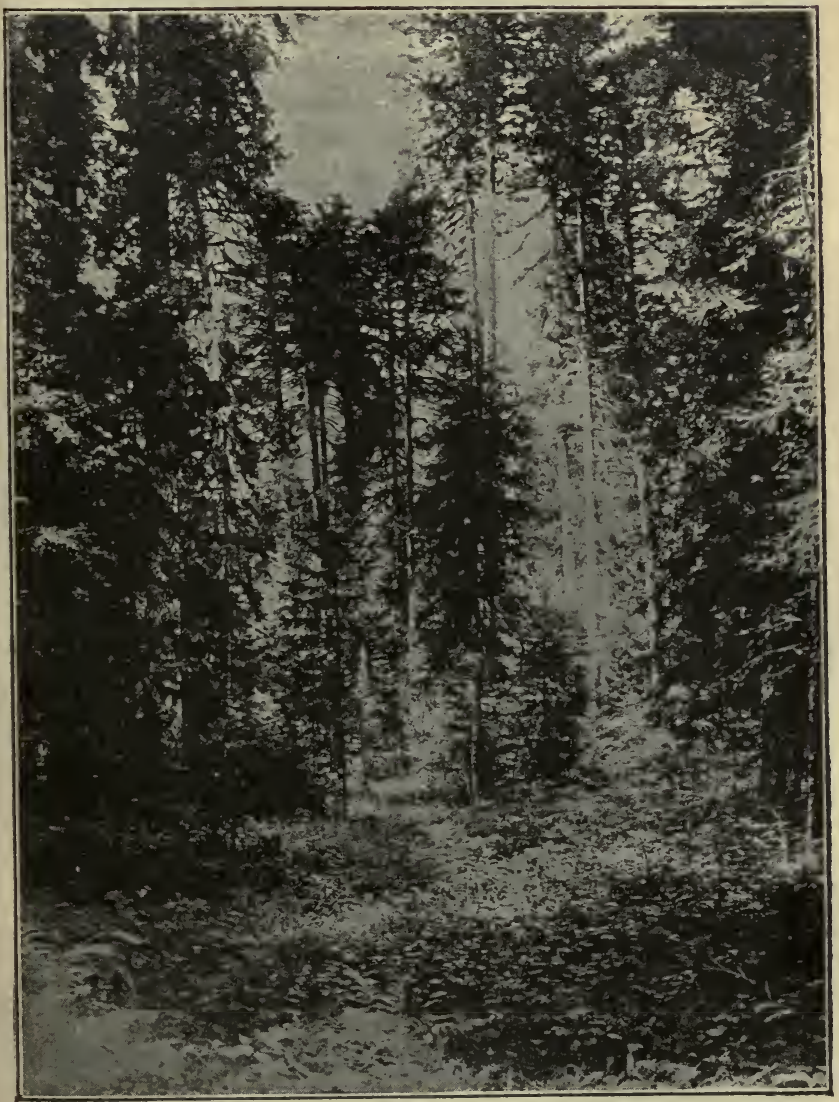

Fig. 17. - Une futaie jardinée dans le Jura.

mieux qu'ils ne l'auraient fait dans une futaie régulière. Ainsi, dans la conduite d'une futaie jardinée, le fores- 
tier imite la nature, mais avec cetle différence qu'au lieu d'agir au hasard, il cherche à maintenir ou à faire naître au besoin toutes les circonstances propres, d'une part, à assurer la régénération de la forèt au moment voulu, el d'autre part, à améliorer la croissance. A part cette influence, la régénération est abandonnée à la nature, et c'est principalement elle qui donne naissance à la futaie jardinée et qui la perpétue.

Toulefois, si la régénération naturelle est incomplète, on peut, dans une certaine mesure, lui venir en aide par des semis ou des plantations fails en temps voulu.

Les forêts jardinées, dit M. Gayer présentent généralement une grande diversité de formes, et, en fait, on constate loujours une prédominance marquée de certaines classes d'âge sur les autres. Dans tel peuplement, les arbres exploilables et les jeunes plants seront en majorité, tandis que les âges intermédiaires seront très peu représentés; tel autre présentera plutôt le caractère de perches, parsemées de groupes de vieux arbres; cela dépend du but que l'exploitation et l'administration de la forèt auront eu en vue, de la régularité des périodes d'exploitation, des intervalles qui les ont séparés, etc.

On tombe encore de nos jours, ajoute le même auteur, dans les plus étonnantes confusions au sujet de la dénomination de futaie jardinée; outre que l'on confond entre elles la futaie à coupes jardinatoires, la futaie sur taillis et la futaie jardinée, on désigne parfois sous celle dernière appellation les forêts soumises au brigandage, plutot qu'à l'exploitation et privées de tout traitement cultural que l'on rencontre dans certains pays de montagne et mème ailleurs; ce sont là des forèts, non pas jardinées mais dérastées, qui excluent toute idée de culture intelligente.

$2^{\circ}$ Dangers extérieurs. - Par l'effet de son couvert continu, la futaie jardinée tend à empêcher les effets désastreux de la gelée et de la sécheresse; elle s'oppose à 
l'envahissement de la végétation herbacée; par le caractère de constance et d'uniformité inhérent à tous les phénomènes de son existence, elle modère dans une large mesure les écarts extrèmes de température et d'humidité de l'atmosphère et du sol. Enfin, mieux que les peuplements réguliers, elle résiste aux effets désastreux des ouragans et des invasions d'insectes. C'est donc justement aux causes de destruction les plus actives que la futaie jardinée oppose la plus grande force de résistance.

$3^{\circ}$ Production totale. - La qualité des produits peut ètre inférieure dans les futaies jardinées où les conditions très diverses de croissance d'un mème arbre pendant son existence, occasionnent des accroissements variables et irréguliers; mais, en revanche, les sujets d'élite y sont mieux dégagés que dans les peuplements réguliers, par suite, particulièrement développés, et la forêt jardinée est la forme la plus apte à produire des gros bois d'œuvre.

C'est enfin la variété des produits qui caractérise cette forme, car tous, depuis le plus menu bois de chauffage jusqu'au plus gros bois d'curre, y sont constamment disponibles.

$4^{\circ}$ Influence sur la fertilité de la station. - La futaie jardinée est essentiellement conservatrice des qualités du sol ; le peuplement conserve toujours le mème caractère, car toutes les classes d'âge y sont constamment représentées; l'espace libre entre le sol et les couronnes des arbres n'y existe pas ; il est occupé par le feuillage des jeunes sujets de tout âge, sinon d'une manière absolúment continue, du moins en bouquets nombreux; cette circonstance fait que, mème dans les situations très exposées, le vent est arrêté et n'exerce pas d'action néfaste sur le sol et sur la litière qui le recouvre; enfin les bouquets de fourré disséminés dans le peuplement concourent puissamment, quand le terrain est en pente, 
à retenir les eaux pluviales, et ils forment avec la couche de litière qui recouvre d'une façon permanente le sol, l'obstacle le plus efficace contre le ruissellement, les glissements des terres et des neiges, et les érosions ou ravinements du sol.

\section{3. - Réserve sur coupe définitive. Sous-étage.}

$1^{\circ}$ Réserve sur coupe définitive. - Nous avons vu dans les formes précédentes de peuplements, qu'à un moment donné, la jeune génération destinée à perpétuer la forêt a besoin, pour se développer, d'air et de lumière; la coupe définitive est l'exploitation qui a pour résultat de faire tomber, au moment voulu, tout ce qui se trouve au-dessus de cette jeunesse.

En opérant cetle coupe définitive, on peut ètre conduit à soustraire à l'exploitation certains éléments du peuplement supérieur, soit sujets isolés, soit bouquets ; el à les laisser subsister pendant une durée déterminée; on se propose de bénificier du surcroilt de production qu'est susceptiblede donner, à ces arbres conservés, l'isolement en pleine lumière. M. Gayer fait remarquer que pour que cette réserve donne de bons résultats, il faut en général que les sujets réservés soient particulièrement vigoureux, que le sol soit fertile et conserve sa fertilité, que le dégagement des arbres qu'on se propose d'isoler ainsi soit graduel, et souvent enfin, que ces réserves soient réunies en groupes ou bouquets.

Le mode de traitement par coupes jardinatoires permet de réaliser facilement toutes ces conditions essentielles; pour cela, il suffit, dans les exploitations successives de chaque groupe ou bouquet, de faire sortir peu à peu du massif les sujets de la réserve fulure, et de ne les dégager complètement qu'à une époque où la jeune génération est suffisamment développée autour d'eux pour 
exercer elle-mème son action conservatrice du sol.

Le mode de traitement par coupe unique ne permet de réaliser aucune de ces conditions; isolement brusque des sujets de réserve, qui passent au moment de la coupe de l'état de massif à l'état isolé; disparition totale du couvert du sol, qui perd de ce fait une partie de ses qualités; appauvrissement progressif du sol pendant la période de vieillesse et par suite, perte de vitalité des sujets qui constituent le massif dès que l'âge d'exploitation d'un peuplement régulier devient élevé; tout concourt à rendre la situation défavorable pour de telles réserves; elles ne pourront gagner réellement à rester sur pied que s'il s'agit d'essences peu exigeantes et surtout si le terrain est bon, profond, à sous-sol humide.

Dans des conditions moins favorables et souvent lorsqu'il s'agit d'arbres déjà âgés, cette phase critique d'isolement se traduit par un dépérissement de l'arbre réservé qui meurt en cime et devient impropre au but qu'on se proposait en le gardant.

Il résulte de ce qui précède que dans la futaie régulière, ces réserves sont plus à conseiller dans les courtes révolutions que dans les longues; en général, on les maintient alors pendant toute la révolution suivante.

Les arbres ainsi réservés sur coupe définitive sont exposés à être renversés par le vent, spécialement pendant la première période de leur isolement. A ce point de vue, il est bon de ne pas choisir pour les réserver, en dehors des situations abritées, des arbres à cime trop développée, et d'écarter avec soin de son choix, toutes les essences à enracinement superficiel.

$2^{\circ}$ Sous-étage. - En étudiant les phases successives de la vie des peuplements, nous avons montré combien il était utile de conserver sous le peuplement principal les tiges dominées et accessoires; cet ensemble de tiges constitue, à proprement parler, un sous-étage, toujours à respecter (sauf en temps de régénération) dans les 
peuplements de futaie en raison du rôle important qu'il joue dans la constitution du massif (fig. 18.)

Un sous-étage de ce genre peut être créé artificiellement, par exemple sous un peuplement dont le couvert s'est relâché avec l'âge, soit spontanément, soit par suite d'opérations raisonnées (éclaircies). Divers cas sont à examiner, suivant le but qu'on se propose :

a. On se propose simplement de maintenir le couver't sur le sol. - Certains peuplements de futaie, constitués par des essences de lumière (futaie résineuse de pin sylvestre, par exemple) s'éclaircissent peu à peu a vec l'âge ; le massif, d'abord serré, devient de plus en plus lâche, le couvert devient irrégulier et interrompu, et le peuplement cesse alors d'exercer une action bienfaisante sur la fertilité de la station; le sol tend à se couvrir d'une végétation herbacée et parasite plus ou moins envahissante.

Une plantation effectuée sous ce couvert plein de lacunes, si elle est faite en temps utile, permet d'établir en sous-étage un nouveau peuplement dont le couvert ne tardera pas à protéger le sol et à exercer sur lui une action bienfaisante.

Le sous-étage ainsi introduit constitue ce que M. Gayer appelle un peuplement de protection du sol (1); son installation est à recommander sous tous les peuplements qui perdent dans la vieillesse leurs propriétés conservatrices du sol.

Remarquons qu'à fortori le sous-étage naturel qui s'établit souvent spontanément sous un peuplement de ce genre, est toujours à respecter, au moins tant qu iil n'est pas nuisible aux opérations de réensemencement; tel est par exemple le sous-étage de feuillus qu'il n’est pas rare de rencontrer sous les plantations résineuses, dès qu'elles deviennent un peu desserrées.

(1) Il vaut mieux qu'il soit réparti en groupes et bouquets que formé en massif continu, surtout quand il est constitué par une essence résineuse (Gayer). 
$b$. On se propose de faroriser l'acroissement de tiges d'élite du peuplement principal par la mise en lumière.

Dans un peuplement en voie de croissance, l'état de massif peut ètre desserré ou interrompu intentionnel. lement par des opérations culturales qui auraient pour but

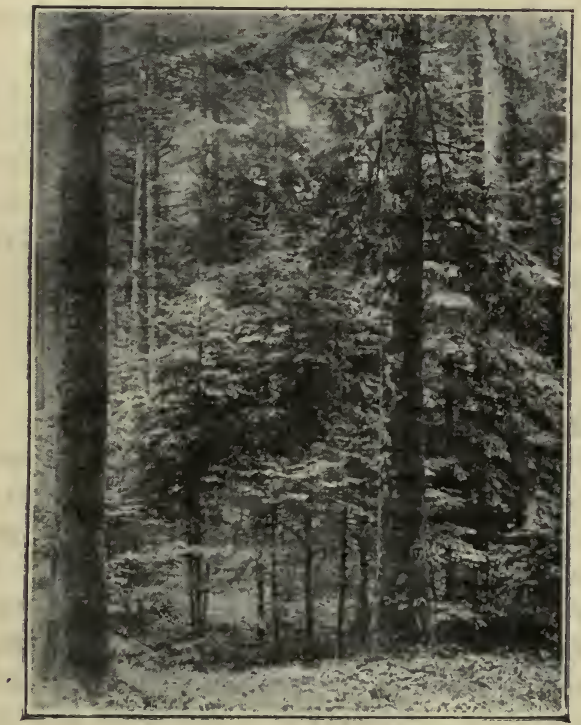

Fig. 18. - Essence d'ombre (sapin) se constituant à elle-mème un sous-étage (forèt des Elieux, Meurthe-et-Moselle).

de mettre en lumière les cimes des tiges d'élite, afin de favoriser leur accroissement, en diamètre surtout(1). De telles opérations se justifient pleinement pendant la période de perchis, alors que les arbres sont en pleine croissance.

On admet comme vérité incontestée, dit M. Gayer, que le dégagement progressif des sujets à croissance vigou-

(1)_Cette opération consiste à faire de fortes éclaircies. 
reuse, a pour résultat une accélération souvent notable de l'accroissement; mais c'est à la condition que l'activité des fonctions du sol s'intensifiera dans la même mesure que l'uccès de la lumière.

Pour que cette dernière condition soit remplie, il peut ètre utile de provoquer l'établissement d'un sous-étage sous le peuplement principal dont les cimes desserrées n'assurent plus au sol un couvert suffisant.

c. Introduction d'essences de mélange. - La création d'un sous-étage faite dans l'une des conditions indiquées précédemment peut avoir pour objet d'introduire ultérieurement dans le peuplement principal une ou plusieurs essences de mélange; sa situation s'y prête évidemment et ce n'est qu'une question de culture.

\section{I. - TAILLIS}

Premier type. Taillis simple régulier.

$1^{0}$ Orjigine et caractères. - Le régime du taillis ne s'applique qu'aux essences feuillues; il est basé essentiellement sur la propriété que possèdent les essences feuillues seules et à des degrés différents : $1^{\circ}$ de donner des rejets si elles sont coupées à fleur de terre ou à certaine distance du sol; $2^{\circ}$ de donner, en outre, chez un grand nombre d'essences feuillues, des drageons, véritables rejets de racines qui proviennent de bourgeons adventifs nés sur les racines.

Rejets de souche et drageons forment, en se dévelopjant, des perches et parfois des arbres, et donnent naissance à une nouvelle forèt, née sur les souches et racines de l'ancienne forèt.

Le peuplement est ainsi, non pas régénéré comme dans la futaie, mais simplement rajeuni à chaque exploitation, et ce rajeunissement peut se répéter plusieurs fois, aussi longtemps que les souches et les racines de la plante primitive continuent à vivre. 
Cet ensemble, souche et racines, conserve une vitalité plus ou moins longue suivant les essences, le sol, le climat et les dommages qu'on leur cause pendant l'exploitation; mais il est à remarquer que dans le végétal arbre ainsi traité, il y a périodiquement rupture d'équilibre entre la partie aérienne et la partie souterraine; le rajeunissement fatigue alors les arbres dans une mesure très variable et se traduit par la mort définitive de quelques souches anciennes au passage de chaque exploitation.

Le peuplement ainsi conduit ne peut durer indéfiniment et il tendrait à disparaître progressivement si deux causes ne renaient modifier cet état de choses :

$a$. Un certain nombre de rejets, spécialement ceux qui proviennent de bourgeons proventifs, ainsi que les drageons, sont susceptibles de se créer dans la partie où ils sont en contact du sol, un enracinement propre ; peu à peu ils s'isolent de la souche mère et virent d'une façon indépendante; aux exploitations suivantes, leurs nouvelles souches et racines, émettent des rejets et drageons, remplaçant ainsi par leur fonctionnement les souches trop âgées qui pourrissent et disparaissent.

$b$. Des semences apportées sur le sol qui porte le taillis, soit naturellement par dissémination d'arbres portegraines voisins, soit accidentellement par les mille moyens de la nature (oiseaux, rongeurs, etc., vent et météores) donnent naissance par places à de jeunes brins de semence qui suivent l'évolution du taillis ; à l'exploitation suivante, les souches et l'enracinement de ces jeunes arbres viennent enrichir le vieux taillis.

Ainsi compris, le peuplement de taillis est, en principe, rajeuni à chaque exploitation et la perpétuité du massif dans la suite des temps est assurée progressivement par l'affranchissement de rejets et de drageons, et par la naissance de jeunes sujets issus directement de semences.

Le peuplement de taillis, constitué dès le printemps A. FRoN. - Sylviculture. 
qui suit la coupe par les rejets et les drageons, nait sur toute la surface à la mème époque, et son ensemble représente un type de peuplement régulier; il a, tout au moins pendant un certain nombre d'années, une croissance beaucoup plus forte et plus rapide qu'une jeune fulaie de mème âge, et cela à cause du plus grand développement de son enracinement.

A l'aspect, les rejets ressemblent à des brins de semence; mais leur groupement autour de la souche qui leur a donné naissance est caractéristique (1); tous ceux qui sont issus d'une mème souche forment ce qu'on appelle une cépée (fig. 19) et le peuplement est composé d'un ensemble de cépées.

L'exploitation d'un taillis simple ayant été faite en hiver, les rejets naissent sur les souches coupées dès le printemps qui suit l'exploitation; les jeunes pousses émergent d'abord par groupes ou îlots de régétation, franchement isolés les uns des autres, chaque groupe formant une cépée, et les cépées laissant entre elles la plus grande partie du terrain à découvert; ces jeunes pousses naissent en grand nombre autour d'une mème vieille souche, mais toutes nes'élèvent pas verticalement; il $\mathrm{y}$ en a qui poussent obliquement; d'autres sont même complètement rejetées par les pousses mieuk placées et arrivent à pousser presque parallèlement au sol ; il en résulte que la jeune cépée a l'aspect d'un buisson hémisphérique qui s'accroît peu à peu.

Entre chacun de ces buissons, le sol découvert se tapisse de verdure; aussi, pendant les premières années, malgré la végétation active des rejets, la surface semble

(1) De jeunes plants recépés une première fois dans un taillis, ne donnent généralement qu'un et rarement deux rejets qu'on peut confondre avec des brins de semence, ce qui ne présente aucun inconvénient. Toutefois un œil lıabilué les distingue facilement des brins de semence a leur aspect plus vigoureux, l'empátement et à la légère courbure de leur base. Les brins de semence, moins vigoureux, n'ont d'a lleurs généralement pas l'âge exact du taillis. 


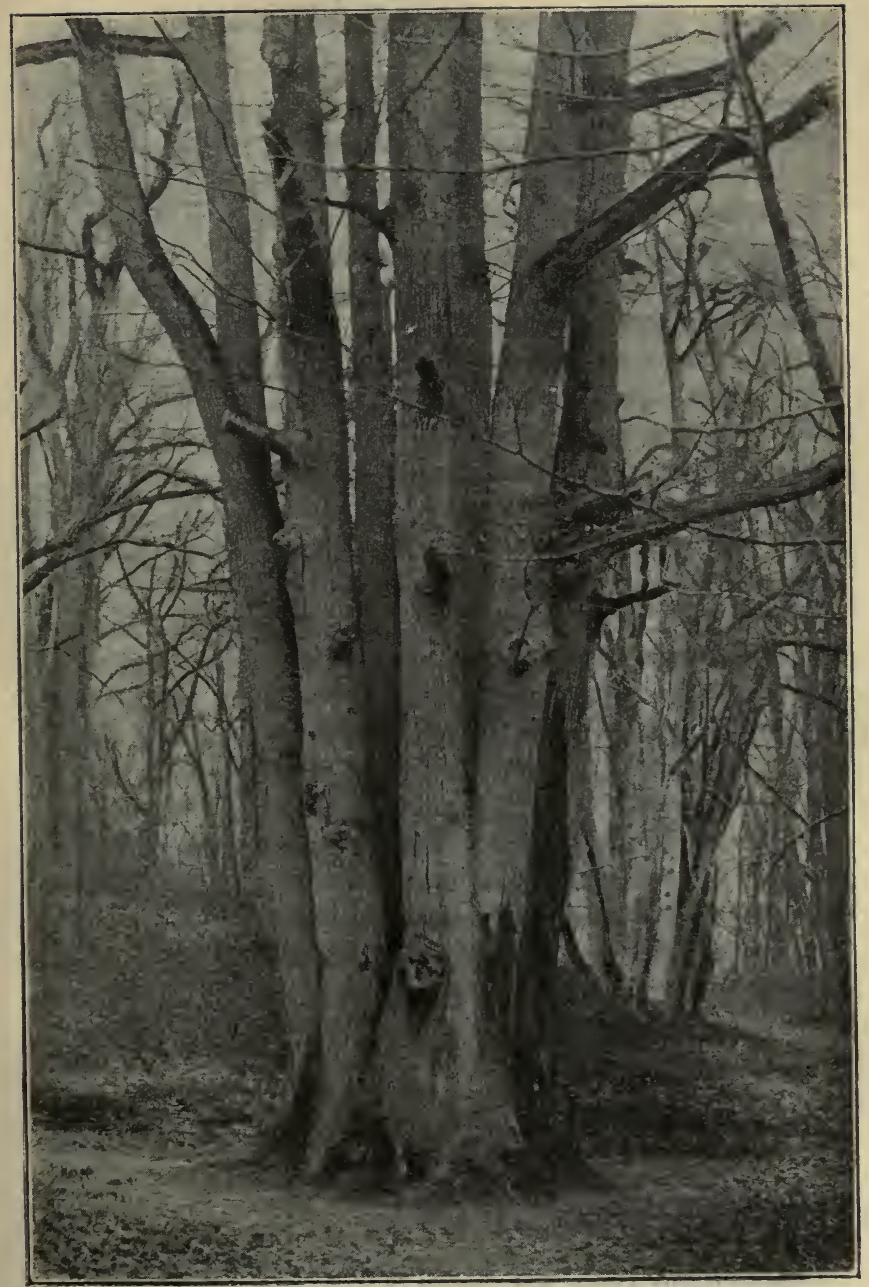

Fig. 19. - " Le Nid de l'Aigle \#, vieille cépée de chêne très âgée dans la forèt domaniale de Fontainebleau (Seine-et-Marne). Photographie de M. H. Roger. 
produire plus d'herbes que de bois. Avec les années, les rejets se développent, les cépées grandissent rapidement grâce à la vigoureuse végétation des rejets, et par suite les vides laissés entre les cépées diminuent, puis sont complètement recouverts; le recru parvient à former massif et étouffe la végétation herbacée.

Cetle reconstitution de l'état de massif demande un temps plus ou moins long suivant les essences, les conditions de la végétation et l'espacement des souches mères. Certaines essences, le charme par exemple, émettent de nombreux rejets, et leurs cépées sont puissamment fournies; un bon sol et des conditions favorables de végétation favorisent le développement des cépées; quant à l'espacement des souches mères, il varie suivant l'âge auquel le taillis a été coupé, devenant d'autant plus grand que la révolution est plus longue. D'après M. Boppe, la constitution de l'état de massif demande environ de six à douze ans suivant les cas (1). Dans les taillis simples réguliers, à révolutions courtes, souvent inférieures à vingt-cinq ans, tous les sujets ont même avenir, et sont appelés à une mème fin prochaine; les cépées existent aux distances que comporte la révolution ; elles s'étalent sans se gêner l'une l'autre; dès lors, si la lutte s'engage, ce n'est pas de cépée à cépée, mais de rejet à rejel dans une mème cépée; cette lutte est toujours de courte durée en raison de la révolution; le peuplement ne dépasse guère l'état de fourré ou de jeune gaulis; mais il n'en est plus de même si les révolutions alteignent ou dépassent trente ans.

Quand le massif est parfait, le feuillage s'élève de plus en plus vers le haut des tiges; il passe peu à peu à l'état

(1) La densité d'un trillis, eu égard au numbre de cépées, est fonction de la révolution; comme chacune de celles-ci se développe avec les années, sa projection occupe d'autant plus d'espace qu'on la laisse vieillir davantage ; par conséquent, le nombie des centres de reproduction est d'autant plus faible, et par suite le fourré véritable (abstraction faite des morts-loois) s'établit d'autant plus tard que les révolutions sont plus longues. (Boppe et Jolyet.) 
de gaulis, puis de bas perchis; dans chaque cépée, les tiges d'élite se dessinent progressivement, au détriment des tiges moins vigoureuses qui restent à l'état dominé. Si on laisse vieillir ce peuplement, il prend alors le caractère des futaies régulières obtenues par voie de semis, et ce caractère s'accentue au fur et à mesure que le peuplement est plus vieux. Il est rare d'ailleurs qu'on conduise le taillis au delà du vieux perchis sur souche.

$2^{\circ}$ Danger's extérieurs. - Le peuplement de taillis, dans son jeune âge, est très sensible à l'action de la gelèe; à ce point de vue il y a lieu de distinguer :

a. Les gelées tardives de printcmps. - Leur action se manifeste très vivement sur de jeunes pousses pleines de sève, à croissance rapide, et par suite à tissus mous et très sensibles, à tel point qu'il n'est pas rare de voir les jeunes pousses du taillis complètement desséchées par la gelée, ayant l'aspect de tiges grillées par le feu. A cet égard, les rejets sont plus sensibles que les brins de semence; si l'action des gelées tardives de printemps n'est qu'accidentelle, elle n'est pas désastreuse pour les taillis, car la souche conserve la faculté d'émettre de nouveaux rejets; mais si ces gelées sévissent fréquemment dans un canton, elles entravent la croissance du taillis qui prend un aspect chétif et rabougri caractéristique; on dirait d'un abroutissement par le bétail.

b. Les gelées hátives d'automne. - Dans leur première année surtout, les rejets peuvent être arrètés dans leur lignification par les gelées d'automne, et disparaitre victimes de l'hiver qui les trouve mal aoittés. C'est alors une année de végétation à peu près perdue, et si l'accident se répète plusieur's années de suite, les souches meurent en grand nombre.

Remarquọns que, dans certaines stations basses et humides, les jeunes pousses, spécialement celles qui se trouvent dans la couche d'air voisine du sol, sont exposées d'une façon permanente à l'action des gelées ; cette situa- 
tion devient sourent désastreuse pour de jeunes taillis.

Remarquons enfin que les rejets de souche ayant dans leur jeune âge une production totale supérieure à celle des brins de semence, demandent une plus forte somme de chaleur, ou une période de végétation plus longue pour mûrir leur bois; mais que, d'autre part, plus la période de végétation est longue, plus le danger de la gelée est imminent; c'est pourquoi le domaine du taillis est beaucoup plus restreint que celui de la futaie; son champ d'application est limité aux climats de plaine, où le taillis trouve des écarts de température peu élerés, une grande somme de chaleur et un temps de végétation suffisamment long.

Quant aux autres causes de destruction (dégâts des (hampignons, insectes, action des vents, etc.), elles sont pour ainsi dire sans action marquée sur les taillis.

$3^{\circ}$ Production. - Le taillis simple régulier produit surtout du bois de chauffage (rondins ou bois à charbon) et des écorces (taillis de chène); comme bois d'œurre, il ne produit que des gaules et perches ou menu bois d'industrie, mais la quantité de ces derniers est subordonnée à la longueur des révolutions; en somme, il produit des jeunes bois.

$4^{\circ}$ Influence sur la fertilité de la station. - L'influence qu'exerce un peuplement de taillis simple sur la fertilité de la station dépend de facteurs agissant dans des sens très différents; leur résultante est variable suivant les régions, le climat, le sol, les essences, l'état du peuplement et l'âge d'exploitation. Nous insisterons sur trois de ces facteurs :

a. Couverlure du sol. - Le peuplement à l'état de taillis simple présente pendant les phases de son existence les caractères du fourré, du gaulis ou du bas perchis. Mlème a vec une rérolution très longue pour un taillis, le peuplement n'arrive qu'à l'état de perchis régulier, mais il n'atteint jamais la période a vancée qui, dans les futaies 
régulières, se caractérise par le desserrement du massif et par un dessèchement dangereux du sol. A ce point de vue donc, il donne plus de garanties que la futaie pour la conservation des qualités du sol, à la condition toutefois qu'on suppose le terrain entièrement occupé par un taillis bien planté et bien entretenu. Sinon, rien n'est plus comparable dans les deux régimes.

b. Coupe blanche à intervalles rapprochés. - Le traitement en taillis simple dénude complètement les surfaces à des intervalles rapprochés; il comporte les inconvènients inhérents à chaque coupe blanche et provoque périodiquement l'appauvrissement de la litière, la détérioration de l'humus, un certain durcissement du sol et linvasion de l'herbe. Toutefois, il est bon de remarquer que la dénudation complète du sol ne dure qu'une année; que dès l'année suivante, en raison de la croissance vigoureuse des rejets, elle n'est plus que partielle, et que dès lor's le massif se rétablit rapidement.

c. Nature de la récolte. - Le taillis ne produit que des bois jeunes, par suite plus riches que les produits de futaie en éléments nutritifs; il fatigue le sol, auquel il n'apporte que des restitutions insuffisantes. Les effets de l'épuisement sont d'ailleurs d'autant plus rapides que le terrain est de nature plus sèche et que la révolution est plus courte.

En résumé, il est admis, d'une façon générale, que les taillis peuvent épuiser le sol quand la coupe en est trop répétée.

Deuxième type. - Taillis simple fureté.

$1^{\circ}$ Origine et caractères. - Le furetage consiste non plus à exploiter à blanc toute la coupe, mais à parcourir cette surface à de courts intervalles, en se bornant à ne couper, sur chaque cépée, que des perches ayant dépassé une grosseur donnée, généralement, 30 à 33 centimètres de tour à hauteur d'homme. Ainsi traité, le peuplement est composé de cépées en forme de buisson, dont les 
rameaux de toutes les dimensions fournissent un amas confus de feuillage et constituent un fourré perpétuel de 6 à 10 mètres de hauteur; il prend un aspect irrégulier avec cette différence que les âges sont distants entre eux d'un nombre d'années en rapport avec celui des intervalles d'exploitation (fig. 20).

Les rejets qui naissent sur les sections d'exploitation ne s'affranchissent pas, et quand une cépée meurt de vieillesse ou par accident, elle doit être remplacée par d'autres qui s'obtiennent à l'aide de marcottes ou de plantations.

$2^{\circ}$ Dangers extérieurs. - Cette forme de traitement procure aux rejets naissants un abri protecteur contre la sécheresse et les gelées, et permet ainsi de faire pénétrer le régime du taillis dans des régions à climat moins doux et moins favorable que celui qui lui convient ordinairement.

$3^{\circ}$ Production. - Le furetage est surtout appliqué au hêtre dans la basse et moyenne montagne. Comme produits, il fournit une plus forte proportion de rondins que le taillis régulier.

$4^{\circ}$ Influence sur la fertilité de la station. - En maintenant le sol continuellement couvert, il entretient bien sa fertilité, et le protège contre les érosions.

\section{III. - TAILLIS COMPOSÉ}

$1^{0}$ Or'igine et caractères. - Un peuplement est à l'état de taillis composé ou de taillis sous futaie, quand il est coinposé de deux éléments :

a. Un étage inférieur, exploité à intervalles égaux, à la façon des taillis simples ;

b. Un étage supérieur, composé d'arbres irrégulièrement disséminés, dans lequel on exploite individuellement, lors du passage des coupes, les sujets devenus exploitables, à la façon de la fulaie jardinée.

En principe, le rajeunissement du taillis est assuré par 
TAILLIS COMPOSÉ.

141

le recépage au moment de la coupe; la régénération de

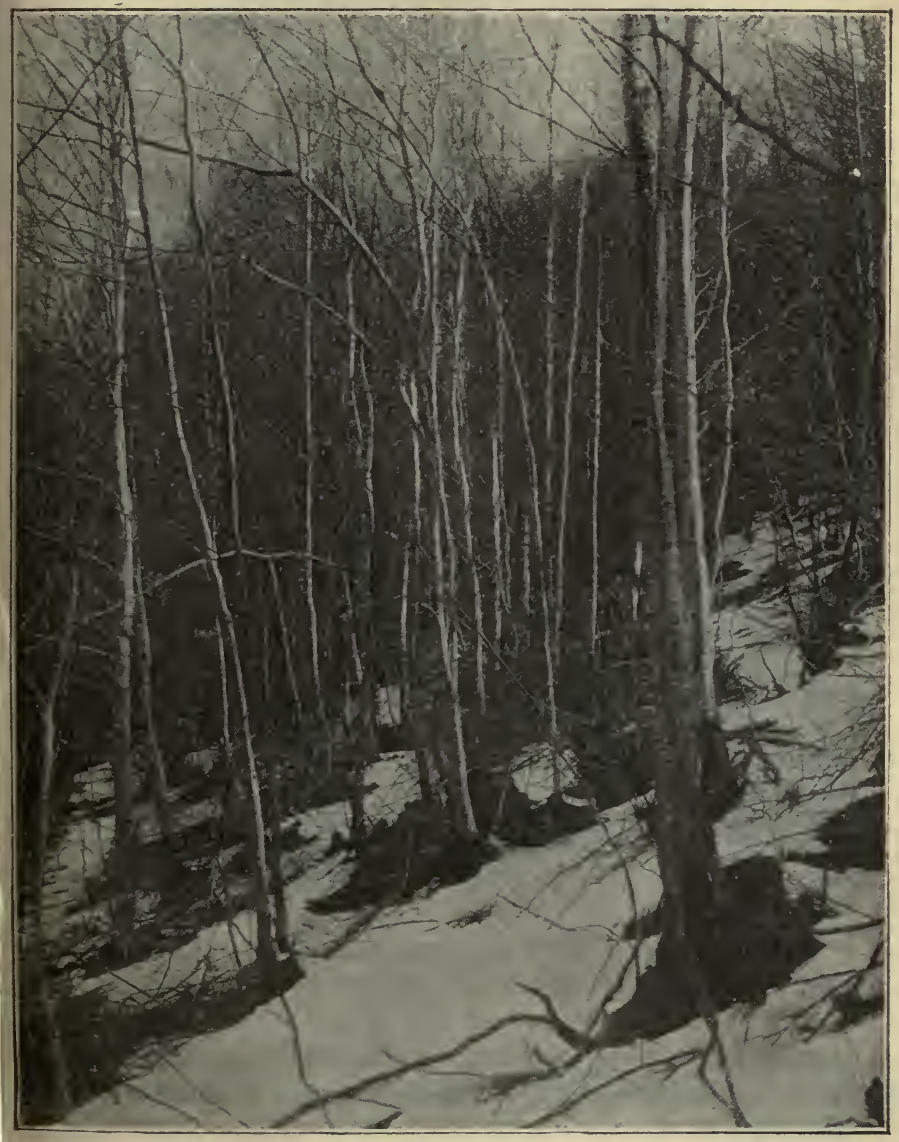

Fig. 20. - Peuplement de hètre à l'état de taillis fureté dans les Pyrénées. Castillon (Ariège).

la futaie est assurée par un certain nombre de brins 
de semence don! la naissance coïncide en général avec celle des brins du taillis, dans le sein duquel ils restent confondus pendant une révolution; à partir de la première exploitation du taillis, ces brins, du moins ceux qu'on conserve, entrent dans l'étage supérieur, et constituent ce qu'on appelle les réserves (fig. 21).

ll résulte de cette constitution que dans l'ensemble, l'étage supérieur se compose d'arbres appartenant à plusieurs classes, dont les âges diffèrent entre eux d'un temps égal à la révolution du taillis, et que, d'autre part, le nombre des classes d'âge de la futaie dépend de la durée de la révolution adoptée pour le taillis, et de l'âge auquel sont exploités les plus vieux sujets.

Autrement dit : si, par exemple, on exploite le taillis à trente ans et les plus vieux arbres de futaie à cent cinquante ans, il se trouvera dans la futaie cinq classes d'arbres, dont les âges différeront entre eux de trente ans, et dont les plus jeunes se confondront a rec le taillis; au moment de l'exploitation, c'est-à-dire tous les trente ans, on réalisera, dans la futaie, individuellement et en jardinant, les arbres derenus exploitables; on recépera le taillis, en ayant soin de conserver, pour les faire entrer comme réserves dans l'étage supérieur, les brins de l'âge du taillis, en nombre suffisant pour venir compléter la futaie et entretenir sa composition. On a donné à ces diverses classes d’âge de la futaie des dénominations spéciales: en général le terme de baliveau est exclusivement attribué aux bring de l'âge du taillis, aussitot après leur isolement; le terme de moderne désigne les arbres de deux âges; le terme d'ancien désigne les arbres de trois âges; le terme de bisancien est attribué aux arbres de quatre âges, et celui de vieilles écorces aux arbres de cinq âges et au delà (1).

(1) II est souvent difficile de déterminer l'age d'unc réserve sur pied, aussi se hase-t-on sur la grosseur pour qualifier un arbre comme moderne et ancirn; dans la pratique, on appelle anciens les sujets de 35 centimétres de dinnetre et nu-dessus. 
11 résulte encore de cette constitution toute spéciale de la futaie, que le nombre des sujets formant chaque classe n'est pas indifférent, si l'on veut que l'exploitation puisse rester soutenue; le bisancien exploité dans la classe la plus âgée doit pouvoir ètre remplacé pendant

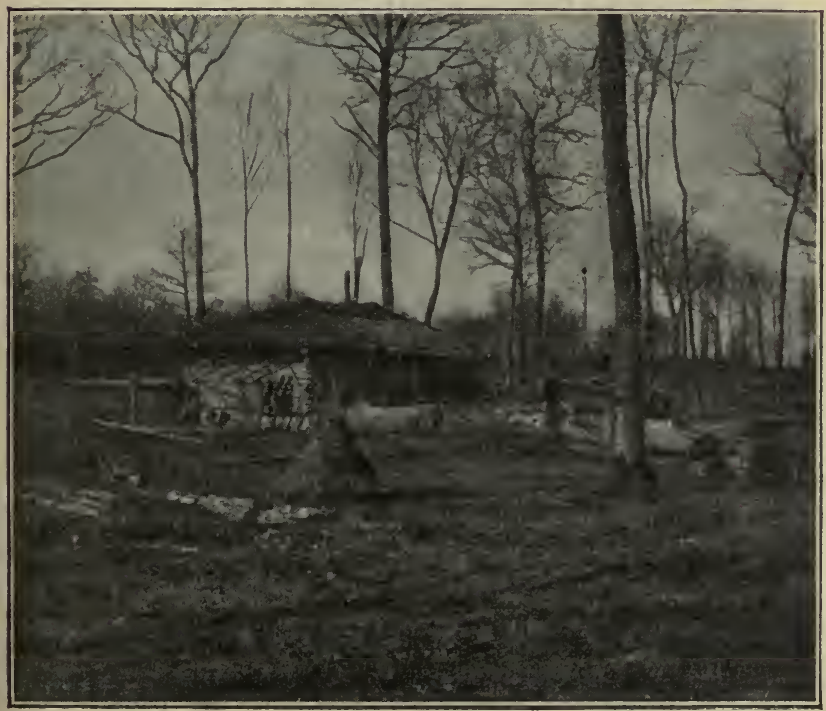

Fig. 21. - Taillis composé après l'exploitation, type à longue révolution, forèt domaniale de Borne (Côte-d'Or).

la rérolution suivante par un sujet de la classe immédi乞tement inférieure, condition nécessaire puisque l'on veut à la fin de cette nouvelle révolution trouver encore un bisancien à exploiter, et ainsi de suite, jusqu'à la plus jeune classe; aucune classe ne peut donc avoir un nombre de sujets inférieurs à celui des sujets de la classe la plus ágée. 
En outre, au fur et à mesure qu'un jeune baliveau vieillit et monte en grade dans les classes successives de la réserve, il est exposé à milles causes qui peuvent l'empêcher de bien se développer, ou le détériorer; il peut, à un moment quelconque, ne plus présenter les qualités voulues pour mériter d'ètre maintenu ; un choix est nécessaire à chaque changement de grade, et, pour permettre ce choix, une classe doit contenir d'autant plus de sujets qu'elle est plus jeune.

Il résulte enfin de cette constitution que l'élément taillis qui forme l'étage inférieur présente vis-à-vis de l'élément futaie le caractère d'un sous-étage; dès lors, il existe une solidarité étroite au point de vue de la crois. sance entre le taillis et la futaie qui le domine ; l'existence et la prospérité de ce sous-étage dépendent de l'accès de la lumière, dont la mesure est déterminée par l'étendue, l'épaisseur et la hauteur du couvert de la futaie. Entre les cas extrèmes, taillis prospère sous futaie claire, et taillis chétif sous couvert épais, se trouve un champ assez large dans les limites duquel on peut concevoir un certain nombre de formes diverses; mais comme toutes les circonstances qui favorisent ou entravent la croissance, agissent tantôt sur le taillis, tantòt sur la futaie, et comme la prépondérance de l'un entraine toujours un certain affaiblissement de l'autre, on comprendra qu'une proportion constante des diverses parties du peuplement ne puisse être obtenue que si des soins intelligents conservent son caractère à cette forme essentiellement mobile (Gayer).

Ce fait de solidarité entre la futaie et le taillis porte à distinguer dans le taillis composé, trois types distincts :

$1^{\circ}$ Type normal, dans lequel futaie et taillis se trouvent sur un pied à peu près égal. Dans cette forme, on se propose d'obtenir une croissance satisfaisante, uniforme et soutenue du taillis, sans négliger la production de la 
futaie; pour cela, il faut avant tout se rendre compte de l'effet produit par le couvert de la futaie sur le sousétage. D'après 11. Gayer, cet effet est très variable selon l'essence, la hauteur des tiges et des cimes de la futaie, la répartition des sujets tant du taillis que la futaie, ainsi que, selon la fertilité des stations, la longueur des révolutions, etc. Pour que le taillis rienne également bien dans toutes ses parties, il faut autant que possible que le couvert soit égal ; aussi doit-on toujours chercher dans cette forme de peuplement à obtenir la répartition à peu près uniforme de la futaie, et pour cela ne pas perdre de vue les classes d'âge les plus avancées.

Mais comme, d'autre part, le couvert des essences de lumière est beaucoup moins épais que celui des essences d'ombre, et nuit moins à la croissance du taillis, il est à désirer, pour la conservation du type normal, que la futaie se compose, au moins partiellement, d'essences de lumière. L'expérience seule peut établir quelle épaisseur le couvert peut prendre pour ne pas trop nuire à la production du taillis. De ces expériences, on pourra déduire, dans une station donnée, quel est le nombre des sujets à admettre dans chaque classe, nombre proportionné à leur couvert; mais ces chiffres n'auront jamais qu'une valeur relative, et devront être considérés comme de simples renseignements, bons à connaître pour la gestion de forèts semblables.

2 Type à futaie prépondérante. - Dans cette forme, on se propose de donner une prédominance marquée à la futaie, dans le but de produire surtout du bois d'œuvre; cette prédominance de la futaie tend à donner plus ou moins au taillis composé le caractère de la futaie jardinée (1) (tig. 22).

(1) Les individus de tous ảges sont dısséminés dans la futaie jardinée; il en est de même dans la partie futaie du taillis composé de ce second type, mais avec cette différence que les âges des individus sont répartis par échelons; entre ces échelons, les âges intermédiaires ne sont pas représentés.

A. Frox. - Sylviculture. 
M. Gayer donne la description de cette forme de peuplement dans les termes suivants: pour produire heaucoup de bois d'cuvre, il faut que les sujets de la futaie soient plus serrés que dans le type précédent, et cela surtout dans les classes jeunes et d'âge intermédiaire. Or, si l'on voulait étabir ce massif serré dans toute l'étendue du peuplement, on devrait, dans la plupart des cas, à cause du couvert très épais, renoncer au sousétage et par suite au caractère du taillis composé. Il s'en suit qu'en règle générale, la futaie doit ètre ici répartie d'une manière irrégulière.

$3^{\circ}$ Type à fulaie cluire. - Dans cette forme, on donne une prédominance marquée au taillis et la futaie se compose d'un petit nombre de sujets disséminés et par suite assez distants les uns des autres. Dans ce type peuvent rentrer: les taillis à longues révolutions, demandant par suile un couvert plus modéré pour pouvoir se développer (fig. 21); les taillis à révolutions ordinaires, surmontés d'une réserve qu'on ne laisse pas vieillir au delà de deux et rarement de trois révolutions de taillis; enfin les taillis avec un très petil nombre d'arbres qu'on laisse vieillir.

Le caractère de ce type est de reléguer la futaie au second plan; cette forme se rapproche beaucoup du taillis simple.

Il est à remarquer que, sous l'influence des abus de pâturage, des abus commis par une mauvaise adminisnistration songeant plus à jouir du présent qu'à réserver l'avenir, et justement en raison de la souplesse de cette.forme de peuplement qui se prête à toutes les exploitations abusives d'un matériel sur pied, heaucoup de taillis composés ont été conduits par la force des choses à cet état, mème dans les régions qui leur présentaient les stations les plus propices.

Les conditions économiques actuelles du marché des lois ne permettent-elles pas de faire micux? Nous 
verrons dans la suite de cet ouvrage qu'une bonne gestion doit souvent tendre à les ramener progressivement aux types précédents, ne fût-ce qu'en vue de conserver, et souvent de restaurer la fertilité de la station, et par suite sa force productive.

Le taillis composé, (spécialement la première el

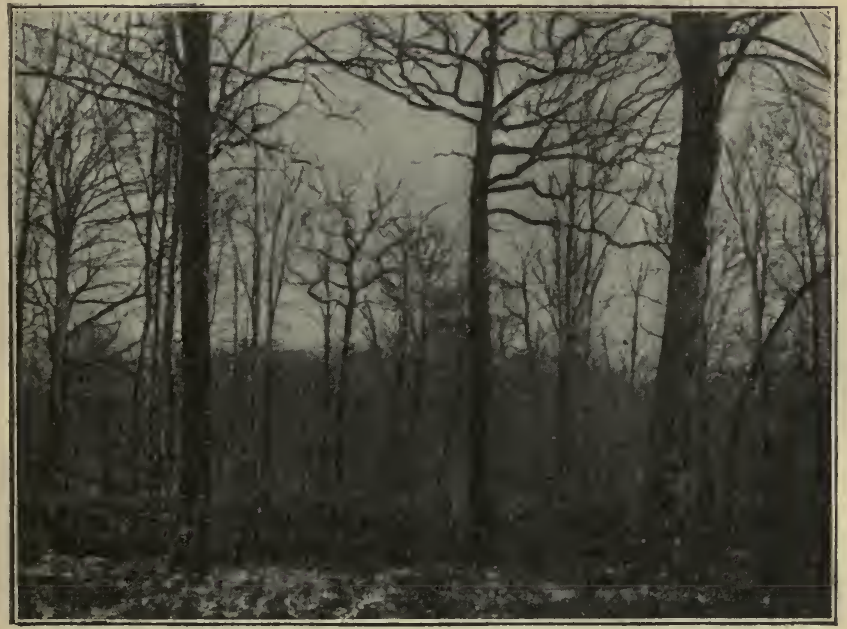

Fig. 22. - Taillis composé, type à futaie prépondérante.

surtout la troisième forme), exige pour les mèmes raisons que le taillis, un climat doux, une longueur súffisante de la saison de végétation, un sol assez frais pour ne pas craindre des découverts souvent répétés, et en plus un terrain dont la fertilité et la profondeur rendent possible la culture des essences précieuses, surtout du chène.

2 - Danger's extérieur's - Il est facile de se rendre comple d'après ce qui a été vu précédemment, que le 
taillis composé présente une immunité presque complète aux dangers extérieurs, ou tout au moins tend à modérer lans une très large mesure leurs effets très dangereux; par le couvert de son étage supérieur, il atténue les écar's de température et les dégâts qu'occasionnerait la gelée ; les vieux arbres de la futaie eux-mêmes, grâce à l'enracinement solide qu'ils doivent à leur état isolé et à la forme conique de leur tronc, offrent une grande résistance au vent; ils servent de protection aux plus jeunes réserves; quant aux insectes et aux champignons, leurs dégâts ne sont jamais que partiels et par suite fort peu à redouter.

En définitive, en toutes circonstances et mieux que la futaie, le taillis composé répare de lui-mème les dégâts dont il a pu être victime; car, quel que soit le sort de la réserve, l'ensouchement du taillis est toujours là pour sauver l'état boisé.

$3^{\circ}$ Production. - Le taillis composé a été très discuté comme production, ce qui s'explique, puisqu'il existe une infinité de types très dissemblables. Trailé d'une manière rationnelle, il est d'un rapport au moins égal à celui de la futaie, et cette comparaison paraîl devoir être encore plus à l'avantage du taillis composé, s'il s'agit de futaies régulières (Gayer).

Quant à la nature de la production, la forme de taillis composé permet d'obtenir du bois d'œurre d'essences feuillues, particulièrement du chêne, dans un temps relativement court; elle présente l'avantage de donner à chaque sujet un espace illimité pour s'y développer, et par suite. de favoriser le développement des cimes; comme elletend à assurer la conservation des qualités du sol, elle a pour effet non seulement.d'augmenter la production totale annuelle, mais encore de donner au bois desqualités intrinsèques qui en font du bois d'ourre.

Notons toutefois que MII. Boppe et Jolyet reprochent au taillis composé de donner du bois d'ouvreà accroissements 
irréguliers, trop dense, trop nerveux et par suite se tourmentant facilement; enfin, ils ajoutent que l'alternative d'isolement et d'enclave, au milieu d'un sous-étage grandissant, dispose les arbres à contracter des tares qui occasionnent un déchet considérable.

En ce qui concerne les fûts, la croissance à l'état isolé tend à leur donner une forme conique inférieure à celle de la futaie, inconvénient qui est compensé, dans une certaine mesure, dans le type à futaie prépondérante.

Quant aux bois de branches et aux ramilles, leur proportion dans les arbres de réserve est très forte et il est rare que ces arbres fournissent plus de 40 à 50 p. 100 de leur volume total en bois d'œuvre; le reste n'est que du chauffage souvent de médiocre qualité.

Quoi qu'il en soit, le taillis composé se recommande au point de rue économique par la variété de ses produits, qui sont de nature à satisfaire les besoins les plus multiples du commerce.

$4^{\circ}$ Influence sur la fertilité de la station. - De ce qui précède, il résulte qu'un taillis composé, soumis à un traitement raisonné, ne dénude que partiellement le sol à chaque exploitation du taillis; dès la première année après l'exploitation, les îlots de rejets opposent déjà à l'assèchement du sol et à l'enlèvement de la litière, un obstacle beaucoup plus efficace qu'un semis, mème âgé de plusieurs années ; dès la formation du massif, le sousétage joue le rôle d'un peuplement de protection.

D’un autre côté, la présence continue de l'étage supérieur, spécialement dans le type à futaie préponrante, constitue un autre excellent élément de conservation, et cette forme de peuplement doit ètre considérée comme d'autant 'plus favorable à la conservation des qualités du sol, qu'elle se rapproche plus du type futaie. 


\section{GINQUIEME SECTION}

\section{État de la forêt.}

\section{GÉNÉRALITÉS}

Toutes les forèts de la propriété privée rentrent plus ou moins directement dans un des types de peuplement que nous venons d'examiner, quelles que soient les méthodes employées pour les gérer.

Mais en raison mème des fautes culturales qui peuvient ètre commises et renouvelées sans cesse sur le mème point, les forèts du même type présentent une variété infinie d'aspects; nous n'en prendrons qu'un exemple: sur les bords de la Saône se trouvent de belles forèts trailées en taillis composé; le sous-étage très complet est formé d'essences de choix; l'étage dominant se compose de superbes réserves de chène. Acôté, dans des conditions identiques, une autre forèt, traitée aussi en taillis composé, est en maurais état; elle ne renferme que des coudriers, des bois blancs, de mauvaises réserves de chène et il est impossible d'y faire un bon balivage ; dans ce cas particulier, la forèt est'ruinée par une série de révolutions trop courtes qu'on lui a imposées. Cet exemple nous permet de concevoir une notion nouvelle, celle de l'état de la foret.

Cet élat de la forèt est trìs complexe à définir. Indépendamment des circonstances locales, des nécessilés, du moment et des produits que l'on veut ohtenir, toutes choses importantes pour le propriétaire, il est en rapport 
non seulement avec la forme du pruplement, mais aussi arec les essences susceptibles de composer ce peuplement, essences qui en font suivant sa forme, une sorte de peuplement tout spécial.

Nous caractériserons donc, dans une certaine mesure, l'état de la forèt : $1^{0}$ par la comparaison entre elles des différentes formes de peuplements̀; $2^{\circ}$ par la composition des peuplements, c'est-à-dire par les essences qui peuvent entrer dans chaque forme de peuplement, pour en constituer un type spécial.

Cette double étude permettra au propriétaire, à qui nous en laissons le soin, en raison des circonstances locales dans lesquelles il se trouve, de délerminer le type général qui correspond au bon état de sa forèt, ou tout au moins d'apprécier, suivant les cas, quelles sont les considérations qui peuvent le conduire à modifier l'état actuel d'une forèt déterminée.

\section{1. - COMPARAISON ENTRE LES DIFFÉRENTES FORMES DE PEUPLEMENTS}

Au point de vue de l'exploitation forestière dans son ensemble, la forme du peuplement qui doit y exister, est fonclion des éléments suivants :

$1^{0}$ Essence. - Certaines essences excluent souvent des formes déterminées de peuplements; telle essence feuillue (aune, micocoulier, robinier, saule, etc.) ne comporte trìs ordinairement que la forme du taillis simple, tandis qu'au contraire les essences résineuses ne se prètent qu'aux formes de futaie, et n'apparaissent comme réserves dans les taillis composés que pour des raisons accidentelles et temporaires.

$2^{\circ}$ Qualités de la station. - La forme du peuplement agit à ce point de vue, soit par ses exigences propres au point de vue de la fertilité du sol, soit par l'action qu'elle exerce sur la conservation de cette fertilité. 
En sol frais et fertile, l'action conservatrice du peuplemient présente un intérèt moins impérieux qu'ailleurs, et les bonnes stations peuvent admettre toutes les formes de peuplement; par contre, ces bonnes stations sont tout indiquées pour les formes de peuplement qui recourent spécialement à l'emploi de la lumière pour forcer en quelque sorte le développement des sujets d'élite (taillis composés, réserves sur coupe définitive).

En sol de fertilité moyenne, en raison d'une constitution physique défavorable, d'une faible profondeur et du peu d'abondance d'éléments minéraux assimilables, ou encore dans les stations plus ou moins exposées aux dangers exlérieurs (pentes fortes et ravinements, vents violents, gelée, neige, etc.), l'action conservatrice du peuplement présente un intérêt souvent très impérieux. Les formes de peuplement préférables sont alors celles qui assurent le mieux le couvert continu et le maintien d'un massif complet (futaie traitée par coupes successives, par coupes jardinatoires, futaie jardinée).

En sol médiocre et mauvais, dans les stationschaudes et défavorables, la question de l'essence à planter prend le pas sur celle de la forme du peuplement, car la plupart des essences en sont exclues, n'y trouvant pas ce qui est nécessaire à leur subsistance; on ne peut que choisir, parmi les formes de peuplement qui conviennent à cette essence, celle qui paraît la plus apte à concourir à l'entretien du sol et à la conservation des qualités qu'il peut avoir, et si les circonstances imposent l'exploitation à blanc étoc, on doit éviter d'opérer la coupe en bloc sur de grandes étendues (Gayer).

D’une façon générale, l'adaptation de la forme du peuplement à la station est d'autant plus nécessaire que la conservation des qualités du sol est plus suborlonnée à l'action protectrice de la forèt, et, d'après. II. Gayer, on ne devrait jamais perdre de vue que c'est une faute de ne pas suivre la nature pas à pas, car nous n'avons pas 
d'autres moyens de production qu'elle-mème. Tous les artifices de l'agriculture ne sont pas à la portée de la sylviculture qui ne peut que concourir à la conservation de la fertilité du sol par les moyens naturels; et le principal de ceux-ci, c'est l'entretien continu du couvert et de la protection du. sol, c'est-à-dire le choix d'une forme de peuplement, en rapport avec les exigences de la situation.

$3^{\circ}$ Dangers extérieurs. - Les peuplements réguliers opposent à presque tous les agents destructeurs (ouragans, neige, gelées) moins de résistance que les autres (fig. 23), ils sont moins aptes à parer, dans certaines situations de montagne, aux effets désastreux des eaux de ruissellement, des avalanches, etc. Par suite, dans les stations fréquemment exposées, d'une manière plus ou moins permanente, à des phénomènes de ce genre, ils sont moins à leur place que les peuplements irréguliers; ces derniers, d'ailleurs, sont toujours plus aptes à produire des peuplements sains, plus susceptibles de résister aux dangers extérieurs.

$4^{\circ}$ Repeuplement. - Certaines méthodes excluent des formes déterminées de peuplements, et inversement, suivant les âges d'exploitation que comporte une forme de peuplement, la création des nouveaux sujets ne peut s'effectuer de la mème manière.

Les formes du taillis simple et du taillis composé ont recours au repeuplement par rejets et drageons, combiné dans une certaine mesure, pour assurer la perpétuité de la forêt, arec d'autres inéthodes de repeuplement; les formes de futaie ont recours à la régénération naturelle ou à la régénération artificielle suivant l'àge d'exploitation (supérieur ou inférieur à l'âge de fertilité des sujets), oubien elles demandent une combinaison de ces deux méthodes (semis naturels incomplets).

Dans les limites des circonstances où cela est possible, il est économiquement plus avantageux d'obtenir la per- 
pétuité de l'état boisé par voie naturelle plutôt que par voie artificielle.

Dans les formes de taillis, les conditions les plus farorables au repeuplement naturel sont :

$1^{0}$ Jeunesse et état sain des souches, qui doivent disparaître, dès qu'elles n'ont plus la vitalité suffisante, et qu'elles occupent sans profit un espace où peuvent venir s'installer des semis naturels ;

$2^{\circ}$ Exploitations faites à des âges relativement peu élevés, c'est-à-dire dépassant rarement trente à quarante ans, pour assurer la formation de rejets nombreux et vigoureux, mais en général suffisamment élevés pour amener la disparition complète des morts-bois et des bois blancs ;

$3^{\circ}$ Présence sur le taillis ou à proximité de portegraines disséminés, susceptibles d'assurer par ensemencement naturel le renouvellement progressif de l'ancien peuplement ;

$4^{\circ}$ Existence, dans une certaine mesure, d'un couvert supérieur ou latéral susceptible, pendant les périodes d'exploitation du taillis, de protéger le sol, la couverture morte et l'humus contre le dessichement et les jeunes sujets (rojets, drageons, semis) contre les danger's extérieurs, tout ou moins pendant la période de toute première jeunesse.

Sauf dans le cas de circonstances exceptionnelles dues au climat (par exemple, climat méditer'anéen ou du midi), au sol et à la fertilité de la station, le taillis comjosé remplira mieux toutes ces conditions et sera prélérable au taillis simple.

Dans les formes de futaie, l'emploi de la régénération nalurelle ne sera possible (qu'avec des iges d'exploilation suffisamment élevés pour permettre aux portegraines de fructilier d'une façon abondante et soutenue, et les conditions les plus favorables à cette régénération naturelle sont : 


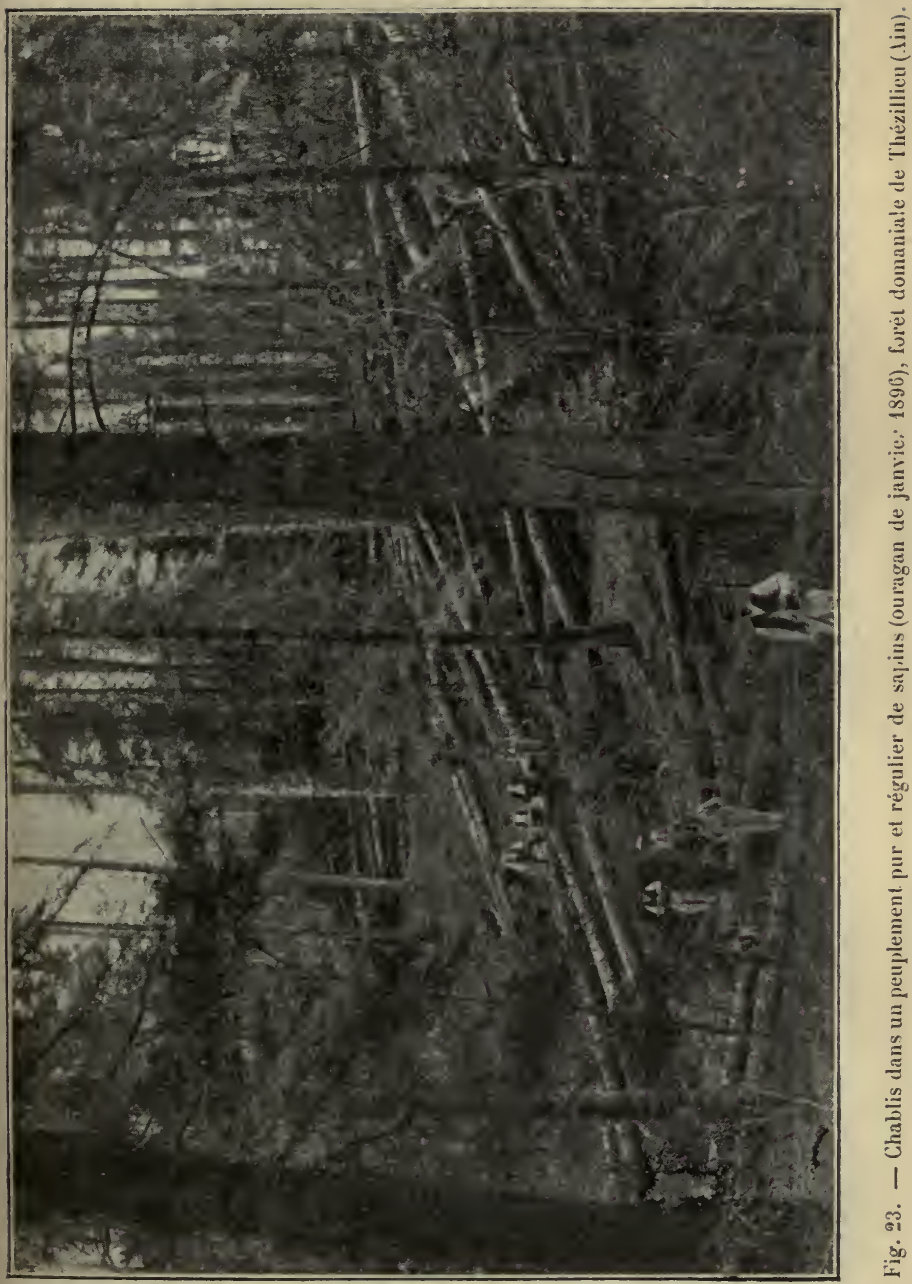


$1^{\circ}$ Sujels arlultes, plus ou moins isolés, ayant par suite une cime développée; recevant plus de lumière, ces sujets produisent plus de graines;

$2^{\circ}$ Sol apte à recevoir la graine et à la faire germer ;

$3^{\circ}$ Jeunes plants plus ou moins protégés par un couvert supérieur ou latéral pendant la première période de leur existence.

Les formes irrégulières de peuplements remplissent ces conditions moins imparfaitement que les formes régulières.

Mais quand toutes les conditions d'une bonne régénération naturelle font défaut, l'emploi de la régénération artificielle est indiqué ; toutefois, on doit souventchercher une forme qui assure l'abri nécessaire à la jeune plantation.

Conclusion. - La comparaison entre elles des formes de peuplement, au point de vue cultural, nous conduit à cette conclusion que la culture forestière doit avoir pour but, non seulement l'exploitation, mais encore la conservation intégrale des forces de production, et que dans bien des cas, une gestion qui n’a en rue qu'un placement pécuniaire aussi élevé que possible est exposée à oublier cette condition.

Nous verrons dans la suite que la comparaison entre le revenu d'une forèt et le capital engagé dans l'exploilation forestière, donne le taux de placement de ce capital argent.

Ciest la recherche du maximum de taux de placement qui conduit le propriétaiı particulier à des exploitations extensives, arec des âges peu élevés; celte exploitation extensive; que peuvent justifier les considérations économiques du moment, doit toujours avoir pour limite les obligations imposées par les règles culturales, sinon le propriétaire appauvrit peu à peu et systématiquement son domaine boisé. Comme il ne fait pas intervenir cet élément essentiel dans son calcul de placement, les 
chiffres qu'il obtient sont trompeurs, quelles que soient les combinaisons savantes qui aient permis de les trouver. Mieux vaut alors demander franchement à d'autres placements, aussi commodes et aussi sûrs, si on les trouve, le taux exigé.

Sans entrer dans le détail des cas particuliers, où le taillis simple peut ètre justifié et où la substitution des essences résineuses aux essences feuillues peut être a vantageuse, nous croyons pouvoir dire que le propriétaire particulier a aujourd'hui intérèt :

$1^{\circ} \mathrm{A}$ préférer, partout où cela est possible pour le traitement des essences feuillues, le taillis composé au taillis simple, sauf dans des circonstances toute spéciales et justifiées;

$2^{\circ} \mathrm{A}$ adopter, dans ces taillis composés, des révolutions assez longues (vingl-cinq à trente-cinq ans) pour l'élément taillis, et à conserver un nombre suffisant de réserves ;

$3^{\circ} \mathrm{A}$ abandonner (sauf exception) les futaies feuillues et à ne pas adopter pour les futaies résineuses de trop longues révolutions, en raison du faible taux auquel fonctionnent ces futaies.

\section{II. - COMPOSITION DES PEUPLEMENTS}

Un peuplement peut ètre composé d'une seul essence, il est dit pur' ou bien il peut être formé d'un mélange de deux ou de plusieurs essences diversement associées, il est dit mélangé.

Peuplements purs. - Dans la généralité des cas, la nature a une tendance à provoquer spontanément des mélanges; les peuplements purs ne seraient donc qu'une rare exception si la nature et l'homme n'étaient intervenus; d'une part la nature, en créant des stations où ne peut vivre qu'une seule essence, soit en raison de l'altitude et du climat (stations rudes, à climat rigoureux 
de l'épicéa, par exemple), soit en raison de situations toutes locales, telles que : excès d'humidité (aune, saule), excès de sécheresse et de stérilité (pin sylvestre!, etc.); d'autre part l'homme, en propageant, à l'exclusion des autres, l'espèce susceptible de répondre à ses besoins, en vue de produits déterminés, tels que perches, bois pour pâte à papier, bois de charbonnage, bois d'œuvre ordinaire, osiers pour la vannerie, écorces à lan, elc.

Enfin de simples erreurs ou des fautes culturales ont pu conduire accidentellement à la création de ces peuplements purs. C'est ainsi, par exemple, qu'après des coupes à blanc, dans les futaies de chène et de hêtre, le sol peut se trouver envahi par des semis de charme pur; que le bouleau ou des essences secondaires se substituent seules aux bonnes espèces dans un sol appaurri par des abus de jouissance, etc.

Peuplements mélangés. - Dans la forèt livrée à ellemème, le mélange des essences s'effectue par les mille moyens dont dispose la nature; les graines légères, ailées, sont disséminées par le vent; les graines lourdes sont transportées au loin par les oiseaux, les rongeurs et mille causes accidentelles; là où elles tombent, elles peuvent trouver des conditions favorables à leur germination et à leur développement ultérieur, et elles constituent un mélange par sujets isolés.

Ailleurs, le mélange s'effectue par groupes ou bouquets, soit que les graines lourdes, tombées au pied de leurs porte-graines, aient trouré des conditions favorables à leur développement, soit que des conditions locales se pretent mieux à l'installation et au maintien d'une essence déterminée, au détriment de toutes les autres; telles peuvent ètre des conditions de sol, un état plus ou moins favorable de la couverture morte, l'humidité, la présence ou l'absence d'un couvert bas el épais, etc.

Ainsi, dans la nature, les essences ont une tendance naturelle à se mélanger par pieds isolés et par bouquets; 
ces deux formes de mélange se présentent sourent simultanément et le mélange peut persister, grâce à la différence des stations, à la différence du tempérament des diverses essences, causes auxquelles s'ajoute une plus ou moins grande rapidité dans la végétation de ces essences.

Dans la forèt cultivée, l'homme qui se propose un but déterminé, intervient pour diriger le rélange, spécialement dans les formes de peuplement qui s'écartent le plus des conditions naturelles. Dès lors, il ne lui suffit pas de créer ou de provoquer ce mélange, il doit savoir maintenir l'équilibre entre des voisins qui luttent à armes inégales. Cette question des mélanges, disent MM. Boppe et Jolyet, est une des plus délicates qui se présentent en sylviculture; il faut toutes les ressources de l'art forestier pour obtenir, soit naturellement, soit artificiellement, la composition voulue; chaque station demande pour ainsi dire un mélange différent; mais en cherchant bien, surtout en interrogeant autour de soi, on trouvera toujours une solution satisfaisante sans sortir des essences spontanées.

Avantages et inconvénients des peuplements mélangés. - Des raisons culturales de premier ordre indiquent que le mélange est l'état de peuplement le plus à désirer dans la plupart de nos stations :

$a$. Le peuplement mélangé, par sa nature mème, s'adapte aux inégalités locales des stations : ses divers éléments qui trouvent, dans la place qu'ils occupent, un sol en rapport a rec leurs exigences, leur enracinement, etc., lui permettent de conserver une croissance également bonne dans toute son étendue, ce qu'il est difficile d'obtenir arec un peuplement pur; par suite, le peuplement mélangé est plus complet dans son ensemble, son état de massif est meilleur, et il utilise mieux la fertilité du sol, ainsi que la lumière disponible.

$b$. L'état de mélange favorise, dans un grand nombre de peuplements, le maintien prolongé du massif, et nous 
avons vu que c'est surtout dans les àges avancés que cette densité du massif est utile en vue des qualités du sol pour une bonne régéuération. Le peuplement mélangé est, à ce point de vue, bien préférable aux peuplements purs qui tous, d'une façon variable, il est vrai, suivant le tempérament de l'essence, ont une tendance à éclaircir leur massif au fur et à mesure qu'ils deviennent plus âgés. En outre, sous un peuplement mélangé, les feuilles qui tombent sur le sol ont des consistances diverses; elles se décomposent plus facilement et la couche de terreau formé est plus épaisse et mieux constituée.

Ainsi, par un couvert plus complet et plus prolongé, par les qualités de la couverture morte et de l'humus, le peuplement mélangé tend à maintenir, bien mieux et plus longtemps que les peuplements purs, la fertilité de la station et l'ameublissement du sol; par suite, il est plus favorable au réensemencement naturel et assure plus facilement la continuité de l'existence de la forèt.

c. Le mélange présente sur les peuplements purs le mème avantage au point de vue de la résistance aux dangers extérieurs, tels que vents violents (essence pivotante adjointe aux essences à enracinement superficiel), bris de neige et de givre (essence à feuillage carluc asscciée à l'essence plus sensible à feuillage persistant), froid (essence résistante jointe à une essence sensible), incendies (essence feuillue avec essence résineuse), insectes et champignons, etc.

d. Le mélange assure au point de vue économique une plus grande variété dans la production; par cela mème, il augmente l'utilité de la forèt dont les produits sont, en général, plus faciles à écouler dans le commerce. Par la concurrence vitale, il tend à améliorer la croissance de certaines essences, modifiant avantageusement la forme de leur fût; c'est ainsi que certaines essences feuillues (le listre par exemple) en mélange avec lles essences résineuses (sapin, épicéa) tendent à suivre la croissance 
en hauteur de ces dernières et à allonger considérablement leurs fûts.

Enfin, le peuplement mélangé, présentant moins de fixité que le peuplement pur, se prète, au moins dans certaines limites, bien mieux que ce dernier à des modifications daus les exploitations.

$e$. Par contre, au point de vue de la gestion, le peuplement mélangé est souvent plus difficile à diriger que le peuplement pur, parce qu'il y a lieu de satisfaire, d'une façon permanente, aux exigences de toutes les essences, et que des fautes culturales répétées peuvent provoquer la disparition d'essences précieuses et l'envahissement d'essences de second ordre.

Exigences des mélanges. - Quatre facteurs essentiels agissent sur le peuplement mélangé et exercent sur les direrses phases de son existence une action prépondérante. M. Gayer les énumère dans l'ordre suivant :

a. Station. - La station doit ètre de nature à pouvoir satisfaire toutes les exigences de chacune des essences du mélange ; sinon l'essence la moins favorisée disparait progressivement du mélange.

b. Fertilité de la station. - Le mélange ne doit pas ètre de nature à ruiner la fertilité de la station, sinon le mélange est pire que le peuplement pur par suite de l'éclaircissement prématuré qu'il subit spontanément.

c. Tempérament. - Chaque essence doit trouver dans le peuplement les conditions d'espace (espace pour la cime dans l'atmosphère et pour les racines dans le sol) et de lumière qui conviennent à son caractère biologique ; et cette situation doit persister pendant toute son existence; sinon l'essence ne pourra pas conserver dans le peuplement une bonne croissance et tendra à être éliminée.

d. Forme du peuplement. - Le peuplement doit être traité dans une forme appropriée aux essences qui le constituent et au but que l'on se propose; le choix judi- 
cieux de la forme du peuplement est une des conditions les plus imporlantes de l'existence des mélanges, et du maintien jusqu'à l'exploitabilité de chacune des essences qui le composent.

Dans un peuplement régulier, mélangé par sujets isolés, les espèces associées n'ont pas la mème activité de régétation à leurs différents âges; ces différences peuvent s'égaliser avec l'âge, mais dans la plupart des cas, le mélange originel tend de plus en plus à se transformer en un peuplement pur; qu'on associe, par exemple, à une essence de lumière une essence d'ombre, dont les tendances envahissantes par sa nature, sont encore exagérées par une meilleure adaptation au milieu; la première succombe toujours victime de la seconde. Il en est ainsi du chêne et du hêtre dans les forèts du nord et de l'est de la France, où le hètre qui se trouve dans le centre de son aire, aura toujours des tendances à dominer le chène, et l'aura bientôt éliminé, si par des éclaircies bien conduites, on ne vient pas sans cesse le cantonner dans le rôle secondaire qui lui est dévolu; ainsi encore du hêtre et du sapin aux altitudes un peu considérables (Boppe et Jolyet).

il résulte de ces considérations qu'on est conduit à accompagner le peuplement mélangé, depuis son origine jusqu'à sa vieillesse, de soins passagers, dans le but de protéger l'essence menacée, intervenant toujours au moment et dans le sens voulu.

Des soins culturaux de ce genre, d'un caractère passager et par suite à renouveler pendant toute l'existence du peuplement, exigent une main d'œurre adroite et sourent assez dispendieuse.

Mais on peut tendre à maintenir le mélange par d'autres mesures spéciales présentant un caractère permanent :

$1^{\circ}$ En faisant préexister, toutes les fois que cela sera possible, l'essence menacée, c'est-à-dire en faisant pren- 
dre au moment de la régénération à un certain nombre de sujets de cette essence l'avance sur les autres essences ;

$2^{\circ}$ En opérant le mélange, non plus par sujets isolés, mais par bouquets, par groupes ou par compartiments plus ou moins étendus, en créant en un mot, suivant l'expression de MM. Boppe et Jolyet, des peuplements de composition zébrée ou mouchetée; dans un tel peuplement, chaque espèce est ainsi isolée sans toutefois perdre les avantages du mélange; elle peut ètre traitée à l'état pur dans chaque bouquet, suivant les besoins qui lui sont propres; les sujets de l'essence menacée sont ainsi préservés de l'influence envahissante et peuvent ètre maintenus jusqu'à leur exploitabilité; si l'étendue des bouquets est réglée sur la susceptibilité de l'essence menacée, l'effet obtenu pourra ètre permanent;

$3^{\circ}$ En faisant préexister à l'essence envahissante les bouquets de l'essence à protéger; l'état du peuplement ainsi mélangé est celui qu'on obtient au moyen du traitement par coupes jardinatoires, qui se prète mieux que tout autre à la conduite et au maintien des peuplements mélangés.

Composition des peuplements mélangés. - Nous avons vu que tout mélange doit être susceptible de se prèter à l'entretien du sol et aux exigences natuelles de chaque essence; accessoirement, il doit prémunir le peuplement contre les dangers extérieurs.

Pour satisfaire à ces conditions, on cherchera en principe et sauf exceptions : $1^{\circ}$ à introduire en majorité dans les mélanges les essences d'ombre; $2^{\circ}$ à associer aux esśences résineuses des essences feuillues.

Remarquons en outre qu'on doit tenir compte dans la détermination du mélange, du but de l'exploitation, en particulier de la valeur industrielle des essences, et dans certaines situations de leur résistance aux agents extérieurs. 
Role du hêtre dans les mélanges. -- C'est le hètre surtout qui, par sa faculté unique de conserver intégralement la fertilité et l'humidité du sol, de se prèter merveilleusement à toutes les combinaisons, mérite de fixer l'attention des sylviculteurs.

MM. Boppe et Jolyet exposent ainsi son rôle dans les mélanges : le hètre vit en plaine comme en montagne; son aire d'habitation, très étendue, englobe toutes les espèces, auxquelles il peut ètre associé comme essence d'ombre. C'est, dès lors, l'espèce indiquée pour faciliter les transitions entre deux stations voisines, dans ces zônes indécises; si délicates à manier, où une espèce va disparaître quand l'autre n'est pas encore bien installée; entre la région du chène et la sapinière par exemple, ou à la limite supérieure de celle-ci, entre la forèt du rendement et le pâturage.

D'ailleurs son tempérament plastique lui permet d'accepter tous les rôles; au gré du forestier, il sera sur le mème point l'essence précieuse atteignant les plus grandes formes ou le modeste buisson végétant en sousbois. Nous connaissons trop de forèts qui ont été viclimes d'une expulsion systématique du hètre, pour ne pas demeurer convaincus qu'on a tout à gagner en lui réservant la grande place qu'il mérite; mais c'est à la condition de rester toujours son maître, sans jamais se laisser dominer par lui.

Conclusion. - 11 résulte des considérations précédentes que, d'une façon générale, les peuplements mélangés doivent ètre préférés aux peuplements purs, et que dans la plupart des cas, ce sont les formes irrégulières de peuplements, et dans ces formes le mélange par groupes, places ou bouquets qui farorisent la constitution et le maintien des mélanges. 


\section{DEUXIÈME PARTIE}

\section{PRATIQUE SYLVIGOLE}

Dans toute exploitation sylvicole raisonnée, l'homme exerce son influence sur la forèt; il la suit dès la naissance et la dirige pendant toutes les phases de son existence afin de l'amener à l'état de production qu'il considère comme le plus avantageux. Il ne peut agir au hasard sans s'exposer aux plus graves mécomptes ; il doit a voir pour guide des règles de pratique sylvicole, tant pour créer artificiellement une forèt, que pour obtenir et diriger le repeuplement naturel; tant pour régler les différentes opérations relatives aux coupes, que pour disposer sur le terrain l'ordre de ces exploitations et en assurer d'une façon permanente la bonne gestion.

Nous nous proposons d'examiner successivement les pratiques de la sylviculture qui permettent d'agir au mieux des intérèts d'une exploitation sylvicole. Nous étudierons dans cette partie le repeuplement, les opérations culturales, et les principales mesures de gestion. 


\section{PREMIERE SEGTION \\ Repeuplement}

Le repeuplement consiste à oblenir la naissance d'un nouveau peuplement ou d'une partie de peuplement; il peut avoir pour but soit de créer de toutes pièces une forêt, soit d'assurer la perpétuité de la forêt existante sur le domaine boisé, perpétuité qui comporte l'enlèvement plus ou moins rapide du vieux matériel exploitable et son remplacement par de la jeunesse. Cette création de sujets nouveaux peut ètre obtenue de différentes manières :

$1^{\circ}$ Par voie artificielle (se mis ou plantation); $2^{\circ}$ par semis naturel (régénération naturelle); $3^{\circ}$ par rejets de souche et drageons $; 4^{\circ}$ par combinaison de ces différentes méthodes.

\section{I. - REPEUPLEMENT ARTIFICIEL.}

Généralités. - Un repeuplement est dit artificiel lorsqu'il est obtenu par l'intervention directe et immédiate de l'homme; les travaux résultant de cette intervention sont désignés sous le nom général de travaux de repeuplement.

Cas ou il faut avoir recours au repeuplement artificiel. - On peut avoir à effectuer le repeuplement artificiel dans deux cas: $1^{\circ}$ dans une iorêt préexistante; $2^{\circ}$ sur des terrains nus ou dépourvus de forêts:

Repeuplement artificiel dans une forêt. - Dans la 
gestion d'une forèt, différents cas peuvent se présenter où il est nécessaire d'intervenir :

a. Dans les futaies, le semis naturel sur lequel on comptait, peut faire défaut partiellement ou même totalement dans un grand nombre de circonstances dont les principales sont : $1^{\circ}$ les porte-graines trop rieux ne donnent plus de graines fertiles; 20 la régénération est manquée sans qu'il soit possible d'y porter remède; $3^{\circ}$ le sol est complètement envahi par de hautes herbes; $4^{\circ}$ la vidange a détruit une partie des jeunes plants, etc.

$b$. Dans le taillis et le taillis composé, les brins de semence nécessaires au balivage ainsi qu'au remplacement des vieilles souches usées, peuvent manquer soit qu'ils aient été étouffés dans leur jeunesse par les essences secondaires, soit que les porte-graines se trouvent trop espacés ou inféconds.

c. Dans beaucoup de forêts, il existe des vides plus ou moins étendus qui peuvent rompre l'état de massif du peuplement.

d. Dans une forèt, on peut avoir à introduire soit une essence nouvelle donnant des produits plus précieux, soit une essence accessoire de mélange utile pour permettre à l'essence principale de se développer plus avantageusement.

$e$. On peut enfin, dans une forèt, substituer complètement la régénération artificielle à la régénération naturelle, soit que les bois soient exploités trop jeunes pour donner des semis, soit que l'on veuille obtenir très rapidement la régénération.

Dans tous ces cas, le repeuplement artificiel s'impose, et sauf peut-être dans le dernier où il s'agit de régénérer artificiellement toute la surface du repeuplement, il présente un caractìre d'ordre général, plutôt qu'un caractère immédiat de spéculation ; autrement dit, l'intervention $d u$ repeuplement artificiel se manifeste dans 
l'économie générale de l'exploitation forestière, moins par les produits bruts qu'il est susceptible de fournir dans un temps donné, que par l'effet qu'aura ce repeuplement sur la reconstitution de l'état de massif et par' suite sur le maintien de la fertilité de la station; à ce titre, des travaux de ce genre sont fréquemment d'une très grande importance et ils se justifient pleinement, à la condition toutefois qu'on agisse avec une grande prudence et en connaissance de cause. Il faut se rappeler qu'en agissant trop vite et sourent mal à propos, on s'expose à dépenser inutilement son argent. Repeuplements artificiels sur terrains nus ou boisements. - Les travaux de repeuplement de ce genre présentent en général un caractère d'intérêt privé; c'est un placement qu'effectue le propriétaire, placement sur lequel il compte réaliser un bénéfice; une bonne administration doit calculer à l'avance les recettes probables et les dépenses de l'opération.

Ghoix des essences. - Avant d'entreprendre un repeuplement artificiel, il est toujours essentiel de déterminer les essences qui s'adapteront le mieux aux conditions de climat, de sol, de station et de situation dans lesquelles on se propose de les placer; et cela est aussi vrai, qu'il s'agisse de reconstituer ou d'améliorer un massif, que de créer une forệt nouvelle. C'est ainsi qu'on ne mettra pas du chêne pédonculé dans un terrain sec ; du sapin en plaine ou à l'exposition du midi; du châtaignier dans des terres calcaires; du pin maritime sous les climats rudes, du hètre dans les plaines de Provence, etc.

En outre, si cela est possible, on doit donner la préférence aux essences qui permettront de réaliser les produits les plus avantageux.

Pour effectuer un repeuplement, on peut disposer: $1^{\circ}$ des essences indigènes; $2^{\circ}$ des essences exotiques.

10 Essexces nimgixes. - Nous possédons dans nos forèts une série d'arbres appropriés à nos différents 
climats, à nos différents sols, susceptibles de donner du bois d'excellente qualité el doués d'une vitalité et d'une résistance parfailes. En les utilisant, nous aurons toutes les chances possibles de réussir; nous les rappelons dans le tableau suivant:

\section{Essences convenant aux diverses natures de terrains à repeupler (1).}

\begin{tabular}{|c|c|}
\hline $\begin{array}{l}\text { Sols. } \\
\text { iliceux, sablonneux } \\
\text { arides. }\end{array}$ & $\begin{array}{l}\text { Essences. } \\
\text { Pin maritime (climats doux), pin syl- } \\
\text { vestre, chêne tauzin, pin d'Alep, chêne } \\
\text { yeuse (climats du midi de la France). }\end{array}$ \\
\hline
\end{tabular}

Siliceux, sablonneux|Résineux en général, chêne rourre, frais. charme, châtaignier, bouleau, robinier.

Légers, granitiques.|Mêmes espèces que ci-dessus; sur les coteaux : le hêtre; dans les vallées : le frêne.

Siliceux-argileux. Pin sylvestre, épicéa, sapin, chênes rouvre et pédonculé, hêtre, chàtaignier, orme, charme, bouleau, írable, frène.

Calcaires.

Pin noir d'Autriche, pin Laricio de Corse, hêtre, érable sycomore, robinier, pin d'Alep (midi), pin sylvestre, épicia; sapin (pourvu que la proportion de calcaire ne soit pas trop considérable).

Marécageuxassainis.|Aune commun, épicéa, pin sylvestre, saule, frêne.

Sols à fonds mouillés, Aune commun, frêne, peuplier, saule. sujets à être inondés.

Bruyères et landes. Pin sylvestre, pin maritime (région sudouest).

Toutefois, il est intéressant de noter qu'on doit dans une certaine mesure tenir compte des influences ayant

(1) Extrait de l'Agenda du forestier.

A. Fron. - Sylvicullure. 
pu modifier les espèces et créer des variélés dans certaines régrions sous l'influence d'un climat spécial.

Bien avant Cieslar (1), il y a plus de soixante-dix ans, M. de Vilmorin, frappé des caractères spéciaux qu'affectait le pin sylvestre dans les différentes régrions d'Europe oì il est spontané, a pu créer aux Barres, entre 1821 et 183\%, une série de places d'expériences, de l'observation desquelles il résulte d'une manière certaine que les variations sont transmissibles par graine. Ces observations, confirmées du reste par celles qui sont actuellement poursuivies en Allemagne et en Autriche, nous amènent à conclure que les essences forestières ne doivent pas être dépaysées et qu'il faut, autant que possible, choisir pour les semer ou les planter des graines ou des sujets provenant de la contrée.

M. Gayer est du mème avis; il conseille cependant de recourir à la variété étrangère lorsqu'on se proposera de faire disparaìtre des tendances fâcheuses, anormales, d'arrêter une dégénérescence résultant notamment d'un mauvais sol.

Pour définir les essences indigènes les plus importantes à utiliser, il y a lieu de faire une distinction, selon qu'il s'agit d'opérer sur un sol nu ou sous une forèt préexistante.

Dans le premier cas, repeuplement en sol $n u$, on ne dispose pas d'abri pour protéger les jeunes arbres au début de leur existence; le choix des essences à employer, dites essences de premier boisement, est alors trìs restreint. On doit se limiter aux essences suivantes, tout en se conformant aux exigences de climat et de sol spéciales à chacune d'elles :

$1^{\circ}$ Sur les sols paurres : les pins;

$2^{\circ}$ Sur les sols plus ou moins fertiles : les pins, l'épi-

(1) Il résulte de nombreuses observations faites par Cieslar à la station d'essais de l'́lcole forestière de Mariabrünn que les végétaux forestiers contractent des labitudes de climat transmissibles par graines. 
céa, le mélèze, et parmi les essences feuillues: le chẻne, le frêne, l'érable, les ormes, l'aune, le bouleau, les saulis, les peupliers.

Remarquons toutefois, en ce qui concerne les essences feuillues et particulièrement le chène, que dans beaucoup de cas, surtout quand il s'agit de reboiser des sols épuisés, il sera généralement indispensable d'employer les pins comme essence de premier boisement ou plutot comme essence transitoire, puis de profiter de leur abri pour introduire ou laisser s'introduire naturellement les essences feuillues.

Comme essences transitoires, on peut choisir des essences rustiques, spontanées elles-mèmes dans la contrée; par exemple, sur bien des points l'aune, le bouleau; dans le sud-est le pin d'Alep; dans le sud-ouest, le pin maritime; mais en général, à défaut de ces essences on emploiera le pin sylrestre (sur tous les sols et dans toutes les stations) et le pin noir d'Autriche (de préférence sur les sols calcaires).

Dans le second cas, repeuplement sous une forét préexistunte, on dispose d'un certain abri pour protéger les jeunes arbres au débul de leur existence.

Le choix des essences à employer est, dès lors, bien moins limité, et on peut, en raison des abris donnés par la forêt, introduire toutes les essences indigènes, en se conformant, bien entendu, aux exigences qui leur sont propres, notamment en ce qui concerne le climat, l'altitude, l'exposition et aussi la lumière nécessaire.

Nous pouvons ajouter à ce qui a été dit précédemment en ce qui concerne nos principales essences, qu'on distingue pour chaque région un climat général et pour chaque point de cette région un climal local.

En tenant compte du climat général seulement, MI. Mathieu a divisé au point de vue forestier notre pays en quatre zones climatériques que nous reproduisons dans le tableau suirant : 
Régions.

Essences :

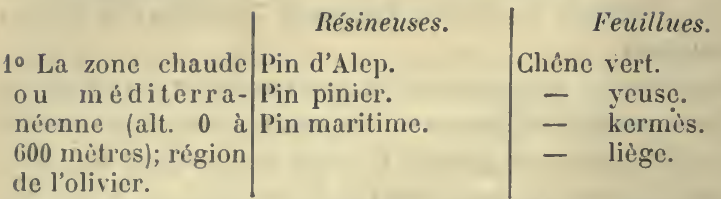

2. Zone tempérée Pin maritime dans|Chêne rouvre. (alt. de 600 à les parties chaudes. - pédonculé. 1000 mètres); ré- Pin sylvestre. gion du chêne. Sapin (exceptionnelFrène, orme, érable, cliarme, peuplier, châtaignier, ete. IIêtre exceptionnellement.

Aune glutineux.

\begin{tabular}{|c|c|c|}
\hline $\begin{array}{c}3^{\circ} \text { Zone froide ou } \\
\text { subalpine (alt. } \\
1000 \text { à } 1800 \text { mètres). }\end{array}$ & $\begin{array}{l}\text { Sapin, épicéa. } \\
\text { Mélèze. } \\
\text { Pin sylvestre. } \\
\text { Pin à crochets. }\end{array}$ & $\begin{array}{l}\text { Hêtre. } \\
\text { Érable sycomore. } \\
\text { Bouleau. } \\
\text { Sorbier des oiseleurs. } \\
\text { Aune blanc. }\end{array}$ \\
\hline
\end{tabular}

$4^{\circ}$ Zone très froide Mélèze. ou alpine (alt. 1800 Pin cimbro. à 3000 mètres).

Aune vert.

Cette division établie d'après les allitudes, n'a rien d'absolu el se trouvera constamment modifiée par une série de circonstances spéciales dont l'ensemble détermine un climat local, climat qui pour la mème altitude sera surtout différencié par l'exposition.

$2^{\circ}$ Essexces exotiques. - Les propriétaires de bois peuvent aussi, dans cerlains cas, faire avantageusement appel à certaines essences forestières étrangères.

Ce serait évidemment une lrès grosse erreur de donner la préférence aux arbres exoliques sur les indigènes; les premiers ne doivent jamais occuper dans les boisements qu'une place restreinte; appelés ainsi à jouer un ròle accessoire, ils peuvent entrer dans les peuplements sous forme de mélange. 
Les proscrire systématiquement serait certainement tomber dans une exagération inverse; il est en effet hors de doute que certains arbres étrangers sont susceptibles de nous rendre de grands services.

Plusieurs d'entre eux ont du reste déjà fait leurs preuves et ont pris place dans notre flore forestière; nous pouvons citer entre autres :

Le pin noir d'Autriche, essence en somme étrangère en France, l'arbre par excellence des terrains calcaires paurres ;

Le pin Weymouth, remarquable par sa croissance rapide lorsqu'il est placé dans les conditions qui lui conviennent;

Le robinier faux acacia, précieux pour la fixation des terres remuées;

Les platanes;

Le marronnier d'Inde;

Le peuplier du Canada, l'espèce actuellement la plus plantée peut-être du genre (fig. 24);

Le chêne rouge d'Amerique, à croissance très rapide, convenant parfaitement pour la mise en valeur des sables de fertilité moyenne;

L'aune cordiforme, qui rend des services dans les terrains calcaires pauvres ;

Et plusieurs arbres fruitiers, notamment l'amandier, le pécher, l'abricotier, le noyer qui, outre ses fruits, donne un bois de première valeur.

Le pin sylvestre lui-mème, n'est-il pas une essence étrangère dans la plus grande partie de notre France? et n'en n'est-il pas de mème du pin maritime dans le centre de notre pays, en Sologne par exemple?

D'autres essences forestières exotiques, encore peu représentées dans nos forèts, se sont, en plusieurs endroits, reproduites naturellement de semences sous nos climats $(1)$.

(1) L. PARDÉ, “ Étude sur les essences forestières exotiques dont la natura lisation semble possible en France "), Bulletin de la Société forestière française des amis des arbres, $1^{\text {er }}$ trimestre 1901. 
Ces arbres sont évidemment susceptibles d'une naturalisation complète dans nos pays; ils méritent de prendre place dans nos boisements, si d'autre part, ils fournissent en quantité et en qualité, des produits utilisables. De ce nombre sont en particulier :

Parmi les essences feuillues :

Le prunier tardif (prunus serolina Ehrh.), qui fournit un bois précieux et peut se contenter de sables relatirement pauvres;

Le noyer noir d'Amérique (juglans nigra, L.) qui, à la différence du noyer commun, peut ètre élevé en massif; il produit un bois de première valeur, mais exige des sols profonds et substantiels de bonne qualité;

Le carya blane (carya alba Nutt.) et le carya des pourceaux (carya porcina Nutt.) qui ont les mêmes mérites et les mêmes exigences;

Le chêne des marais (quercus palustris Michx), le meilleur peut-être des chênes américains pour nos pays ;

Le chêne à feuilles d'yeuse ou de Banister (quercus ilicifolia Wangh. vel Banisleri Michx.), de petite taille, mais précieux pour le boisement des terrains sablonneux arides, et aussi pour la création de sortes de tirés pour la chasse, etc., etc.

Parmi les essences résineuses:

Le thuya géant de Menziès ou de Lobb (thuya gigantea Nutt., vel Menziezii Dougl. vel Lobbii Hort.) qui fournit un bois léger propre à de nombreux emplois spéciaux;

Le faux cyprès de Lawson (chamxcypuris lawsoniana Parl.) qui présente le même intérêt ;

Les pins laricio de Corse et de Calabre, susceptibles de donner un fort rendement que nous aurions pu ranger dans la première catégorie;

Le pin à feuilles rigides (pinus rigida Mill.) qui peut rendre des services dans les sables mouvants humides, notamment dans les dunes du Nord, où le pin maritime ne peut résister; 
Le tsuga de Canada (tsuga canadensis Carr.) ;

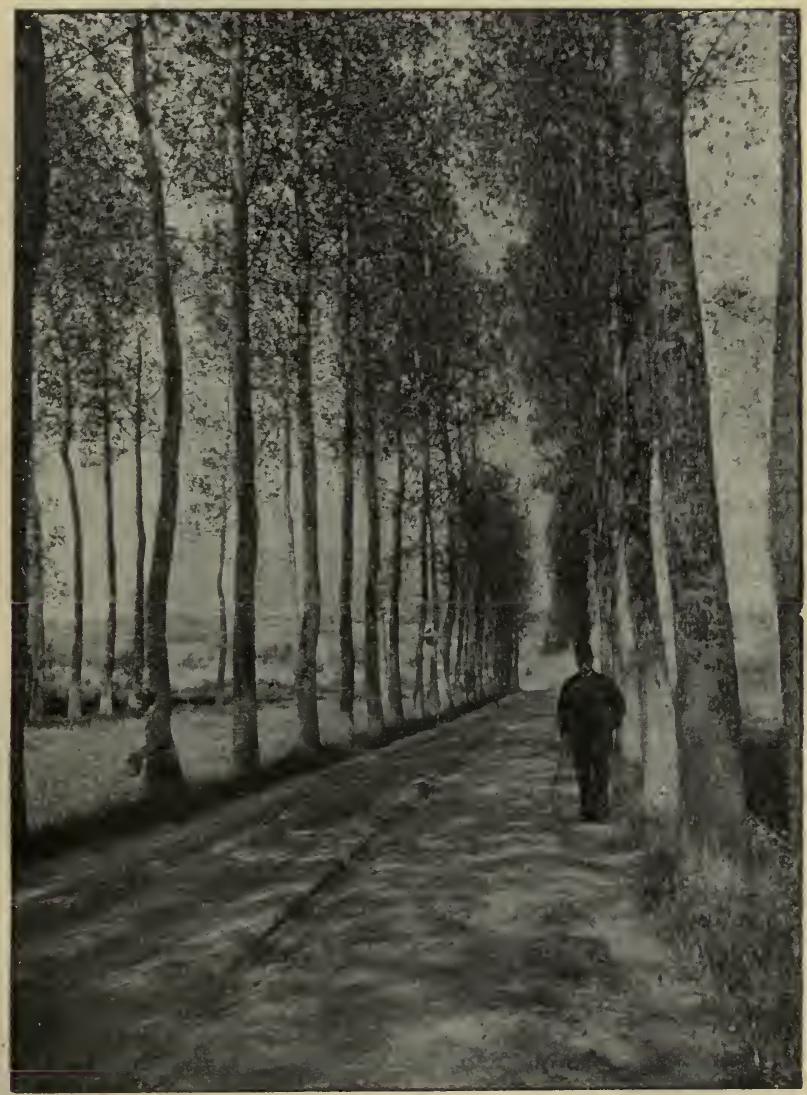

Fig. 24. - Avenue de peupliers âgés de dix-neuf ans. (P. du Canada) à Pontvallain (Sarthe). (Photographie de M. Sarcé.)

Le faux tsuga de Douglas (pseudo-tsuga Douglasii Curr.) 
qui par sa croissance rapide et par la qualité de son bois de cour, mérite certainement au plus haut degré une place, et mème une large place dans nos forêts ;

Les sapins d'Espagne (Abies pinsapo Boiss.), de Cilicie (abies Cilicica Carr.), de Céphalonie (abies Cephalonica Lond.), de Numidie (abies numidica de Lannoy), et de Nordmann (abies nordmanniana Spach) qui, comme l'espèce indigène, peuvent rendre des services dans le midi pour les quatre premières espèces, et mème dans le nord pour le sapin de Nordmann, etc.

Une troisième catégorie d'arbres étrangers, bien que n'ayant pas encore, du moins en nombre suffisant, donné de semis naturels, se comportent de telle façon sous nos climats, qu'on peut espérer leur naturalisation. Ces arbres peuvent aussi, mais à un degré moindre et en plus petit nombre, être plantés dans nos forèts. Tels sont:

Parmi les essences feuillues :

Le tulipier de Vivginie (Liriodendron tulipifera L.) qui donne un bois tendre el léger pouvant avantageusement remplacer célui des peupliers ;

Le cedréla de la Chine (celrela sinensis. A. Juss.) qui fournit un bois de grande valeur mais exige des terrains frais, profonds et meubles ;

Le frêne blanc d'Amérique (fraxinus alba Marsh. vel americana L.), plutot plus rustique et supportant mieux les inondations que notre frêne commun auruel il n'est pas inférieur du reste;

Le bouleau merisier (betula lenta L.) et le bouleau jaune (betula lutra Michx.), dont les bois sont si recherchés par les ébénistes;

Le zelkowa à feuilles crénelées (Zelkowa crenata Spach. vel crenata Desf.) el le zelkowa i feuillèes acuminées (Zelkowa acuminata Planch.), qui produisent un bois de premiẻre qualilé, mais demandent de bons sols; 
Le pterocarya du Caucase (pteraucaria cancasica C. A. Mey.) qui fournit également un bois de valeur, mais veut des terrains profonds et frais, humides mème;

Enfin pour le midi, plusieurs eucalyptus et plusieurs bumbous, notamment phyllostachys aurea A. et C. Rivière; phyllostachys mitis A. et C. Riviere; phyllostachys nigra IIunro; phyllostachys violascens A. et C. Rivière; Bambusa metake Sieb, bambusn fulcata Nees; etc., etc.

Parmi les essences résineuses:

Le libocèdre décurrent (libocedrus decurrens Torr.) qui donne un bois propre à des emplois spéciaux;

Le faux cyprès de Nutka (chamæcyparis nutkænsis spach. vel. thuopsis boreaiis Fisch.), l'analogue du faux cyprès de Lawson;

Le faux cyprès à foruits obtus (chamxcyparis obtusa Sieb. et Zucc.), le fameux hinoki du Japon, au bois si estimé;

Le genévrier de Virginie (juniperus virginiana L.), dont le bois, si recherché pour la fabrication des crayons, commence à manquer en Amérique ;

Le cyprès chauve (taxodium distichum Rich.), qui planté au bord des eaux, prend des dimensions énormes, et fournit un bois spécial;

Le pin de Banks (pinus banksiana Lamb.), susceptible de rendre des services pour le boisement des sables pauvres, dans les hautes régions et sous les climats très rudes;

Le méleze du Japon (larix leptolepis Murr, vel japonica Carr.) qui croìt très rapidement, et semble se comporter mieux que l'espèce indigène' aux altitudes relativement basses ;

L'épicéa de Menziès (picea Menziezii Carr. vel sitchensis Trautv. et Mey.) qui mérite certainement d'ètre planté dans les terrains humides;

Le tsuga de Californie (tsuga mertensiana Carr.) à croissance très rapide, beaucoup plus intéressant au point de vue forestier, que son congénère le tsuga du Canada;

Le sapin concolor (abies Concolor Lindl et Gord.) et le 
sapin élancé («bies grandis Lindl. vel gordoniuna Carr.), remarquables l'un et l'autre par leur belle croisance, etc., etc.

Enfin d'autres essences, moins connues ou d'introduction plus récente, méritent aussi d'ètre plantées dans nos forèts à titre d'essai. La liste, trop nombreuse, ne peut ètre donnée ici (1).

Pour ces dernières essences, il convient évidemment de n'opérer que par petites surfaces, et le plus souvent sous forme de mélange.

Il ne faut pas poursuivre l'acclimatation, qui n'existe guère en matière forestière, mais rechercher quelles peuvent ètre les espèces susceptibles de naturalisation. dans nos pays.

A un autre point de vue, il est inutile de rechercher à multiplier dans nos forèts des arbres exotiques qui fournissent en quantité et en qualité non supérieures, des produits analogues à ceux que donnent des esjèces indigènes.

En somme, - question de climat mise à part, - une essence étrangère mérite d'ètre introduite dans nos forêts :

10. Si elle fournit des produits de qualité supérieure à ceux que donnent les essences indigènes similaires;

2. Si elle produit, de qualité égale ou mème un peu moindre, une plus grande quantité de bois utilisable, dans un temps égal ou plus court;

$3^{\circ}$ Si elle donne des produits spéciaux que ne peuvent fournir les espèces indigìnes et que nous devons faire venir de l'étranger ;

(1) Consulter : L. PAndê, "Les principaux végétaux ligreux exotiques au point de vue forestier "). Congrès international de Sylviculture, Paris, 1900; et Bulletin de la Société foresticire de Franche-Comté et Belfort, décembre 1900. - Sсншаграсн: " Die Ergebnisse der in den preussichen Staatsforsten ausgefuhrten Anbauversuche mit fremdiandischen Ifolzarten ». Berlin, 1901. - Cirsi.an : "Ueber Anbauversuche mit fremdländischen Holzarten in Oesterreich $)$. Vienue, 1901. 
$4^{\circ} \mathrm{Si}$, quand bien mème elle donnerait un bois inférieur en quantité et en qualité, elle se recommande par sa frugalité, par sa résistance au vent, aux gelées, à la sécheresse; en un mot, si elle présente sur les espèces indigènes des a vantages précieux au point de vue de son adaptation à des conditions particulièrement difficiles de climat et de sol.

Il est bien évident qu'il ne faut introduire en forèt que des essences qui, plantées dans les écoles de botanique, dans les parcs et jardins, ont fait preuve d'une rusticité complète dans nos climats (1).

Quant aux conditions de sol et surtout de station, il est assez difficile, pour le moment, de les fixer; il faut pour cela attendre les essais qui doivent ètre faits précisément dans ce but; ces essais sont particulièrement intéressants à faire pour les propriétaires particuliers qui peuvent suivre les résultats plus longtemps que les agents' forestiers de l'État, trop souvent déplacés ; ils doivent naturellement être pratiqués en petit, porter sur de petites surfaces; ils doivent être faits dans un but pour ainsi dire scientifique, plutôt qu'arec une pensée de lucre; toute expérience comporte en effet forcément des chances d'insuccès.

Mode de repeuplement à employer. - Le choix de l'essence ou des essences que l'on peut employer étant déterminé, on peut procéder au repeuplement par semis, ou par plantation. Faut-il semer, faut-il planter? C'est une question qui se pose tout d'abord et on ne peut pas y répondre à priori; il n'est pas possible de prévoir avec rertitude dans tous les cas les résultats qui seront obtenus par le semis ou par la plantation, car ces résultats sont dus au jeu d'un trop grand nombre de facteurs.

(1) L'Arboretum national des Barres au milieu duquel se trouve l'École forestière des Barres, possède une très belle collection d'arbres forestiers exotiques. 
La nature, a-t-on dit, ne fait que semer; puisqu'on ne peut faire mieux qu'en limitant, il faut donc semer. Mais on oublie trop facilement que les forces naturelles ont pour elles le temps, et qu'elles procèdent toujours lentement et par progression. Quand un terrain est livré à lui-mème, il se recouvre de la végétation qu'il peut nourrir; ce sont d'abord des herbes, puis des broussailles, des morts-bois ; puis des essences rustiques qui demandent peu au sol, et ne craignent pas le plein découvert; puis enfin les espèces plus précieuses arrivent quand le sol est plus riche, plus frais et elles s'installent à l'abri des premières; l'abondance des graines assure ensuite le maintien de ces essences précieuses. En pratique, il n'est pas possible d'agir ainsi; ni le temps, ni la quantité de graines dont on dispose ne le permettent.

'Toutefois, le semis direct est possible si on dispose d'un terrain suffisamment profond et riche et si, d'un autre côté, l'essence à introduire est rustique et ne demande pas d'abri pendant sa première jeunesse. Malgré cela, il y a toujours cette différence capitale qu'on ne peut pas semer aussi abondamment que le fait la nature, qu'on ne place pas les graines dans des conditions aussi favorables à la grermination que le fait la dissémination naturelle, enfin que les graines qu'on sème, non seulement peuvent ètre mal conservées et avoir perdu, au moins partiellement, leur faculté germinative, mais encore qu'elles sont exposées à être détruites par un grand nombre d'animaux.

Une autre question intervient; c'est celle du prix de revient; Iongtemps on a pu dire que le semis coûtait moins que la plantation; actuellement, en règle générale, on considère la plantation comme plus expéditive, jolus sủre et mème souvent plus économique (en comptant les frais d'entrelien) que le semis.

Plus expéditive, dit M. Ragnéris, parce qu'on opère 
avec des plants déjà̀ d'un certain âge et qu'on s'aperçoit, dès la première année, des insuccès qu'on peut réparer immédiatement; avec le semis, on ne peut juger de la réussite qu'après plusieurs années; pour le pin sylvestre notamment, on voit beaucoup de semis et des plus beaux, périr par la défoliation entre la troisième et la sixième année.

Plus sûre, parce que la réussite d'un semis dépend de la qualité des graines; le plus souvent on est obligé de se les procurer par le commerce, et on est exposé à recevoir des semences vieilles, échauflées ou desséchées, ou recueillies avant la maturité; parce que, en supposant la graine de bonne qualité, on a à redouter toutes les circonstances défavorables à la germination, à craindre l'envahissement des plantes herbacées, etc.

Plus économique, parce que dans l'incertitude où l'on est de la qualité des semences, on sème toujours plus de graines qu'il n'en faut, et si toutes réussissent, le semis trop dru est exposé à languir ; parce que, s'il se produit des vides, il faut les repeupler à l'aide de plantations, qui ne s'exécutant pas d'une manière suivie, coûtent quelquefois autant que le semis primitif.

Malgré celte supériorité incontestable en général de la plantation, il faut recourir au semis quand on reut boiser de grandes surfaces avec les moindres frais, si on dispose d'une grande quantité de semences à bas prix, si la saison favorable aux repeuplements est de courte durée; enfin si la main d'œuvre nécessaire pour une plantation est trop dispendieuse. Le semis sera préféré en particulier: $1^{\circ}$ pour reboiser en pin maritime; $2^{\circ}$ pour reboiser en pin sylvestre, lorsque le sol, suffisamment, meuble et frais, est couvert d'une bruyère courte qui procure un abri aux jeunes plants, les préserve du déchaussement et permet de semer à la volée sans préparation du sol ; $3^{\circ}$ en plaine, pour reboiser en chène, lorsqu'il y a une glandée abondante

A. Frox. - Sylviculture. 
dans la région; on peut alors donner un labour à la charrue et semer les glands en mème temps qu'une demisemaille de seigle ou d'aroine; la récolte des céréales devra se faire à la faucille; $4^{\circ}$ en pays montagneux pour le chène, dans les taillis sartés; le semis de glands se fera la seconde anné; $5^{\circ}$ en général pour les essences franchement pirotantes.

La plantation devra être préférée dans les cas ci-après :

$1^{\circ} \mathrm{Si}$ la graine à employer est rare et ch ̀re; $2^{\circ} \mathrm{si} \mathrm{la}$ reprise des plants est facile; $3^{\circ}$ si le semis direct est difficile à réussir (bouleau, acacia); $4^{\circ}$ si le sol est compact ou très humide; $5^{\circ}$ si l'on peut avoir à craindıe une inondation; $6^{\circ}$ si le sol est envahi par les herbes, par les ajoncs ou par de hautes bruyères, ou s'il est susceptible de s'enherber; $7^{\circ}$ dans le fond des vallées ou encore sur les hauteurs, les plateaux élevés, en un mot, partout ò̀ les gelées ou les brouillards sont le plus à craindre; $8^{\circ}$ si le sol est crayeux, calcaire, granitique ou léger, parce qu'il est exposé à ètre soulevé lors des gels et dégels successifs, action à laquelle ne résistent pas les radicelles des jeunes semis; $9^{\circ}$ si les travaux doivent ètre exécutés sur des pentes rapides; $10^{\circ}$ s'il s'agit de repeupler des vides ou clairières de petite étendue entourées d'un peuplement constitué; ou si l'on reut introduire une essence nouvelle ou multiplier une essence déjà existante dans un peuplement; $11^{\circ}$ s'il s'agit de reboiser des terrains exposés à la dent du bétail ou du gibier. Dans ces deux derniers cas, il est toujours bon d'employer de forts plants, et la plantation devient très coûteuse; mieux vaut, cependant, dans ces cas particuliers, faire peu et bien que de s'exposer à n'obtenir aucun résultat.

\section{I. - Boisement par semis direct.}

Récolte et conservation dés graines. - Si on veut étre sůr de la qualité el de la provenance des semences, 
il faut les récolter soi-mème, et choisir convenablement les arbres; c'est ainsi qu'on recueillera de préférence les graines sur des arbres franchement fertiles, mais sains et vigoureux; les sujets trop jeunes ou trop vieux ne conviennent pas. La récolte se fait, autant que possible, par un temps sec; on gaule généralement les semences lourdes, au moment de la dissémination naturelle, après avoir ramassé les plus belles parmi celles qui sont déjà tombées, et avoir balayé les autres. On cueille à la main, aussitôt qu'elles sont mùres et avant qu'elles ne se détachent les semences légères, de même que les cônes des résineux. Mais cette récolte demande une main-d'œurre dont on ne dispose pas toujours; la conservation des graines récoltées exige pas mal de soins, aussi le propriétaire s'adresse-t-il en général au commerce. Nous donnons toutefois, sans entrer dans plus de détails, un court résumé sur la récolte des principales graines:

Alisier. - Maturité automne; semer immédiatement ou stratifier dans le sable avec le péricarpe et semer au printemps suivant.

Aune. - Maturité automne; le cône s'ourre, mais ne se désarticule pas comme celui du bouleau; dissémination hiver sur la neige ou au premier printemps ; conservation facile, trois ans environ.

Bouleau. - Maturité automne; le cône se désarticule; dissémination jusqu'en novembre. Éviter d'acheter la graine au commerce; la récolter plutôt soi-même; la conservation en est très difficile. Semer à l'automne ou bien très dru au printemps suivant.

Cèdre. - Maturité décembre; les cônes recueillis sur les arbres sont plongés dans l'eau de vingt-quatre à trentesix heures et la désarticulation s'opère sans difficulté. La graine, très chargée en résine, est d'une conservation difficile. Semer au printemps suivant. (La transplantation du jeune plant se fait très difficilement; transplanter en motte).

Cerisier. - Maturité été; semer immédiatement ou conserver les noyaux dans du sable frais. 
Charme. - Maturité automne; semer immédiatement ou stratifier dans la terre pour semer au printemps.

Châtaignier. - Maturité automne; semer automne si on ne redoute pas les rongeurs, sinon, conserver en silos et semer printemps.

Chêne. - Maturité automne; semer immédiatement si on ne redoute pas les rongeurs; sinon, conserver en silos et semer printemps.

Coudrier-noisetier. - Maturité automne ; semer immédiatement. Conservation très difficile.

Cytise. - Maturité automne; graine d'une conservation facile, à semer au printemps. (Plante aimant le calcaire el réussissant sur les calcaires arides).

Épicéa. - Maturité automne; récolte des cônes en septembre-octobre; extraction au soleil sans frais (ne pas amonceler les cônes pour éviter l'échauffement des graines). Conservation assez facile quelques années, mais semer de préférence au prinlemps suivant. 124000 graines au kilogramme.

Érable. - Maturité septembre-octobre; semer automne ou printemps suivant.

Frêne. - Maturité automne; dissémination: hiver et printemps suivant; semer automne de l'année ou stratifier les graines et semer au printemps.

Genévrier. - Maturité automne; semer en automne ou au printemps suivant; elle germe en un ou deux ans.

Hêtre. - Maturité automne (gaulage sur draps). Conservation très difficile; semer automne ou mettre en silos et semer printemps suivant.

If. - Maturité automne; semer immédiatement (elle lève en deux ans) ou après (elle lève tardivement).

Mélèze. - Maturité à la fin de l'automne; dissémination pendant l'hiver, surtout en février; récolter sur la neige gelée où elle s'amasse dans les sinuosités, ou bien gauler sur diaps. Se conserve quelques années. 130000 à 200000 graines au kilogramme dont un grand nombre mauvaises.

Orme. - Maturité juin; semer immédiatement. Conservation difficile et alors germination lente.

Peuplier. - Graine abondaute, mais généralement mauvaise; les graines perdent en quelques jours leur 
faculté germinative; le peuplier est multiplié par boutırage.

Pin sylvestre. - Maturité décembre (les cônes ne devraient jamais être récoltés avant cette époque). L'extraction des graines se fait au soleil ou à l'étuve (température inférieure à $\left.38-44^{\circ}\right)$. Conservalion facile quelques années. 140000 à 160000 graines au kilogramme.

Robinier. - Maturité automne; graine longtemps bonne.

Sapin. - Maturité septembre, commencement octobre. Cueillir cônes arant désarticulation, les placer dans un lieu sec où elle se produit. Semer en automne autant que possible; elle se conserve jusqu'au printemps suivant, pas au delà. Cette graine s'échauffe facilement, on devra l'épandre en couche peu épaisse en la laissant avec les écailles et la remuer souvent. 31000 graines au kilogramme.

Sorbier. - Maturité automne; semer immédiatement ou stratifier dans le sable (avec le péricarpe) et semer au printemps.

Saule. - Les graines de saule sont généralement mauvaises et celles qui sont bonnes perdent, en deux à quatre jours, leur faculté germinative. Ces végétaux sont multipliés par bouturage.

Tilleul. - Maturation automne; semer automne ou conserver dans le sable et semer printemps suivant, mais pas au delà.

Remarquons que, mème lorsque le temps est sec au moment de la récolte, il est généralement bon d'étendre immédiatement les graines, surtout les plus grosses, dans un grenier sec et bien aéré, et de les remuer de temps en temps pendant une quinzaine de jours.

On évitera d'amonceler sur de grandes épaisseurs, les graines sujettes à la pourriture et à l'échauffement; on les remuera une fois par jour pendant les quinze premiers jours qui suivent la récolte, puis ensuite une fois par semaine jusqu'au semis.

Quant aux graines sujettes à se dessécher, on les pla- 
cera immédiatement après la récolte dans un lieu froid plutot qu'humide (cellier de préférence à une cave).

Détermination de la valeur culturale des graines employées. - Achat degraines au Commerce ; garanties à exiger du fournisseur. - Lorsqu'on entreprend un semis forestier, il est essentiel de connaitre la valeur culturale des graines employées; cette valeur culturale exprimée par un coefficient, 90 p. 100 par exemple, en représente la véritable valeur marchande.

Un exemple fera comprendre toute l'importance de celte détermination : pour effectuer un semis de pin, nous employons de la graine parfaite; sur 100 graines semées, 100 peuvent ètre susceptibles de germer et dans ces conditions nous devons en semer 6 kilos par hectare par exemple; au contraire, nous ne disposons que d'une graine de qualité inférieure, déjà vieille, dont la conservation a été effectuée dans des.conditions défectueuses, qu'on a laissé s'échauffer ou qui a élé plus ou moins falsifiée; 10 graines seulement sur 100 sont susceptibles de germer; il est très mauvais d'effectuer un semis dans ces conditions, mais si on ne peut faire autrement, ce n'est pas 6 kilos de graine qu'il fuut semer par hectave, mais dix fois plus, soit 60 kilos, sinon le semis sera forcément manque.

Cette considération, si simple qu'elle est évidente par elle-mème, n'est plus discutée aujourd'hui (1); en Allemagne, en Autriche, en Suisse, des stations de contrôle et d'essai de graines forestières sont organisées, soit par le service forestier, soit par les services agricoles, et sont ouvertes au public qui en use très largement. L'établisse-

(1) C'est l'administration forestière française qui, la première, est entrée dans l'essai systématique des graines forestières en créant en 1872 au Domaine des Barres une station d'essai des graines forestières, où sont soumises à des essais minu ieux aussi bien les graines achetées par l'administration au commerce que celles qui proviennent des sècheries domaniales. (Schwappach).

Malheureusement, cette station forestiêre n'a pas été ouverte au public. 
ment suisse de Zurich, d'après M. le Dr Schwappach (1), joue au point de vue de l'essai des graines forestières un rôle d'une importance tout à fait exceptionnelle; ainsi par exemple, du $1^{\text {er }}$ juillet 1900 au 30 juin 1901, on a essayé 10257 échantillons, dont 2261 , soit 22 p. 100, de graines forestières, et on lit dans le compte rendu de cet établissement que la plus grande partie de la récolte des pins de l'Europe, récolte dont la valeur est de plusieurs millions de francs, est essavée à Zurich.

En France, le propriétaire particulier n'est en général pas encore entré dans cette voie, et nous pourrions citer plusieurs faits d'où il résulte que des propriétaires, après avoir effectué des semis importants dans de bonnes conditions culturales, ont perdu plusieurs années à attendre la naissance des jeunes plants qui ne sont apparus que par points isolés et disséminés, évidemment parce que les graines employées étaient de mauvaise qualité. Aussi est-il très à désirer, que les propriétaires français se munissent toujours, avant d'effectuer un semis important, d'un certificat d'essai d'une station française. Il nous paraît inutile d'ajouter qu'en agissant ainsi, on évitera dans la mesure du possible, de longues pertes de temps, et mille frais accessoires souvent considérables nécessaires pour regarnir une surface dont le sernis est imparfaitement réussi.

Essai des graines récoltées directement par le propriétaire. - Lorsque le propriétaire récolte la graine sur son domaine, il peut vérifier lui même la qualité de cette graine; pour les grosses graines, comme les glands, châtaignes, faînes, etc., la vérification est facile et il suffit d'un examen rapide et de quelques essais au couteau, pour voir si ces graines sont pleines, fraîches et sans moisissures. Le contrôle des petites graines, comme celles de la plupart des essences

(1) Sсншартасн, Directeur dela station d'essai de graines forestières d'Eberswalde. (Allemagne). 
lésineuses couramment employées, et de quelques essences feuillues (bouleaux, aunes, etc.) n'est pas aussi simple; en principe, on peut se rendre compte de leur valeur en faisant germer un nombre de graines déterminé dans une caisse remplie de terre ou de sable stérilisé qu'on arrose avec de l'eau tiède, afin de conserver toujours une humidité suffisante, et qu'on maintient dans une atmosphère tempérée; un autre moyen assez simple consiste à faire germer les échantillons d'expérience entre deur bandes de papier buvard maintenues légèrement humides à une température de 20 à 28 degrés, dans une étuve par exemple; en comparant le nombre de graines mises en expérience à celui des graines germées, et en rapportant ces résultats à 100 graines, on obtient la faculté germinative de la graine. Dans cet essai, il est important que l'expérience porte sur des graines séparées de leurs impuretés, et on doit alors déterminer au préalable un coefficient de pureté; pour cela, prélevant sur l'échantillon d'expérience un poids $P$, on en sépare à la main avec une spatule ou un canif les impuretés (graines étrangères, fragments de graines, débris d'écailles. terre, pierrailles, etc.) dont le poids est 1 ; le rapport $\frac{P-1}{P}$ est le coefficient de pureté; le produit du coefficient de pureté par la faculté germinative donne la valeur culturale, ou valeur marchande de l'échantillon.

Remarquons que ces essais, simples en théorie, sont très délicats à effectuer dans la pratique; pour obtenir des résultats sérieux, il faut non seulement avoir la pratique de ces opérations, mais encore disposer d'appareils lien installés; afin de donner aux essais une durée aussi restreinte que possible, on doit pouvoir placer les échantillons d'expérience dans les conditions de chaleur, d'humidité et d'aération les plus favorables à leur germination. - $\Lambda$ notre avis, le propriétaire aura loujours inlérêt à s'adresser à une station d'essai. 
Contróle des graines achetées au commerce. - Lorsqu'on achète des graines au commerce, et spécialement lorsqu'il s'agit de commandes importantes comme peuvent en faire des syndicats agricoles (1), l'acheteur doit s'adresser à une des maisons placées sous le contrôle de la station officielle d'essais de semences et se faire garantir sur facture l'espéce, la varièté, la pureté et la faculté germinative des semences (2); en opérant de cette manière, il dispose (gratuitement dès que la fourniture dépasse 5 kilos de chaque espèce) du moyen de contrôle suivant que nous extrayons des instructions de la station officielle d'essai de semences du Vinistère de l'agriculture.

Délais accordés pour les réclamations au vendeur. Les maisons placées sous le contrôle de la station indemnisent l'acheteur ou reprennent à leurs frais la marchandise qui n'est pas conforme aux garanties données. Le droit du client à une indemnité quelconque cesse lorsque celui-ci néglige d'envoyer un échantillon à la station dans les huitjours qui suivent la réception des semences, ou s'il n'a pas adressé de réclamations au vendeur dans les huit jours qui suivent la réception du bulletin d'analyse. Analyses de contrôle. - ll arrive fréquemment qu'une marchandise ne répond pas aux garanties fournies; de là, la nécessité d'une analyse de contrôle. Les maisons placées sous le contrôle de la station prennent à leur charge les frais de contre-analyses pour toute livraison d'au moins 5 kilos de chaque espèce. (La liste des maisons placées sous le contrôle de la station est adressée gratuitement aux personnes qui en font la demande.)

(1) Certains syndicats agricoles se chargent de réunir en temps utile toutes les commandes des propriétaires d'une région, et se font expédier en bloc les graines qu'ils répartissent ensuite selon les commandes. Nous ne saurions trup recommander cette méthode de procéder, car elle permet d'exiger des fournisseurs des garanties sérieuses qu'il est facile de contróler.

(2) Une garantie de tant pour 100 de germination en bloc, n'est pas suffisante, et ne renseigne que d'une façon très imparfaite et empirique sur la qualité de la semence. 
Prise des échantillons. - Les échantillons sont prélevés, en double, avec le plus grand soin, dès l'arrivée de la marchandise, en présence de deux témoins impartiaux, puis cachetés; l'un des échantillons est adressé à la station, arec un certificat des témoins, l'autre demeure entre les mains de l'expéditeur pour servir à une contre-analyse en cas de contestation.

Il est recommandé de bien mélanger les semences avant de recueillir les échantillons d'analyse; lorsque celles-ci se trouvent dans des sacs, on peut faire des prises à différentes hauteurs dans un certain nombre de sacs, puis les réunir et prélever les échantillons d'analyse. Le poids minima des échantillons à prélever varie de 50 à 200 grammes suivant la grosseur des graines.

Durée des essais. - La détermination du coefficient de pureté et du poids absolu, sont faites dans un très court délai à partir de la réception; la recherche de la faculté germinative exige en général un maximun de vingt-huit jours pour les semences d'espèces ligneuses (pin sylvestre, pin noir d'Autriche, épicéa, mélèze, etc.); toutefois elle peut demander un temps plus long (pin d'Alep, etc.).

Composition moyenne d'une bonne semence. - Un acheteur est en droit d'exiger. - $1^{\circ}$ En ce qui concerne la pureté, que les semences qui lui sont livrées soient débarrassées autant que possible des graines vides, cassées ou détériorées, ainsi que des matières étrangères telles que débris d'ailes et d'écailles, pierrailles, terre, poussière, etc. $-2^{\circ}$ En ce qui concerne la faculté germinative que les semences soient en forte proportion susceptibles de germer rapidement.

Parmi les essais que nous arons exécutés en 1902 à la station forestière du domaine des Barres, sur germoir en papier burard placé dans une étuve et comparativement sur sable, nous relevons les résultats suivants : 
BOISEMENT PAR SEMIS DIREGT.

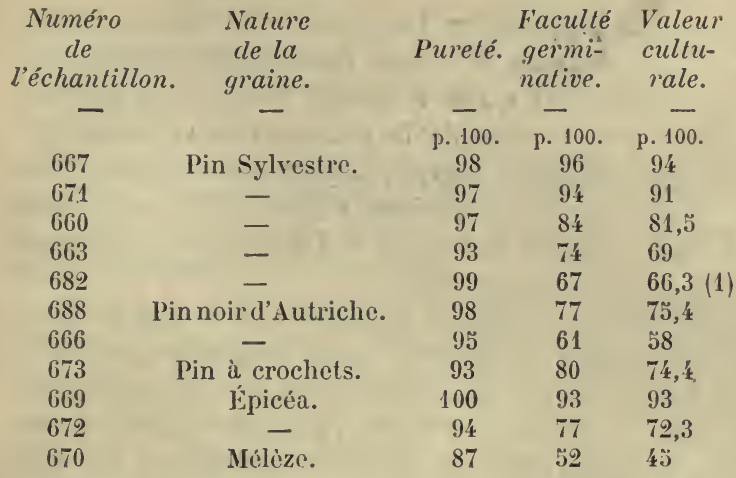

Dans la pratique on peut admettre pour apprécier la qualité d'une graine résineuse l'échelle suivante :

Pins, Epicéa, et la plupart des graines résineuses.

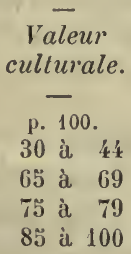

Qualité
cle la graine.

mauvaise. assez bonne. très bonne. parfaite.
Mélèze.

Valeur culturale.

p. 100 .

10 à $1^{\prime}$

30 à 34

40 à 49

60 à 100

Divers modes de semis. - D'après M. Bouquet de la Grye (Dictionnaire d'Agriculture, Barral et Sagnier), il existe plusieurs manières de procéder :

Semis en plein à la volée sur terrain non préparé. Le mode de semis le plus simple est celui qui consiste à répandre à la volée la graine sur le sol, sans lui faire

(1) Les résultats des essais nos 667 et 682 montrent que pour opérer un semis dans les mèmss conditions avec les graines de ces deux échantillons, si on sème 2 kilos de la graine $n^{\circ} 667$, on devra semer 3 kilos de la graine n 682 sur la même suriace; la quantité de graine à employer pour un mème semis varie alors dans la proportion de 2 dे. 3 . Il est évident que ces deux échantillons ne doivent pas avoir la mème valeur commerciale. 
subir aucune préparation. Ce procédé ne réussit que sur les sols couverts de bruyères courtes ou d'herbes peu serrées. C'est ainsi qu'on a reboisé en pins de grandes surfaces dans le centre de la France. Ces reboisements exigent peu de main-d'cuvre ; la principale dépense consiste dans l'achat des graines, soit 8 à 10 kilogrammes par hectare. Le prix de revient de l'hectare ainsi semé ne lépasse pas 40 francs.

Si la bruyère est haute et serrée, si le gazon et les mousses forment un feutrage épais, beaucoup de graines jetées à la volée n'arrivent pas jusqu'au sol, et si quelques plants viennent à lever, ils sont étouffés avant d'arriver au jour ; dans ce cas, il faut écobuer à feu courant, deux ou trois ans d'avance, le terrain à reboiser; puis quand le sol est regarni des bruyères qui doivent servir d'abris aux jeunes plants, on y répand la graine à la volée. On peut encore réduire la dépense quand on se borne à déposer entre les touffes de bruyères, dans le sol préalablement débarrassé de la mousse et ameubli à l'aide d'une petite houe à manche court, quelques graines de pin ou d'épicéa.

Ces procédés simplifiés ne peuvent s'appliquer qu'aux semis d'essences qui ne redoutent pas la pleine lumière; ils ne sauraient convenir au sapin qui ne vient bien que sous le couvert. On ne pourrait non plus les employer sur des terrains dénudés. Là, il faut préparer par une culture préalable le sol à recevoir les graines.

Semis en plein à la volée sur terrain préparé. Quand le terrain est peu accidenté, peu pierreux et qu'il ne contient pas de grosses racines, on y pratique, à six mois d'intervalle, deux labour's en plein à angle droit. Ces labours exécutés à la charrue rompent les gazons et les soulèvent en mottes entre lesquelles les graines trouvent l'abri et la fraicheur qui assurent leur germination. Le sol ameubli est d'ailleurs plus aisément pénétré par les faibles racines des plants naissants. 
Dans les terrains légers, non embarrassés, et pour les semis de graines légères, on peut se contenter d'une préparation superficielle à l'aide d'une herse lourde à dents de fer.

Remarquons que dans toute préparation du sol pour un semis, il faul éviter de localiser la meilleure terre dans la zone profonde et de ramener à la surface un terrain moins favorable à la germination.

Semis à la volée par bandes alternes. - Le labour en plein est coûteux, aussi dans beaucoup de cas, on lui substitue le labour par bandes, qui consiste à labourer, sur la surface à reboiser, des bandes de terre de $0^{\mathrm{m}}, 30 \mathrm{au}$ moins à 1 mètre de largeur, séparées par des bandes incultes plus larges, de 1 à 2 mètres environ. En terrains accidentés, les bandes doivent être tracées perpendiculairement aux lignes de la plus grande pente et les débris provenant de la culture sont placés à l'aval des bandes cultivées. Ce mode de préparation a, sur le labour en plein, de nombreux avantages : il est moins coûteux; il ne facilite pas l'érosion du sol; il procure, par les herbes et les arbustes qui croissent sur les bandes incultes, un abri aux jeunes plants; enfin, il donne aux peuplements une régularité qui facilite l'exécution des travaux d'entretien.

Semis à la volée par bandes brisées. - Dans les cas où la charrue ne peut être employée, soit en raison de la nature rocheuse du sol, soit parce qu'il est accidenté, les bandes sont préparées à la houe; mais alors, au lieu de les faire continues, on les interrompt tous les 4 à 6 mètres, en laissant entre elles, dans le sens de la longueur, un intervalle de 2 à 3 mètres qui reste en friche. Ces bandes brisées ont tous les avantages des bandes continues et coûtent bien moins cher.

Semis par places ou par potets. - Enfin, sur les terrains très accidentés dont la surface est hérissée d'obstacles, roches, buissons, etc. ou bien lorsque la terre 
régétale est rare et qu'on est obligé de chercher les points où l'on peut semer, on prépare par potets les emplacements où doivent ètre déposées les graines. Ces potets sont défoncés à la houe; on leur donne en moyenne $0^{\mathrm{m}}, 40$ à $0^{\mathrm{m}}, \breve{0} 0$ de côté, ils sont disposés aussi régulièrement que possible et espacés depuis 1 jusqu'à 3 mètres.

Semis sur une culture agricole. - Il est souvent avantageux de livrer le terrain à l'agriculture avant d'y semer les graines forestières. Le sol ameubli par la culture, amélioré par la fumure qu'elle exige, se trouve parfaitement préparé pour recevoir les graines et favoriser la croissance des jeunes plants qui en proviennent. Les récoltes qu'on tire des terrains ainsi traités couvrent, et au delà, les frais de l'opération (1). C'est par ce procédé qu'on a reboisé, en Sologne, lant de friches qui sont devenues de belles forêts.

Voici la série des travaux que nécessite ce mode de procéder, assez avantageux quand on l'applique à des terrains couverts de bruyères, d'ajoncs et de fougères, végétalion spontanée qui prouve qu'ils sont siliceux et qu'ils ont une certaine profondeur. Le sol est défoncé à la charrue pendant l'hiver. Ce défoncement est suivi de deux hersages donnés en juillet et en septembre. Après le second hersage, on répand la graine d'aroine ou de seigle mélangée avec des engrais phosphatés. Un dernier coup de herse recouvre le lout. La seconde année, on donne deux labours après la récolte, et on sème de nouveau du seigle ou de l'avoine avec une demi-fumure d'engrais. La troisième année, on cultive des plantes sarclées sans fumure, et la quatrième on

(1) Nous n'en sommes plus au temps oủ l'homme pouvait, en agricullure, disséminer ses forces, le fumier et la main-d'œurre sur de grandes surfaces; In cullure intensive nécessaire aujourd'hui l'oblige a donner tous ses soins aux honnes terres et à abandonner à la culture forestière les nédiocres et surtout les mauvaises. Pour reboiser ces terrains, le semis sur une deruière culture agricole est souvent commode. 
sème arec l'avoine les graines forestières en y joignant une demi-fumure.

Saison la plus favorable pour semer. - L'époque où il convient de répandre les graines varie suivant l'espèce, et aussi suivant le climat du lieu où le semis doit ètre fait. On peut dire qu'il faut, en général, choisir le printemps de préférence, spécialement pour les graines résineuses, en tenant compte des conditions de température et d'humidité que présente l'année où on opère. Quant aux graines dont la conservation est difficile, telles que glands, châtaignes, faines, etc., il vaudrait mieux les semer au printemps pour éviter de les roir manger pendant l'hiver par les mulots et les sangliers, mais on est obligé souvent de les semer dès l'automne.

Soins à prendre pendant et après le semis. - Les soins à prendre pour enterrer la graine ou la faire adhérer au sol,varient avec la grosseur de cette graine et la nature de la terre. Les grosses graines sont généralement semées par potets. Les glands, châtaignes peuvent ètre enfouis à 3 centimètres dans les sols lourds, à 6 centimètres dans ceux qui sont légers. La profondeur du trou où sont semées les faines, et les graines de charme varie de 1 à 3 centimètres. Les graines légères d'orme, de bouleau, d'aune, sont à peine recourertes; il suffit qu'elles soient appliquées sur le sol, de manière à ne pas ètre enlevées par le vent.

De toutes les graines résineuses, celles du pin cembro qui sont un peu grosses peurent seules ètre recourertes d'une épaisseur de terre de 1 centimètre, les autres ne doivent pas ètre recouvertes de plus d'un demi-centimètre.

Quand le semis a été fait en plein, soit sur bruyères, soit après labour, il est bon de faire passer sur le terrain un troupeau de moutons afin de faire entrer dans le sol les graines qui, sans cette pression, resteraient sur les mousses ou les souches de bruyère ; à défaut de moutons, on peut faire passer le rouleau ou un fagot d'épines. 
Après le semis, il faut attendre, car la réussite ne peut être assurée qu'au bout de cing ou six ans. Toutefois, il est indispensable d'empècher l'accès des animaux sur le terrain reboisé, et il est souvent utile de préserver les jeunes plants contre l'envahissement des herbes et contre la sécheresse par quelques sarclarges et binages faits à propos. Les sarclages, utiles pour les semis résineux, doivent ètre faits avec soin, après la pluie au mois de juin et à la main, de façon à ne pasdéraciner les jeunes plants.

Ajoutons enfin que toujours, dans un semis, il faut, vers la quatrième ou cinquième année, combler les vides qui se produisent à l'aide de jeunes plants qu'on peut, à la rigueur, prendre là où les semis sont trop serrés. On en profite ainsi pour effectuer un premier desserrement; c'est vers la deuxième année environ après le semis, sourent mème avant, qu'il y a lieu de procéder à un véritable desserrement dans toutes les parties où le semis est trop dense : cette opération est très utile pour que lesjeunes plants puissent se développer avec toute leur énergie; elle est spécialement indispensable dans les semis d'essences de lumière (pin sylvestre, mélèze) qui sont toujours trop compacts. On procède à celte opération à l'aide d'un sécaleur ou de forts ciseaux plutôt que par arrachage.

Quantité de graines à employer. - La quantilé de graines à employer varie pour chaque espèce avec la qualité de la graine et le mode de semis adopté; M. Bouquet de la Grye domne les chiffres suivants:

Pour les semis en plein sur bruyère, on emploie 8 à 10 kilogrammes de graine désailée de pin par hectare ; cette quantité se réduit à 6 kilos si le semis se fait par places; elle est de 7 kilos pour le semis par bandes. Le semis par bandes nécessite l'emploi de 33 kilogs de graine de sapin, de 10 kilos de graine d'épicéa ou de pin noir, de 14 kilos de graine de mélèr.

Les semis de glanıls ou de faînes, qui se font généra- 
lement par potets, emploient : pour les glands : hectolitres et pour les faìnes 3 hectolitres à l'hectare.

\section{II. - Boisement par plantations.}

Les plantations se font arec des plants de haute tige ou des plants de basse tige.

Les plantations de haute tige sonl rarement employées en sylviculture; quand elles s'appliquent à des essences forestières, elles ne diffèrent pas de celles des arbres fruitiers et d'ornement, qui rentrent dans le domaine de l'horticulture, et on doit y procéder avec les mèmes soins.

Les plantations de basses tiges ou jeunes plants sont plus généralement usitées dans la pratique forestière.

Origine des plants. - On pourrait à la rigueur extraire en forêt les semis de l'année, et les repiquer en pépinière en automne ou au printemps. Cette manière de procéder est très onéreuse et, en général, ne doit pas ètre employée. Dans certaines circonstances toutefois on peut faire exception pour le sapin et le hêtre qui ont, dans leur jeune âge, un tempérament délicat, spécialement s'il s'agit de les transplanter en forèt. Les jeunes sapins provenant dessemis naturels âgés de quatre à cinq ans sont extraits arec la motte et transportés à quelque distance arec la certitude de réussir. Ce mode de repeuplement coûte assez cher néanmoins ; il ne saurait être trop recommandé lorsqu'on reut multiplier le sapin, en raison des difficultés que l'on éprouve à élever cette essence en pépinière (1). Les jeunes hêtres âgés de deúx ou trois ans au plus peurent également ètre extraits et transplantés en forèt. Dans les années où la faîne est trìs abondante, on peut extraire en forèt à peu de frais des semis d'un an, et les repiquer en pépinière ou en

(1) Remarquons toutefois qu'il n'est pas impossible d'élever le sapin en pépinière, à la condition que les jeunes semis soient bien à l'ombre. 
forèt en ayant soin de les enterrer jusqu'à la hauteur des feuilles cotylédonaires.

Pour les essences à tempérament robuste, qui s'accommodent mal du couvert, comme pour celles qui dans les premières années développent peu de chevelu à moins d'y être forcées artificiellement, les plants élevés en pépinière sont toujours préférables à ceux pris en forèt, et dans tous les cas, ils reviennent à un prix beaucoup moins élevé.

En général donc, il y a lieu de se servir de plants élevés en pépinière. S'il s'agit de repeuplements importants, et si on dispose du personnel nécessaire, il est possible d'établir chez soi, soit une pépinière fixe, soit des péjinières volantes destinées à fournir les plants nécessaires. Mais il ne faut pas oublier que la création et l'entretien d'une pépinière demandent de grands soins, qu'on doit s'y prendre à l'avance, éviter la surproduction. Pour un propriétaire particulier qui n'est pas outillé spécialement à cet effet, tous les travaux accessoires demandent des frais de main-d'œuvre qui augmentent singulièrement le prix de revient des plants. Aussi, en général, s'adresse$\mathrm{t}$-on au commerce. C'est le seul cas que nous en visagerons.

Age et quaiité des plants à employer. - Les plants doivent être normalement constitués, sains et vigoureux.

M. Demontzey pose en principe que jour une mème essence, on obtient un succès d'autant plus assuré que les plants employés sont plus jeunes; il ajoute que la jeunesse de ces plants doit avoir pour limite une conformation de leur's organes suffisante pour se bien déveJopper et lutter contre les dangers qui peuvent les menacer. Il fixe pour les pins d'Alep, maritime, pinier, l'âge d'un an; pour le piun sylvestre et d'Autriche, un à trois ans suivant leur développemenl; pour le pin à crochets, leux à Irois ans; pour l'épicéa et le pin cembro, aux grandes altitudes, trois, quatre el cinq ans; pour le 
mélèze, deux et trois ans au maximum. Dans ces conditions, ajoute-t-il, le repiquage des résineux ne paraît a voir aucune raison d'ètre employé pour la production des plants destinés au reboisement des montagnes, parce que l'expérience a démontré que les plants résineux non repiqués produisent des peuplements aussi complets, aussi bien venants que ceux formés avec des plants repiqués et que le repiquage entraine sans profit à des dẻpenses considérables. M. Gayer est aussi affirmatif que II. Demontzey; s'occupant spécialement du repeuplement artificiel tel qu'il se pratique en Allemagne, il déclare que le repiquage a été avec raison complètement supprimé dans un grand nombre de cantonnements pour les plants résineux ordinaires; quant à l'âge de plantation des résineux, il donne sensiblement les mèmes chiffres que M. Demontzey.

Dans quelques régions, dans le Loiret et notamment en Sologne, les propriétaires particuliers ne veulent employer que des plants résineux repiqués: ces plants leur coûtent cher et ne sont pas meilleurs que les autres, à la condition, bien entendu, de n'employer que des plants normalement constitués comme le prescrivent du reste MM. Demontzey et Gayer; nous pensons qu'ils peuvent faire aussi bien sinon mieux et plus économiquement.

Si le repiquage doit ètre supprimé chez les résineux en général, il n'en est plus de mème chez les feuillus dont la destination et le mode de plantation exigent l'emploi de plants d'une certaine taille, d'une reprise rapide et facile qui sera favorisée par la présence d'un chevelu serré, abondant; ce chevelu ne peut être obtenu que par le repiquage.

En résumé, nous admettons que les plants feuillus seront un peu plus âgés que les résineux et repiqués; les premiers, d'ailleurs, peuvent être recépés après la reprise complète, s'il y a intérèt à obtenir des rejets vigoureux. Le sapin, en raison de son tempérament délicat, et 
l'épicéa, en raison de ses très faibles dimensions dans un tout jeune âge, demandent à être élevés plusieurs années en pépinière.

Les pins, les mélèzes seront pris jeunes en pépinière et non repiqués.

En ces questions, d'ailleurs, il n'y a rien d'absolu, et ce sont les dimensions des plants et leur vigueur qui seront surtout à envisager.

Voici l'âge auquel sous le climat tempéré de l'arboretum des Barres (Loiret) on peut employer les plants :

Essences feuillues. - Robinier, de deux à trois ans. - frène, orme, érable, aune, bouleau, charme, de deux à quatre ans; - hètre, chène, de deux à trois ans.

Essences résineuses. - Pin sylvestre, mélèze, pin Laricio, noì d'Autriche, pin à crochets, de deux à trois ans; - sapin, épicéa, pin cembro, de trois à cinq ans.

Remarquons qu'une plantation sera d'autant moins onéreuse (indépendamment du prix d'acquisition) que les plants seront plus petits; il est donc avantageux à ce point de vue d'employer de jeunes plants. Mais il existe des cas où il y a intérèt à employer des plants déjà forts, ayant $0^{\mathrm{m}}, 23$ à $0^{\mathrm{m}}, 30$ de hauteur et par suite repiqués. Nous en citerons quelques-uns :

$1^{\circ}$ Sur un sol meuble, se soulevant facilement sous l'aclion de la gelée; $2^{\circ}$ sur un sol envahi par des herbes un peu hautes; $3^{\circ}$ sur un sol déjà planté antérieurement où on ne fait qu'un repiquage pour garnir les vidles; $4^{\circ}$ dans les vides de petite étendue entourés de peuplements plus âgés, et dans toutes les situations où le climat local ou bien des conditions de lempérament nécessitent d'agir vite et d'une façon difrérente.

Époque la plus favorable à la plantation. - Nous distinguerons, s'il s'agit d'essences à feuilles persistantes (résineux) ou d'essences à feuilles caduques.

a) Essences à feuilles persistantes. - M. Demontzey 
est d'avis, et il est généralement admis, que pour les essences à feuilles persistantes l'époque la plus favorable à la plantation est le printemps, au moment mème où la végétation va entrer en action. Ce fait constaté par une longue expérience a été du reste parfaitement expliqué par Ebermayer au point de rue physiologique : les plants à feuillage persistant évaporent constamment une assez grande quantité d'eau; leurs racines complètes, bien étalées, sont en bon contact arec les particules terreuses et elles trouvent à leur disposition toute l'eau qui leur est nécessaire; après une transplantation le contact des racines est imparfait et beaucoup de radicelles sont brisées; que le sol se dessèche, qu'il survienne une forte insolation, la plante ne tarde pas à jaunir et à périr; toutefois si la transplantation est opérée au moment de la reprise de la végétation, il se forme de nouvelles radicelles qui alimentent la plante.

Remarquons que, dans la plantation sous le courert le danger est moindre, de mème qu'il est moindre aussi lorsque le sol est constamment humide.

b) Essences à feuilles caduques. - Le mème danger n'existe pas pour les sujets à feuilles caduques qui peuvent ètre plantés en tout temps. Mais la sécheresse, les mauvaises herbes et surtout le déchaussement peurent toujours compromettre les plantations d'automne.

M. Gayer est du même avis et il s'exprime ainsi : la plantation de printemps vaut mieux en général que celle d'automne, surtout pour les conifères; la régétation commence immédiatement après la plantation; les plants ne sont pas exposés à souffrir des fortes gelées, du déchaussement (qui sur certains sols calcaires est extrêmement à redouter), du desséchement; le sol possède généralement un degré de chaleur et d'humidité nécessaire à la prompte reprise de racines; l'envahissement des mauvaises herbes est moins actif; les jours déjà assez longs permettent d'abattre reaucoup de travail. 
On devrait donc toujours planter au printemps, et cependant c'est en automne que sont effectuées toutes les plantations résineuses faites par exemple dans la Sologne,

- le Loiret, etc. Aussi ajouterons-nous qu'il y a peut-être de bonnes raisons pour agir de la sorte; dans ces régions les hivers sont habituellement humides, les printemps fréfuemment secs, les gelées sans importance. Il ne faut donc pas avoir de parti pris et il peut être bon, un peu partout, de se baser sur l'expérience.

En tout cas, quelle que soit la saison adoptée, il ne faut jamais planter sur une terre trop détrempée, se prenant en boue collante, et impossible à disposer convenablement autour des racines.

Expédition et conservation des plants. - Les plants, livrés par le fournisseur en bon état, doivent ètre emballés avec soin pour le transport, dans une caisse à claire-voie de préférence aux paniers, de façon à éviter l'échauffement, surtout au printemps. Ils redoutent pendant les transports à de grandes distances les excès de chaleur et de froid; à cet effet, il est utile de les disposer par couches horizontales dans la caisse, préalablement tapissée de mousse humide et de les recouvrir d une dernière couche de mousse. Dès leur réception la caisse doit ètre immédiatement défaite; les plants à racine nue ne doivent ètre exposés ni au soleil, ni à un vent sec, ne fủt-ce que pendant dix minutes; ils doivent être soigneusement protégés; à cet effet, on peut les envelopper de mousse humide, mais le mieux est de les mettre en jauge; pour cela, on défait les paquets, on creuse un fossé peu profond, on y introduit les racines sans trop les serrer, et il suffit ensuite de recouvrir ces racines avec de la terre fine bien émieltée.

Du soin apporté dans ces opérations dépend en partie la réussite de la plantation; on y procède aussitó que jossible.

Habillage des racines. - En principe, un plant n'a 
jamais trop de racines, et beaucoup de forestier's pour ce motif ne taillent jamass les plants avant de les employer. Cela paraît ètre le meilleur procédé, car il importe toujours de ménager soigneusement le chevelu. On peut toutefois sans inconvénient couper les racines qui sont dépourvues de chevelu lorsqu'elles sont cassées ou abìmées ou bien qu'elles sont trop longues et gènent la mise en terre.

Habillage des branches. - Il existe un rapport harmonique entre l'enracinement et les branches d'un régétal; la transplantation ayant toujours pour effet de supprimer des racines, on est porté à supprimer également des branchages. Nous ferons une distinction :

$1^{\circ}$ Lorsqu'on plante des résineux, on doit s'abstenir de toucher à leurs branches ou à leur tige, à moins toutefois qu'ils ne soient de forte taille, auquel cas on peut enlever' ou plutôt raccourcir les branches basses.

$2^{\circ}$ Lorsqu'on plante des feuillus, si les plants sont jeunes, on peut se dispenser de les tailler, surtout si le sol où on les place est frais et fertile; si au contraire le terrain est pauvre, on peut couper quelques branches ou mème une partie de la tige; si les plants sont plus forts, on pourra les tailler davanlage.

Quant au recépage total, certains planteurs l'exécutent immédiatement après la plantation à quelques centimètres du collet; nous ne sommes pas partisan de ce système, surtout si les plants sont mis en terre en automne, et préférons a ttendre deux ou trois ans ; pendant ce temps, le système des racines se sera développé, le végétal aura fonctionné en place, et lorsqu'on rabattra le plant, il émettra des rejets vigoureux. Ce système est applicable aux plantations en essences feuillues exécutées pour enrichir un taillis.

Espacement à donner aux plants. - On ne peut rien fixer d'absolu sur l'espacement à donner aux plants. D'une part, les raisons d'économie obligent à espacer les plants le plus possible; les raisons culturales exigent 
d'autre part que les jeunes sujets soient suffisamment rapprochés pour qu'ils se protègent les uns les autres, et que l'état de massif se forme de bonne heure. Mais d'un autre côté, il ne faut pas planter trop serré, car cn dehor's de la dépense exagérée, les plants se gènent mutuellement et souffrent; leur végétation est moins active jusqu'au jour où les plus vigoureux prennent l'avance et étouffent les plus chétifs.

On plante très rarement plus de 10000 plants par hectare; ce chiffre doit ètre considéré comme un maximum; les espacements variant entre $1^{\mathrm{m}}, 25$ et $1^{\mathrm{m}}, 50$, donnant en chiffres ronds de 6500 à 4300 plants par hectare sont généralement adoptés par les forestiers. Enfin lorsqu'on plante des sujets très forts, on peut espacer à 2 mètres, ce qui correspond à 2300 plants à l'hectare.

En principe, il faut très bien planter, sauf à planter très clair. On a souvent intérêt à ne pas donner aux plants un espacement égal dans tous les sens; ainsi les files ou rangées de plants peuvent ètre écartées de $1^{\mathrm{m}}, 50$ ou de 2 mètres, tandis que les plants dans chaque file seront à 1 mètre ou $1^{\mathrm{m}}, 50$ l'un de l'autre. Cette disposition est économique, car pour l'écartement de $1^{\mathrm{m}}, 50$ en largeur et de 1 mètre dans le sens des lignes, il ne faudra ouvrir que 6700 potets, et si l'écartement en largeur est de 2 mètres, celui en longueur restant de 1 mètre, il n'en faudra que 5000 .

Mise en place de plants. - Quelque simplifié que soit le procédé de mise en place des plants, cette opération doit toujours ètre faite avec le plus grand soin, c'est une des conditions essentielles de réussite.

Nous recommanderons tout d'abord d'éviter que pendant celle opération les ouvrier's ne laissent les racines des plants exposées au soleil ou à un vent sec. Pour cela, chaque ouvrier planteur peut transporter avec lui de place en place un petit panier dans lequel il conserve sa 
provision de plants, les racines toujours couvertes de terre fraìche.

La réussite d'une plantation dépend en grande partie des conditions météorologiques sur lesquelles l'homme ne peut exercer aucune influence; mais elle dépend en outre de la préparation du terrain qui doit recevoir les plants, des soins apportés à leur choix et à leur mise en place.

Il est rare qu'on prépare le terrain sur toute sa surface ; la culture en plein n'est praticable que sur des terrains peu accidentés où il n'y a ni pierres, ni racines; d'ailleur's elle est coûteuse ; elle est généralement remplacée par une piéparation partielle qui consiste soit dans l'ouverture de sillons parallèles entre lesquels on laisse des bandes incultes, soit dans le défoncement de petites surfaces diversement espacées.

Les sillons sont ouverts à la charrue ou à la houe, suivant la configuration et l'état dv sol ; ils doivent être tracés suivant les lignes du niveau, lorsque le terrain présente une pente accentuée. Les plantes qui courrent les bandes incultes soutiennent les terres et abritent les jeunes plants.

Enfin quand on reut planter avec économie, on renonce à préparer à l'avance le terrain.

Voici queiques-unes des méthodes les plus employées quand il s'agit d'éxécuter des plantations.

Plantation directe à la pioche. - Ce genre de plantation consiste à soulever d'un coup de pioche la couche superficielle du sol, à engager dans cette fente les racines du jeune plant, puis à tasser le tout avec le pied.

Ce mode de procéder est très économique, mais il ne doit être employé que lorsqu'on a à planter dans un sol naturellement très meuble ou léger et frais, c'est-à-dire en état de permettre aux racines de se développer librement. Dans les sols compacts, quelle que soit leur nature, en procédant ainsi, on échouera.

A. Fron. - Sylvicullure. 
Pour agir dans de bonnes conditions, l'ouvrier doit avoir soin d'enlever d'un coup de pioche superficiel le gazon, puis d'ameublir le sol ; cela fait, il enfonce vigoureusement sa pioche verticalement, puis en l'inclinant il dégage un ride dans lequel il place le plant en étalant les racines dans leur position naturelle, aussi bien que possible; arec le pied, il tasse ensuite fortement la terre de manière à fermer l'entaille.

Notons que dans la plupart des cas il est préférable de faire la plantation par trous.

Plantation par trous ou potets. - Dans la plantation par trous, on doit faire le trou d'autant plus spacieux que le terrain est plus compact, plus argileux et que le climat est plus chaud.

Les trous sont faits à la bêche ou à la pioche; les dimensions à leur donner peuvent être, par exemple, de $0^{\mathrm{m}}, 20$ en longueur et largeur sur $0^{\mathrm{m}}, 20$ de profondeur, où bien de $0^{\mathrm{m}}, 20$ sur $0^{\mathrm{m}}, 30$, de $0^{\mathrm{m}}, 30$ sur $0^{\mathrm{m}}, 30$ avec une profondeur de $0^{\mathrm{m}}, 2 \mathrm{~s}$ à $0^{\mathrm{m}}, 30$.

L'ouvrier commence par peler le gazon ; puis il enlève la terre régétale chargée d'humus, qu'il met à part; il creuse ensuite le trou en rejetant la terre et l'épierrant avec soin.

Le planteur prend un plant dans son panier (où les racines sont protégées avec de la terre fine); il abat dans le fond du trou la motte de gazon retournée, c'est-à-dire racine en l'air, puis la terre végétale, la disposant en taupinière; de la main gauche, il saisit le plant, étale ses racines dans leur position naturelle sur la motte de terre, puis le. la main droite ramène la bonne terre bien fine, secouant légèrement une ou deux fois le plant, afin de permettre à la terre de pénétrer et de se tasser entre les racines.

Le trou comblé il tasse arec le pied et s'il trouve quelques pierres à sa portée, il les couche à plat autour du plant de préférence en aval du plant si le terrain est 
en pente; ces pierres ont pour effet de tenir le sol frais et de paralyser l'effet des gelées (soulèvement, déchaussement).

Il faut avoir soin de ne pas enterrer trop le plant; le collet de la racine doit affleurer à la surface du sol ou plutôt être légèrement en dessus, en raison du tassement qui se produira plus tard.

Ce mode de plantation est de tous le meilleur et celui qu'il faut préférer, mais il a le défaut d'ètre le plus cher.

Une très bonne pratique consiste à faire ouvrir les trous à l'avance afin de permettre au terrain de se déliter et de s'aérer, au fond et tout autour du trou, sous l'action des phénomènes atmosphériques (pluies, gelées, etc.).

Plantation à la bêche demi-circulaire. - La bèche demi-circulaire est surtout employée dans les terres meubles et légères.

En deux coups de bêche opposés, l'ourrier enlève un cylindre de 2 à 30 centimètres qu'il dépose à côté du trou et qu'il divise en deux portions, la partie herbeuse et la terre. Le planteur ou plutot la planteuse met le plant en terre comme il a été dit précédemment, puis tasse fortement et recouvre la terre remuée à l'aide d'une pierre s'il y en a à proximité.

Plantation par touffes et par motte. - Lorsqu'on ne dispose que de jeunes plants âgés d'un ou deux ans non repiqués (certains résineux surtout) situés sur des pépinières volantes, au milieu mème des terrains à planter, on peut les extraire avec la motte de terre que l'on dépose dans les paniers des planteurs; ceux-ci séparent une petite motte comprenant plusieurs plants, les déposent avec soin sans détacher la terre dans le trou préparé et achèvent la plantation dans les conditions habituelles.

Ce procédé donne d'excellents résultats, mais n'est pas applicable si la pépinière est éloignée.

Plantation par bouquets sans motte. - Lorsque les 
plants dont on dispose sont également petits, mais avee les racines dégarnies de terre, on assure les chances de succès en réunissant ensemble 3 à 5 petits plants que l'on met en terre en étalant les racines, en les éloignant un peu les uns des autres, et en les enterrant arec soin comme il a été dit précédemment.

I'lantation au plantoir Prouvé. - M. Prouvé, ancien inspecteur des forèts, a fait construire un plantoir qui est assez avantageusement utilisé pour planter le chène dans les terrains compacts.

Le plantoir Prouvé se compose: $1^{\circ}$ d'un plantoir proprement dit qui, armé d'un étrier, permet de faire facilement un trou de 25 à 30 centimètres de profondeur ; $2^{\circ}$ d'un appareil ayant la forme d'un pistolet dans le canon duquel on place les racines des plants; on introduit le tout dans le trou préparé, puis d'une main fixant le plant que l'on tient près du collet, de l'autre main on retire l'appareil en laissant le plant; le trou reste béant; on enfonce de noureau le plantoir à quelques centimètres de là et on écrase le trou dans toute sa hauteur serrant ainsi les racines entre deux terres.

Le plantoir Prouvé est surtout utilisé pour les plantations d'essences fortement pirotantes non repiquées, pour le chène en particulier, ce qui permet d'enfoncer profondément les racines sans les retourner, et en mème temps de conserver toutes les radicelles situées à l'extrémité du pirot.

Cette méthode de plantation ne serait pas à conseiller pour les plants ayant beaucoup) de chevelu (plants repiqués), ni pour les résineux; elle est économique, mais eı somme elle place les racines dans un milieu de terre tassé el comprimé; elle les resserre, alor's qu'elles demandent pour prospérer une terre ameublie autant que possible.

Plantation par butles. - II y a lieu de dire deux mots d'un procédé préconisé en Sase et dans l'Allemagne du Nord, la plantation en buttes. 
Lorsque le terrain est maréeageux, couvert d'une épaisse couche de mousse, Ia plantation par trous peut devenir fort difficile, sinon impossible; on a imaginé d'élever' sur les points les plus propices de petites buttes de terre sur lesquelles on plante le jeune sujet. Ces buttes sont formées soit avec du terrain apporté, soit avec de la terre prise sur place en creusant un trou dans le sol marécageux; cette terre est déposée sur la mousse en forme de tas conique au milieu duquel se fait la plantation arec tous les soins désirables.

On peut également, lorsque le terrain le permet, tracer à la charrue deux sillons relevant la terre en sens contraire et se joignant deux à deux ; on obtient ainsi des lignes de terre en ados, dans lesquelles on fait la plantation; la butte est assainie par le creusage de la charrue.

Remarquons que ces plantations exceptionnelles en terrains marécageux ne peuvent s'appliquer qu'à certaines essences spéciales; les diverses espèces de peupliers, les aunes, le chène pédonculé, les frènes et les ormes prospèrent dans ces conditions : le cyprès chauve (Taxodium distichum Richard) est tout indiqué pour le repeuplement de ces sols marécageux; mais cet arbre ne résiste pas aux fortes gelées, il ne faut l'employer que dans les climats tempérés.

Dépenses qu'entraine la plantation. - Les dépenses que nécessitent les plantations sont très variables suivant la nature du sol (terrain facile à creuser ou non, sol rocheux, etc.), le mode de procéder, le prix de revient des plants, leur taille, l'habileté des ourriers et enfin suirant le prix de la main-d'œurre.

a. Plantation à la pioche. - - Sans avoir besoin d'un auxiliaire (femme ou enfant) un bon ouvrier peut dans sa journée planter de 800 à 1000 plants au maximum, en prenant les précautions qui ont été indiquées plus haut.

b. Plantation par trous. - II sera toujours préférable et plus économique de faire ourrir les trous à la tâche en 
réglant d'avance leurs dimensions en tous sens, et en exigeant que l'uuvrier sépare et dépose à part la terre végétale et la terre du fond. Dans une journée et avec une terre très facile à travailler, un homme peut faire jusqu'à 500 trous de $0^{\mathrm{m}}, 20$ sur $0^{\mathrm{m}}, 20$ et de $0^{\mathrm{m}}, 2 \mathrm{2}$ de profondeur. Un planteur peut mettre en terre 600 plants, correspondant à 600 trous en une journée.

Dans les terres mélangées de pierrailles et difficiles à travailler, on comple qu'une journée d'ouvrier correspond au prix de creusage des trous, et au travail de plantation de 100 plants, les trous ayant $0^{\mathrm{n}}, 30$ sur $0^{\mathrm{m}}, 40$ et $0^{\mathrm{m}}, 30$ de profondeur.

Ces données ne sont du reste que très approximatives et il est toujours bon de se rendre compte par expérience du travail qu'on peut exiger par jour dans une région.

Lorsqu'il s'agit de reboisements importants, Ies ouvriers sont disposés en chantiers mixtes; d'une part les ourriers creusent les trous, et derrière eux marchent lesplanteurs, généralement des femmes. On peut admettre qu'un planteur suffit pour deux ourrierscreusant les trous.

c. Plantatic $n$ ì la bêche demi-circulaire. - Ici encore les chantiers peuvent ètre mixtes; un ouvrier ouvre les trous, et derric̀re lui marche une femme qui effectue la plantation. Dans les terrains où ce mode de plantation est applicable, c'est-à-dire dans les terrains suffisamment meubles, dépourvus de pierrailles, un homme et une femme peuvent planter 1000 plants par jour; ce mode de plantation revient donc à très hon marché.

d. Plantation par motte. - La plantation par motte nécessite la présence de la pépinière au milieu des travaux, en raison du transport des moltes. Ce mode de plantation revient toujours très cher à cause des précautions multiples nécessaires pour extraire les plants, transporter les moltes et les défaire avec soin pour conserver de bonnes mottes. Ce procédé ne peut donc atre employé que dans des cas exceptionnels; on peut l'estimer 
approximativement à 20 ou 23 francs par 1000 de plants.

e. Plantation par bouquets. - Lorsqu'on ne dispose que de plants de très petite taille, de pins d'un an par exemple, la plantation par bouquets est préférable à la précédente. Le prix de revient est sensiblement le même que celui de la plantation par trous.

\section{III. - Repeuplement par boutures et par marcottes.}

Le bouturage et le marcottage ne sont pas à proprement parler des opérations forestières, mais bien plutôt des procédés employés en horticulture. Cependant, on se sert du bouturage, de préférence au semis, pour propager les saules et les peupliers (moins toutefois le saule marceau et le peuplier tremble), pour conserver de belles variétés, pour des essences exotiques qui ne sont pas complètement naturalisées, comme le platane, etc. Il peut s'appliquer dans les terrains en pente, dans les sols aquatiques ou encore pour fixer des sables légers.

On distingue deux sortes de boutures: la bouture en plançon et la bouture à bois de deux ans.

Le plançon ne réussit bien qu'avec les saules de grande taille, le saule blanc, le saule osier; c'est une branche de 3 à 4 mètres de haut sur $\ddot{3}$ centimètres environ de diamètre; on la dépouille de tous ses rameaux et on la taille en biseau aux deux bouts ou au moins à son extrémité inférieure. On obtient ainsi une plus grande surface d'absorption et on assure mieux la reprise. Pour la mettre en terre, on creuse soit avec la bèche, soit, dans les terrains humides, avec un pieu, un trou d'à peu près 0 centimètres et on rebouche avec de la terre émiettée. On se sert des plançons pour établir des tètards.

La bouture à bois de deux ans est employée pour les petits saules, les peupliers et les platanes; c'est une 
branche de l'année à laquelle on laisse une portion de bois de deux ans, de trois ans au plus. On en réduit la longueur à 40 ou š 0 centimètres, et on la taille en biseau par le bas, puis on l'enfonce en terre à 30 ou 40 centimètres de profondeur. Quand le terrain est léger, on peut l'entrer directement; sinon, on se sert d'une lige en fer ou bien on cultive le terrain de manière à ne pas arracher l'écorce. Pour plus de facilité, on peut l'entrer obliquement. C'est sourent en pépinière qu'on élève ces boutures; alors on les dispose en lignes, après avoir ameubli le sol.

La marcotte consiste en une jeune tige qu'on courbe de manière à lui faire toucher le sol en la laissant adhérente à la souche. On la maintient en place avec de forts crochets en bois, et on recouvre de terre les jeunes rameaux dont on redresse les extrémités. Ceux-ci ne tardent pas à s'enraciner directement et quand ils peurent vivre d'une manière indépendante, on les sèvre, c'est-àdire qu'on coupe la branche-mère généralement au bout de deux ou trois ans.

Si la tige est trop grosse pour la plier sans qu'elle casse, on l'entaille, sauf à recourrir la plaie afin d'éviter le desséchement.

Ce procédé pourrait sarvir à reboucher de petites vides, à regarnir des clairières dans un taillis après l'exploitation, mais il est plus a vantageux de recourir à la plantation, qui permet d'introduire des essences précieuses là où clles font défaut (Bagnéris).

\section{1. - REPEUPLEMENT PAR SEMIS NATUREL.}

\section{I. - Régénération par coupes successives.}

Dans le repeuplement par semis naturel, le nouveau peuplement nait de la semence qui tombe spontanément des arbres; l'homme n'intervient pas d'une façon directe et immédiate; toutefois il doit combiner les 
diverses opéralions d'exploitation du vieux peuplement avec les nécessités d'assurer la régénération.

En sylviculture, on donne à ce mode de repeuplement le nom de régénération naturelle.

Observation des phénomenes naturels. - Pour qu'une graine germe, il faut qu'elle se troure dans un milieu aéré, présentant un certain degré de chaleur' et d'humidité; ces trois éléments (air, eau, chaleur) agissant simultanément, sont nécessaires et suffisants. La lumière est utile, mais non nécessaire et lorsque la chaleur qui l'accompagne est trop forte, elle peut amener le desséchement et empècher la germination.

Le sol n'est pas non plus nécessaire à la germination, mais il la favorise parce qu'il distribue aux graines, et ensuite aux jeunes sujets dans une proportion convenable les éléments dont ils ont besoin ; pour cela, il faut qu'il soit suffisamment meuble; il faut de plus qu'il soit substantiel pour nourrir les plants après la germination.

Tant que le sol n'est pas bien couvert, que les racines sont petites et restent dans la partie de la terre végétale qui peut se dessécher, les jeunes plants ont besoin d'abri contre la chaleur'; plus tard, l'insolation complète devient nécessaire à une bonne régétation, et elle doit ètre amenée progressivement.

Si l'on joint à ces faits d'observation la nécessité d'obtenir un repeuplement complet, on en déduit les conditions suivantes, relatives à la régénération naturelle et à la végétation des premières années :

Un sol meuble et substantiel; un ensemencement complet; un premier abri aux jeunes plants ; une participation successive aux influences atmosphériques selon le tempérament des jeunes plants.

Deux conditions essentielles doirent tout d'abord ètre remplies, lorsqu'on reut obtenir sme - régénération naturelle ; elles se rapportent à l'état dú sol et à la fertilité des porte-graines. 
État du sol. - Le sol destiné à recevoir la graine est un sol forestier, c'est-à-dire un sol dans cet état spécial de terre à bois que nous avons défini; il en résulte que les conditions favorables à l'installation du jeune plant sont autrement assurées qu'en terrain nu. L'état du sol varie toutefois avec le caractère du peuplement qui le recouvre et il dépend de l'épaisseur du couvert, de l'abondance des débris régétaux, notamment des feuilles qui tombent sur le sol et de leur plus ou moins grande aptitude à se décomposer.

Certaines feuilles comme celles du liètre oa les aiguilles d'épicéa, de consistance coriace, se décomposent très lentement et s'accumulent sur plusieurs lits superposés avant de se dissocier et de se transformer en terreau; d'autres au contraire, celles du charme notamment, persistent peu longtemps et se décomposent rapidement; la nature du sol, un excès d'eau ou d'humidité, l'absence de chaleur et de lumière sous un couvert épais peuvent ralentir la décomposition de ces débris végétaux et Ia rendre très incomplète, provoquer mème la formation de combinaisons acides; de telles situations peurent se présenter sous des peuplements peu âgés d'essences d'ombre ; si elles ne nuisent pas, en général, à la germination, du moins constituent-elles un milieu défavorable à la conservation et au développement ultérieur d'un jeune plant pendant la phase où ses radicelles ne sont pas encore insérées dans le sol minéral.

Dans le mème ordre d'idées, nous avons vu que les peuplements réguliers n'exercent pas la même influence bienfaisante sur les qualités du sol pendant toutes les phases successives de leur existence; dans ces peuplements, il existe toujours une période plus ou moins longue et qui se produit à un âge plus ou moins avancé, où le sol est maintenu en bon état par le peuplement; avant cette période et après, il présente des conditions moins favorables à la naissance d'un jeune recru. Si on 
est conduit à exploiter et par suite à régénérer un peuplement après cette période, en opérant par exemple sur des peuplements en massif clair, les couches de litière peuvent être devenues très faibles, et la reprise du jeune recru moins bien assurée.

Les nécessités qu'impose le but de l'exploitation ne permetlent généralement pas à l'homme de choisir le moment le plus favorable pour provoquer le réensemencement naturel d'un peuplement ou d'une partie de peuplement. Le sol dans ce cas peut ètre plus ou moins apte à remplir le rôle qu'on lui assigne; l'homme doit y veiller, car il peut ètre indispensable, a vant de songer à jrovoquer le réensemencement, d'obtenir par un procédé naturel ou artificiel l'amélioration du sol en rue d'assurer la reprise des semis futurs.

Fertilité des porte-graines. - Pour qu'un peuplement soit susceptible de se régénérer naturellement, il faut qu'il renferme en nombre suffisant des arbres portegraines, susceptibles de donner des semences en grande abondance. Or, toutes les essences ne sont pas fécondes le la mème manière.

D’après MII. Boppe et Jolyet, on peut les classer en deux groupes : celles à semences lourdes et celles à semences légères; et tandis que l'arbre à semences légères fournit presque tous les ans de la graine en très grande quantité, l'arbre à semences lourdes au contraire est rarement fertile deux années de suite. Le degré de fertilité varie donc suivant les caractères biologiques de chaque essence.

Il varie aussi pour une même essence avec les conditions plus ou moins favorables de climat et de sol (1) et avec

(1) Dans le bassin de l'Adour, les chènes, par exemple, donnent des semences tous les deux ou trois ans, tandis que dans les régions du centre et de l'ouest, les glandées abondantes se produisent seulement tous les quatre ou huit ans, et que dans le nord et l'est de la France, elles se font parfois attendre quinze ans et plus. De mène, la fertilité du sapin diminue sensiblement aux grandes altitudes. 
l'effet direct de la lumière et de la chaleur. A ce dernier point de vue deux phénomènes importants sont à noter :

$1^{\circ}$ Dans les régions méridionales, les arbres forestiers fructifient plus tot et plus abondamment que dans les contrées du Nord froides et brumeuses.

20 Les arbres isolés ou les réserves des taillis sous fulaie dont la cime est bien étalée au soleil, sont toujours beaucoup plus fertiles que ceux qui croissent en massif serré; ils donnent plus tôt et en plus grande abondance des semences fertiles.

En raison de ces considérations il est difficile de fixer, l'âge mojen de fructification des arbres forestiers; on peut dire qu'en général cet âge correspond à leur maximum de développement en hauteur; c'est à l'àge adulte qu'ils fructifient avec autant d'abondance et de régularité que le comporte leur situation et l'espèce à laquelle ils appartiennent. Dès que l'arbre devient vieux ou très vieux, sa fécondité diminue progressivement.

Il résulte de ce qui précède que le repeuplement par semis naturel ne s'applique qu'au régime de la futaie, avec celte restriction toutefois que l'âge d'exploitation du peuplement principal aura pour minimum celui qui correspond à l'état adulte de ce peuplement.

Par suite, toutes les futaies exploitées à un âge inférieur à celui de la fructification, par exemple certaines plartations résineuses qui ne sont destinées qu'à donner des produits de faibles dimensions devront ètre repeuplées artiliciellement.

Remarquons enfin que les arbres de réserve des taillis composés, ainsi que les réserves sur coupe définitive, seront susceptibles de donner des semences et par suite d'enrichir, si les circonstances s'y prètent, le peuplenrent inférieur.

Mode de procéder. - Pour régénérer naturellement une futaie on est conduit à réaliser le vieux peuplement 
en un cerlain nombre de coupes successives. Ces coupes sont dites préparatoires el de régénération.

J'hase des coupes préparatoires. - La phase des coupes préparatoires précède celle d'ensemencement. Dans les peuplements serrés, on se propose à ce moment d'amener progressivement le sol à un état favorable au réensemencement par le desserrement du couvert; ce desserrement a pour but de rendre accessible à l'air et à la chaleur les accumulations de litière non décomposée el d'humus brut, et de hâter dans la mesure voulue leur décomposition et leur tassement, sans toutefois interrompre le massif; on se propose en oulre de préparer des porte-graines adultes, susceptibles de donner en grande abondance des semences fertiles et pour cela de légager dans la mesure du possible, par des exploitations partielles, les cimes de ces arbres.

Dans les peuplements dont le couvert est morns épais et surtout élevé, les couches de litière peuvent ètre devenues très faibles, et la phase préparatoire perd à ce point de vue son importance; elle devient même inutile ou plutot change de caractire si l'on se trouve en présence de vieux peuplements où l'état de massif a cessé d'exister ; la lumière qui pénètre jusqu'au sol sous de tels massifs a provoqué la naissance d'une régétation herbacée qui appaurrit le lerrain, le rend dur, desséché et feutré; en présence d'une telle situation, qu'on doit prévoir à l'avance, toutes les mesures de jrolection et de soin général du sol jointes au respect scrupuleux de la couche de litière, et au maintien de toute la régétation ligneuse ou herbacée qui courre le sol, sont les seuls moyens que l'homme ait à sa portée pour préparer le sol à l'ensemencement.

De ce qui précède il résulte que les coupes préparaIoires, si elles sont utiles, ont pour résultat de desserrer le couvert, là où il est trop compact pour une bonne préparation du sol; mais il faut remarquer, en outre, que

A. Fron. - sylvicullure. 
dans les sols riches en éléments minéraux, humides, où la couche d'humus reste épaisse et abondante, l'envahissement des herbes est à redouter dès qu'on desserre un peu trop le massif, spécialement vers la fin de la phase préparatoire; ces conditions seraient mauvaises, de sorte yłue pour améliorer le sol en vue de la régénération, tout en ne provoquant jas lenvahissement de la couverture vivante herbacée, il y a une limite de desserrement, difficíle souvent à atteindre progressivement.

Sur les sols calcaires, la décomposition de la litière s'effectue rapidement et le desserrement du massif doit ètre beaucoup plus prudent que sur les sols frais et troj) humides.

Chaque cas différent exige donc une attention toule particulière si l'on veut établir dans les conditions voulues un milieu propre à la germination. L'entretien de ce milieu est indispensable; d'après M. Gayer, le succès d'une régénération naturelle dépend de la bonne direction donnée aux travaux préparatoires, absolument comme le succès d'un semis artificiel dépend en bonne partie de la fréparation rationnelle du sol.

Cette phase des coupes préparatoires à l'ensemencement comporte donc des opérations délicates qui ont pour triple but :

$1^{\circ}$ De mettre le sol dans un état favorable à linstallation des jeunes sujets, en diminuant peu à peu et progressivement l'épaisseur du couvert ;

$2^{\circ}$ 1'isoler les cimes des porte-graines, de façon à leur donner progressivement l'espace et la lumière ;

$3^{\circ}$ De maintenir toujours le couvert sur le sol pour l'empêcher de se dessécher, de se dígrader', ef par suite le perdre sa fertilité.

Les exploitations que comportent ces coupes préparatoires seront plus ou moins intensives suivant les cas; souvent mème, elles n'auront pas de raison d'ètre; elles devont être eflectuées dans l'élage dominant, afin 
que leur effet soit double : desserrer les cimes des portegraines et restreindre dans une certaine mesure le couvert du sol.

Ajoutons qu'il pourra y aroir lieu pour compléter ces mesures préparatoires de venir en aide à la nature au moment de la chute des graines, si l'état du sol l'exige; suivant les cas, on travaillera par places le sol, râtissant par bandes les amoncellements de litière non décomposée, arrachant et écartant la mousse, enlevant l'humus brut trop abondant, creusant des fossés et des sillons, etc. Toutefois ces moyens, qui n'ont pour but que de rendre le sol apte à recevoir les semences, doivent n'ètre mis en ruvre qu'immédiatement avant la chute des graines, pour remédier à une situation défavorable.

Pliase des coupes de régénération. - Les coupes successives de régénération présentent des caractères différents el on distingue:

a. Coupe d'ensemencement. - Lorsque la phase préparatoire est terminée, le peuplement ou partie de peuplement à régénérer se trouve généralement dans un état de massif encore complet; les années à graines se succèdent à des intervalles plus ou moins rapprochés; mais si les graines peuvent germer, il arrive généralement que les jeunes plants ne persistent pas sous le massif, faute d'une lumière suffisante; si l'essence est à couvert très épais, il peut même arriver que la chaleur fasse défaut au moment voulu pour la germination et que les graines soient tombées inutilement sur le sol. On est alors amené à entr'ouvrir les massifs par une coupe d'ensemencement.

La coupe d'ensemencement doit réaliser les trois premières conditions indiquées précédemment, savoir : maintenir le sol meuble et substantiel, assurer le semis complet, procurer le premier abri aux jeunes plants.

Les exploitations que comporte cette coupe d'ensemenrement doivent porter d'abord sur l'étage dominé, le sous- 
étage el toute la régétation qui, se trouvant à la surface du sol, est susceptible de nuire à l'ensemencement; elles porlent ensuite sur l'étage dominant ou l'on cherche à isoler d'une façon plus ou moins prononcée, suirant les cas, les cimes des arbres porte-graines, afin de rendre leur couvert assez clair pour permetlre laccissur le sol

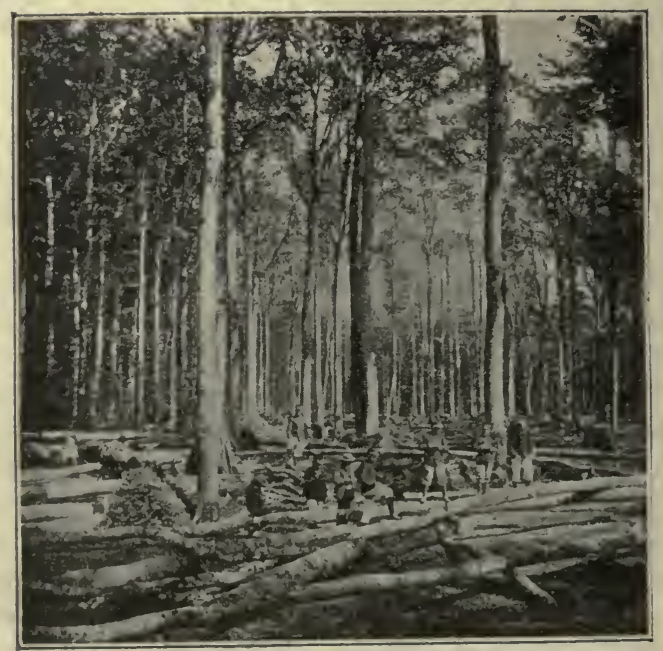

Fig. 25. - Futaie de chène pur à l'état de coupe d'ensemencement. (Forêt domaniale de Bercé, Sarthe). (Pendant l'exploitation.)

de la lumière et des précipilations atmosplériques (fig. 2:ï).

La coupe d'ensemencement se distingue toujours des coupes préparatoires en ce qu’elle provoque une interruption prononcée du massif. A ce point de vue, elle diffire fortement de la dernière coupe préparatoire; dis qu'elle est faite, le semis doit avoir lieu, car lesolest dìs ce moment mal protégé et exposé à l'enrahissement des mauraises herbes. 
L'état du sol, les conditions du climat et le tempérament des jeunes plants conduisent à interrompre plus ou moins le massif à ce moment, et c'est ainsi qu'on distingue la coupe sombre et la coupe claire ou espacée.

Une coupe d'ensemencement est dite sombre quand les branches latérales des cimes des réserves' se touchent

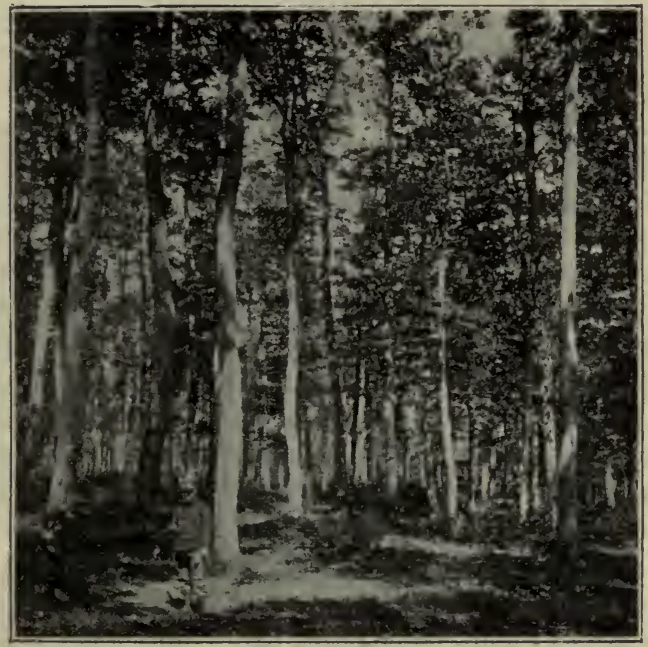

Fig. 26. - Futaie de chène pur à l'état de coupe d'ensenencement. (Forét domaniale de Bercé, Sarthe). (Après l'exploitation.)

lorsqu'elles sont agitées par le vent; l'exploitation dans une coupe sombre ne fait tomber indépendamment de l'élage dominé et du sous-étage qu'un nombre relativement restreint d'arbres de l'élage supérieur, de telle sorle que le couvert du vieux peuplement reste assez épais mais élevé (fig. 26).

Dans la coupe claire, au contraire, l'intervalle entre les cimes peut aller de deux à cinq ou six mètres; l'exploi- 
tation fait tomber indépendamment de l'étage dominé et du sous-étage un nombre plus considérable d'arbres de l'étage supérieur, de telle sorte que le couvert du vieux peuplement reste clair ou très clairiéré après l'opération.

Les circonstances seules peuvent permettre de déterminer l'importance qu'il y a lieu de donner à la coupe suivant la nature de la station, suivant les essences et la hauteur de fût des arbres porte-graines el enfin suivant le tempérament du jeune recru.

La coupe sombre est celle qu'on a le plus souvent occasion d'appliquer; elle est nécessaire toutes les fois que la semence est lourde et s'écarte peu du pied de l'arbre qui l'a produite, que le tempérament du jeune plant est délical, que le sol est exposé à s'enherber fortement ou à se dessécher, qu'on opère sur les lișières: des forêts ou dans des endroits exposés aux rents. Dans toutes ces circonstances, en effet, on peut craindre que le semis ne soit pas suffisamment complet, ou que les graines ne germent pas, ou que le jeune plant ne disparaisse faute d'abri, ou enfin que les réserves ne soient renversées avant d'avoir produit le semis, ou a vant que les jeunes plants ne puissent se passer d'abri.

C'est seulement lorsque toutes les conditions contraires sont réunies qu'on peut faire une coupe d'ensemencement plus ou moins espacée. C'est dire que la coupe sombre est la règle, la coupe espacée l'exception (Gayer).

Dans la pratique des opérations, deux précautionsimportantes sont à prendre :

$1^{\circ} \mathrm{On}$ doit chercher à réserver les plus beaux portegraines, c'est-à-dire ceux qui possèdent une cime ample fortement développée, mais dont le fût est on mème temps élevé; cette hauteur de fût a pour but de diminuer l'action du couvert, et de permettre à la chaleur en mème temps qu'à la pluie d'arriver en quantité suffisante sur le sol. 
En agissant ainsi on contribue à relever le couvert et cette opération est tellement importante qu'elle suffit dans certains cas à assurer l'ensemencement (1); on peut mème provoquer le relèvement du couvert en augmentant artificiellement la hauteur de fût par des élagages, à la condition qu'on opère sur des essences à couvert épais ou sur des chènes destinés à disparaitre au plus tard lors de la coupe définitive.

$2^{\circ}$ En mème temps qu'on recherche des fûts élerés ou qu'on relève le couvert par des élagages, il faut avoir' bien soin de nettoyer le sol de la végétation basse qui peut le recouvrir ; cette précaution, dit M. Bagnéris, est de toute nécessité pour éviter la moisissure des graines pendant l'hiver, en mème temps que pour assurer, en temps utile, le degré de chaleur nécessaire à leur germination; elle est nécessaire pour relever le couvert et permettre le maintien des jeunes plants qui disparaitraient rapidement sous un couvert aussi bas.

b. Coupes secondaires. - Quand le semis est formé et paraît complet, il ne reste plus qu'à assurer le développement de la jeune génération. Le moment est venu de faire tomber progressivement les arbres du peuplement porte-graine, dès qu'ils ne sont plus indispensables au maintien de la fertilité du sol, d'une part, et d'autre part dès que leur couvert commence à devenir nuisible à la croissance des jeunes plants.

Les exploitations ayant pour but de faire tomber ainsi peu à peu les porte-graines, portent le nom de coupes secondaires, et la dernière de ces coupes qui abat tout le vieux peuplement restant constitue la coupe définitive.

(1) Sous des peuplements fornant massif, mais composés d'un petit nomlre d'arbres très branchus dès le bas, le semis ne se produit pas; mais si on élague les branches basses, on voit apparaitre, sous les mèmes arbres, dès la première année de semence, de jeunes senis nombreux et bien venants. Remarquons que l'élagage ne présente dans ce cas qu'un faible inconvénient, car il est opéré sur des sujets destinés à être exploités très rapidement. 
('es coupes secondaires (fig. 27) sont plus ou moins nombreuses et par suite plus ou moins prudentes suivant le tempérament des jeunes plants, et aussi suivant le degré d'humidité du sol, les dangers auxquels est exposé le recru, etc. Le sylviculteur doit mettre tout

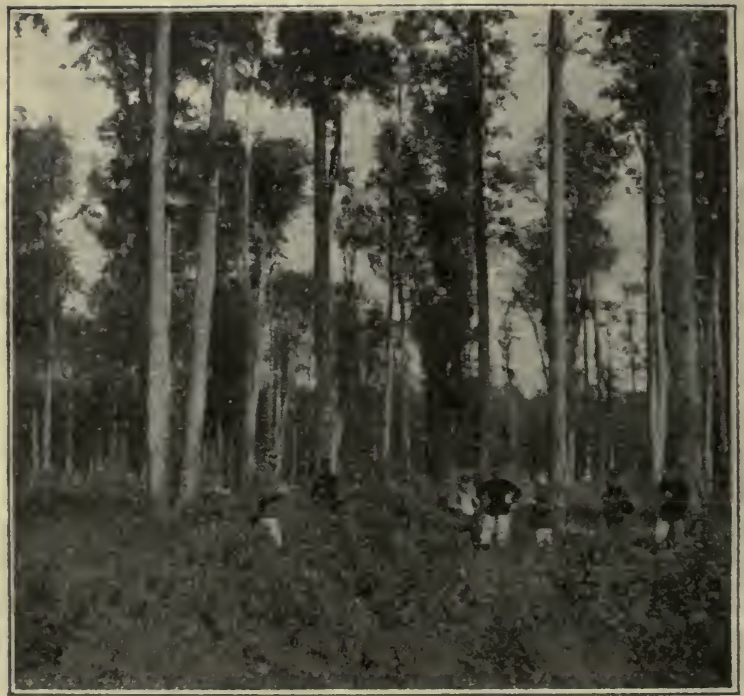

Fig. 27. - Futaie de chéne pur à l'élat de coupe secondaire. (Forêt domaniale de Bercé, Sarthe).

son art à déterminer le degré de couvert le plus favorable à la bonne croissance du recru; il désigne pour etre abaltus les arbres qui recouvrent les semis les plus complets et les plus vigoureux; il laisse inlactes les places dégarnies de plants, car pendant celle période des coupes secondaires, les porte-graines continuent à donner des semences fertiles yui viennent peu à peu completer le repeuplement. 
Dans la pratique, il peut arriver qu'à la suite d'une coupe d'ensemencement sombre, il se passe plusieurs années sans semis et que le massif se referme complètement; il devient dès lor's nécessaire de rétablir le premier état, et la coupe qu'on opère présente le caractère d'une nouvelle coupe d'ensemencement.

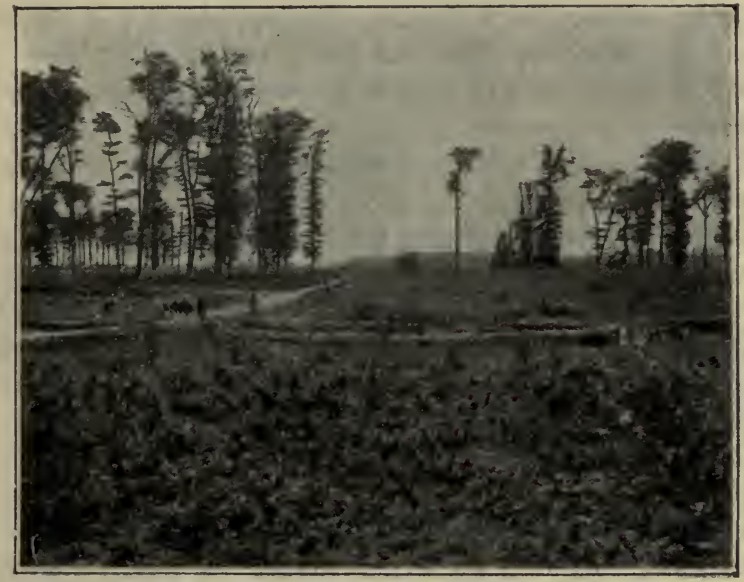

Figr. 28. - Futaie de chène pur à l'état de coupe définitive. (Forèt domaniale de Bercé, Sarthe).

De mème, si dans une coupe espacée les semis se font attendre, le sol peut ètre envahi par des morts-bois ou des arbustes dont la présence devient nuisible pour l'installation des semis; il est alors nécessaire de venir enlever loute cette végétation dès qu'on prévoit une année de simence.

c) Coupe définitive. - Au point de vue de la régénération, la coupe définitive peut se faire quand les jeunes plants, couvrant bien le sol, forment massif à l'état de fourré (figr. 28). 
Mais il ne faut jamais se hâter de passer à la coupe définitive, parce que l'abri des réserves disséminées est le seul moyen de soustraire le jeune peuplement à l'action des gelées printanières dans les endroils qui y sont exposés; parce que tant que le fourré n'est pas bien constitué, le sol n'est pas suffisamment abrité et se délériore; parce qu'enfin, la production ligneuse de la surface est augmentée de tout le développenent que prennent les réserves, sans que les jeunes plants aient à souffrir d'un courert disséminé en même temps que relevé.

Dans la pratique, cette méthode de réensemencement naturel est appliquée de différentes manières. Nous distinguerons deux cas, suivant qu'il s'agit de créer un peuplement régulier, ou un peuplement irrégulier. $V$

$1^{\circ}$ Régénération des peuplements réguliers. - Futaie régulière. - On se propose en régénérant un peuplement régulier, d'obtenir simultanément le semis naturel sur toute la surface de ce peuplement (parcelle en régénération) de telle sorte que la nouvelle génération se compose de sujets ayant tous sensiblement le mème âge et forme un peuplement régulier.

Dañs ce but, on procède par coupes s'étendant uniformément sur toute la surface du peuplement à régénérer, et on distingue la phase préparatoire et la phase de régénération (coupe d'ensemencement, coupes secondaires ef définitive).

La phase préparatoire suit les dernières éclaircies pratiquées dans le peuplement et les continue, car les coupes préparatoires ont le mème hut et emploient les mêmes moyens. Il n'y a donc pas de durée spéciale à leur assigner, elles font suite naturellement et sans modilication aux opérations d'éclaircie. D'intensité très inégale suivant les cas, elles varient suivant les peuplements; dans certaines circonstances, un simple élagage suffit; ailleurs il n'est possible de faire qu'une seule coupe 
préparatoire modérée; ailleurs plusieurs coupes préparatoires progressives et prudentes sont nécessaires; la durée de la phase préparatoire en dépend; elle peut atteindre dix ans et mème davantage.

Dès la coupe d'ensemencement, qui est effectuée uniformément sur toute la surface, les semis doivent se produire et se conserver; on doit s'attacher à obtenir une égale distribution du feuillage plutôt qu'à répartir régulièrement les réserves, afin d'obtenir un semis bien uniforme sur toute la surface. En principe, la régénération doit ètre obtenue en bloc, c'est-à-dire en une année de semence et le semis complet doit recouvrir entièrement le sol.

Il est rare qu'un tel semis se produise en une seule année, et il faut compter au moins sur deux années à graine pour obtenir un semis complet; la période d'ensemencement dure ainsi plusieurs années jusqu'à dix et quinze ans dans certaines régions; toutefois, il y a lieu de remarquer qu'en attendant ainsi une nouvelle année de semence on s'expose, pour les essences à tempérament robuste, à voir les jeunes plants disparaître successirement sous le courert et le semis rester toujours incomplet; aussi pour ces essences faut-il le plus souvent se contenter d'un semis partiel, pourvu que les jeunes plants, d'ailleurs bien répartis, soient assez nombreux pour se constituer à l'état de fourré au bout de peu d'années (dix ans par exemple). Les essences à tempérament délicat, dont les plants peuvent persister sous le couvert d'une année de semence à l'autre, permettent seules d'attendre un semis complet, c'est-à-dire réparti très uniformément et en grand nombre à la surface du sol (1).

Aussitôt qu'on dispose de ce semis, on doit commencer

(1) On appelle semis préexistants ceux qui existent sur la surface à régénérer et qui ont pu persister, soit en raison de leur tempérament, soit pour des causes accidentelles, jusqu'à la coupe d'ensemencement. Souvent, ces semis sont susceptibles de se raccorder avec l'ensemble des autres senis. 
le desserrement progressif des porte-graines; c'est la phase les coupes secondaires qui s'échelonnent jusqu'à la coupe définitive. Leur durée absolue est très variable; elle dépend des essences et des stations; iei la coupe définilive aura lieu au bout de trois à cinq ans après la chute des graines; ailleurs elle n'aura lieu qu'au bout de dix ou quinze ans et plus.

Du fait qu'on a mis simultanément toute la surface en ensemencement, il résulte que la phase des coupes secondaires ne peut se prolonger; si la régénération n'a été obtenue que partiellement, elle peut se compléter pendant les premières coupes secondaires, mais à partir le ce moment il ne faut plus compter sur les portegraines disséminés pour la terminer, parce que le sol a dû se détériorer sous l'action du découvert là où le semis n'est pas assuré, et qu'il ne reste pas apte à un ensemencement naturel. Si les vides présentent une grande élendue, la régénération y est manquée; on doit faire tomber les arbres qui les surnontent et procéder à des repeuplements artificiels.

Dans les bonnes stations, rien n'empèche de conserver assez longtemps sur la coupe définitive les plus heaux porte-graines, spécialement ceux qui sont les plus élanrés, pour les faire bénéficier de la mise en lumière, et leur permettre d'atteindre des dimensions exceptionnelles. Ce cas se présente surtout pour l'essence chène, dont les fortes pièces deviennent de plus en plus rares, et qui sont si recherchées par la consommation. Les arbres ainsi conservés doivent ètre rigoureusement débarrassés de leurs branches gourmandes; si on néglige cette pré(aution, beaucoup de chènes, ainsi laissésen réserve, sont exposés à mourir en cime et il devient nécessaire de les exploiter.

Observations sur le martelaye des coupes. - L'époque. la plus favorable pour effectuer le martelage de ces différentes coupes rost géuciralement le commencement de 
l'automne, époque où l'on peut le mieux se rendre compte de linfluence du couvert sur le sol et sur le recru.

Dans les coupes secondaires, le but principal de l'opération est de découvrir le recru là où il est le plus fort et où il paraît menacé par le couvert, et de laisser au conlraire sur place les porte-graines là où il est peu abondant ou n'existe pas. En général, ce recru est disséminé par taches plus ou moins réparties sur toute la surface; c'est en recherchant ces taches qu'on se guidera, dans la pratique, pour effectuer un bon martelage; dès lors la régularité absolue du peuplement tend à disparaitre, mais comme on opère en fait toujours assez rapidement pour que les différences d'âge ne soient pas Irès accentuées, la régularité se rétahlira très vite dans le jeune peuplement.

Remarquons enfin que l'expérience permet de considérer, spécialement pour les essences à tempérament robuste, un semis comme réussi, s'il existe au moins un plant par mètre carré de surface; ce ne sont pas d'ailleurs, en général, les semis trop serrés qui donnent les meilleurs résultats.

\section{Régénération de peuplements irréguliers. -} Futaie traitée par coupes successives ayant un caractère jardinatoire. - On se propose en régénérant un peuplement irrégulier d'obtenir successivement dans ses différentes parties le semis naturel, de telle sorte qu'au bout d'un temps donné, il existe un semis complet 'mais irrégulier sur toute la surface de ce peuplement (parcelle à régénérer).

Les opérations successives de régénération ont pour but de créer un nouveau peuplement irrégulier, dans lequel les différences d'âge varieront dans une moyenne de vingt, trente à quarante ans.

II en résulte qu'au lieu de n'utiliser en principe qu'une seule année à graine pour régénérer toute la surface du 
peuplement, on est conduit à utiliser sur des places isolées et disséminées dans le peuplement à régénérer, successivement et presque au hasard, un grand nombre d'années à graine.

Le peuplement à régénérer doit donc être supposé divisé, sans aucun ordre ni suite, en un certain nombre de groupes ou bouquets disséminés les uns à côté des autres.

Remarquons que si l'on opère sur un peuplement régulier, rien n'est plus facile que de commencer les opérations par places disséminées pour arriver au mème but.

Dans chaque groupe ou bouquet, le repeuplement partiel de ce bouquet s'effectue au moyen de la semence d'une ou de deux années fertiles successives; l'irrégularité est donc créée par le fait qu'on n'opère pas simultanément la régénération sur les différents bouquets, el dès lor's le peuplement dans son ensemble se présente sous les aspects les plus divers; on y trouve des parties où le repeuplement est opéré, des bouquets en voie de régénération plus ou moins avancée, des places où le vieux massif complet persiste encore et va entrer dans la période de régénération; tout est mélangé, et il appartient à l'opérateur de déterminer le moment où chaque bouquet doit être traité, et quelle opération il y a lieu de faire sur chaque point particulier. D'un côté, il trouve quelques semis déjà installés et de bonne qualité, et un sol aple à recevoir la semence; l'opération désignée est une coupe locale d'ensemencement; d'un autre côté, co sont l'âge, la croissance du peuplement, son état de massif ou bien les essences qui le composenl, qui décidenl l'opérateur à faire tomber de vieux sujets de fortes dimensions et à commencer ainsi une régénération urgente; ailleurs, il fait de simples coupes préparatoires; en un mol, se laissant guider par la nature et les lois nalurelles, il agit indépendamment dans chaque groupe 
ou bouquet, qui constitue pour lui un peuplement à part, et il opère la régénération dans ce groupe en se conformant entièrement aux nécessités locales et en suirant les règles que nous avons établies précédemment. Toutefois, en raison même du but qu'on se propose, deux préoccupations doivent guider les exploitations : provoquer la formation de bouquets de recru, ou conserver ceux qui existent déjà; agrandir les taches de jeune repeuplement.

Nature des coupes ayant pour objet la création ou la conservation des bouquets de recru.

Il est facile de se rendre compte que suivant les cas, ces coupes présenteront le caractère de coupes préparatoires, là où il y a lieu de rendre le sol apte à recevoir la semence; de coupes d'ensemencement, là où le sol est en bon état et où existent déjà quelques jeunes semis qu'il est urgent de décourrir; de coupes secondaires, là où un jeune recru bien venant est déjà installé; enfin de coupes définitives, là où il est possible d'enlever tout ce qui n'est plus indispensable à la protection du sol et du jeune fourré, et où il est utile de décourrir complètement un bouquet de jeune recru préexistant en bonne roie de croissance.

b. Nature des coupes ayant pour objet d'agrandir les taches de jeune repeuplement. - Dès qu'on possède dans la parcelle en régénération des bouquets de repeuplement formés en massif, les exploitations qui concernent ces bouquets changent de caractère; la coupe définitive a fait table rase au-dessus d'eux et ils se présentent alors comme une tache de eune recru enclos dans le peuplement porte-graine et entouré par lui de tous côtés. D’après II. Gayer, la lisière de ce peuplement reçoit assez de lumière oblique pour permettre à un nouveau recru d'y prendre racine et de se souder étroitement au bouquet déjà existant. Aussitôt que la reprise en est assurée, on pratique dans ces parlies des coupes sccondaires circu- 
laires, puis la coupe définitive; on peut d'ailleur's réserver parplaces des sujets d'avenir en vue de leur accroissement par la mise en lumière. La première année à graine qui se présente ensemence sous le vieux peuplement les farties contiguës à la lisière du bouquet; ces parties son t soumises aux mêmes coupes, et ainsi de suite. Ainsi le semis se fait toujours sur les lisières des bouquets et 'nvahit plus ou moins profondément le vieux peuplement qui se retire au fur et à mesure.

A la fin de la période fixée pour la régénération totale du peuplement, il ne reste plus du vieux peuplement que les sujets convenablement répartis qu'on a pu réserrer sur les coupes définitives, et le jeune repeuplement se compose par bouquets épars d'un jeune recru, et de bouquets d'àges gradués, depuis zéro jusqu'à vingt, trente ou quarante ans, suivant le temps qu'on a mis à obtenir la régénération totale. Le peuplement est irrégulier et conservera ce caractère pendant le cours de son existance.

Observation sur la marche génẻrale des opérations. En principe, il y a lieu de commencer le travail de régénération à l'intérieur du peuplement et de terminer par les lisieres; toutefois, les bouquets de semis hien constitués qui se trouveraient à proximité des lisières ne doivent. pas ètre négligés.

Quant à la surface occupée par chaque bouquet, elle peut varier de quelques mètres carrés à plusieurs ares, mais, en général, l'étendue moyenne des bouquets est réglée par le tempérament des essences et par le but quion se propose d'ohtenir, notamment dans les forèts mélangées où l'on peut répartir le mélange par bouquets de mémes essences, et traiter chacun de ces bourquets séparément comme il vient d'être dit. Dans ce cas particulier, la surface de chaque bouquet présente un maximum au delà duquel le peuplement perd les avantages du mélange, et un minimum délerminé par les exigences de chayne essence. 
En faisant varier ainsi les étendues de chaque bouquet, suivant les nécessités locales et les essences qui les composent, on obtient une immense variété de combinaisons qui se prètent à tous les cas particuliers.

Cette méthode de régénération par groupes ou bouquets, destinée à créer un peuplement irrégulier se rapproche le la méthode naturelle à qui elle emprunte ses multiples a vantages; le passage de l'ensemble du peuplement d'une génération à l'autre se fait lentement, d'une façon progressive, et la forèt évite ainsi d'une façon absolue, le temps de crise qui accompagne la régénération en futaie régulière.

Dans un très grand nombre de cas, cette méthode de régénération, ainsi que les conséquences qui en résultent (peuplement irrégulier, réparti par bouquets d'essences de mélange), parait conduire au type de forèt le plus à conseiller dans la propriété boisée particulière, bien entendu lorsqu'il s'agit d'un peuplement de futaie.

La forme de peuplement qu'on obtient ainsi (futaie mélangée exploitée par coupes jardinatoires) présente l'immense avantage de ne pas soumettre la forèt à des exigences déterminées, et à un cadre fixe dont on ne peut s'écarter, mème si les nécessités l'exigent ou si des parlages viennent en rompre subitement l'harmonie.

Suivant les cas, la durée totale de la période de régénération peut ètre courte, plus ou moins longue, enfin mème égale à l'âge d'exploitation du vieux peuplement; ainsi la forèt se rapproche, tantôt de la futaie régulière (période de régénération courte), tantôt de la futaie jardinée (période de régénération très longue); se pliant aux́ diverses exigences des stations, elle permet d'utiliser' en tous lieux, au maximum, les forces naturelles.

Ajoutons toutefois que, comme tout peuplement irrégulier, cette forme de peuplement demande une plus grande surveillance, des soins plus assidus et plus nombreux, intelligemment dirigés; qu'enfin les exploitations ont 
besoin d'ètre réglées avec grand soin afin d'éviter de trop enrichir ou de trop appauvrir la forèt en matériel sur pied et par suite d'en changer le caractire.

Futaie jardinée. - La période de régénération cmbrasse ici toute la durée de la révolution; on se propose, pendant toute l'existence du peuplement, d'obtenir suc:cessivement et par places le semis naturel, de telle sorte qu'il existe toujours sur toute la surface de la torêt et disséminés sans aucun ordre $: 1^{\circ}$ des points isolés, des places ou bouquets en régénération; $2^{\circ}$ d'autres places présentant isolément tous les degrés de développement depuis le jeune âge jusqu'à l'état de vieux peuplement ou d'arbre exploitable. Dès lors toutes les années à graine sont mises à profit, les semences qui se produisent naturellement pendant toute la phase de l'existence du peuplement ne donnent de résultats utiles que sous le courert des vieux sujets isolés ou réunis par groupes, dans. les bouquets de vieux perchis espacés, dans les lacunes quand il y en a ; en un mot, partoutoù l'état du sol et du couvert est favorable à l'installation du jeune recru.

C'est donc par taches irrégulièrement disséminées sur toute la surface de la forèt que se présentent les semis uaturels.

L'unique préoccupation de l'opérateur qui marque ses coupes d'exploitation est de stimuler le développement ultérieur de ce recru, par des coupes présentant généralement le caractère de coupes secondaires et définitives, rarement de coupes préparatoires; il est ainsi amené naturellement à faire tomber sur des points quelconques de la forèt les arbres du vieux peuplement, et en mème temps, les arbres tarés mal renants ou dépérissants (1).

Mieux que loute autre, la futaie jardinée se rapproche. de la forèt naturelle; elle en présente plus ou moins,

(1) Dans les futaies jardinées, la préoccupation de l'opérateur est souvent bien plus de disposer des arbres exploitables que de faire de la régénération. Et, en effet, celle-ci laisse souvent à désirer daus les forêts jardinées. 
suivant la manière d'opérer, les avantages et les inconrénients.

L'emploi de cette méthode de régénération est utile partout où il est indispensable de maintenir constamment le couvert pour protéger le sol contre les dégâts des eaux de ruissellement, de la neige et lu vent, surtout dans les situations exposées de hautes montagnes; la futaie jardinée convient bien aux petites exploitations qui ne sont pas susceptibles de combinaisons d'aménagement, et auxquelles on se contente de demander, à des dates indéterminées et suivant les besoins, les ressources disponibles.

\section{II. - Régénération par coupe unique.}

Les trois méthodes générales de repeuplement naturel que nous venons d'examiner ont toutes pour objet d'obtenir. la régénération sous le couvert des arbres porte-graines, et se conforment aux principes généraux que nous avons posés dès le début de cette étude.

Dans certains cas particuliers, soit qu'il s'agisse de stations spéciales, soit qu'il s'agisse d'essences plus aptes à se reproduire et à se disséminer, le repeuplement naturel peut être obtenu d'une façon plus simple.

Tel est le cas de la régénération par coupe unique ou par ensemencement latéral.

Examinons la situation d'une parcelle de forèt, coupe de forme indéterminée ou bande, qu'on exploite à blanc: étoc au milieu d'un peuplement; à peu de chose près (1), elle est la même que celle d'une grande clairière, d'une lacune ou d'une terre bordant la forèt.

Si les circonstances s'y prêtent (transport des graines

(1) L'état du sol est plus favorable au réensemencement sur une surface ou le terrain se présente à l'état de terre à bois, que partout ailleurs, à la condition que ce sol ne soit pas envahi trop rapidenent par une végétation parasite exubérante. 
légères par le rent ou par toute autre cause accidentelle, humidité et conditions climatologiques favorables, puissance plus ou moins enrahissante des herbes, vigueur des jeunes semis dès leur premier âge, etc.), il suftira Ique cette surface se trouve à proximité d'arbres portegraines pour qu'elle puisse se boiser naturellement.

Ajoutons toutefois que dans le cas où l'on veut obtenir la l'égénération à l'aide d'une coupe unique, il existe au moment de l'exploitation à blanc étoc du peuplement supérieur une grande quantité de semences fertiles qui viennent de tomber sur le sol et sont susceptibles de germer.

Si les jeunes plants peurent se développer dans ces conditions, la méthode est acceptable; elle est appliquée dans certaines circonstances, en montagne, pourl'épicéa, dont la semence légère se dissémine facilement au loin, et dont le jeune plant ne craint pas, en climat humide, un découvert total dès la première jeunesse.

On procède dans ce cas par bandes longues et étroites, disposées de telle façon que les rents dominants tendent à apporter naturellement sur ces bandes les semences fertiles du vieux peuplement voisin.

C'est par un repeuplement du mème genre qu'on voit en montagne se reboiser naturellement et d'une façon progressive, en épicéa, les prés - bois ou pâtures voisins de la forèt.

Observation générale. - Nous avons détini un celtain nombre de manières d'opérer le repeuplement pa. voie de réensemencement naturel. En pratique, dans la nature, entre chacune de ces méthodes existe une fusion qui ne permet pas de définir leurs limites respectives; c'est ainsi que la méthode des coupes jardinatoires peut lendre vers la création de peuplements réguliers, ou jardinés; que la méthode de régénération par coupes sueressives peut tenir compte, dans une certaine mesure, de lensemencement latéral, sous l'influence duquel des 
peuplements purs peuvent se transformer progressirement en peuplements mélangés.

En raison de cet ensemencement latéral et quelque soin qu'on prenne, il arrive sourent, surtout dans les sols riches et sous les climats tempérés, que des essences à semences légères, notamment des bois blancs, s'emparent du terrain pendant les coupes de régénération. Nous verrons qu'il appartient à des opérations culturales bien conduites de s'en servir dans une certaine mesure, pendant qu'elles sont utiles à la constitution du massif, puis de les éliminer progressivement au fur et à mesure qu'elles deviennent nuisibles.

\section{1. - REPEUPLEMENT PAR REJETS DE SOUCHES ET DRAGEONS.}

Lorsque les forèts sont exploitées avant l'âge de fructification du peuplement, elles ne peurent se reproduire par voie de semis naturel. Pour les essences résineuses, la seule méthode pour obtenir la création d'un nouveau peuplement est le repeuplement artificiel.

Pour les essences feuillues, le repeuplement se fait par roie de rejets de souche et drageons.

Rejets et drageons. - Dans les méthodes de repeuplement par voie artificielle ou par semis naturel, nous ne nous sommes pas préoccupé de la souche et des racines des arbres exploités; ces souches sont laissées dans le sol si l'exploitant ne peut en tirer parti, ou bien elles sont partiellement extraites. En raison de l'âge toujours arancé de l'exploitation, elles ne sont en général plus susceptibles de donner des rejets, tout au moins des rejets utilisables; elles n'ont plus, par suite, aucun rôle à jouer.

Il n'en est pas de mème dans les exploitations de peuplements jeunes qu'on veut régénérer par rejets ou drageons. Toutes les souches et racines de l'ancien peuple- 
ment doivent rester incorporées au sol, et ce sont elles qui émeltent les rejets et drageons indispensables pour la reconstitution naturelle du nouveau peuplement. Le repeuplement par voie de rejets de souches et drageons est ainsi un simple rajeunissement du peuplementinitial, plutôt qu'un repeuplement proprement dit.

Dès lors, on doit admettre que les, mèmes souches, après avoir fonctionné pendant'de longues révolutions, pourront être qualifiées de vieilles ; à ce moment elles auront perdu peu à peu leur vitalité, et par suite leur faculté d'émettre des rejets vigoureux.

Le taillis perpétué uniquement par rajeunissement"des mèmes souches d'un peuplement initial, est infailliblement roué à un dépérissement progressif s'accentuant au fur et à mesure des exploitations successives.

Perpétuité du peuplement. - Dans la pratique, il n'en est pas ainsi et à côté du simple rajeunissement, il y a effectivement véritable création de sujets nouveaux, lue aux causes suivantes :

$1^{\circ}$ Affranchissement des rejets et drageons. - Les rejets issus des bourgeons proventifs qui existent 'sur le pourtour de la souche apparaissent en terre, ou au moins en contact avec elle, si la souche a été bien exploitíe rez terre; ils y trouvent d'abord un point d'appui, puis un grand nombre d'entre eux ne tardent pas à développer des racines qui en font des pieds indépendants` de la souche mère. A l'exploitation suivante, leurs souches jeunes et indépendantes sont susceptibles de donner des rejets vigoureux, aptes à remplacer ceux de la vieille souche qui perd sa vitalité. C'est de celle manière qu'on peut expliquer la durée pour ainsi dire indéfinie de certains taillis simples, sans aucune réserve, ne pouvant, far conséquent, so reconstituer à l'aide de brins de semence el dans lesquels on n'a jamais fait de repeuplements artiliciels. Ce fait, dit M. Bagnéris peut du reste s'observer dans tous les taillis; on y voit fréquemment 
les cépées indépendantes les unes 'des autres, mais disposées suivant une circonférenée d'un diamètre variable ; ces cépées proviennent d'une suuche primitivement unique et dont la partie centrale s'est détruite; ce phénomène peut se suivre depuis la formation d'une cépée, au centre de laquelle on retroure encore l'ancienne souche, jusqu'au moment où les rejets se séparent par la décomposition de plus en plus complète de cette souche; le cercle ainsi formé qu'on appelle quelquefois le cercle de fées s'agrandit jusqu'à ce qu'il y ait assez d'espace à l'intérieur pour permettre la production de rejets et de nouvelles souches; alors la disposition devient diffuse et on a peine à la reconnaître.

Lorsque l'essence possède la faculté de drageonner, les drageons sont susceptibles de s'affranchir de la mème manière.

$2^{\circ}$ Création effective de nou veaux sujets. - L'apparition de noureaux sujets est due à des semences fournies par des arbres porte-graines voisins; ces brins de semence, recépés arec le taillis dont ils font partie, constituent de nouvelles souches jeunes qui viennent remplacer les vieilles souches épuisées.

Ainsi s'explique la perpétuité des massifs repeuplés par voie de rejets et de drageons.

Laissant de coté ces causes de véritable repeuplement, qu'il était toutefois indispensable de signaler, en raison de leur grande influence sur l'arenir de la forèt, nous nous occuperons spécialement du rajeunissement par rejets de souche $(\mathbf{1})$.

Les conditions exigées pour obtenir le meilleur résullat possible avec le repeuplement par rejets de souche,

(1) Les drageons jouent, chez certaines essences dites drageonnantes, un role prépondérant et fournissent alors au taillis une partie des tiges qui le constituent; il ne paraft pas utile d'en faire une étude spéciale, car les souches mises dans les conditions les plus favorables à la production des rejets, sont aussi celles qui chez les essences drageonnantes donnent les meilleurs drageons. 
dépendent des pratiques de l'exploitation. Dès lor's il est nécessaire de considérer successivement l'âge d'exploitation, l'élat et la vitalité des souches au moment de l'exploitation, la saison la plusfavorable à l'exploitation el l'opération mécanique de l'abatage.

Age d'exploitation, ou révolution du taillis. - S'il s'agit d'un arbre de franc pied, d'après M. Gayer, l'âge auquel les souches conservent la faculté de donner naissance à des rejets après abatage de l'arbre, varie suivant les essences et les stations; en moyenne, on peut admeltre que c'est pendant la période du plus grand accroissement en hauteur que la faculté de rejet atteint sa plus grande intensité; elle persiste mème plus longtemps, dans une mesure qui varie selon les essences. Dans l'intérêt d’une bonne régénération, il est désirable que la coupe se fasse à ce moment, car de fortes souches bien développèes assureront un massif vigoureux susceptible de devenir riche en fortes perches; il est impossible d'obtenir le mème résultat au moyen d'un nombre plus considérable de souches moins âgées (1).

Quand il s'agit de souches déjà plusieurs fois recépjées, on ne peut admettre des révolutions aussi longues; la faculté de rejeter de souche se perd plus ou moins rapiment suivant les essences et les stations'; l'expérience a nettement établi une limite supérieure générale audessus de laquelle on ne peut plus être certain de la régénération par rejets; il n'est jamais prudent de dépasser l'age de quarante ans pour aucune essence.

(1) Cetle remarque peut s'appliquer i la transformation en taillis d'une jeune futaie par recépage pur et simple; elle inontre en outre que dans les taillis, spécialement dans les taillis à courte révolution, il est bon, mème lorsqu'on ne fait pas de taillis sous futaie proprement dit, de réserver avec soin comme baliveaux tous les brins de semence qui se trouvent mélangés au taillis; lexploitation ultérieure de ces arbres comme baliveaux ou petits moderues, enrichit leaucoup plus le taillis en souches bien développées que ne le ferait leur exploitation immédiate nvec le taillis lui-même. Inutile d'ajouter que ces arbres fructifieront, et que dès lors leur influence bienfaisante sera slouble. 
Quant au minimum d'âge d'exploitation, on peut dire qu'il n'y en a pas au point de rue de la régénération. En effet, plus le taillis est coupé jeune plus on a de chances de trouver les bourgeons proventifs encore virants ef rigoureux, et par suite susceptibles de se développer.

État et vitalité des souches. - Les souches doirent ètre saines pour donner de bons rejets au moins chez un grand nombre d'essences, telles que l'orme, le tremble, l'aune; chez le chène et le charme, les rejets paraissent moins souffrir de la pourriture partielle de la souche. Cet état sain est plus ou moins lié d'ailleurs à la vitalité de. la souche, et cette vitalité varie beaucoup avec l'essence d'abord et aussi a vec la qualité de la station; le chène et le charme conservent longtemps, pour ainsi dire indéfiniment, la faculté de rejeter de souche après un grand nombre d'exploitations; le frêne, le bouleau, la plupart des bois blancs et mort-bois ne la conservent guère plus de deux à trois révolutions.

De ce qui précède, il résulte que pour avoir un bon taillis, il sera préférable d'arracher à temps les souches gâtées qui tiennent inutilement une place où il vaut mieux voir se réinstaller naturellement ou mème artificiellement des semis de bonnes essences; et qu'en outre un recépage fréquent (et intentionnellement mal fait) de certaines essences non précieuses tendra à provoquer la disparition de ces essences secondaires, et par suite à favoriser la bonne composition d'un taillis (1).

Saison la plus favorable à l'exploitation. - En principe la saison la plus favorable à l'exploitation du taillis est, la tin de l'hiver, quelques semaines avant le gonflement des bourgeons. La première condition pour que les rejets se produisent c'est que l'écorce soit bien adhérente au bois; si elle en est séparée, le bourrelet sur lequel se

(1) Remarquons toutefois que pour agir ainsi, il faut bien connaltre son peuplement, les conditions de végétation et surtout le but à obtenir, car en agissant au hasard, des opérations de ce genre deviendraient très scabreuses.

A. Fron. - Sylvicullure. 
développent les bourgeons adventifs ne peut se former dans de bonnes conditions; de mème si les bourgeons proventifs, dont l'extrémité est engagée dans toute l'épaisseur de l'écorce ont été brisés, la production des rejets en est singulièrement amoindrie. Pour conserver cette adhérence, il est nécessaire de ne pas exploiter avant les grands froids, car les pluies d'automne peuvent s'infiltrer dans les tissus ligneux, entre l'écorce et le bois, et provoquer un décollement de l'écorce si les gelées surviennent à ce moment; de même il faut éviter de couper pendant les grands froids, parce que, à ce moment, l'adhérence de l'écorce au bois est moins grande, ot que celle-ci peut sauter sous le choc de l'instrument. D'un autre côté, on reproche ‘à l'exploitation faite en temps de sève de donner des rejets moins nombreux, moins vigoureux, exposés aux gelées d'automne avant d'ètre bien lignitiés et surtout de faire perdre l'accroissement d'une année. Il ne resterait ainsi pour l'exploitation qu'un temps assez restreint, compris entre le commencement de février et la fin de mars, et en théorie c'est l'époque la plus favorable, car la cicatrisation peut commencer immédiatement après, dès le départ de la sève, ce qui contribue à la bonne conservation de la souche. Mais en pratique ce délai est trop restreint; pour de grandes coupes, il exigerait trop de main-d'œuvre en un mème moment, et aurait le grand inconvénient de supprimer le travail d'hiver pour tous les ouvriers ilu métier qui se font bûcherons pendant le chômage des travaux. Aussi, en fait, exploite-t-on pendant toute la morte-saison et mème à l'automne comme l'exigent souvent les conditions de l'exploitation, sauf peut-itre dans la zone parisienne où les dégradations dues à l'influence de l'humidité et de la gelée sont à redouter. II est nécessaire que les bois abattus soient tous enlevés de la coupe ou tout au moins façonnés avant le départ de nouveaux rejets, et ce n'est qu'exceptionnellement, lors- 
qu'il s'agit de rendre possible l'écorçage, qu'on doit exploiter en temps de sève.

Abatage. - L'abatage doit se faire rezterre et la section doit être aussi nette que possible; il est nécessaire en effet, d'obtenir des rejets de souche susceptibles de s'affranchir et non des rejets de tige. La section doit ètre nette et bombée; on l'exécute à l'aide d'instruments tranchants (hache et serpe pour les brins n'ayant pas ciny centimètres au moins de diamètre), prohibant l'emploi de la scie qui donne une section rugueuse et fibreuse (1); puis on la ravale afin de lui donner de préférence la forme convexe d'un verre de montre, sans esquille, ou celle d'un toit à double pente et non la forme défectueuse d'une gouttière centrale ; en opérant ainsi, on évite d'avoir une surface mâchonnée, par la scie, ou en goutlière permettant à l'eau de séjourner sur la souche et d'en accélérer la décomposition; une telle section amènerait la pourriture des rejets dans la partie de leur pied qui englobe la souche; elle présenterait ainsi l'inconvénient d'empècher, à l'exploitation suivante, la production de. rejets, ou du moins d'altérer leur vitalité. A ce point de vue le chène, le charme, le tilleul sont moins sensibles que d'autres essences telles que le hètre, l'érable et les bois tendres.

\section{IV. - COMBINAISON DES DIFFÉRENTES MÉTHODES DE REPEUPLEMENT.}

Dans la pratique, on n'emploie pas d'une façon absolue une méthode de repeuplement à l'exclusion de toute autre, et b́ien souvent dans les opérations forestières il y a lieu

(1) L'emploi de la scie exige beaucoup trop de temps quand il s'agit de perches de la grosseur de celles d'un taillis. C'est seulement pour des arbres pouvant fournir du bois d'œuvre que l'emploi de la scie reprend ses avantages, parce qu'on économise tout le bois qui tomberait dans la taille d'abatage. Mais alors il ne présente pas d'inconvénients, car on ne se propose plus d'obtenir des rejets de souche. 
de combiner entre elles les différentes méthodes de repeuplement.

Cas des futaies. - Dans les futaies régénérées naturellement, on peut être amené à se servir du repeuplement arlificiel de deux manières:

$1^{\circ}$ En le faisant précéder sur des points déterminés la naissance du semis. Par exemple:

a) Si le sol, dans certaines parties du peuplement à régénérer, ne peut être amené à un état favorable au succès du repeuplement, s'il s'appauvrit, est envahi depuis longtemps par un épais feutrage de gazon ou devient inarécageux, etć., l'introduction immédiate d'une nouvelle essence, par voie de plantation, est utile à tous points de vue.

b) Si par suite d'un manque d'abri, le sol ne peut plus ètre amené à un état favorable au succès du repeuplement; l'introduction par voie de plantation d'un peuplement accessoire de protection peut permettre de l'améliorer, en vue du réerisemencement naturel.

c) Si on veut introduire dans un peuplement, soit homogène, soit mélangé, de nouvelles essences à l'état de bouquets préexistants.

$2^{\circ}$ En complétant une régénération naturelle incomplète, soit par semis, mais plus généralement par plantation artificielle.

Cas des taillis. - Dans les taillis, il peut ètre utile d'a voir recours au repeuplement artificiel pour entretenir le peuplement dans de bonnes conditions de massif et maintenir la proportion des bonnes essences principales. Suivant l'essence, et aussi suivant la station, les souches perdent plus ou moins leur faculte de rejet, et la disparition de ces centres de production donnne naissance it des lacunes dans le peuplement. L'apparition de semis arcidentels vient souvent fermer les vides ainsi créés. mais il est à remarquer que.les essences dont lc rent dissémine au loin les graines, sont généralement de qua- 
lité inférieure. Il est dès lors avantageux de combler artificiellement ces lacunes, au moyen de forts plants repiqués, qu'on peut utilement recéper, dès qu'ils sont bien enracinés. On remplit ainsi les vides de jeunes souches vigoureuses, fort utiles pour l'avenir du taillis, et pour le bon entretien de la fertilité du sol.

Cas du taillis composé. - La combinaison de l'ensemencement naturel avec le repeuplement par rejets de souche est habituelle dans la régénération des taillis composés.

La régénération de l'élément taillis est identique à celle d'un taillis simple régulier en semblable condition ; il y a lieu de prendre les mèmes précautions d'exploitation pour l'assurer, et il n'y a pas à compter sur les rejets généralement sans avenir que peuvent donner les souches des réserves exploitées.

D'après MM. Boppe et Jolyet, il n'y a pas lieu d'autre part de trop s'attacher à la prédominance des bois durs dans le sous-étage ; aujourd'hui bois durs et bois tendres, (les premiers moins recherchés qu'autrefois pour le chauffage, les seconds plus employés dans la fabrication (les pâtes à papier), se vendent aussi bien ou aussi mal l'un que l'autre. D'après M. Gayer qui se place au point le vue du maintien de la fertilité de la station, les eflorts de la culture doivent tendre à obtenir dans le sous-bois une prédominance des essences d'ombre, tout au moins Jes bois durs, tels que le charme, le frène, le hètre, l'orme, etc. Mais à côté d'eux, les bois blanes et les morthois, comme le tremble, le saule, le noisetier, l'aubépine, etc., se développent très rapidement et forment de larges buissons qui tendent à les écarter. Les taillis comjosés, mal soignés, se reconnaissent en général à l'envahissement des mort-bois, phénomène qu'on ne doit jamais perdre de vue. Malgré cela, dans un traitement rationnel, la régénération du taillis ne présente pas de difficultés. 
Le point le plus important, pour ainsi dire le plus essentiel, est le bon entretien de la futaie, et pour cela il est nécessaire de veiller au maintien, en nombre suffisant des essences qui doirent assurer un bon recrutement de la réserve. D'après la constitution même du taillis composé, tous les arbres de futaie doivent vivre, à l'étal de brins de semence, arec le sous-étage, dans le sein duquel ils sont confondus pendant une révolution, puis ils sont réservés à l'état de baliveaux, et sont acquis dès lors à la réserve qu'ils viennent renforcer de façon à entretenir sa composition et sa production aussi constantes que possible.

Si les brins de semence font défaut, on peut réserver comme baliveaux des rejets de souche jeunes et vigoureux, choisis de préférence parmi les rejets de jeunes souches, les drageons et enfin les brins isolés détachés des cépées; il y a lieu d'éviter de choisir les baliveaux sur les grosses cépées, parce que ces cépées donnent d'abondants rejets et sont nécessaires pour entretenir en bon état la consistance du taillis; elles en forment la véritable richesse el il ne faut pas en priver le taillis. Mais, en principe, la futaie doit se composer exclusirement de brins de semence; on est en droit de considérer comme tels les rejets généralement uniques qui naissent du premier recépage d'un sujet de franc pied; ces rejets ont à peu près la mème valeur que lui; ce sont d'ailleurs eux qui, en général, fournissent les meilleurs baliveaux, car il est rare qu'un lrin de semence soit assez fort pour ètre isolé à la fin do la révolution au début de laquelle il est né; c'est seulement après avoir été recépé qu'il s'élance avec assez de vigueur. pour marcher arec, le sous-étage.

La présence des semis dans le peuplement est duc à l'ensemencemen' des arbres de l'étage supérieur qui produisent de la graine; dès lors, on doit toujours avoir soin de réserver dans la futaie assez de porte-graines à larges cimes parmi lés classes d'âge le plus élevé, pour 
assurer cet ensemencement; si on ne veut pas conserver ces réserves, il est possible de venir les enlever encore un ou deux ans après la coupe du taillis, lorsqu'elles ont produit leur effet utile.

L'ensemencement qui se produit immédiatement après la coupe donne des brins de semence qui croissent simultanément avec les rejets de souche, mais qui, généralement, restent toujours en arrière pendant toute leur période de jeunesse; lorsque ces brins se trouvent disséminés à l'état isolé au milieu des rejets, ils persistent en très petit nombre; mais si, au contraire, ils sont réunis par petits bouquets (1) et s'ils sont l'objet de soins nécessaires, notamment s'ils sont préservés de l'envahissement des rejets et drageons, on peut les conserver, même dans un taillis formé en massif.

Quant à l'ensemencement qui se produit pendant la croissance du taillis, durant l'intervalle de deux coupes, il est sans avenir et ne persiste généralement pas. Toutefois dans certaines circonstances et surtout grâce à des soins spéciaux, on peut assurer la conservation de quelques bouquets ayant pris naissance vers la fin de la révolution et peu de temps avant la coupe.

La régénération artificielle peut, elle-mème, interveniı dans les taillis composés, au même titre que dans les taillis; en venant combler artificiellement, à l'aide de. plantations, les lacunes existant dans le peuplement, on peut entretenir en bon état la consistance de ce taillis; mais ces travaux complémentaires ont généraiement pour objet la futaie, et pour cela on procède par plantation de lorts plants, de hautes tiges mème, en ayant soin de choisir des essences susceptibles de derenir utilement des arbres de futaie; on les plante dans les meilleures parties du'sol afin de favoriser leur croissance, et on

(1) C'est souvent dans des bouquets de tremble et de bouleau que se reproduit ainsi le chêne par des semis qui persistent. 
cherche à les disposer autant que possible par groupes régulièrement espacés.

II est inutile d'ajouter que de tols travaux peuvent devenir très coûteux, en raison de la longue échéance”à laquelle se fera la récolte, et y sont parfois à peu près sans résultat; il vaut mieux leur préférer, dans toutes les circonstances où cela est possible, l'emploi judicieux du repeuplement naturel. 


\section{DEUXIÉME SEGTION}

\section{Opérations culturales}

Dans un exploitation agricole, les opérations culturales proprement dites consistent exclusivement à donner au sol les soins nécessaires pour placer le végétal dans des conditions favorables à son développement; avant l'ensemencement, ce sont le labour, les hersages, la fumure et l'apport d'engrais ; après l'ensemencement une culture intensive et sérieuse continue ces soins par des binages, des sarclages, etc. et souvent par l'apport de nouveaux engrais complémentaires. Par contre, le végétal lui-même, dont les différentes phases de végétation s'effectuent rapidement dans l'espace d'une année, et beaucoup plus rarement dans l'espace de deux ou de quelques années, n'est l'objet, en général, d'aucun soin spécial.

A une époque plus reculée que la nôtre, l'exploitation sylvicole consistait à assurer le repeuplement de la forêt, puis à abandonner à lui-même le peuplement jusqu'à la date de la récolte. Mais aujourd'hui, la forèt cultivée exige d'autres soins et sa croissance ne peut se maintenir vigoureuse que si le sol reste fertile et si le peuplement est innvenablement dirigé.

Nous nous proposons d'examiner quelle est, en sylviculture, l'action de l'homme sur ces deux facteur's.

\section{I. - ACTION DE L'HOMME SUR Le SOL.}

Le sol est la portion superficielle de l'écorce terrestre qui est accessible aux racines des végétaux; il repose 
sur le sous-sol, masse minérale inaccessible à ces racines.

Le sol provient de la désagrégation des roches par les agents physiques et mécaniques et de leur décomposition par les agents chimiques; il se compose :

$1^{\circ}$ de la courerture, formée de débris organiques diver's non encore décomposés;

$2^{\circ} \mathrm{Du}$ terreau, qui n'est autre chose que la couverture décomposée ;

$3^{\circ}$ De la terre végélale, formée par les débris des roches plus ou moins imprégnés de matières organiques.

Quant au sous-sol, il se compose :

10 De la terre minérale formée par les débris de roches, non encore imprégnés de matières organiques;

$2^{\circ}$ De la base minéralogique constiluée par les roches sous-jacentes, en place.

'Tandis qu'en agriculture, l'homme exerce sur le sol une action directe, en sylviculture cette action, sauf dans des cas spéciaux, est toujours indirecte et l'homme ne peut en général chercher à entretenir et même à améliorer les qualités du sol que par l'action lente des végétaux eux-mèmes.

Les principaux facteurs qui constituent celte qualité du sol sont : la profondeur du sol; sa consistance et son degré d'humidité; sa richesse en éléments nutritifs.

Profondeur du sol. - La profondeur du sol est l'épaisseur de la couche accessible aux racines (fig. 29); sous ce rapport un sol est dit : Superficiel, si l'épaisseur de la couche accessible aux racines est inférieure à 0,15 centimitres.

Peu profond, si celle épaisseur est de $0^{\mathrm{m}}, 1$ :̈ à $0^{\mathrm{m}}, 30$. Assez profond,

Profond,

Très profond,

En ce qui concerne la profondeur du sol, les conditions rxistantes sont inhérentes a la nature géologique de la 
station; l'homme ne peut les modifier; dans un sol profond, est-à-dire dans les conditions les plus favorables, il peut tout demander et tout obtenir; mais au contraire, dans un sol peu profond, c'est-à-dire en pré-

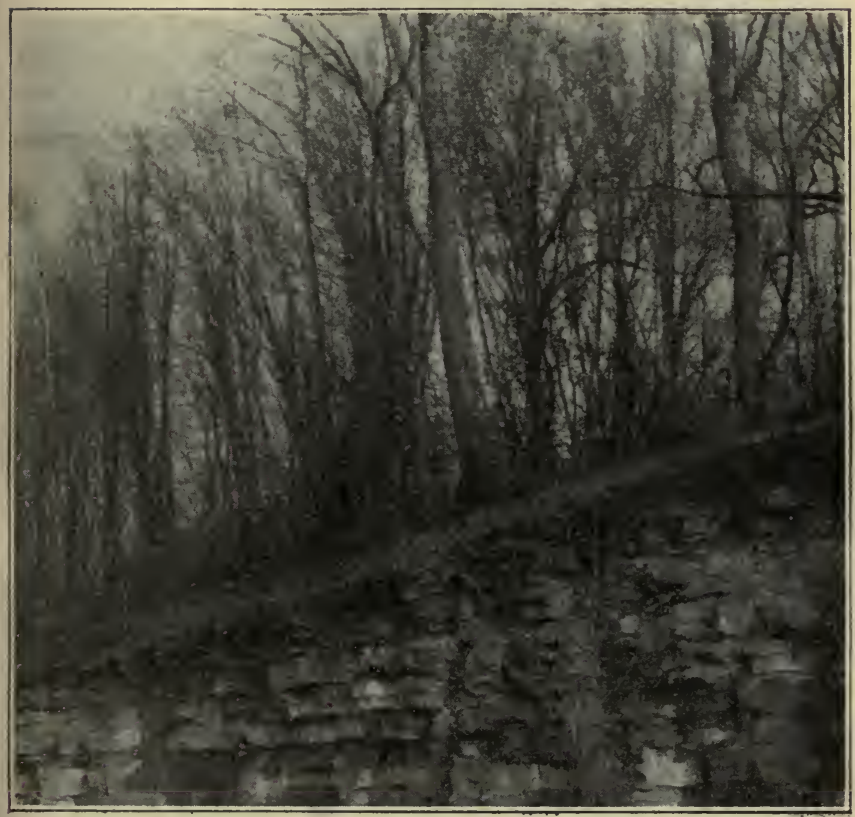

Fig. 29. - Taillis composé sur roche calcaire fissurée.

(Sol peu profond).

sence de conditions mauvaises, il doit, par des mesures d'entretien, soit améliorer ces conditions, soit les empècher d'empirer. Examinons quelques-uns des cas principaux :

$1^{\circ}$ Dans un sol manquant de profondeur, que le peuplement qu'il porte soit bon ou médiocre, ou doit éviter 
loute exploitation à blanc étoc, afin de maintenir le plus possible dans le sol l'humidité, les conditions les plus propices à la formation de l'humus, toutes choses nécessaires et essentiellement favorables à la désagrégation lente du sous-sol minéral.

Par suite dans un tel sol, dit M. Gayer, on régénérera lentement et sous le couvert, on limitera l'exploitation, on soignera au besoin les bouquets préexistants bons ef mauvais, et mème les broussailles sans valeur quand elles seront nécessaires pour abriter le sol.

Dans de tels terrains, la base géologique qui constitue le sous-sol peut ètre compacte ou fissurée, disposée par couches horizontales ou inclinées, et par suite plus ou moins pénétrable aux racines; cet état influe sur la profondeur; et les conditions les plus défarorables à cr point le vue sont données par les éboulis à croûte végétale mince des terrains calcaires et dolomitiques; par les sols rocheux nus et crevassés où la roche est disposée par couches horizontales ou peu inclinées ; dans de tels sols, lorsque l'état boisé disparaît, toute végétation et mème toute terre végétale disparaît aussi, spécialement lorsque par suite de leur inclinaison les eaux pluviales peuvent entraîner continuellement tous les débris minéraux provenant de la délitation superficielle de la roclie elle-mème.

Dès lor's, pour remédier à des situations aussi mauvaises, pour reconstituer peu à peu une nouvelle couche le terre végétale, il faut rétablir la végétation boisée; mais en général, a vant de pouvoir obtenir un peuplement de quelque valeur, on derra parcourir lentement toute l'échelle de la végétation, en commençant par les lichens et les mousses, les mauraises herhes, puis les arbrisseaux et les arbustes de peu de valeur. L'entretien de cette première végétation que ne doit venil détruire aucun bétail, la conservation des moindres buissons isolés est de toule nécessité. Les semis ou la plantation, 
ajoute l'auteur que nous venons de citer, ne peuvent domner que très lentement un certain résultat dans les dépressions et les crevasses remplies de terre, et il peut se passer un grand nombre d'années avant que la formation de la couche végétale, dans les autres parties du terrain, soit assez avancée pour justifier de nouvelles lentatives de boisement. Il est vrai que ce sont là des situations extrèmes, mais leur existence mème est un avertissement (fig. 31).

Quand les conditions sont meilleures, c'est-à-dire

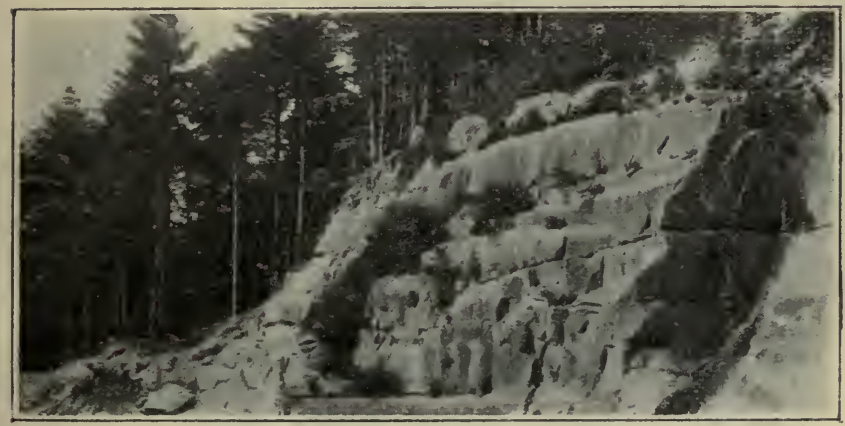

Fig. 30. - Futaie résineuse (sapins) sur to grès des Vosges.

qu'on a aflaire seulement à un sol manquant de profionleur, mais non encore dégradé, on doit, en le maintenant complètement boisé, lui éviter toute déperdition; car, exposé aux intempéries, il peut en peu de temps, de mérliocre qu’il est, se transformer en un désert improluctif (Gayer).

$2^{\circ}$ L'instabilité de certains sols exposés aux ravinements et aux aflouillements les eaux, specialement dans les rígions de haute et de moyenne montagne, nécessile l'autres soins et l'entretien de la profondeur se présente alors sous un autre aspect ; c'est la lutte contre les eaux

A. Frox. - Sylviculture. 
de ruissellement et d'infiltration, contre la puissance affouillante des torrents et contre les glissements. La conservation des forèts, la lutte contre le déboisement, le gazonnement et le reboisement sont seuls susceptibles d'arrêter le mal à sa naissance, et les mesures préventives les jlus simples dans ces situations dangereuses sont: maintien des forêts, spécialement à l'état jardiné, fixation du sol par le maintien de la végétation herbacée, suppression ou tout au moins réglementation sévère du pâturage, captation ou détournement des eaux rui s'accumulent dans des dépressions de haute montagne et y constituent un danger permanent, etc.

Si on agit trop tard, le mal se transforme vite en fléau, - et on ne peut plus alors le combattre que par de véritables travaux de défense qui ont pour but la correction lente et progressive des torrents. Les plus importants de ces travaux consistent dans la construction de barrages pour arrêter la puissance affouillante des eaux, dans l'établissement de drains sur les pans des montagnes en voie de glissement pour fixer les terrains, et dans le reboisement des bassins de réception, des berges fixées, elc. L'étude de ces importants travaux est en dehors du cadre de notre ouvrage.

Toutefois, si dans la basse el la moyenne montagne le sol est instable, il y a lieu de prévenir le commencement des ravinements, des éboulis et des glissements par de petits travaux simples de fixation; ces Iravaux peuventconsister en barrages en pierressèches, lascinagr's ou clayonnages, et en reboisements qu'on effectue souvent par boutures ou plantations en saules ou autres régélaux; souvent on doit compléter ces travaux par la créalion de quelques rigoles d'assainissement, 'n ayanl soin de ne pas leur donmer une forte pente, et par le maintien de la végélation ligneuse sur tous l's terrains. en pente forte.

$3^{\circ}$ Dans cerlains lrrains sablonneux, une action 


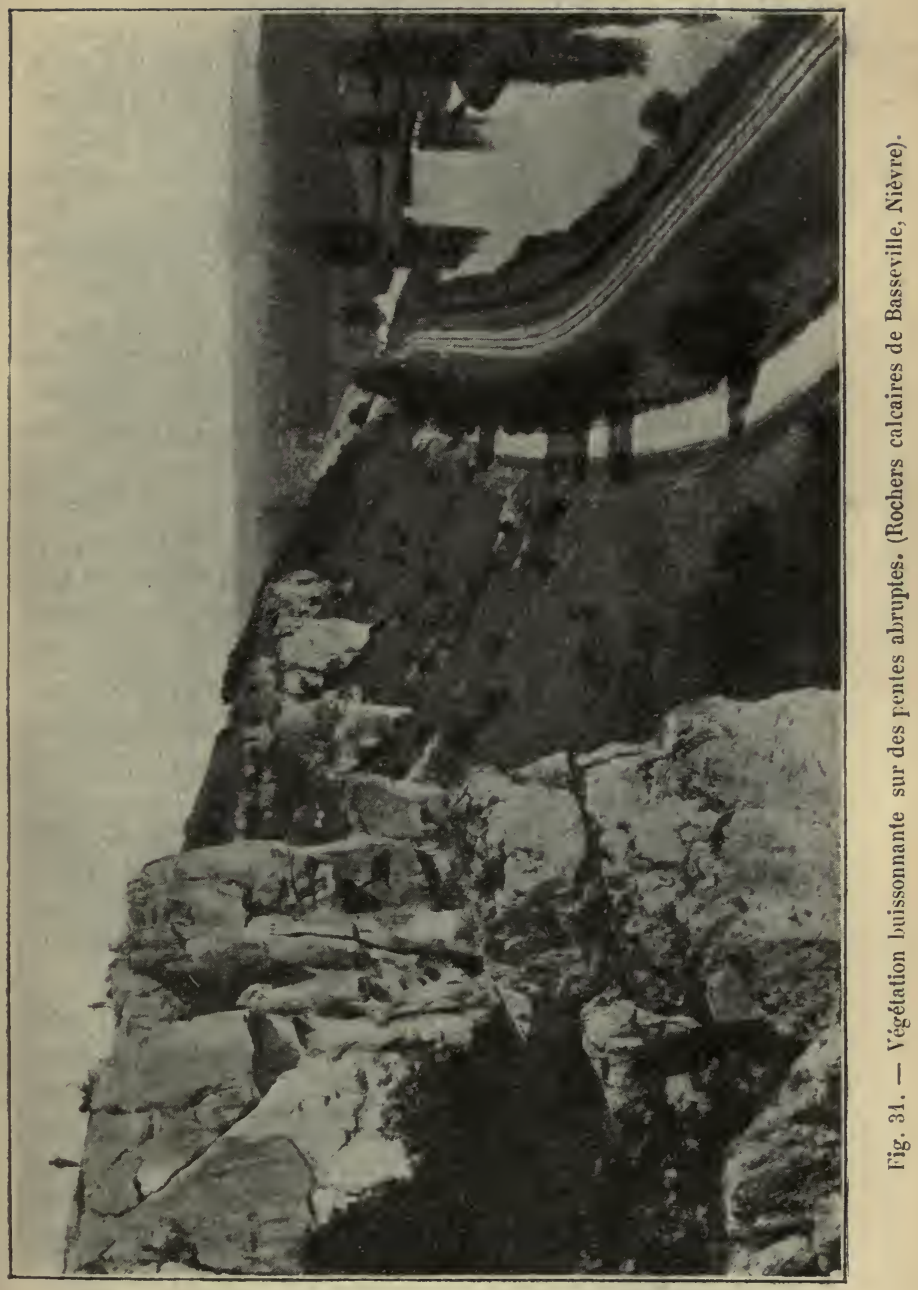


spéciale vient agir sur la profondeur des sols et donne naissance à des formations connues sous le nom d'alios. L'alios est une eouche plus ou moins dure ou plus ou moins épaisse, généralement impénétrable aux racines formée de 80 à 95 p. 100 de sable agglutiné par de l'humus de bruyire ou des ciments ferrugineux; des couches de ce genre s'élendent sur de vastes espaces, tantôt près de la surface, tantôt à une certaine profondeur, et sont plus ou moins susceptibles d'ètre ramollies par l'eau du sol. Outre l'obstacle mécanique qu'elles opposent au développement des racines, ces formations peurent devenir très nuisibles en ne permettant pas l'aération des couches inférieures du sol.

En certains points de la forèt domaniale de Bercé (Sarthe), le sol, constitué par un sable à gros grains, est complètement acidifié par les bruyères; il n'a guère que 30 centimètres de profondeur et repose sur un sous-sol de sables agglutinés par l'humus de bruyère et un ciment forrugineux, formant une couche impénétrable aux racines, analogue à l'alios; sur ce terrain, la végétation rst languissante et mauvaise, les arbres ne peuvent acquérir une grande hauteur; ils dépérissent rapidement et le sol se recouvre de bruyères qui l'envahissent, l'acidifient peu à peu et le rendent complètement infertile; le pin sylvestre lui-mème, planté sur un tel sol, commence par croitre, puis son développement se trouve subitement arrèté et il dépérit.

Dans ce cas tout spécial, des travaux d'amélioration ont pu remédier partiellement à une situation aussi défectueuse; la couche imperméable d'alios se trouve, en c:ffet, n’àvoir dans cette région que 10 centimètres environ d'épaisseur; en creusant des rigoles de 30 à 30 centimètres de profondeur, et en brisant par places celle couche imperméable, .I. I'lnspecteur Roulleau a pu effectuer dans le fond de ces rigoles des plantations de pin sylvestre qui ont parfaitement réussi. 
En général, de tels traraux sont rares dans la culture forestière proprement dite.

Consistance $d u$ sol. - La consistance d'un sol est en relation directe arec sa ténacité, sa cohésion et sa perméabilité (1). A ce point de rue, une terre est dite :

Compacte, quand sèche elle prend la dureté de la pierre.

Liante, quand sèche elle se brise sous la moindre pression.

Légère, quand elle n'a un peu de cohésion qu'à l'éta humide.

Meuble, quand elle n'a aucune cohésion, même à l'élat humide.

On sait que quand un sol est assez meuble, sa productivité s'en troure augmentée, par le fait qu'il est plus pénétrable aux racines; dans un sol compact aucontraire, les racines se trouvant dans un milieu insuffisamment aéré pourrissent rapidement et ne peurent pas se dérelopper autant en profondeur.

En agriculture, pour obtenir un état meuble du sol, le terrain est soumis à des travaux de culture qui sont absolument nécessaires. En sylviculture, il est fort rare qu'on effectue un travail d'ameublissement de ce genre; le degré de consistance et de perméabilité convenable du sol s'établit et se maintient spontanément lorsque la régétation forestière qui le recouvre se trouve dans des conditions conformes aux lois naturelles. Le sol abrité par une forêt et courert par une couche d'humus et de litière ne peut être tassé et durci par l'action mécanique des pluies, comme celui qui est complètement à découvert; grâce à l'humidité plus uniforme entretenue par le couvert et par la litière, il reste meuble; en outre, le travail de décomposition ininterrompu des mycéliums,

(1) La ténacité est la faculté qu'ont les particules de terre d'adhérer entre elles. La cohésion est la faculté qu'elles ont d'adhérer à des corps étrangers; la perméabilité est la faculté que possède un sol de se laisser pénétrer par les liquides et les gaz. 
ainsi que l'action incessante des insectes, des larves, myriapodes, lombrics et de tous les ètres vivants qui l'habitent, l'empèchent de se durcir; aussi la culture forestière ne comporte-t-elle ni labour, ni culture dur sol. L'action de l'homme se borne, en général, à maintenir les conditions favorables ou à faire usage des moyens dont se sert la nature dans ce but. Cesmoyens, dit II. Gaver, consistent à tenir le sol constamment abrité, soit par le peuplement formé en massif, soit par le couvert d'ensemencement lors de la régénération, puis à respecter aussi rigoureusement que possible les couches de litiere et d'humus, dans leur ordre naturel de superposition.

Exceptionnellement, certains travaux d'ameublissement peuvent ètre utiles dans des sols argileux très compacts, riches en éléments nutritifs, mais qui restent trop peu pénétrables alors mème qu'ils sont soumis à un traitement forestier bien entendu; mais partout ailleur's, (hercher à pousser plus loin la productivité d'un sol forestier en le travaillant périodiquement dans le cours de l'existence d'un peuplement, serait effectuer une mauraise opération; dans les sols de fertilité ordinaire, de tels travaux conduiraient rapidement à l'épuisement, en provoquant une consommation beaucoup trop rapide de la réserve organique et des éléments minéraux assimilables du sol, sans qu'on puisse, comme dans la cullure agricole intensive, venir remédier à cet épuisement par un apport d'engrais appropriés.

Humidité du sol. - Les propriétés physiques du sol, ì ce point de vue, sont: $1^{0}$ l'hygroscopicité, c'est-à-dire l'aplilude du sol à retenir l'eau, la faculté qu'il a de simbiber; $2^{\circ}$ l'aptitude à absorber les vapeurs d'eau de l'atmosphère; $3^{\circ}$ l'aptitude à la dessiccation, c'est-à-dire la faculté qu'a le sol de perdre par évaporation l'eau absorbée. Ces qualités physiquẹs régissent l'humidité des sols, et sous ce rapport d'humiditi, un sol est dit: 
Mouilleux, quand l'eau séjourne à la surface en tout temps ;

Humide, quand l'eau ne séjourne à la 'surface que pendant la saison des pluies, et qu'en tout temps elle tend à maintenir le terrain au moins frais ;

$S e c$, quand il n'y a jamais d'eau stagnante ;

Aride, quand il n'y a jamais assez d'eau.

Le maintien constant de l'humidité du sol est une condition nécessaire de la culture for'estière. L'homme n'a d'autre action que de se conformer aux principes généraux suivants, qu'énonce M. Gayer :

Éviter autant que possible l'interruption du couvert, choisir dans chaque cas la forme de peuplement adéquate aux circonstances, proscrire les grandes coupes blanches, régénérer sous le couvert quand c'est possible, mettre en auvre tous les moyens propres à conserver au sol forestier la couche de litière et d'humus qui lui est indispensable.

Dès que l'intégralité des massifs est atteinte, on est conduit à respecter et à propager les essences de couverture, essences qui prennent pied spontanément ou sont introduites à temps sous le peuplement principal et se présentent sous forme de semis préexistants, de rejets, drageons et sous-bois de toute nature; on les recherche dès qu'elles peuvent ètre utiles pour abriter le sol, pour modérer l'écoulement des eaux sur les pentes, pour proléger l'intérieur des peuplements contre l'action du vent.

A ce dernier titre, on ne peut que conseiller le maintien et mème l'établissement de rideaux de protection, le long des lisières des peuplements adultes, partout où ces abris sont utiles pour fermer la forèt, et pour détruire l'action asséchante des déplacements d'air et l'action néfaste du vent sur la couverture morte. L'intérieur de la forèt doit ètre masqué aux regards indiscrets par des bandes de plusieurs mètres de largeur qui garnissent les lisières des parcelles du côté des vents dominants. Pour 
établir ces rideaux, on peut se servir d'essences à feuillage persistant, en ayant soin d'observer, dans leur plantation, un large espacement entre les sujets, afin que leur feuillage descende aussi bas que possible; on doit veiller au maintien de ces conditions; l'épicéa, le sapin, le pin noir conviennent, suivant les cas, pour cet usage.

Remarquons que dans les peuplements composés d'essences feuillues, encore aptes à rejeter de souche, on arrive à un résultat analogue en recépant et en traitant en taillis une hande de 5 à 10 mètres de large sur la lisière du peuplement.

Les propriétaires particuliers peuvent aroir intérèt, dans bien des cas (bois isolés), à soigner ainsi les lisières de leurs exploitations; sous une forme ou sous une autre, ils paraissent avoir intérêt à constituer des rideaux de protection autour de leurs massifs.

En ce qui concerne l'humidité nécessaire au sol foorestier, nous avons précédemment signalé le danger des fossés d'assainissement dont on abuse quelquefois; mais par contre, l'ouverture de quelques fossés horizontaux de 30 à 50 centimètres de profondeur et de largeur, sur :i à 6 mètres de longueur, à parois aussi verticales que possible, peut être utile pour maintenir une certaine humidité dans le sol; on les dispose dans ce cas, en * échiquier, en les séparant de 3 à 5 mètres en largeur; N. Gayer est d'aris que des fossés de ce genre peuvent, dans certains cas, exercer une influence bienfaisante sur la croissance des peuplements; leur emploi peut ètre utile, lorsqu'il s'agit de se débarrasser d'un excès d'eau local nuisible; à l'aide de petits fossés horizontaux ou de simples sillons, on peut faciliter l'évacuation de celle eau et en faire bénéficier une autre partie du pecuplement.

Notons enfin que dans certaines forèts de plainc, lirrigation peut ctre utile, el que sourent elle y serait fort possible. 
Fertilité $d u$ sol. - Les sols n'ont qu une propriété chimique générale, mais elle est importante ; c'est le pouvoir absorbant ou faculté que possède une terre de fixer et de retenir certains éléments essentiels mis en contact a vec elle sous une forme soluble.

Quant aux caractères et propriétés des divers sols, ils dépendent des éléments qui les constituent, dont les quatre principaux sont: sable, argile, calcaire, humus. Ces éléments ne se trouvent presque jamais complètement purs et indépendants les uns des autres. Ordinairement, ils sont associés en proportions variables et unis en outre à une plus ou moins grande quantité d'éléments minéraux divers.

Lorsque ce mélange a lieu dans des proportions conrenables, on a une terre franche, renfermant approximativement les éléments suivants : silice, 50 à 70 p. 100 ; argile, 20 à 30 p. 100 ; calcaire, 5 à 10 p. 100 ; humus, 10 p. 100.

Mais le plus souvent, l'un ou l'autre de ces quatre éléments domine et donne un caractère spécial à la terre qu'on ramène alors à quatre types principaux:

Terres siliceuses, qui renferment plus de 70 p. 100 de silice ;

Terres argileuses, qui renferment plus de 30 p. 100 d'argile;

Terres calcaires, qui renferment plus de 30 p. 100 de calcaire ;

Terres humifères, qui renferment plus de 30 p. 100 de terreau.

Le tableau suivant, dressé par M. Pardé(1), résume les caractères et les propriétés principales de ces quatre types fondamentaux.

L'action de l'homme ne peut s'exercer sur la composition minérale du sol, mais elle peut agir sur sa teneur

(1) Agenda du forestier. 


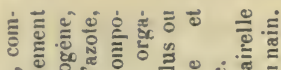

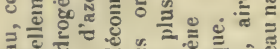

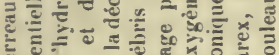

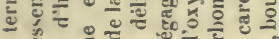
亏

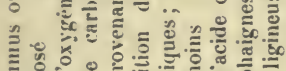

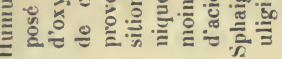

|.

월

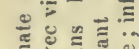

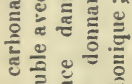

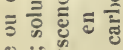

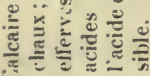

垔

㭋言

흘.

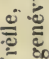

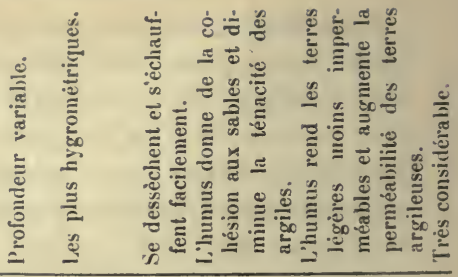

1.

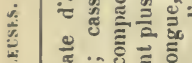

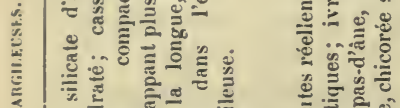

:

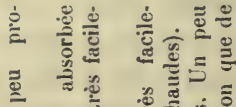

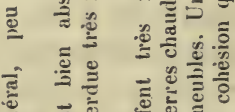

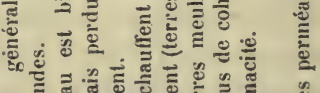

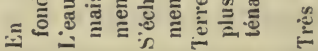

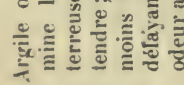

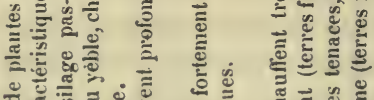

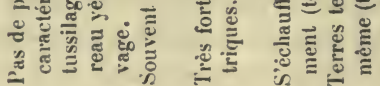

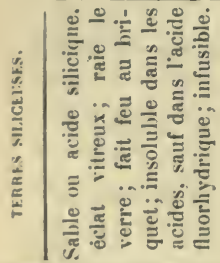

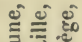

픈

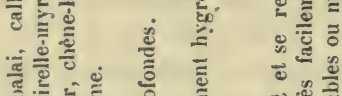

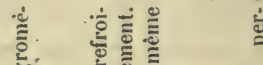

离

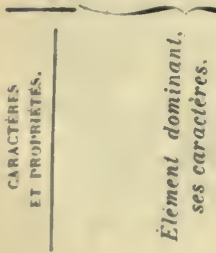

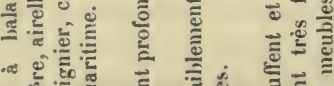

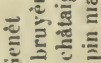

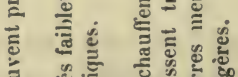

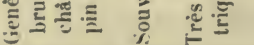
型 
en humus, et en général, tous les soins de la culture devront avoir pour objet de maintenir et d'améliorer les conditions existantes en conservant intégralement la litière et tous les corps organiques qui vont au sol ou qui en proviennent, telles que les feuilles mortes, le bois mort, les rémanents des exploitations, écorces, racines, etc., les herbes, les mousses, etc.

Nous avons assez insisté sur le ròle et la formation de l'humus, pour ne pas avoir à y revenir, sinon pour l'épéter ce principe primordial qu'en sol forestier', l'entretien de la fertilité du sol est intimement liẻ au maintien aussi constant que possible du couvert des cimes, et à la conservation intégrale de la couverture de litière el d'humus.

Remarquons toutefois que le terreau ne se constitue pas toujours dans les conditions idéales que nous avons indiquées, certaines feuilles se décomposent beaucoup moins rapidement que d'autres.

D'autre part, d'après MM. Boppe et Jolyet, quand l'eau est en excès, l'action de l'oxygène et de la chaleur se trouve diminuée; la décomposition des débris organiques devient extrèmement lente et reste incomplète ; les combinaisons acides se produisent abondantes, et il en résulte un résidu analogue à la tourbe dans lequel, parmi nos grandes essences forestières, l'aune, le bouleau, le tremble, le pin de montagne peuvent seuls résister.

Inversement, quand il y a excès de sécheresse, le terreau se brûle et devient charbonneux, poudreux ou fibreux. Cette poussière brune ou noire, de décomposition ultérieure très difficile, est une réritable lourbe sèche avec tous ses inconvéntents; elle se rencontre surtout dans les sols siliceux, auxquels elle se mélange pour donner les terres dites de bruyère.

Conclusion. - Il résulte de ces considérations que l'action de l'homme sur le sol peut se résumer par les conseils suivants que nous empruntons à MM. Boppe el 
Jolyel: respecter tous les détritus végétaux qui recouvrent le sol ; maintenir les massifs complets en respectant les sous-étages, et éviter les découverts trop fréquents; conserver, tout autour des enceintes, des arbres de lisière qui tiennent le peuplement bien clos à l'abri des coups de soleil et aussi des coups de rent; créer des forêts mélangées où la décomposition des leuilles se faisant beaucoup mieux, on évite la formation de ces litières de feuilles de hêtre, ou de feutrages d'aiguilles d'épicéa parfois si gènants dans les forèts de la montagne.

- Dans les sables grossiers, secs et brûlants: exagérer encore le principe du couvert bas et continu; faire tous ses efforts pour maintenir les espèces à feuillages épais, qui ont trop de tendance à fuir les régions oủ l'humidité fait défaut; dans ce but, préférer les peuplements irréguliers aux peuplements réguliers.

Au contraire, dans les argiles froides et très humides : relever le courert pour faciliter l'accès de la chaleur ef mème dans quelques cas choisir des traitements qui découvrent périodiquement le sol et au besoin préférer le taillis composé à la futaie pleine.

En résumé, dans toutes les circonstances et en tous lieux, maintenir le sol à l'état de saine fraîcheur que commande l'hygiène de la forêt, et qui convient aux lombrics et autres animaux sur le rôle desquels on ne saurait trop insister.

\section{II. - ACTION DE L'HOMME SUR LES PEUPLEMENTS.}

Généralités. - L'action de l'homme a pour but de maintenir le peuplement convenablement constitué pendant toute la durée de son existence, afin de lui conserver toute sa vigueur et de le diriger vers le but économique cherché. 
Nous supposons le peuplement créé; l'homme est déjà intervenu lors de cette création, soil en dirigeant le repeuplement naturel, soit en le complétant, soit en agissant par repeuplement artificiel.

A partir de cette période de première jeunesse et jusfu'à l'exploitation, un certain nombre de soins culturaux sont, sinon toujours indispensables, du moins utiles, soit pour supprimer à temps les obstacles qui entravent la croissance du repeuplement, soil pour remédier aux causes qui tendent à modifier sa composition dans un sens favorable, soit enfin pour stimuler directement la croissance des sujets d'élite.

Cies soins culturaux ne doivent jamais avoir pour but un profit immédiat plus ou moins élevé ; mais toujours un résultat précis de culture. Souvent, il est vrai, la vente des produits accessoires ainsi obtenus sera rémunératrice; mais souvent aussi, l'opération couvrira à peine les frais de main-d'œurre ou mème ne donnera pas de produits utiles; alors la crainte d'une dépense ou l'insouciance du propriétaire la font négliger; ;lans certains cas, e'est un tor't.

L'importance de ces soins culturaux varie avec les différentes formes de peuplements, et aussi arec chaque type; mais leur nature varie surtout avec l'àge de ce peuplement.

Nous suivrons donc le peuplement dès sa naissance, pour étudier ces opérations dans leur ordre naturel.

Répartition des jeunes sujets. - Nous avons vu, à propos de la formation en massif, que l'état le plus favorable à une bonne croissance n'est ni le massif trop serré, ni le-massif trop clair; en tenant compte, bien entendu, des exigences des divers âges, l'homme peut intervenir dès l'origine pour tendre vers cet élat parfait, soit par des desserrements, soit par une opération inverse.

a. Desserrement. - On procède à des desserrements, partout où les jeunes plants sont trop serrés, fait qui se 
produit fréquemment dans les semis artificiels, comme lans les semis obtenus par régénération naturelle; lopération culturále consiste à supprimer un certain nombre de sujets, de manière à augmenter la place disponible pour ceux qui restent.

Si on intervient à temps, si l'on a soin d'agir dès les premières années et de recommencer' souvent l'opération, au fur et à mesure des besoins, on arrive à stimuler d'une façon considérable la végétation, spécialement chez les essences de lumière.

Si on commence à intervenir trop tard, surtout lorsqu iil s'agit d'essences d'ombre, sur des sols paurres, les sujets trop serrés sont déjà arrêtés dans leur croissance, ou bien ayant filé trop rapidement en hauteur, ils sont constitués par des tiges tellement grèles qu'ils ne sont plus capables de se soutenir si ce n'est par l'appui mutuel qu'ils se prètent à cet état serré.

Dans de tels gaulis âgés de dix à vingt-cing ans, on ne peut plus agir qu'arec une extrême prudence; il faut n'enlever à la fois qu'un très petit nombre de sujets afin d'amener progressivement ceux qui restent à corriger leur forme défectueuse en développant plus de feuillage, et à prendre plus de résistance en fortifiant leur tige; le desserrement, dans ce cas, doit se faire lentement.

D'après M. Gayer, les essences feuillues et parmi elles plus spécialement le chène, doivent être soumises à ces premiers soins, non seulement dans les futaies, mais aussi dans les taillis; en réduisant avec prudence un nombre excessif de rejets, on favorise singulièrement la croissance de ceux qui restent; ajoutons toutefois que dans les taillis surtout, il ne faut pas oublier quion a pour but non pas d'avoir un massif clair, mais simplement de ne pas aroir un massif trop serré (1); qu'il faul,

(1) Des semis qui paraissent espacés, mais qui sont régulièrement répartis, sont souvent très suffisants et le massif ne s'en formera pas moins d'une facon trik convenable. 
en outre, que les sujets soient aples à profiter rapidement de l'espace qu'on leur donne, ce qui se produit surtout dans les bons sols.

b. Regarnissage des vides. - Une méthode inverse permet de compléter à temps un massif très clair par des plantations; et souvent on se contente d'utiliser pour cela les jeunes plants arrachés ou plutôt extraits en motte des parties trop serrées.

On peut toutefois se servir pour faire ces regarnissages d'essences auxiliaires telles que le pin sylvestre, le mélèze, le bouleau et, en général, de toutes les essences de lumière robustes. En fermant ainsi de bonne heure les vides, on prévient l'envahissement de la végétation herbacée, des genèts, de la bruyère ou des mort-bois; on stimule la croissance de l'essence principale, et on prévient le ralentissement de la végétation et le dépérissement progressif du peuplement, conséquence fatale d'un état de massif clairiéré dès le jeune âge, surtout lans les sols pauvres et amaigris à la surface.

Dégagements dans les jeunes peuplements. L'opération qu'on désigne souvent sous le nom de nettoiement a toujours en vue, dans des peuplements (léjà formés en massif, de venir dégager une essence précieuse de l'étreinte de sujets moins précieux qui tendent à la dominer, à nuire à son développement et par suite à l'éliminer.

Lorsqu'on efrectue un repeuplement, le terrain sur lequel on travaille est souvent déjà occupé par une végélation ligneuse d'arbustes ou d'arbrisseaux, de semis préexistants, etc., auxquels viennent s'ajouter peu à peu des rejets de souche et des mort-bois. Cette végétation

Quant aux semis trop serrés, spécialement s'il s'agit d'une essence d'ombre (semis en brosse du sapin ou de l'épicéa), ils végèten l mal, surtout sur les sols pauvres, et la croissance des jeunes plants s'arrête de bonne heure. Chez les essences de lumière, au contraire, le tempérament mème de ces jeunes plants corrige en général assez rapidement cet état de choses par une élimination spontinée. 
est, suivant les cas, tantôt ulile, tantôt nuisible au repeuplement.

Les opérations culturales successives qui ont pour but d'enlever en temps utile parmi cette végétation accessoire tous les sujets qui gênent le développement du nouveau peuplement, prennent le caractère de dégagements.

Suivant les peuplements, et même dans un même peuplement suivant les emplacements, un très grand nombre de cas peuvent se présenter:

a) Le dégugement porte sur des semis préexistants. - II faut supposer que ces semis préexistants n'ont plus d'avenir, et qu'on les a conservés pendant la période de toute jeunesse, soit pour faire bénéficier le jeune recru d'un abri latéral dans des situations exposées, soit pour contribuer à le préserver de la dent du bétail ou du gribier, soit enfin pour compléter le massif. Dans ces divers cas, on ne peut les enlever que graduellement au fur et à mesure que leur concours devient superflu; leur enlèvement progressif a pour but de dégager peu à peu le jeune repeuplement; il peut s'effectuer au fur et à mesure des besoins, par un simple ébranchement, par un étètement et enfin par coupe ou extirpation.

b. Le dégagement porte sur des rejets de souche. - Toutes les fois que sur le terrain à repeupler se trouvent des souches saines, l'évolution de rejets vigoureux, à croissance rapide et envahissante, vient entraver le développement des sujets de repeuplement. Les brins de semence de 10, $1: 3$ et même 20 ans ont une évolution plus lente que les rejets de même âge, et il est souvent utile de les: dégager; l'opération consiste simplement à casser le sujet gènant ou à enlever d'un coup de serpe diverses parties de sa cime. Dans un travail de ce genre, l'objectif ne doit pas ètre la chose à détruire, mais le brin à légager.

c) Le dégagement porte sur des mort-bois et des essences secondaires. - Nos grandes aspeces ligneuses ont souvent 
une croissance lente, si on la compare à l'érolution rapide des mort-bois et essences secondaires qui se jettent au milieu d'elles; il en résulte que partout où l'accès de la lumière le permet, on voit s'introduire peu à peu et s'élever rapidement des essences à graine légère, comme le bouleau, le saule marceau, le tremble, el parfois le tilleul, l'aune, l'orme, etc.

Le propriétaire particulier n’a pas toujours intérèt à extirper ces essences, dont au moins quelques-unes peuvent avec avantage entrer dans un mélange, spécialement aujourd'hui où elles se vendent parfois plus cher que les essences principales; il doit mème, au contraire, leur donner accès dans le peuplement, ne fût-ce que temporairement et à titre d'essences secondaires. Mais si res essences tendent à dominer et à supplanter les autres, leur suppression partielle est nécessaire, et il faut les empêcher de se dérelopper à l'excès. Le plus envahissant de ces mort-bois est le saule marceau, qui s'étale en largeur; le tremble et le bouleau le sont moins; quant au tilleul et à l'aune, ils produisent un courert épais, mais il est rare qu'ils se multiplient dans des proportions inquiétantes; leur groupement d'ailleurs est important à considérer, et en principe il faut éviter de les conserver en groupes et bouquets formés en massif.

Les coupes de dégagement ont done pour but à ce point de vue de faire disparaittre les mort-bois gênants, tout en en laissant par pieds isolés un certain nombre çà el là dans le peuplement; elles doivent ètre répétées plusieurs fois, spécialement là où ces opérations s'effectuent sur des emplacements déjà trop enrahis. On procède par élêtage, coupe ou simple ébranchage, comme il a déjà été dit.

Des opérations de ce genre, très importantes dans les jeunes fourrés, peuvent ne pas être à négliger dans les taillis où le dégagement des semis d'essences précieuses, et mème de roiets de bonnes essences, contre l'enrahissement des mort-bois, est quelquefois une opération du 
plus haut intérèt pour conserver la forèt en bon état.

(l) Les dégagements portent sur des essences envahissantes clans un mélange. - Présentent encore le caractère de dégagements les opérations culturales qui ont pour but de maintenir dans un peuplement mélangé, certaines essences qui, dès la période de jeunesse, se trouvent dans une situation moins favo:able vis-à-vis d'autres essences envahissantes.

Les soins culturaux prennent une très grande importance quand il s'agit de maintenir un mélange de deux ou plusieurs essences principales dans un peuplement régulier. Mieux que cela, le mélange intime sujets par sujets ne peut en général persister sans soins culturaux. En effet, dans la période de jeunesse de tels peuplements, l'une ou l'autre des essences du mélange tend à prendre l'avance sur les autres, qui dès lors sont menacées dans leur existence, et résistent plus ou moins longtemps suivant leur tempérament.

Les soins culturaux ont pour but de venir dégager les sujets qui en ont besoin, dans la mesure qu'on juge ulile.

Si l'essence envahissante est une essence d'ombre, à croissance rapide, comme cela se présente dans certains ras avec le hètre, l'épicéa, etc., en mélange intime avec le sapin, le chène, etc., les dégagements doivent ètre commencés de très bonne heure; ils consistent à supprimer la pousse terminale el quelques pousșes latérales des sujets envahissants, quitle à revenir plusieurs fois reeommencer l'opération si cela est utile.

Si l'essence envahissante est une essence de lumière à croissance rapide, telle que le pin sylvestre dans un mélange avec le sapin, l'épicéa, le hètre, le chène, ete., "t si celte essence, en élalant trop largement sa cime, devient gènante pour les sujets dominés, l'opération de digagrement peut consister simplement à diminuer ou à raler le couvert de celte essence en supprimant les 
verticilles inférieurs, opération que le pin sylvestre supporte, mème jeune, dans les terrains frais.

La nature du mélange qu'on se propose d'obtenir règle limportance de ces opérations; il est à remarquer d'ailleurs que le dégagement doit rarement porter sur les sujets vigoureux et élancés d'une essence, sujets désignés par ce fait même pour persister dans le peuplement comme arbres d'élite.

Dans un peuplement régulier, mélangé par groupes ou bouquets, les soins culturaux sont beaucoup plus simples et plus faciles à apporter; car ils ne concernent que les bords des bouquets, où l'essence de croissance lente peut ètre dominée par l'autre. Si le bouquet est suffisamment étendu, on peut ouvrir tout autour un layon d'un mètre, et recommencer l'opération quand le besoin s'en fait sentir; sinon, on se contente d'exploiter ou d'étèter les sujets dominants dans la zone voisine. Quoi qüil en soit, dans de telles opérations M. Gayer recommande de n'agir que graduellement, pas à pas, et de ne jamais faire plus que ne le commandent les nécessités du moment; on cassera les branches latérales trop envahissantes, on élaguera peu à peu, on étêtera, on pratiquera des incisions circulaires qui produisent un effet plus ou moins rapide suivant les essences (1), enfin on enlèvera complètement les sujets envahissants; un mème sujet peut ètre soumis successivement à plusieurs de ces opérations, voire même à toutes.

Ajoutons les deux conseils suivants que donnent MII. Boppe et Jolyet et qu'on ne peut trop répéter:

Le premier est de donner ses soins toujours aux mémes indiviulus; il est regrettable, en effet, de prendre la peine de dégager une première fois un brin, puis de l'oublier pour s'occuper d'un autre, qui, noyé jusque-là dans le fourré, a perdu toule vilalité.

(1) L'épicéa et le pin sylvestre meurent en général au bout de deux à trois ans, si l'incision est assez profonde. 
Le second est de fuire exécuter, dans la mesure du possible, les degagements par les gardes de la forêt. - In bon garde doit défendre les semis confiés à sa surveillance comme il les défend contre les délinquants. Des primes l'encourageant dans cette voie sont un argent mieux placé que des salaires donnés à des tâcherons ignorants des choses forestières qui agissent sans méthode et sans suite ef saccagent tout autour d'eux.

Si mème le propriétaire veut prècher d'exemple, el quand il se promène dans son domaine, s'armer d'un croissant au lieu d'une canne, il sera fout étonné du nombre très respectable de jeunes chênes ou de jeunes épicéas qu'il tirera d'affaire dans un temps relativement court.

Les dégagements les mieux faits sont ceux dont le prix ne dépasse pas la valeur de deux ou trois journées par hectare.

Mesures propres à stimuler la croissance. Coupes d'éclaircie. - Dans la période de jeunesse, les soins culturaux ont eu pour but de constituer le massif en bon état, et d'assurer le maintien dans le peuplement de's essences voulues, au détriment des mort-bois et d'autres essences envahissantes. Ils ont pu ètre appliqués jusquà la fin de la période de fourré. Au delà de celte période, dès celle de gaulis ou has-perchis, plus ou moins tot suivant les cas, les opérations culturales changent de nature; tout en continuant dans une certaine mesure ì diriger la formation des peuplements et leur composition, elles tendent à meltre ces peuplements en élat de doinner les produits qu'on en altend, et peuvent se proposer pour but de stimuler la croissance des sujets d'élite.

L'opération culturale prend le nom de coupe d'éclaircie, parce qu'en faisant tomber des bois déjà plus gros, elle cause un trouble momentané dans les conditions d'existence du peuplement, trouble qui, d'après M. Gayer, peut chre suivi de la réaction la plus salutaire si les opérations 
sont conduites avec discernement et soin, comme il peut, en cas de traitement négligent et routinier, ètre suivi des conséquences lesplus fàcheuses pour l'existence ultérieure du peuplement.

C'est pourquoi, ajoute le mème auteur, les soins eulturaux réclament ici toute la surveillance, l'application et la réflexion du préposé, et cela d'autant plus impérieusement que l'état du peuplement et la qualité de la station sont moins bons.

Aussi, dans la majorité des cas, la prudence commande-t-elle de marcher lentement ver's le but voulu, et le l'atteindre pas à pas.

Coupe d'éclaircie. - Une coupe d'éclaircie est une vérilable exploitation partielle, donnant des produits marchands; mais ici encore, comme dans toute opération culturale, l'idée de récolte ne doit pas intervenir dans la conduite de l'opération.

Nous arons déjà établi que dans tout peuplement en voie de croissance, il se crée naturellement, par le fait de la concurrence vitale, une séparation en plusieurs parties distinctes et nous avons distingué le peuplement principal et le peuplement accessoire (fig. 32) (1).

La partie la plus vigoureuse du peuplement se trouve dans le peuplement principal; dès lors la coupe d'éclaiıcie ne s'occupe que du peuplement principal; elle ne touche pas au peuplement accessoire : elle respecte la tolalité de ces tiges dominées dont le maintien tend à assurer d'unc part la couverture du sol, et de l'autre le remplarement, en cas de besoin, d'éléments qui viendraient à manquer dans le peuplement principal par suite d'erreurs ou d'accidents. A fortiori, elle respecte lous les sous-

(1) Dans la figure 32, page 27ö, l'ensemble des tiges $a a$ et des tiges $b b$ constitue le peuplement principal; dans ce peuplement principal, les tiges $a$ a sont les plus vigoureuses, les mieux conformées et par suite les tiges d'élite à conserver; les sujets $b b$ nuisent aux tiges d'élite et c'est sur eux que porte l'éclaircie.

L'ensemble des sujets $c$ e constitue le peuplement accessoire à conserver. 
bois plus ou moins buissonnants à la surface du sol

Dans le peuplement principal composé de sujets dominants, il existe en général des tiges d'élite (tiges $a, u$, fig. 32) reconnaissables à leur aspect plus sain, à leur diamètre plus gros, à leur cime plus fournie (1). Ces liges, serrées de près par les autres sujets moins bien doués du peuplement principal (tiges $b, b$ ) ne peurent occuper que l'espace disponible, et se trouvent à un moment donné trop serrées et retardées dans leur développement; c'est le moment où doit intervenir une coupe d'éclaircie. Si l'on se rend compte que la même lutte pour l'espace existe dans le sol pour les racines, il esl facile d'admettre, ce que d'ailleurs confirme l'expérience, que la suppression de quelques-uns des arbresqui serrenl les sujets d'élite, doit stimuler le développement el l'accroissement de ces derniers.

Ur c'est là, précisément, le but des coupes d'éclaircie ; elles consistent à venir procurer aux sujets d'élite, dans le peuplement principal, l'espace nécessaire, et à donnel' a chacun de ces sujets le pouvoir d'utiliser sans limiles l'air et la lumiere par les organes de leur cime, et les ressources du sol par-leurs racines. Les coupes d'éclaircic consistent dès lors à venir enlever, dans ce même peuplement principal, à côté des sujets d'élite, les arbres: moins beaux dont la cime est gênante pour les premiers, et cela dans la mesure prescrite par la nécessité du maintien de l'état massif. Q̨uant aux sujets dominés (liges $c$, fig. 32) ils sont ì conserver.

Comprenant ainsi la coupe d'éclaitcie, il parait très facile de venir desserrer les sujets précieux, dans la région où leur cime manque d'espace, et cela progressivement.

(1) S'il en est autrement, ce qui arrive quelquefois, surtout dans les peuplements d'origine artificielle, Ia première éclaircie tend à rompre cette uniforinité trop grande; on choisit au lıasard, à des distances convenables, des perches A desserrer, nuxquelles ce premier travail donne sur leurs voisines un avanlage qu'il ne reste plus qu'à maintenir dans la suite. 
ACTION DE L'HOMME SUR LES PEUPLEMENTS. 275

Dans la pratique, la coupe d'éclaircie est toujours une opération délicate, en raison de la grande variété que présente cette opération, suivant les conditions de sol, de climat, d'exposition et d'essence; elle change de forme et d'intensité, disent MIM. Boppe et Jolyet, suivant

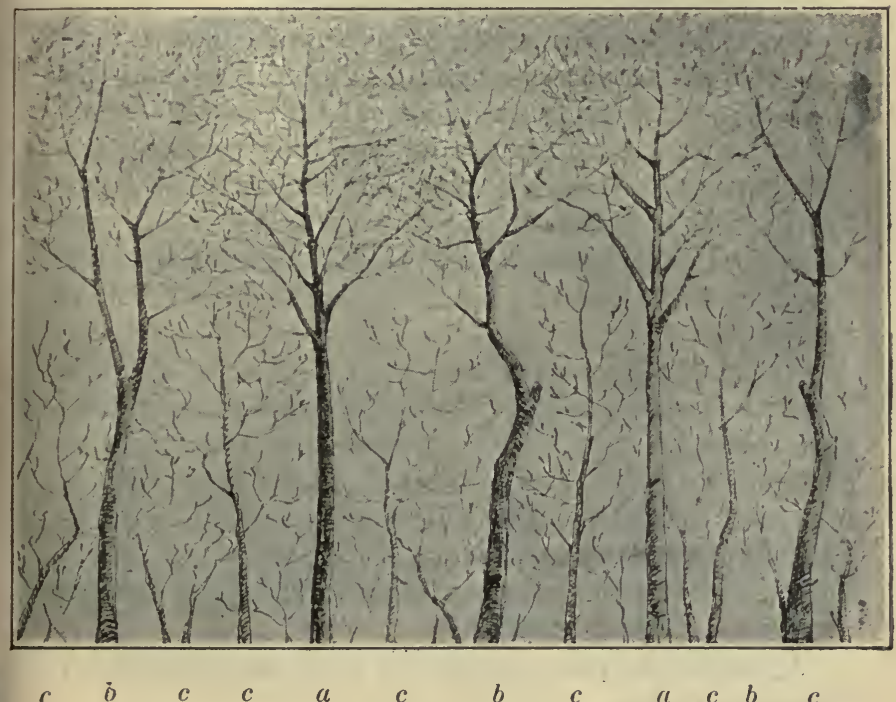

Fig. 32. - Schéma d'un peuplement à éclaircir. (MM. Boppe et Jolyet, " les Forêts ").

les essences, suivant leur état de mélange, suivant le bul poursuivi, suivant les idées et le hon vouloir.de celui qui les dirige ; toutefois, si l'on prend pour point de départ l'arbre d'élite, et si l'on agit autour de lui arec prudence, de façon à ne pas détruire l'état de massif, on sera toul au moins toujours sûr de ne pas faire une mauvaise opération. 
Dans les peuplements purs, la première éclaireie se fait plus tòt quand il s'agit d'essences de lumière que quand il s'agit d'essence d'ombre, mais il est important dans tousles cas d'opérer prudemment; en général, cette première éclaircie doit avoir lieu, dans les futaies, aussitot que les peuplements sont arrivés à l'état de gaulis ou au plus tard de bas perchis (Boppe et Jolyet).

Dans les peuplements mélangés, l'éclaircie, tout en conservant ses caractères propres, emprunte un nouveau 'aractère aux coupes de dégagement; elle doit alors leur succéder sans qu'un intervalle trop long permette aux essences envahissantes de reprendre leur œuvre de dégradation; d'après MII. Boppe et Jolyet, la grosseur des sujets et la réalisation des produits marehands fait r'hanger l'étiquette de l'opération, mais son but reste le mème.

Quant à la périodicité des coupes d'éclaircie, elle varie surtout avecl'âge etl'état de croissance des peuplements; on reviendra tous les six à douze ans dans les forèts traitées en futaie, depuis l'état de gaulis jusquà la fin de celui de haut perchis; puis seulement tous les douze à vingt ans dans les hautes futaies constituées; on reviendra plus fréquemment dans les sols fertiles que dans les sols médiocres ou maurais.

Ajoutons quelques conseils que nous empruntons aux inèmes auteurs.

Dans la pratique, un bon critérium pour juger de l'opportunité d'une éclaircie est l'aspect mème des arbres du peuplement dominant; si, par exemple, des chènes de futaie se couvrent de branches gourmandes, sans que leur âge très arancé ou toute autre cause accidentelle explique cette évolution anormale, c'est que leur cime manque de lumière dans les régions élevées; il en esi ainsi encore des chènes dont la cime apparait étriquée, souffreteuse, à frondaison chlorotique; de mème, dans un perchis, un pin, un sapin dépourvu de hranches 
ACTION DE L'HOMME SUR LES PEUPLEMENTS. 27?

vivantes sur plus des deux tiers et surtout des trois quarts de la hauteur de sa tige, réclame d'urgence qu'on le desserre.

Nous n'avons pas de restriction à faire à ce qui vient l'ètre dit, lorsqu'il s'agit d'exécuter des coupes d'éclaircie dans des peuplements en taillis; remarquons toutefois que les coupes d'éclaircie ne sont admissibles que si la révolution du taillis est suffisamment longue.

Dans les taillis composés, oṇ ne fait en général qu'une seule éclaircie, six à huit ans avant la coupe principale; pourtant, dans certains taillis à longue révolution et sur certains sols, on peut avoir intérèt à réaliser plus tôt, dans une première éclaircie, les bois tendres parrenus à maturité. 


\section{TROISIEMME SECTION}

\section{Mesures de gestion}

\section{1. - PLAN DU DOMAINE. - LIMITES}

Tout propriétaire d'un domaine boisé doit en connaitre exactement la situation, la contenance et les limites. A cet effet, il peut employer les trois moyens suivants :

$1^{\circ}$ Relevé cadastral. - A titre d'indication, relever sur' le plan cadastral des communes sur le territoire desquelles se trouve le domaine boisé, un calque des parcelles cadastrales constituant ce domaine, et des parcelles limiIrophes.

Ce plan, souvent incomplet, peut ètre facilement rectifié à vue, et il est facile d'y mentionner l'état des limites existantes, la nature des propriétés riveraines, ainsi que lenom de leurs propriélaires, d'y indiquerles principaux chemins portés au cadastre et les voies naturelles de vidange, l'orientation générale, etc.

Ce plan, généralement fait à l'échelle de 1 à 2500 ou de 1 à : 5000 , peut donner, à l'aide de la matrice cadastrale une indication approchée de la contenance de la propriété, el sa disposition générale.

20 Plans d'arpentage. - Juxtaposer 'les plans d'arpentage des anciennes coupes exploitées, que le propriétaire peut posséder', plans sourent faits à la mème échelle que le cadastre. Les plans, rapportés approximativement sur le calque cadastral, sorvent à indiquer la répartition des anciennes exploitations et par suite celle du matériel sur pied. 
3० Plan exact. - Notions succintes d'arpentage.La méthode suivante permet de faire dans la petite et moyenne propriété un plan du domaine boisé avec une "xactilude suffisante.

Pour ètre à même de dresser un plan, il faut piqueter à l'aide de jalons les différents sommets de la figure qu'on veut reproduire (1) (pourtour du périmètre, chemins intérieurs, etc.), mesurer les longueurs de toutes les lignes ainsi établies, et déterminer leurs directions.

Pour mesurer une ligne, on se sert de la chaîne d'arpenteur (10 mètres en général); au début de la mesure de chaque alignement les chaîneurs doivent s'assurer que l'homme qui marche en avant a bien les dix fiches réglementaires; si le terrain est incliné, le chaìnage doit ìtre fait en descendant, et la chaine doit ètre toujours tendue horizontalement. L'opérateur inscrit au fur et à mesure les résultats du chainage sur le croquis qu'il tient comme l'indique la figure 33.

Pour determiner la direclion d'une ligne, on se sert de la boussole (2) que l'on place à son origine; à l'aide de la lunette on vise le jalon placé verticalement à son autre extrémité; on lit sur le limbe divisé le numéro de la graduation où s'arrète la pointe bleue de l'aiguille, et on note cette lecture sur le croquis.

Opérations sur le terrain. - Soit A B CDE, une portion de polygone à lever; on place la boussole au point $\mathrm{A}$, on vise $\mathbf{B}$, on fait la lecture sur la boussole, on rhaine $\mathrm{AB}$; on transporte la boussole en $\mathrm{B}$, on vise $\mathrm{C}$ et ainsi de suite, en ayant soin de viser et de cheminer de proche en proche et toujours dans le mème sens, c'est-àdiré en laissant par exemple toujours l'intérieur de la

(1) Le plan est par définition une figure géométrique semblable à la projection horizontale de cette figure sur le terrain.

(2) Boussole forestière, conjposée essentiellement d'un limbe gradué sur lequel est mobile une aiguille aimantée, et d'une lunette servant à faire les visées. 
forèt à sa droile (ou à sa gauche), ce qui facilite le rapport du plan. On opère ainsi jusqu'à ce qu'ayant fait tout le tour du polygone, on soit revenu exactement au point de départ A. On opère de mème pour les lignes intérieures (chemins, cours d'eau, lignes de crète, elc.)

Si une partie EA du périmètre se trouve très sinueuse, il est arantageux de la remplacer par quelques grandes directions rectilignes qui seront généralement prises en dehor's du périmètre de la forèt, par exemple EM et MA. On lève ces lignes absolument comme le reste du périmètre. Il suffit ensuite de rattacher le périmètre sinueux à ces directrices par des perpendiculaires menées à l'équerre d'arpenteur; pour cela, on chaîne et on note sur le croquis les longueurs E $p ; p q ; q \mathrm{M}$, etc., el $p p^{\prime}, q q^{\prime}$, etc., dont le rapport sur le plan se fait d'un' facon très simple.

Rapport du plan. - La lecture faite à la pointe bleur de l'aiguille indique l'angle que fait la ligne de visér avec la direction nord-sud de l'aiguille aimantée; rol angle s'appelle un orientement; il s'agit donc tout simplement de tracer sur le papier une ligne faisant arec retle direction nord-sud, et dans le sens convenable, un angle correspondant à l'angle lu.

Pour tracer cette ligne, on se sert d'un rapporteur, demi-cercle en corne, gradué comme le limbe de la boussole; on commence par tracer sur le papier unc ligne droite représentant la direction fixe nord-sud d. l'aiguille aimantée; on place le rapporteur de telle façon que cette ligne passe simultanément par la division du rapporteur correspondant à la lecture faite, ol par le point de croisement des diamètres passant par les divisions $0^{\circ}$ et $90^{\circ}$ du rapporteur; un trait de crayon tracé suivant le bord rectiligne du rapporteur donne une ligne parallèle à la ligne cherchée. Il est facile de comprendre que si l'on a tracé d'avance une série de parallibes à la ligne nord-sud, on trowera aisément le moyen 
de placer le rapporteur de telle façon que cette ligne à

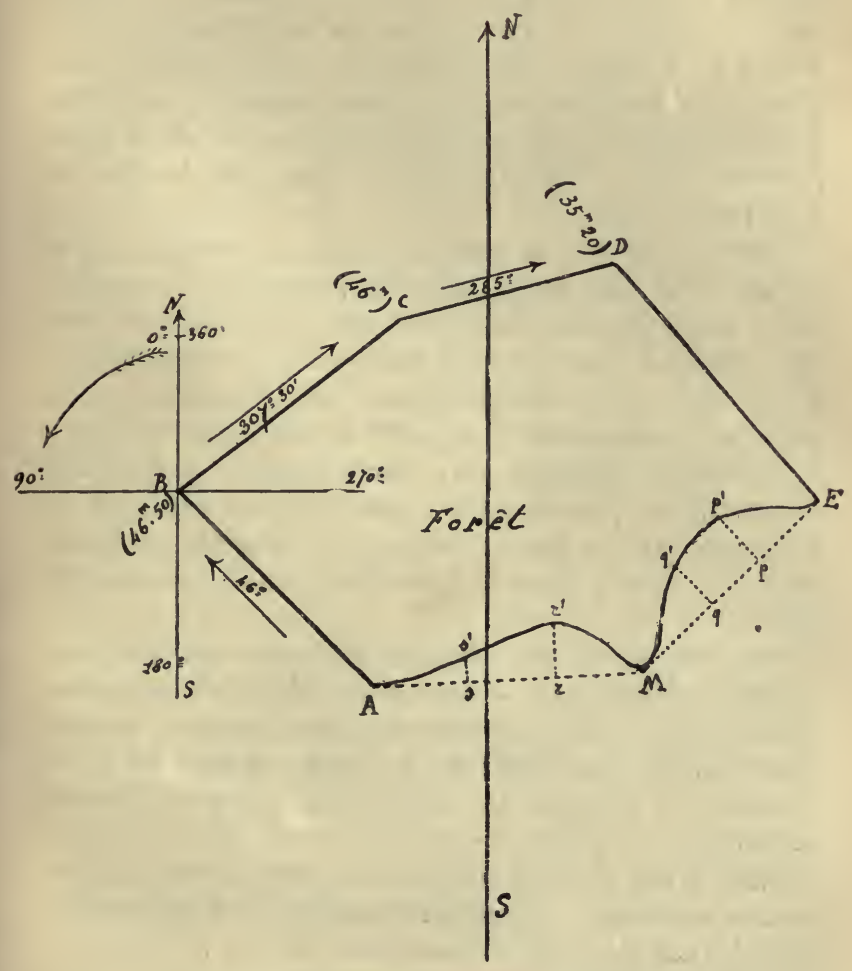

Fig. 33. - Arpentage du périmètre d'une forèt.

trácer passe par le point mème qui doit être son origine sur le plan (1).

Pour déterminer le sens dans lequel on doit tracer la

(1) En se servant d'un papier d'archilecte, soit quadrillé, soit simplement a lignes parallèles, l'opération est très facile. . 
ligne à partir de son point d'origine, on supposera dessiné en ce point d'origine la figure que nous avons faite au point B (fig. 33) et on partira de ce principe que les angles se comptent en partant du nord dans le sens inverse du mouvement des aiguilles d'une montre; de telle façon que, si par exemple l'orientement de la ligne à tracer est de $307^{\circ} 30^{\prime}$, la ligne sera dirigée dans l' sens $\mathrm{BC}$.

Pour donner sur le plan la longueur voulue à cetle ligne, on se sert d'une échelle de réduction, c'est-à-dire d'une règle graduée à l'échelle du plan. On prend sur cette règle, à l'aide des deux pointes d'un compas, la longueur mesurée horizontalement sur le terrain; on la porte sur la ligne tracée, à partir de son point d'origine, et on obtient la position du sommet suivant; on continue de proche en proche. Si le périmètre ainsi rapporté ne lerme pas dans les limites tolérables, c'est qu'on a commis une erreur, soit dans le lever, soit dans le rapport du plan.

Pour caleuler la surface ainsi arpentée, on décompose le polygone rapporté en figures géométriques (triangles, (rapèzes, etc.) dont on mesure graphiquement les éléments sur le plan quand ils n'y sont pas déjà cotés, el on fait la somme de toutes ces surfaces élémentaires.

Dans le cas où on a substitué des lignes droites à des parties sinueuses, il reste à retrancher ou à ajouter suivant les cas les surfaces comprises entre le périmètre el ces directrices (1).

Le plan d'un donaine boisé doit indiquer les principaux mouvements de terrain, les roules, les cours l'eau, les habitations voisines, etc.

Il est bon de profiter de l'établissement de re plan

(1) Pour calculer rapidement les surfaces, on peut se servir d'un planimètre, ou du système des quadrillages. 
pour reconnaitre et fixer les limites de la forèt sur les points incerlains (1).

Ces limites peurent ètre désignées par des bornes numérotées qu'on place aux angles (saillants et rentrants) du périmètre. Partout où des chemins, ruisseaux de bordure, etc., ne désignent pas nettement la limite, il est utile de la préciser artificiellement par des fossés, des rigoles, des cordons ou murs en pierres sèches, des liaies, etc., se rappelant qu'une forèt ne restera en bon état que si elle est suffisamment close, partout où il y a lieu de redouter les divagations du bétail voisin.

Reproduction du plan à plusienr's exemplaires. Lorsque le propriétaire possède un bon plan de sa forèt, sur lequel il a mentionné la situation des coupes, ou l'aménagement, il peut le faire autographier. A défaut de plan autographié, nous conseillons le procédé suivant :

Le propriétaire possède un exemplaire de son plan, tracé avec soin sur une toile calque d'architecte; les traits et l'écriture, faits à l'encre de Chine, sont nettement visibles; il lui suffit d'avoir une glace et du papier photographique au ferro-prussiate pour en tirer facilement un certain nombre d'exemplaires comme on tire un cliché photographique (2).

Ces exemplaires, distribués aux gardes, aux marchands de bois, aux chasseurs, font connaitre le domaine et rendent de très grands services.

(1) Voir Code civil, article 64.6. Et en ce qui concerne les propriétés contiguës à des bois soumis au régime forestier, les articles 8 à 14 du Code forestier, et les articles 57 à 66 de l'ordonnance réglementaire du 1 or aoủt 1827 .

(2) Certains papiers spéciaux pour le tirage des plans donnent le trait en noir. Il n'est pas nécessaire de posséder, comme la plupart des administrations (oủ cela est justifié par le grand usage qu'on en fait), un immense châssispresse. Il suffit de laisser dépasser hors de l'unique glace qui recouvre le plan, une bande de papier assez large, sur laquelle on tire simultanément et dans les nièmes conditions des écritures analogues. En coupant de temps en temps quelques inorceaux de cette bande, et en les fixant, on arrive très facilement à régler le tirage du plan. 


\section{II. - ORDRE DES EXPLOITATIONS. - ASSIETTE DES COUPES.}

Définitions. - On appelle coupe, toute étendue de terrain délimitée dans une forêt pour y abattre tout ou partie des bois qui garnissent cette surface; coupe en usunce, les coupes qui sont en exploitation ou en vidange; le mot exploitation est souvent employé dans le sens de coupe ou de vente; l'assiette d'une coupe est la désignation de l'emplacement que doit occuper cette coupe; l'opération d'asseoir une cou, $e$ consiste donc à déterminer l'endroit où celle-ci doit être faite, et sourent à en lever le plan, afin de lui donner la contenance voulue.

Ordre des exploitations. - Règles d'assiette. - Dans une bonne exploitation, il est préjudiciable à l'intérêt du propriétaire de disséminer sans raison les exploitations au hasard dans une forèt.

Au point de vue cultural, il est indispensable de suivre un certain ordre, déterminé par les règles suivantes, dont les deux premières concernent spécialement les taillis, et les trois autres visent plus particulièrement les futaies.

Première règle. - Les coupes doivent être assises de manière à se succéder de proche en proche, et recevoir la forme la plus régulière possible. - La succession des coupes de proche en proche crée une graduation suivie entre les diver's peuplements d'àges différents qui s'élèvent ensemble; elle leur permet de se protéger mutuellement contre les rents violents, l'action de la neige, etc., et diminue les chances de chablis; elle lacilite la surveillance des coupes en usance. Quant à la régularité des coupes, elle est très commode; elle simplifie les opérations d'assiette, celles d'abalage el de vidange des arbres de lisière, et diminue l'action nuisible du vieux peuplement restant, sur le jeune recru des bords de la coupe. 
Deuxième règle. - Les coupes seront assises de telle sorte que le transport des bois d'une coupe en exploitation puisse s'effectuer sans qu'on soit obligé de traver'ser les coupes précédemment exploitées. - Cette règle s'explique d'elle-mème, en raison du préjudice que causeraient les nombreux charrois nécessaires pour la vidange de toute une coupe en passant au travers de jeunes recrus ou de repeuplements en voie de croissance. L'inobservation de cette règle est souvent la cause de nombreux vides plus ou moins élendus qu'on est obligé de repeupler artificiellement.

Il y a donc lieu, dans l'opération d'assiette d'une coupe, de tenir compte de l'existence de laies sommières (1).

Troisième règle. - Les coupes doivent être assises à l'encontre des vents dangereux. - Dans la plus grande partie de la France, les vents dangereux sont ceux du sud et de l'ouest, en raison de leur violence et de la pluie qui les accompagne presque toujours et qui détrempe le sol. Par suite, on doit en général, tendre à asseoir successivement les coupes en allant du nord vers le sud et de l'est vers l'ouest; les exploitations laissent ainsi du côté exposé aux vents dangereux, le massif plein; ce massif sert d'abri, et protège les arbres de réserve qui sont moins résistants contre l'effort du vent, surtout pendant les premières années de leur isolement. Pour procurer le mème abri aux dernières coupes, il peut ètre utile, dans les situations très exposées, de laisser du côté des vents dangereux, un rideau de protection de 20 à 30 mètres d'épaisseur, constitué au besoin par le vieux mássif qu'on n'exploitera alor's que plus tard quand son ahri ne sera plus utile.

L'application de cette troisième règle devient surtout importante dans les futaies, pour les essences résineuses,

(1) Lignes de division ou de séparation des coupes, assez larges pour permettre la vidange. 
à cause de leur hauteur considérable, et spécialemenl jour les essences à enracinement superficiel; elle est indispensable sur les versants et les portions élevées des régions monlagneuses.

Elle ofrre encore l'avantage, quand les essences ont des yraines légères ou munies d'ailes, de faciliter le réensemencement naturel, car les semences sont emportées par les vents, des massifs non encore exploités, ver's les coupes en usance (E. Muel).

Quatrième règle. - Ein montagne, les exploitations doivent êtr'e dirigées de bas en haut. - L'eflet du massif supérieur vers lequel se dirigent les exploitations est protecteur, comme dans le cas précédent, contre les vents généralement plus violents des régions élevées. On conslitue d'ailleurs, en général, sur le sommet des montagnes, des zones protectrices plus importantes formées par des peuplements jardinés et l'on a soin d'y réduire à dessein les exploitations pour y interrompre le moins possible le massif. Souvent, alors, la vidange des coupes s'effectue forcément dans le sens de la pente, au travers les coupes récemment exploitées; on doit, dans ce cas, chercher à réduire les dégâts dans la mesure du possible, "n installant de bonnes voies de vidange bien réparties, des lançoirs ou plutôt des chemins de schlitle, des ràbles aériens, etc.

Cinquième règle. - E'n montagne, les coupes seront antant que possible longues et étroites, et assises de manière à offrir aux vents violents leur moindre profondeur. - La coupe ainsi établie offrira moins de prise aux rents dangereux et sera mieux protégée jar le massif voisill.

Observation. - Dans l'application, ces riggles conduisent jarfois à des résultals contradictoires l'un avec l'autre; il est nécessaire alor's de comparer leur raison l'ètre et leur utilité respective, atin d'en déduire la marche la plus avantageuse ì suivre. Hans tous les ras, 
le but à rechercher est d'éviter les nombreux chablis qui viennent jeter le désordre et sourent la ruine dans des peuplements de valeur.

Manière d'asseoir une coupe sur le terrain. Pour asseoir une coupe d'une contenance donnée sur le terrain, autrement dit pour la détacher du vieux massif restant et la délimiter, il est nécessaire de procéder à un arpentage. Cet arpentage donne lieu à l'ouverture de filets ou lignes de délimitation qui séparent la coupe du surplus du bois. Nous envisagerons successirement le rôle de l'agent arpenteur et celui des gardes.

Rôle de l'agent arpenteur. - En général, la ligne à ouvrir doit avoir uné direction déterminée à l'avance, soit pour être parallèle aux lignes prúcédentes, soit en raison de la vidange de la coupe et de la pente du terrain.

On commence par lever et rapporter le périmètre de la forèt dans la partie où se trouve la coupe à asseoir, comme il a été dit précédemment (page 279); puis on trace sur le plan une ligne provisoire ayant la direction donnée. On calcule la partie ainsi détachée, et en ajoutant ou retranchant des trapèzes, on arrive par approximations successives à détarher sur le plan la contenance voulue (1).

Il reste alors à ouvrir la ligne sur le terrain. D'après les indications du plan, on détermine l'orientement de cette ligne et son point de départ sur le terrain; plaçant la boussole en ce point, on met la pointe bleue de l'aiguille en coïncidence avec la division du limbe correspondant à cet orientement; on fixe la boussole dans cette position; la ligne de visée de la lunette donne la direction de la ligne à ouvrir. L'agent arpenteur dirige avec sa

(1) Dans le cas particulier où les côtés opposés du périmètre sont sensiblement parallèles. la surface à ajouter ou à retrancher pouvant être assinilée à un parallélogramme dont on connait lı surface et les hases, il est facile d'en calculer immédiatement la hauteur. 
lunette les premiers jalonneurs, qui se guident ensuite pour la continuation de l'ouverture de la ligne sur les premiers jalons placés.

La ligne une fois ourerte, on s'assure que son tracé sur le terrain correspond bien avec les indications du plan, comme point d'arrivée et comme longueur.

Róle des gardes. - a. Avant l'operration. - Le garde ronnaissant la position que doit occuper la coupe, visite la ligne sur laquelle la coupe doit s'appuyer, la rafraichit, la jalonne, en recherche les anciens cornier's et parois; puis il dégage le périmètre à arpenter, découvrant les hornes, fossés, murs, etc; il prépare des jalons bien droits, taillés en pointe aux deux bouts, des piquets en bois dur appointis à un bout, et prend ses mesures pour que, au jour de l'opération, il y ait des serpes et haches entre les mains des auxiliaires.

b. Pendant l'opération. - Le garde exécute scrupuleusement toutes les instructions qui lui sont données par l'arpenteur. Il donne tous ses soins à un bon jalonnage el spécialement au chainnage pour lequel on ne saurait prendre trop de précautions (vérification des fiches à chaque chaînage partiel ; mesurer toujours en descendant, par continuité, bien horizontalement, par cinq mètres si la pente est forte, etc., etc.).

Des arbres parois sont marqués au pied du marteau de l'opérateur, du coté et en regard de la ligne qu'ils déterminent; des arbres corniers sont choisis à un angle lormé par la rencontre de deux lignes, et marqués sur deux faces, en regard et du côté de ces lignes. Les piquets d’angle sont, en l'absence de corniers, rattachés i des arlres témoins, dont on note l'essence et la grosseur; les blanchis de ces arbres témoins sont faits légìrement sur l'écorce et à deux mètres de hauteur.

c. Apris l'operation. - Bien dégager la ligne ouverte, l'entretenir, veiller sur les piquets, les remplacer šils dispraraissent, ceinturer d'un lien les arbres corniers, 
parois et témoins; s’il existe plusieurs lots, indiquer le numéro de chaque lot sur un blanchis fait aux arbres de limite (Agenda du Forestier).

\section{III. - NOTIONS SUCCINCTES D'AMÉNAGEMENT.}

Généralités. - Aménager une forèt, c'est régler la marche des exploitations de manière à obtenir des produits périodiques, annuels si c'est possible, aussi soutenus et aussi avantageux que possible.

Les végétaux ligneux, par la lenteur de leur croissance, ne donnent que des produits périodiques, à période assez longue; raccourcir ces périodes, chercher même à obtenir des produits annuels, rentre dans les conditions des exploitations ordinaires et dans l'idée courante qu'on se fait d'avoir des revenus.

Pour rendre plus fréquentes les récoltes, il faut diviser la forêt en coupes au lieu de tout exploiter d'un seul coup; pour rendre les produits aussi avantageux que possible, il faut couper à l'âge le plus convenable.

Nous indiquons dans le cours de cet ouvrage les considérations qui peuvent guider pour déterminer l'âge auquel il faut couper ; connaissant cet âge, il reste, si l'on veut des produits annuels, à diviser la forèt en autant de coupes équivalentes en production qu'il y a d'années dans le nombre qui exprime l'âge auquel on veut couper; c'est-à-dire à former ce qu'on appelle, en style d'aménagement, le plan d'exploitation.

Plan d'exploitation. - Établir un plan d'exploitation, c'est effectuer un travail qui consiste à régler la quotité et la marche des coupes.

Suivant la méthode d'exploitation adoptée, le plan d'exploitation s'établit de manières différentes et nous examinerons successivement les divers cas qui peuvent se présenter :

Premier cas. - Taillis simple. - Considérons d'abord A. Frox. - Sylviculture. 
une forèt dans laquelle tous les arbres parcourent simultanément les phases successives de leur existence (bois taillis de petile étendue); elle ne donne de produits marchands qu'après un certain nombre d'années d'existence, nombre d'années égal à l'âge des arbres qu'on coupe. Une fois l'exploitation faite, le propriétaire doit attendre un temps égal pour faire une nouvelle récolte, et ainsi de suite.

Soit, par exemple, 30 hectares de taillis que le propriétaire exploite à l'âge de trente ans; en 1902 le taillis est âgé de cinq ans; le plan d'exploitation est évidemment le suivant:

En 1927 : exploitation de trente hectares âgés de trente ans;

En 1957 : exploitation de trente hectares âgés de trente ans, etc.

On dit que la forêt est à exploitalion périodique.

Mais on peut concevoir dans la mème forèt une méthode meilleure, ou tout au moins plus commode, d'effectuer la récolte; subdivisant la forèt en un certain nombre de coupons, le propriétaire dirige la formation des peuplements de telle sorte que l'âge d'exploitation de chaque coupon ne tombe plus à la mème époque. Pendant la mème période de temps, il exploitera encore toute la forèt, mais par parties el non en bloc, et à des intervalles déterminés.

Dans l'exemple précédent, nos trente hectares de taillis sont divisés en trois coupons de dix hectares, et les arbres sont toujours exploités à trente ans; mais les âges sont différents sur chacun des coupons, ils sont, par exemple, en 1902 de vingt-neuf ans dans le coupon $n^{\circ} 1$, de vingt ans dans le coupon $n^{\circ} 2$ et de cinq ans dans le coupon $\mathrm{n}^{\circ} 3$.

Le plan d'exploitation est évidemment le suivant :

En 1903, exploitation du coupon $n^{\circ} 1$ composé de dix hectares âgés de trente ans. 
En 1907, exploitation du coupon $n^{\circ} 2$ composé de dix hectares âgés de trente ans.

En 1927, exploitation du coupon $n^{\circ} 3$ composé de dix hectares âgés de trente ans.

En 1933, exploitation du coupon $n^{0} 1$ composé de dix hectares âgés de trente ans, etc.

I.a forêt constituée par une série de coupons à exploitation périodique, permet au propriétaire d'obtenir des récoltes partielles, à intervalles plus rapprochés.

Cette première amélioration nous conduit à la notion d'aménagement par contenance.

Pour que la récolte forestière, essentiellement périodique sur un point donné, puisse devenir annuelle, comme l'exigent les besoins de l'homme, il est nécessaire de réaliser les produits successivement sur autant de surfaces différentes qu'il faut d'années aux arbres pour atteindre les dimensions requises. La forèt, ainsi économiquement constituée, est dite aménagée.

Dans l'exemple précédent où il s'agit d'un taillis exploitable à l'âge de trente ans, si le propriétaire veut pouvoir réaliser tous les ans un hectare de taillis âgé de trente ans, il devra posséder une forêt de trente hectares, composée de trente coupons d'un hectare, et il est nécessaire en outre que les âges des bois de chaque coupon diffèrent entre eux d'une année, depuis les sujets naissants jusqu'à ceux qui ont vécu le nombre d'années que comporte la récolte.

Quand la forèt est dans cet état, elle est dite normalement constituée (1).

Dès lors, pour établir le plan d'exploitation, on commence par fixer la quotité des coupes annuelles, en di-

(1) Si les différences de sol et d'état de végétation du peuplement sont assez importantes pour que la valeur d'un hectare de taillis de trente ans sur un point soit notablement différente de la valeur d'un hectare de taillis de même Age sur un autre point, pour obtenir un revenu constant les coupons devront avoir, non des surfaces égales, mais des surfaces équivalentes. 
visant la surface de la forêt en autant de parties sensiblement égales qu'il y a d'années dans la révolution adoptée, puis on limite ces différentes coupes sur le terrain en les arpentant, ce qui s'appelle aménager la forèt par contenance.

L'aménagement par contenance est le plus simple, le plus pratique, celui qui permet le mieux le contrôle ; il est toujours applicable au taillis.

Il ne reste plus qu'à numéroter les coupes obtenues, en donnant le numéro 1 à celle à exploiter la première, le numéro suivant à la coupe voisine et ainsi de suite dans l'ordre des âges, les derniers numéros étant donnés aux coupes les plus jeunes.

Remarquons qu'en procédant ainsi dans une forêt très anormale, on sera amené à exploiter quelquefois des bois un peu plus âgés ou un peu plus jeunes que l'âge qu'on s'était fixé, mais cela pendant la première révolution seulement. Ces sacrifices une fois faits, on aura ramené la forêt à un état normal.

leuxième cas. - Taillis fureté. - La quotité et la marche des coupes réglées par contenance comme pour les taillis simples, on partage la révolution en un certain nombre de rotations (2 à 4 ) et on divise la forêt en autant de coupons qu'il y a d'années dans la rotation, en observant les règles prescrites pour la division en coupes des taillis simples.

On indique l'ordre dans lequel ces coupons seront parcourus, et en même temps on détermine les dimensions minima des perches à exploiter à chaque passage (dimension des bois à l'âge normal d'exploitabilité).

Troisieme cas. - Taillis composé. - Le taillis composé est constitué par deux éléments distincts: le taillis et les réserves.

La quolité et la marche des coupes se règlent par contenance, en vue de l'élément taillis, comme pour un taillis simple. 
Ce plan d'exploitation se complète ensuite par des prescriptions spéciales à l'élément réserve; ces prescriptions portent le nom de plan de balivage et nous en parlons au cours de notre ouvrage ( $\mathrm{III}^{\circ}$ partie). Ce plan de balivage indique pour chacune des essences de la réserve :

$1^{\circ}$ L'intérèt qu'il y a à conserver des réserves;

$2^{\circ} \mathrm{La}$ proportion à observer dans le recrutement des baliveaux;

- $3^{\circ}$ Le terme d'exploitabilité des réserves et la dimension moyenne à cet âge ;

$4^{\circ}$ Les signes de la maturité.

Il n'est pas possible de déterminer à l'avance le nombre des arbres de chaque catégorie à conserver lors du balivage des coupes.

Le traitement en taillis composé peut comporter, pour le bon recrutement de la réserve, des coupes d'amélioration (dégagements de semis, éclaircies); on peut en régler, s'il y a lieu, la périodicité et la marche.

Quatrieme cas. - Futaies exploitées par coupe unique. - Sont dans ce cas toutes les futaies repeuplées artificiellement, et même quelques futaies régénérées par voie naturelle, comme par exemple certaines futaies de pin maritimes, de pin d'Alep, d'épicéa en région montagneuse.

Le plan d'exploitation se fait alors comme dans un taillis simple; on divise la forèt en autant de coupes qu'il y a d'années dans la révolution (1) choisie, et on exploite chaque année une de ces coupes, en commençant par la plus âgée.

Quand on assied ces coupes sur le terrain, il y a lieu, autant que la disposition des lieux le permet, de leur donner une forme étroite et allongée; on peut ainsi bénéficier, dans une certainemesure, de l'ensemencement

(1) La révolution, d'une façon générale, est le laps de temps adopté pour la régénération successive de tous les peuplements qui constituent la forêt. 
latéral, si des graines proviennent des peuplements voisins, et il est toujours facile de se conformer aux principales règles d'assietle.

Cinquième cas. - Futaies régénérées par coupes successives où la période de régériération est courte (inférieure à vingt ans). - Forêts de plaines et de coteaux (Méthode de M. Parade).

Dans ces futaies, le plan d'exploitation se divise en deux parties bien distinctes : le plan d'exploitation des coupes principales; le plan d'exploitation des coupes d'amélioration.

a. Plan d'exploitation des coupes principales. - Par suite des exigences culturales de la méthode de régénération par coupes successives, il est difficile de concilier une marche de coupes par contenances avec rapport soutenu, et les exigences culturales de coupes successives faites chacune en temps opportun.

Aussi, en pratique, un des maitres de la sylviculture, M. Parade, a divisé le problème :

Suivant sa méthode, on divise la révolution en un certain nombre de parties égales que l'on nomme périodes; les périodes doivent être plus longues que le temps nécessaire à la régénération d'une surface donnée ; elles doivent ètre au moins du double (environ vingt à quarante ans) pour permettre, d'après la pratique, d'assurer les exigences du rapport soutenu tout en se conformant aux exigences de la culture.

La division en périodes fixée, on partage la surface de la forêt en autant de parties égales qu'il y a de périodes dans la révolution. Ces parties sont des coupes périodiques par contenance; elles ont reçu le nom d'affectations.

Chaque affectation correspond à une période, et doit être régénérée pendant cette période.

La division de la révolution en périorles et le partage de la forèt en affectations, porte le nom de règlement général d'exploitation. 
Le règlement général d'exploitation formé, il ne reste plus qu'à dresser la quotité et la marche des coupes dans l'intervalle de chaque période. Pour régler cetle quotité et cette marche, on a recours à la possibilité par volume; c'est l'objet de ce qu'on appelle le règlement spécial d'exploilution.

Ce règlement spécial d'exploitation se dresse au début de chaque période et seulement pour la période qui s'ouvre; il ne porte en principe que sur l'affectation à régénérer au cours de cette période.

Pour régler la quotité des coupes annuelles dans cette affectation, on procède alor's de la manière suivante : on cube le matériel existant sur l'affectation, matériel qui ne comprend que des tiges déjà âgées; par suite de l'âge de ces tiges, on peut admettre que pendant le cours de la période où on les exploite, l'accroissement qu'elles prennent chaque année est sensiblement égal à leur accroissement moyen passé (1). Connaissant cet accroissement moyen passé, on calcule l'accroissement que prendront les bois pendant la moitié de la période. On ajoute ce volume au volume déjà cubé, et on divise la somme par le nombre d'années de la période. Le quotient ainsi trouvé donne le nombre de mètres cubes à enlever chaque année (2).

On indique ensuite l'ordre dans lequel les diverses parties de l'affectation seront mises en coupes d'ensemencement (3). On ne peut faire plus, les exigences culturales ne pouvant permettre de prévoir à l'avance l'ordre dans lequel se produiront les semis, et les époques où il sera opportun de les découvrir par les coupes secon. daires successives.

(1) Cet accroissement moyen est égal au volume du matériel existant sur l'affectation divisé par l'âge moyen des bois qui composent ce matériel.

(2) Il est évident que pour cuber le volume annuel à enlever on devra faire usage des mèmes tarifs que ceux qui ont servi à dresser l'inventaire.

(3) En pratique, l'affectation est divisée en coupons ou parcelles désignèepar les lettres $A, B, C$, etc... . 
En ayant soin de prendre chaque année le volume prévu, on doit exploiter tout le matériel pendant la période, et d'autre part on a assez de matériel pour continuer les exploitations pendant toute la période.

Il est facile de se rendre compte qu'en principe, les coupes d'ensemencement doivent avoir parcouru toute l'affectation pendant la première moitié de la période.

b. Plan d'exploitation des coupes d'amélioration. - On dresse au commencement de chaque période un tableau indiquant, pour chaque année de la période, les contenances à parcourir en coupes d'éclaircie. Si les éclaircies passent tous les douze ans (1), on divise par douze la contenance totate des parties de la forèt à parcourir en coupes d'éclaircie pendant la durée de chaque rotation de douze ans; puis on cornmence les éclaircies aux points où l'opération est le plus utile.

Quant aux dégagements, ils ne peuvent ètre prévus; ils sont effectués quand on les reconnaît nécessaires.

Cette méthode, pour ètre appliquée, exige des forèts ayant déjà une certaine étendue, environ 150 hectares au minimum. A ce titre, elle n'est guère pratique dans la propriété particulière ; elle exige d'ailleurs un matériel superficiel très important, ce qui est peu compatible arec le fonctionnement économique de la propriélé privée.

Exemple. - Nous considérons une futaie de sapin et d'épicéa située sur le troisième plateau du Doubs, à l'altitude de 900 mètres. La contenance est de 148 hectares; la durée de la révolution adoptée est de cent quarante ans; la durée choisie pour la période est de vingt ans (2).

Il s’ensuit que la révolution se trouve partagée en sept

(1) On prend pour durée de rotation un sous-multiple du chiffre exprimant la durte de la périodo.

(2) Celle duréc de période, empruntée à l'aménagement d'une forèt existante, est un peu courto. 
périodes, et parallèlement que la forêt a été divisée en sept affectations de 21 hectares en moyenne.

Règlement général d'exploilation . Affectations. Nature des peuplements. Contenances.

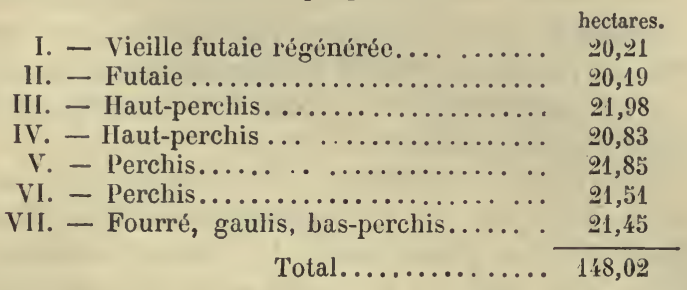

Règlement spécial pour la première période.

a. Coupes principales. - Quotité des coupes. - Matériel existant au début de la période : 16107 mètres cubes.

Age moyen des bois $\left(\frac{6 \times 140}{7}+\frac{20}{2}\right)=130$ ans.

Accroissement annuel moyen jusqu'à cet âge $\frac{16107}{130}$ $=123^{\mathrm{mc}}, 9$. Multipliant ce nombre par la moitié de la durée de la période, et y ajoutant le cube primitif, on a le cube total : $16107+123,9 \times 10=17346$ mètres cubes.

Et divisant par 20, on obtient 817 mètres cubes, volume à enlever chaque année pendant la période (1).

Marche des coupes. - On commencera les coupes d'ensemencement par le nord-est de l'affectalion à régénérer.

b. Coupes d'amélioration. - Rotation adoptée : dix ans. Contenances à éclaircir pendant la période $: 127^{\mathrm{h}}, 81$, soit les affectations II, III, IV, V, VI et VII.

Étendue moyenne à parcourir chaque année : $12^{\mathrm{h}}, 78$.

(1) Généralement, en pratique, pour éviter des mécomptes ou des accidents, on ne tient pas compte de l'accroissement pendant la période. Le volume à enlever chaque année serait alors simplement de $\frac{16107}{20}=\$ 0$ m mètres cubes. 
Marche des coupes (ou état d'assiette).

$$
1902 \ldots \ldots \ldots \ldots \ldots, \text { II (partic)...... }
$$

Sixième cas. - F'utaies régénérées par coupes successives où la période de régénération est longue (par exemple supérieure à vingt ans); forêts de montagne (méthode nouvelle).

$1^{\circ}$ Division en parcelles. - On divise la forèt en parcelles s'appuyant sur les lignes de crète, lignes de fond, lignes de plus grande pente, les voies de vidange existantes où à créer, et on établit ainsi sur le terrain une division fixe et permanente, en rapport avecles conditions topographiques de la forèt. Ces parcelles doivent être régulièrement limitées et d'une bonne assielte; la contenance de chaque parcelle ne doit pas dépasser 20 à 23 hectares; elles sont exactement reportées sur le plan, et sont désignées en allant de l'est à l'ouest et du nord au sud, par la suite non interrompue des nombres.

$2^{\circ}$ Inventaire du matériel. - On procède dans chaque parcelle au dénombrement et au cubage de tous les arbres à partir de 20 centimètres de diamètre.

$3^{\circ}$ Iièglement d'exploitation. - Muni du plan parcellaire et des tableaux de cubage, on dresse un règlement d'exploitation pour un laps de temps assez court, de dix à vingt ans; il comprend les parties suivantes :

a. Dimension d'exploitabilité. - On détermine les dimensions en diamètre que l'on se propose de réaliser dans la forèt (1).

b. Révolution. - On cherche à se rendre compte du nombre d'années nécessaires aux arbres de la forêt pour acquérir cette dimension; cette durée, allongée de quel-

(1) Pour raison économique, le propriélaire particulier coupe, en général, bien avant l'ḋge où les peuplements de la forèt donnent des sigues de dépérissement ou d'un ralentissement de croissance trop accentué. 
ques années pour tenir compte des retards possibles dans la production de la régénération, est ce qu'on appelle la revolution.

c. Détermination de la possibilité. - Le diamètre que l'on se propose de réaliser, et la révolution ainsi entendue fixée, on répartit les arbres dénombrés en trois groupes:

Les vieux bois, formés par tous les arbres ayant dépassé les deux tiers de la dimension d'exploitabilité;

Les bois moyens, formés des arbres dont les dimensions sont comprises entre les deux tiers et le tiers de la dimension d'exploitabilité ;

Enfin les jeunes bois comprenant les arbres dont la dimension est inférieure au tiers de celle d'exploitabilité.

On compare le cube des vieux bois à celui des bois moyens; si le premier est au second dans la proportion de 5 à 3 , on admet que la proportion des deux groupes est normale, el on considère le groupe des vieux bois comme devant être réalisé pendant le premier tiers de la révolution. En ajoutant au cube des vieux bois son accroissement moyen probable jusqu'à l'époque de l'exploitation et en divisant le total obtenu par le tiers de la révolution, on obtient l'expression de la possibilité annuelle (1).

Si le volume des vieux bois, n'est pas à celui des bois moyens dans le rapport considéré comme normal, 5 à 3 , deux cas peuvent se présenter.

$1^{\circ}$ Les vieux bois sont en excès. - $\mathrm{Si}$ on applique la formule précédente $\mathrm{P}=\frac{2,25 \mathrm{~V}}{n}$ qui suppose que le volume

(1) Dans le cas de peuplement normalement constitué, on peut sans grande erreur appliquer la formule suivante :

$$
\mathrm{P}=\frac{2,25 \mathrm{~V}}{n}
$$

dans laquelle $\mathrm{P}$ représente le nombre de mètres cubes à enlever chaque anné', $V$ la somme des volumes des vieux bois et des bois moyens, et $n$ le nombres d'années de la révolution. 
des vieux bois et celui des bois moyens sont entre eux dans le rapport 5 à 3 , on doit s'attendre à une baisse de rendement après l'exploitation des vieux bois.

Pour maintenir le rendement soutenu, il faut s'y prendre de la manière suivante : on diminue le cube des vieux bois d'un volume $a$ que l'on transfère au volume des bois moyens, de telle sorte que l'on ait :

formule qui donne :

$$
\frac{\mathrm{V}_{1}-a}{\mathrm{~V}_{2}+a}=\frac{5}{3}
$$

$$
a=\frac{3 \mathrm{~V}_{1}-5 \mathrm{~V}_{2}}{8}
$$

dans laquelle $V_{1}$ représente le volume inventorié des vieux bois, $V_{2}$ le volume inventorié des bois moyens; le volume (a) déterminé par cette formule est constitué par les tiges classées d'abord dans les vieux bois, et ayant dans cette classe les plus faibles diamètres.

On calcule la possibilité, c'est-à-dire le nombre de mètres cubes à exploiter chaque année pendant le laps de temps considéré, en ajoutant au volume $\left(V_{1}-a\right)$ définitivement classé parmi les vieux bois, l'accroissement probable pris par ce volume jusqu'à l'époque de l'exploitation, et en divisant le total obtenu par le chiffre exprimant le tiers de la révolution.

En agissant ainsi, on enrichit la forèt, mais il faut s'assurer que l'état de végétation des arbres ayant les catégories de diamètre qui dépassent le moins les deux tiers de la dimension d'exploitabilité, leur permet de vivre encore plus du tiers de la révolution.

$2^{\circ}$ Il y a manque de vieux bois. - Si on applique la formule donnée précédemment : $\mathrm{P}=\frac{2,2: \mathrm{V}}{n}$ qui suppose que $\frac{V_{1}}{r_{2}}=\frac{5}{3}$, on doit s'attendre à ce qu'il y ait plus tard une élévation de rendement. 
Pour maintenir le rendement soutenu, on peut agir d'une façon analogue, mais inverse; il faut s'y prendre de la manière suivante : on augmente le cube des vieux bois d'un volume $b$ que l'on distrait des bois moyens, pour le transférer aux vieux bois, de telle sorte que l'on ait :

$$
\frac{\mathrm{V}_{1}+b}{\mathrm{~V}_{2}-b}=\frac{5}{3},
$$

formule qui donre :

$$
b=\frac{5 \mathrm{~V}_{2}-3 \mathrm{~V}_{1}}{8} .
$$

Le volume $b$, déterminé par celte formule, est constitué par les tiges classées d'abord dans les bois moyens, et ayant dans cette classe les dimensions les plus fortes.

On calcule la possibilité en ajoutant au volume $\left(V_{1}+b\right)$ définiti vement classé parmi les vieux bois l'accroissement probable pris par ce volume jusqu'à l'époque de l'exploitation, et en divisantle total obtenu par le chiffre exprimant le tiers de la révolution.

En agissant ainsi, on appauvrit la forèt en gros bois, et il y a lieu d'examiner' si les catégories des bois d'âge moyen, présentant les plus fortes dimensions, peuvent ètre à la rigueur exploitées sans faire de trop grands sacrifices dans le temps affecté à la réalisation des vieux bois.

d. Marche des exploitations. - La possibilité calculée, il faut régler la marche des exploitations. On classe les parcelles en deux groupes:

Premier groupe. - Parcelles dont on entreprend ou poursuit la régénération, c'est-à-dire dans lesquelles on se propose de faire pendant le temps que concerne le plan d'exploitation (dix à vingt ans) des coupes d'ensemencement secondaires ou définitives; ces parcelles ne forment pas nécessairement un seul tenant.

Deuxieme groupe. - Parcelles dans lesquelles on ne 
doit faire, pendant le mème temps, que des coupes d'amélioration (1).

Après aroir effectué la répartition des parcelles en deux groupes, on fixe dans chacun d'eux la succession probable des opérations, d'après une reconnaissance faite sur le terrain et on s'occupe de régler la marche des coupes dans chaque groupe; dans le premier groupe, on doit se borner à indiquer l'ordre probable des coupes de régénération; on ne peut faire plus, puisque la production et le déreloppement plus ou moins rapide des semis sur tel ou tel point, ne peuvent ètre déterminés àl'avance.

Dans ces parcelles du premier groupe, il est généralement bon de prévoir des coupes d'extraction de vieux arbres ou de bois dépérissants, surtout dans les forêts résineuses. On dresse alors, à cet effet, un tableau indiquant année par année les parcelles de ce premier groupe devant être parcourues par ces coupes d'extraction.

Dans les parcelles du second groupe soumises à des coupes d'amélioration, la marche des coupes est réglée par contenance; on indique donc, année par année, les parcelles à parcourir.

e. Applicution de la possibilité (2). - Chaque année, on commence par reconnaître les chablis qui ont pu se produire dans toute la forèt; on déduit leur volume de la possibilité; ce qui reste indique le nombre de mètres cubes à enlever pendant l'année en coupes d'amélioration ou de régénération.

On se rend alors dans les parcelles désignées par le tableau de la marche des coupes d'amélioration, et on fait dans ces parcelles les éclaircies voulues par les rìgles de culture, el les extractions des vieux bois qui doivent tomber en raison de leur état; on défalque le volume ainsi abaltu de ce qui reste de la possibilité.

(1) Eclaircies et extractions de vieux arbres.

(2) Pour ap a dresser l'inventaire. 
Enfin, le surplus de la possibilité est recruté en coupes de régénération, c'est-à-dire en coupes d'ensemencement, en coupes secondaires ou définitives suivant l'état des parcelles en régénération.

A la fin des dix ou vingt ans pour lesquels le règlement d'exploitation a été dressé, on procède dans les parcelles, et sans les modifier, à de nouveaux comptages ; à l'aide de ces nouveaux comptages, on détermine à noureau la possibilité, et on refait la répartition des parcelles en deux groupes, remettant dans le second groupe les parcelles dont la régénération se trouve terminée et les remplaçant dans le premier groupe par des parcelles dont la régénération doit être entreprise d'après l'état des peuplements.

La comparaison des nouveaux comptages arec les comptages effectués précédemment permet de faire les opérations avec plus de certitude et de précision.

Application de la méthode. - Nous prendrons comme exemple, une belle forêt de sapin et épicéa, située à 800 mètres d'altitude sur les plateaux du Jura.

Cette forêt, d'une étendue de 380 hectares, est divisée en 25 parcelles d'une étendue moyenne de 15 hectares.

Inventaire du matériel. - Les arbres sont dénombrés à partir de $0^{\mathrm{m}}, 80 \mathrm{de}$ tour à $1^{\mathrm{m}}, 30 \mathrm{du}$ sol.

Le nombre des arbres dénombrés est de 95159 ; leur volume est de 200022 mètres cubes.

Pour obtenir ce cube, on a opéré de la façon suivante : après avoir mesuré tous les arbres ayant $0^{\mathrm{m}}, 80$ et plus de circonférence à $1^{\mathrm{m}}, 30 \mathrm{du}$ sol, on a apprécié leur liauteur moyenne pour chaque catégorie de circonférence; puis, leur appliquant le tarif en usage pour la forêt, on a déterminé le volume des tiges qui a été augmenté de $1 / 10$ pour tenir compte du houppier. Les petits bois ayant moins de $0^{\mathrm{m}}, 80^{\circ}$ de tour à $1^{\mathrm{m}}, 30$ du sol n'ont pas été compris dans le dénombrement.

Règlement d'exploitation. - Ce règlement d'exploitation est établi pour une durée de vingt ans. 
Dimension d'exploitabililé. - Dans la forêt considérée, l'arbre ayant atteint $2^{\mathrm{m}}, 40$ de circonférence mesure prise à $1^{\mathrm{m}}, 30 \mathrm{du}$ sol est considéré comme exploitable, et l'expérience prouve que dans cette forêt la moyenne de l'âge des arbres qui ont atteint cette dimension est de cent soixante ans. Ce chiffre 160 est celui qui est adopté pour la durée de la révolution.

Détermination de la possibilité. - D'après les chiffres précédents, sont classés : dans la catégorie des jeunes bois, toutes les tiges ayant moins de $0^{\mathrm{m}}, 80$ de tour à $1^{\mathrm{m}}, 30 \mathrm{du}$ sol; dans la catégorie des bois moyens, les arbres qui ont de $0^{\mathrm{m}}, 80 \mathrm{à}^{\mathrm{m}}, 40$ de tour; dans la catégorie des vieux bois, ceux qui ont plus de $1^{\mathrm{m}}, 40$. Les comptages ont permis de déterminer les chiffres suivants :

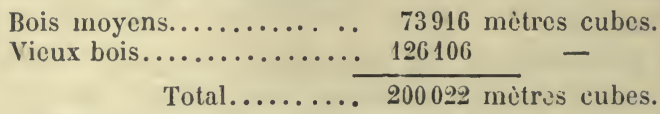

Si on compare le volume des vieux bois au volume des bois moyens, on trouve que le rapport est de $\frac{126106}{73916}=1,70$ soit sensiblement égal à celui de 5 à 3 qui est 1,666 .

Mais dans le cube des vieux bois figure un certain nombre de tiges de 3 mètres et plus de circonférence dont l'exploitation est urgente; le cube de ces bois a été mis à part sous la rubrique: vieux bois surannés à exploiter d'urgence en dehors de la possibilité, et il résulte de cette situation que l'inventaire doit être établi de la façon suivante :

Bois moyens................. 73916 mètres cubes.

Vieux bois proprement dits (ayant plus )

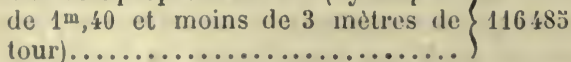

Bois surannés (ayant plus de 3 metres de tour à $1^{\mathrm{m}}, 30$ du sol).............

Total........... 200022 mètres cubes.

La comparaison est dès lors faite seulement entre le volume des vieux bois proprement dits et celui des bois 
moyens : $\frac{116485}{73916}=1,5$, rapport inférieur à $\frac{5}{3}$ (soit 1,6666$)$.

Pour calculer la possibilité, on doit faire passer de la classe des bois moyens dans celle des vieux bois un volume a calculé de la façon suivante :

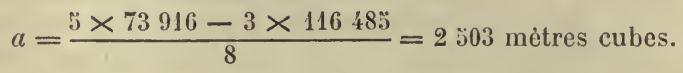

et ce volume a est obtenu en prenant les tiges de plus forte dimension dans la classe des bois moyens.

Le total des arbres de $1^{\mathrm{m}}, 40$ de tour, bois les plus gros de la classe des bois moyens étant de 2015 mètres cubes, on adopte ce chiffre et dès lors le volume des arbres classés définitivement dans les vieux bois, est de :

$11648 \ddot{+}+2315=119000$ mètres cubes.

C'est ce volume qui est à exploiter pendant le premier tiers de la révolution pour assurer un rendement soutenu.

Le calcul de la possibilité s'effectue dès lors de la façon suivante :

10 Calcul de l'accroissement moyen annuel passé : l'accroissement moyen annuel passé est le quotient du volume total des arbres classés définitivement comme vieux bois, par l'âge moyen de ces bois ; or, cet âge moyen ne peut s'établir qu'en se reportant aux calepins de dénombrement et à la constitution de la classe définitive de vieux bois; il est variable suivant les circonstances; pour l'obtenir, il faut, en réalité, prendre la moyenne arithmétique des âges de tous les bois de chaque catégorie de diamètre qui entrent dans le cube total de tous les arbres, définitivement classés dans les vieux bois.

- Dans l'exemple que nous avons choisi, le calcul précédent, fait sur le relevé des opérations, donne pour tous les arbres définitivement classés dans les vieux bois, un âge moyen de cent trente-trois ans.

Dès lors, l'accroissement annuel moyen passé est de 119000 
L'accroissement probable des vieux bois s'en déduit pour un sixième de la révolution, il est de :

$$
894 \times \frac{160}{6}=23840 \text { mètres cubes. }
$$

La somme du volume des arbres classés définitivement dans les vieux bois, 119000 mètres cubes, et de leur accroissement probable jusqu'à leur exploitation 23840 mètres cubes donne 142840 mètres cubes; c'est le volume qui doit tomber pendant le premier tiers de la révolution.

La possibilité annuelle, ou volume à couper chaque année est de $\frac{142840}{1 / 3 \times 160}=2678$ mètres cubes.

Mais en outre de ce volume, on doit réaliser dans les vingt premières années, les 9621 mètres cubes de bois surannés mis à part, soit par an $\frac{9621}{20}=481$ mètres cubes en chiffres ronds.

Pendant les vingt premières années, on a donc à exploiter par exception un total de 3159 mètres cubes. C'est la possibilité annuelle prévue par le règlement d'exploitation pour une durée de vingt ans.

Marche des exploitations. - Le tableau de la marche des exploitations classe les parcelles en deux groupes.

Premier groupe. - Parcelles $n^{0 s} 6$ à 15, qui sont celles dans lesquelles doit être achevée, continuée ou entreprise la régénération.

Deuxième groupe. - Parcelles 1 à ǰ et 16 à 23.

Marche des coupes d'amélioration. - Les coupes d'amélioration sont de deux sortes : ce sont des éclaircies et des enlèvements de vieux bois.

Ces coupes sont effectuées suivant l'ordre indiqué dans un tableau dressé à cet effet.

Observation. - Notons ici, à titre de renseignement approximatif, que les frais d'inventaire d'une forèt bien peuplée alteignent rarement 1 franc par hectare, si l'on ne compte pas les arbres ayant moins de $0^{\text {in }}, 20$ de diamitre. 
L'opération peut ètre effectuée par quatre préposés et un pointeur; dans une futaie renfermant de $350^{\circ}$ à $4: 30$ mètres cubes à l'hectare, on estime que cette équipe peut opérer le dénombrement de 20 hectares par jour.

Septièmecas.-Futaies jardinées. - L Laménagement des forèts jardinées se fait tantôt par volume, tantôt par pied d'arbres.

$1^{\circ}$ Méthode par volume. - a. Division en parcelles. - Pour aménager par volume une futaie jardinée, on commence par la diviser en parcelles comme dans le procédé précédent.

b. Révolution. - Comme pour les forêts précédentes, on détermine la dimension (diamètre ou circonférence) de l'arbre exploitable, et on se rend compte du tempsnécessaire aux arbres de la forèt pour acquérir cette dimension. Ce nombre d'années donne ce qu'on appelle la révolution.

c. Inventaire. - On dresse l'inventaire du matériel sur pied à partir des arbres ayant atteint $0^{\mathrm{m}}, 20$ de diamètre, et on divise les bois inventoriés en catégories comme dans le cas précédent.

d. Pussibilité. - On fixe de même la possibilité comme dans le cas précédent. Les notes de l'administration des eaux et forèts prescrivent cependant, par mesure de prudence, de ne pas tenir compte de l'accroissement des vieux bois pendant la durée de leur exploitation.

e. Marche des coupes. - La possibilité calculée, il convient de fixer la durée de la rotation, c'est-à-dire le temps qu'on mettra à parcourir entièrement la forèt en jardinant. La rotation sera autant que possible un sous-multiple du tiers de la révolution (soit de six à quinze ans).

On règle ensuite la marche des coupes pendant la première rotation, c'est-à-dire que l'on indique l'ordre dans lequel les parcelles seront atteintes par les coupes annuelles pendant cette première rotation.

f. Application de la possibilité. - Chaque année au 
printemps, on commence par dénombrer les chablis sur toute l'étendue de la forèt, ne faisant entrer dans le cubage que les tiges ayant atteint le tiers de la dimension d'exploitabilité. On déduit ce cube de la possibilité et le surplus se recrute dans la coupe de jardinage de l'année.

Observation. - Le rìglement des exploitations ne s'établissant que pour une rotation, à l'expiration de cette première rotation, on refait l'inventaire et on procède à nouveau à la délermination de la possibilité.

Enfin, en appliquant l'aménagement, il faut avoir soin de diriger les opérations de manière à parcourir toute la forêt pendant la durée de la rotation.

2. Méthode par contenance et par pieds d'arbres. - On détermine le nombre d'arbres à enlever en jardinant, par hectare et par an; on fixe la durée du temps nécessaire pour parcourir toute la forèt, c'est-à-dire la durée de la rotation; on délimite ces coupons sur le terrain et on détermine l'ordre dans lequel seront parcourus les coupons à raison de un par an.

Nous renvoyons, en ce qui concerne l'application de cette méthode, au "traitement des bois en France " par M. Broilliard, où cet auteur définit, suivant les cas, le nombre d'arbres à enlever.

3o Méthode par volume et par contenance (procéde Gurnaud)(1). - Le matériel de la forêt est divisé en matériel principul, qui se compose des tiges des arbres de futaie à partir de $0^{\mathrm{m}}, 20$ de diamètre à $1^{\mathrm{m}}, 30 \mathrm{du}$ sol, et audessus, et matériel accessoire, comprenant le sous-bois, et le branchage des futaies.

Les coupes doivent revenir souvent sur le mème point (par périodes de six à dix ans), et le cube total à exploiter dans la forèt pendant la durée de chaque période doit étre égal à l'accroissement du matériel principal pendant toute la période précédente. Cet accroissement est cons- 
taté dans la pratique par des mesurages directs du matériel principal.

Pour faciliter l'application du procédé, on partage la forêt en divisions de contenances sensiblement égales, dont les inventaires sont établisséparément au commencement de chaque période. Chaque année on parcourt un nombre entier de ces divisions (de façon à tenir toute la forèt pendant la période) et on y enlève, en matériel principal, un cube égal à l'accroissement annuel moyen de la forêt pendant la période précédente (quotient de l'accroissement total du matériel principal par le nombre d'années de la période).

Opérations communes à tous les aménagements (1). - A. Plan. - On ne peut entreprendre l'aménagement d'une forêt sans en avoir le plan.

B. Statistique. - ll est indispensable de connaittre d'une façon générale les faits qui intéressent l'exploitation de la forêt; ces renseignements constituent la statistique générale et sont classés sous les rubriques suivantes:

$1^{\circ} \mathrm{Nom}$, situation et origine de la propriété; $2^{\circ}$ contenance générale; contenance du sol boisé ; vides et clairières; $3^{\circ}$ limites; $4^{\circ}$ droits d'usage et servitudes; $5^{\circ}$ configuration du terrain et hydrographie; $6^{\circ}$ sol; $7^{\circ}$ climat; $8^{\circ}$ nature et état du peuplement; $9^{\circ}$ nature $d u$ traitement $; 10^{\circ}$ produits ligneux : volume et valeur pendant les dernières années; $11^{\circ}$ routes, chemins et moyens de vidange; $12^{\circ}$ lieux de consommation.

On complète cette étude par tous les renseignements utiles sur: les impôts; les frais de garde, de gestion; les délits habituels; les prix du bois, des travaux dans la localité, etc.

C. Division en séries. - Une série est un groupe de peuplements d'âges convenablement gradués, pouvant comporter le mème mode de traitement.

(1) Résumé des instructions de ladministration forestière; F. DERox (Agenda du forestier de 1900). 
Il faut donc commencer par faire le choix du régime à adopter, puis le choix du mode de traitement.

Le partage de la forèt en séries permet d'obtenir, dans la mesure du possible, le rapport soutenu, et de rapprocher les produits des divers centres de consommation. Ce partage en séries ne s'effectue que lorsqu'il s'agil de grands massifs boisés.

Les limites des séries doivent être naturelles; sinon elles seront établies de façon à pouvoir servir à la vidange.

D. Dispositions complémentaires. - Tout aménagement doit ètre complété:

$1^{\circ}$ Par l'exposé des améliorations désirables pour son assielte, pour les repeuplements, établissement de fossés, travaux nécessaires pour la vidange, etc.;

$2^{\circ}$ Par l'examen comparé des produits annuels en matière et en argent dans l'état actuel et après l'aménagement.

Comptes d'aménagement et de gestion. - Tout aménagement doit être contrôlé, et les résultats qu'il donne doivent être enregistrés; il est donc indispensable d'ouvrir, dès sa mise en vigueur, des états ou registres, comptes ou carnet d'aménagement et de gestion, sur lesquels sont consignés chaque année ces divers résultats.

Le propriétaire particulier pourra utilement consulter à ce sujet les modèles publiés par la librairie Radenez, à Montdidier (Somme), sous le patronage de la Société forestière de Franche-Comté et de Belfort.

\section{IV. - OPÉRATIONS RELATIVES AUX COUPES.}

Role de l'agent opérateur. - La gestion d'une forèt implique divers acles extérieurs ou operrutions qui ont pour but, les unes de désigner les arbres ì ahandonner à l'exploitation et les arbres à réserver, lesautres d'exercer un controle et une vérification des exploitations.

La direction de ces opérations appartient à l'agent opé- 
rateur, propriétaire ou régisseur qui seul connaît réellement le but qu'il se propose; la manière d'agir varie avec les circonstances et avec les peuplements; nous avons indiqué, au cours de notre ouvrage, les principes qui doivent le guider en toutes circonstances ; pour diriger l'opération, il doit connaître les règles et leséléments de la sylviculture; nous n'avons pas d'autre conseil à lui donner.

Quant à la pratique des opérations, l'agent doit employer des aides, gardes ou auxiliaires dressés au métier (1). Pour éviter des erreurs et des mécomptes, il est nécessaire de procéder avec ordre; c'est à ce point de vue que nous appellerons l'attention sur le rôle et le devoir des gardes pendant les opérations.

Róle des gardes auxiliaires. - D'une façon générale, les gardes qui collaborent aux opérations, doivent se pénétrer des instructions qui leur sont données, les comprendre et s'appliquer à les exécuter ponctuellement. Arant de commencer, ils doivent s'assurer que les marteaux, les griffes, les compas, les chaînes ou roulettes, elc., sont en bon état, car rien n'est mauvais comme de roir un garde faire arrêter une opération pour des détails de ce genre; il est souvent d'usage, pour des martelages un peu longs, d'avoir en poche une pierre à aiguiser ou une lime pour réparer les brèches qui seraient failes au taillant des marteaux. Un bon garde, d'ailleurs, n'est ‘amais pris au dépourvu par des accidents de ce genre.

D’une façon générale aussi, les gardes ne doivent

(1) Un agent qui dirige une opération exécutée par quatre gardes par exemple, ne peut surveiller lui-mème tous les détails de l'opération et diriger quatre hommes inexpérimentés; mieux vaudrait faire le travail tout seul que d'avoir à rectifier sans cosse des fautes conmises. Dans un bon service, les jeunes gardes sont formés au cours de leurs tournées par un vieux garde ou un bon brigadier, et tous doivent connaltre leur métier. L'agent qui dirige peut alors donner tous ses soins à l'opération proprement dite, c'est-d̀-dire au choix des arbres et à l'application des règles culturales. Le reste va tout seul et c'est une condition indispensable, si l'on veut que le personnel ne soit pas énervé par des opérations trop longues et par suite fastidieuses et mauvaises. 
jamais marcher au hasard; si le travail n'est pas bien réparti, on laisse des places entières sans les visiter. A cet effet, la méthode employée consiste à diviser la surface à parcourir en virées, c'est-à-dire en bandes parallèles, limitées sur la droite et sur la gauche par des lignes tracées à l'avance; ces lignes doivent autant que possible être droites et jalonnées (chaque. jalon est rendu visible à l'aide d'un morceau de papier qu'on fixe à son extrémité supérieure). Sous bois, elles sont indiquées par de très légers blanchis faits aux perches du taillis; souvent mème on se contente de couper des tiges de petite dimension, ou de briser ou courberquelques branches secondaires. Ces virées sont, autant que possible, dirigées dans le sens de la longueur, et en montagne, perpendiculairement à la pente; elles présentent une largeur dépendant de la nature de l'opération et du nombre de préposés qu'on y emploie, et on doit s'arranger pour que le travail soit fait en une seule fois dans chaque virée par les préposés espacés et marchant en ligne. Dans les balivages, on peut compter en moyenne une largeur de 8 à 10 mètres par marteau.

Pour que l'opération, faite par plusieur's gardes, s'effectue sans erreur, il est nécessaire que ceux-ci malchent en ordre, non pas de front, mais en écharpe. A cet effet, l'un des gardes (celui de droite ou de gauche suivant les cas' est désigné comme guide; il part le premier au début de l'opération et s'appuie contre une ligne de coupe ou une ligne jalonnée, qu'il est même bon de faire suivre par un jalonneur quand on dispose d'un homme de plus; il tra vaille sur un espace d'en viron 8 à 10 mètres en largeur, et sert de guide à son voisin, en le devançant légèrement; cedernier est ainsi à mème de savoir ce qu'a fait son guide, et ne s'occupera pas des arbres examinés par lui; les gardes suivants opèrent de mème, restant légèrement en arrière de leur voisin du côté du guide. En outre, il est indispensable que les blanchis de réserve ou d'abandon, 
ou toute autre marque, griffages, etc., soient faits sur les arbres du côté opposé au guide, de manière qu'un garde quelconque roie nettement ce qu'a fait son voisin du côté du guide. Quand une virée est acherée, les gardes font une conversion, pivotent de manière que les bords des deux virées consécutives soient parcourus par le mème homme; cet homme se rappelle alors, le cas échéant, ce qu'il a marqué dans la précédente virée, chose utile lor'squ'il y a lieu de choisir entre deux arbres rapprochés, non compris dans la mème virée.

En opérant autrement, on s'expose à oublier des arbres, ou même, chose plus grave dans un balivage par exemple, à appeler deux fois un mème arbre de réserve.

Opérations relatives à la désignation des arbres. Dans une opération, on peut désigner soit les arbres à réserver, soit les arbres à abattre; la marque est dite dans le premier cas en réserve, et dans le second en délirrance. Cette marque s'effectue à l'aide d'un marteau el l'opération consiste à faire un ou plusieurs blanchis sur l'arbre, à des endroits désignés à l'avance, et à apposer sur ces blanchis l'empreinte du marteau (1).

En général, on appelle balivage l'opération qui consiste à frapper du marteau les divers arbres qu'on réserve dans un taillis composé, et martelage l'opération qui consiste à frapper du marteau les arbres des futaies. Le martelage se fait, suivant les cas, en réserve ou en délivrance. Enfin les désignations d'arbres, soit à réserver, soit à abattre, soit à compter, s'effectuent parfois à l'aide d'une simple griffe; l'opération devient alors un griffage.

(1) Les blanchis doivent être fails avec soin; assez profonds pour faire sauter l'écorce, et permettre de mettre l'empreinte du marteau en plein bois, mais pas assez pour détériorer l'arbre. Plusieurs propriétaires préfèrent au marteau un signe apparent fait à la peinture autour de l'arbre, spécialement pour la marque de jeunes réserves. Si la surveillance est suffisante, et si le propriétaire n'a pas ù redouter la fraude, le procédé est bon; il a l'avantage de ns jras détériorer la patte des jeunes arbres.

A. Fron. - Sylvicullure. 
Dans toute opération de ce genre, le garde doit avoir soin de ne jamais appeler un arbre, avant de l'aroir marqué des empreintes convenues; sinon, il s'expose à oublier de le marquer après l'avoir appelé, si son attention est distraite à ce moment; l'inverse, il est vrai, serait possible, mais en opérant toujours de la mème façon, le garde est beaucoup moins exposé à commettre une erreur; d'ailleurs on doit absolument exiger que tuut arbre marqué soit immédiatement appelé (1).

Balivages. - Le garde du triage doit visiter sa coupe en tous sens, de manière à la connaître à fond; il doit pouvoir fournir sur elle tous les renseignements que lui demandera l'agent directeur des opérations (voir Estimations). Le balivage consiste à marquer en réserve les arbres à conserver; en règle générale, on adopte les procédés conventionnels suivants : les brins de l'âge, appelés baliveaux, sont marqués d'une empreinte au pied; pour éviter de détériorer des arbres d'avenir encore très jeunes, il est bon de se contenter de les griffer. Les modernes sont marqués à l'aide de deux blanchis rapprochés, mais distincts et séparés par deux doigts d'écorce; on appose sur chaque blanchis l'empreinte du marteau; les anciens, bisanciens, etc., sont marqués à l'aide d'un long blanchis sur lequel on appose trois empreintes distinctes du marteau. Ces diverses marques, bien que prenant du bois, sont réduites au strict nécessaire afin de ne pas endommager la tige destinée à rester sur pied. Les blanchis doivent être faits aussi bas que possible sur la patte de l'arbre, et de préférence sur l'empâtement des premières racines, lorsque cela est possible.

Les arbres non réservés, c'est-à-dire abandonnés à l'exploitation (ce qu'on appelle abandon), sont désignés par un large blanchis au corps, c'est-à-dire à $1^{\mathrm{m}}, 50$ au-dessus du sol; mieur encore et pour les rendre plus visibles, par

(1) Cette manière de procéder évite aussi les fraudes, ou tout au moins elle permet à un brigadier attentif de les réprimer immédiatement. 
deux miroirs opposés; nous conseillons mème, dans le but d'éviter la fraude, d'apposer sur ces blanchis l'empreinte du marteau, ce qui n'allonge pas l'opération et rend efficace la surveillance au moment des exploitations.

Tenue du calepin. - Chaque pays a ses habitudes pour appeler ou crier les arbres réservés ou abandonnés; il y a lieu toutefois d'exiger que tous les gardes adoptent la même méthode d'appeler. Les dimensions des arbres abandonnés doivent être appelées en mème temps que l'arbre; quant aux réserves, nous ne saurions trop conseiller de profiter des balirages pour noter le diamètre (ou la circonférence) des modernes et des anciens. Les calepins ainsi tenus sont très précieux à consulter plus tard quand on veut se rendre compte du matériel existant en forèt. Certains propriétaires vont mème jusqu'à faire numéroter ces arbres, tout au moins les anciens, ce qui rend les contrôles plus faciles. Enfin, nous répétons qu'un garde ne doit crier qu'après avoir marqué et mesuré l'arbre. Il doit appeler distinctement en se tournant du côté de l'agent pointeur, et en évitant d'appeler en même temps qu'un voisin. Si, pour une cause quelconque, il appelle une seconde fois le mème arbre (par exemple sur la demande du pointeur), il doit toujours ajouter le mot " répété " afin de prévenir le pointeur.

Les calepins destinés à inscrire les résultats de l'opération doirent être préparés à l'avance, afin que l'opérateur n'ait plus qu'à pointer au fur et à mesure des appellations. Des calepins tout préparés pour ce genre d'opérations existent dans le commerce (1). Le pointage s'effectue par dizaines, suivant la méthode courante.

Après l'opération, l'agent directeur doit arrèter ses ca-

(1) L'imprimerie administrative Radenez (Montdidier, Somme), édite des calepins préparés en vue de l'annotation de ces renseignements, ainsi que tous les imprimés qui peuvent être utiles pour les opérations et la gestion d'un domaine boisé. 
lepins, puis procéder à l'estimation comme il sera dit ultérieurement; enfin, il doit noter sur son calepin tous les renseignements relatifs aux limites, aux chemins de vidanges à imposer à l'adjudicataire, aux travaux à imposer sur la coupe, etc. Ces renseignements doivent lui ètre fournis à ce moment par le garde du triage, et il est d'usage que ce garde prépare à cet effet une note qu'il remet à l'agent.

Martelage. - Les règles principales du martelage en réserve sont les mèmes que "pour le balivage, sauf qu'on ne fait pas de marques distinctes suivant la grosseur des arbres: tous sont marqués d'une seule empreinte à la patte.

Quant au martelage en délivrance, certaines précautions sont nécessaires; dans ce cas, la marque doit ètre représentée après l'exploitation; si donc l'arbre à marquer est en versant, la marque à la racine doit ètre en dessous; si cet arbre est près d'une route, d'un glissoir, d'un passage fréquenté par les attelages, il faut éviter que les blanchis soient du côté de cette route, de ces passages, elc. C'est une dérogation à la règle qui consiste à marquer du côté opposé au guide.

Dans les futaies, tout arbre marqué en délivrance doit ètre frappé du marleau sur un large blanchis, à la racine d'abord, puis au corps ensuite; c'est une sage précaution d'opérer toujour's ainsi, car on a vu des gardes marquer au corps, puis s'en aller en oubliant la racine; les blanchis doivent mordre dans l'aubier et les copeaux délachés, mutilés au besoin, sont jetés au loin afin de ne pouvoir servir à dissimuler la marque.

Tout garde, avant de marteler un arbre, doit l'examiner avee soin, pour voir si, d'après les instructions qu'on lui a données, il est bien désigné pour ètre exploité; jamais un garde ne doit se presser pour procéder ì de telles opérations, et les meilleures opérations sont les plus lentes. 
$\mathrm{Si}$ on délivre un arbre double, c'est-à-dire formé de deux tiges confondues en partie par le pied, il faut, s'il y a deux cœurs, double martelage et double criée.

Les arbres que l'on marque comme témoins sont frappés au corps seulement, à $1^{\mathrm{m}}, 50$ ou 2 mètres de hauteur, et sur un léger blanchis fait à l'écorce; il est souvent d'usage de les entourer d'un anneau en paillis ou en mousse, pour les rendre bien visibles.

La manière d'appeler varie suivant les pays. Tantôt on appelle les circonférences, tantôt les diamètres. Parfois le garde apprécie la hauteur de chaque arbre; parfois l'opérateur les estime seul, et par catégories de grosseurs. Dans certains lieux, on évalue le rendement approximatif de la tige; dans d'autres, celui du houppier, etc.; tout dépend du but qu'on se propose et des habitudes locales.

Estimation d'une coupe. - L'estimation d'une coupe est ordinairement faite par les agents ou régisseurs, d'après les données du balivage et du martelage. Nous traitons cette question dans la quatrième partie. Notons toutefois que c'est au moment de l'opération que l'agent estimateur doit réunir les éléments de son estimation et les mentionner sur son calepin.

Pour l'aider dans cette tàche, un garde sérieux doit lui fournir certains éléments d'appréciation ou de contrôle, qu'il recueille pendant l'année en suivant les exploitations des coupes de son triage. A cet effet, le garde peut tenir note exacte et détaillée des produits qu'on tire de ces coupes, par nature, par catégories de marchandises ; il peut prendre note des marchés intervenus, des lieux d'expédition, des frais d'abatage, façonnage, empilage, transport, des prix de vente en forèt et hors forèt, etc.; en un mot, il recueille sur place tousles renseignements qui peuvent permettre d'apprécier la valeur des produits d'une coupe, ainsi que le rendement vrai d'opérations connues.

La comparaison du rendement d'une coupe avec l'esti- 
mation qu'il en a faite préalablement constitue, pour un estimateur, le moyen le plus pratique de se former le coup d'wil et d'arriver dans une région donnée à des eslimations aussi approchées que possible.

En pratique, avant tout balivage, l'agent opérateur doit se laire remettre pour chaque coupe à titre d'indication el sous forme de note (1), réponse à tout ce qui est énuméré ci-après :

Forêt de....; canton de....; lot $n^{0} \ldots .$. ; âge.... ; vides..... ; essences par dixième..... ; limites au nord....; à l'est.....; au sud.....; à l'ouest.....; chemins de vidange à désigner.....; produits présumés du taillis à l'hectare.....; produits présumés pour toute la coupe.....; écorce : rendement.... ; qualité.... ; futaies : qualité....; prix à appliquer aux diverses unités pour les bois façonnés sur le parterre de la coupe.....; prix d'abatage et de façonnage des divers produits de la coupe.....

Comptage d'un matériel sur pied. - Les complages s'effectuent pour apprécier exactement le matériel ligneux qui couvre une étendue déterminée. Ils servent généralement de base à l'aménagement des futaies, à l'estimation de la superficie d'une forêt.

Ces travaux varient suivant les circonstances; tantôt ils portent sur toutes les tiges, tantôt seulement sur une jartie, par exemple sur celles qui constituent le peuplement principal, qu'on désigne à partir d'une certaine grosseur; parfois sans distinction d'essences, parfois (:a les distinguant; tantôt en appréciant les circonlérences, tantôt les diamètres, soit exactement, soit par catégories. (Ies circonférences de $0^{\mathrm{m}}, 20$ en $0^{\mathrm{m}}, 20$ ou de $0^{\mathrm{m}}, 2: 5$ en $0^{\mathrm{m}}, 2: 3$; les diamètres de $0^{\mathrm{m}}, 03$ en $\left.0^{\mathrm{m}}, 0: 3\right)$, elc., elc. Aussi est-il impossible de donner des instructions gúnérales.

(1) I. a librnirie administrative Badenez à Montdidier, a ces imprimés pour ces surtes de notes. 
En ce qui concerne les gardes, ils doivent : $1^{\circ}$ bien se pénétrer des instructions et indications qui leur sont données par l'opérateur; $2^{\circ}$ s'ils sont plusieurs compteurs, marcher non pas de front, mais en écharpe ainsi qu'il a été dit précédemment ; $3^{\circ}$ suivre dans ce travail l'ordre ci-après : mesurer la grosseur, puis griffer ou blanchir du côté opposé au guide, et en dernier lieu appéler l'arbre en question.

Quant aux pointeurs, leurs calepins doirent être préparés à l'avance, en prévision des diverses appellations des gardes. Nous donnons ci-dessous un exemple, en supposant que les gardes appellent les diamètres moyens à $1^{\mathrm{m}}, 50 \mathrm{du}$ sol, de 5 en 5 centimètres, et les hauteurs. Pour tenir le calepin, on prend note des dimensions de chaque arbre par un point sur la ligne et dans la colonne correspondant au diamètre et à la hauteur de l'arbre; afin d'éviter toute confusion, ce qui est très important, attendu que chaque point représente une certaine valeur, on donne toujours aux points la disposition qu'ils ont dans le spécimen du carnet que nous reproduisons cidessous, en sorte que les arbres d'une même catégorie sont groupés par dizaines et se comptent ainsi.

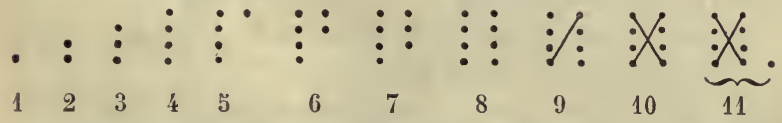

Les huit points et les deux barres forment une dizaine; dès lors il est très facile de compter le nombre d'arbres compris dans chaque colonne sans en omettre, et par suite de relever les résultatś du carnet.

Dénombrements de produits. - Le dénombrement n'est qu'un comptage de produits exploités et façonnés d'après un certain. nombre d'unités déterminées à l'avance. La préparation des calepins varie suivant les classifications et les catégories adoptées.

Nous nous bornerons à signaler que les gardes ne 
doivent appeler les diverses unités qu'après les avoir désignées d'une façon apparente, soit par un numéro, soit par un signe quelconque (par exemple, retourner en travers une des bûches d'un moule de bois qu'on va appeler). Les erreurs et omissions sont dès lors faciles à vérifier sans avoir à recommencer toute l'opération.

Récolements. - Le récolement est une opération qui a pour but dès qu'une coupe vendue est vidée, de s'assurer si l'exploitation est bien faite, s'il n'y a pas eu de délit commis et si l'arjudicataire a rempli toutes les clauses, charges et obligations de son marché. Cette opération change d'aspect suivant que la coupe a été marquée en réserve ou en délivrance. Il existe cependant certaines précautions communes à ces deux sortes d'opérations : le récolement doit ètre fait par le propriétaire ou son régisseur, avec l'assistance des gardes, en présence ou en l'absence de l'adjudicataire quil est utile de convoquer à l'avance.

Pour ces opérations, comme pour les martelages, les gardes doivent marcher avec ordre, par virées successives et légèrement en écharpe; ils doivent suivre, pour les vérilications, appels, etc., les instructions qui leur sont données par l'opérateur.

Pour la préparation et la marche de l'opération nous avons à distinguer deux cas :

a. Coupes en réserve. - Le garde a dû, avant l'opération, dégager le périmètre de la coupe, le jalonner, surtout du côté des jeunes coupes, entourer d'un lien tous les arbres réservés (corniers, parois, témoins, anciens, modernes; on néglige parfois les baliveaux); les modernes ont deux liens.

L'opération consiste à vérifier si chaque tige sur pied est bien marquée, puis à enlever le lien, la griffer et l'appeler, non d'après sa grosseur et son âge, mais conformément à sa marque. Pour faciliter les rérifications, il est d'usage de marguer les baliveaux et anciens d'un 


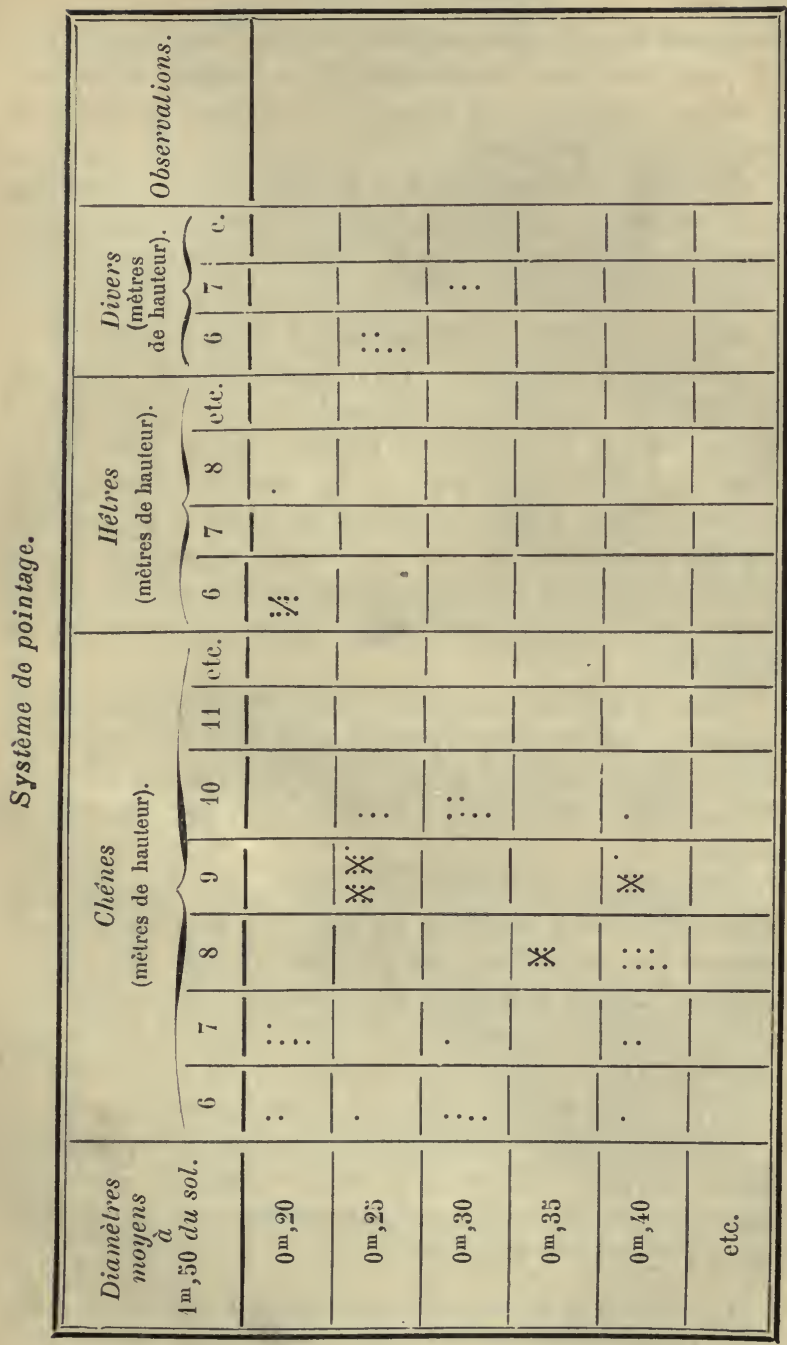


seul coup de griffe; les modernes de deux coups parallèles. $\mathrm{Si}$, pour une cause quelconque, on est obligé de recommencer l'opération, on reproduit les mêmes marques en croix arec les précédentes.

Ce griffage est toujour's fait du côté opposé au guide; il est nécessaire de signaler dans les criées les arbres non marqués de ceux qui le sont, les tiges brisées, renversées ou chablis ; le pointeur réserve sur son calepin des colonnes spéciales à cet effet, correspondant à chaque catégorie.

b. Coupes en délivrance. - Il faut admettre que, pendant le cours de l'exploitation, le garde l'a suivie de près, et qu'au fur et à mesure de l'abatage, il a contrólé les souches, vérifié si l'empreinte du marteau existait réellement. Toute souche reconnue en règle a dû recevoir de lui, au-dessus de l'empreinte du martelage, sur la section même de la souche et non sur un blanchis latéral, un coup de son marteau particulier, noirci au besoin sur un tampon.

Avant le jour du récolement, l'adjudicata ire ou le garde plante à côté de chaque souche et en face de l'empreinte du marteau, un léger jalon, rendu apparent, s’il y a des broussailles, des grandes herbes, etc., par un carré de papier.

Liopération consisle à vérifier de nouveau les empreintes des souches, au fur et à mesure que les gardes les rencontrent, en marchant en ligne, jar virées ; chaque souche reconnue est marquée d'une empreinte du marteau sur la souche ; le garde enlève le jalon, puis appelle; s'il y a doute sur la nature de l'empreinte, il convient d'en référer de suite à l'agent opérateur.

Une partie des renseignements qui précèdent relativement au rôle et devoir des gardes pendant les opérations, sont extraits textuellement des articles faits par M. Pardé dans l'A genda du forestier (1).

(1) Nous profitons de cette occasion pour signaler à l'attention des propriétaires jarticulier's l'Agenda du forestier, publié sous les auspices de la Su- 


\section{V. - NOTIONS GÉNÉRALES SUR LES VENTES DES COUPES.}

M. Broilliard pose, en matière de ventes de coupes de bois, un principe essentiel : le propriétaire doit, avant de discuter un marché, déterminer d'une manière précise les produits à vendre, qu'ils soient sur pied ou abattus, et arrèter d'une manière sûre le chiffre mème de leur estimation en argent.

Cela fait, il peut procéder à la vente en connaissance de cause, quitte à tenir compte, dans la mesure qu'il jugera utile, de l'état du marché au moment de la vente (cours du commerce local, et plus ou moins grande concurrence). Au contraire, si les bois à vendre ne sont pas nettement déterminés, soit par le balivage et par les limites de la coupe, soit par un lotissement très simple du matériel abattu, le propriétaire est, en principe, à la merci de l'acheteur qui connaît en général mieux que lui, en raison même de son métier, les bois, leur valeup et l'état du commerce.

Il y a lieu de distinguer deux cas généraux suivant que le propriétaire exploite lui-mème ou qu'il vend sur pied les coupes à effectuer.

Premier cas. - Exploitation directe. - Le propriétaire exploite lui-mème et vend ses produits soit en grume, soit après le premier façonnage.

II semble a priori, que ce procédé soit avantageux, parce qu'il supprime l'intermédiaire et quelques propriétaires l'essayent. Mais, en pratique, le propriétaire ne gagne pas grand'chose à se subtituer au marchand de bois; il ne possède pas les connaissances spéciales nécessaires pour bénéficier du façonnage, du débit en lui-même

ciété forestière de Franche-Comté et Belfort jar M. P. Jacquin, imprimeuréditeur à Besançon (Doubs). Cet agenda, constitué par une série d'articles faits par les agents forestiers les plus éminents sur toutes les questions intéressant la propriété boisée, est le Vade-Mecum de tout propriétaire de bois. 
et de l'état actuel d'un marché variable; en faisant exploiter avant la vente, il arrive rarement à façonner les bois avec économie et pour le mieux; il s'expose en outre à ètre forcé de vendre après l'exploitation du bois qui une fois abattu, ne peut que se détériorer.

Aussi croyons-nous que c'est avec raison que le propriétaire emploie une autre méthode.

Deuxième cas. - Ventes sur pied. - Le propriétaire n'exploite pas lui-même et vend ses produits sur pied. Il y a une distinction à faire. La vente peut être faite sur pied à l'unité de produits, ou sur pied en bloc:

a. Vente sur pied à l'unité de produits. - Dans la vente sur pied par unité de produits, on débat simplement à l'avance le prix d'un certain nombre d'unités de produits dont les dimensions (en volume ou autrement) sont arrêtées d'avance. La quantité de marchandises qui proviendront de la récolte à faire est inconnue au moment du marché ; le propriétaire marque sa coupe, en principe, au fur et à mesure de l'exploitation; les produits sont façonnés sur le parterre de la coupe par l'adjudicataire dans les conditions prévues au marché et ils ne doivent être enlevés que lorsqu'il en a été fait un dénombrement contradictoire permettant d'établir quel est le nombre d'unités de chaque catégorie de produits qui ont été façonnés.

Pour établir le prix de la vente, il suffit d'appliquer à chacune de ces catégories le prix convenu.

Ce mode de procéder présente des avanlages culturaux, si l'on ne désigne les arbres à abattre qu'au fur et à mesure de l'exploitation; il est encore justifié si l'olojet de la vente n'est pas suffisamment net pour qu'on en fasse d'avance une estimalion précise (bois très disséminés dans une coupe d'éclaircie). Mais en revanche il présente, en pratique, de notables inconvénients : l'adjudicataire n'est pas libre de faire ce qu'il veut 
suivant les circonstances; il ne peut pas consentir à payer cher du bois dont il ne connait bien ni la quantité ni la qualité, et il devient dès lors maître du marché; il y a toujours des lacunes dans le cahier des charges, des dispositions sujettes à interprétation, ce dont un comimerçant cherche à profiter lors de la découpe, du façonnage et aussi du dénombrement qui est nécessaire pour arriver au règlement de la somme due; pour trancher ces difficultés à l'amiable, le vendeur est amené à faire đes econcssions préjudiciables à son marché. Enfin, suivant I expression de M. Broilliard, ce mode prête entre tous à ce que, dans le commerce, on appelle un coulage incessant, sans parler mème des coulages autrement sérieux qui peuvent se produire par fraude ou par erreurs voulues dans l'exploitation de sujets désignés par un simple griffage.

En tout cas, ce mode de procéder exige une surveillance vigilante et fidèle, et de la part du vendeur et de la part d'un adjudicataire consciencieux, car, qui peut affirmer que pendant l'exploitation, des bois ne disparaissent pas, le jour ou la nuit, qui ne seront jamais dénombrés ni payés? Il est préférable, quand on le peut, de ne pas avoir recours à ce procédé.

- Vente sur pied en bloc. - La vente de produits sur pied en bloc est généralement le mode le plus simple et le plus désirable, tant pour le propriétaire qui n'a ni frais ni risques à sa charge, que pour l'adjudicataire qui y trouve toute liberté de façonner les produits et d'en disposer comme il l'entend (Broilliard). Dans ce mode de vente, l'acheteur ou adjudicataire achète le droit d'exploiter et d'enlever tous les bois désignés à l'avance qui sont dans la coupe, sous certaines réserves ou conditions protectrices du sol forestier.

En dehors de ces deux cas généraux, il existe d'autres modes de vente; mais ils sont plus ou moins inférieurs à da vente sur pied en bloc.

A. Fron. - Sylviculture. 
Un système mixte consiste par exemple à vendre à tant l'unité de produits les bois sur pied, à exploiter et à façonner par les soins du propriétaire.

D'après M. Broilliard, ce procédé est usité par un grand propriétaire de l'Est, M. Vieillard-Migeon; les divers produits d'une mème coupe trouvent des acquéreurs différents; ainsi les corps d'arbres forment un lot de chènes, un lot de hètre, etc; le chauffage un lot de bois tendres (blancs), un lot de bois durs, un lot de bois écorcés; le charbon est vendu livrable, les ramiers en bloc: les écorces seules sont à exploiter par l'acquéreur.

Suivant les cas et les usages locaux, un propriétairc peut exploiter et vendre de façon très différentes; tous les modes passés dans les habitudes commerciales sont plus ou moins bons; l'important, d'ailleurs, pour qu'un marché se fasse bien, c'est que la vente provoque une certaine concurrence; à ce point de vue, on ne peut trop conseiller aux propriétaires particuliers de se grouper dans une région pour mettre en vente le même jour, par l'intermédiaire d'une mème personne, un certain nombre de lots; par ce moyen, ils s'assurent presque sans frais une assez grande publicité ; la vente attire un plus grand nombre de marchands de bois, ce qui donnea chaque propriétaire, en ce qui le concerne, la faculté de se mouvoir librement.

Différentes manières de passer le contrat de vente. - Le contrat peut être passé de gré à gré ou par adjudication publique.

a. Ventè de gré à gré. - La vente non publique est urr contrat amiable, pour lequel il est inutile de recourir à l'intervention d'un officier ministériel.

Le systime des ventes par soumissions directes est en faveur, à juste titre, pour des ventes de ce genre. La soumission n'est autre chose qu'une lettre de négociant faisant une offre; si le propriétaire a provoqué la concur- 
rence par une publicité suffisante, il accepte le marché le plus avantageux.

La vente amiable peut être constatée d'une manière quelconque: par témoins, si la valeur est moindre que 150 francs; sinon par acte sous seing privé (1) ou par acte notarié. L'acte notarié a l'avantage d'être exécutoire et de présenter ainsi des facilités spéciales pour le recouvrement du prix. Tout acte de vente, mème sous seing privé doit être enregistré, et les droits d'enregistrement sont de 2 p. 100 du montant de la vente, plus deux décimes et demi (soit un quart en sus); on doit payer en outre le timbre de l'acte et les droits de l'officier ministériel qui y a concouru, quand ce concours a eu lieu, soit, en somme, 3 à 4 p. 100 du prix de vente. Les frais sont à la charge de l'acquéreur sauf clause contraire (Broilliard).

b. Vente par adjudication publique. - En cas de marché assez important, le système d'adjudication publique est préférable, surtout quand plusieurs propriétaires peuvent s'entendre pour vendre leurs divers lots le même jour par l'intermédiaire d'une même personne; la publicité est faite au moyen d'affiches qui sont publiées et placardées à l'avance, et d'un cahier de ventes où les divers lots à mettre en vente sont détaillés; les cahiers sont adressés personnellement aux négociants et' amateurs, qu'ils invitont à venir prendre part à la vente.

Cette vente a lieu dans une salle publique; elle ne peut être faite que par le ministère d'un notaire, sauf pour les coupes de bois taillis seulement et pour les ventes de bois abattus, pour lesquels la loi admet l'intervention des commissaires-priseurs, huissiers et greffiers de justice de paix. On met en adjudication sous diverses formes; encore aujourd'hui, beaucoup de propriétaires

(1) Voir les imprimés de la librairie Radenez (Montdidier, Somme). 
vendent aux enchères, à l'extinction des feux; mais la meilleure forme est la vente au rabais qu'adopte l'administration des Eaux et Forêts; le crieur énonce une valeur bien supéricure à celle qu'a le lot mis en vente suivant l'estimation du propriétaire (estimation inconnue des marchands de bois qui ont fait chacun la leur); il descend progressivement en appelant des chiffres inscrits sur un tarif qui est connu à l'avance et public. Au chiffre qui lui convient, l'acquéreur arrête la vente par un " je prends ". Mais si, avant qu'il y ait preneur, le propriétaire ou son représentant veut arrêter la vente (ce qu'il fait s'il n'y a pas preneur à son estimation, ou même un peu en dessous, suivant les cas), il arrête le crieur; la coupe est retirẹe et renvoyée à l'année suivante.

Ce mode de vente donne une très grande latitude au vendeur qui reste toujours libre de ne pas vendre, si les estimations des acquéreurs présents sont insuffisantes ; il crée la concurrence entre les personnes présentes à la vente, et enfin tout marchand peut y prendre part librement, pourvu qu'il soit solvable, sans crainte de coalition ni d'écrasement. Ainsi le commerce fait les prix d'après l'état des affaires.

Le système de vente aux enchères n'oflire pas cetaranlage; en principe, il crée la concurrence, mais pas plus (qu'un autre mode de vente; par contre, le propriétaire vendeur fixe une mise à prix et dès lors il est obligé d'adjuger, même sur une seule enchère; s'il met à prix au-dessus de son estimation, il risque de ne pas rendre ; s'il met à prix en dessous, il risque, faule de concurrence, d'être obligé d'adjuger sa coupe à une valeur qu'il ne juge pas suffisante; le commerce n'établit plus les prix d'après l'état les affaires. Enfin, dans certains cas exceptionnels, quelques acquéreurs, menacés d'une surenchère et écrasés à l'avance peuvent se laisser écarter de l'adjudication au détriment de la concurrence. 
Conditions de la vente. - Les obligations du vendeur et de l'acheteur sont définies dans un cahier des charges dont les clauses sont acceptées par l'adjudicataire, au moment de la signature du contrat de vente. ll est souvent d'usage de s'en rapporter expressément au cahier des charges de l'administration des Eaux et Forêts, pour toutes les clauses générales relatives aux garanties de contenance et de qualité (art. $1^{\text {er }}$ du cahier des charges générales de l'administration des Eaux et Forèts), aux garanties de paiement (art. ๖े et suivants), au paiement du prix de vente, des frais d'adjudication et des droits d'enregistrement (art. 10 et suivants), et enfin, pour les clauses générales relatives à l'exploitation, à la vidange et au récolement (art. 16 et suivants).

Toutefois, il y a lieu de remarquer que le propriétaire vendeur ne peut pas user contre l'acheteur de tous les moyens de coercition dont l'administration des Eaux et Forèts dispose contre les adjudicataires en vertu des textes spéciaux du Code forestier, et il ne dispose pas d'autres sanctions que celles qui sont édictées par le droit commun.

En ce qui concerne les exploitations, toute la section IV du titre III du Code forestier (art. 29 à 46 du Code forestier) est inapplicable; cette section vise les infractions aux clauses et conditions relatives à la délivrance d'un permis d'exploiter (art. 30 du Code forestier); au travail de nuit (art. 36 Code forestier); au mode d'abatage des arbres et au nettoiement des coupes (art. 37 du Code forestier); à la désignation d'emplacement où l'adjudicataire pourra établir des fosses ou fourneaux pour charbon, des loges ou des ateliers (art. 38, C.f.); à la traite des bois et aux chemins de vidange (art. 39, C.f.); aux prorogations de délai d'exploitation et de vidange (art. 40, C.f.); à l'inexécution des travaux imposés aux adjudicataires (art. 41, C.f.); à l'interdiction d'allumer du feu ailleurs que dans les loges ou ateliers (art.42, C.f.); 
au dépôt par les adjudicataires dans leurs ventes de bois étrangers à la coupe (art. 43, C.f.);

Dans les bois soumis au régime forestier, ces infractions sont érigées à l'état de délits, et justiciables des tribunaux correctionnels; dans les bois non soumis au régime forestier (bois particuliers), les sanctions du droit commun sont seules applicables, et il en résulte que l'inexécution d'une obligation imposée par le cahier des charges ne peut, absolument comme une clause quelconque d'un contrat, que donner lieu à une action en dommagesintérêts devant les tribunaux civils. Pour éviter toute discussion devant le tribunal, et aussi des frais d'expertises souvent délicates, le seul remède est de régler d'avance les indemnités qui seront dues en cas de non exécution des prescriptions fixées par le cahier des charges; de cetle manière, le tribunal saisi est obligé d'allouer la somme convenue, et le cahier des charges enregistré faisant corps avec l'acte de vente permet de sauvegarder ainsi les intérêts du vendeur et la conservation de la forêt.

Pour fixer ces indemnités, on peut se placer à différents points de vue; le principe est de déterminer le dommage effectif causé et de baser l'indemnité sur ce dommage.

En matière de prorogation de délai d'exploitation de vidange par exemple, l'adjudicataire, en restant sur le parterre de la coupe au moment où le jeune recru ou les rejets devraient partir, cause un préjudice sensible qui peut se traduire par la perte totale de production pendant une année; en sylviculture, on appelle feuille cette production. La perte de la première feuille dans un bois normalement exploité à $n$ ans, représente pour le propriétaire qui sera forcé d'exploiter à la coupe suivante un bois âgé de $n-1$ ans, la différence qui existe entre la valeur vénale de la coupe à $n$ ans, et celle du même bois à $n-1$ ans; c'est ce chiffre qui paraîl devoir entrer 
dans le calcul du dommage causé, comme donnant exactement la valeur de la feuille perdue. D'une façon approchée, on peut admettre que la première feuille s'acquière du 15 avril au 15 juillet, soit pendant une durée de trois mois; il appartient dès lors au propriétaire de voir, d'après les conditions de la prorogation de délai qu'il accorde, s'il doit faire son calcul pour toute la surface de la coupe, ou seulement pour une fraction de son étendue, et s'il doit évaluer son dommage proportionnellement au temps, en adoptant comme base que la feuille est perdue avec un délai de trois mois.

Parmi les clauses du cahier des charges, on impose souvent aux adjudicataires divers travaux d'amélioration ou d'entretien; la méthode est bonne, car l'adjudicataire, ayant un fort personnel sous la main, sait les exécuter à bon compte et c'est un moyen pratique pour le propriétaire de faire exécuter divers petits travaux (curage de fossés, mise en état des limites, etc.) au fur et à mesure du passage des coupes, et d'entretenir ainsi sa forêt en bon état. Toutefois, il convient d'être modéré quant au montant de ces mises en charge, et d'éviter de dépasser 3 à 5 p. 100 de la valeur de la coupe.

D'une façon générale, d'ailleurs, on doit se montrer plutôt libéral que très strict au sujet de certaines clauses accessoires relatives à des frais supplémentaires (frais d'enregistrement du contrat ou autres) et aux diverses latitudes dont pourra jouir l'adjudicataire dans son exploitation (latitudes relatives aux places à feu, à la coupe de harts pour lier les écorces, etc.), car les acheteurs ont toujour's tendance à s'exagérer les frais accessoires ainsi que les petits ennuis qui s'ensuivent; le propriétaire a souvent intérêt à en imposer le moins possible aux adjudicataires. 


\section{VI. - TRAVAUX FORESTIERS.}

Nous donnons les chiffres suivants à titre d'indication (1):

A. Travaux à la journée. - Dans une journée moyenne de dix heures, un ouvrier bûcheron peut :

Débroussailler, soit couper un taillis de 8 â 10 ans............................ sur 5 à 10 ares. Abattre un taillis moyen de 15 à 18 ans....... 4 à 8 -

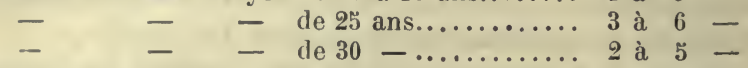

Élaguer feuillus de 1 à 2 mètres de tour. de 10 à 12 arbres.

- résineuxde $1^{\mathrm{m}}, 40$ à $2 \mathrm{~m}$. de tour.

- $\quad$ - de 2 à 3 mètres de tour.

Abattre arbre (petit bois) de $1 \mathrm{~m}$. cube.

(bois moyens) de $2 \mathrm{~m} . \mathrm{c}$.
(à 2 ouvriers)........
(gros bois) de $4 \mathrm{~m} . \mathrm{c}$. (à

- $\quad$ (bois moyens) de $2 \mathrm{~m} . c$.
(à 2 ouvriers).........
$-\quad$ (gros bois) de $4 \mathrm{~m}$. c. (a

- $\quad$ (bois moyens) de $2 \mathrm{~m}$. c.
(à 2 ouvriers).........
$-\quad$ (gros bois) de $4 \mathrm{~m} . \mathrm{c}$. (à 2 ouvriers).

Écorcer sapin moyen de $1 \mathrm{~m}$. de tour.

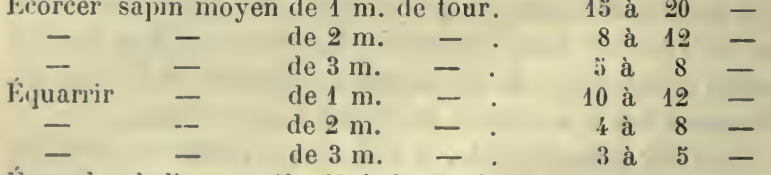

Émonder baliveaux $\left(0^{\mathrm{m}}, 40\right.$ à $0^{\mathrm{m}}, 60$ de tour)...........

- modernes $\left(0^{\mathrm{m}}, 60\right.$ à $1 \mathrm{~m}, 20$ de

8 à $12 \quad-$

6 à $10 \quad-$

8 à $14 \quad-$

8 à $14 \quad-$ tour)..........

100 à 140 tiges.

60 à $80 \quad-$

B. Prix de l'unité.

Façon d'un stère quartier :

francs.

francs.

Abatage, sciage......... de $0,6 i$ à 1 »)

Fendage.............. de 0,20 à 0,300 de 1 » à 1,50

limpilage............. de 0,15 à 0,20 )

Façon d'un stère rondin :

Abatage, sciage........ de 0,65 à 0,80$\}$ de 0,80 à 1 n

Empilage............ de 0,15 à 0,20

(1) Emprunté à l’Agendu du forestier. Besançon, Paul Jncquin. 
Façon de 100 lagots, de $1 \mathrm{~m}, 33$ de haut sur

1 mètre de tour.................. de $\dot{t}$ » à 6 \#

Façon de 100 bourrées, de $1^{\mathrm{m}}, 33$ de haut, sur

1 mètre de tour.................. 2,50 à 3 »

Élagage d'un feuillu de 1 à 2 mètres de tour.. 0,50 à 1 ”

- d'un résineux de $1^{\mathrm{m}}, 60 \mathrm{à} 2^{\mathrm{m}}, 20$ de tour. $\quad 0,80$ à 1,20

- - de 2 w,40 à $3 \mathrm{~m}$. de tour. 1,50 à 2 ”

(Les élagueurs, exerçant un métier pénible et dangereux, demandent à gagner à la journée de 6 à 12 francs).

Abatage d'un taillis de 8 a 10 ans (ou

débroussaillement)............. àl'hectare, de 30 à 60

Abatage d'un taillis moyen de 15 à

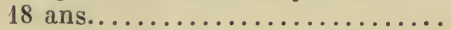

Abatage d'un taillis moyen de 25 ans. -

- $\quad$ - $\quad$ - de 30 ans. - $\quad 60$ à 150

francs.

Abatage d'une perche de $0^{\mathrm{m}}, 40$ à $0^{\mathrm{m}}, 60$ de tour. de 0,05 à 0,15

- d'un arbre (petit bois) de 1 mètre

cube environ............... 0,60 à 1 ”

- d'un arbre (bois moyen) de 2 mètres

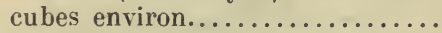

- d un arbre (gros bois) 4 mètres cubes

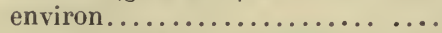

Écorcement, sapin moyen de 1 mètre de tour.

$\begin{array}{llllll}- & - & \text { le } 2 & - & . & 0,60 \text { à } 1,20 \\ - & - & \text { de } 3 & - & . & 1 \text { » à } 1,50\end{array}$

- façon d'une botte d'écorce, de $\mathbf{1 6}$

à 18 kilos.................

façon de 1000 kilos d'écorce......

Équarrissage d'un sapin moyen de $1 \mathrm{~m}$. de tour.

$\begin{array}{lllll}- & - & - & \text { de } 2 & -\end{array}$

Émondage d'un baliveau $\left(0^{\mathrm{m}}, 40\right.$ à $0^{\mathrm{m}}, 60$ de tour $)$.

- moderne $\left(0^{\mathrm{m}}, 60 \dot{a}^{\mathrm{m}}, 20\right.$ de tour $) .$.

- à l'hectare (100 baliveaux, $40 \mathrm{mo-}$

dernes environ)............

1,50 à 2,25

2,วั0 à 3,15

0,25 à 0,40

0,50 à 0,60

30 » $\dot{a} 40$ »

0,40 à 1,20

1,20 à 2,25

2,50 à 3,50

0,02 à 0,04

0,04 à 0,06

3 » 6 »

En ce qui concerne les travaux forestiers (travaux d'amélioration et d'entretien', ils consistent en travaux de bornage (bornes, écussons, fossés, cordons, etc.); en travaux de clôture (murs, fossés, etc.); en travaux d'irrigation ou d'assainissement (fossés, rigoles, etc.); 
en travaux pour l'assielte de l'aménagement (ouverture de lignes, placement de bornes, etc.); enfin en travaux d'ouverture ou d'amélioration des voies de vidange.

Le prix de revient de ces divers travaux varie beaucoup d'une région à une autre, et il convient de les débattre avec les ouvriers du pays; nous renvoyons à leur sujet aux indications générales mentionnées à l'Agenda du forestier. 


\section{TROISIEME PARTIE}

\section{PRINCIPAUX MASSIFS FORESTIERS}

\section{PREMIÉRE SEGTION}

\section{Étude spéciale des taillis simples.}

\section{I. - GÉNÉRALITÉS.}

Le taillis simple est très commun en France où il est appliqué sur une assez grande échelle, spécialement dans la propriété particulière. On trouve des taillis simples dans les régions méridionales comme dans les régions septentrionales, et cette catégorie de peuplements couvre plusieurs millions d'hectares (1). Les communes en possèdent environ 300000 hectares, mais ce n'est qu'exceptionnellement que ce mode de traitement est appliqué par l'État.

Produits et débouchés du commerce. - Le taillis simple n'est susceptible de donner que du bois de chauffage, et exceptionnellement des produits spéciaux; aussi son importance a-t-elle singulièrement diminué pendant la seconde moitié du siècle dernier, principalement à

(1) Il est regrettable que des statistiques sérieuses n'existent pas en ce qui concerne la propriété boisée particulière. 
cause de la diminution progressive de la consommation du bois de feu.

Autrefois, dans notre pays, le combustible presque universellement employé était le bois, qu'on utilisait tel quel, ou sous forme de charbon de bois. Une révolution s'est effectuée depuis lors, et tient aux causes suivantes :

$1^{\circ}$ La consommation du bois de chaufage (1) a diminué de nos jours dans une très forte proportion, car on tend de plus en plus à se servir, même dans les campagnes, d'autres combustibles d'origine minérale, tels que le charbon de terre, le gaz, les huiles et essences de pétrole, l'alcool, etc. Le bois brûlant à l'air libre, donne en effet, à poids égal, beaucoup moins de chaleur; son feu demande plus de soins d'entretien; c'est une marchandise encombrante, payant pour la même valeur beaucoup plus de frais de transport et de manipulation, et par suite, affectée davantage par la hausse générale des salaires des ouvriers. Depuis ringt ans par exemple, la consommation à ce point de vue a diminué d'un cinquième à Paris alors que celle des autres combustibles a augmenté dans une très forte proportion.

$2 \circ$ L'industrie actuelle, qui utilise des méthodes de chauffage perfectionnées et a besoin de températures élevées, n'emploie plus le bois comme autrefois; anciennement en effet, les forges, poteries, verreries, etc., ne s'alimentaient qu'au bois et la répartition de ces établissements industriels était intimement liée à celle des forèts dont elles assuraient la consommation des produits; il n'en est plus ainsi aujourd'hui.

$3^{\circ} \mathrm{La}$ fabrication du charbon de bois en forèt a diminué progressivement; toutefois la distillation du bois en vase clos, dans le but d'obtenir des produits industriels tels que des acides pyroligneux, acétales ou acide acétique,

1) D'une façon générale, on distingue comme donnant un bon chauffage. les hois suivants : alisier, charme, chêne, érable, frène, hêtre, noyer, orme, platane. Et comme donnant un médiocre chauffage, le merisier, le tilleul, etc. 
goudrons, etc., et accessoirement des charbons de bois, a pris une certaine extension dans diverses régions boisées, dans la Nièvre par exemple (usine de Prémery, etc.), et a créé pour les produits des taillis des débouchés locaux importants.

$4^{\circ}$ D'autres débouchés spéciaux ont eux-mèmes disparu; c'est ainsi que l'extraction de la potasse des cendres de bois n'est plus un procédé industriel, depuis qu'on a découvert l'utilisation des sels de Strassfurth.

эo Enfin, un débouché nouveau, très important s'est créé pour l'utilisation du bois, mais il ne s'adresse pas spécialement aux produits donnés par le taillis; c'est la fabrication de la pâte cellulosique (1) dont on fait aujourd'hui surtout du papier ordinaire et des cartons; on a estimé qu'en 1895, sur 2300000 tonnes de papier fabriqué, 1500000 l'avaient été avec cette pâte, ce qui représente une consommation d'environ dix millions de mètres cubes de bois; on tend à faire un peu de tout avec ce produit qui semble extrèmement plastique; on le malaxe à volonté; on le polit comme l'ivoire, on lui fait acquérir par la compression et les procédés chimiques toutes sortes de propriétés mème l'incombustibilité.

Mais cette nouvelle industrie ne consomme pas en France d'énormes quantités de produits forestiers, en raison du faible prix de revient que coûte la pâte cellulosique, fabriquée dans les pays septentrionaux où les forèts ont peu de valeur, et surtout en raison du prix peu élevé des transports; en 1897, il a été importé chez nous 116 millions de kilogrammes de pâte à papier, et 130000 tonnes de pâte brute valant 28 millions.

Une foule d'autres utilisations chimiques et mécaniques du bois est annoncée, mais souvent il ne s'agit que d'essais sans lendemain (soie de bois); quelques-unes toutefois semblent déjà entrées dans la pratique (bois

(1) Pour la pâte à papier, les bois les plus usités aujourd'hui sont l'épicéa, le bouleau, le peuplier, le pin sylvestre, le sapin, le tilleul, le tremble. 
tranché, bois fondu et durci, etc.). Bref, nombre de débouchés d'avenir de la matière ligneuse semblent ètre dans ces multiples transformations. (1).

Quoi qu'il en soit, le bois de chauffage, produit principal du taillis, est dès maintenant, pour la propriété boisée, un débouché important en voie de disparition, et malgré toutes les utilisations possibles de l'avenir, le propriétaire particulier doit tenir compte qu'aujourd'hui les menus bois et la charbonnette ont baissé de valeur d'une façon très considérable dans toute la France; le prix des fagots est presque nul, et les bourrées paraissent inutiles sur le parterre des coupes. Du reste, là où cette haisse ne s'est pas encore accusée très nettement, ce ne paraît être qu'une question de temps, souvent une simple question de voies et moyens de pénétration; de tous côtés, on signale l'envahissement des combustibles minéraux, et avec leur pénétration, l'avilissement de la marchandise fournie par le taillis.

Les écorces à tan, qui étaient autrefois un produit très rémunérateur, sont aujourd'hui atteintes de la même manière.

11 résulte de cet aperçu très général que l'intérêt hien entendu des propriétaires particuliers, les conduit à installer le taillis composé pour obtenir des produits plus demandés, et cela partout où existent les éléments d'un balivage en bonnes essences. Ce n'est plus, dit M. Bagnéris, que par des raisons d'essences, de sols ou de produits spéciaux, que le taillis simple peut se justifier.

Par exemple, ajoute le même auteur, le chêne yeuse n'arrive pas en France à des dimensions utiles comme bois d'œuvre; au contraire son écorce jeune est très riche en tannin et de toute première qualité pour la préparation des cuirs; le taillis s'impose. De même le châtaignier possède un aubier peu abondant qui acquiert, heaucoup

(1) I'aprés Vaxutbengue, Exploitation commerciale des foréts. 
plus vite que le chène, les qualités de bois parfait ; mais en revanche, conservé comme bois d'œurre il est sourent altéré au cœur; aussi l'exploite-t-on en taillis simple à courtes révolutions pour en faire des cercles de tonneaux et des échalas dans les pays vignobles. Dans les mèmes conditions de culture agricole, ou bien aux environs des minières, il est encore possible qu'on ait arantage à traiter des forêts en taillis simple, soit pour produire des échalas (chêne, châtaignier) ou des piquets et perches de mine (toutes essences), soit pour tirer parti de l'écorce (chène); mais il est en général bien rare que les bois d'œurre ne soient pas assez recherchés pour qu'on n'ait pas intérêt à faire plutôt encore du taillis composé; il faudrait pour cela que le sol très peu profond, ne permît pas d'obtenir une hauteur de fût suffisante, au moins six mètres. Sans doute alors, dans l'intérèt général, il vaudrait mieux, comme aux expositions très chaudes, faire de la futaie arec des essences appropriées au sol et au climat. Mais il y a là, une considération économique de capital engagé très importante pour le propriétaire particulier (voir $\mathbf{I V}^{\mathrm{e}} \mathrm{par}$ tie), considération qui peut obliger ce dernier à préférer le taillis simple.

Traitement en taillis simple. - Les points essentiels à étudier pour le traitement en taillis simple, concernent tous le repeuplement naturel par rejets de souche et drageons, et par suite la perpétuité de l'essence. Nous arons vu en traitant du repeuplement (page 237) qu'ils se rapportent essentiellement au mode d'exploitation. Nous avons à compléter ici cette étude.

Rejets et drageons. - La base du traitement en taillis est la formation de rejets de souche et de drageons, dont un grand nombre sont susceptibles de s'affranchir du pied; les rejets sont en général de beaucoup les plus fréquents, et leur nombre ainsi que leur vitalité dépend surtout de l'aptitude de l'essence à rejeter; quant aux drageons ou rejets de racines, il est à remarquer que certaines essen- 
ces, peu aptes à donner des rejets de souche, produisent de nombreux drageons, et sont ainsi précieuses pour assurer le maintien du taillis.

Les essences qui rejettent le plus abondamment de souche sont les chènes (moins le chène yeuse), le charme, les ormes, les érables, le châtaignier, l'aune glutineux, le frène, les saules, etc. Les essences les plus aptes à drageonner sont : le chène yeuse, tauzin et liège; le tremble, l'aune blanc, le robinier, etc.

Un certain nombre rejettent et drageonnent à la fois, tels que les ormes, la plupart des fruitiers, le frène, les chènes à feuilles persistantes, le chène tauzin; le hètre drageonne très rarement, et la plupart de ses rejets proviennent de bourgeons adventifs; encore ne se produisent-ils que jusqu'à un âge peu avancé.

Les résineux ne rejettent pas de souche, et par suite ne sont pas aptes à être traités en taillis.

Révolution. - Au point de vue du repeuplement, nous avons fixé une limite supérieure qu'il ne paraît pas possible de dépasser en général pour aucune essence (quarante ans au maximum) et nous avons vu qu'il n'y a pas de limite inférieure; néanmoins, il y a dans la pratique un âge d'exploitation au-dessous duquel on ne saurait descendre; c'est, dit M. Bagnéris, le moment où les bois ont une valeur commerçable et représentent un placement avantageux. On conçoit que cette limite varie arec les essences, et que si une oseraie peut êfre coupée tous les ans ou tous les deux ans, il n'en est déjà plus de mème d'une châtaigneraie qu'on ne peut exploiter utilement avant douze ou quinze ans; un taillis simple d'aune ou de chène rouvre et pédonculé ne doit pas se couper avant vingt ou vingt-cing ans. L'article 69 de l'ordonnance réglementaire de 1827 (1) fixe pour les forêts de l'Élat la durée minima de la révolution à vingt-cinq ans, à l'excep-

(1) Ordonnance réglementaire du ler aoui 1827, complétaut les dispositions du Code forestier. 
lion des forèts dont les essences dominantes sont le châtaignier et les bois tendres (blancs). L'article 134 de la même ordonnance rend ces dispositions applicables aux forêts des communes et établissements publics (bois soumis au régime forestier). C'est une excellente mesure, que les particulier's eux-mémes auraient le plus souvent intérét à adopter; ces derniers devront se rappeler toutefois que si dans les sols excellents on peut à la rigueur adopter une courte révolution, il est au contraire nécessaire dans les mauvais sols de la choisir longue.

Saison d'abatage. - En vue du repeuplement, l'abatage ne devrait être fait, ni avant les grands froids, ni pendant les grands froids, ni en temps de sève.

Il ne resterait ainsi pour l'exploitation qu'un temps assez restreint compris, en moyenne, entre le commencement de février et la fin de mars; un délai aussi court, aurait le double inconvénient d'exiger, à un moment donné, un nombre de bras impossible à trouver, et en outre de ne pas permettre d'utiliser pendant l'hiver tous les ouvriers de métier qui se font bûcherons pendant le chômage de leurs travaux. Aussi, en fait, admet-on en France, où le climat général est un climat tempére, qu'on peut sans danger sérieux commencer les exploitations en automne, aussitot l'arrêt de la sève, et les continuer pendant tout l'hiver, sauf au moment des fortes gelées, et ce n'est seulement que dans les situations exceptionnelles qu'il faut agir arec plus de prudence.

Quant à la coupe en temps de sève, elle présente desinconvénients, spécialement quand elle est suivie d'étés exceptionnellement chauds, et de sécheresses prolongées; mais si la révolution du taillis est assez longue, le retard occasionné est insensible; son grand inconrénient parait consister dans la moindre qualité des produits récoltés.

Mode d'abatage. - Les précautions à prendre en ce qui concerne l'abatage sont autrement essentielles au point de vue du repeuplement, et nous avons indiqué 
que la section devait être faite rez terre, à la serpe pour les tiges de moins de 5 centimètres de diamètre, arec un instrument tranchant plus lourd (hache ou cognée) pourles tiges d'une dimension supérieure; que la surface de section devait être ravalée, pour empêcher le séjour de l'eau sur la souche.

La coupe rez terre doit être prescrite et observée rigoureusement; il n'y a d'exception que pour les endroits plats et bas, exposés à de fréquentes inondations; alors il faut exploiter un peu au-dessus de la hauteur qu'atteigent habituellement les eaux pour empêcher leur séjour sur les souches, ou tout au moins il convient d'exploiter tard, après l'hiver, quand on n'a plus guère à craindre l'eau.

Enfin, dans certain cas cas spéciaux (furetage du hêtre ; exploitation en têtards), on peut être conduit à faire la section dans le jeune bois, laissant ainsi des tronçons de rejets sur lesquels vont s'en former de nouveaux.

Façonnage. - Le façonnage doit être fait rapidement pour ne pas entraver le développement des rejets, et surtout pour éviter de les endommager, alors qu'ils se détachent encore facilement de la souche. Nous avons signalé cette opération comme condition essentielle d'un bon et rapide repeuplement; pour la même raison, les ramiers ne doivent pas rester longtemps épars sur le parterre de la coupe. Si la main d'œurre manque, on peut tout au moins exiger qne tous les produits soient façonnés arant l'apparition des rejets, et que les ramiers soient toujours rassemblés sur les endroits vides et mal garnis; on en sera quitte pour effectuer sur ces places, après la vidange, quelques plantations.

Vidange. - Lne bonne pratique consiste à faire transporter les produits aussitot qüils sont façonnés le long de chemins désignés à l'avance, soil sur le parterre de la coupe, soit plutot en deliors de la coupe sur une place de dépot. On évite ainsi des dégâts souvent importants, 
occasionnés par le passage des voitures dans l'intérieur de la coupe, et par l'abroutissement des bêtes de somme qu'il est nécessaire d'y introduire ; si la surveillance n'est pas constante et très sévère, ces dégâts sont souvent très importants.

Le cahier des charges de l'administration des Eaux et Forêts (1) trace à cet égard un certain nombre de règles adoptées, tant pour les délais d'exploitation et de vidange, que pour les prescriptions à imposer aux adjudicataires ou entrepreneurs; il prévoit le cas où on pourra y déroger, à l'aide de clauses spéciales, lorsque des circonstances particulières le demanderont.

Il est bon toutefois de remarquer que le propriétaire particulier a intérêt à ne pas exagérer les obligations qu'il impose aux adjudicataires, afin de ne pas les écarter de sa forèt; il ne devra exiger que les précautions indispensables pour assurer l'avenir du taillis.

Balivage. - Le traitement en taillis simple n'exclue pas forcément la réserve de baliveaux qui seraient destinés à parcourir seulement deux révolutions du sousbois; spécialement si les révolutions sont courtes, les souches de ces baliveaux ne perdent pas leur faculté de rejeter. Nous avons dit en parlant du repeuplement que ces réserves peuvent être très utiles, sinon indispensables, pour assurer la perpétuité du taillis; leur rôle principal est de disséminer quelques semences, pour maintenir le taillis complet; toutefois leur nombre doit être assez restreint si l'on ne veut pas que leur couvert vienne nuire au développement du taillis simple; le meilleur moyen de les distribuer est de les disposer pour la plupart en cordons le long des lignes et chemins tout autour de la coupe. Abuser de ces réserves, dans un taillis simple, et leur demander la production de bois de

(1) Tout propriétaire particulier peut se procurer un exemplaire du cahier des charges générales de l'administration des Eaux et Forêts. Il lui suffit de le demander à l'agent forestier de la circonscription. 
charpente, serait changer la nature de l'exploitation, et dans ce cas le propriétaire a tout intérèt à adopter franchement le taillis composé.

Développement du taillis. - Opérations culturales. Iès l'année qui suit l'exploitation, le peuplement se trouve formé de jeunes cépées ne couvrant que très imparfaitement le sol, et entre ces cépées, les herbes se développent en grande abondance. La production ligneuse est faible malgré la vigueur de végétation des jeunes pousses; elle augmente d'année en année, au fur et à mesure que l'espace se restreint entre les cépées, que les feuillages se développent pour arriver à se toucher et à former massif plein.

C'est dans le début de cette période qu'on peut avoir intérèt : $1^{\circ}$ à effecter des repeuplements artificiels sur les vides qui se produisent toujours par places, spécialement lans les taillis où il ny a pas de porte-graines; $2^{\circ}$ à effectuer des dégagements, c'est-à-dire des opérations culturales ayant pour but spécial de dégager d'abord les brins de semence qui pourraient exister, et les plantations dont la végétation, lente au début, ne peut lutter avec la végétation rapide des rejets de souche de toute nature; ces dégagements peuvent porter ensuite, s'il y a lieu, sur les rejets de souche, les morts hois et mauvaises essences (saule marceau, tremble, etc.) et aussi, mais exceptionnellement, sur les ronces, les épines et autres morts bois, toujours envahissants, qui tendent à étouffer les rejets des bonnes essences, et qui ne tardent pas, dans bien des cas, à les déposséter du terrain; ils consistent généralement en étètements; leur but est lonc de favoriser la végétation des chènes, hètres, charmes, frènes, érables et autres arbres à bois dur, dont la culture est généralement plus avantageuse. Celte opération doit ètre faite dis que l'on s'aperçoit que les essences secondaires gènent la bonne croissance de celles que l'on a intérèt à conserver et à propager; elle doit, quand cela 
est possible, ètre répétée à plusieurs années d'intervalle Il est essentiel dans ce cas de ne pas aller au hasard dans chaque opération, afin de continuer à protéger les mèmes sujets qu'on a précédemment dégagés ; il est difficile d'indiquer l'âge auquel le dégagement doit être entrepris, puisque c'est l'inspection des lieux qui doit en décider; disons cependant que très souvent cette opération est utile dès l'âge de six à dix ans.

Cette opération essentiellement culturale doit être effectuée avec une très grande prudence; elle ne doit jamais avoir pour effet d'interrompre le massif; il vaut mieux conserver des trembles, des saules, tilleuls et mort-bois şà et là, que de provoquer des vides dans le taillis par leur extraction complète. Elle consiste donc le plus généralement à étêter quelques bois tendres (blancs) afin de dégager les semis ou cépées du voisinage, sans les priver entièrement de l'appui que leur prètent les tiges ainsi conservées dans leurs parties inférieures.

Dès l'âge de dix, quinze ou vingt ans, la production annuelle peut s'élever à 4 ou 5 mètres cubes par hectare et par an par exemple, plus ou moins suivant les forèts. Le peuplement passe alors de l'état de fourré à l'état de gaulis ou plutôt de bas perchis (1); parmi le très grand nombre de tiges qui formaient le massif, un certain nombre seulementse sont développées, et ont pris l'avance pour constituer le peuplement principal ; les autres, a près avoir végété quelque temps à l'état de tiges minces, ont été éliminées progressivement. Plus la station est fertile, plus le peuplement principal se sépare rapidement du peuplement accessoire et prend un accroissement vigoureux.

Eclaircies dans les taillis. - A l'état de bas perchis, le massif reste constitué et le volume ligneux augmente

(1) Le peuplement de taillis, constitué par un ensemble de cépées, formant tout d'abord des ilots isolés qui s'élargissent peu à peu jusqu'à la constitution du fourré, ne présente pas le facies du gaulis; il passe plutôt insensiblement du facies fourré à celui de bas perchis. 
rapidement; si on laisse vieillir un peuplement ainsi constitué, il peut devenir utile de l'éclaircir, et l'éclaircie a pour but de venir en aide à la nature en desserrant les cimes du peuplement principal, afin de leur permettre de prendre plus rapidement un développement favorable; ce que les dégagements ont commencé pour les brins de semence, les éclaircies doivent le continuer, mais d'une façon différente. Cette opération culturale, d'après II. Broilliard, n'est opportune que quand le massif est principalement composé, non plus de gaules, mais de petites perches ayant au moins 10 centimètres de diamètre à hauteur d'homme (inutile de dire qu'elle ne doit pas porter sur l'étage dominé, ni sur le sous-étage, mais bien sur certaines tiges dont la cime pénètre dans l'étage (lominant); on passe alors assez facilement sous bois; alors aussi l'éclaircie est rémunératrice; mais pour qu'elle soit utile, il faut que le taillis ait encore quelques années pour se développer, huit ou dix ans par exemple, ce qui suppose une révolution d'une trentaine d'années au moins.

Cette opération délicate serait absolument mauvaise si le propriétaire en abusait pour faire une récolte prématurée de produits, et dans ce but la dirigeait au hasard, ne craignant pas de desserrer ou d'interrompre le massif, ou bien de faire disparaître, sous prétexte d'éclaircie, des tiges franchement dominées dont le maintien est indispensable pour le bon état du sol et l'entretien de la fertilité de la station.

Dans les opérations de ce genre, on se rappellera qu'il y a toujours avantage à ce que le sol reste le plus garni el le mieux abrité possible.

Toujours exploité à un âge relativement jeune, en raison de la nécessité qu'il y a d'obtenir des rejets de souche, le peuplement ainsi conduit n'atteint pas la phase que nous avons signalée dans la futaie régulière, où il commence à s'éclaircir fortement et où par suite son 
état, de moins en moins'serré, exerce une influence fàcheuse sur la fertilité de la station.

Mais par contre le sol est dénudé régulièrement à chaque exploitation, et en outre la récolte présente un caractère spécialement épuisant.

Il en résulte que, pour que les exploitations successives puissent se perpétuer indéfiniment sur le mème sol, sans exercer une influence néfaste sur sa fertilité, ce mode de traitement exige qu'on apporte un soin permanent à entretenir le bon état de massif. Plus que partout ailleurs, la moindre faute entraine des conséquences fàcheuses; plus l'état d'un taillis est mauvais, plus il tend à accentuer ce mauvais état, et à ruiner complètement la fertilité des stations. L'augmentation de la révolution est alors, bien souvent, un des meilleurs remèdes à conseiller.

Le repeuplement artificiel peut intervenir, et est appelé à rendre de grands services dans le taillis simple; il consiste à venir reboiser les vides qui se produisent souvent dans un taillis où il n'y a pas de porte-graines. Cette introduction de sujets qui, après recépage, sont destinés à fournir de jeunes cépées, permet de maintenir l'état de massif complet. Partout où il est possible, le propriétaire a intérèt à l'effectuer, fût-ce lentement et progressivement, au passage des coupes. Ajoutons que de tels travaux sont parfois onéreux; ils exigent en outre des opérations de dégagements faites avec soin. M. Broilliard conseille, si les vides sont nombreux mais petits, d'en attendre le repeuplement naturel en laissant vieillir le taillis en ceinture autour d'eux, sur une faible largeur, pendant toute une révolution; il peut suffire de garder une ceinture formée de l'épaisseur d'une large cépée, et il est facile d'en griffer les perches à conserver; au retour de la coupe, ces cordons sont beaux et riches, et le vide qu'ils enserrent change d'état en s'améliorant.

S'il s'agit au contraire de grands vides, tout en les ceìn- 
turant, M. Broilliard conseille de les repleupler en essences à végétation rapide, pins, bouleaux ou aunes suivant les sols; sous ces essences, à courert léger, le chêne rient s'établir naturellement.

\section{II. - APPLICATIONS DU TAILLIS SIMPLE.}

Le rôle et la composition des taillis diffèrent sensiblement d'une région à l'autre. Le chêne yeuse en Provence, le chêne tauzin dans l'ouest, spécialement dans le pays de Bayonne, les chènes rouvre et pédonculé dans le centre de la France et en Sologne, le chêne rourre dans les Ardennes et les Vosges méridionales, sont fréquemment les essences presque uniques, ou tout au moins prédominantes, de certains taillis simples. Un peu partout aux chênes se mélangent.le bouleau sur le: sables pauvres, les bois blancs dans les stations fraîches, le charme, l'érable champêtre, le coudrier dans les sols secs et moins profonds.

- En un mot, les taillis sont généralement formés d'essences mélangées, mais la très grande prédominance d'une essence leur donne parfois le caractère de peuplements purs ou homogènes. Nous avons donc à examiner plus en détail quelques-uns des types principaux de taillis simple.

Taillis simple d'essences mélangées. - Il existe en France d'immenses surfaces couvertes de taillis simples d'essences mélangées; ce sont par exemple, d'après M. Broilliard: sur les calcaires rocheux, des taillis formés de charme, de coudrier et autres arbrisseaux, d'érables, d'alisiers et de fruitiers diver's, de chêne et de hèlre disséminés, de saule, de tilleul, enfin d'une foule d'essences; dans les sables frais, on trouve des bouleaux, du tremble, du charme, du hêtre avec les chẹenes rouvre et pédonculé; dans les dépressions humides, de l'aune et quelques chènes; dans les parties riches, du tremble, du frène, de 
l'orme et du chène ainsi que d'autres essences diverses suivant la nature et l'humidité du terrain; souvent une ou plusieurs essences sont dominantes suivant le climat, le sol et la manière dont sont dirigées les exploitations ; les autres restent à l'état subordonné.

Quand le mélange est bien proportionné, un tel peuplement se présente dans des conditions arantageuses,

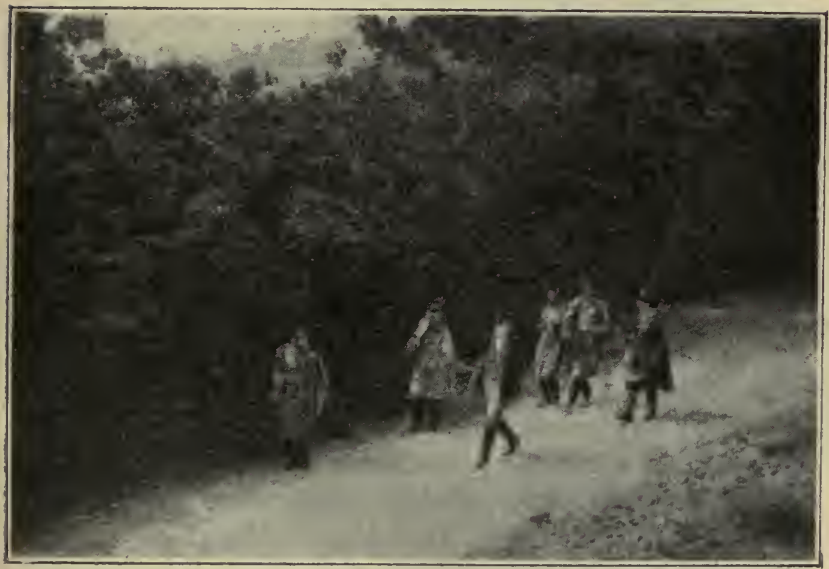

Fig. 34. - Taillis simple (chêne et essences mélangées).

tant pour donner une production variée que pour assurer par un couvert suffisant le bon état du sol; aussi le taillis à essences mélangées permet-il en général d'obtenir les peuplements les plus complets et les plus grands rendements en matière (fig. 34).

Les dégagements, tels que nous les avons définis, deviennent des opérations nécessaires, ayant pour but de maintenir le mélange, de l'améliorer et de diriger la production vers les produits les plus utiles; ces dégagements ou simples étêtements, permettent le plus sou-

A. Fron. - Sylviculture. 
vent d'assurer le maintien du chène tout en y utilisant pour le mieux lesautres essences ; on doit les commencer de bonne heure, et les répéter fréquemment dès que le besoin s'en fait sentir.

Les éclaircies deviennent dans un tel peuplement des opérations utiles, spécialement là où il y a lieu de favoriser le chène; pour tendre à multiplier cette essence, on fait cinq ou six ans avant le terme de la révolution une éclaircie forte, qui en relevant le couvert et en desserrant les perches dechêne, favorise la fructification de ces perches et par suite la production de semis naturels; ces semis, recépés avec soin lors de l'exploitation du taillis, enrichissent alors le taillis en jeunes souches de chène, et plus tard les opérations de dégagement ne doivent pas les oublier.

Pour que ces opérations culturales donnent le résultat (qu'on en attend, il faut opérer sur de longues révolutions.

Dans chaque cas particulier, la longueur de la révolution dépendra de l'essence à multiplier et des produits à obtenir; on n'oubliera pas que plus les révolutions sont. courles, plus elles sont favorables au développement des mauraises essences, notamment des bois tendres.

Bien traiter un taillis d'essences mélangées en vue d'obtenird'une part une production soutenue en matériaux de bonne qualité, et d'assurer d'autre part la conservation de la fertilité de la station, n'est pas chose aussi facile qu'on le pense à première vue. La preuve en est dans ces pauvres taillis qui existent un peu partout 'et qui sont en voie d'appauvrissement très accentué ; rien de triste à voir comme certains terrains dits boisés, où les exploitations sè répètent sur les mêmes surfaces à des intervalles de dix, quinze ou à peine vingt ans, pour donner des produits de peu de valeur, où les bonnes essences ont presque complètement disparu et sont remplacées par des morts bois et des essences de qualité inférieure, où le massif est entrecoupé et interrompu par des vides et des 
friches incultes abandonnées par la culture forestière ou déjà livrés au bétail. Il est nécessaire de remédier à cette situation. Nous empruntons à M. Broilliard les conseils suivants:

En sol frais ou profond, les bois tendres (blancs), dont la végétation est rapide, ou les essences à couvert épais propres à la station, s'emparent souvent du terrain en lieu et place du chêne, plus précieux qu'elles, tant par le bois et l'écorce que par la qualité des produits. Or, seul le chêne, quand il a une belle végétation, réunit tous les avantages; c'est donc lui qu'il importe de maintenir ou de multiplier dans ces terrains. Les moyens dont nous disposons à cet effet se trourent dans la durée de la révolution, dans les éclaircies et dans les repeuplements.

Pour diriger les exploitations, il faut se rendre compte que l'état de la forêt montre toujours les fautes commises et les résultats acquis; les conclusions qui découlent de cet examen sont les suivantes:

a. Si le chêne est bien représenté et bien venant, s'il est en bonne proportion dans le mélange et dominant, il convient le plus souvent de s'en tenir à la révolution en vigueur, à moins que des considérations locales (utilisation de produits spéciaux, etc.) ne portent à la modifier dans un sens favorable.

$b$. Si les arbrisseaux ou les bois blancs abondent, il est utile de reculer de quelques années l'âge d'exploitation du taillis ; une éclaircie des bois blancs faite cinq ou six ans avant le terme de la révolution est toute indiquée pour favoriser la production de semis de chêne en sousétage.

c. Si au contraire ce sont les essences à couvert épais, ormes, charmes, érables ou hêtres qui occupent le terrain, il convient de prolonger notablement une première fois le maintien des taillis sur pied; au lieu de les exploiter à dix-huit ans, on y fait une éclaircie, récoltant la plupart des arbrisseaux, dédoublant le nombre des 
rejets sur chaque souche, desserrant les bois tendres (1), légageant les perches de chène, et enlevant les brins trainants ainsi que les rejets grêles de cépées à couvert épais, de charme notamment. Le chêne se multiplie dès lors par brins de semence sous le couvert de taillis ainsi traités; recépés avec soin au moment de la coupe du taillis, ces brins donneront des rejets qui feront plus tard de nombreuses cépées et d'excellents baliveaux.

Si le chène est devenu trop rare, on peut compléter ces semis par des plantations faites après l'éclaircie, el quelques années seulement avant la coupe du taillis.

Notons enfin que dans de tels sols, la réserve de baliveaux bien choisis, et de bons cordons lelong des lisières, avec une révolution convenable, suffit en général pour ramener l'essence précieuse.

En terrain calcaire, sur les plateaux ou versants rocheux, les taillis d'essences mélangées couvrent de grandes étendues; parmi les essences variées qu’ils présentent, les unes sont peu longévives (arbrisseaux, coudriers, saules); d'autres ont une végétation rapide mais un bois lendre (tilleul); d'autres un bois dur mais une régétation lente (charme, petit érable); le chêne et le hètre en sont les essences précieuses (Broilliard).

Arec des révolutions courtes, la production de ces laillis consiste en fagots ou charbonnelte; si au contraire on les laisse sur pied jusqu'à trente et quarante ans ils continuent à prospérer. d'une manière soutenue; ceux yui fournissent 100 stères à vingt-cing ans en domnent généralement 160 à trente-six ans et alors c'est surtout du rondin ou bois de moule, de sorte que la valeur du

(1) Pour prospérer, chaque essence exige une place plus ou moins grande au soleil. Celles qu'il faut desserrer le plus sont: le tremble, le frêne, les grands érables, les ormes, le merisier, qui ne prospèrent qu'avec la cime "ntièrenent libre; puis le bouleau, l'aune, le chêne, le tilleul, l'érable champêtre, qui se développent mieux à l'état libre qu'en massif; enfin le charme -t le hètre qui prennent en massif de très lelle's dimensions, pourvu que les cimea nient quelque ampleur (Broilliard). 
rendement est double ou triple ; ils passent par exemple, d'après $\mathrm{M}$. Broilliard, de 200 francs à l'hectare à vingtcing ans, à 500 francs vers l'âge de trente-six ans. $A$ vingt-cinq ans, ces taillis entrent à peine en valeur; ils ne sont qu'à la fleur de l'âge. C'est de vingt-cinq à quarante ans qu'ils s'enrichissent, repeuplent leurs clairières, étiolent leurs morts bois, et donnent la prédominance aux bonnes essences.

Dans de tels taillis à longue révolution, le hètre est une essence précieuse, et M. Broilliard conseille sur ces plateaux calcaires de réserver la plupart des hètres, arbres, baliveaux ou faibles brins, au lieu de recéper ces derniers, si grèles qu'ils soient.

Notons enfin, avec le même auteur que, sur les plateaux calcaires, l'éclaircie n'est guère praticable avant l'âge de trente-six à quarante ans ; c'est-à-dire qu'elle y est peu applicable aux taillis; mais en revanche, les cordons continus, pleins, formant des haies d'arbres ou de hauts taillis, fournissent un excellent abri, très favorable à la végétation forestière surles plateaux; on peut leur donner une largeur de 4 à 5 mètres, et les disposer soit au pourtour des coupes, soit mème à l'intérieur de trop grandes coupes, pour partager chacune d'elles en compartiments clos de 4 à 3 hectares au plus.

Quant aux vacants et aux grands vides, ils tiennenc généralement aux abus de pâturage ; souvent aussi sur certains sols sablonneux ou argilo-siliceux, ils sont provoqués par un état fortement acide du sol, état dû à une décomposition incomplète des feuilles de chêne et de bouleau. Si les vides ainsi créés sont de grande étendue, on doit y remédier en reboisant le terrain ainsi découvert, et le concours des essences résineuses, comme essences transitoires de reboisement, est alors généralement indispensable.

Taillis où domine le chêne. - Les écorces à tan, d'un produit autrefois rémunérateur, souffrent aujourd'hui 
d'une baisse considérable, aussi l'existence de taillis où le chène constitue pour ainsi dire l'essence unique du peuplement, taillis généralement exploités à de courtes révolutions (1) en raison de leur production spéciale, ne paraît plus aussi justifiée.

Le chêne yeuse ou chêne vert et le chêne tauzin sont cultivés presque uniquement en vue de la production des écorces et à des révolutions très courtes; souvent très clairiérés en raison d'un pâturage abusif, et formés de tiges grêles ou même debroussailles naines, ils produisent peu par hectare et par an. Il n'est pas douteux qu'il y a beaucoup mieux à faire (2).

Dans la Sologne et le centre de la France, arec le chêne rouvre et le chêne pédonculé on adopte une révolution plus longue; de vingt à vingt-cinq ans. Aussi les taillis y donnent-ils, outre de bonnes écorces, des produits ligneux assez importants, et même des perches utilisées comme menus bois d'œurre. Là où le chêne pédonculé est seul, il couvre très mal le sol, et les taillis sont d'un entretien difficile; il est alors préférable de le voir associé au charme.

Dans les Ardennes, les taillis simples de chêne rouvie et pédonculé, tantôt purs, tantôt mélangés entre eux ou avec des bouleaux, des charmes et des noisetiers, sont soumis au sartage (3); on applique généralement aux taillis sartés une révolution de vingt-quatre ans. Les produits obtenus en dehors de la récolte de céréales, consistent en étançons de mines, en charbonnette et en bois de chauffage et en écorce; tous ces produits réunis donnent un revenu net moven de trente francs par hectare

(1) En laissant de côté le chêne-liège, l'écorce de chéne est d'autant meilleure pour le tannage des cuirs, qu'elle est récoltée sur des arbres plus jeunes et plus vigoureux,

(2) Voir Traitement des taillis de chene vert: Brollunnd, Traitement des bots en France.

(3) Après la coupe, on lirùle les ranilles et tous les rémanents de l'exploitation, et on cultive des céréales pendant un an. 
et par an environ; la pratique du sartage n'est plus justifiée aujourd'hui avec la facilité actuelle des communications, et tend à disparaître.

Les taillis de chêne se rencontrent souvent sur les terrains siliceux (sables, schistes, granites, grès), sols sur lesquels paraissent la bruyère, le genèt à balais; le bouleau se présente alors habituellement a rec le chêne. Ils se rencontrent aussi sur les terrains calcaires, et ce sont alors des épines noires, des églantiers, des cornouilliers et autres mort-bois qui se trouvent répandus dans la forêt.Généralement, aussi, le charme et les fruitiers s'y montrent en mélange.

Dans de tels taillis, si les massifs sont complets, il n'est pas indispensable de modifier la révolution; mais si les vides se montrent nombreux, la première chose à faire est d'allonger la révolution en la portant au moins de vingt à trente ans par exemple.

Les semis de chêne se multiplieront ensuite, les autres essences de la région viendront s'y adjoindre naturellement, et la forêt ira s'améliorant d'elle-même.

On favorisera cet enrichissement du taillis, en même temps que la création d'un mélange toujours avantageux dans ces conditions, en réservant le long des laies qui séparent les coupes des cordons de brins ou rejets de toutes essences, en gardant par pieds isolés les beaux sujets qui se présenteront sur le parterre de la coupe, ou en conservant, comme on le fait dans certains taillis de la Nièvre, des cépées de charme et de hêtre dites volières, destinées à parcourir deux révolutions.

Les éclaircies, opérations nécessaires dans de tels taillis, dès que les rejets montants, les lances, ont une grosseur moyenne de $0,{ }^{\mathrm{m}} 10$ au moins, ne produisent un effet utile que si le peuplement doit rester sur pied environ huit ou dix ans au moins après l'opération. Dans ce cas elles doivent avoir pour objet tout d'abord de desserrer les lances les plus vigoureuses, tout en maintenant le sol 
couvert et frais. On peut enlever dans chaque céjée, dit II. Broilliard, une ou plusieurs tiges, parmi celles qui percent vers le ciel et qui nuisent à la tige la plus vigoureuse; mais on doit respecter tout ce qu'il n'est pas utile d'enlever (tiges franchement dominées, rejets étalés, branches basses, etc.) comme nous l'avons exposé précélemment. Quant aux essences mélangées au chène, l'éclaircie peut servir à en régler la proportion, mais on doit se souvenir que le bouleau, le tilleul, le tremble, le saule mème sont alors souvent une vraie richesse, à la condition qu'ils restent. subordonnés au chène, et qu'on ne doit pas en appauvrir systématiquement le taillis.

Taillis fureté de hêtre. - En dehors de ces types généraux, certaines régions spéciales se prêtent au taillis de hêtre, généralement soumis à un traitement particulier, le furetage, en raison de la faible faculté que possìde le hètre de rejeter de souche.

On trouve des tallis furetés principalement dans le Morvan, dans le Jura, sur le rersant suisse, et au pied des Pyrénées (fig. 35).

Les taillis furetés sont ordinairement des taillis simples, sans aucune réserve, dans lesquels on coupe dans une cépée les tiges les plus grosses en laissant subsister les plus faibles; on revient sur le mème point tous les huit ou dix ans, etsi on coupe à l'àge de vingt-quatre ou trente ans, les trochées sont formées de tiges de trois âges; c'est le jardinage appliqué aux taillis.

Dans l'Ariège, la révolution des taillis furetés, variable suivant les régions el la vigueur de la végétation, est en moyenne de trente ans, el on la divise en deux rotations de quinze ans ou trois rotations de dix ans; la formule yui sert à diriger les exploilations est un peu différente de la précédente; on doit enlever au passage de chaque coupe les perches exploilables et rúserver: $1^{0}$ tous les brins isolés; $2^{\circ}$ tous les rejets trainants ; $3^{\circ}$ tous lesautres 
rejets ayant moins de 20 centimètres de tour au collet de

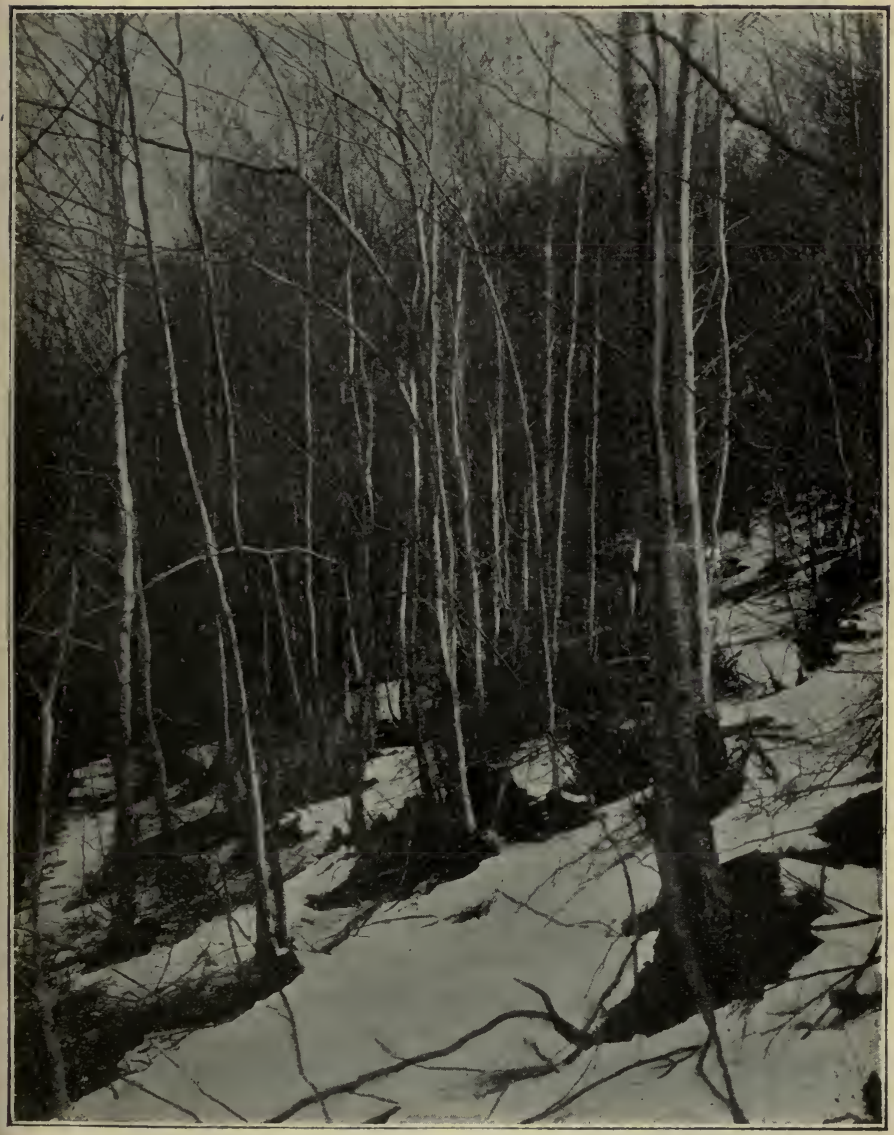

Fig. 3ă. - Peuplement de hètre à l'état de taillis fureté. Castillon (Ariège).

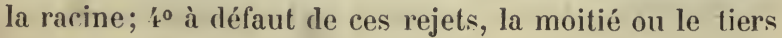


des tiges de plus de 20 centimètres de tour dans toutes les cépées qui ne présentent pas de rejets de dimension inférieure.

Ce mode de traitement, qui.s'est longtemps expliqué par la nécessité d'exploiter exclusivement du bois de moule, ne semble pas devoir être généralisé; il paraît préférable de traiter le hêtre en futaie, et, pour les particuliers qui possèdent des forêts de hêtre, en taillis composé afin de produire du bois d'œuvre.

Pour transformer en taillis composé de tels peuplements, le point de départ paraît être de déterminer de suite, le plus simplement possible, et d'asseoir sur le terrain la division des nouvelles coupes de taillis composé. Suivant la rotation adoptée pour les coupes de furetage, chaque coupe ancienne comprendra deux, trois coupes nouvelles ou plus, et si on dispose dès le début de bois exploitables, on commencera les coupes de taillis composé par ces parlies; si au contraire la forêt n'offre absolument aucun bois immédiatement exploitable, on sera évidemment forcé de reculer l'application du plan d'exploitation, quitte à faire en attendant quelques éclaircies dans les plus vieux bois ; c'est une question de tact, et pour agir avec méthode on devra se pénétrer du but que l'on poursuit et bien comprendre le résultat rers lequel on tend; c'est ainsi, par exemple, que les éclaircies devront ètre conduites dans un esprit contraire à celui du furetage ; on se propose de laisser vieillir l'ensemble du taillis fureté, et dès lors l'opération culturale (qu'on ne doit faire d'ailleurs, que si l'on tient absolument à réaliser de suite quelques produits disponibles) ne doit porterque sur les mauraises tiges, au lieu des meilleures, dans le but d'améliorer l'étage supérieur au lieu de le dégrader.

II parait inutile d'ajouter qu'on peut trouver dans de tels taillis du chêne et d'autres essences, même du charme en mélange, et qu'il convient de les soigner fet de les 
surveiller pour conserver et améliorer le mélange; c'est ainsi qu'au passage des exploitations, les jeunes brins et sous les rejets de chène, de charme et d'autres essences eront soigneusement recépés, et que, s'il est nécessaire, des dégagements ultérieurs viendront rabattre les brins de charme, d'érable, etc., qui tendraient à devenir envahissants au détriment du chène et du hètre.

Taillis de châtaignier. - Le châtaignier n'est pas spontané en France; quand on veut le conduire àun âge avancé, il se carie au coeur, et ne donne que des produits très médiocres en raison du déchet. Au contraire, quand on l'exploite jeune, son bois est sain et durable; très recherché pour les échalas de vigne, il est d'un grand produit.

Pour établir une châtaigneraie, il faut d'abord se rappeler que le châtaignier se refuse à croître dans les terrains calcaires; le sol qui lui convient le mieux est un sol graveleux et siliceux, comme celui qui résulte de la décomposition du granit. Le châtaignier est exposé aux gelées printanières sur les versant sud et ouest, et il craint beaucoup l'envahissement des herbes ; sa croissance est très rapide ; il rejette facilement.

D'après M. Bagnéris (1), il est bon de commencer par cultiver le terrain pendant deux ou trois ans, en choisissant de préférence des plantes sarclées, des pommes de terre, par exemple. Le sol est ainsi parfaitement nettoyé des mauvaises herbes, et il peut recevoir les jeunes plants qu'on a préalablement élevés en pépinière. On espace ces plants à 2 mètres en tous sens, et on peut encore cultiver pendant un an ou deux des pommes de terre dans l'intervalle. $\mathrm{Au}$ bout de six ou huit ans, on fait une première coupe dont les produits couvrent seulement les frais; puis on exploite en taillis simple sans réserves, tous les douze ou quinze ans. Un assez bon indice de

(1) G. Bagnéris, Manuel de Sylvicullure. 
l'âge auquel il convient de couper' le taillis est fourni par l'apparition de rejets au pied de certaines tiges.

Une châtaigneraie se maintient ainsi pendant environ un siècle et demi, après quoi il est opportun de la renouveler. Située auprès de vignobles, dans de bonnes conditions de sol et d'exposition, c'est une forèt d'un excellent rapport et parfaitement placée entre les mains des particuliers (Bagnéris).

Taillis de micocoulier. - Le micocoulier vient bien dans les plaines et rallées des bords de la Méditerranée et dans une certaine mesure dans les régions tempérées de la France, spécialement sur les bons sols et dans les terrains divisés.

Pour établir un taillis de micocoulier, d'après M. Broilliard, on plante à 2 ou 3 mètres d'espacement, en sol bien ameubli, des sujets de trois ans qui ont été protégés en pépinière contre les vents froids du nord; on cultive des plantes sarclées entre ces jeunes brins; on élague graducllement et rez-tronc les branches basses pour former les fûts, et on émonde en mai les branches gourmandes qui rejoussent après ces élagages; on bine le terrain chaque année une ou deux fois; on irriguequand on peut le faire, surtout au printemps, et au moins une fois par semaine.

La première coupe a lieu à quinze ans, et les coupes suivantes tous les huit, dix ou douze ans, quand les barres ont 8 à 12 centimètres de diamètre à hauteur d'homme, et 3 mètres sous branches. On ne conserve que deux ou trois rejets sur chaque souche, pour que les liges soient plus droites et l'accroissement plus rapide.

Dans de bonnes conditions de sol el d'exposition, ces taillis sont d'un excellent rapport, et parfaitement placés entre les mains des particuliers.

Remarquons que dès qu'on s'éloigne des bords de la Méditerranée, il faut avoir soin de ne planter les jeunes sujets qu'à l'age de cinf ou six ans seulement, et de 
garantir les jeunes tiges des froids trop vifs pendant toute leur jeunesse.

Taillis de robinier. - Le robinier ou faux acacia, n'est pas spontané en France.

Pour établir des taillis de cette essence, il faut se rappeler que le robinier craint le vent, exige beaucoup de lumière, redoute le mélange arec d'autres essences, et réussit médiocrement dans les terrains compacts ou très secs; les sols légers et frais lui conviennent bien, et il est susceptible, en raison des qualités de son bois, à aubier trìs mince, de donner de riches produits.

La plantation doit ètre faite à 2 mètres environ d'intervalle ; en général après avoir recépé à trois ou quatre ans, on exploite rez terre tous les dix, douze ou quinze ans. On peut conserver plus longtemps le robinier à l'état de massif, à la condition de l'éclaircir souvent et très fortement; tant qu'il prospère, le propriétaire peut avoir intérèt à le garder sur pied pour obtenir les produits de luxe qu'il donne dès l'âge de quarante à cinquante ans. D'ailleurs, à quelque âge qu'on l'exploite, il se reproduit facilement par rejets et surtout par drageons.

Taillis d'aune. - L'aune se prète très bien au taillis simple ; c'est une essence qui rejette vigoureusement et longtemps; elle est précieuse pour rendre productifs des terrains mouilleux.

L'aune est rarement en mélange avec d'autres essences, tant en raison des terrains qu'il habite que de sa croissance très rapide, mais c'est une essence très bonne à titre temporaire pour préparer l'installation d'essences plus utiles, propres à la station, telles que le chène pédonculé, le frêne, etc.

Selon la nature des produits à obtenir, la révolution à appliquer est plus ou moins longue et varie entre vingt et vingt-cinq ans; il peut mème ètre intéressant de garder sur ces taillis quelques réserves destinées à fournir des perches de plus fortes dimensions.

A. Fron. - Sylviculture. 
Culture de losier (1). - Les taillis de saule ou oseraies sont plutot des cultures industrielles que des cultures forestières. Tous les saules ne se prêtent pas également bien à cette culture, et ne fournissent pas un bon osier. Nous citerons parmi les espèces les plus employées :

L'osier vert ou saule viminal, qui aime les terrains aquatiques, légers, et donne des produits abondants, mais grossiers; l'osier brun, ou saule amandier ou saule à trois étamines, qui préfère les terrains humides et donne une bonne production; l'osier rouge ou saule pourpre, qui vient bien sur les terrains simplement frais, sur les bords des eaux courantes, et donne un osier très fin; le saule. blanc qui forme des oserais très productives, et est susceptible d'ètre traité en tétards; le saule fragile qui peut supporterles terres fortes, et donne des osiers de seconde qualité.

La création d'une oseraie demande trois années, et l'oseraie se trouve en plein rapport à la quatrième

Cette culture, souvent très rémunératrice, sort du domaine des exploitations forestières proprement dites, car elle peut coûter à l'hectare 1200 francs environ de frais d'établissement, et demande 200 à 300 francs de frais annuels d'entretien.

(1) Voir au sujet de la création d'une oseraie et des soins à lui donner, l'ouvrage de M. Brous.und, Traitement des bois en France. 


\section{DEUXIEME SEGTION}

\section{Étude spéciale des taillis composés.}

\section{I. - GÉNÉRALITÉS.}

Caractère et produits. - Le taillis composé présente l'avantage de réunir à la fois les mérites du taillis simple (régénération simple, facile, presque toujour's certaine) et une partie de ceux de la futaie. Il permet d'élever des arbres d'âges gradués sur des espaces très restreints et d'obtenir dans un temps relativement court des produits d'assez gros calibre; il fournit des produits très variés, bois de feu et bois d'œuvre, susceptibles de satisfaire dans une très large mesure à la demande du commerce (fig. 36).

Mieux que le taillis simple, il procure l'utilisation du sol jusqu'à une certaine profondeur. Découvrant moins complètement le sol, il est plus susceptible d'assurer la permanence de la forêt par le maintien de la fertilité de la station. Enfin les différentes formes du taillis composé sont susceptibles de se plier aux direrses exigences d'une exploitation forestière.

A tous points de vue l'emploi de ce mode d'exploitation est à conseiller, dans la majorité des cas, dans la propriété boisée particulière, quand il s'agit de tirer le parti le plus avantageux possible d'essences feuillues (1).

Le taillis composé (fig. 37) diffère, comme nous l'avons

(1) A condition toutefois que le terrain ne manque pas de profondeur, et qu'il ne soit pas mal doté sous le rapport du sol et du climat. 
vu, du taillis simple, en ce qu'il comprend toujours une réserve d'arbres àgés au moins de deux ou trois révolutions et généralement davantage; on se propose de produire à la fois des bois de faible dimension, qui se coupent à des époques peu éloignées, et des arbres de futaie qui restent longtemps sur pied et acquièrent une grosseur plus ou moins considérable. Le taillis proprement dit ou sous-bois, qui est essentiellement constitué par les cépées, c'est-à-dire par les rejets et les drageons et aussi par des brins de semence, n'est plus l'élément principal de la forèt; c'est la réserve, nommée aussi futaie, qui en forme la partie la plus importante; c'est elle dont la valeur pécuniaire est de beaucoup la plus considérable, et dont les produits sont, en général, les plus utiles et les plus recherchés (E. Muel).

Ces deux éléments, sous-bois et réserve, par leur nature mème, s'entravent, se contrarient réciproquement. D'une part le sous-bois est gèné dans son développement par le couvert des réserves; d'autre part les réserves, isolées périodiquement au moment des exploitations successives du sous-bois, peuvent donner naissance à un grand nombre de branches gourmandes; de plus, en raison de cet isolement, elles se développent latéralement au détriment de leur hauteur, et peuvent derenir branchues et noueuses, en restant relativement peu élevées; cet inconvénient toutefois est atténué dans une certaine mesure par l'accroissement considérable en diamètre que prennent ces réserves, spécialement pendant les ๆuelques années qui suivent l'exploitation du sous-bois (1).

(1) Pendant les premières années qui suivent l'exploitation du taillis, l'isolement des réserves favorise l'action de la lunière, le renouvellement de lintnosphẻre autour des cimes, conditions favoraliles à l'action chloropliyllienne ef $\mathbf{l}$ la chlorovaporisation; il en résulte que les fonctions vitales: v'activent; cette activité, qui a pour conséquence une plus grande absorption par les racines, un mouvement plus actif de la sève, est d'ailleurs favorisée jar le fait que, pendant celle période, les racines des réserves sont pour ainsi dire maftresses du sol 
Enfin le sol, partiellement découvert à des intervalles assez courts, s'améliore difficilement.

Quoi qu'il en soit, il y a toujours antagonisme entre la réserve et le sous-bois, et dès lors dans un traitement rationnel il faut chercher le moyen de concilier autant que possible ces deux éléments. On y arrive en tenant compte des considérations générales suivantes, pour fixer d'une part la révolution du taillis, et d'autre part le choix des essences à réserver, leur répartition, leur nombre et leur âge d'exploitation.

1. Révolution du taillis. - En principe, la révolution du sous-bois doit être plus longue que celle qui aurait convenu à un taillis simple situé dans les mèmes conditions, et cela pour deux raisons: a) plus la révolution du sous-bois est longue, en se tenant bien entendu dans les limites indiquées précédemment pour obtenir la régénération par rejets de souche, plus le sol reste couvert, et par suite en bon état, et moins il a à souffrir du découvert occasionné par les exploitations; $b$ ) plus la révolution du sous-bois est longue, plus la hauteur de fût des baliveaux, et par suite de toutes les réserves, est considérable; il est en effet démontré par l'expérience que le fût des perches qui ont ainsi crû en massif, n'est plus susceptible de s'allonger d'une façon sensible une fois que l'arbre a été isolé, bien que leur cime continue à s'accroître, ainsi d'ailleurs que le diamètre du tronc.

Comme conséquence de ce deuxième fait (hauteur de fût des réserves), plus les révolutions sont longues, plus le couvert des réserves est élevé au-dessus du taillis naissant, et par suite, moins son effet (bien entendu à nombre égal de réserves) est préjudiciable à la croissance de ce taillis.

Ainsi le mode de traitement en taillis composé exige, en raison mème de sa constitution propre, des révolutions assez longues; celles de vingt ans et même de vingtcing ans, qu'on rencontre pourtant si fréquemment, ne 


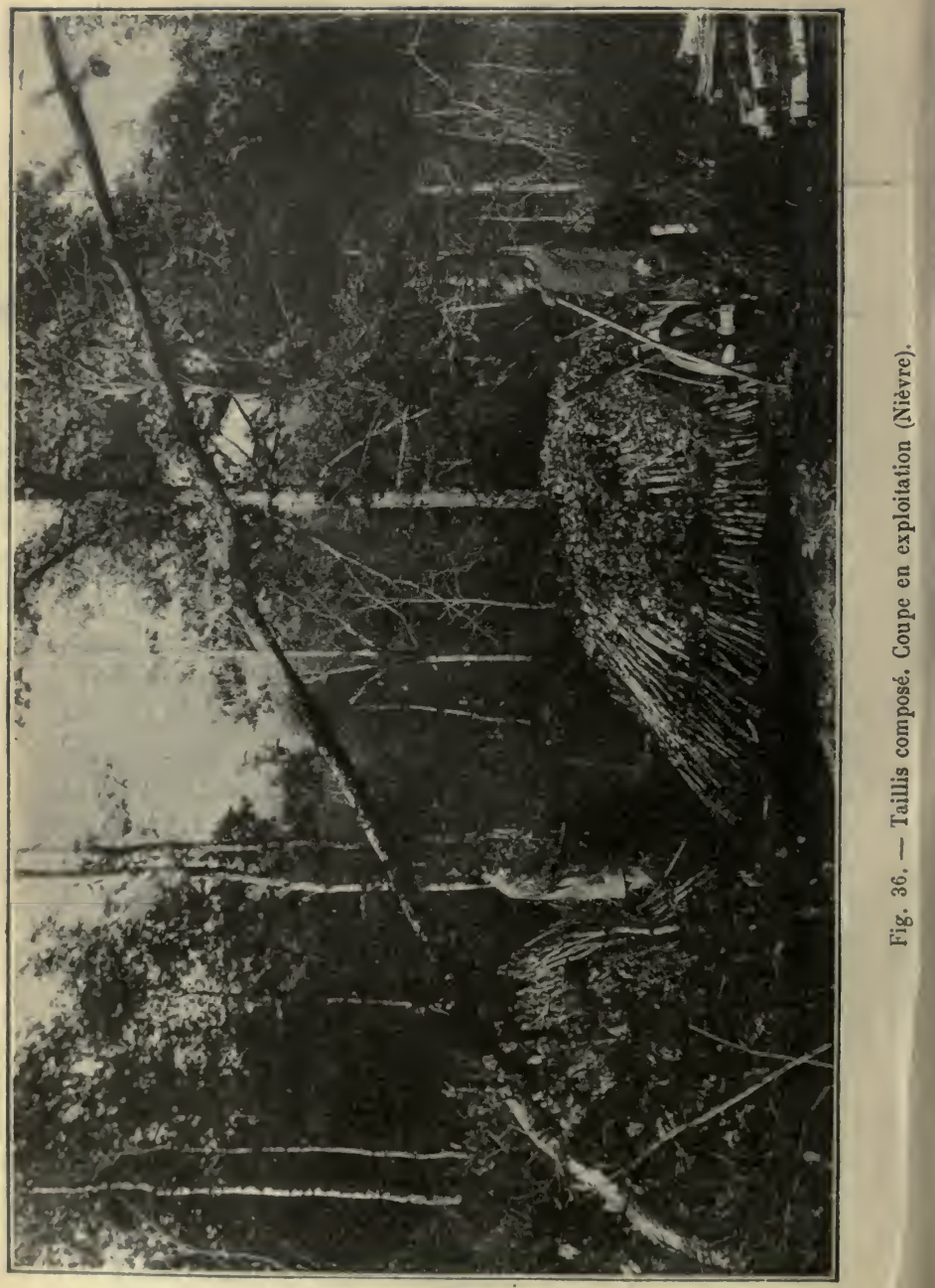




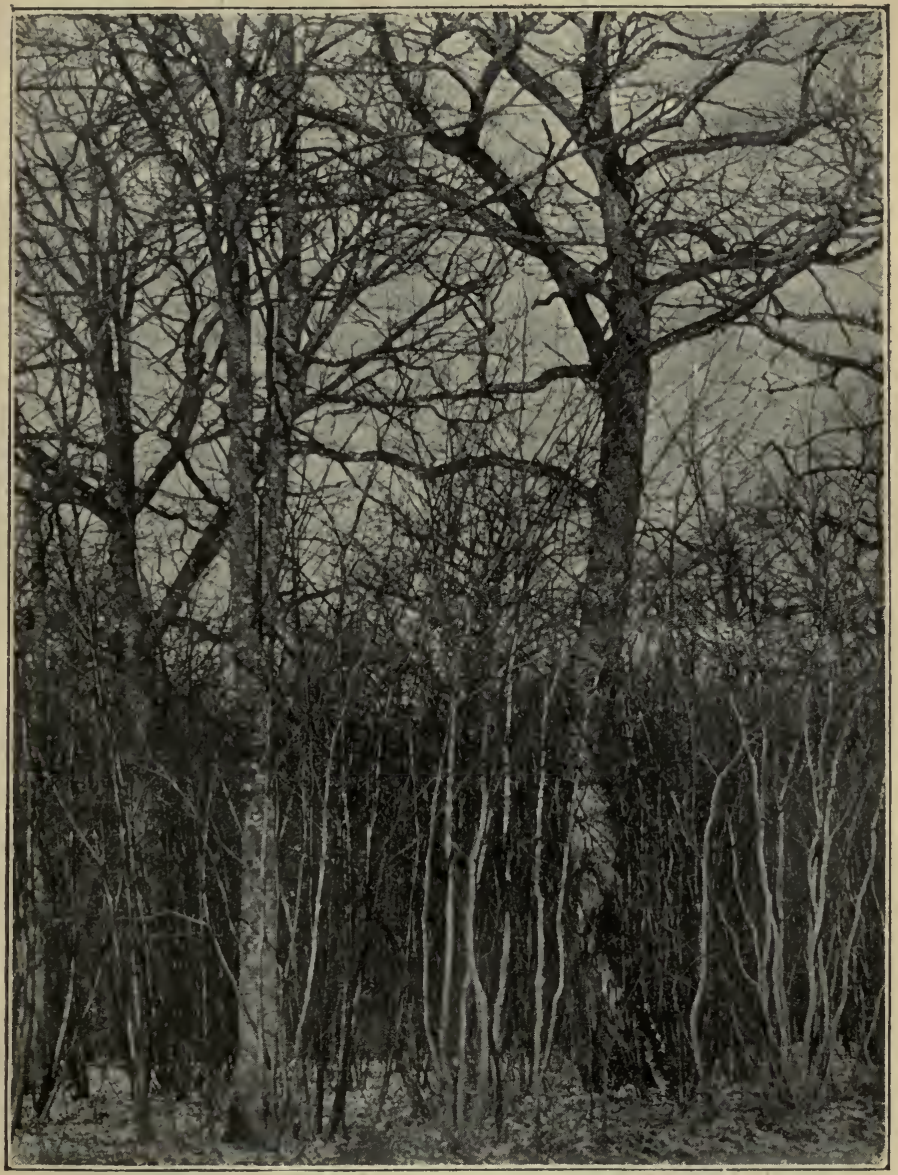

Fig. 37. - Taillis composé avant la coupe. Forèt de Champenoux (Meurthe-et-Moselle). "Les Forèts " par M.M. Beppe et Jolyet. 
sont généralement pas suffisantes pour atteindre le but proposé. A notre avis, il faut proscrire d'une façon presque absolue la révolution de vingt ans; on peut adopter celle de vingt-cing ans quand la végétation, eu égard au sol (sol excellent), au climat ou à l'exposition et à l'essence cultivée, offre une vigueur suffisante. Mais lor'sque les conditions de végétation sont insuffisantes et mauvaises, spécialement dans les mauvais sols, la révolution doit être portée à trente ou trente-six ans.

$2^{\circ}$ Choix des essences à réserver. - Avant tout on préférera les essences de lumière, puisque le besoin qu'elles ont d'espace pour étaler leur cime, les dispose tout naturellement à croître à l'état isolé plutôt qu'en massif, et que leur couvert gẻnéralement léger exercera une influence moins préjudiciable sur le développement du taillis.

l'armi elles on choisira tout d'abord le chène (chêne rouvre et chêne pédonculé), auquel on associera, suivant les stations, des essences disséminées telles que le frène, l'orme champêtre, les grands érables (érable plane, érable sycomore) le châtaignier (1), l'alisier, le sorbier (notamment le cormier) et des fruitiers, tels que les merisiers, dont les fruits sont précieux à tous égards. Là où manqueront les essences précieuses, le propriétaire particulier trouvera intérêt à réserver quelques bois tendres (blancs), tels que le tremble et le bouleau, dont le couver't très léger entrave peu la croissance des cépées qu'elles dominent. Toutes ces essences sont susceptibles de donner d'excellents produits.

Quant aux essences d'ombre, leur emploi ne vient qu'en deuxième ligne, en raison de leur couvert épais; parmi elles on réservera le hêtre et le charme, spécialement à titre de porte-graines;'le hêtre s'accommode assez mal de l'état isolé; il rejette mal de souche; aussi,

(1) Les réserves de chátaignier se carient rapidement au cour, et on ue peut guére les conserver utilement au-delà de soixante ans. 
dans les bons sols, tend-il à disparaitre; dans les sols médiocres, au contraire, il persiste plus longtemps, et souvent, n'ayant pas mieux à obtenir à sa place, on devra chercher à le maintenir dans le taillis, à l'aide de brins de semences donnés par les porte-graines. Le charme rejette abondamment et est par excellence une essence précieuse dans le sous-étage; mais il présente en outre l'avantage de donner une semence légère, qui réussit presque chaque année; à ce titre, quelques réserres de charme sont souvent précieuses pour combler les vides du taillis. Néanmoins ces deux essences ne doivent en principe être réservées qu'en nombre restreint; le couvert bas et épais de ces arbres, surtout quand ils deviennent un peu âgés, "tue le plus grand nombre des cépées qu'ils recouvrent, aussi ne leur laisset-on généralement parcourir qu'un nombre restreint de rérolutions; le hètre à peine trois à quatre révolutions, et le charme deux, c'est-à-direqu'en général on est conduit à les exploiter dès qu'ils sont devenus franchement fertiles.

Enfin, dans des conditions spéciales, la conservation de l'aune et du tilleul, dont le couvert est assez épais, peut être utile; pour l'aune, sur les bords des ruisseaux, dans les parties mouilleuses et franchement humides; pour le tilleul, dans les pierrailles amoncelées, les débris de carrières, où aucune autre essence ne prospère souvent aussi bien que lui.

Le choix des baliveaux, dont dépend la constitution de la réserve future, doit se baser, non seulement sur les considérations d'origine que nous avons exposées à propos du repeuplement, mais encore sur leur forme; on ne doit en principe accepter que des baliveaux de bonne forme, c'est-à-dire cenx qui joignent à une hauteur' de fût suffisante, un diamètre proportionné et une cime bien déreloppée ; le développement de la cime, pour les baliveaux de l'âge qui se choisissent en plein massif, 
peut toujour's s'apprécier par l'inspection du pied; un arbre dont le pied s'érase bien, et en tous sens, à l'endroit où il pénètre dans le sol, est un arbre vigoureux. Ajoutons enfin que les baliveaux de l'âge doivent être pris exclusivement dans les tiges droites, et qu'on doit écarter ceux dont la tige se partage, à peu de hauteur, en deux ou trois branches s'élevant à peu près parallìlement.

$3^{\circ}$ Répartition des réserves. - La répartition des réserves, comme nous l'arons déjà exposé précédemment (page 145), dépend essentiellement du type de taillis composé qu'on est susceptible d'adopter; nous avons distingué trois types: le type à futaie claire, le type normal et le type à futaie prépondérante.

$1^{\circ}$ Le type $\dot{a}$ futaie claire se rapproche beaucoup du taillis simple; la futaie y est peu nombreuse et disséminée ;

$2^{\circ}$ Dans le type normal on se propose d'obtenir une croissance satisfaisante, uniforme et soutenue du taillis, sans négliger la production de la futaie. Dès lors, les réserves de toutes catégories, choisies au moins en grande partie parmi les essences de lumière, doivent en général ètre régulièrement distribuées, afin de répartir l'ombrage et le couvert sur toute la surface de la coupe; toutefois, si cette uniformité est utile en principe, elle ne doit pas ètre acquise au détriment de considérations culturales de premier ordre, qui imposent: 10 de tenir compte des différences qui peuvent exister dans la nature du sol et de l'exposition; $2^{\circ}$ de prendre l'arbre bon à réserver là où il se trouve, et non de lui préférer un sujet médiocre susceptible d'ètre réservé précisément à l'endroit où on le désirerait; c'est ainsi que dans les parties les plus fertiles on pourra serrer lavantage la futaie; qu'aux expositions chaudes on tendra, autant que possible, à rendre la réserve plus nombreuse, en la composant principalement de baliveaux et de modernes; 
toutefois, autant que le permettront la qualité du sol et les ressources présentées par les peuplements, les différentes classes de réserve doivent se succéder et se mélanger sur les différentes parties de la coupe, dans la proportion déterminée par le nombre total des arbres à conserver pour chaque catégorie, et il y a lieu, en principe, de s'abstenir d'accumuler les arbres âgés sur le mème point.

Dans la pratique, disent MM. Boppe et Jolyet, nous engageons les opérateurs à se pénétrer de cette vérité que sur chaque point, l'arbre le meilleur à conserver, est toujours celui qui, eu égard à l'espèce à laquelle il appartient, à sa vigueur, à son état sain, à ses dimensions, à sa valeur actuelle, travaille le plus utilement dans l'intérèt du propriétaire; ici nous réserverons un chène ancien, là un hêtre moderne, ailleurs autre chose, et par suite notre attention se porte tout d'abord sur la grosse réserve.

D'après M. Broilliard, nous ajouterons le conseil suivant: avec le mérite de l'essence et l'absence de tares, toutes choses à constater en examinant chaque sujet, la végétation donne le critérium essentiel des arbres à réserver dans les taillis composés; aussi peut-on résumer en deux mots les conditions du balivage des arbres de réserve : enlever les mauvais, garler les bons. C'est pour'quoi, au lieu de chercher d'abord les sujets à réserver, est-il bien préférable de prendre pour point de de départ la recherche des arbres à réformer. Quels sont-ils? premièrement, les arbres dépérissants, couronnés, dégradés ou affectés d'une tare grave; en mème temps il y a lieu d'abandonner les arbres exploitables par suite de leurs dimensions; enfin il convient de disposer des arbres surabondants qui entravent le développement d'autres sujets plus précieux; on procure, par cela mème aux arbres et aux sous-bois, une place suffisante. Tout en procédant ainsi, on marque en réserve les arbres non réformés. 
C'est seulement quand le choix de cette réserve est bien arrêté qu’on s'occupe des baliveaux, en les répartissant dans les espaces où les arbres manquent, en évitant surloul de les marquer trop près des modernes et des anciens, ou comme cela se fait trop souvent, sous leur projection. Ce serait d'ailleurs se faire illusion que d'en exagérer le nombre pour masquer l'indigence d'une réserve trop pauvre en arbres constitués. Il ne faut pas non plus, sous prétexte d'éviter de grands trous et de répartir également l'ombrage, réserver un grand nombre de baliveaux de l'âge d'essence charme, ou d'une autre essence inutile comme bois d'œuvre; de tels baliveaux ne donneront toujour's que du chautrage quand ils seront devenus modernes, et en les réservant on se sera privé de cépées au moins aussi productives qu'eux, et qui auraient mieux assuré l'état complet du sous-bois.

$3^{\circ}$ Duns le type à futaie prépondérante (fig. 38), la direction de la culture tend à donner à la futaie une place prépondérante, et dès lor's il est impossible d'éviter l'action préjudiciable du couvert sur le sous-bois. Dès lors, M. Gayer donne le conseil de répartir la futaie d'une manière irrégulière. Cette disposition de la futaie, tantôt par groupes (spécialement pour les classes jeunes et d'âge intermédiaire), tantôt par sujets isolés, est non seulement imposée par les circonstances, mais encore de nature à favoriser l'objet de ce genre d'exploitation; elle donne la faculté de profiter des conditions de terrain pour donner aux sujets d'élite tout l'espace nécessaire à leur développement, et de stimuler, par le groupement, la croissance en hauteur des arbres choisis à cet effet; ces réserves sont alor's plus utiles, et sous le couvert de chênes ainsi réservés, on est bien certain de trouver des semis qui assureront en temps utile les besoins du balivage.

Celle distribution inégale amène la mème inégalité dans la répartition et le développement du taillis, qui 


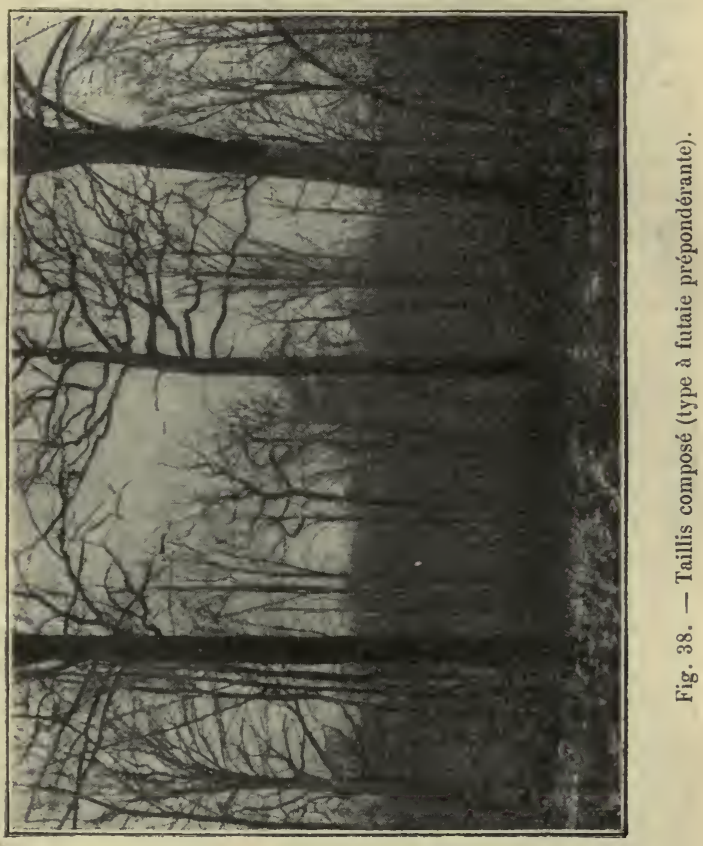


d'ailleurs au point de vue de la production totale, demeure relégué à l'arrière-plan. Là où la futaie se groupe en bouquets plus ou moins formés en massif, le taillis végète, on lui cède complètement le terrain; il forme dans l'ensemble un peuplement très irrégulier, interrompu par places, et prend plutot le caractère d'un peuplement de protection, caractère qui ne doit pas ètre perdu de rue; du reste sa conservation est assurée, en mème temps que la production totale de la forèt est stimulée, si celle-ci se compose surtout d'essences de lumière.

Ajoutons enfin qu'une très bonne mesure, quels que soient les cas, consiste à réserver le long des lisières de la forèt ou des coupes, le long des tranchées ou des chemins, des cordons plus ou moins épais d'arbres de futaie, qui bénéficient de la mise en lumière, et rendent le double service de servir de rideaux de protection du plus heureux effet, et d'assurer une riche épargne pour le propriétaire ; ce dernier, le cas échéant, peut toujours en disposer, dût-il, après exploitation, procéder à un repeuplement artificiel des parties exploitées (1).

$4^{\circ}$ Nombre des réserves. - La question du nombre des réserves à maintenir dans un taillis composé, ne se pose en fait, que dans le cas du taillis composé normal, où l'on se propose de rechercher le nombre de réserves de toutes catégories que l'on peut conserver par hectare, en se basant sur la quantité du couvert qui peut ètre imposé au taillis, sans nuire trop fortement à son développement. Ce nombre de baliveaux, modernes et anciens, dépend d'une foule de circonstances; il ne peut ètre donné d'une 'façon absolue, puişu'il doit varier d'une forêt à l'autre, dans les divers cantons d'une mème forèt, souvent sur les différents points d'une mème coupe,

(1) Nombre de propriétaires particuliers enrichissaient ainsi autrefois leurs forèts, tout en les embellissant; aujourd'hui cette coutume semble se perdre, et c'est regrettable. 
et enfin suivant les ressources qu'offrent les peuplements.

I. Rivet énonce trois lois à cet égard (1):

10 A surface égale, l'intensité du couvert est proportionnelle à l'épaisseur du feuillage des essences de réserve ;

$2^{\circ} \mathrm{A}$ surface égale et pour une mème essence, l'intensité du couvert est en raison inverse de la hauteur des fûts ;

$3^{\circ}$ L'intensité du couvert est proportionnelle à l'épaisseur de la cime, et par conséquent à l'âge des réserves (puisque la cime s'allonge en hauteur avec l'âge, et que la hauteur de fût reste sensiblement stationnaire après la première révolution du taillis).

De ces trois règles découlent les conclusions suivantes énumérées par M. Muel (2): les essences à couvert léger, telles que le chène, frène, bouleau, nuisant moins au sous-bois, pourront ètre réservées en plus grand nombre que celles à couvert épais, comme les hêtres et les charmes; - dans les forèts et les cantons fertiles, la réserve pourra ètre plus serrée, parce que les arbres ayant plus de hauteur de fût, donneront un couvert moins dommageable au taillis, et que celui-ci sera, grâce à la bonne qualité du sol, plus à mème de résister à l'action du couvert; - pour la mème raison (hauteur des fùts), le nombre des réserves peut augmenter avec la durée de la révolution; - un taillis formé d'essences supportant bien le couvert, comme les charmes, les hètres et certains morts bois, pourra ètre surmonté avec moins d'inconvénients d'une réserve plus abondante; à surface égale, le couvert des arbres âgés est plus nuisible parce que leur feuillage est plus épais; on se gardera donc d'en réserver une trop forte proportion; - aux expositions chaudes, le nombre des réserves devra ètre assez grand pour protéger le recru contre les ardeurs du soleil; mais on conservera peu d'arbres âgés, parce que

(1) Extrait du cours professé à l'Institut national agronomique, par M. Rivet.

(2) E. Muel, Notions de Sylviculture. 
leur couvert plus étendu et plus épais serait plus nuisible qu'utile au taillis, et que d'ailleurs leur croissance plus lente, et leur longévité généralement moindre à ces expositious chaudes, rendent leur maintien sur pied peu avantageux. La même recommandation est applicable aux sols secs, peu profonds et de médiocre qualité; - enfin, dans les sols très pauvres et aux expositions trè's chaudes, c'est-à-dire dans les plus mauvaises conditions, si le propriétaire n'est pas apte à faire de la futaie arec les essences spontanées, il est obligé de préférer le taillis simple au taillis composé; c'est le conseil que donne M. Bagnéris aux propriétaires de tels terrains.

Le nombre et l'importance des réserves à conserver dans un taillis composé, varie encore dans les mêmes situations, avec l'intensité que le propriétaire veut donner' à sa culture; selon qu'il conserve peu ou beaucoup de réserves, et notamment des arbres âgés, son capital fonctionne à des taux très différents, et cette considération est pour lui une des plus importantes; nous l'examinerons dans la quatrième partie de cet ouvrage.

Malgré ces causes d'incertitude, il peut être bon d'aroir un type moyen de balivage, sauf à s'en écarter plus ou moins dans chaque cas particulier. Si nous tenons compte que dans la pratique, afin de faciliter l'opération et d'arriver à une plus grande régularité, on ne distingue en général que trois catégories de réserves, baliveaux, modernes, anciens, pour chacune des essences principales qui se trourent dans la coupe, et que lon établit ces catégories plutôt sur la grosseur des arbres, que sur le nombre exact des révolutions qu'ils comptent, nous pourons adopter le type de balirage moyen que signale M. Muel.

Ces chiflres qui représentent le nombre d'arbres à réserver par hectare répartis parcatégories et par essences, constituent ce qu'on appelle le plan de balivage. 


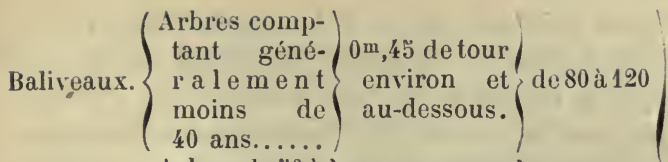

Nombre d'arbres

Modernes. $\left.\left\{\begin{array}{c}\text { Arbres de } 50 \text { à } \\ 100 \text { ans en- } \\ \text { viron....... }\end{array}\right\} \begin{array}{c}0 \mathrm{~m}, 50 \text { à } 1 \mathrm{~m}, 10 \\ \text { de tour.... }\end{array}\right\}$ de 50 à 90 à réserAnciens.. $\left.\left\{\begin{array}{l}\text { Arbres } \\ \text { plus } 1 \text { d e } \\ 100 \text { ans..... }\end{array}\right\} \begin{array}{r}1^{\mathrm{m}}, 20 \text { de tour } \\ \text { et au delà.. }\end{array}\right\}$ de 6 à 15 ver par hectare.

De l'examen du plan de balivage, il est facile de déduire le nombre d'arbres de futaie à abandonner en principe à l'exploitation si l'on veut conserver le matériel de la forèt dans le même état; on peut le calculer de la façon suivante :

\begin{tabular}{|c|c|c|c|}
\hline $\begin{array}{c}\text { CATÉGORIES } \\
\text { DE } \\
\text { RÉSERVES. }\end{array}$ & $\begin{array}{c}\text { Arbres existant } \\
\text { imnédiatementavant } \\
\text { l'exploitation } \\
\text { (d'après l'ancien } \\
\text { balivage). }\end{array}$ & $\begin{array}{c}\text { Plan de } \\
\text { balivage (ou } \\
\text { arbres } \\
\text { à marquer } \\
\text { en réserve). }\end{array}$ & $\begin{array}{c}\text { Arbres à abandonner } \\
\text { à l'exploitation. }\end{array}$ \\
\hline $\begin{array}{c}\text { Baliveaux. } \\
\text { Modernes. } \\
\text { Anciens. }\end{array}$ & 80 & $\begin{array}{c}80 \\
50\end{array}$ & $\begin{array}{c}80-50=30 \\
50-6=44\end{array}$ \\
\hline
\end{tabular}

Cies chiffres n'ont rien d'absolu; ils varient, comme nous l'avons exposé, dans de grandes limites, suivant les circonstances. Ajoutons que lorsqu'une classe de réserves est rare, on doit y suppléer en augmentant le nombre des réserves d'une autre catégorie, à raison de trois ou quatre baliveaux pour un moderne, et de deux ou trois modernes pour un ancien.

De tels chiffres, réglés à l'avance, ne peuvent ètre qu'une simple indication; il serait tout aussi puéril, dit M. Bagnéris, de prétendre atteindre le nombre règlementaire à l'aide d'essences quelconques, que de 
s'arrèter sous prétexte qu'on a le nombre voulu, eu égard à l'étendue totale de la coupe, quand on n'en a jarcouru que la moitié ou les trois quarts.

Remarquons enfin, a vec M. Broilliard, que dans la plupart les cas, à chaque exploitation une coupe change d'état; riche en vieux arbres avant le dernier passage de la hache, elle n'offre guère ensuite que de jeunes baliveaux ou inversement; aussi essayer de maintenir un taillis composé dans un élat constant, c'est poursuivre une chimère; prescrire dans un règlement de jouissance un nombre déterminé de inodernes et d'anciens, c'est aller trop loin. On doit tendre, autant que le permettent les circonstances, à se rapprocher d'un balivage moyen, mais on ne peut se fixer une règle absolue, et l'on doit se laisser guider par les circonstances, par les règles culturales et par le but qu'on se propose. Enfin on doit surlout se rendre compte de ses actes; nous reviendrons plus tard sur cette importante question.

:0 Age des réserves. - En principe on devrait, dans les taillis composés, conserver les arbres de réserve aussi longtemps qu'ils restent sains, vigoureux, et que leur accroissement se maintient en bonne roie; c'est le moyen d'obtenir les produits les plus utiles et les plus demandés aujourd'hui par le commerce des bois.

En se plaçant au point de vue du plus grand rendement en argent, on arrive à un résultat du même genre; jour voir si l'on a intérêt à ce point de vue, à conserver un arbre de réserve, par exemple un ancien, ou à l'abattre, il suffit de comparer la valeur qu'acquiert cet arbre en restant sur pied une révolution de plus, au prix qu'on pourrait en tirer actuellement augmenté des intérèts composés de cette somme jendant une révolution, el augmenté en outre de la valeur du recru qui se serait produit si l'arbre considéré avait été abattu immédiatement ; c'est un calcul à faire, et nous empruntons à M. Muel l'exemple suivant : 
Supposons qu'un chène de cent ans mesure $1^{\mathrm{m}}, 20$ de tour (à $1^{\mathrm{m}}, 50 \mathrm{du}$ sol) sur 8 mètres de hauteur, qu'il cube $0^{\mathrm{mc}}, 743$, de bois d'œuvre et vaille à 40 francs le mètre cube la somme de 30 francs; à l'âge de cent vingtcinq ans ses dimensions pourront être de $1^{\mathrm{m}}, 60$ de circonférence sur 9 mètres de hauteur propres au service; le volume du bois d'œuvre serait de $1^{\mathrm{mc}}, 485$ et sa valeur à 50 francs le mètre cube atteindrait 74 francs. (On néglige le bois de feu à tirer du houppier, celui-ci représentant à peu près lles frais d'abatage, de façon, de vidange et de vente). En restant sur pied pendant vingtcinq ans, cet arbre est passé de la valeur de 30 francs à celle de $\pi$ francs.

Or ces 30 francs placés à intérêts composés à 3 p. 100 pendant vingt-cinq ans acquièrent une valeur de $62^{\text {fr }}, 82$ (1). Ajoutons à ce chiffre le montant du dommage causé aux cépées qui n'ont pu prendre le mème accroissement que si cet arbre avait disparu, soit environ 8 francs, le total deviendra $62^{\mathrm{fr}}, 82+8=70^{\mathrm{fr}}, 82$. Mais pendant ce temps notre ancien a acquis une valeur de 7 tr francs; il y a donc profit à conserver ce chène jusqu'à cent vingt-cinq ans.

Par un calcul analogue, on pourra savoirs'il y a encore avantage à le conserver jusqu'à cent cinquante ans, ce qui se réalise très souvent dans les sols de bonne qualité.

A moins de circonstances exceptionnellement favorables, M. Muel estime qu'en moyenne, lorsqu'on cherche à obtenir la production en argent la plus considérable, on arrive aux résultats suivants (2):

(1) Chiffre obtenu par la formule des intérèts composés; la valeur à la fin de $n$ années d'un capital C placé à intérêts composés au taux $t$ est $\mathrm{C}(1+t) n$. Le facteur $(1+i) n$ pour toutes les valeurs de $t$ et de $n$ est donné dans la pratique par la table I de Cotta (Voir Agenda du forestier).

(2) Ces chifres ne peuvent être que de simples indications, car ils dépendent de l'activité de la végétation. Or, il arrive souvent dans les taillis composés que les chênes ont de $1^{\text {m }}, 50$ à $1^{\text {m }}, 80$ de tour avant cent ans, et on peut alors avoir grand intérêt à les conserver. Pour chaque arbre, c'est l'activité de la végétation qui doit guider. 
$1^{\circ}$ Les chênes de $1^{\mathrm{m}}, 50$ à $1^{\mathrm{m}}, 80$ de circonférence (dont l'âge peut varier de cent vingt à cent cinquante ans) ne doivent plus ètre réservés, sauf peut-être dans les bas fonds, où la conservation d'un ou deux de ces arbres par hectare peut achalander une coupe;

$2^{\circ} 11$ n'y a pas avantage à conserver, du moins en thèse générale : des frênes, des érables, de plus de 1 mètre à $1^{\mathrm{m}}, 20$ de tour (âgés d'environ soixante-quinze à quatrevingt-dix ans);

$3^{\circ}$ Les bouleaux sont exploitables dès l'âge de cinquante à soixante-dix ans;

$4^{\circ}$ Enfin pour les hètres et les charmes, réservés sur les taillis composés à défaut d'essences plus précieuses, et qui ne se débitent la plupart du temps qu'en bois de feu, on ne leur laisse guère dépasser $1^{\mathrm{m}}, 20$ de tour pour le hêtre (quatre-vingts à cent ans) et 80 à 90 centimètres pour les charmes (soixante-quinze à quatre-vingt-dix ans).

Remarquons ici qu'en ce qui concerne le nombre d'arbres à réserver dans les taillis composés, ainsi que l'âge de ces réserves, le propriétaire fait intervenir la considération du capital engagé dans l'exploitation, capital qui devient de plus en plus considérable avec le nombre et la valeur des réserves engagées dans son exploitation; que dès lors il tient compte du taux de placement de son capital engagé, beaucoup plus que du revenu brut à obtenir, et que cette considération vient modifier pour lui, dans une certaine mesure, les considérations précédentes. Un ancien, représente un capital engagé considérable, en raison du temps qu'il met à devenir exploitable, et ce capital fonctionne à un taux de placement financier moins élevé que le même capital représenté par de jeunes réserves ou simplement du taillis.

Dès lors apparait une difficulté nouvelle; dans les limites imposées par les règlesculturales, le propriétaire, 
suivant l'état de sa fortune, sera maitre de faire fonctionner son taillis composé suivant le type à futaie claire, suivantle type normal ou enfin suivantle type à futaie prépondérante; dans chacune de ces catégories il peut conserver des futaies plus ou moins âgées, ce qui crée encore de nouveaux types de peuplements, et à chacun de ces types correspondront des sortes de placement argent, différentes et variables suivant les cas.

Nous donnerons dans la quatrième partie de cet ouvrage quelques règles générales, se rapportant à ces considérations économiques; mais nous pouvons en léduire immédiatement cette conséquence, que le trailement en taillis composé se prête, dans la généralité des cas, à toutes les fortunes, à toutes les exigences, voire même, dans une certaine mesure, à tous les placements et à toutes les épargnes. C'est un de ses plus précieux avantages pour le propriétaire particulier.

Opérations culturales. - Les dégagements et éclaircies dont nous avons parlé à propos du taillis, sont des opérations culturales justifiées à fortiori dans les taillis composés, et l'on peut s'y préoccuper spécialement d'assurer la conservation des brins de semence d'essences précieuses. Dirigées dans ce but, ces opérations culturales tendent à assurer un bon recrutement de baliveaux. Dans les éclaircies, dit M. Broilliard, on néglige presque toujours et à tort de dégager les cimes des modernes et des anciens gênées par les perches du taillis qui tendent à les embrasser; cependant les arbres restent exposés ainsi à perdre quelques-unes deleurs branches principales, ce qui amène la dégradation de leur tige; bien souvent c'est surtout aux arbres menacés que l'éclaircie est utile, à la réserve donc plutôt qu'au sous-bois, mais à la condition qu'elle soit opérée autour des cimes, mais non pas en dessous d'elles.

Si l'on joint à ces opérations quelques repeuplements artificiels bien conduits, on aura contribué à entretenir 


\section{2 ÉTUDE SPÉCIALE DES TAILLIS COMPOSÉS.}

le bon état de la forèt, et à la rendre susceptible du plus grand rendement en matière et en argent.

Une excellente pratique consiste à arracher toutes les souches mortes ou impropres à rejeter vigoureusement, pour repiquer sur leur emplacement 3 ou 4 plants; l'extraction des grosses souches peut ètre permise à l'exploitant, ou concédée à des ouvriers à qui l'on donnera le bois comme salaire de leur travail, en leur imposant mème l'obligation de reboiser l'emplacement de la soucne ; quelquefois même le propriétaire peut retirer de ce travail une redevance de $0 \mathrm{fr}$. 50 à $1 \mathrm{fr}$. 50 par stère de bois de souches. Dans tous les cas, on devra toujours choisir a vec soin les essences à replanter, en adoptant celles qui sont le mieux appropriées au sol, au climat, et aussi aux produits à obtenir.

Ces repeuplements artificiels s'imposent aussi pour reboiser les vides occasionnés par l'envahissement des épines et morts bois, ou pour restreindre l'envahissement des bois tendres (blancs).

Enfin, souvent, ils peuvent être effectués dans le but de créer des ressources, ou de venir compléter les éléments du balivage à la prochaine exploitation; il n'est pas rare en effet de rencontrer fort peu de brins de chênes propres à former des baliveaux, mème dans certaines coupes où la vieille réserve en chène est abondante; ce fait d'ailleur's s'explique facilement par le tempérament de cette essence, qui ne tarde pas à languir ou à succomber sous le couvert.

Dans la pratique, on fait presque toujours la plantation au moment de la coupe du taillis; ce mode de procéder coûte cher si l'on plante des hautes tiges; il est incertain si l'on opère avec des hasses ou moyennes tiges qu'il est difficile de venir dégager à temps, et qui dès lors sont exposées à être étouffées par le recru.

Il est préférable d'effectuer les repeuplements artificiels quelques années a rant l'exploitation, au plus cinq ans et au 
moins deux ou trois ans a vant la coupe principale, à la suite d'une dernière coupe d'éclaircie ; on plante alors de basses tiges qu'on dispose régulièrement sur des places dégarnies, ou sur celles où le couvert est le plus relevé; ces plants, sans se développer beaucoup, prennent possession du terrain, et quand vient l'exploitation du taillis on les recèpe, si leur végétation laisse à désirer; des baguettes fichées en terre à côté des plants permettent de les retrouver et de les dégager au moment du passage des coupes de dégagement.

Enfin une dernière opération utile dans la plupart des taillis composés est l'émondage des réserves. Il y a lieu de ne pas confondre sous ce nom deux choses essentiellement différentes par leurs résultats :

$1^{\circ}$ La coupe des branches gourmandes qui se déreloppent sur le fût des arbres après leur isolement, et qu'on enlève avant de leur laisser prendre un certain développement; c'est l'émondage.

$2^{\circ} \mathrm{La}$ coupe de branches vives ayant déjà acquis un certain développement et qu'on est porté à effectuer à la partie inférieure de la cime pour releverle couvert de l'arbre; c'est l'elagage proprement dit.

Emondage des réserves. - L'émondage est une opération utile, sans inconvénient sensible pour l'arbre, si elle est faite à temps (1). L'opération consiste à enlever les branches gourmandes dès leur apparition, ou au plus tard un ou deux ans après; la section doit être faite rez tronc, à l'aide d'instruments tranchants (serpe ou émondoir), arec assez de précautions pour ne pas détacher l'écorce qui doit rester bien adhérente tout autour de la plaie. II y a lieu en outre de procéder à cette opération

(1) Quelques forestiers considèrent cette opération, les uns comme inutile, d'autres même comme nuisible. MM. Boppe et Jolyet recommandent de ne pas en généraliser l'application, et de reslreindre cette opération à un petit nombre de cas particuliers; il y a lä, disent-ils, pour les propriétaires particuliers qui en abusent par tradition, de sérieuses économies à réaliser. 


\section{4 ÉTUDE SPÉCIALE DES TAILLIS COMPOSÉS.}

dans le courant de l'été afin d'éviter, dans une certaine mesure, ce qui pourrait favoriser la repousse des branches; néanmoins il arrive généralement qu'après la coupe de ces branchettes, il s'en reproduit d'autres, et dans ce cas il ne faut pas hésiter à recommencer l'émondage; il est rare quon soit obligé d'intervenir une troisième fois. Les petites plaies qui résultent d'un tel émondage se ferment rapidement; elles sont sans influence ou peu nuisibles.

Dans la pratique, pour émonder les arbres de réserve on se sert d'échelles légères qu'on transporte facilement l'arbre en arbre, et il est indispensable de prohiber d'une façon absolue l'emploi des crampons dont certains ouvriers se servent pour monter sur les arbres.

Si lescirconstances n'ont pas permis d'effectuer l'émonlage en temps utile, et si les branchettes ont déjà acquis plus de 2 à 3 centimètres de diamètre, il paraît préférable derenoncer à l'opération, quitte à faire tomber ces arbres à l'exploitation suivante, si la présence des branches mortes dans le faite de la cime indique quils n'ont plus d'avenir.

Élagage. - L'élagage au contraire est une opération dangereuse, que dans la plupart des cas il est nécessaire d'éviter. C'est, disent MII. Boppe et Jolyet, une sorte de taille appliquée aux arbres, en vue de leur donner en une seule fois une forme plus avantageuse au moyen d'amputations totales ou partielles des rameaux réputés inutiles ou nuisibles. Invariablement et quelles que soient les jrécautions prises, cette opération se traduit par une blessure vive faite à l'arbre, une plaie nuisible par ellemème, et souvent désastreuse par les vices qu'elle occasionne dans le corps de l'arbre.

Toute opération de ce genre doit donc ètre proscrite des forêts; toutefois elle peut être lolérée sans grant inconvénient sur les branches basses, appartenant à la cime des baliveaux de l'âge et des jeunes modernes, dont le 
calibre ne dépasse pas 3 à 8 centimètres de diamètre à la base, car d'aussi petites plaies se recouvrent bientôt, et ne laissent plus tard au centre mème de l'arbre qu'une très faible solution decontinuité presque insignifiante (1).

Lorsqu'on procède à un élagage il est nécessaire de prendre quelques précautions : $1^{\circ}$ interdire l'usage de crampons pour monter le long des arbres; $2^{\circ}$ ne procéder qu'à l'élagage des branches basses, appartenant à la cime des baliveaux et des jeunes modernes, et n'ayant pas plus de 5 à 8 centimètres de diamètre à la base; $3^{\circ}$ faire la section verticale, rez tronc, bien nette et unie ou légèrement bombée, à l'aide d'un instrument tranchant ; pour atteindre ce but, commencer toujours l'abatage de la branche par sa face inférieure, afin d'éviter le décollement de l'écorce, et procéder avec assez de soin pour que l'écorce reste bien adhérente tout autour de la plaie; se servir dans ce but d'instruments bien tranchants, tels que hachette à manche court, serpe assez lourde à tranchant droit, et prohiber l'emploi de la scie; $4^{\circ}$ recouvrir la section d'élagage avec du coaltar (2), dans le but de soustraire, autant que possible, le bois mis à nu à l'action de l'air et de l'humidité pour le préserver de la carie et de la pourriture ; $\breve{5}^{\circ}$ ne jamais couper à la fois plusieurs branches un peu importantes sur un mème arbre, et éviter avec soin que deux sections voisines en se réunissant ne constituent une large plaie, plus difficile à cicariser.

Remarquons enfin que les branches mortes doiventêtre traitées comme les branches vivantes, et qu'en général, spécialement quand il s'agit de branches ayant un dia-

(1) Ne jamais élaguer les arbres résineux; ils ont plus besoin encore de toutes leurs branches et de leurs organes foliacés que les essences feuillues; en outre, les écoulements de résine causés par l'amputation des branches amènent fréquemment des chancres sur leur tige.

(2) Résidu bitumeux de la houille dont on a extrait le gaz.

A. Frox. - Sylviculture. 
mètre supérieur à ş ou 8 centimètres, il est préférable de ne pas y toucher; on surveille l'arbre, quitte à le faire tomber s`il est exposé à se dégrader.

Dans certains cas, et spécialement dans les futaies, l'élagage peut avoir pour but de relever le couvert de vieux peuplements afin de favoriser la production et le développement des jeunes semis; effectué sur des arbres dont la date d'exploilation est arrètée et prochaine, il ne présente plus d'inconvénients sérieux.

\section{II. - APPLICATIONS DU TAILLIS COMPOSÉ.}

Les observations recueillies par M. Mathey, dans le bassin de la Saône, et les conclusions souvent d'ordre très général qu'il en déduit, intéressent les propriétaires de taillis composés de toute la France. Nous ne pouvons que renvoyer le lecteur à cette intéressante étude, dans laquelle l'auteur répartit les taillis composés de cette région en six catégories, définies par les qualités du sol et caractérisées par leur flore ligneuse et herbacée.

MM. Boppe et Jolyet résum ent ainsi, d'après M. Mathey, ces diver's groupes: les trois premier's groupes comprennent les terres à chênes, celles où le taillis composé donne son plein rendement; à tout seigneur, tout honneur... la futaie est constituée en chêne. Pourtant, sur les colmatages dont la fertilité est exceptionnelle, on lui associe des essences disséminées · frêne, orme champètre, sans toutefois donner une trop grande prépondérance à ces espèces dont l'accroissement n'est supérieur à celui du chêne que pendant les deux premiers âges, dont la valeur marchande est variable, et qui sont très épuisantes. Dans les sables argileux et siliceux fins, une petite place est faite au hêtre, auquel on ne laisse pas dépasser la dimension d'ancien de $1^{\mathrm{m}}, 50$ de tour. Enfin, tans les murnes compactes mais profondes. et fertiles, les baliveaux et modernes de bouleau et de tremble, essences à cou- 
vert très léger et d'un bon rapport, sont utilement associés au chène quand celui-ci est insuffisant. On cède trop souvent sur de pareils sols à la fâcheuse habitude de réserver des charmes.

Le quatrieme groupe englobe des argiles oxfordiennes ou autres, des conglomérats calcaires ou siliceux, terres de composition variée, mais toujours compactes, froides et acides. Le chène seul, dit M. Mathey, doit y constituer la futaie, mais au milieu des maigres taillis que décime la bruyère, il végète mal. Aussi le forestier doit-il se préoccuper, avant tout, de resserrer la trame ordinairement trop lâche et trop uniforme du sous-bois.

Quant aux deux derniers groupes, leur caractéristique est la profondeur de plus en plus faible du sol, et comme corollaire, le rôle de plus en plus prépondérant du hètre. Sur les calcaires marneux des pays de collines et de basse montagne, la terre est mélangée de plaquettes calcaires ou de rognons marneux; le chêne décline; il devient logique d'accepter largement le hêtre, qui est, en fait, l'essence la plus productive; il ne faut pas craindre de le multiplier en modernes, et d'en garder les plus beaux anciens, les plus longs; quelques chènes, là où la profondeur sera suffisante, quelques alisiers torminaux, enrichiront la réserve et achalanderont les coupes. Sur les arènes provenant de la décomposition des granites ou des porphyres, sur la terre rouge que recouvrent certains calcaires jurassiques, sols éminemment superficiels tous deux, reposant tous deux sur des roches dures, la réserve du chêne ne compense, à aucun âge, la perte du recru qu'elle entraine ; le hêtre, jusqu'aux dimensions d'ancien, est l'essence fondamentale et exclusivement rémunératrice de la futaie.

De ce qui précède, il résulte que nous avons à examiner:

$1^{\circ}$ Les taillis sous futaie d'essences mélangées;

$2^{\circ}$ Les taillis sous futaie où domine le chène; 
$3^{\circ}$ Les taillis sous futaie où domine le hètre.

$1^{\circ}$ Taillis sous futaie d'essences mélangées. - C'est le cas général, dans les stations qui ne présentent rien d'exceptionnel, et la prédominance de l'une ou de l'autre des essences du mélange s'accuse en bien ou en mal suivant la gestion.

Les règles générales à leur appliquer sont celles que nous arons données précédemment; revenons sur quelques points :

Dégagements - Les semis ou brins très faibles d'essences précieuses (chène par exemple) peuvent ètre noyés dans un recru de charme qui les anéantit d'une façon complète, et au moment du balivage des coupes, les baliveaux chêne ne sont pas en nombre suffisant; dès lor's des dégagements partiels et réitérés sont nécessaires pour les sauver; si ces brins de semence sont répartis sur loute l'étendue du terrain, et si la proportion de chène est réellement compromise par l'abondance du recru de charme, le moyen le plus sûr, dit M. Broilliard, est de recéper ce dernier seul à l'âge de quatre, cinq ou six ans, selon que la végétation est plus ou moins rapide, en ayant soin toutefois d'agir avec prudence dans les places lépourvues d'arbres de réserve.

Eclaircies. - Avec une révolution suffisamment longue, l'éclaircie est utile huit ou dix ans avant la coupe ; elle a pour but, principalement, de préparer le balivage en desserrant les cimes des sujets intéressants, tant dans la réserve elle-mème qui peut être gênée par des perches du taillis, qu'autour des sujets d'avenir disséminés par pieds isolés dans le taillis; quand cette opération aura desserré ainsi 50 à 60 sujets de l'âge par hectare, le nécessaire sera fait.

Balivage. - Le balivage est effectué, autant que possible, en automne et en hiver, pendant la périorle où le feuillage ne gêne pas l'opérateur qui a besoin d'examiner les cimes de toutes les réserves. 
Dans le choix des bonnes essences, on évite de garder simultanément deux sujets de l'âge qui se touchent, par' exemple deux rejets sur une jeune souche; le meilleur des deux, conservé seul travaillera mieux (1). Entre deux chênes d'âges différents qui se gênent l'un l'autre, c'est en général le plus gros, s'il est sain, qu'il y a intérêt à conserver au point de vue du travail qui s'effectuera pendant la rérolution suivante.

En principe, dans les taillis mélangés, il y a intérèt à porter d'abord son attention sur les réserves chêne; après le chêne, sur les essences précieuses disséminées (frène, orme, etc.); puis, à défaut de ces sujets d'élite, sur les arbres bien renants des autres essences intéressantes quelle que soit leur grosseur. car ici le mètre cube de bois fabriqué n'augmente pas sensiblement de valeur arec le diamètre.

Exploitation. - L'abatage des arbres abandonnés peut ètre effectué en mème temps que l'exploitation du taillis; cette méthode facilite la vente des coupes. Toutefois, si les conditions du marché le permettent, un propriétaire particulier peut trouver avantage à n'exploiter les arbres de futaie, y compris dès lors toutes les perches de l'âge du taillis susceptibles de donner des baliveaux, qu'après exploitation du sous-bois; il est alors nécesssaire que le taillis abattu soit façonné et enlevé, ou transporté hors de la coupe avant le $1^{\text {er }}$ novembre au lieu du 15 avril suivant.

Avant l'abatage, les arbres de futaie doivent ètre ébranchés, pour éviter les dégats qu'occasionne leur chute, soit sur l'arbre lui-mème, soit sur ceux qui l'entourent.

Exploitation du taillis. - L'exploitation du taillis

(1) Deux chênes contigus de $0^{m}, 30$ de diamètre et de 8 mètres de tronc valent par exemple 10 francs chacun, et au total 20 francs. Tandis qu'un seul chêne de 0 m,40 et de mème hauteur vaut à lui seul 24 francs, et il a plus d'avenir (Broilliard). 
doit ètre faite avec soin, comme il a été indiqué, rez terre, sauf peut-être pour le hètre. On doit recéper avec soin tous les brins de semence (le hètre excepté) qui se trouvent dans la coupe et qui sont trop faibles pour être désignés immédiatement comme baliveaux; le rejet de ces jeunes souches, généralement unique, donnera à l'exploitation suivante d'excellents baliveaux.

Conservation du mélange. - Dans certains taillis sous futaie, le chêne tend à disparaître, envahi par le charme, et cette question du retour du chène sur un terrain occupé par le charme est, dit M. Broilliard, une des plus difficiles à résoudre.

On peut mettre à profit la régénération naturelle, obtenue grâce à la réserve de chênes bien venants; mais il est bon d'agir aussi par repeuplements artificiels ; on fera planter par exemple environ 200 plants par hectare de chênes de trois, quatre ou cinq ans, deux ans avant l'exploitation; ce travail peut être fait par un bon garde qui opère avec soin, et place les plants sur tous les pointsoù le couvert du taillis est le moins épais, en ayant soin de les espacer d'au moins 5 mètres, et de ne jamais les placer à proximité de réserves chène où ils sont inutiles; on profite du passage de l'exploitation pour recéper ces plants avec soin, et il ne reste plus qu'à les soigner à l'aide de dégagements successifs pour éviter (qu'ils ne soient étouffés par les rejets voisins pendant la première croissance du taillis.

En sol humide ou très riche, M. Broilliard conseille de planter, immédiatement après l'exploitation du taillis, quelques chênes de haute tige, de 2 mètres environ, qui reprennent alor's facilement, et s'élancent avec le recru.

Enfin, s'il y a à lutter contre l'envahissement du hètre, il est nécessaire de ne réserver que très peu de portegraines de cette essence; on doit avoir soin surtoul de ne pas réserver de hêtre sur des brins ou rojets de chène, 
si faibles, si déjetés ou si misérables qu'ils soient. $2^{\circ}$ Taillis composé où domine le chêne. - Si la prédominance du chène s'accuse dans le peuplement, c'est en général le chêne rouvre, qui existe tant dans le sous-bois que dans la réserve, car le chêne pédonculé ne se trouve guère naturellement à l'état pur (1). De tels peuplements se rencontrent soit sur la roche, soit dans l'argile, soit dans le sable; ils se présentent en général sous un aspect clairiéré, malvenant; le taillis a une végétation peu vigoureuse, sauf pendant quelques années après- la coupe; les baliveaux y languissent et meurent en cime.

En présence d'un état de végétation aussi mauvais, le propriétaire tend souvent à restreindre la durée des révolutions, sous prétexte que le bois ne pousse plus.

Avec M. Broilliard, nous sommes d'avis que c'est là une faute culturale de première importance, car plus les révolutions sont courtes, plus le sous-étage est exposé à se clairiérer, à prendre une végétation languissante, et plus aussi les autres grandes essences, telles que le hètre, le charme, le tilleul mème, tendent à disparaitre. On doit au contraire, partout où cela est possible, allonger la révolution, la doubler au besoin, peut-être même la porter jusqu'à quarante ans. Au lieu de la coupe ordinaire, faite à l'âge de vingt à vingt-cinq ans par exemple, dit M. Broilliard, on se contentera d'opérer une éclaircie pour desserrerles rejets des cépées, en conservant soigneusement tous les charmes, érables et hêtres disséminés, et on pourra recommencer cette opération dix ou douze ans plus tard, quelques années avant l'exploitation.

Partout où ce sacrifice immédiat n'est pas possible, on doit se rappeler que pour améliorer la forèt, trois choses sont utiles; $1^{\circ}$ tendre à allonger et non à restreindre la durée de la révolution; $2^{\circ}$ favoriser la végétation du taillis par une éclaircie opérée uniquement dans les

(1) En raison des sols frais ou riches qu'il affectionne, il est accompagné d'essences diverses plus ou moins nombreuses (Broilliard). 
cimes; $3^{\circ}$ rechercher arec soin les hêtres, charmes, tilleuls, coudriers mème, toutes essences fertilisantes, pour en favoriser le développement ou pour les conserver comme baliveaux, même en cépées ou volières; mieux encore, en situation mal abritée, dans les grandes coupes, l'action des cordons pleins réservés autour des coupes, ou même en travers, afin de les diviser en compartiments bien abrités, paraît ici indispensable.

Sur certains terrains froids et humides, sur des sols argilo-siliceux plats, où l'eau n'a pas d'écoulement, la lécomposition incomplète des détritus organiques, des feuilles dechêne et de bouleau rend le sol acide, tourbeux, apte à ètre envahi par la bruyère, et le mauvais état des taillis y est caractéristique. En pareil cas, M. Broilliard conseille d'avoir recours au coudrier pour changer ces conditions mauraises; on plante par exemple, aussitôt après l'exploitation du taillis, un millier de coudriers en ayant soin au préalable de remuer la terre du trou par quelques coups de pioche, de manière à la mèler et à l'aérer; aprẻs les coudrier's, des charmes dans les argiles, des aunes dans les bas-fonds, peuvent être utiles. La couverture morte, améliorée par les débris végétaux que donnent ces essences, est d'une décomposition plus facile; les lombrics reviennent cultiver le sol; ils abondent sous les coudriers, et le chéne sur coudrier végèle toujours aussi bien que possible en taillis et en arbres.

$3^{\circ}$ Taillis composé où domine le hétre. - Si la prédominance du hêtre s'accuse dans le peuplement, le taillis composé prend un nouvel aspect; et il doit en être ainsi, si toutefois le climat convient au hètre, dans les sols secs, peu profonds, ou bien dans les terrains formés de pierrailles ou de sable grossier, terrains dans lesquels le chêne se développe rarement et ne devient qu'exceptionnellement beau et vigoureux.

Malheureusement, sur de tels terrains, nous dit 
M. Broilliard, l'exploitation hâtive en taillis a substitué les cornouilliers, coudriers, épines, petits érables, etc., ou bien des bouleaux rachitiques, la bourdaine et la bruyère, au hêtre mélangé de chênes, de frênes, de grands érables; la broussaille y occupe la place de la forêt. L'auteur que nous venons de citer indique le remède qu'il faut apporter à cette situation: allonger la révolution, et la fixer à trente-six ou quarante ans; réserver en modernes ou en baliveaux de l'âge tous les sujets bien venants, quand même les cimes se touchent, pourvu qu'elles ne soient pas étriquées; y adjoindre les chênes d'élite, mais en les tenant écartés des hêtres, de mème que les plus beaux sujets des autres essences. C'est, en un mot, tendre ver's le type de taillis composé à futaie prépondérante dans lequel on cherche à constituer la futaie, d'abord avec des chênes bien constitués, réservant les anciens bien sains et vigoureux, ainsi que les modernes bien venants, en ayant soin d'isoler leurs cimes; ensuite avec des hètres, groupés même par petits bouquets de modernes et de jeunes anciens, et formant entre eux non un massif serré, mais un massif clair; enfin accessoirement, là où cela est utile, avec des charmes, sorbiers, alisiers, à la condition que ces arbres soient bien venants. Quant au choix des baliveaux, il doit porter sur les chênes et les hêtres, n'acceptant que ceux qui sont vigoureux et bien constitués.

Sous une telle forêt, qui prend en quelque sorte l'aspect d'une futaie claire, inégale et entrecoupée avec sousétage de taillis, les semis ne feront pas défaut; on aura soin alors, au moment des exploitations, de conserver intacts pour les recéper tous les semis de hêtre, et de recéper a vec soin ceux des autres essences.

Si le sol est calcaire, et s'il est envahi par les mortbois, coudriers, cornouilliers, troënes, saules, épines, viornes, ronces, etc., le mieux est de laisser vieillir ces mort-bois au lieu de les recéper, et d'aider ensuite à 
l'éclaircie qu'ils donnent, afin de permettre aux semis de hètre de venir peu à peu s'installer à leur place.

Si au contraire le sol est siliceux, sablonneux, s'il devient tourbeux ou tout au moins acide et est envahi par la bourlaine, les genèts, la bruyère, on peut avoir recours au coudrier, comme nous l'avons indiqué précédemment; sous les plantations de coudrier, le terreau redevient doux, et le semis de hêtre s'installe naturellement.

Partout enfin, s'impose le respect des hêtres, grands et petits; les arbres porte-graines doivent rester pour donner de la semence, les jeunes hêtres ne doivent pas ètre recépés; seuls les jeunes brins de cette essence qui sont courbés ou brisés doivent être coupés, mais encore vaut-il mieux ne les recéper qu'à 2 ou 3 centimètres audessus du sol, et mème leur laisser une branchette, ou tout au moins un bourgeon bien formé.

De cette élude forcément restreinte, dont nous avons puisé une partie des éléments dans l'intéressant ourrage de $\mathrm{M}$. Broilliard, il résulte que le taillis composé, méthode de traitement très en faveur, et à juste titre, dans la propriété boisée particulière, présente une variété infinie d'aspects. Des fautes culturales fréquemment répétées. des balivages défectueux, et surtout l'adoption de trop courtes révolutions pour les exploitations du taillis ont tries souvent provoqué dans ces peuplements l'envahissement des mort-bois et des mauvaises essences au détriment des bonnes essences, l'appauvissement progressif de l'état de massif, la diminution de la fertilité du sol, et un ralentissement marqué dans la végétation du taillis et de la réserve.

Une telle situation est la note caractéristique des taillis composés mal gérés.

Nous dirons qu'un taillis composé est en mauvais état, quand toutes ces causes réunies ont tendu à faire disparaitre, lout au moins en grande pairtie, les bonnes essences. liemarquons qu'un grand nombre de taillis composés se 
trourent aujourd'hui en maurais état; il y a lieu de les restaurer progressirement.

\section{III. - RESTAURATION DES TAILLIS COMPOSÉS EN MAUVAIS ÉTAT.}

Lorsqu'une forêt, traitée en taillis composé, est en mauvais état, deux méthodes sont à la portée du propriétaire particulier pour la restaurer progressivement, et nous distinguerons : $1^{\circ}$ la restauration proprement dite, qui a pour but de reconstituer un bon état de peuplement sans abandonner la forme du taillis composé; $2^{\circ}$ la conversion en futaie résineuse.

$1^{\circ}$ Restauration proprement dite. - La restauration proprement dite des taillis composés en mauvais état varie avec les circonstances, et en fait, nous avons traité cette question en examinant successivement, dans le chapitre précédent, les divers types de taillis composé.

L'opération de restauration consiste à augmenter la durée de la révolution, à faire un balivage raisonné approprié à la situation et à favoriser l'installation d'un mélange d'essences justifié par les circonstances où l'on se trouve. En général, ces trois opérations sont intimement liées l'une à l'autre.

Pour remédier à des situations très défavorables, nous avons signalé l'emploi très utile qu'on peut faire du coudrier, essence très facile à planter en sous-étage, et dont les débris végétaux tendent à améliorer un terreau trop acide.

Dans les régions de plaines et de coteaux, voire même en montagne (bien qu'en montagne une véritable substitution d'essences paraisse plus justifiée), les résineux peuvent fournir une aide des plus utiles; l'introduction par voie de plantation du pin sylvestre, si le sol est sablonneux, du pin noir, sile terrain est calcaire, est toute indiquée pour rétablir l'état de massif dans des taillis 
clairiérés, entrecoupés de vides, où la végétation devient languissante. Il nous paraît inutile d'insister à nouveau sur le rôle que jouent ces résineux; l'amélioration de la fertilité de la station par la reconstitution progressive de la couche d'humus, suffit à justifier leur présence, et ces arbres, par leur croissance rapide, ont vite compensé les frais de plantation. Leur introduction. d'ailleurs n'est faite qu'à titre temporaire, et nous savons que sous ces essences, sur le sol amélioré par leurs aiguilles, apparaissent spontanément, dès que le massif s'éclaircit, les essences feuillues de la région, et notamment les chênes dont les glands sont apportés par les oiseaux et les rongeurs.

$2^{\circ}$ Conversion en futaie résineuse. - La restauration de l'état boisé peut se proposer un autre but; s'il s'agit par exemple de mauvais taillis, incapables de donner une production avantageuse, le propriétaire peut être conduit, même si la forèt ne renferme pas de vides, à recourir aux résineux pour faire une forèt plus riche et plus productive.

Il peut agir par voie de plantation artificielle; faisant coupe rase du taillis, il plante des pins sylvestres sur les sables, des pins noirs sur les calcaires, et d'après M.M. Boppe et Jolyet, l'opération est en général fructueuse. Mais ce mode de procéder demande qu’on exécute un véritable reboisement; il demande en outre que des opérations culturales viennent pendant un certain temps légager les jeunes plantations et les protéger contre l'envahissement des rejets des essences feuillues.

Pour réduire la dépense, et procéder d'une façon progressive et plus lente, il peut suffire d'appliquer le système que préconise M. Runacher dans son mémoire présenté au Congrès international de sylviculture en 1900 : planter à chaque révolution du taillis cent épicéas ou sapins (1), mème deux ou trois cents, qu'on exploitera

(1) L'épicéa se recommande par la facilité de sa reprise; le sapin par une uplitude â se réensemencer naturellement au milieu des taillis. Rappelons tou- 
vers l'âge de quatre-vingt-dix ans; ces arbres finiront probablement, à la longue, par ensemencer les taillis en résineux; ajoutons mème que rien n'empèche d'utiliser par places le pin Weymouth et le mélèze qui, dans les situations qui leur conviennent, méritent de coopérer à cette œuvre de reslauration.

En montagne, si la futaie résineuse doit prendre la place du taillis composé, il peut ètre avantageux d'opérer plus radicalement. Le but qu'on se propose alor's est de créer un massif plein en résineuxx, à la place du taillis composé, dans le temps le plus court et a rec le moins de frais possible.

Suivant les cas, suivant les situations, plusieurs manières d'opérer peuvent être admises (1), mais le propriétaire qui veut réunir toutes les chances de réussite fera bien d'employer concurremment l'épicéa et le sapin, donnant, suivant les régions, la prédominance à l'une ou l'autre de ces essences ; il devra a voir soin en outre de conserver du hètre, et à son défaut du chène, des divers ou des bois blancs afin d'obtenir un mélange dans cette forèt naissante, mettant ainsi en pratique le conseil suivant de M. Broilliard (2): les épicéas ne durent pas bien longtemps sous le couvert de leurs frères; aussi se rencontre-t-il en sous-étage des sujets d'essences feuillues, hètre, coudrier, chène ou divers; ils sont fort utiles, gardez-les soigneusement; et si par hasard il se trouvait en mélange quelques feuillus en arbres, mème élancés

tefois que le sapin, tout en acceptant de vivre assez loin des montagnes où il est spontané, exige néanmoins des stations suffisamment fraíches, accidentées ou maritimes. Le plus sage sera, le plus souvent, de planter des épicéas en majorité, avec une faible proportion de sapins destinés à servir plus tard de porte-graines (Boppe et Jolyet).

(1) De très utiles conseils pour les opérations de ce genre sont résumés dans les trois mémoires présentés par MM. Runacher, Rosemont et Lamiable au Congrès de la société forestière de Franche-Conté et Belfort, tenu ả Gérardmer en 1902 et publiés au bulletin de la Sociélé. Nous ne pouvons que renvoyer le lecteur à ces intéressants travaux (Paul Jacquin, éditeur, Besançon).

(2) Brollliard, Revue des Eaux et Foréts, 10r janv. 1902).

A. Fron. - Sylviculture. 
au-dessus des épicéas, ainsi des bouleaux, un cerisier, un hètre, à moins qu'ils ne soient très nombreux, conservezles tels quels; ces feuillus sont les amis de nos amis, les oiseaux et les lombrics, si utiles et si rares dans les massifs d'épicéa.

Il résulte de ces considérations que les propriétaires particuliers ont souvent grand intérèt à se servir des résineux pour l'amélioration deleurs forèts, et qu'ils peuvent avec profit utiliser ces essences, beaucoup plus qu'on ne l'a fait jusqu'à présent. 


\section{TROISIEME SECTION}

\section{Étude spéciale des futaies.}

Peu de propriétaires particuliers possèdent des futaies d'essences feuillues, parce qu'un placement en futaie représente de longues épargnes accumulées, par suite un capital argent élevé, fonctionnant à un taux relativement faible, et parce que les essences feuillues se prêtent à d'autres méthodes d'exploitation (taillis el taillis composé) qui répondent mieux pour eux aux conditions économiques de placement qu'ils recherchent.

Nais par contre, les arbres résineux, ne rejetant pas de souche, sont tous forcément élevés en futaie, à révolution plus ou moins longue, suivant les cas et le but qu'on se propose.

L'étude des futaies feuillues ne peut toutefois ètre négligée, d'abord à cause des mélanges entre feuillus et résineux qui donnent très souvent d'excellents peuplements ; et ensuite parce que c'est en ne se confinant pas dans sa forèt, mais en regardant autour de soi, chez les autres, et en comprenant ce qui s'y passe, qu'on devient capable de gérer un peuplement quel qu'il soit.

Nous étudierons donc, en général, les divers types de peuplements qui peurent se présenter; nous avons pris pour guide l'ouvrage de $\mathbf{M}$. Gayer, auquel nous avons emprunté une partie des renseignements qui suivent. 


\section{PREMIÉRE SOUS-SECTION}

\section{PEUPLEMENTS PURS.}

Les peuplements purs, uniformes ou homogènes, sont constitués, au moins en très grande majorité, d'une essence unique, qui donne seule son caractère propre au peuplement.

\section{I. - ESSENCES fEUILLUES.}

Chêne rouvre et chêne pédonculé. - Futaie régulière. - Le peuplement de chène pur prend naissance par semis, plantation (1) ou par régénération naturelle, et dans ce dernier cas, le semis général ne peut être obtenu qu'à la condition de voir toutes les réserves de futaie qui dominent les semis enlevés plus ou moins rapidement avec les années de semence.

Suivant son origine (2), suivant les qualités du sol ou re la station (climat, etc.), les conditions de croissance et de développement de ce peuplement sont très inégales, et ces différences s'accentuent plus ou moins avec l'âge. Le peuplement, toutefois, tire de sa constitution mème des caractìres spéciaux, qui permettent de résumer les phases successives de son existence.

a. Première jeunesse. - Croissance vigoureuse en hauteur pendant les deux premières années, si le repeuplement est suffisamment serré. (On admet que dans une

(1) Dans l'introduction du chène par semis ou plantation, on doit apporter une grande attention au choix, soit du chène rouvre, soit du chène pédonculé, suivant la qualité de la station; ces deux essences présentent en effet des exigences très différentes $\mathrm{a}$ ce point de vue.

(2) Le peuplement qui provient d'une plantation est, spécialement dans le jeune Age, toujours moins serré que celui qui provient d'un senis artificiel, ce dernier btant moins serré que celui qui provient d'un senis naturel. 
régénération naturelle, le repeuplement est assuré par la présence d'un jeune plant par mètre carré en moyenne, mais généralement le semis naturel est beaucoup plus serré, tout au moins par places.)

b. Formation en massif. - Dès la troisième année, la croissance en hauteur se ralentit, et toute la vigueur de la végétation se porte sur le développement des branches latérales, développement qui hâte la formation du fourré. Cet état persiste jusqu'à l'âge de huit à dix ans, souvent plus, spécialement en sol pauvre, en situation exposée aux gelées, etc. Puis la production foliacée devient abondante, l'état de massif est obtenu, et le peuplement se présente à l'état de fourré épais et bien constitué, à couvert complet et très bas (fig. 39).

c. Relèvement du couvert. - A cette phase succède le relèvement progressif du couvert par l'élagage naturel; les tiges se poussent en hauteur, et cette croissance en hauteur dure plus ou moins, suivant la qualité de la station; en bonne station (climat tempéré, sol profond, frais, etc.), le maximum d'accroissement en hauteur est atteint dès l'àge de trente à quarante ans; en station médiocre ou mauraise (climat rude, sol maigre, etc.), ce maximum n'est atteint que plus tard (trente-cinq à soixante ans), à la condition toutefois que l'état de massif puisse persister jusque-là.

Le massif qui peut être assez dense jusqu'à létat de bas perchis, tend ensuite à s'éclaicir plus ou moins fortement (fig. 40).

d. Desserrement du massif. - En station médiocre ou mauvaise (sol peu frais et peu fertile), l'élimination qui se produit dès l'état de bas perchis s'accentue rapidement, le massif s'éclaircit, le couvert et l'abondance des détritus végétaux (feuilles, etc.) diminue, et le sol perd de sa fraîcheur et de sa fertilité; les herbes l'envahissent, les fûts se couvrent de mousses et de lichens, et à part quelques sujets vigoureux qui persistent dissé- 


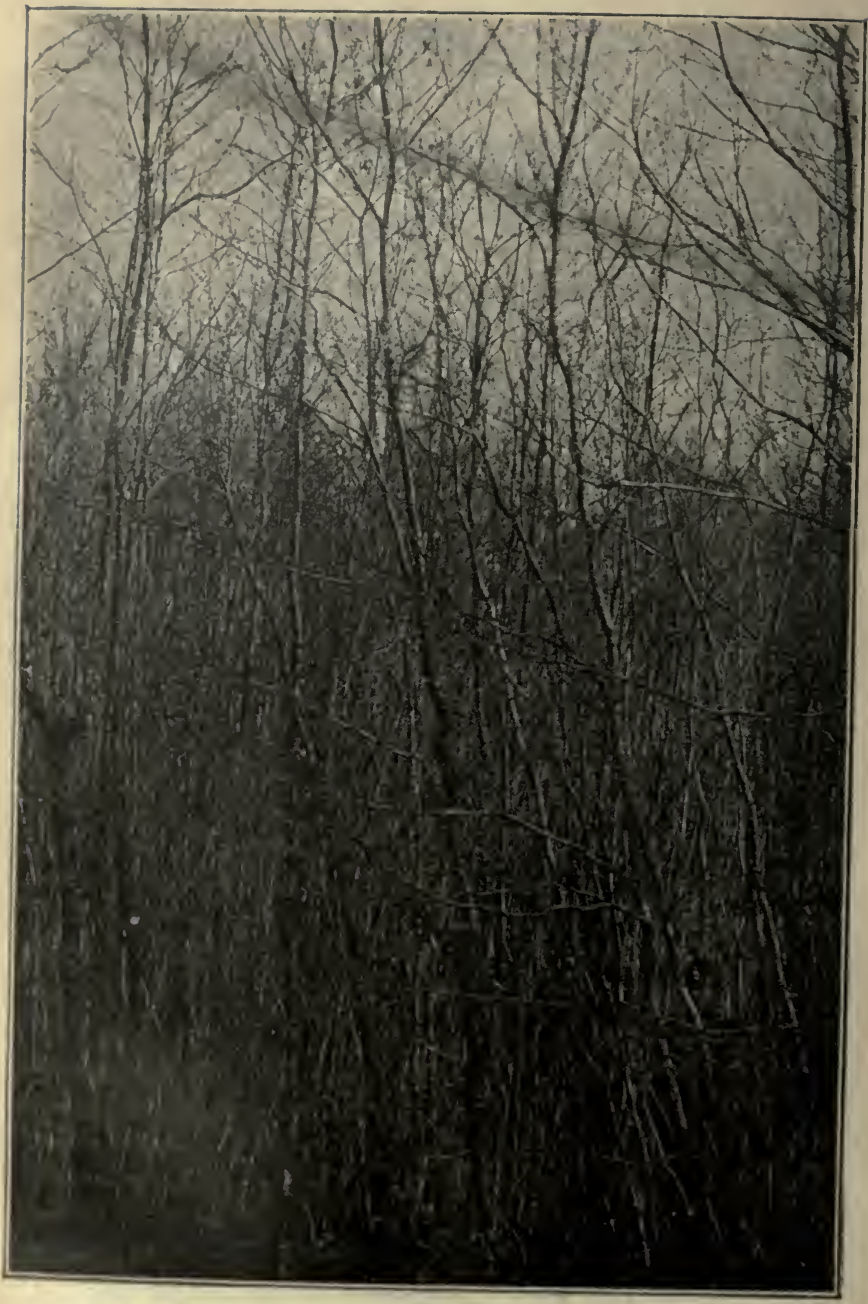

Kig. 39. - Peuplement de chêne pur à l'état de fourré. Forêt domaniale de Blois (Loir-et-Cher). 


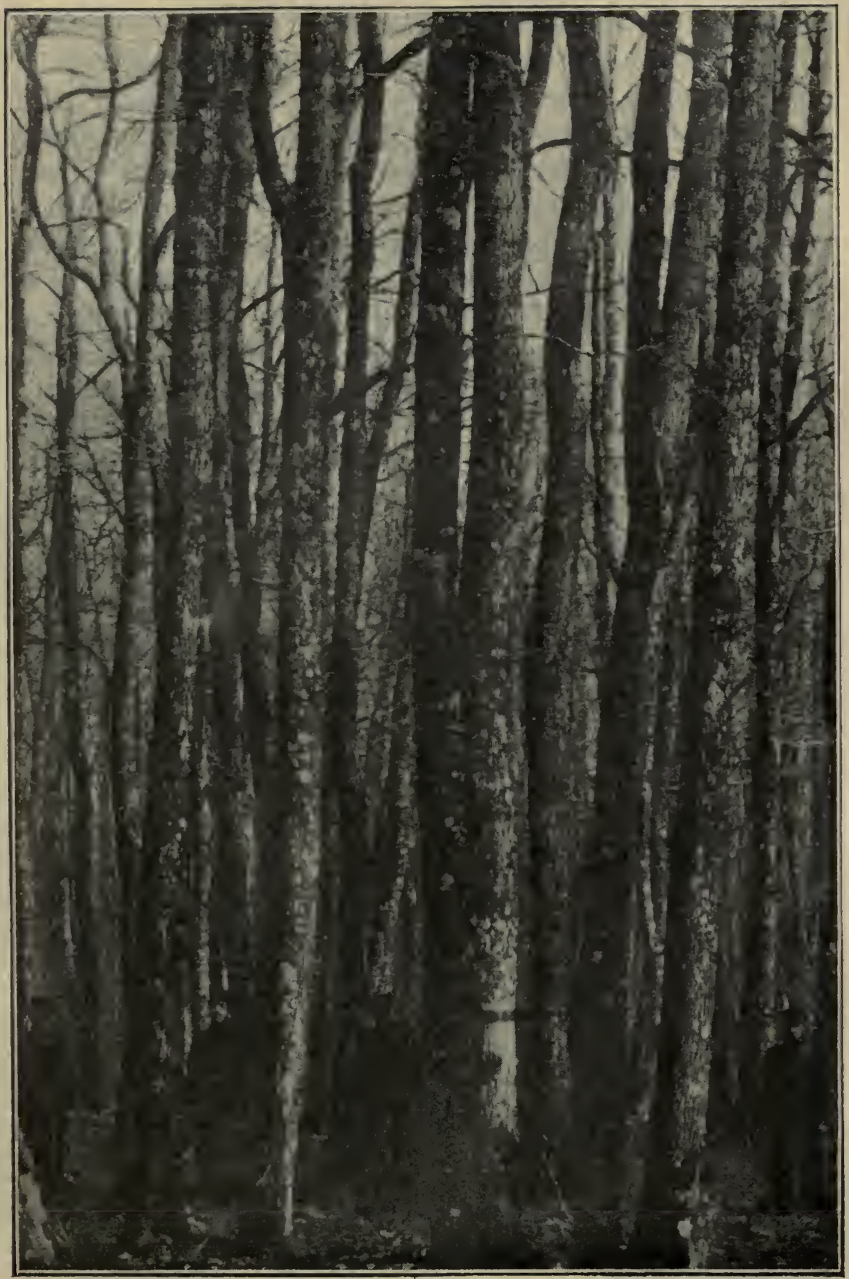

Fig. 40. - Peuplement de chêne pur à l'état de haut perchis. Forèt domaniale de Blois (Loir-et-Cher). 
minés, la régétation se ralentit et devient mauraise. Les conditions dès lors sont défavorables pour obtenir la régénération naturelle du peuplement.

En station favorable (sol fertile, humeux, abondamment pourvu d'humidité, climat favorable, etc.), par exemple sur les alluvions des grandes vallées fluviales (1), la tendance à l'éclaircissement s'accentue dès l'âge du perchis, et peut aller jusqu'au complet isolement et à la rupture entière du massif, mais cette mise en lumière progressive des sujets d'élite a pour effet, non seulement de stimuler vigoureusement leur croissance et de provoquer la production d'un fort volume te bois, mais encore de maintenir cette croissance pendant une longue période et de donner au bois une bonne qualité. Dans de telles stations, suivant que le sol sera ou ne sera pas profond et fertile, les arbres seront hauts et élancés ou ceurts et étalés (Gayer).

Les chènes provenant de peuplements purs ne présentent pas les formes régulières et élancées qui caractérisent ceux des futaies mélangées; dans tout le nord et l'est de la France, et de moins en moins, au fur et à mesure qu'on s'avance vers le centre, vers l'ouest et la région girondine, les massifs purs de chène sont exposés à souffrir pendant feur période de jeunesse des effets de la gelée; les jeunes plants déeouverts sont alors tourmentés et retardés; ils s'élèvent difficilement au-dessus de la zone dangereuse et conservent souvent pendant toute leur existence les traces de cette lutte.

Il résulte de ces diverses considérations, qu'à tous points de vue, il y a lieu de préférer, dans toute station, à la futaie régulière de chène pur, un peuplement d'essences mélangées, où le chène pourra èlre, suivant la station, l'essence principale et la plus abondante.

c. Repeuplement naturel. - Le chène se régénère

(1) Futaies de chêne de l'Adour. 
facilement par ensemencement naturel, tout au moins dans les stations qui lui conviennent. En raison de l'éclaircissement prononcé du massif de chène pur, dès qu'il arrive à un âge un peu élevé, les coupes préparatoires ne sont pas nécessaires; les coupes de régénération s'effectuent en général assez rapidement; la coupe d'ensemencement, toujours sombre, est souvent inutile, si ce n'est pour faire disparaître les sous-bois et l'étage dominé, c'est-à-dire relever le couvert (fig. 41); on doit nettoyer radicalement le sol, excepté pourtant dans les régions où les années de semence sont rares ou très rares ; en pareil cas, on attendra pour faire l'opération que la glandée soit certaine. Partout et toujours il y a lieu d'effectuer un crochetage au moment de la chute des glands.

La semence lourde du chêne tombe au pied des portegraines, qui dès lors doivent être nombreux et régulièrement répartis sur toute la surface à régénérer. Le semis général ne peut être que le résultat d'une glandée complète, car les résultats partiels disparaissent rapidement sous un couvert prolongé (1). Aussitôt après la glandée générale, dès qu’on juge la régénération acquise, on est conduit à faire très rapidement les coupes secondaires et définitives, et on les effectuera dès la deuxième ou la troisième année par exemple, si le climat s'y prête, si l'on ne redoute pas les gelées, et si l'on n'a aucun motif de ronserver le peuplement primitif une fois qu'il a rempli ses fonctions de porte-graine; on y procédera dans les six ou dix années' qui suivent l'ensemencement, parfois même encore plus lentement dans certaines stations humides et mouilleuses, si l'on redoute l'abondance de

(1) MM. Boppe et Jolyet font remarquer que souvent les jeunes semis de chêne, dont la tigelle semble morte, conservent dans les régions avoisinant le collet de la racine, une vitalité \&uffisante pour émettre des rejets qui s'élancent de nouveau dès que la lumière leur parvient. Aussi dit-on que les jeunes chênes se recèpent sous le couvert. 
l'herbe, les effets de la gelée, l'envahissement des mortbois, etc. D'après MII. Boppe et Jolyet, dans la région girondine, une coupe secondaire ou deux au plus, précèdent la coupe définitive; dans le centre et l'ouest, deux ou trois coupes secondaires sont nécessaires ; enfin, dans le nord et l'est de la France, on doit procéder plus lentement pour éviter les accidents de gelée et pour permettre aux essences de remplissage de combler les lacunes d'une régénération trop souvent incomplète.

Dans les bons sols, il peut ètre avantageux de laisser quelques beaux sujets isolés bien hauts de fût, comme réserves au-dessus de la coupe définitive afin de leur faire acquérir, par la mise en lumière, un développement et par suite une valeur supérieure.

Remarquons qu'un propriétaire particulier peut trouver un gros inconvénient à jeter sur le marché, aussi rapidement, une grande quantité de gros bois de chêne ; dès lors, il peut ètre amené à n'effectuer la régénération que par parties, en profitant de glandées successives; chacune des glandées lui donne un certain nombre de bouquets, petits ou grands, répartis irrégulièrement. Si en procédant de cette manière on a soin de favoriser, fût-ce mème artificiellement, la création d'un certain mélange, la méthode de traitement devient, dans un grand nombre de cas, plus sûre et plus commodé pour le propriétaire particulier.

f. Soins culturaux. - Les dégayements de semis sont presque toujours indispensables, sauf peut-être dans la région girondine; leur nécessité s'impose partout oủ la rareté des années de semence permet aux mort-bois et au hètre de s'installer avant le chêne.

Pendant la période de jeunesse, il est plus nécessaire d'entretenir le massif et la fertilité du sol que de stimuler la croissance, et jusqu'à l'état de bas perchis, le chène pur peut former des massifs assez denses; les premières éclaircies seront donc modérées et très prudentes; mais à partir 


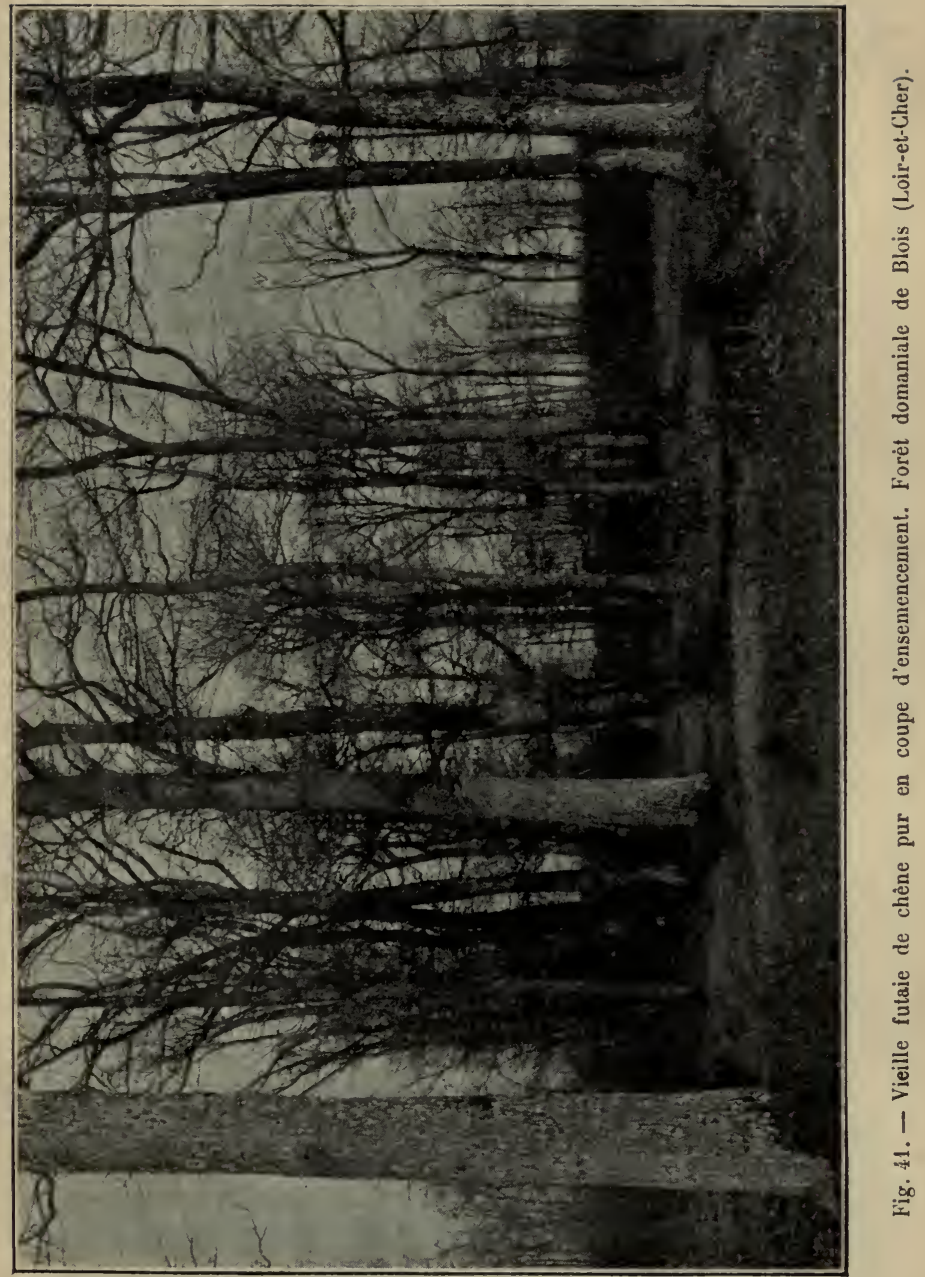


de la période de perchis, un tel peuplement, constitué par une essence delumière et d'espace, demande de plus fortes éclaircies: les cimes ont besoin d'ètre desserrées, et dès qu'on voit les tiges se couvrir de gourmands on peut-ètre certain que les arbres soufrent et qu'il faut intervenir; les éclaircies deviennent rapidement plus fortes, et il faut alors procéder hardiment en fareur des tiges d'arenir qui s'affirment; on peut revenir tous les dix ans et mème plus souvent, jusqu'à l'état de haut perchis; MI. Boppe et Jolyet conseillent d'enlerer au passage de ces éclaircies certains chènes dominés qui sont voués à une mort inévilable, mais de respecter tous les sous-bois de hètre ou d'essences diverses qui peuvent exister. Dans les hautes et vieilles futaies, l'éclaircie peut ne revenir sur le même point que tous les vingt ans.

Notons enfin que, dans de tels peuplements, il n'est permis d'agir dès le début avec moins de prudence, que lorsqu'il s'agit de sols très fertiles et de massifs très serrés.

Hêtre. - Futaie régulière ou quasi-régulière. - Le hètre a la semence lourde, le couvert trìs épais etle jeune plant très délicat; il en résulte que les forèts, dont il est l'essence dominante, se régénèrent facilement par la semence, et que le hêtre devient sourent, si on n'y met ordre, l'essence unique de la futaie.

En fait, ce n'est en général qu'au moyen de la régénération naturelle, complétée au besoin par des plantations, qu'on obtient des massifs purs de hitre. Le jeune peuplement nait ainsi sous l'abri de porte-graines yu'on fait tomber au fur et à mesure que le semis est constitué. En raison de son tempérament qui lui permet de résister longlempss sous le couvert, et du poids des faines, le jeune semis est géméralement réparti par bouquets; sa croissance reste assez lente pendant une dizaine d'années, tant que le fourré n'est pas complètement constitué et que - l'élat de massif n'est pas aequis. Puis l'accroissement en 
hauteur saccentue de plus en plus, pendant toute la période de gaulis, et bien souvent, en France, le hêtre croit plus vite que le chène pendant presque le premier demisiècle de son existence; mais au delà, sa végétation reste relativement faible et assez lente. L'état de perchis, entre la trentième et la cinquantième année, correspond ordinairement au maximum de croissance, et dans les bonnes stations, les pousses s'allongent de 30 à 50 centimètres par an; cette vigueur de la végétation dépend toutefois de la fertilité du sol et de la densité du massif. C'est pendant la période du perchis, dit $\mathrm{M}$. Gayer, que le sol se recourre d'une abondante couche de feuilles mortes, et que grâce à la densité du peuplement et au peu d'élévation des cimes, les effets du vent et de la sécheresse sont le plus atténués; c'est le moment où la fertilité du sol est le plus active, et où le peuplement concourt le plus efficacement à son entretien; c'est alors aussi que se présente le maximum de son accroissement en volume, en général au milieu de la phase du perchis, entre quarante et soixante ans.

Un tel peuplement de hêtre pur ne restera en bon état, surtout dans les terrains de fertilité et d'humidité médiocres, qu'à la condition de conserver rigoureusement la couche de feuilles mortes et de terreau qui recouvre le sol et d'en empêcher la destruction soit par un enlèvement continu de litière, soit par l'action du vent qui dans les peuplements exposés est néfaste, tout au moins pendant la deuxième moitié de l'existence du peuplement; sous de telles influences, le sol tend à se dénuder et à se dessécher; les conditions d'une bonne croissance cessent d'exister, car la feuille du hêtre, toujours lente à se décomposer et à se pourrir, exige plus que d'autres, pour former une bonne couverture et de l'humus, une grande humidité et une atmosphère calme; dans de mauvaises conditions cette couverture se dessèche, le sol perd par évaporation son humidité et se durcit; on voit dès lors 
apparaitre la myrtille et la bruyère qui deviennent facilement envahissants, et le nombre de plus en plus considérable des arbres morts et dépérissants dénote une végétation languissante; le peuplement s'éclaircit jusqu'à disparaître complètement.

Dans les conditions moyennes, le peuplement de hètre pur est exploitable vers l'âge de cent à cent vingt ans, et on peut y faire les coupes régulières de futaie. D'après M. Gayer, un bon massif moyen, obtenu dans de bonnes conditions, peut renfermer 550 à 750 arbres par hectare à l'âge de cent vingt ans, et donner un produit de 500 à $7 \% 0$ mètres cubes.

D'après M. Broilliard, qui conseille pour le hêtre des éclaircies fortes et hardies, dans un sol où la production du massif plein est de 5 mètres cubes à l'hectare par an, la futaie de hêtre peut donner en un siècle 150 à 200 mètres cubes de produits accessoires dans les éclaircies, et 300 mètres cubes de produits principaux, dont moitié en bois d'œuvre dans les coupes de régénération; à raison de 10 à 13 francs le mètre cube, cela représenterait au moins 6000 francs, soit un produit moyen de 60 francs par hectare et par an.

Une grande partie des produits des futaies de hêtre est encore aujourd'hui employée comıne bois de chauffage; toutefois le hêtre donne un. excellent bois de travail qui trouve de nombreux emplois dans la menuiserie, l'ébénisterie, le charronnage, la carrosserie, la boissellerie, le sabotage, etc. ; la compagnie de l'Est l'emploie actuellement pour faire des traverses de chemin de fer après l'avoir injecté, et cet essai parait donner de bons résultats. Ce serait une faute que de se désintéresser aujourd'hui de tels massifs qui peuvent exister sans mélange et à des âges les plus diver's chez plusieurs propriétaires particuliers. En général, des forêts de celte nature conslituent d'excellentes stations où on peut songer à introduire avec succès, et grand avantage, le chène en mélange 
dans le peuplement; le hètre est susceptible en effet de constituer, associé au chène, au sapin, à l'érable, au pin sylvestre, etc., d'excellents mélanges.

Régénération naturelle par coupes successives. - Dans la régénération naturelle du hètre, le point essentiel est. de tenir compte des conditions de la station où l'on opère, conditions qui sont très variables en raison de la diversité des stations de cette essence, et de tenir compte aussi des phénomènes qui influent sur la première croissance du hètre en variant d'intensité d'un endroit à un autre. On n'oubliera pas, dit M. Gayer, que le jeune hètre demande un sol délité, suffisamment meuble et humeux, mais libre d'acide et frais; que la lumière lui est très favorable, à la condition qu'elle ne lui soit pas donnée à trop haute dose, c'est-à-dire avec chaleur trop forte, qu'il est très sensible à la sécheresse et plus sensible encore à la gelée jusqu'à l'âge de gaulis; enfin qu'il supporte bien un couvert prolongé.

C'est dans les formes régulières que nous devons user avec le plus grand soin des moyens qui sont à notre disposition pour favoriser cette régénération (coupes bien dirigées, adaptées aux circonstances, bonne préparation du sol, etc.)

En général, on peut dire :

$1^{\circ}$ Les coupes préparatoires, si elles sont nécessaires mème dans des peuplements déjà éclaircis, doivent être effectuées lentement; si leur action est par places insuffisante pour la préparation du sol, on doit les compléter par l'intervention d'une certaine culture; ainsi, suivant les cas, on pratiquera un ratissage des couches trop épaisses de feuilles mortes, on effectuera un crochetage des surfaces tassées, de la couverture vivante, des mousses et plantes diverses qui tapissent le sol, retournant partiellement et par places la terre par grosses mottes, ouvrant quelques bandes ou sillons; ou bien on pourra se contenter d'introduire des troupeaux de 
porcs sous bois pendant la période préparatoire, etc.

$2^{\circ}$ La coupe d'ensemencement doit ètre faite d'une façon très prudente, très sombre, disent MM. Boppe et Jolyet, formant un abri régulièrement réparti si la station est fertile, interrompu par petites trouées si le sol est paurre et superficiel; on laissera donc les cimes des, portegraines se toucher presque, mais avec lintention de faire tomber assez rapidement une partie de cet étage supérieur dans les coupes secondaires dès que le semis sera formé ; on ne craindra pas de profiter des semis préexistants s'ils n'ont pas été trop longtemps dominés, et on saura attendre que les faînées partielles complètent une régénération insuffisante au début (fig. 42).

$3^{\circ}$ Les coupes secondaires seront très prudentes, au nombre de quatre à cinq, limitées chacune à l'enlèvement d'un arbre sur trois ou quatre arec retour tous les cinq à six ans. Toutefois, suivant les stations, la première de ces coupes pourra devenir rapidement urgente; les suivantes, ainsi que la coupe définitive le seront moins en raison du tempérament du hêtre, et on n'a plus intérèt, en général, à les conduire rapidement; il est au contraire bon d'assurer aux jeunes plants pendant quelque temps l'abri et la protection contre les gelées.

Notons enfin, qu'en principe, chez le hètre, il est utile ¿'opérer par coupes restreintes, et non par surfaces de trop grande étendue.

Opérations culturales. - a. Dégagements de semis. - Le hètre se défend contre les semis de toutes les autres grandes essences; parfois des dégagements peuvent s’imposer pour le protéger contre l'envahissement des rejets, des morts bois et des ronces.

Éclaircics. - Le hètre se plaît en massif serré; la première éclaircie n'est nécessaire qu'une fois l'élat de bas perchis bien affirmé. Celle essence possède la faculté d'allonger rapidement ses branches du côté du jour, et de refermer ainsi très promptenent des trouées faites 
dans le massif; il en résulte qu'un jeune perchis de hêtre peut ètre éclairci sans grandes précautions; toutefois il est encore nécessaire d'agir prudemment pour favoriser l'élagage naturel, et éviter l'envahissement des ronces sur le sol; on respectera d'une façon absolue l'é-

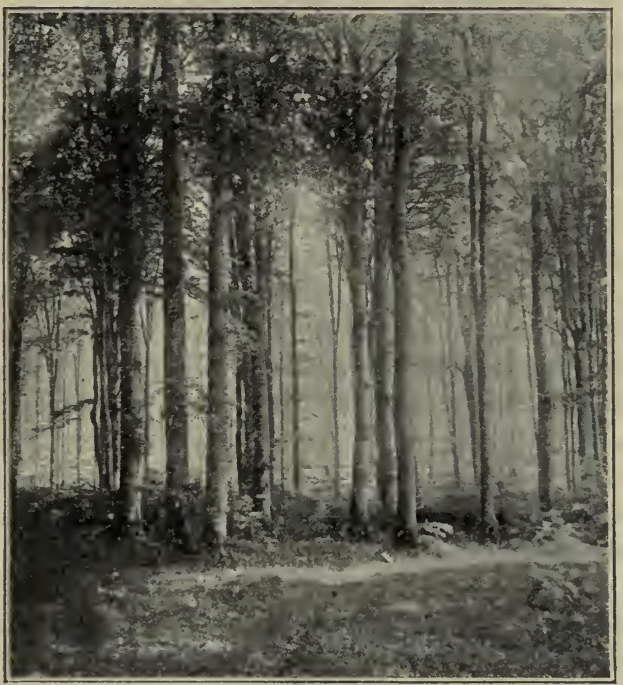

Fig. 42. - Coupe d'ensemencement et, au dernier plan, coupe secondaire dans une futaie de hêtres, forêt de Lyons-la-Forêt (Seine-Inférieure). (Photographie de MM. J. George. "Les Forêts ", par MM. Boppe et Jolyet.)

tage dominé, et on profitera du passage des éclaircies pour enlever systématiquement tous les sujets branchus ou tarés qui ne paient pas la place qu'ils occupent.

Dès que les sujets du peuplement principal commenceront à croître avec vigueur, les éclaircies se suirront à de courts intervalles et seront de plus en plus fortes; à partir del'âge de quarante ans, d'a près $M$. Broilliard, on 
peut, en se bornant à desserrer les plus belles cimes par l'éclaircie, enlever tous les dix ans 30,40 ou כ̋0 stères à l'hectare, suivant le sol, sans dégrader le peuplement. Si l'on a soin de conserver tous les brins dominés encore vivants, on peut mème isoler pour ainsi dire, dans chaque éclaircie, les cimes des sujets d'arenir, car le massif se referme rapidement; les arbres grossissent trìs vite, et le capital engagé dans la futaie se trouvant périodiquement réduit par un prélèvemenf sensible; le taux du placement reste longtemps suffisant pour satisfaire l'intérèt des propriétaires particuliers.

Pour tendre à la futaie mélangée, toujours préférable à la futaie de hêtre pur, il y a lieu, dans toutes ces opérations d'éclaircie, de ménager, en les desserrant largement, les chènes bien venants qui se rencontrent, de conserver les beaux pieds de bouleaux et de bois blancs, qui gènent très peu le hètre, et de mème les frènes disséminés, les charmes nécessaires pour compléter le peuplement, et les sujets de toutes essences quand ils sont plus utiles que nuisibles. Néanmoins le hètre devient peu à peu très prédominant, parce que le régime de la futaie lui est plus favorable qu'à tout autre arbre feuillu (Broilliard).

Hêtre. - Futaic à coupes jardinatoires. - Dans les vieilles futaies de hètre, à massif irrégulier, et à croissance variable, sur les lisières des grandes étendues boisées, sur les plateaux des montagnes moyennes, ou dans les bouquets de bois isolés au milieu de la plaine, il est très bon d'obtenir des peuplements constitués par des bouquets ou groupes formés en massif et d'àges divers; l'opération du repeuplement doit s'étendre alors sur une assez Iongue période, d'environ vingt à trente ans, et la régénération s'effectue pendant toute cette période par groupes et bouquets.

A ce point de vue, d'après M. Gayer, la première chose à prendre en considération, ce sont les bouquets de 
semis préexistunts utilisables, comme il s'en présentètoujours dans les lacunes et les massifs clairs; ce qui est considéré comme utilisable doit ètre amené à bon développement au moyen de coupes secondaires ou définitives convenablement dirigées; on doit conserver les semis préexistants mème s ïls ont atteint les dimensions de perchis, s'ils sont susceptibles de se former bientot en massif continu avec des semis avoisinants; on doit profiter de toute faìnée, mème peu abondante, pour provoquer la formation de nouveaux bouquets, et on dégage progressivement ceux qui existent; en mème temps, on ne doit pas perdre de vue les lisières sans abri et les endroits exposés à perdre leur fertilité. Les bouquets peuvent en général ètre tenus assez grands, mais on peut aussi procéder par groupes plus petits.

Pendant que les opérations de la régénération se transportent lentement d'un point à un autre, les parties non encore entamées doivent être maintenues en massif plein, si elles ne sont pas encore soumises aux coupes préparatoires.

Chêne-liège, futaie régulière. - Le chène-liège, répandu autour de la Méditerranée, et sur les versants de l'Atlantique ne s'accommode que de terrains franchement siliceux, de granite, porphyre, schistes, grès ou sable, ce qui en limite le domaine d'une façon très étroite. Il n'acquiert pas de grandes dimensions, soit en hauteur, soit en diamètre, et se traite en futaie régulière très clairiérée, dans un but d'utilisation industrielle tout spécial.

A l'état naturel, l'écorce du chène-liège, très rugueuse, fortement crevassée, et qui peut devenir très épaisse, est sans élasticité et sans valeur; mais si l'on a soin de l'enlever au préalable, l'arbre se recouvre d'un liège de reproluction, d'une grande valeur industrielle et commerciale. L'opération qui consiste à enlever ce liège est ce qu'on appelle le démasclage. Nous renvoyons à des 
ouvrages spéciaux pour l'étude de cette production industrielle (1).

D’après M. Broilliard, sous les forèts clairiérées de chène-liège, arbre à couvert léger, se développe un sousbois plus ou moins épais; ce sont au nord de hautes bruyères, des myrtes, des arbousiers, des philarias à larges feuilles que des ronces et des smilax épineux enguirlandés aux arbres peuvent rendre impénétrables.

Sur les coteaux exposés au soleil se trouvent des fourrés moins élevés formés surtout de bruyères. Dans les clairières, la broussaille est composée de lentisques, myrtes, philarias à feuilles étroites, oliviers sauvages, palmiers nains, genèts épineux et cistes, lavandes, etc. Au printemps, se montre une herbe abondante; en élé, la vie semble s'éteindre, et le sol est couvert d'herbes et de feuilles desséchées, aliments tout prêts pour un feu rapide. De là d'effroyables incendies, danger permanent pour les forèts de chène-liège.

\section{II. - ESSENCES RÉSINEUSES.}

Sapin pectiné, futaie régulière ou quasi régulière. - Le jeune plant de sapin présente la faculté de se maintenir longtemps sous un couvert épais, et de continuer à végéter lentement, pouvant attendre ainsi, plus ou moins longtemps suivant la qualité du sol, qu'on vienne: lui donner la lumière nécessaire à son développement. Il en résulte que les futaies dont le sapin est l'essence dominante se régénèrent facilement par la semence.

Le jeune peuplement de sapin pur, naît sous l'abri des porte-graines qu'on fait tomber au fur el à mesure que le semis est constitué. Le jeune plant commence par étaler ses branches latérales; il devient rerticillé ver's l'âge de six à huit ans, et dès la dixième année il allonge

(1) Voir Bnoniınd, Trailement des bois en France, et Lamey, Le chéne. liège, 1893. 


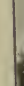

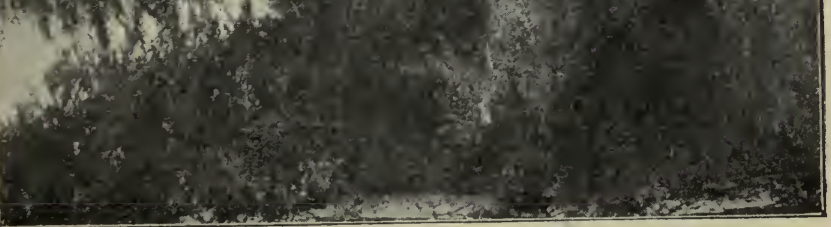


vigoureusement sa pousse terminale. De l'état de fourré, il passe à celui de gaulis et de perchis (fig. 43) avec des accroissements annuels en hauteur de plus en plus forts ; rette croissance en hauteur atteint de bonne heure son maximum; dans de bonnes conditions entre vingt et trente ans, ordinairement entre trente et quarante ans, et dans des circonstances très défarorables, entre soixante et soixante-dix ans (Gayer); elle se maintient vigoureuse jusqu'à un âge avancé (fig. 44 et 4 5̆).

Régénération sous le courert par coupes successives. - Dans la régénération naturelle du sapin, il est bon de tenir compte des conditions de la station où l'on opère; privé prématurément d'abri, le jeune plant est exposé à soufrir de l'abondance des mauvaises herbes, de la gelée et de la sécheresse; la mousse très épaisse lui nuit aussi, en arrêtant les. précipitations atmosphériques; mais sur un sol nu, ce sont surtout la gelée et la sécheresse qui lui sont funestes.

L'exploitation en bloc d'un massif de vieux sapins ne peul être admise que s'il s'agit d'une petite surface, d'un bouquet de bois à exploiter en un ou deux ans, mais dans ce cas le repeuplement du sol doit en général être complété dans une large mesure par voie artificielle; l'abri latéral de massifs voisins peut dès lors ètre indispensable pour faciliter l'opération.

L'exploitation graduelle s'impose dès qu'il s'agit de surfaces plus au moins étendues, pour obtenir la régénération naturelle par coupes sous le couvert.

En général, les coupes préparatoires sont inutiles; tout au plus peurent-elles se borner à faire tomber quelques sujets chancreux, car le massif est suffisamment préparé par les éclaircies successives.

La cuupe d'ensemencement sera en général très sombre, sauf peut-être dans les bonnes stations où elle peut indifféremment ètre moins sombre; la présence d'une couche de mousse de peu d'épaisseur, alternant arec 
38.

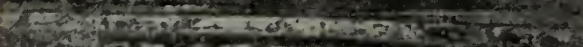

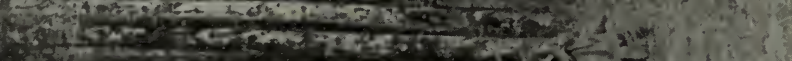

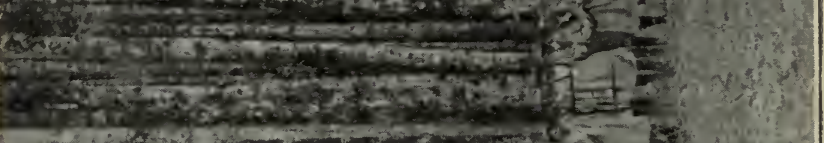

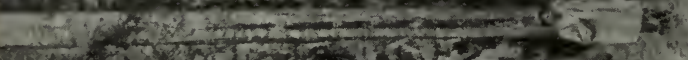

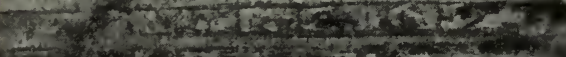

$x^{2} x^{2}$ o

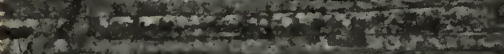

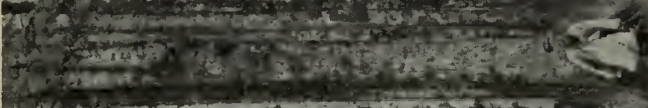

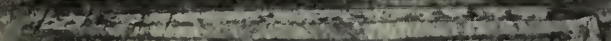

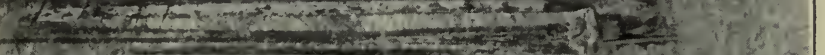

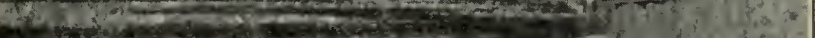

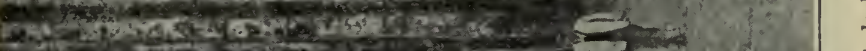

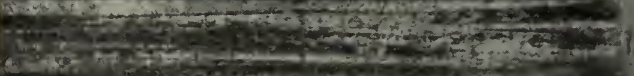

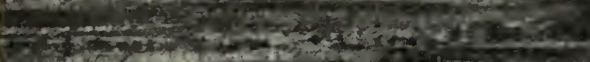

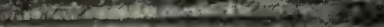

Clin ab

A A.

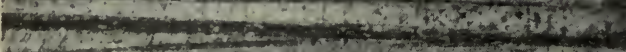

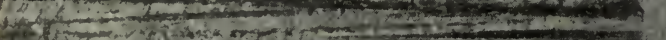

$2013 T^{2}$.

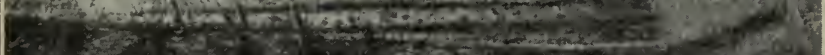

1.5.

Shat $\rightarrow$,

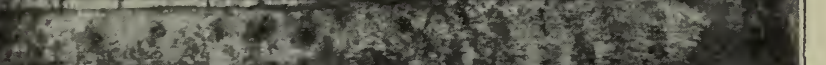
exply:

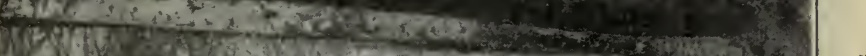
7. 1 . -

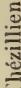

Ð

$\frac{0}{5}$

:

बाँ

(5)

$\frac{\dot{m}}{\frac{10}{Z}}$

.

Е

d)

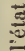

$\approx$

㶓

ஓ्

.

ڤั)

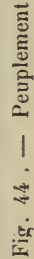

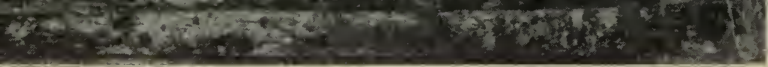


une végétation claire de myrtilles n'est pas défavorable à l'installation du semis; toutefois, si cette couverture vivante est trop abondante, il peut y avoir lieu de la détruire (herbes, myrtille, bruyère) et de mettre le terreau à nu par bandes. Il est inutile de recéper les mort-bois feuillus (sureaux, coudriers, etc.) qui envahissent fréquemment le parterre des coupes ; car arec le temps le sapin s'installera, sous leur couvert devenu moins épais, dans l'excellent terreau formé par leurs détrilus.

Les coupes secondaires se commencent en général quand le sapineau est verticillé, c'est-à-dire àgé de six à huit ans; les premières ont avant tout pour objet de mettre en lumière les bouquets de recru bien formés, el les semis préexistants dont l'avenir ne paraît pas compromis; les suivantes sont conduites avec lenteur; les semis préexistants, qui n'ont plus d'avenir, sont détruits, ou bien conservés pendant un certain temps comme abri et dès lors élagués peu à peu a vant de disparaître.

Aux grandes altitudes, où il $\mathrm{y}$ a lieu de craindre les chablis, il est permis de laisser la régénération s'installer solidement pendant un certain temps, puis après dix ou douze ans, de passer sans transition à la coupe délinitive.

Le maintien sur coupe définitive de quelques beaux sujets d'élite peut ètre une bonne opération en station fertile; l'arhre est propre, d'une façon très évidente, à être mis peu à peu en lumière, el à condition qu'on l'isole d'une facon progressive, il est susceptible de résister et de donner des produits de valeur supéricure.

Opérations culturales. - Dégugements de semis. - Le jeune sajuin arrive en général à percer, par ses seuls moyens, des fourrés épais de hètres ou do mort-bois. Néanmoins, afin d'éviter que les sujets dominés soient déshonorés par la perte de leur fliche, il est toujours utile d'intervenir par des dégagements de semis sobres et par suite peu couteux (Boppe et Jolyet). 


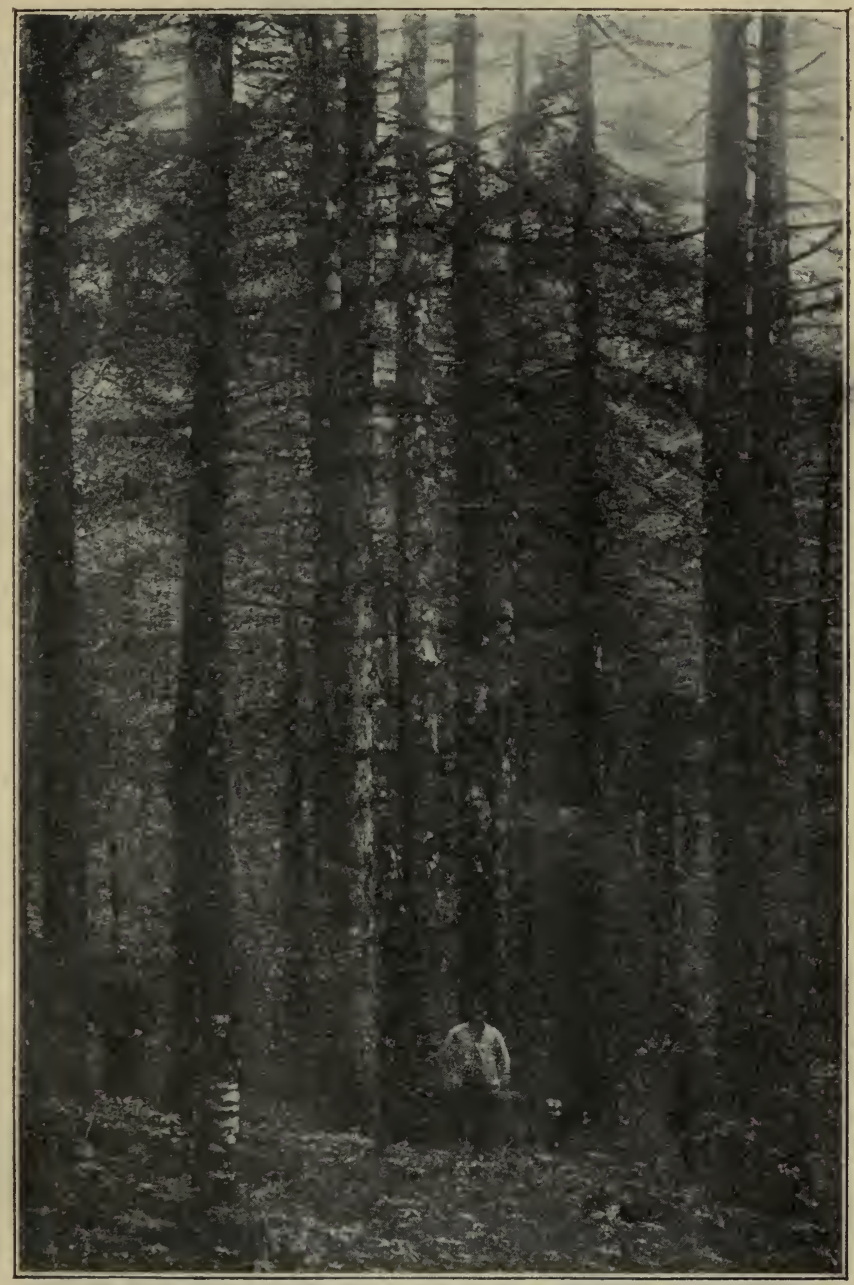

Fig. 43. - Futaie régulière de sapin. Forêt domaniale de Thézillien (Ain). A. Fron. - Sylviculture. 
Eclaircies. - Dans un jeune peuplement régulier ainsi obtenu, les sapineaux, très nombreux d'ordinaire, se pressent, et dès lor's le massif ne tarde pas à se diviser en deux ou plusieur's étages. Dans le peuplement dominant les sujets d'arenir se dessinent, et il est temps d'intervenir par des éclaircies pour desserrer les plus hautes cimes, en diminuant leur nombre. M. Broilliard appelle cette éclaircie, qui consiste à venir prendre des tiges une à une, et d'un point à un autre, une éclaircie jardinatoire ; il est bon d'y revenir fréquemment, tous les six à huit ans par exemple. L'éclaircie jardinatoire ainsi comprise permet d'obtenir de beaux arbres, assez rapidement et en maintenant l'état de massif ; elle peut se continuer indéfiniment, à la condition d'ètre prudente, et de ne jamais porter sur l'étage dominé. Lnfin on doit profiter du passage de ces éclaircies pour enlever avant tout les arbres champignonnés, chaudronnés, ou mal conformés.

Sapin. - Futaieà coupes jardinatoires. — Les formes irrégulières conviennent beaucoup mieux que les autres à la nature du sapin pectiné, et M. Gayer, à qui nous empruntons la description qui suit, les préconise à tous points de rue: considérons d'abord l'aspect du peuplement pendant sa jeunesse; le peuplement antérieur exploitable et présentant des différences d'âge de dix à cinquante ans est en usance depuis vingt à trente ans; il est très clariéré el très irrégulier; ici, il est encore formé en massif, le sol porte un recru peu abondant et qui échappe souvent à l'xeil, perdu dans la mousse et les myrtilles; là, il vient d'ètre enlevé, à l'exception de quelques vieilles réserves encore vigoureuses, dans le. but de donner aux jeunes bouquets de cinq à dix ans, déjà formés en massif, la lumière nécessaire ; ailleurs encore, ce sont des perchis, massifs de vingt à trente ans et plus, en pleine croissance. Ainsi l'aspect général est des plus variés; Ies classes d'àge les plus 
jeunes jouissent de l'abri des bouquets plus avancés, et de celui des restes de l'ancien peuplement; réparties d'abord en groupes isolés, elles s'étendent de plus en plus, grâce à l'enlèvement progresssif des vieilles classes qui les entourent et grâce à l'occupation graduelle des lacunes qui divisent les bouquets de perchis; enfin les innombrables groupes de repeuplement arrivent à se mettre en contact étroit, et le sol est entièrement recouvert par le massif ondulé et inégal des cimes; du peuplement primitif, il ne reste que des sujets réservés, plus ou moins nombreux, et le nouveau peuplement prend possession_du terrain. Rien ne peut mieux donner l'ilée de l'exubérance de la nature et de la vigueur de la végétation arborescente, qu'une jeune forêt de sapin traitée par la méthode des coupes jardinatoires.

Pendant toute la phase suivante, qui correspond à la période moyenne de son existence, le peuplement passe par l'état de gaulis et de perchis pour arriver à l'âge adulte. Par suite du peu de besoin d'espace de l'essence, et de la facilité avec laquelle elle supporte la privation de lumière, le massif reste serré pendant toute cette phase; aucun rayon de lumière n'arrive jusqu'au sol, la mousse elle-mème disparait complètement et fait place à la litière et aux restes abondants du peuplement accessoire. Des coupes judicieusement dirigées dégagent les plus vieilles parties du perchis pour stimuler leur croissance; les sujets ont les dimensions de la futaie en sortant de la période, et donnent même parfois, suivant la plus ou moins grande densité du massif, de la graine fertile. Pendant ce temps, les classes les plus jeunes entrent dans la phase active du perchis.

A l'âge de quatre-vingts ans environ, commence pour les plus vieilles classes la période de haute futaie; dans les bonnes stations, le massif reste serré; la croissance terminale se ralentit, l'insertion de la cime remonte à une grande élévation, l'enlèvement des bois morts et 
chancreux éclaircit peu à peu le massif, et la lumière, en réapparaissant sur le sol, y fait renaître peu à peu la mousse, au sein de laquelle le repeuplement commence lentement à se montrer.

Quand le besoin de régénération se montre ainsi dans les vieilles parties de la forêt, c'est signe que le moment de l'exploitation et des coupes d'ensemencement approche; on retombe au point de départ.

La culture ainsi comprise se distingue de la culture en peuplement régulier, en ce que le principal accroissement n'est pas recherché dans la jeunesse de l'arbre et dans le massif, mais bien au moment de la plus forte croissance à l'état dégagé, et en effet, chez certains sujets, le résultat est véritablement prodigieux.

Mais pour obtenir cet accroissement par la mise en lumière, un choix attentif des sujets et des stations est nécessaire; la spécialisation de la culture trouve là un champ d'opération des plus rémunérateurs; c'est là que se produisent ces pièces de dimension et de haute valeur, que le sapin est si apte à fournir en raison de sa forme et de la qualité de son bois (Gayer).

Régénération sous le couvert par groupes ou bouquets. - Dans la régénération du sapin par groupes ou bouquets, on se donne pour règle de tirer tout le parti possible des semis préexistants, et le choix à faire est de la plus haute importance.

En principe, d'après M. Gayer, on doit conserver tous les bouquets suffisamment formés en massif (de tout âge, jusqu'au bas perchis) et même ne pas dédaigner des sujets isolés s'ils sont vigoureux; on les écartera au contraire s'ils sont rabougris, mais, mème dans ce cas, ils doivent ètre provisoirement respectés comme bouchetrous et abris. Les coupes dès lor's prennent par places sur toute l'étendue du peuplement, le caractère de coupes préparatoires, de coupes d'ensemencement, secondaires ou définitives, ou encore de simples éclair- 
cies; dans les parties de peuplement encore formées en massif, l'enlèvement des sujets chancreux et mal venants, constitue une coupe préparatoire; dans les parties déjà entamées ou naturellement plus claires, mais non encore ensemencées, l'opération est une coupe d'ensemencement; enfin dans les recrus gagnant constamment du terrain, et dans les parties ensemencées, c'est une coupe définitive; et avec le temps des bouquets bien formés en massif finissent par occuper tout le terrain.

La durée de la régénération de tout le peuplement est fort variable; elle est de cinq à quinze ans pour les bouquets non préexistants, et en y ajoutant l'âge des semis préexistants, elle est de vingt, trente à quarante ans et plus, pour tout l'ensemble du peuplement, condition nécessaire pour le maintenir à l'état irrégulier.

Si l'on combine cette forme de peuplement avec le mélange d'essences (sapin avec hêtre, épicéa, chêne, etc., suivant les cas), on obtient des massifs irréguliers et mélangés, excellents à propager et avantageux pour un propriétaire particulier qui fait de la futaie de sapin.

Sapin. - Futaie jardinée. - Lejardinage vrai ne convient qu'aux essences d'ombre, et en fait c'est dans la sapinière qu'il a pris naissance, c'est à elle seule qu'il peut s'appliquer sans réserves (1).

D’après M. Gayer, dans la sapinière jardinée, les vieux arbres qui ont crû en bouquets se répartissent plus ou moins uniformément dans tout le peuplement, séparés par les groupes encore nettement tranchés de perchis el de fourré. Malgré ce groupement des différentes classes d'âge, les vieux sujets, considérés seuls, semblent dis-

(1) La présence du hêtre ne s'oppose en rien à l'application d'une méthode grâce à laquelle le mélange se maintient en d'excellentes proportions (Boppe et Jolyet). 
tribués d'une manière assez égale dans l'ensemble.

Mais il est évident, ajoute le mème auteur, que cette forme type n'est pas constante en tous temps et en tous lieux; selon la nature de l'exploitation et les perturbations de toute nature qui peuvent se produire, les unes ou les autres classes pourront prendre le dessus. Les forèts,jardinées de sapin de la petite propriété privée, assez soignées, mais exploitées d'après les principes les plus divers, nous montrent, tout en conservant leur caractère, des variétés de forme, qui vont depuis.la vraie forêt jardinée jusqu'à la culture plutôt jardinatoire.

Et en fait, si dans la culturejardinatoire, nous étendons la durée de la période de régénération à quarante ans et plus, au' lieu de la restreindre à une limite variant entre vingt et quarante ans, nous tendons vers la forme jardinée.

Conduite des coupes. - Dans la sapinière jardinée, la pratique des opérations est guidée par les principes suivants: agissant sur toute l'étendue de la forèt, on fait tomber sous les premières coupes les sujets malades, dépérissants et de fortes dimensions; en mème temps on se préoccupe des semis préexistants et des bouquets de recru qui sont dégagés par des coupes d'éclaircie plus ou moins fortes; enfin, on tend à conserver les arbres moyens, à bonne cime et vigoureux, en les répartissant irrégulièrement pour servir de porte-graines.

Epicéa. - Futaie régulière ou quasi régulière. - La futaie pure et régulière d'épicéa est rare dans la propriété privée; elle ne se trouve que dans les situations labritées de haute et de moyenne montagne, et en so frais et fertile.

Le peuplement prend uaissance, soit par semis ou plantation en terrain nu, soit par régénération naturelle au moyen de porte-graines, soit par ensemencement naturel et latéral au moyen de la semence venue d'un peuplement voisin. 
La caractéristique de l'épicéa est qu'il demande à ètre maintenu en massif sombre, mais que, de tempérament assez robuste, il végète mal à l'état dominé et se constitue naturellement en un seul étage. Sa cime, qui reste toujours conique, lui permet de vivre à l'état très serré, et de former des peuplements très riches en matériel.

Tant que le massif d'épicéa n'est pas formé, les jeunes épicéas s'étalent horizontalement et se développent peu en hauteur; dès la formation en massif, la croissance terminale saccentue; le massif se constitue naturellement en un seul étage, auquel le grand nombre de tiges conserve toute sa densité, et dès le commencement de la période de gaulis, le sol est aussi abrité que possible.

Parfois, à la suite d'un semis trop abondant, la densité mème du peuplement peut devenir un obstacle à sa bonne croissance, et si l'on n'y porte remède, les arbres dépérissent, mais en général l'élimination spontanée du peuplement accessoire se produit suffisamment, et le peuplement principal entre alors, avec des pousses terminales de plus en plus fortes, dans la période du perchis.

D'après M. Gayer, le maximun de la croissance de la pousse terminale se présente dès la première moitié de la période du perchis (pousses annuelles $0^{\mathrm{m}}, 40$ à $0^{\mathrm{m}}, 60$, entre la vingtième et la vingt-cinquième année); il se présente plus tôt dans les bonnes stations que dans les mauraises.

Le ralentissement de la pousse annuelle reste longtemps peu sensible, et sa croissance annuelle se maintient à $0^{\mathrm{m}}, 2 \mathrm{~s}$ pendant quatre-vingt-dix ans environ dans les bonnes stations, pendant soixante-dix ans dans les médiocres.

Pendant toute cette période de végétation vigoureuse, le peuplement reste très dense, les fûts sont cylindriques, serrés et très élancés; il en résulte que le peuplement est très sensible dans les situations exposées aux bris de la neige et à l'action des vents. 
Pendant la période de futaie, l'accroissement en hauteur se ralentit notablement, et de plus en plus avec l'âge ; le massif se relàche peu à peu, l'espace occupé par chaque arbre s'élargissant de plus en plus; mais dans les bonnes stations, le massif ne devient clair que trìs tard; le sol est envahi peu à peu par la mousse, puis par une régétation herbacée où dominent les airelles.

Dans les conditions normales, le massif peut devenir exploitable dès l'âge de quatre-vingts à quatre-vingt-dix ans; mais dans les hautes régions montagneuses des Alpes, la lenteur de la croissance devient parfois extraordinaire, et elle a pour effet de donner au bois d'épicéa une qualité toute spéciale (bois dle résonnance).

Les peuplements purs et réguliers d'épicéa (fig. 46), sont toujours plus ou moins exposés suivant les stations, aux dangers de la neige (massif très serré), des rents (enracinement superficiel), et des insectes, notamment des bostriches.

Réyénér'ation. - Suivant l'état du massif et du sol, les coupes préparatoires pourront être plus ou moins utiles; continuant les effets des éclaircies, elles ont pour résultat d’augmenter la résistance des sujets deslinés à devenir des porle-graines, en les dégageant graduellement.

Elles deviennent inutiles si le peuplement est déjà interrompu ou suffisamment éclairci, si le sol a une lendance à être envahi par la mousse ou par une végétation herbacée trop abondante.

La coupe d'ensemencement, faite de préférence au commencement d'une bonne année à graine, sera plutôt claire, enlevant environ, d'après M. Gayer, le tiers ou la moitié de la masse totale des bois existant dans le peuplement; son intensité sera réglée par la nécessité d'enrayer l'envahissement de la régétation herbacée et des mort-bois; toutefois, d'apris M.M. Boppe et Jolyet, les chablis étant à redouler, il vaut mieux procéder par 


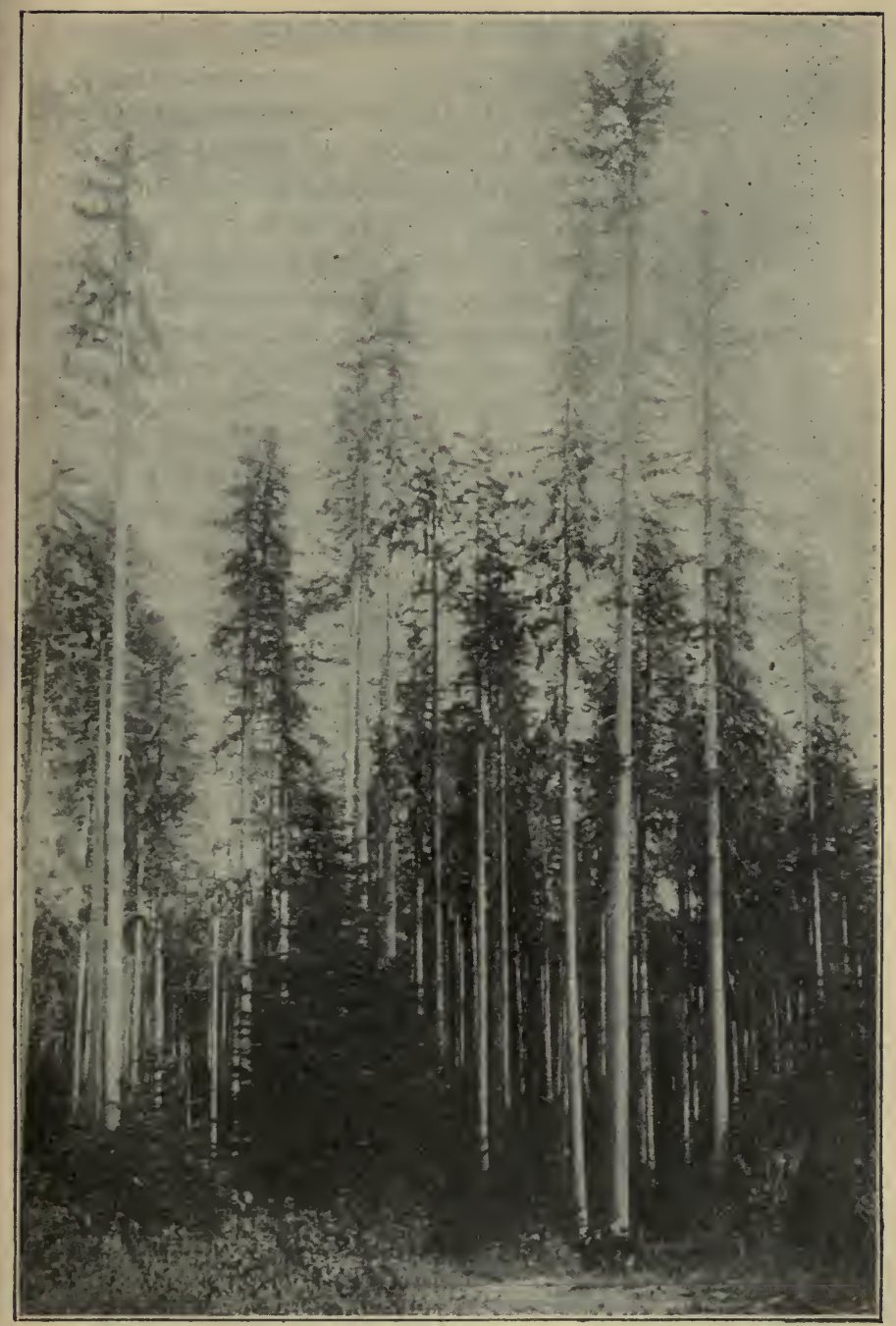

Fig. 46. - Epicéas de Gilley (Doubs). Arbres atteignant 50 mètres de hauteur. (Photographie de MM. Thiollier, "Les Forêts ") par MMinoppe et Jolyet). 
trouées éparses, en enlevant trois ou quatre arbres sur le mème point.

On doit considérer une couche de mousse forte et compacte sur le sol, ayant par exemple plus de 3 centimètres d'épaisseur, comme un obstacle à la germination, spécialement dans les sols qui ne sont pas suffisamment humides, ainsi que les couches épaisses d'aiguilles non décomposées; un ratissage par bandes ou par places peut dès lors être nécessaire pour faciliter l'installation des semis; au contraire, une couche de mousse peu serrée et peu épaisse, entremèlée d'aiguilles en partie décomposées, constitue un milieu favorable à la germination. La semence légère et ailée de l'épicéa se dissémine très facilement et le semis se répartit assez régulièrement.

Une fois le semis installé, les coupes secondaires et défnitives le découvrent rapidement; il est bon de remarquer, toutefois, que les fourrés d'épicéa peuvent rester vigoureux sous un couvert léger, et ne subir par ce fait qu'un faible retard, sans conséquence pour l'avenir.

Si l'on a procédé par trouées, la régénération se produit sous le couvert par groupes ou bouquets; les coupes secondaires viennent alors élargir les premiers vides et en créer de nouveaux.

Opérations culturales. - Les dégagements sont utiles à l'épicéa pendant sa première jeunesse, mais les premières éclaircies ne devront guìre commencer avant l'àge de trente à trente cinq ans; elles seront très prudentes, c'est-à-dire faibles, mais fréquemment répétées; plus tard, ces éclaircies peuvent devenir de plus en plus fortes, à la condition, toutefois, de tenir comple du caractère de l'essence, et de la station ainsi que de l'état du sol.

Au passage de ces éclaircies, doivent tomber tous les arbres tarés el dépérissants.

En résumé, dans ses stations naturelles, et en silua- 
tion favorable, le peuplement pur et régulier d'épicéa est un bon mode de culture.

D'après M. Gayer, il donne, dans les sols de fertilité moyenne, de 600 à 850 mètres cubes de bois par hectare à l'âge de cent à cent vingt ans; dans les stations les plus fertiles, il peut donner jusqu'à 1000 ou 1200 mètres cubes par hectare à cent quarante ans, et son action conservatrice sur le sol est considérable ; toutefois, il paraît avantageux de préférer au peuplement pur d'épicéa un mélange avec le sapin et le hêtre, ou tout au moins de constituer sous de tels peuplement un sousbois de ces essences.

Stations anormales de l'épicéa; plantations. - La simplicité et la facilité de la plantation ou du semis d'épicéa en terrain nu, ont contribué à propager la culture de l'épicéa à l'état de plantations ou jeunes futaies en dehors de ses stations naturelles, jusque dans les plaines basses et les parties de collines, dont le climat se caractérise par la douceur de la température et la longueur de la période de végétation; mais dans ces stations anormales, la vigueur de la végétation ne s'y maintient généralement pas longtemps; elle se paralyse souvent dès l'àge de quarante à soixante ans; la résistance du peuplement aux agents destructeurs, neige, insectes, champignons, etc., y est moindre que dans les stations normales. La croissance, très rapide, a pour effet de donner un bois tendre et peu résistant, putrescible et sujet à la destruction; aussi le massif se disloque-t-il de bonne heure, et, si l'on n'y prend garde, la fertilité de la station diminue; la plupart de ces peuplements doivent ètre exploités entre quarante et soixante ans.

Mélèze. - Futaie régulière. - Le mélèze peut constituer, dans la région qui lui est propre (à partir de 1000 mètres dans les Alpes), de charmante futaies régulières pures de tout mélange.

Le peuplement homogène de mélèze se caractérise par 
l'espacement considérable des tiges, dès le commencement de la seconde moitié de son existence; d'après II. Gayer, les phases successives de son existence sont les suivantes:

Croissance forte, dès les premières années, supérieure mème à celle de presque toutes les autres essences, ce qui permet aux jeunes plants de mélèze de lutter en général victorieusement contre l'envahissement de la régétation herbacée. Dès la formation en massif, qui a lieu au bout de cinq à six ans, si le jeune repeuplement est assez serré, commence la période du plus grand accroissement en hauleur. Dans de bonnes conditions de station, spécialement dans les sols frais et profonds, cette période de forte croissance se maintient parfois jusqu'à l'àge de trente ou quarante ans, avec des pousses annuelles de $0^{\mathrm{m}}, 60$ à 1 mètre, et le peuplement atteint alors, en un temps relativement court, une hauteur considérable, plus considérable que chez toute autre essence. Dans les stations médiocres, au contraire (basses altitudes, sol peu frais et peu profond), la croissance terminale se ralentit très vite; dès l'âge de vingt. à trente ans elle devient inférieure à celle de presque toutes les autres essences, et peut mème sourent ètre considérée comme arrètée. II en est de mème de la densité et de l'état de massif du peuplement; le mélèze étant avide d'espace et de lumière, l'élimination des tiges trop serrées ou dominées se produit très vite et le massif se desserre rapidement.

Il en résulte que le; meilleures stations seules, celles qui par suite de leur situation à de hautes altitudes, et de la nature de leur sol, peuvent se passer du couvert, et ne pas ressentir les effets de l'éclaircissement, permetlent au mélèze d'arriver à un àge arancé; dans ces stations, la lutte pour l'espace el la lumière commence très vive dès le commencement de la période de perchis; soutenue prar l'énergie de la eroissance terminale elle élimine 
rapidement un très grand nombre de sujets, et cet éclaircissement s'accentue arec l'âge. Les arbres cependant prennent des fûts élancés et sans nœuds; le sol, recerant chaque automne des aiguilles abondantes et molles, reste frais et s'enrichit; l'herbe même, au lieu de se montrer dense et touffue de très bonne heure, comme il arrive entre de jeunes sujets isolées, se fait attendre quelques années, mais elle se développe mieux dans la suite, sous les cimes élevées.

D'après M. Broilliard, de tels massifs d'un même âge, incomparablement meilleurs que les massifs jardinés, offrent le grand avantage de permettre le pâturage des vaches dans d'excellentes conditions; or la forêt de mélèze nourrit une vache à l'hectare pendant tout l'été, une petite vache des Alpes françaises, et le revenu du pâturage égale ou dépasse le revenu fourni par le bois; il importe done de conserver ces beaux massifs à l'état régulier, et sur les grands versants des hautes Alpes, et partout où le mélèze aura été introduit (dans ses bonnes stalions), on en obtiendra de riches produits en herbe et en bois.

Dans les stations médiocres ou mauvaises, mème de qualité moyenne et aux basses altitudes, l'éclaircissement s'accentue rapidemment pour arriver au point où chaque cime est complètement dégagée de ses voisines, et n'a aucun point de contact avec elles; le développement rapide et la fin prématurée sont dès lors le caractère presque général de tels peuplements de mélèze à l'état pur ; il est rare qu'on puisse les maintenir plus de quarante à cinquante ans, et souvent l'exploitation doit être faite dès l'âge de vingt-cing à trente ans. Dans de telles stations, le mélange avec d'autres essences est nécessaire à la bonne croissance du mélèze.

Repeuplement. - Le peuplement pur et régulier de mélèze prend naissance en terrain nu par semis ou plantation artificielle ou par ensemencement latéral d'arbres

A. Fron. - Sylviculture. 
voisins; il n'est pas rare, dit M. Broilliard, de voir le mélèze se reproduire à découvert sur les terrains voisins de la forèt, cultivés ou enherbés, et même si les prairies avoisinantes n'étaient pas fauchées annuellement, elles passeraient bien vite à l'état boisé. Le semis prend aussi naissance sous les vieux peuplements entrecoupés ou interrompus par ensemencement naturel sous le couvert le porte-graines.

Dans la région du mélèze, cet arbre tend ainsi à se répandre partout, en sol frais, comme le chène de nos plaines.

Aussi, d'après le mème auteur, pour obtenir en forèt un semis général et immédiat, il suffit d'établir une coupe d'ensemencement, ne gardant que des arbres espacés, en enlevant par exemple deux arbres sur trois du peuplement complet; on peut en outre sillonner le sol de petites rigoles, larges d'un fer de bêche sur les points où il est fortement enherbé; il est inutile que le semis soit abondant et bien égal; on peut donc se borne* à ouvrir des rigoles écartées de 2 mètres environ ; quelques brins naîtront d'ailleurs intercalés. Mais il est indispensable de mettre rigoureusement en défens les parties à repeupler et de les y maintenir jusqu'à la formation du perchis.

Le semis de mélèze devenu général et haut de $0^{\mathrm{m}}, 50$ au moins, on peut opérer hardiment la coupe secondaire, partout où l'on n'a pas à craindre des éboulements et des avalanches; dans ce dernier cas, le jardinage des arbres morts est le seul mode de traitement à conseiller (Broilliard).

Opérations culturales. - Dégagements de semis. - En raison de la croissance rapide des jeunes sujets, les dégagements sont peu nécessaires, spécialement aux hautes altitudes où la végéfation basse est peu redoutable; il suffit d'opérer un dépressage dans les semis trop serrés. Eclaircies. - Le mélèze étant franchement une essence 
de lumière se trouve mal à l'état serré; les éclaircies doivent avoir pour but de venir en aide à la nature en favorisant l'élimination naturelle; dans les bonnes stations, les éclaircies peuvent ètre faites de bonne heure et hardies, afin d'empêcher la végétation de devenir languissante dans un massif trop serré; toutefois elles doivent se borner à suivre les indications naturelles, c'est-à-dire à n'enlever dans l'étage principal que les cimes les plus faibles, sans isoler celles qui sont conservées, afin de ne pas entraver le fonctionnement de l'élagage naturel; elles ne doivent pas toucher à l'étage dominé et au sous-bois, afin de conserver au sol le plus d'abri possible.

Pin sylvestre. - Futaie régulière ou quasi régulière. - Le pin sylvestre est une essence très rustique et il ne se trouve peut-être pas de station où il ne puisse vivre ou du moins végéter; il en résulte une allure très diverse dans les différents phénomènes de sa végétation, de sorte qu'il est très difficile de donner une description générale de ce peuplement (fig. 47).

Le peuplement régulier et pur de pin sylvestre prend naissance par plantation ou semis artificiel en terrain découvert, ou par régénération ṇaturelle. En bonne station et dans des conditions favorables, la première croissance du pin sylvestre est très rapide, et le massif peut ètre formé au bout de cinq à six ans. Mais dans un grand nombre de cas, notamment dans les stations médiocres ou mauvaises où on l'installe si souvent, il n'en est plus ainsi; au moment de leur reprise et de leur première croissance les jeunes plants ont à lutter contre de multiples obstacles dont les principaux sont le climat, les insectes, certaines maladies et souvent aussi la nature de la station. La germination des graines, l'installation et le premier développement des jeunes plants, exigent une certaine humidité du sol; or, les terrains occupés par le pin sylvestre sont souvent maigres et secs; si à ces condi- 
tions défavorables s'ajoutent des vents secs et persistants au printemps, de fortes chaleurs et un été sec, la première croissance est très ralentie, et un très grand nombre de plants sont exposés à périr; si dès lors on ne vient pas immédiatement combler ces clairières el ces lacunes souvent très considérables, les plants croissent isolés ou en petits groupes, ils ne se forment que très lentement en massif et cette situation est très défarorable, car la formation en massif est la principale condition d'une croissance vigoureuse dans la jeunesse de cet arbre. Ce fait est important à signaler aux nombreux propriétaires particuliers qui laissent leurs jeunes plantations de pin sylvestre très souvent beaucoup trop clairiérées et entrecoupées ; des regarnissages sont indispensables, tant que l'état de massif n'est pas acquis; ils doivent être d'autant plus soignés que les conditions sont plus mauraises.

D'après M. Gayer, quand le peuplement échappe à ces diverses causes de destruction il met environ huit ans à devenir fourré, et alors commence la période de la plus forte croissance terminale dont le maximum arrive entre la dixième et la vingt-cinquième année; à cette époque, les pousses annuelles dépassent 60 centimètres ; c'est la phase du bas perchis; les cimes très touffues empiètent largement les unes sur les autres (1); le couvert parfait, les aiguilles s'accumulent sur le sol et ne tardent pas à le couvrir d'une couche protectrice de litière qui élève sa fertilité au maximum; les détritus d'un tel pe'uplement sont plus abondants qu'on ne pourrait le croire tout d'abord; ils produisent annuellement un poids de litière sensiblement égal à celui que donnent les forêts: d'épicéa el de hètre, c'est-à-dire un peu plus de 3000 kilos: de matière absolument sèche par hectare; ils sont pour le sol un excellent engrais, d'une décomposition lentr cependant, en raison de la consistance coriace, de la struc-

(1) Dans les stations h̀ climat rude, cet état serré constitue parfois un danger assez sérieux, car le jeuplement résiste mal au poids du givre et de la neige. 


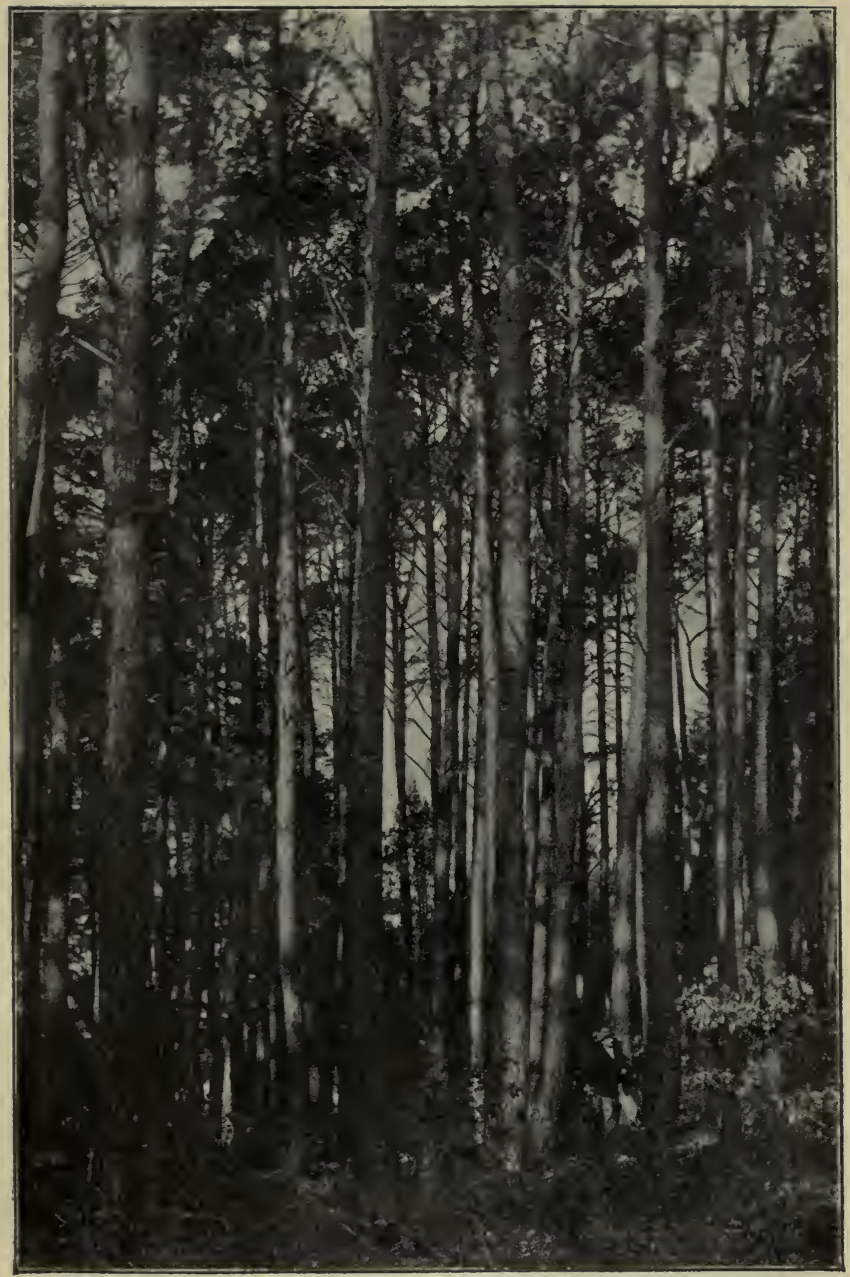

Fig. 47. - Peuplement de pin sylvestre de Haguenau à l'état de haut perchis. Arboretum national des Barres. 
ture fibreuse des aiguilles, et souvent aussi du manque de fraîcheur superficielle qui se fait sentir dans les forêts de cette essence (1) (Mathieu).

La fertilité du sol se maintient aussi longtemps que, durent les dites conditions; bien plus, pendant toute cette période, si l'on a soin de ne pas toucher à la litièr. de la forèt, le peuplement possède la facultè de ranimer un sol autrefois sterile, et de relever sa force productrice.

Le massif favorable au développement du pin sylvestre ne doit pas avoir le même degré d'intensité dans toutes les stations; en sol fertile, l'état de massif serré farorise l'accroissement en hauteur et facilite l'élimination du peuplement accessoire; mais dans les sols maigres et peu profonds, il n'en est plus de même, et l'état serré du massif rend cette élimination difficile; il peut occasionner un arrêt ou un retard dans la croissance et parfois même le rabougrissement des arbres; il appartient alors à des éclaircies bien dirigées de régler cet état de massif.

Durant cette courte période où le massif reste serré, le peuplement principal se développe sans manifester de très grandes exigences, particulièrement en ce qui concerne l'espace; mais à mesure que le pin prend de l'accroissement, ses besoins augmentent, et la caractéristique de cet arbre devient un grand besoin de lumiere et d'espace; il faut alors que la plus grande parlie des arbres disparaisse et le peuplement de pin sylrestre s'éclaircil rapidement. Cet espacement des sujets prend une lelle intensité qu'il ne peut plus ètre question de massif et de conservation du sol, et cela, d'autant moins que les cime: arrondies et étroites remontent jusqu'au sommet des arbres, s'aplatissent en laissant complètement vide l'es-

(1) Dans les grandes plaines sablonneuses de la région du pin, cette litière d'une valeur égale et même supérieure à celle dı hois est, mallieureusement pour la forêt, trop fréquemment enlevée par les habitants des communes riveraines, dont les terres participent à la nature ingrate au point de vue agricole du sol des pineraies. On estime que le funier de feuilles de pin sọlvestre équivaut a moitié de son joids de fumier de paille (T. Hartig). 
pacequi les sépare du sol. A cette époque, l'accroissement en hauteur s'est progressivement ralenti.

Cette phase d'éclaircissement spontané est plus ou moins précoce selon la qualité du sol; en bonne station, en sol sablonneux, fertile, profond et frais, en sol argileux, riche en humus, le massif persiste souvent jusqu'à soixante-dix et quatre-vingts ans; en sol calcaire et peu profond, l'éclaircissement spontané se produit dès l'âge de quarante à cinquante ans; il s'ensuit que la rérolution à adopter est très variable, que certains peuplements sont exploitables à cinquante ou soixante ans, d'autres à soixante-dix ou quatre-vingts ans, et que dans les meilleures stations ils peuvent vieillir au-delà de cent et cent vingt ans; leurs rendements varient en conséquence. L'aspect de tels peuplements est particulier ; généralement purs de tout mélange d'autres essences, en massif clair et diffus, ils restent ouverts à la lumière; le sol n'est couvert que d'aiguilles mortes et de longues herbes aux tiges grêles; le bouleau et le chène peurent se trouver mélangés au pin sylvestre, mais ils sont, comme lui, insuffisants pour bien couvrir le sol (Broilliard).

Opérations culturales. - Dégagements de semis. - Si les dégagements de semis ne sont que rarement nécessaires, du moins est-il important, d'après MM. Boppe et Jolyet, de pratiquer des dépressages toutes les fois que les semis trop nombreux se constituent en fourrés très denses, où l'évolution des champignons parasites est à craindre. Les dépressages s'imposent dans les pineraies de création artificielle.

Éclaircies. - L'éclaircie est la base du traitement des essences de pleine lumière, comme le pin sylvestre, dont la cime, franchement desserrée dès le jeune âge, isolée même à partir de l'etat de haut perchis, doit alors occuper au moins le tiers de la hauteur totale du sujet; sinon la croissance est ralentie, l'arbre ne forme pas de bois de cœur, prend une forme étriquée et devient la 
proie des insectes ou des champignons (Boppe et Jolyet).

Pour réaliser ces conditions, les massifs de pin sylvestre ou pinatelles demandent à ètre desserrés de bonne heure, sans quoi les sujets s'alanguissent. VI. Broilliard recommande, pour opérer d'une manière sûre et bonne, l'éclaircie forte mais partielle et répétée souvent, tous les six, huit ou dix ans par exemple.

II importe, en effet, dans les éclaircies successives du pin sylvestre, non pas d'isoler les cimes, mais d'exposer progressivement à la lumière toute la pyramide des branches, et pour cela on se borne au passage de chaque éclaircie à dégager les belles perches d'un côté de l'arbre seulement, ou tout au plus de deux côtés sur quatre, quilte à revenir sur les autres côtés des mèmes arbres lors des prochains passages d'une nouvelle éclaircie. Dans les peuplements artificiels, ces éclaircies peurent itre commencées dès l'àge de dix ans; dans les peuplements naturels, on peut les retarder quelque peu.

Au passage de ces éclaircies, les pins dominés et les sujets mal conformés doivent ètre enlevés systémaliquement; au contraire, on doit s'abstenir d'une façon absolue, conseil qu on ne saurait trop répéter, d'élaguer les branches basses, et de faire disparaitre, sous prétexte d'éclaircie, les essences feuillues qui peuvent se présenter en mélange dans le massif, les mort-bois ou bonnes essences (bouleaux, chênes, etc.), qui se trourent en sousétage à l'état de buissons, de broussailles ou de jeunes sujets. La présence de ces éléments est très utile à tous les points de vue; elle conserve au sol un peu de fraicheur dans le sable, diminue par ses détritus l'acidité naturelle du terrain sous les pins sylvestres, comble les vides, courre le sol, attire les oiseaux, ctc.

Le peuplement de pin sylvestre est d'une utilité multiple, et donne en peu de temps un certain nombre de produits appréciés; il est peu exigeant et facile d'entretien; e'est pourquoi la culture de celle essence s'est 
beaucoup propagée en France, un peu dans toutes les stations, et elle y rend de grands services.

11 résulte de cet examen que le peuplement de pin sylvestre possède la faculté de relever la fertilité du sol pendant sa jeunesse, et aussi longtemps qu'il reste formé en massif, mais qu'il perd d'autant plus cette faculté qu'il est laissé plus longtemps à l'état espacé; au point de vue de l'amélioration du sol, un tel peuplement ne se justifiera que jusqu'au moment où l'éclaircissement commence à se manifester.

Dès lor's, le propriétaire d'un massif de pin sylvestre peut se proposer deux choses : relever le degré de fertilité d'un sol ou produire du bois.

$1^{\circ}$ Il se propose de relever le degré de fertilité d'un sol, d'améliorer le terrain pour permettre d'y faire ensuile soit une culture agricole, soit une culture forestière plus exigeante. - Il est bon, dans ce cas, d'exploiter le massif à l'âge de trente-cinq à quarante ans; c'est à ce moment que le sol est le mieux préparé par les détritus abondants de la forèt de pins. M. Broilliard indique dans les termes suivants la manière de créer ce massif et de le conduire jusqu'à cet âge: dans un sol convenable au pin sylvestre, où l'on peut espacer les sujets de $1^{\mathrm{m}}, 40$, on en plante 5000 à l'hectare. Quand ces pins ont en moyenne 10 centimètres de diamètre à hauteur d'homme, vers l'âge de vingt ans, on en retrouve 4000 valant 50 centimes pièce, soit environ 2000 francs; on en coupe dès lor's et en deux fois les trois quarts peut-ètre, 2000 d'abord à vingt ans, puis 1000 à vingt-cinq ans, les plus laids, donnant par exemple 1000 francs en tout. Quand les autres ont acquis 20 centimètres de diamètre, vers l'âge de trente ans, il en reste encore un millier, cubant chacun 2 décistères et pouvant valoir à $12 \mathrm{fr}$. 50 le mètre cube, $2 \mathrm{fr}$. 50 l'un, soit en somme $2 \% 00$ francs; on en prend encore en deux fois par exemple 350 d'abord à trente.ans, puis 150 à trente-cinq ans, donnant peut-ètre de nouveau 
1000 francs pour l'ensemble. Enfin quand les survivants ont 30 centimètres, vers l'âge de quarante ans, il n'en reste plus guère que 500 , cubant 5 décistères en moyenne, et pouvant valoir à 16 francs le mètre cube, 8 francs pièce, soit en somme 4000 francs. On peut les exploiter en bloc, et entreprendre immédiatement soit une culture agricole, soit une nouvelle culture forestière améliorante.

$2^{\circ}$ Le propriétaire se propose simplement de produire $d u$ bois, et trouve avantageux de maintenir les pins jusqu’à l'âge de fertilité, pour obtenir un repeuplement par voie naturelle.

Il est nécessaire de maintenir les pins au delà de quarante ans, en continuant à les éclaircir tous les dix ans par exemple; mais dans ce cas, l'installation, voire mème la création artificielle d'un sous-bois de sapin ou mieux encore de hêtre, sous les perchis de pins arrivés à l'âge de trente ou quarante ans, ne saurait être trop recommandée.

Tant que les pins restent bien renants, ce qui dépend surtout de la station et du climat, le revenu annuel ne fait que s'accroître ; ainsi, d'après M. Broilliard, la pineraie qui a produit 4000 francs dans les quarante premières années, en produira peut-être autant dans les vingt années suivantes, à moins de quelque dégradation accidentelle.

Dans de tels massifs, il y a lieu vers l'âge de soixante ans, ou même beaucoup plus tard suivant l'état de la végétation, de commencer les coupes de régénération. La coupe d'ensemencement est faite très claire ou plutôt par trouées, et il peut être nécessaire de donner une culture au sol souvent durci, tassé et couvert de sous-arbrisseaux de grande taille et envahissants (bruyères, genêts, etc.); on peut au besoin procéder par arrachis, suivant des bandes plutôt espacées et larges $\left(0^{\mathrm{m}}, \$ 0 \mathrm{et}\right.$ plus) que nombreuses et étroites; mais en général il est facile d'éviter en partie tout au moins cette dépense, en 
effectuant l'extraction des souches des arbres exploités.

En bonne station, le pin sylvestre se prête très bien à l'accroissement par la mise en lumière, et la réserve des plus beaux sujets sur coupe définitive, disséminés audessus de la nouvelle forèt naissante peut ètre une bonne opération.

Nous avons vu au cours de cette étude que l'avenir des massifs de pin sylvestre purs et uniformes dès leur jeunesse est étroitement restreint; les inconvénients du pin sylvestre à l'état pur, suffisent à faire ressortir l'utilité des sous-bois et l'avantage d'un mélange avec d'autres essences, en peuplements plus ou moins irréguliers. Dans toutes les stations moyennes, le peuplement pur depin sylvestre ne devrait être employé que là où le sol ne peut pas produire autre chose que du pin sylvestre, à moins qu'il ne s'agisse de s'en servir uniquement comme essence transitoire de reboisement.

Pin laricio d'Autriche, ou pin noir d'Autriche. - Plantation. - Le pin laricio d'Autriche plus connu sous le nom de pin noir d'Autriche, qu'il doit à la couleur d'un vert sombre de ses aiguilles, n'est pas spontané en France, et son introduction remonte seulement à 1834. Son tempérament robuste, la rapidité de sa croissance, le rendent précieux pour le repeuplement des terrains où peu d'autres essences pourraient croître. Ce pin peut en effet végéter dans les sols calcaires les plus ingrats ; les terrains argileux et humides lui sont défavorables ainsi que les sables purement siliceux.

I réussit mieux que le pin sylvestre dans les plaines crayeuses de la Champagne et sur les côtes arides des montagnes jurassiques. A ce titre, il est très précieux pour le reboisement des terrains calcaires et crayeux (fig. 48).

Pin maritime. - Futaies régulières et plantations.Le pin maritime est une essence de lumière; sa cime peu fournie donne un couvert léger; ses graines abondantes se disséminent facilement. Dans les pays où cet arbre 
prospère, il n'y a guère à se préoccuper du repeuplement qui s'opère naturellement ; les semis, qu'ils soient naturels ou artificiels, sont généralement trop serrés; il convient de les desserrer vers la dixième année, et de réitérer cette opération tous les cinq ans. Quand le peuplement a atteint sa vingtième année, on l'éclaircit en gemmant à mort les sujets qui doivent disparaitre; une seconde éclaircie est faite jar le mème procédé cinq ans après. Enfin, quand les arbres qui constituent le peuplement définitif ont atteint leur trentième année, on commence a les gemmer à vie, et l'on continue à les soumettre à ce traitement jusqu'à leur soixantième ou leur soixantedixième année. Arrivés à cet âge ils sont gemmés à mort et abàttus.

Toutes les pignadas ainsi créées par semis artificiels, of traitées en vue de la production de la résine peuvent ètr' considérées comme des exploitations industrielles, nécessitant des méthodes de production et d'exploitation particulières; nous renvoyons à leur sujet à l'ourrage de M. Broilliard (1) et à des traités spéciaux.

Nous avons vu dans l'étude spéciale de cette essence (p. 8i) que le pin maritime est quelquefois employé au delà de son aire d'habitation normale jusque dans le centre et même l'est de la lirance, comme essence de reboisement. Cette extension est dangereuse, et nous n'en rappellerons qu'un exemple célèbre concernant la Sologne. Les premiers travaux de reboisement, eflectués depuis 1830 en Sologne, avaient eu pour conséquence de provoquer un véritable enthousiasme; on choisit à ce moment de préférence le pin maritime, parce que cell. essence s'accommodait bien de ce terrain sablonneux ('I humide, qu'elle présentait sur les autres pins l'avantage d'être d'un ensemencement facile, d'ume croissance tris rapide, et par suite d'une exploilation assez rémunéra-

(1) Bnondand, Le Traitement des bois en France, nouvelle édition, 1894. 


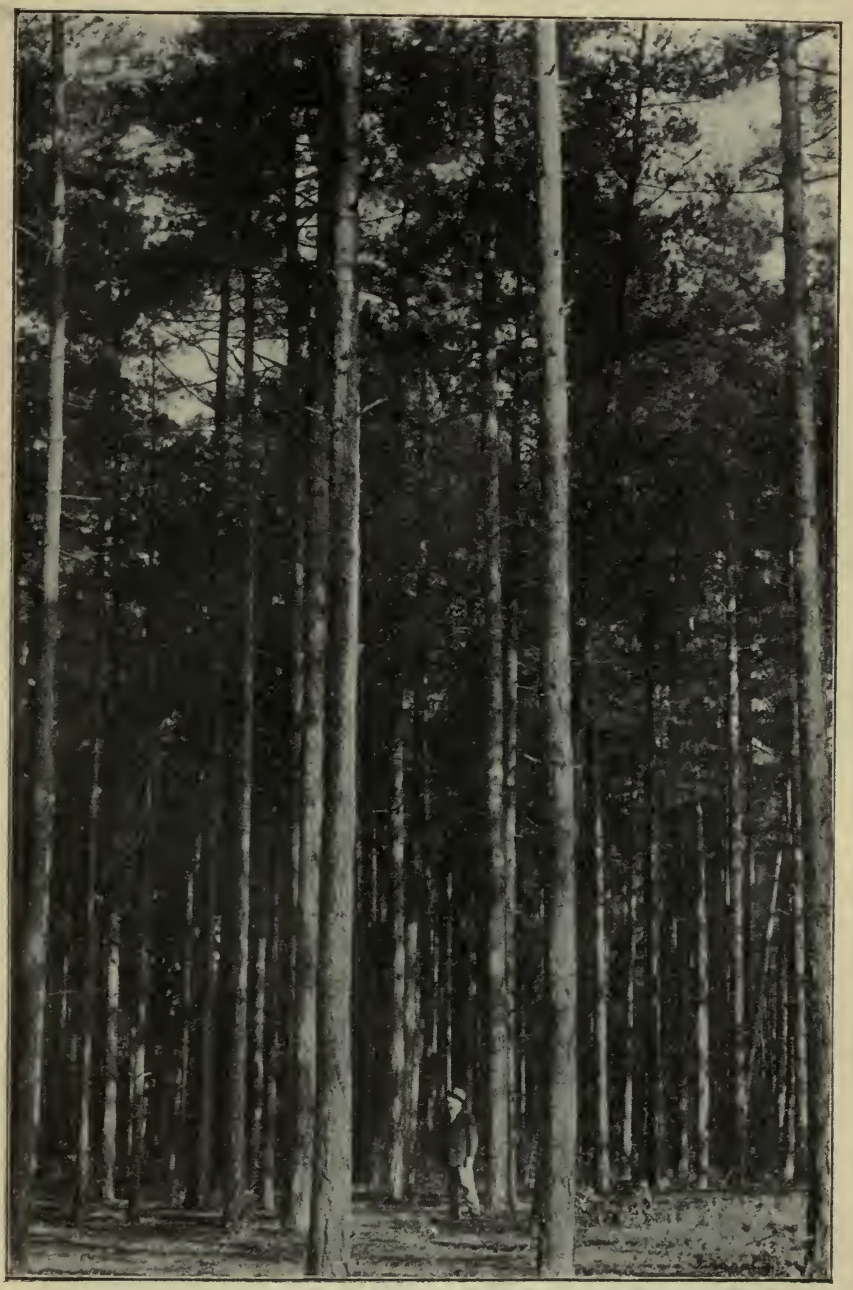

Fig. 48. - Peuplement de pin laricio de Corse à l'état de laut perchis. A:boretum national des Barres. 
trice. De tous côtés on se mit à l'œurre arec une prodigieuse activité ; l'État et plus tard le Comité central favorisèrent cet essor en faisant distribuer, par les agents forestiers, des milliers de jeunes plants, en ourrant des concours, et en facilitant aux propriétaires la première installation; les établissements modèles de la MotteBeurron expérimentaient et indiquaient les meilleures méthodes (1).

Un cruel désastre faillit tout perdre; pendant l'hiver de 1879-1880, des froids comme on n'en avait jamais vus de mémoire d'homme atteignant 30 et 3 ¡ degrés au-dessous de zéro, gelèrent toutes les pineraies de pins maritimes; la perte causée par la gelée a été estimée par un inspecteur des forêts, M. Caquet, à quarante millions de francs. II y avait de quoi décourager pour longtemps d'une entreprise encore si près de ses débuts.

Mais une remarque fut faite à ce moment. Partout où les pins maritimes avaient succombé, le pin sylvestre qu'on avait moins prôné jusque-là, parce qu'il donnail moins de revenu, avait résisté. Beaucoup de propriétaires se résignèrent donc à replanter du pin sylvestre. Beaucoupd'autres ont replanté en pin maritime; il est évidenl qu'ils sont exposés aux mêmes désastres, et la spéculation est peut-ĉtre dangereuse; d'un autre côté, des hivers rudes ne se reproduisent qu'à de longues échéances, peut-être seulement tous les siècles; el alors on ne peul désapprouver ces propriétaires de courir le risque, pour profiter de celte essence, qui à tous les autres points de vie est avantageuse, et leur procure, en vingt, vingt-cing ou trente ans, des produits beaucoup plus rémunérateurs, arec moins de peine et moins de soins.

Pin d'Alep. - Futaie quasi jardinée. - Sur les calcaires de Provence, on trouve encore de vastes forèts de pin d'Alepen futaie rlaire, a vec sous-ótage dechêne kermex,

(1) Marcer. Monvarcue, Eturle sur la Sologne. 
de chène vert et de chène blanc; souvent on exploite les feuillus en taillis et on se borne lors du passage des coupes à réserver les plus beaux pins qui constituent l'essence la plus précieuse. M. Broilliard conseille de subordonner la coupe du taillis à celle des pins, et d'exploiter ceux-ci par jardinage. La forêt, dit-il, est divisée en vingt ou vingtcinq coupes; au passage de chaque coupe on peut se borner à enlever successivement les pins mûrs ou dominant des semis, et les tiges trop serrées, en mème temps qu'on recèpe avec soin le taillis de chêne. Ce recépage, effectué sous des pins isolés pour la plupart, entretiendra un sousbois des plus utiles par son couvert et par ses produits, tout en permettant la reproduction de la pineraie; le jardinage des pins, réduit aux bois mùrs et surabondants et assurant ainsi le développement des arbres, donnera bientôt la prépondérance à l'essence la plus précieuse.

Le mème auteur ajoute deux conseils : $1^{\circ}$ n'exploiter les pins que lorsqu'ils sont assez grospour fournir de bonnes planches, ou des bois de marine d'un certain prix, dont le débouché est assuré par Marseille et Toulon; $2^{\circ}$ interdire le pâturage des moutons et des chèvres, ou tout au moins ne le permettre que dans les parties les plus âgées de la forêt, et ne jamais en abuser. La suppression du pâturage à elle seule, permettrait la constitution en vingt ans à peine de peuplements de chène et de pin beaucoup trop rares aujourd'hui; le meilleur état de massif ainsi obtenu, l'amélioration du sol, et par suite une plus forte production, sont susceptibles de compenser dans une large mesure les faibles bénéfices que donne le pâturage bien peu rémunérafeur sur de tels rochers.

\section{DEUXIEMME SOUS-SECTION}

\section{PEUPLEMENTS MÉLANGÉS.}

Généralités. - Nous donnerons moins d'extension à I'étude des peuplements mélangés, d'abord en raison du 
cadre restreint de notre ouvrage, et ensuite parce que dans les mélanges par groupes ou bouquets, chaque essence se comporte, dans une certaine mesure dans chaque groupe ou bouquet, comme nous l'arons examiné dans les chapitres précédents.

Dans un mélange, les associations simples d'essence à essence peurent ètre groupées d'après leur caractère cultural en trois catégories: essences d'ombre entre elles; essences d'ombre avec essences de lumière; essences de lumière entre elles.

Quant au nombre, à la proportion et au tempérament des essences à introduire dans un mélange, il dépend de la qualité de la station, c'est-à-dire non seulement de sa fertilité, mais aussi de la mesure dans laquelle l'action conservatrice du peuplement est nécessaire; il dépend aussi de la forme du peuplement (1) et des soins qu'on peut leur donner.

Nous avons conseillé en principe et sauf exceptions, en parlant des peuplements mélangés (p. 158), les règles suivantes :

$1^{\circ}$ Chercher à introduire en majorité dans les mélanges les essences d'ombre, et souvent le hètre;

20 Associer aux essences résineuses des essences feuillues, spécialement le hètre;

$3^{\circ}$ Domner aux peuplements ainsi constitués des formes irrégulières, et dans ces formes irrégulières, constituer le mélange par groupes, places ou bouquets.

\section{I. - Étude SUCCINCTE DES DIVERS MÉLANGES.}

1. - Mélange des essences dombre entre elles.

Caractères du mélange. - Les peuplemments qui résultent du mélange d'essences d'ombre entre elles sont

(1) Les formes irrégulières admettent unẹ plus grande variété d'essences que les autres, surtout lorsque les essences sont de tempérament très différent (Gayer). 
composés d'essences se ressemblant par leur caractère cultural, et parsuite généralement aptes à s'accommoder d'une mème station. De tous les mélanges c'est celui qui réclamera le moins le concours de l'art pour se mainlenir.

Dans les bonnes stations, les formes régulières ou quasi régulières prospèrent en général par les moyens ordinaires de la culture, et la répartition du mélange par bouquets; ailleurs, dans les stations médiocres ou mauvaises, il sera bon de tenir compte des qualités de la station, mais la forme du peuplement n'y est pas aussi strictement imposée que dans les autres espèces de mélange.

Sapin et Épicéa. - Dans les régions moyennes de son aire d'habitation le sapin peut ètre traité à l'état pur; ınais sur les limites supérieures de celle-ci, aux altitudes élevées, il semble indiqué d'avoir recours à l'épicéa et de donner dans le mélange, au besoin par voie artificielle, une large part à cette essence, comme cela se présente spontanément dans le Jura et dans les Alpes.

Cette association constitue un très bon mélange, surtout dans les zones moyennes des montagnes, et présente de notables avantages culturaux sur le peuplement d'épicéa. Pour prendre un développement prospère, un tel mélange demande un sol satisfaisant aux exigences du sapin pectiné, c'est-à-dire profond, fertile, sans ètre détrempé.

En peuplement régulier, mélangé par sujets isolés, le sapin tend, dans une certaine mesure, à supplanter l'épicéa, et le mélange intime est parfois maintenu difficilement.

Tout en conservant le peuplement régulier, M. Gayer est d'a vis qu'il est plus sùr de procéder par bouquets entremélés, lesquels ne doivent pourtant pas excéder l'étendue d'un are environ, si l'on ne veut pas s'exposer à perdre les avantages du mélange.

Mieux que la futaie régulière, la futaie à coupes jardina- 
toires se prête à une croissance prospère des mélanges de sapin et d'épicéa, et assure la conservation du mélange. D'après M. Gayer, cette forme de peuplement permet non seulement de satisfaire de la manière la plus naturelle aux exigences que chacun des deux arbres manifeste pendant sa jeunesse, à l'égard de la lumière et de l'éspace, mais encore d'obtenir de très beaux résultats, au moyen de l'accroissement par la mise en lumière.

Sapin et hêtre. - Sur les limites inférieures de l'aire d'habitation du sapin, un mélange rationnel avec les essences qui le précèdent est souvent indispensable; à ces altitudes basses du sapin, dans la région moyenne des montagnes, la nature lui associe très souvent, on pourrait dire de préférence, le hêtre.

Un tel mélange est très favorable au hètre dont il allonge les fûts; il est aussi très utile au sapin.

Dans la forme régulière, la période de première jeunesse est souvent la plus critique pour l'existence du mélange, spécialement si le hêtre est en forte proportion et en mélange intime, et si le sol n'est pas très fertile; à 'ce moment le sapin, dont la croissance est lente les premières années, risque d'ètre étouffé par l'accumulation des feuilles de hêtre; puis il est environné de toutes parts par un épais fourré de hêtre et tend à disparaître. Quand le mélange a échappé à ce double danger, le sapin commence à croître vigoureusement en hauteur, rattrape le hêtre qu'il pousse en hauteur pendant la période de gaulis et de perchis; souvent alors, le hêtre s'efforçant de se maintenir au mème niveau, devient élancé et se dépouille de branches jusqu'à une grande hauleur. Plus tard le sapin laisse en arrière le hêtre qui ne le suit plus dans son développement; le hêtre se borne dans le mélange adulte à occuper les dépressions du niveau géné ral des cimes, et souvent même il reste dans le sousetage.

Tout en conservant la forme régulière, il parait plus 
súr de procéder par bouquets entremélés, dans lesquels mème on aura tout arantage à donner une certaine avance au sapin. Le mélange par bouquets, même petits, est susceptible de donner des massifs très bien formés et de longue durée.

Mieux que la futaie régulière, la futaie à coupes jardinatoires se prête à une croissance très prospère du mélange du sapin et du hêtre.

Épicéa et hêtre. - Une certaine proportion de hêtre, à titre auxiliaire, dans les peuplements d'épicéa, est une condition très utile pour leur bonne croissance et leur conservation, de même que l'introduction d'une certaine proportion d'épicéa dans les peuplements de hêtre permet d'améliorer sensiblement la croissance du hêtre, et l'état général du peuplement.

Ces deux essences n'ont presque rien de commun comme port, ni même comme exigences de terrain; l'épicéa prend place volontiers dans les endroits détrempés, fortement humides, et le hêtre recherche ceux qui ne sont que frais.

Dans de tels peuplements jeunes, en voie de croissance, le hêtre, d'après M. Gayer, reste généralement en arrière sous le couvert de plus en plus épais et envahissant de l'épicéa, et en est éliminé de bonne heure, si le mélange est régulier et intime. Aussi le mélange n'a-t-il d'existence durable qu'à la condition d'être réparti par groupes de moyenne grandeur ( 50 centiares à 2 ares) qui mème à âge égal se maintiennent assez facilement, et le peuplement reste composé de petits groupes de hêtre répartis au sein du peuplement d'épicéa.

Toutefois, le maintien du hêtre est plus assuré quand on lui donne une certaine avance sur l'épicéa, avance qu'on peut obtenir, soit en régénérant de bonne heure le hêtre d'après le principe des coupes jardinatoires, soit en comblant après coup, à l'aide d'épicéas, les lacunes qui se seraient produites dans les peuplements de hêtre. 
Épicéa, sapin et hêtre. - A tous points de vue, le mélange de ces trois essences est avantageux; aux altitudes élevées, il parait nécessaire d'y donner, au besoin par voie artificielle, une large part à l'épicéa, comme cela se présente spontanément dans le Jura et dans les Alpes.

Dans de tels mélanges, l'épicéa est toujours quelque peu dominant par rapport aux espèces associées, ou du moins il tend à les dépasser en hauteur; on ne peut que se féliciter d'un pareil état de choses.

\section{2. - Mélange des essences dombre avec les essences de lumière.}

Les peuplements qui résultent du mélange d'essences d'ombre avec des essences de lumière (fig.49) sont composés d'éléments très dissemblables par leur caractère cultural; les différences très marquées qui existent entre ces deux catégories d'essences, tant au point de vue de leur port, de leur croissance en hauteur, de leur longévité et de leur tempérament, qu'au point de vue de l'influence de la station, donnent à ce mélange un caractère artificiel.

Dans l'appréciation d'un tel mélange, il y a lieu de tenir compte essentiellement des deux considérations suirantes:

10 L'essence de lumière a un impérieux besoin de lumière et par suite d'air et d'espace pendant toutes les phases de son existence; le concours d'une essence d'ombre dans le mélange rend cette condition difficile à maintenir.

$2 \circ$ L'essence de lumière seule ne peut entretenirla densité du massif et la fertilité du sol pendant toutes les phases de l'existence du peuplement, et le concours d'une essence d'ombre est très utile pour obvier à cet inconvènient.

Pour de tels peuplements, d'après .1. Gayer, la forme régulière en mélange intime n'est possible que dans cer- 


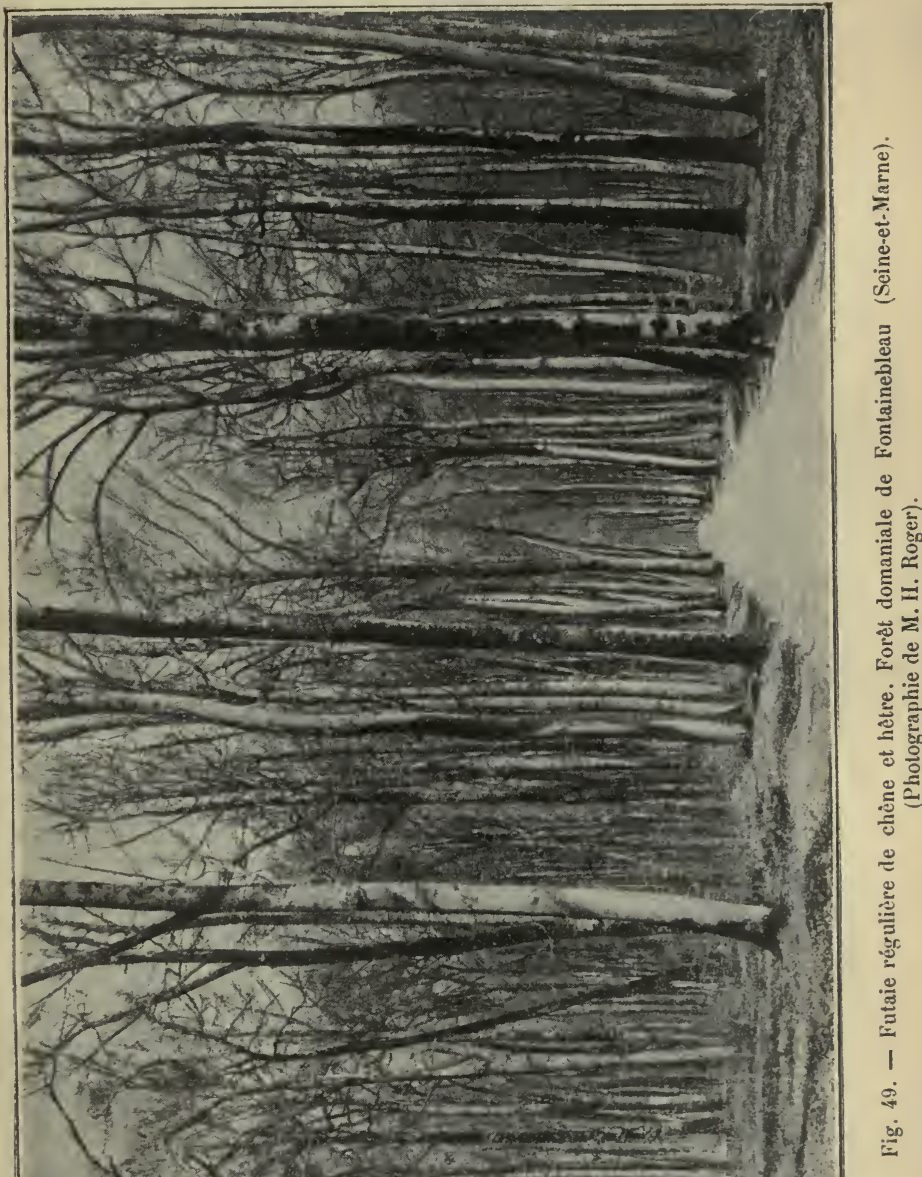


taines conditions déterminées, mais, par contre, c'est ici que les formes irrégulières paraissent atteindre toute leur valeur pratique.

D'après M.M. Boppe et Jolyet, quand les tendances envahissantes qu'a par sa nature l'essence d'ombre, sont encore exagérées par une meilleure arlaptation au milieu, l'essence de lumière succombe toujours victime de l'essence d'ombre. 11 en est ainsi du chène et du hètre dans les forêts du nord et de l'est de la France, où le hètre, qui se trouve dans le centre de son aire, a toujours des tendances à éliminer le chêne (fig. 50) et le mélange ne peut être conservé qu'à la condition de venir sans cesse cantonner par des éclaircies bien conduites, l'essence d'ombre, au rôle secondaire qui lui est dévolu.

\section{3. - Mélange des essences de lumière entre elles.}

Ce mélange se justifie rarement, car dans de tels peuplements, l'état de massif tend de plus en plus à se relâcher au fur et à mesure que le peuplement vieillit. Le desserrement lu massif est d'autant plus caractérisé et hâtif que le besoin de lumière des essences du mélange est plus accentué et que la fertilité du sol est moindre.

C'est donc spécialement dans les circonstances exceptionnelles seules que de tels mélanges pourront st justifier, par exemple :

10 Dans les stations de qualité tellement supérieure que leur fertilité est indépendante de l'état du massif ;

2 Dans les sols maigres, et alor's dans un but spécial et temporaire, le peuplement transiloire ainsi constitué présentant moins d'intérêt que le but à atteindre.

Dans la plupart des cas, c'est la futaie régulière seule qui sera admissible.

\section{Observation.}

En terminant cette étude des principaux massifs, ne serait-il pas bon de metlre en garde le propriétaire fores- 


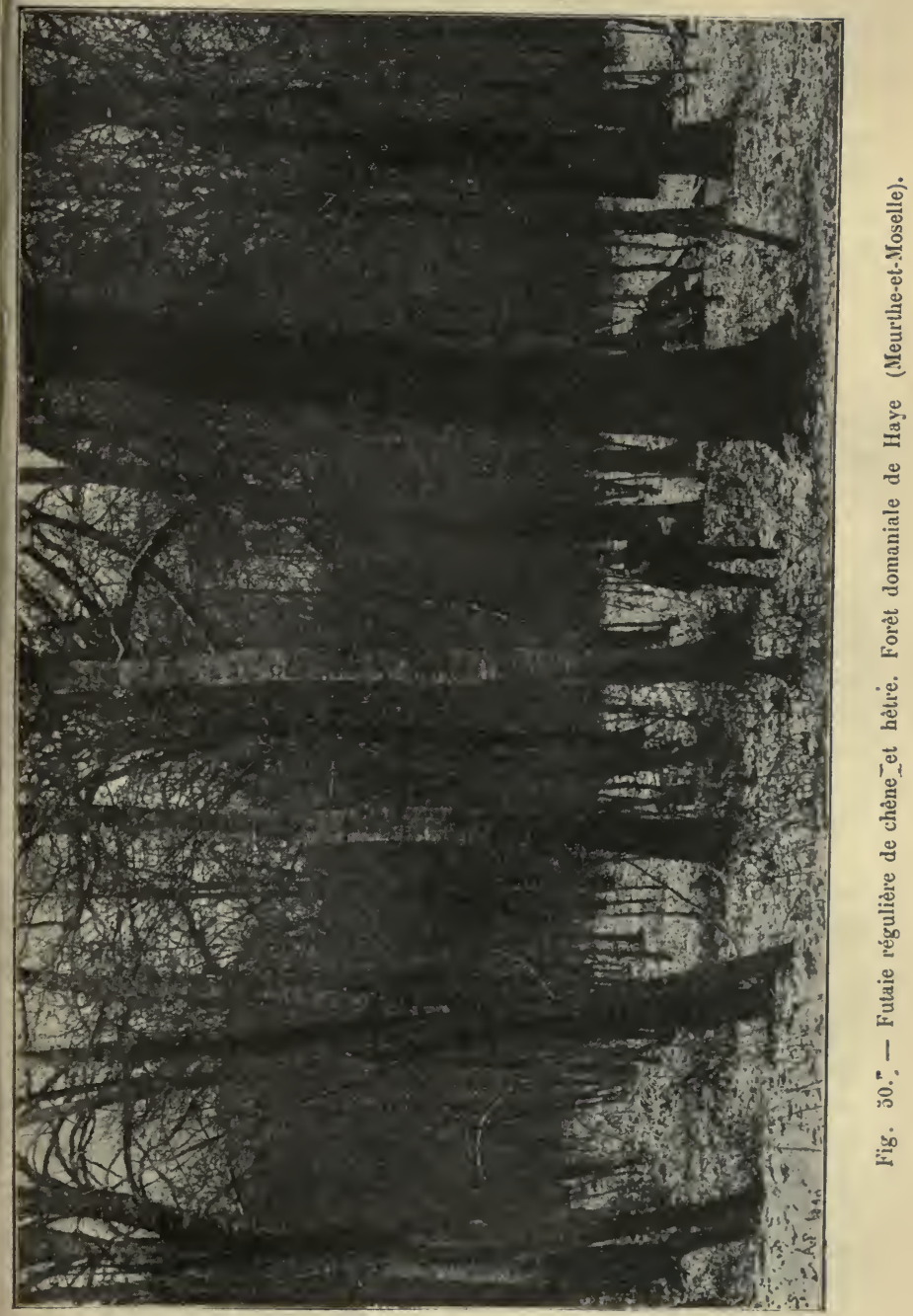


tier contre l'abus de la hache? La coupe élant le grand moyen de traitement des forêts, nous en parlons presque. à chaque ligne dans ce livre; mais sur le terrain, il asl sage de l'économiser.

En gestion forestière, il est nécessaire de se rappeler qu'après chaque coupe, c'est l'action lente de la régélation qui restaure le peuplement.

\section{APPENDIGE}

\section{DES PRÉS-BOIS.}

Un pré-bois est un terrain mi-partie en prairies ou en pâturages à peu près dépourvus d'arbres, et mi-partie à l'état de bouquets ayant une certaine consistance; les arbres isolés n'y sont qu'exceptionnels. Celte culture mixte, très avantageuse dans certaines régions montagneuses, permet de concilier la production du bois el l'entretien du bélail.

M. Broilliard éludie trois types de prés-bois :

$1^{\circ}$ Les prés-bois du Jura, dont le vrai type se rencontre surtout dans le haut Jura, où l'essence forestière dominante est l'épicéa.

$2^{\circ}$ Les prés-bois des Alpes, prés-bois fauchables et pâtures isolées qui n'apparaissent guère que comme des taches isolées, comme des oasis de verdure au milieu des pâturages dénudés et des ravinements du sol; l'essence forestière dominante est le mélèze.

$3^{\circ}$ Les prés-bois du plateau central. - Sur les terres dénudées des montagnes du centre de la France, au climat 'n quelque sorte intermédiaire entre le climat des Alpes $\leadsto 1$ celui du Jura, existent d'immenses étendues de terrain granitique à grains grossiers, ou formé par la désagrégation d'autres roches siliceuses; là rxistent près de 400000 hectares de terrains improductifs auxpuels on pourait faire produire de l'herbe ou du bois. Le moyen est simple; 
c'est de créer des forèts claires, des prés-bois de pin sylvestre et de mélèze.

Nous renvoyons le lecteur à l'ouvrage de M. Broilliard où l'auteur étudie de la façon la plus instructive ce mode de culture' spéciale, et aux intéressants travaux de M. E. Cardot sur le reboisement des Hautes-Alpes et les prés-bois.

\section{ÉDUCATION DES ARBRES ISOLÉS.}

L'éducation des arbres isolés, permet d'en obtenir à peu près partout des produits importants, à la condition qu'on choisisse avec soin les essences, et qu'on prenne grand souci de la plantation.

Le chêne, l'orme champêtre, le frêne, le cormier, le mélèze et le pin, essences à couvert léger, parfois le hêtre, les grands érables, l'épicéa et le sapin malgré leur couvert épais, le noyer, le châtaignier et tous les arbres fruitiers, les peupliers, les saules, enfin certaines essences exotiques, telles que le robinier et le platane actuellemen I bien acclimatés, et peut-être quelques autres moins bien connues, tous ces arbres peuvent être plantés utilement à l'état isolé, chacun dans le milieu qui lui convient.

Pour donner des produits utiles, ils ne doivent pas ètre mutilés, et la culture doit leur réserver une place suffisante.

M. Broilliard, dans son ouvrage " Traitement des bois en France, " leur a consacré quelques pages, bien utiles à lire, surtout aujourd'hui où l'homme exploite, mais bien souvent n'a pas le soin de replanter pour ses enfants. Que de terres nous connaissons où quelques arbres, fûtce seulement en bordure, ne feraient pas grand mal à la culture, et seraient susceptibles de donner de beaux produits! Nous souhaitons que cet appel soit entendu de ceux qui ne plantent pas, uniquement par insouciance de l'avenir.

A. Frox. - Sylviculture. 


\section{QUA'TRIÈME PARTIE}

\section{LE DOMAINE BOISÉ ET SES ÉLÉYENTS CONSTITUTIFS}

\section{PREMIERE SEGTION}

\section{Notions générales d'économie forestière.}

L'économie forestière, par cela mème qu'elle est la science des richesses forestières, est en mème temps la science des rapports, des harmonies, des proportions, des solidarités qui déterminent les conditions d'équilibre et de bonne distribution des forces productives de la sylviculture.

Elle considère, dit M. Puton (1), l'arbre comme un ouvrier fabriquant du bois, le peuplement comme une association d'ourriers unis pour une fabrication commune, la forèt comme une entreprise, une sorte d'usine destinće à produire la matière ligneuse.

L'économie forestière, ainsi d'ailleurs que l'économie rurale, se rattache à l'économie politique, en ce que comme cette dernière, elle observe et décrit les milieux économiques; mais elle revèt nettement le caractère d'une science distincte dans l'étude de la constitution des.

(1) A. P'uton, Trailé d'économie forestière. Paris, 1888. 
entreprises forestières. C'est surtout par cette seconde partie que nous l'aborderons.

\section{I. - AGENTS dE LA PRODUCtion fOREstiére.}

L'économie politique enseigne qu'on ne peut rien produire sans le concours de trois éléments : le capital, le travail et les agents naturels. Nous nous proposons d'examiner à ce point de vue la constitution de l'exploitation forestière.

\section{1. - Capital d'exploitation.}

L'exploitation forestière présente un caractère tout spécial, dù à ce que les fonds boisés se distinguent des autres domaines, en raison mème du mode de jouissance qu'ils comportent, par la constitution de leur capital d'exploitation.

Mode de jouissance. - La récolte forestière est essentiellement périodique sur un point déterminé. Pour atteindre les dimensions qu'on lui demande, l'arbre doit occuper le sol pendant un certain nombre d'années, très variable suivant les circonstances; dans certains taillis il lui suffit de huit ou dix ans; il demande au contraire souvent plus d'un siècle dans les futaies; et pour donner sur le même point la récolte suivante, le sol doit être ensuite occupé pendant une nouvelle période aussi longue; le temps devient dès lors un facteur prépondérant en économie forestière.

En fonctionnant ainsi, en ne livrant pas tous les ans, comme en agriculture une récolte, l'arbre ou le peuplement accumulent leur production annuelle; ils sont des réserves, des sortes de greniers conservant des économies annuelles et les faisant fructifier. Susceptibles de créer ainsi-un capital et d'augmenter une fortune, ils ont 
l'inconvénient de ne pas donner un revenu annuel pour les besoins de la vie.

Mais cet inconvénient n'est qu'apparent; nous arons vu que pour obtenir d'un domaine boisé un revenu annuel, il suffit de réaliser les produits successivement sur autant de surfaces différentes qu’il faut d'années aux arbres pour atteindre les dimensions requises. La forêt ainsi économiquement constituée est dite aménagée; elle présente sur des surfaces égales ou équivalentes, surfaces qui sont indépendantes l'une de l'autre ou intimement mélangées, une suite non interrompue d'arbres ou de peuplements, différant entre eux d'une année d'âge, lepuis les sujets naissants jusqu'à ceux ayant vécu le nombre d'années que comporte l'exploitation ; ces derniers seuls constituent la récolte ou le revenu, et tous les bois qui occupent le sol sur les surfaces autres que celles qui portent la récolte, font nécessairement partie d'un matériel en croissance, c'est-à-dire non encore exploitable, nécessaire dans son intégralité pour assurer le revenu annuel.

Le matériel en croissance, ou capital générateur, devien I dìs lors un facteur prépondérant en économie forestièrr.

Remarquons que la forêt aménagée peut se proposer pour but, non plus d'obtenir une récolte annuelle, mais simplement d'avoir des récoltes se suivant à des intervalles égaux ou inégaux, et plus ou moins rapprochés ; c'est une exploitation intermédiaire entre les deux précédentes; les âges se suirent alors, non plus d'année en année, mais ell gradins successifs, séparés par des intervalles égaux ou inégaux, supérieurs à une annéc, et inférieurs à l'âge d'exploitation.

Signalons enfin que dans toute exploitation aménagée, chacune des parties, considérée isolement, se comporte comme une exploilation périodique.

Fonds, propriété foncière. - La terre est l'agent frimordial de la production forestiere, et le sol appartient 
soit à des individus (propriété privée), soit à des collectivités, telles que l'État, les départements, les communes, les établissements publics, ou les associations diverses dont les domaines sont soumis à la taxe des biens de mainmorte.

D'après l'enquète agricole faite en 1882, la surface possédée par les diverses catégories de propriétaires se répartit ainsi en France :

1. Propriétés de l'État. $\left\{\begin{array}{lr}\text { Bois et forèts.... } & 12.300 \\ \text { Donıaines divers. } & 998.834\end{array}\right\} \begin{gathered}\text { Hectares. } \\ 1.011 .153\end{gathered}$

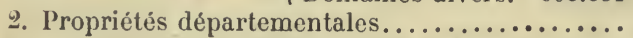

6.513

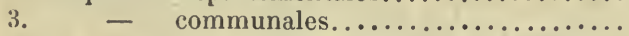

4. - des établissements publics et autres (hospices, fabriques, séminaires, couvents, bu-) reaux de bienfaisance, congrégations religieuses, chemins de fer, sociétés anonymes

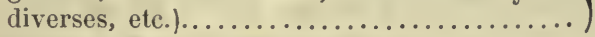

5. Propriétés des particuliers............... 45.025 .598

6. $\quad$ - non définies............... 1.810.885

Superficie totale de la France.... ....... $\overline{52.857 .199}$

D'après la même enquête, la répartition des cultures en France en 1882 peut être représentee par les chiffres suivants :

p. 100.

p. 100.

Terres labourables......... $\quad 49,60$

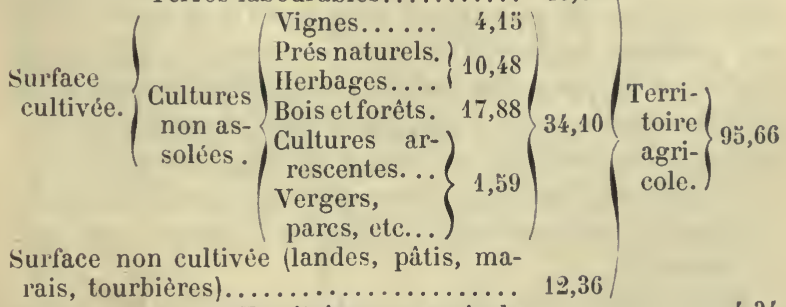

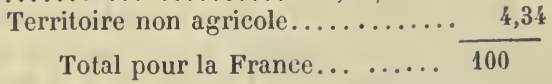

Les bois et forêts couvrent une surface de 17,88 p. 100 du territoire de la France; ils occupent une superficie de 


\section{NỌTIONS GÉNÉRALES D'ÉCONOMIE FORESTIÈRE.}

.9149000 hectares, c'est-à-dire la sixième partie de la superficie totale de la France.

Sur ces 9149000 hectares, 1020106 hectares appartiennent à l'État ; 1923137 hectares appartiennent aux communes et établissements publics, etc. Le surplus, soit en chiffres ronds 6200000 hectares, est possédé par les particuliers. Ce dernier chiffre est loin d'avoir le même caractère d'authenticité que ceux de la contenance des forêts de l'État et des communes, parce qu'il résulte seulement d'évaluations faites en $18 \% 9$ pour une revision du cadastre.

ll résulte de ces chiffres que les forèts des propriétaires particuliers forment la grande majorité des forêts françaises.

Au sujet de cette catégorie de propriétés, nous ne possédons malheureusement pas encore aujourd'hui des pocuments statistiques exacts et complets, et il est impossible de donner des chiffres sérieux sur la division de cette propriété en grande, moyenne et petite exploitation forestiêre ; remarquons toutefois que l'établissement dụ Code civil et la transmission par héritage tendent à favoriser le morcellement de la propriété; le père de famille ne peut en effet disposer en toute liberté que d'une faible partie de sa fortune, et le reste doit ètre partagé par portions égales entre tous ses enfants. En ce qui concerne la propriété boisée, ce partage fréquent des grandes forêts tend à diminuer leur importance, rend onéreux l'assiette sur le terrain de longues et savantes combinaisons d'aménagement, ainsi que les modifications incessantes qu'il entraîne au cours de l'existence des peuplements.

Nous avons réuni dans le tableau suivant, les renseignements statistiques concernant la répartition de la propriélé boisée en France; les chiffres de ce tableau sont extraits de l'Atlas de statistique forestière publié en 1878 par l'administration des eaux et forêts. 


\begin{tabular}{|c|c|}
\hline 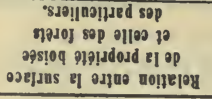 & $\begin{array}{l}\dot{\delta} \\
\dot{\delta} \\
\dot{0}\end{array}$ \\
\hline 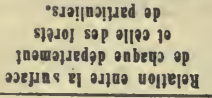 & $\begin{array}{l}\dot{8} \infty \\
\dot{0}\end{array}$ \\
\hline 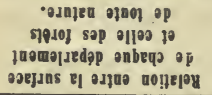 & $\begin{array}{l}\dot{\delta} \\
\text { வ }\end{array}$ \\
\hline
\end{tabular}

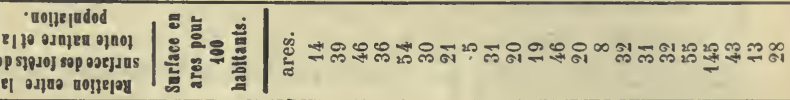

\begin{tabular}{|c|c|}
\hline : & 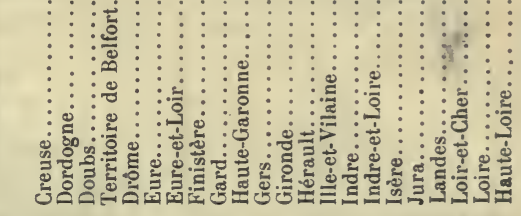 \\
\hline 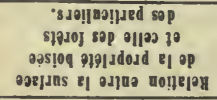 & 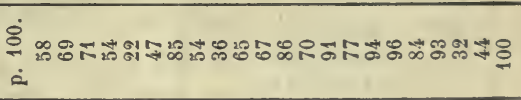 \\
\hline 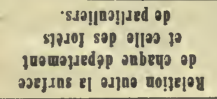 & 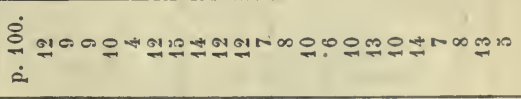 \\
\hline 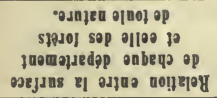 & 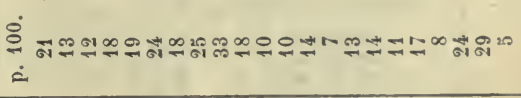 \\
\hline
\end{tabular}

\begin{tabular}{|c|c|c|}
\hline 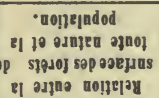 & 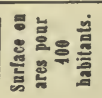 & 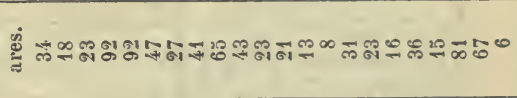 \\
\hline
\end{tabular}

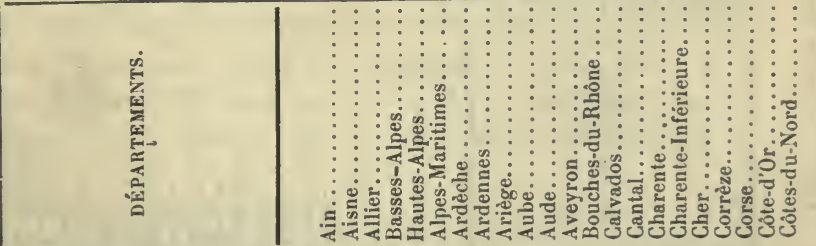




\section{6千 NOTIONS GÉNÉRALES D'ÉCONOMIE FORESTIÈRE.}

\begin{tabular}{|c|c|}
\hline 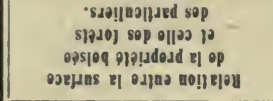 & 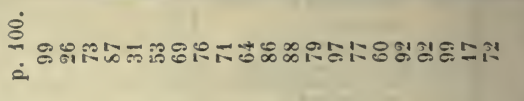 \\
\hline 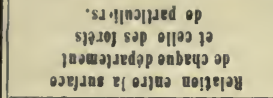 & 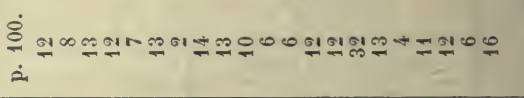 \\
\hline 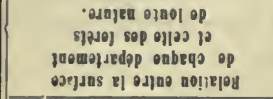 & 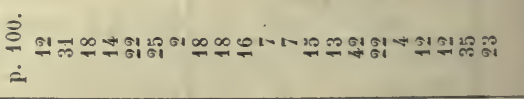 \\
\hline 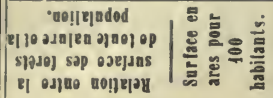 & 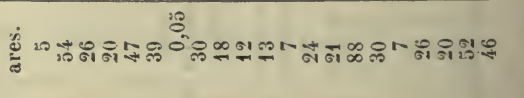 \\
\hline 点 & 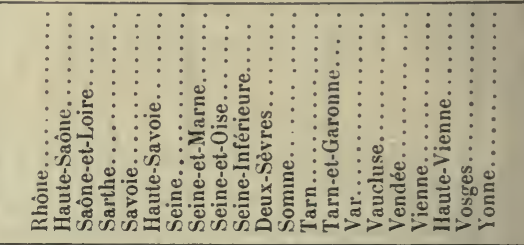 \\
\hline 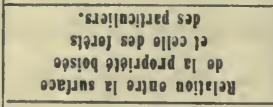 & 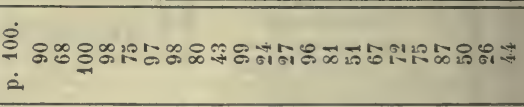 \\
\hline 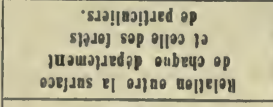 & $\begin{array}{l}\dot{\delta} \\
\dot{\delta} \\
\dot{\dot{1}}\end{array}$ \\
\hline 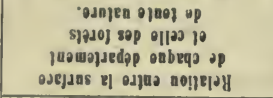 & 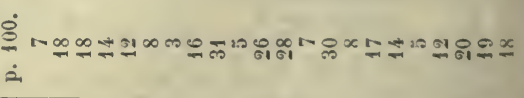 \\
\hline 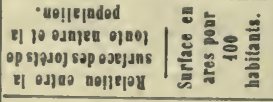 & 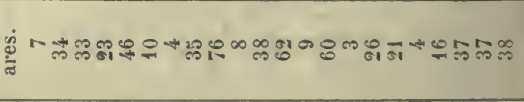 \\
\hline 造 & 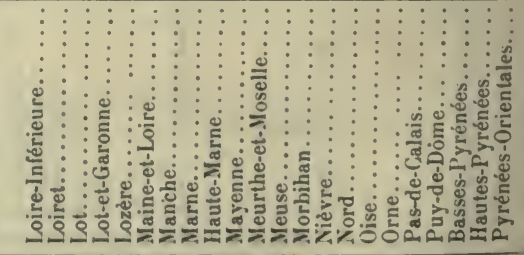 \\
\hline
\end{tabular}


L'examen de ce tableau, et celui de la carte dressée pal' les soins de l'administration, montre que les diverses régions de la France sont fort inégalement boisées ; nous empruntons au Dictionnaire d'agriculture (1) et aux rapports présentés par les instituteurs à la Société forestière française des amis des arbres (2) les renseignements suivants sur la propriété forestière en France.

Les départements du Nord, du Pas-de-Calais et de la Somme contiennent peu de forèts ; ces pays de grande culture et très industrieux présentent l'aspect de vastes plaines au milieu desquelles s'élèvent les masses sombres de forèts peu nombreuses, mais richement peuplées: Mormal, Saint-Amand, Crécy, Nieppe, sont les principaux massifs de cette région.

En parcourant de l'ouest à l'est la zone comprise entre les $48^{\mathrm{e}}$ et $50^{\mathrm{e}}$ degrés nous trouvons à l'ouest la Bretagne et la Normandie, pays en grande partie granitique où les forèts sont rares et disséminées; la Bretagne accidentée et pittoresque a quelque analogie, dit M. Cardot, avec le plateau central, sinon par l'élévation de ses collines qui atteignent à peine l'altitude de 300 mètres, du moins par' la nature du sol formé de roches granitiques ou schisteuses, par l'importante surface qu'occupent encore les terres incultes, les landes d'ajonc, de bruyère et de genél, enfin par la faible teneur en bois ou forèts qui caractérisent son territoire; les haies d'arbres, chènes, châtaigniers, hètres, essences diverses qui entourent les champs donnent bien à certaines parties de la Bretagne l'aspect ou l'illusion d'un immense bocage ; mais sur bien des points, les arbres ont disparu sans être remplacés, et les bois de haute futaie n'existent guère que dans quelques forèts domaniales peu étendues et dans les parcs des châteaux.

(1) Dictionnaire d'Agriculture, par J.-A. Barnal et H. Sagnier.

(2) Rapport de M. Cardot sur le concours forestier entre les instituteurs (Bulletin trimestriel, $\mathrm{n}^{\circ} 38,1902$, de la Société forestière frangaise des amis des arbres). 
Aussi arrive-t-il que dans certains cantons particulièrement déboisés, comme l'extrémité occidentale du Finistère, la plage du Conquet, etc., où l'abri des arbres serait pourtant si nécessaire, l'habitant n'a pour combustible que desfagots d'ajonc ou de genêt, roire même des bouses de vache que l'on fait sécher au soleil en les appliquant contre les murs.

Mais quand on s'éloigne de la mer, en allant vers Paris, on rencontre de puissants massifs comme ceux de Paimpont, d'Audaine, d'Ecouves, de Conches, de Breteuil, de Senonches, et sur les rives de la Seine ceux de Bretonne, Lyons, la Londe.

Dans le voisinage de Paris Ies forêts deviennent plus nombreuses et plus importantes; celles de Retz, de Rambouillet, de Compiègne, de Saint-Gobain, d'Halatte, les bois de Meudon, des Fausses-Reposes, de Saint-Germain, situés aux portes de la capitale, les forêts de Fontainebleau, de Montargis et d'Orléans, font de cette région. pourtant la plus peuplée de France, une desmieux boisées.

En poursuivant notre route vers l'est, nous rencontrons les forêts d'Othe, de Traconne, d'Enghien et de la montagne de Reims; puis des plaines de Champagne, si dénudées autrefois, mais qui se garnissent maintenant le bois de pins, nous entrons dans la région forestière de l'est qui s'étend depuis l'Ardenne jusqu'aux Vosges, ef qui comprend les massifs de l'Ardenne et de la Thiérache, ceux de l'Argonne, de la Voëvre, du Val, du Der et de Soulaines, et les forêts de Vaucouleurs, de Ligny, de Charmes, Darney, etc., qui se relient à la grande forè vosgienne.

Les montagnes vosgiennes, dit M. Cardot, passent à bon droit pour les mieux boisées de France; dans le département des Vosges, l'élat boisé occupe plus de 35 p. 100 de la superficie; les forêts y produisent annuellement un revenu de plus de huit millions de francs; cette région renommée par 'ses forêts est riche aussi en her- 
bages; à l'entour des ballons assombris par les forèts résineuses se déroule, d'après M. Cardot, le riant tapis des prairies dont la verdure et les fleurs sont incessamment rafraichies parles mille courants d'eau claire qui découlent en cascades des pentes de grès ou de granit.

Il semble qu'il n'y ait plus place dans ces montagnes privilégiées pour les terres incultes; pourtant elles y occupent encore plus de 16000 hectares, et M. l'instituteur Dagneaux nous apprend que dans sa commune il y a 400 hectares de terrains communaux improductifs (1).

Dans cette mème région de l'est, en Lorraine, existent également des friches, et des terres incultes du mème genre ; dès 1878, M. Henriet Joseph, instituteur à Vacqueville (Meurthe-et-Moselle) s'est mis à acheter de petites propriétés, pour la plupart en friches, et à y faire des semis et des plantations forestières; le plus souvent il commençait par labourer le terrain et l'ensemencer d'avoine; sur le sol ainsi préparé, des graines de pin sylvestre étaient répandues qui germaient sous l'abri des chaumes; on y repiquait aussi des plants de bouleau, d'aune, parfois d'épicéa, sapin, mélèze, etc. ; quelques années plus tard, le hêtre et le chène étaient introduits en sous-étage pour constituer les peuplements d'avenir, après que les pins et bouleaux avaient été employés pour la papeterie, et les aunes pour la fabrication de jouets d'enfants. En agissant ainsi avec de la persévérance et de la ténacité, il a préparé une petite fortune à ses enfants, ses économies ont fructifié mieux que dans une caisse d'épargne, et l'exemple est bon à suivre.

(1) Dans cette population de montagnards vivant des produits de la culture et de l'industrie du bois, les familles nombreuses émigrent vers la ville. C'est bien le cas de chercher à les retenir par des travaux, des salaires, et par le développement des ressources forestières et pastorales. C'est ce qu'a fait déjà M. Chipon, instituteur à Lesseux, en créant une petile Société mutuelle forestière; il a montré comment de petits écoliers peuvent contribuer à la fortune de leur pays (Cardot). 


\section{NOTIONS GÉNÉRALES D'ÉCONOMIE FORESTIĖRE.}

La partie occidentale de la zone comprise entre le $46^{\circ}$ et le $48^{\mathrm{e}}$ degré est très pauvre en forêts; citons, dans la Loire-Inférieure, la localité de Saint-Colombin dont la description nous est donnée par M. l'instituteur Bonhier; le terrain de la commune est en plaine et en petits coteaux; dans la plaine: les céréales, les prairies naturelles et artificielles; sur les coteaux: des vignobles que le phylloxéra a en grande partie détruits, mais qu'on s'occupe à reconslituer; aucune forêt, si ce n'est quelques petits taillis qu'on coupe tous les huit à dix ans pour obtenir du bois de chauffage; les champs sont séparés les uns des autres par des fossés plantés de haies d'aubépine, de ronces, de genêts et d'ajoncs parmi lesquelles poussent des essences forestières, principalement le chêne, l'orme et le frêne; dans les lieux humides ou sur le bord des eaux, croissent abondamment des aunes, dont on se sert pour faire des fagots; sur le bord des rivières ou des ruisseaux, on plante quelques peupliers, mais pas assez, car cette plantation est peu coûteuse, se faisant par boutures. Dans l'arrondissement de Châteaubriant, d'après M. Ponvreau, on ne produit pas de vin, mais on fait beaucoup de cidre ; en général les champs sont de véritables vergers, renfermant de nombreuses variétés de fruits. Quant aux massifs forestiers, les forêts de la Guerche, d'Ancenis, de Chizé, de Bercé, de Perseigne, de Milly, méritent d'être signalées dans cette partie de la Bretagne, de l'Anjou et du Poitou; la rareté des grands massifs est compensée par les haies de grands arbres qui environnent les champs et les prairies. La Touraine contient les belles forêts de Blois, de Marchenoir, d'Amboise, de Boulognes, de Loches, de Fontevrault; celles de Vierzon, de Châteauroux, d'Allogny, de Maulne et de Tronçais rompent la monotomie des plaines du centre de la France. Sur la rive droite de la Loire, les forêts de la Nièvre présentent une masse imposante qui occupe les montagnes du Morvan, s'étend 
au nord dans les plaines de la Puisaye, au sud dans celles du Bourbonnais.

Un autre massif forestier, partant de Chaumont-enBassigny, couvre les coteaux calcaires de la HauteMarne et de l'Aube, se continue dans le Châtillonnais et se rattache par de nombreuses ramifications aux forèts jurassiques parmi lesquelles nous citerons celles de Chaux, de la Joux et de Levier; toute la haute montagne du Jura forme de grands rideaux de noirs sapins qui encadrent les vallées, et qui donnent à cette contrée un aspect un peu sévère et mélancolique; mais ces ombrages qui recouvrent d'une façon si régulière et un peu monotone les longues chaines de collines, recèlent de véritables trésors; ces immenses forèts de sapins donnent aujourd'hui, nous dit M. Cardot, et donneront indéfiniment, si on continue à les gérer avec prudence, des revenus de 100,150 et mème 200 francs par hectare et par an; on y trouve des parcelles où la valeur des arbres sur pied atteint par hectare 20000 , 25000 et parfois mème 30000 francs. Ce pays est renommé aussi pour ses prés-bois, pour ses pâturages où paissent pendant la saison d'été de nombreux troupeaux de vaches; mais il tend à déchoir sous ce rapport et le regard est assez souvent affligé par la vue de vastes espaces où les pierres éparses, les dénudations rocheuses, es taupinières moussues, les buissons raboůgris, remplacent les pelouses et bosquets de grands bois qui existaient autrefois (Cardot). Sur les plateaux inférieurs, du Jura, par-dessus les escarpements calcaires qui en forment les assises, on ne trouve plus de sapins. Quant aux pâturages, l'instituteur d'Aiglepierre, M. Tournier', nous en donne la description: d'immenses surfaces, des centaines d'hectares d'une seule pièce sont incultes et improductifs; ces terrains, communaux pour la plupart, produisent sur le touriste une impression de pénible tristesse; on voit çà et là poindre quelques rochers

A. Fros. - Sylviculture. 
rougeàtres qui s'effritent; quelques buissons rabougris de buis, de houx, de genévrier; quelquefois ce sont d'épais fourrés d'épines, de ronces, où il est absolument impossible de pénétrer. Que produisent ces terrains qui devraient être une source de richesses pour les communes? peu de chose; le bétail que l'on y conduit ne peut y vivre, il s'y fatigue, dénude le sol tous les jours davantage; la pâture, envahie par les broussailles et les mauvaises herbes, ravinée par les eaux et par le pied du bétail, se dégrade ou ne produit plus que quelques buissons où les malheureux viennent piller chaque hiver un maigre supplément d'affouage (1). Cette situation tend à s'améliorer, sous l'influence des efforts de l'administration des Eaux et Forèts, de la Société forestière de Franche-Comté et Belfort, et des sociétés scolaires forestières, nombreuses aujourd'hui dans la région.

La zone comprise entre les $44^{\circ}$ et $46^{\circ}$ degrés est bordée du côté de l'ouest par les grandes pineraies des landes de Gascogne; mais si, continuant par notre tour de France nous nous éloignons de l'océan, nous arrivons en Dordogne, pays qui contient beaucoup de forêts plus importantes par leur étendue que par leur richesse, et où l'on peut souvent tirer parti d'une culture spéciale, la trufficulture. M. Cardot résumant à la Société forestière française des amis des arbres le mémoire de M. Bordes (2),

(1) Bulletin de la Sociélé forestiére des amis des arbres, n 38, 1902.

(2) Ce ménıoire est la reproduction d'une conférence faite à des habitants et conseillers municipaux pour les engager à mettre en valeur leurs' friches et les communaux incultes par des plantations forestières et truffigènes. Combien l'ngriculteur's, dit M. Bordes dans sa conclusion, coudoient la inisère, et pourraient vivre dans une aisance relative en utilisant la partie de leur bien qu'ils ne travaillent pas, ou qu'ils laissent en jachẻre pour cause d'infertilite: Counlien d'autres plus aisés se créeraient des revenus considérables par une flantation peu coúteuse de coteaux dénudés, qui servent de sortie h̀ leurs troupeaux ! et eufin que de conmunaux, dans un complet abandon aujourd luui pourraient fournir demain aux communés rurales les ressources qu'elles epronvent tant do difficultés à se procurer!

A l'appui de ces aflimntions, M. Cardot cite une commune de Vaucluse qui a pergu jusqu'à 60000 el 80000 francs pour In location de ses trullières. 
instituteur à Orléaguet, donne à ce sujet les renseignements suivants : la truffe est un petit champignon végétant et se multipliant sous terre comme les autres champignons à la surface du sol; elle est beaucoup plus répandue qu'on ne le suppose, car on la trouve dans 53 de nos départements français. On croit généralement qu'elle se produit exclusivement sous des taillis de chène; c'est une erreur, près de 40 espèces d'arbres concourent à la formation de truffières; mais il y a truffe et truffe. La meilleure variété de truffe est la truffe noire d'hiver (tuber melanosporum); on la trouve dans la région méridionale, dans la Dordogne, les Charentes, le Lot-et-Garonne, dans le Lot et ses confins, dans la Corrèze, en Vaucluse et les contrées voisines; elle exige un terrain calcaire avec sous-sol perméable, se plait sur les plateaux et les pentes inférieures bien ensoleillées des montagnes ou des collines, à des altitudes ne dépassant pas 700 mètres. La qualité de la truffe dépend beaucoup aussi de l'espèce d'arbres qui courre la truffière: l'épicéa et les pins lui donnent, parait-il, un goût de résine; l'orme et le tilleul, d'après M. Bordes, la rendent nauséabonde et souvent immangeable ; le chêne vert ou chêne yeuse est l'essence truffigène par excellence.

Les truffes ne se développent pas sous un couvert trop complet; pour la création d'une truffière, il convient de planter les arbres à un écartement de à à 6 mètres en moyenne; pour que la production soit abondante il faut que la ramification de l'arbre s'étale en largeur au lieu de se développer en hauteur; on peut obtenir artificiellement cette forme en empèchant le pivot de s'allonger dans le sol, et d'autre part en pratiquant sur la cime elle-mème une taille spéciale, dès qu'elle a une tendance à s'élancer.

Quittant cette région, nous arrivons aux premiers contreforts du plateau central qui présentent un autre 
aspect; on ne voit plus là que quelques bouquets isolés dans les montagnes de la Harche, du Limousin, de l'Aurergne, du Velay et du Vivarais; le Forez lui-mème est, malgré son nom, presque entièrement déboisé. Un instituteur, M. Jean-Baptiste Lavialle (1) donne la description suivante du territoire de la commune de Treignac (Corrèze): des montagnes recouvertes de bruyères et de genèts, entrecoupées parfois de champs de seigle, de pommes de terre et de blé noir ; çà et là de rares touffes de chènes, de bouleaux et de hètres, et des arbres isolés; un petit nombre de prés passables aux abords des villages; de mauvais pâturages, au milieu de la plaine; des moutons sur les Puys; de maigres vaches dans les bas pacages; d'immenses espaces incultes! tel est le spectacle qui de tous côtés se déroule sous mes yeux jusqu'à l'horizon. Il est triste de dire que cette situation si bien décrite se reproduit à quelques variantes près pour la plupart des conmunes de la région montagneuse du plateau central ( $(2)$.

Il faut passer sur la rive gauche du Rhône pour trouver quelques massifs comme ceux de la Chartreuse, du Vercors, de Lente, du Ventoux, el les bois épar's sur les versants des Alpes; mais dans celte région encore, spécialement dans les Alpes de Provence et les Alpes du Dauphiné, on peut constater tous les résultats de la destruction des gazons, et des bois qui étaient la sauregarde

(1) Mémoire de M. Lavialle. D'après M. Gandor (Bulletin société forestière française des amis des arbres, $\mathrm{n}^{\circ} 38,1902$ ).

(2) Cette situation inspire à M. Lavialle le désir d'y remédier; il essaie de déraciner la routine et tente de faire un peu de bien autour de lui; il enseigne ce qu'il appelle la culture arborale et la culture pastorale ot cet enseignement porte ses fruits; quelques propriétaires commencent d'abjo à drainer les mauvaises parties des prés, à planter des aunes et des saules le long dés ruisselets; ils sément du pin sylvestre et des épicéas; ils créent des taillis, des futaies, et des garennes de chène, de hệtre, de bouleau, de châtaignier ; ils plantent et greffent de bomes variétés d'arbres fruitier's, etc. Aujourd'hui on ne reconnatrait plus le pays, tant y ont progressé l'agriculture et l'hygiène qui ont tout embelli, amélioré el enriehi. Ciest par plusieur's centaines d'liectares que se sont effectuées ces heureuses modifications (Cardot). 
et la richesse des villages; dans cette région des avalanches et des torrents, on a trop oublié, que le pàturage, et surtout le pâturage commun doit ètre soigné et entretenu, et que dans la montagne, suivant l'expression de M. Cardot, si l'on veut avoir des pelouses, il faut savoir conserver des arbres. Les Alpes de Savoie sont en meilleur état, mais que d'améliorations il reste encore à y faire!

$\mathrm{Au}$ sud $\mathrm{du} \mathbf{4}^{\mathrm{e}}$ degré, la végétation tout à fait méridionale dans les plaines de la Gascogne et du Languedoc, prend un caractère plus septentrional quand on s'élève sur la chaîne des Pyrénées où l'on trouve une zone assez boisée qui s'étend de Quillan à Bagnères-deBigorre, de l'Aude à l'Adour. Les forèts de la plaine de Sault, d'Auzat, de Seix, de Bordes, de Bethmale et de Belesta sont les plus importantes de cette région; mais sur bien des points les pâturages commencent à se dénuder, à se dégradèr; sur bien des points aussi le déboisement a accompli son wuvre néfaste, notamment dans la partie orientale de la chaine où la destruction du sol végétal est presque complète, et où les vallées ou plaines inférieures sont sous la menace des crues torrentielles. A l'exception du massif de la Montagne noire, où il reste quelques lambeaux de bois, on ne voit que des plaines cultivées ou des montagnes dénudées dans les départements du Gard, de l'Hérault et des Bouches-duRhône. Il faut aller jusqu'au Var pour retrouver de vraies forèts. Celles des Maures et de l'Esterel donnent à cette région un caractère bien accentué; malheureusement elles sont exposées d'une façon permanente au danger des incendies.

En ce qui concerne spécialement les massifs boisés, nous pouvons conclure de cet aperçu général que la répartition des forèts est très irrégulière en France. Les causes qui ont amené cette répartition sont extrèmement complexes, et nous pouvons classer parmi les plus 


\section{$47 \pm$ NOTIONS GÉNÉRALES D’ÉCONOMIE FORESTIÈRE.}

importantes celles qui concernent la question des débouchés et la constitution géologique du sol. Dans bien des cas, dans le Morvan, dans les Vosges par exemple, la forèt s'est conservée parce que la proximité de grands marchés assurait aux produits ligneux fabriqués des débouchés suffisants.

Mais si l'on fait abstraction de cette influence, on peut dire que les forêts ne se sont maintenues que sur les sols les moins fertiles. Les massifs les plus importants, comme ceux de l'Ardenne, de l'Argonne, du Jura, de Bassigny, de la Puisaye, des Landes, de l'Esterel, reposent sur des sols trop maigres, trop peu profonds ou trop accidentés pour que la culture y soit profitable.

Et M. Bouquet de la Grye ajoute ailleurs (1): Dans noz vieux pays de l'Europe, ce n'est pas seulement l'accroissement de la consommation du bois qui a amené l'appauvrissement et la ruine des forêts, c'est aussi la nécessité de pourvoir, en augmentant la surface des terres cultivées, à l'alimentation d'une population devenue de plus en plus nombreuse; cette nécessité a donné naissance à une lutte sans trève, dans laquelle les forèts ont toujours reculé devant l'agriculture; celles que la charrue ou les troupeaux n'ont pas détruites, ont été reléguées sur les sols les plus infertiles.

Le défrichement des forèts des plaines a été quelquefois avantageux, lorsqu'il portait sur des terrains profonds et fertiles; mais si l'on envisage la situation aujourd'hui, il est facile de constater que ces terrains ont été presque tous défrichés et livrés à la culture depuis bien des siècles, et qu'il ne reste plus, en plaine, de forêts que sur les sols argileux et froids, d'une culture difficile, siliceux ou caleaires, secs, sans profondeur et partant peu propres à la culture; dès lors, dans ces deux cas, le résultat le plus net du défrichement, c'est qu'aprìs

(1) Dictionnaire d'Agriculture, de J.-A. Barnat. el II. Sagner. 
la réalisation de la superficie et de quelques maigres récoltes, la forèt est infailliblement remplacée par une lande improductive.

De ce qui précède, il résulte qu'aujourd'hui les forèts occupent le plus souvent, en France, les terres que ne peut occuper la culture agricole. La valeur foncière de la propriété boisée doit s'en ressentir.

Valeur foncière. - Sol. - Le sol, support de toute entreprise, est un véritable capital, puisqu'on ne peul l'acquérir qu'en échange d'un capital argent. En matière forestière, la valeur foncière ou valeur vénale du sol est difficile à définir; on vend bien rarement des sols forestiers exploités, dépouillés des éléments de production ligneuse qui font corps avec eux, et il est alors délicat, inutile d'après beaucoup d'auteurs, de chercher à avoir une idée de la valeur vénale d'une sol forestier nu.

M. Puton, néanmoins, est d'avis d'évaluer le sol nu d'après l'usage qu'on en pourra faire; terres à mettre en labour, parties à transformer en prairies, parcelles à bâtir, simples pâturages, terres à bois (cas le plus fréquent puisque la culture agricole a déjà pris la plupart des terrains utilisables); tout cela sera estimé d'après les prix résultant des ventes de la localité, selon qualité, et déduction faite, s'il y a lieu, des frais de défrichement, et aussi suivant les probabilités qui existent dans les débouchés, en terres à culture ou à bois. La règle, pour M. Puton, est d'estimer le sol d'après les ventes de terres de mème qualité dans les environs; son application est affaire d'appréciation et de sagacité. b'après ce même auteur, il suffit qu'il y ait eu dans la localité quelques ventes de forêts, pour qu'un estimateur intelligent déduise la valeur $\mathrm{du}$ sol de la connaissance des bois qu'il supportait; là par exemple, une forèt de 10 hectares a été vendue 12500 francs; si la visite de la forêt permet d'évaluer la superficie boisée à 7500 francs, c'est que le sol a été compté 5000 francs, ou 500 francs 
l'hectare; des renseignements de cette nature suffisent à éclairer les experts, s'ils y joignent la connaissance des terres voisines, agricoles ou pastorales, faites dans la localité.

Dans le cas de plus en plus rare aujourd'hui où le sol sera estimé en vue de lui demander des cultures agricoles, il y aura lieu d'examiner si le domaine boisé tombe sous le coup de l'article 219 du Code forestier (1); si l'opposition au défrichement est absolue, c'est-à-dire sans aucune chance d'ètre levée, on estimera le sol comme terrain boisé, uniquement destiné à la culture forestière, sans tenir aucun compte d'utilisations plus fructueuses, impraticables par suite de cette interdiction; si celle opposition n'est que temporaire, elle constitue une charge, une servitude plus ou moins certaine et durable; on estimera le sol comme terrain à cultiver ou à bâtir, en déduisant une certaine somme, un cinquième ou un quart par exemple, pour la charge dont l'éventualité permet une évaluation de celte nature.

Quant aux acheteurs qui veulent conserver l'immeuble en nature de forêt, et que l'interdiction de défricher laisse fort insensibles, ils estimeront le sol comme terre à bois, suivant les indications des ventes de la localité.

Le plus souvent, les uns el les autres arriveront à un résultat presque identique, car les défrichements ont donné tant de mécomptes que le goût s'en est perdu, et que les terres de médiocre qualité, étant plus ou moins ahandonnées par l'agriculture, les marchés faits sur les terres avoisinantes fourniront la meilleure et la plus pratique indication de la valeur à donner au sol, sauf déduction du gros, nécessaire ici, comme pour les mar-

(1) Art. 219, Code forestier. - Aucun particulier ne peut user du droit d'arracher ou de défricher ses bois qu'après en avoir fait la déclaration à la sous-préfecture, au moins quatre mois d'avance, durant lesquels l'administration peut faire signifier au propriétaire son oplosition au défrichement. Voir art. 210 a 226 du Code forestier. 
chandises, si la forêt est de grande étendue (Puton).

Couverture morte. - Humus. - Le sol des forèts n'est pas un terrain vierge; tandis que la terre des champs doit ètre préparée par des cultures onéreuses pour recevoir la semence et dotée d'éléments fertilisants par l'apport de fumier ou d'engrais appropriés, la forèt, dans la majorité des cas, se présente comme un bien spontané dont on prétend obtenir perpétuellement des récoltes par la simple utilisation des agents naturels; or nous avons vu qu'en sol forestier l'action de ces agents naturels est mise en valeur par la courerture morte et l'humus.

Un sol forestier, c'est-à-dire un sol couvert depuis longtemps par des végétaux forestiers, présente à ce point de vue un état tout spéciul; la couche arable, et la qualité de terre à bois sont des épargnes antérieures dont la présence est spécialement utile pour le propriétaire qui veut maintenir ce sol à l'état boisé; mais cette richesse est tellement incorporée au sol, que si on doit la distinguer du terrain vierge, pour l'analyse deschoses, on ne saurait la disjoindre pour évaluer la valeur vénale du terrain (Puton).

Ensouchement. - Semis. - Le caractère de la forèt en général est de se perpétuer plus ou moins identique à elle-même; après les récoltes successives le massif boisé se reconstitue par l'ensemencement naturel, c'est-à-dire par les graines de toute nature qui sont tombées sur le sol et sont prètes à germer ou par l'enracinement du peuplement ancien, souches et racines qui restent dans le sol et sont susceptibles de donner des rejets et des drageons. Tous ces éléments sont susceptibles, dès l'exploitation et sans nouveaux frais, de donner les jeunes plants, et le recru qui reconstitueront naturellement un nouveau peuplement; ils sont incorporés au fonds et assurent sa mise en valeur et sa force productive. Un sol forestier boisé présente à ce point de vue un état tout spécial ; la présence de l'ensouchement susceptible de donner après 
4:8 NOTIONS GÉNÉRALES D'ÉCONOMIE FOORESTIÈRE.

exploitation les rejets de souche, celle des semis, des drageons, etc., est pour le propriétaire qui veut maintenir le sol à l'état de bois, une richesse toute particulière. Peut-on l'évaluer directement? M. Puton est d'avis que cette richesse spéciale a une valeur incontestée, sans que cependant ce soit un élément vendable, et il l'apprécie par la comparaison du prix des sols boisés avec celui des sols nus d'égale qualité, par l'évaluation de ce que coûterait un semis ou une plantation dont le succès serait assuré ; cette estimation est évidemment délicate et sujette à caution; c'est une affaire d'appréciation.

Inversement, pour le propriétaire qui ne reut pas maintenir le domaine à l'état de bois, la présence de l'ensouchement nécessite un travail important de défrichement; il est obligé d'extraire toutes les souches et leurs racines, une ane, avant de pouvoir mettre le terrain en culture; cette opération plus ou moins difficile suivant les terrains, l'âge du peuplement, etc., représente une dépense afférente au fonds lui-mème.

Avec M. Puton, nous pouvons appeler mise en ètat de forét, cet élat tout spécial d'un sol forestier transformé en terre à bois par l'action prolongée de la forèt, et occupé par tout un système de racines vivantes ou de graines prètes à germer.

Le sol nu, auquel on ajoute la mise en état de forèt que nous venons de définir, constitue le fonds, première partie du capital d'exploitation.

Superficie. - Sur ce londs, dans une exploitation forestière, on trouve un matériel bois; pour analyser économiquement ce matériel, nous examinerons successirement la forèt à exploitation périodique, la forèt aménagée, et la forèt à arbres d'âges mélangés, seuls cas qui puissent se présenter.

Premier cas. - For'ct à exploitation périodique revemant toutes les n années sur toutc la surface. -- Nous avons à distinguer deux types: 
Premier type. -- Exploitation de peuplements d'un seul àge (taillis simple, plantations, futaies régulières). - A l'âge prévu pour la récolte, les bois sont exploilés en bloc, sur toute la surface de la forèt, autrement dit, toute la superficie est coupée; elle constitue à ce moment la récolte, le revenu $\mathrm{R}$ fourni par la forèt toutes les $n$ années. Rien dans la superficie ne doit entrer dans le capital d'exploitation; tout le matériel bois sur pied fait partie de la récolte qu'on obtiendra à $n$ ans. C'est un cas très simple; il n'y a pas de confusion possible entre le capital et le revenu.

Deuxième type. - Exploilulion périodique à arbres de réserve, dont le type est le taillis composé. - Dans la superficie boisée, il faut distinguer la partie du matériel bois qui appartient au revenu, et celle qui est incorporée au capital d'exploitation. Pour analyser économiquement ce matériel, il est nécessaire d'établir le plan de balivage probable, d'après les indications du terrain ; ce plan de balivage est destiné à faire connaître le nombre d'arbres de réserve qu'on sera conduit à marquer lor's du passage de la coupe, et par suite, le nombre d'arbres qu'il y aura lieu d'abandonner, tout au moins en principe, en supposant qu'on se propose de conserver la forêt dans le même état.

Pour établir ce plan de balivage à un moment quelconque avant l'exploitation, on compte toutes les réserves, auxquelles on donne le nom qu'elles auront sur la coupe exploitable (1).

Dans un taillis composé exploité périodiquement tous les trente ans par exemple, les résultats de l'opération de comptage sont pointés sur un tableau à deux entrées, l'une pour les diamètres, l'autre pour les hauteurs ; c'est

(1) Un arbre marqué en réserve comme baliveau, lors du passage d'une coupe, sera au moment du comptage fait ultérieurement, considéré comme moderne, puisque, au passage de la coupe suivante, il sera appelé comme moderne à réserver ou à abandonner. De mème pour les autres caté. gories. 
une bonne mesure d'ordre, utile pour l'estimation du matériel sur pied.

Le total général de l'opération a donné par exemple:

Modernes 64 ; anciens 35 ; bisanciens 13 ; soit en tout 112 arbres sur toute la surface; autrement dit la réserve, ou balivage actuel, est composée de 112 arbres répartis en 64 modernes, 35 anciens et 13 bisanciens.

Pour conserver après l'exploitation la réserve dans le mème état, il est évident qu'on devra être guidé par les indications suivantes :

a. Le nombre de baliveaux à prendre dans le taillis et à marquer.en réserve, lors du prochain balivage, doit ètre au moins égal au nombre des modernes qui se trouvent sur la coupe avant cette opération (1).

$b$. Le nombre des modernes à marquer en réserve lor's du prochain balivage doit être au moins égal au nombre des anciens qui se trouvent sur la coupe avant cette opération.

c. Le nombre des anciens à marquer en réserve lors du prochain balivage doit être au moins égal au nombre des bisanciens qui se trouvent sur la coupe avant cette opération.

d. Quant aux bisanciens, on n'a pas à en marquer, si on ne garde pas dans la réserve d'arbres au-dessus de cette catégorie.

En ce qui concerne les arbres qui normalement doivent être abandonnés, le plan de balivage établi d'après les indications du terrain permet d'en calculer le nombre; ce nombre est égal dans chaque catégorie, à la différence qui existe entre le nombre d'arbres existant, et celui des arbres à réserver.

Dans l'exemple choisi, il est donné par le tableau suivant:

(1) Il est nécessaire en pratique de conserver en surplus quelques baliveaux, jour tenir compte dans une certaine mesure du déchet, et permettre aussi un choix futur; de même pour les autres catégories. 


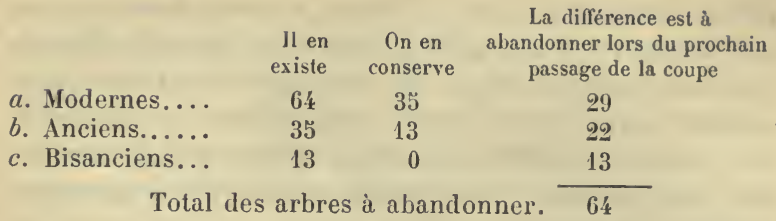

On est bien certain qu'en marquant en délivrance lors du prochain balivage les différences entre chaque classe de futaies, c'est-à-dire 64 arbres ( 29 modernes, 22 anciens, 13 bisanciens), on laissera en réserve sur la coupe 48 arbres (3马 modernes et 13 anciens) qui, alimentés par 64 baliveaux de l'âge du taillis (avec quelques-uns en plus), reconstitueront les 112 tiges du début, et laisseront la coupe garnie de la même manière pour l'exploitation suivante (1).

Le revenu en matière à trente ans, c'est-à-dire la récolte à effectuer lors de l'exploitation, est dès lors constitué par les arbres de futaie abandonnés à l'exploitation (29 modernes, 22 anciens, 13 bisanciens) et par le taillis, dont il faut déduire les 64 baliveaux (et quelques-uns en plus) nécessaires pour assurer le maintien des arbres de réserve.

Le restant de la superficie, c'est-à-dire 64 baliveaux à prendre dans le taillis, 35 modernes et 13 anciens, appartient au capital d'exploitation; la présence de ces arbres est toujours nécessaire pour maintenir la forêt dans le mème état.

Dans ce deuxième type, une partie de la superficie appartient au matériel d'exploitation; cette partie est ce qu'on appelle en économie forestière le balivage.

Si un propriétaire modifie ce balivage, soit pour des

(1) Dans la pratique, on n'est pas toujours maftre de faire ce balivage théorique; l'analyse économique, basée sur la théorie, est nécessaire toutefois, pour permettre de se rendre compte du résultat d'un balivage, quel qu'il soit. 
raisons culturales, soit pour des raisons économiques, par exemple s'il marque en délivrance plus de réserves que ne le prescrit le plan de balivage, il doit se rendre compte, qu'en augmentant aujourd'hui sa récolte, il diminue son capital ; l'opération, si elle est faite dans un but raisonné, peut être justifiée ; sinon, elle consiste à manger son fonds avec son revenu.

Dans ce deuxième type, il y a confusion possible entre le capital et le revenu, et la distinction ne peut ètre faite qu'en se reportant au plan de balivage ; la détermination de ce plan de balivage est importante, et souvent délicate.

Deuxième cas. - Forêts aménagées. - Dans les forèts aménagées, nous avons à considérer la suite non interrompue du matériel en croissance, et la coupe annuelle; nous distinguerons deux types :

Premier type. - Exploitation aménagée de peuplements d'un seul âge; récolte à l'âge $\mathrm{n}$. - Les $(n-1)$ coupes âgées de 0 à $(n-1)$ ans font nécessairement partie du matériel en croissance ; elles appartiennent au capital d'exploitation; il est nécessaire de les conserver dans leur intégralité pour assurer le revenu annuel; la $n^{\text {e }}$ coupe âgée de $n$ années, constitue la récolte et par suite le revenu; il n'y a pas de confusion possible entre le capital et le revenu.

II est facile de se rendre compte que ce capital d'exploitation devient d'autant plus important que l'âge d'exploitation est plus élevé.

Deuxieme type. - Exploitation aménagie à arbres de réserve, dont le type est le taillis composé exploité à la révolution de $\mathrm{n}$ années. - Dans la superficie boisée, autrement dit dans les $n$ coupes, il faut distinguer le balivage (baliveaux, modernes, etc.); on peut le définir dans chacune des $n$ coupes, en considérant chacune de ces $n$ coupes comme une exploitation périodique exploitée toutes les $n$ années. Dans chacune de ces $n$ coupes, le balivage néces- 
saire pour assurer le maintien des arbres de réserve, appartient au capital d'exploitation.

Dans les $(n-1)$ coupes, où le taillis n'a pas encore atteint l'âge d'exploitation fixé par la révolution, le restant de la superficie (déduction faite du balivage) appartient au matériel en voie de croissance; il doit encore être conservé intégralement, et fait partie du capital d'exploitation. Ce restant de la superficie comprend: le taillis, déduction faite des baliveaux qu'on y marquera en réserve, au moment de l'exploitation de chaque coupe ; les arbres de réserve qu'on abandonnera au moment de l'exploitation de chaque coupe, et dont le nombre est donné par le plan de balivage de cette coupe.

Enfin dans la $n^{\text {e }}$ coupe, c'est-à-dire la coupe exploitable, la superficie, déduction faite du balivage, appartient à la récolte de l'année, et celte récolte comprendra : le taillis de cette $n^{\text {e }}$ coupe, déduction faite des baliveaux qu'on y marquera en réserve, et les arbres de réserve à y abandonner, dont le nombre est fixé par le plan de balivage de la $n^{\text {e }}$ coupe.

Dans ce deuxième type, une partie de la superficie constituée par les récoltes en croissance dans les $(n-1)$ coupes jeunes, et le balivage dans les $n$ coupes, appartient au capital d'exploitation.

L'autre partie de la superficie constituée par les bois de la $n^{\text {e }}$ coupe (taillis et arbres de réserve), déduction faite du balivage de cette $n^{e}$ coupe, appartient à la récolte et constitue le revenu de la forèt. Dans ce deuxième type il y a confusion possible entre le capital et le revenu.

Il est facile de se rendre compte que le matériel d'exploitation devient, toutes choses égales d'ailleurs:

$1^{\circ}$ D'autant plus important que la révolution adoptée est plus longue ;

$2^{\circ}$ D'autant plus important que la valeur des réserves conservées sur le taillis est plus grande.

T'roisième cas. - Exploitation à arbr'es d'âges mélangés. 


\section{8: NOTIONS GÉNÉRALES D’ÉCONOMIE FORESTIÈRE.}

- Ce type de forêts, renfermant un mélange de liges de tous âges, depuis le brin naissant jusqu'à l'âge exploitable, échappe à l'analyse. Ce n'est qu'en déterminant le revenu de la forèt par le contrôle, c'est-à-dire par des inventaires successifs permettant de déterminer exactement l'accroissement, lequel d'ailleurs est très variable suivant l'état du peuplement, qu'on pourra arriver à analyser économiquement le matériel réparti sur la superficie de la forêt.

Dans ce cas, il y a toujours confusion possible entre le capital et le revenu. Des exploitations qui ne sont pas réglées sur la production normale de la forèt peuvent tendre soit à exploiter plus que la possibilité, et par suite à attaquer le capital ou matériel nécessaire pour assurer cette production normale, soit à un résultat inverse.

Capital d'exploitation. - Les deux facteurs que nous venons d'examiner successivement, fonds et superficie en tant qu'elle ne fait pas partie de la récolte, constituent le capital d'exploitation. Leur valeur, eu égard à l'emploi qu'en veut faire le propriétaire, forme le capital engagé dans l'exploitation forestière.

Le caractère particulier du capital ainsi constitué est d'ètre toujours en partie mobilisable, ce qui expose à des confusions fàcheuses avec le revenu, et par suite à des abus de jouissance; par contre, toute fraction du revenu qu'on ne réalise pas, s'incorpore à ce capital et fonctionne avec lui, naturellement et sans frais.

Il est donc très important dans une exploitation forestière de bien distinguer ce qui est le revenu, qui doit ètre coupé, et ce qui est valeur génératrice, qui doit être laissé sur pied si l'on veut maintenir le statu quo dans la jouissance. Car autrement, si on réalise plus qu'autrefois, sans considérer cet excès de revenu comme semblable à un capital qu'il faudrait placer ailleurs, on use, à proprement parler, le fonds avec le revenu, tandis qu'au contraire, si on réalise moins gu'autrefois, on laisse accu- 
muler une valeur qui dans certains cas, rapporte peu, tandis que réalisée et placée ailleurs, elle aurait pu mieux fructifier.

En matière de gestion forestière il est essentiel:

$1^{\circ}$ De savoir distinguer ce qui est le maintien du statu quo, et par là méme, d'apprécier quand il y a excès de réserve ou de coupe;

$2^{\circ}$ D'avoir, dans ces deux cas, conscience de la nature et de l'importance de son acte.

\section{Travail. - Mode d'exploitation du sol.}

Le travail, qui est le grand facteur de toutes les entreprises agricoles ou industrielles, n intervient pas dans la fabrication de la matière ligneuse. La forèt est un immeuble qui travaille tout seul; là, point de labours, de fumures; rien de ce travail agricole qui oblige à chercher soit un entrepreneur, soit un métayer, soit un fermier, suivant les différents modes d'exploitation usités (Puton); le plus important travail que nécessite la forêt, c'est celui de la récolte.

Faire valoir direct. - On entend par faire valoir direct le mode de jouissance du sol par le propriétaire mème, qui dirige personnellement l'exploitation de son domaine; nous pouvons distinguer deux cas, suivant que le propriétaire vend les coupes à effectuer, ou qu'il les fait lui-mème.

Premier cas. - Le propriétaire se borne à gérer des peuplements sur pied, et il vend ses coupes sur pied. La vente des bois sur pied est généralement le mode de gestion le plus simple et le plus désirable, tant pour le propriétaire qui n'a ni frais, ni risques à sa charge, que pour l'adjudicataire qui y trouve toute liherté de façonner les produits et d'en disposer comme il l'entend (1). La

(1) La vente sur pied, en bloc, est généralement la plus avantageuse et la plus commode. 
gérance est alors simple; elle consiste à donner la direction générale, à baliver ou marteler les coupes, à faire les estimations, à récoler et à préroir, s'il y a lieu, quelques opérations culturales; enfin à passer un traité arec le marchand de bois pour exploiter la coupe.

Dans ce cas, le faire valoir direct est possible et toujours préférable ; il est mème particulièrement facile ; la gestion du domaine boisé n'exige pas l'habitation à la campagne, puisque la main-d'œuvre est étrangère à la fabrication des produits, et qu'elle n'intervient que pour leur réalisation.

Deuxième cas. - Le propriétaire exploite lui-q:ême, et vend ses produits, soit en grume, soit après premier façonnage. - ll semble qu'on réalise ainsi le bénéfice que retire le marchand de bois, et nombre de propriétaires l'essayent; mais cela n'est pas toujours vrai, car le métier de marchand de bois exige des connaissances spéciales relatives non seulement au façonnage et au débit en lui-mème, mais encore à l'état d'un marché variable. En ce qui concerne l'exploitation, il est nécessaire de surveiller activement le travail des ouvriers, de dénombrer les produits fabriqués par les bûcherons, en un mot, de s'occuper personnellement et en tout temps de la gestion de son domaine boisé ; un traité peut être passé arec des bûcherons, braves gens que l'on retrouve chaque année parce qu'ils travaillent en morte-saison. Lorsqu'on a un garde dont l'utilité principale n'est pas seulement de surveiller, mais de servir d'intermédiaire entre le propriétaire et les populations riveraines de la forêt, lorsque la coupe façonnée est ouverte aux acheteurs le mème jour chaque année, le public en prend vite l'habitude; il sait quau même jour, le propriétaire vient lui-mème vendre ses hois; en quelques heures les marchés sont faits avec des clients accoutumés ; s'il restequelques produits invendus, le garde peut avoir mission de les céder au prix moyen. En fait, le propriétaire exploitant n’a pas besoin d'y consacrer tout son temps. 
Régie. - Le propriétaire a recours pour lagestion de son domaine à un régisseur. Pour que la régie présente les mêmes avantages que le faire valoir direct, il est nécessaire que le régisseur soit capable, consciencieux, et rétribué par un salaire fixe. Nous empruntons à ce sujet, à M. Broilliard, les lignes suivantes : L'histoire du gérant qui s'enrichit, tandis que le propriétaire se ruine n'est que trop fréquente ; et ce n'est pas seulement cet homme qui est condamnable; c'est d'abord le propriétaire qui néglige entièrement son domaine. Il faut donc absolument que le propriétaire d'une forêt, grande ou petite, la visite et plusieurs fois par an plutot qu'une seule, qu'il la parcoure, qu'il l'observe, qu'il en connaisse les cantons et les exploitations; à la première visite, il ne fera pas grande découverte; à la dixième, il se sentira chez lui, il sera connu dans le pays, et la propriété deviendra sienne en réalité, au lieu d'ètre la chose du gérant ou de tout le monde.

Cependant, ajoute le mème auteur, un grand propriétaire ne peut laisser sa forèt sans qu'elle ait un administrateur permanent, et les forestiers honnêtes ne manquent pas; mais il faut les trouver. Le moyen le plus simple, et peut-être bien le plus sûr, est de s'adresser aux conservateurs des Eaux et Forêts qui connaissent les gardes, brigadiers, agents et autres personnes aptes à gérer les bois, et peuvent, en désignant aux propriétaires des serviteurs éprouvés et sûrs, procurer à ces derniers une situation meilleure que leur poste dans l'administration. Quoi qu'il en soit, il faut éviter de payer le gérant à tant pour cent du prix des ventes des coupes, ce qui est encore en usage dans quelques lieux; c'est l'intéresser à tout vendre, et il n'y a pas à s'étonner, une vingtaine d'années après, qu'il n'y ait plus d'arbres dans la forèt (1).

(1) On rencontre quelquefois pis encore; certaines personnes confient au marchand de bois le soin de baliver leurs forèts ; c'est organiser à plaisir un conflit entre l'honnêteté et l'intérèt (Broilliard). 
Dans certaines régions il existe des spécialistes à qui le propriétaire qui ne peut effectuer les opérations et s'occuper de la vente des coupes, peut confier ce soin ; c'est ainsi que M. Broilliard cite M. Duret Paul qui rend des coupes en bloc à Besançon, à Vesoul, à Mouchard et à Troyes; son cahier d'affiches, transmis aux marchands de bois, assure la publicité; il vend sur offres écrites, sur soumissions. Quand il est chargé de toutes les opérations relatives à la coupe, arpentage, balivage, vente et récolement, sa rétribution est fixée comme il suit :

5 p. 100 pour les ventes de 5000 francs et au-dessous; 4 p. 100 pour les ventes de 5000 à 20000 francs; 3 p. 100 pour les ventes de 20000 à 60000 francs.

Pour la coupe, quand il est simplement chargéde vendre sur données fournies, la rétribution n'est que de moitié, soit respectivement de 2,5 -1. et 1,5 p. 100.

Fermage, etc.-Desessais de fermage des forêts domaniales, faits en France sous la Restauration n'ont pas réussi; cette disposition des forèts par d'autres que par le propriétaire ou son représentant est singulièrement délicate, à cause de la facilité d'une réalisation possible de tous les instants, et de la nécessité qu'il y a de ne pas confondre involontairement ou non, ce qui est valeur génératrice ou simplement revenu. Un tel mode d'exploitation du sol est fort rare, sauf en ce qui concerne quelques petits bois isolés, annexés comme accessoire à un domaine agricole.

De mème tout contrat analogue (concession, etc.) est. dangereux, en matière d'immeubles boisés.

Organisation du travail. - Attributions des gardes ou préposés. - Indépendaminent des ouvriers bûcherons employés pour l'exploitation des coupes et le façonnage des produits (vente de produits façonnés), le propriétaire utilise des gardes ou préposés dont les attributions sont complexes.

a. IRépression des délits. - Les gardes particuliers 
d'un propriétaire forestier ont tout d'abord des attributions de surveillance; ces attributions sont justifiées par ce fait que les délits sont particulièrement faciles à commettre en forèt. Il est vrai que les bois particuliers sont placés sous la surveillance des officiers de police judiciaire, et principalement des gardes champêtres et des gendarmes (Code forestier, art. 188) (1); mais en fait cette surveillance est peu efficace; il appartient donc au garde particulier de l'exercer. Le propriétaire doit faire assermenter son garde particulier conformément à l'article $117 \mathrm{du}$ Code forestier (2). Mais pour assurer la répression des délits, M. Broilliard conseille au garde particulier d'aller requérir le garde champêtre jour lui faire dresser procès-verbal, dès qu'il y a un délit à constater; une fois que le procès-verbal est dressé, le propriétaire a intérèt, s'il veut que l'affaire soit poursuivie, à prendre l'initiative et à se porter partie civile (Code d'instruction criminelle, art. 182) soit au parquet; soit au moyen d'une citation directe ; il doit communiquer celleci au procureur en s'informant du jour de l'audience, afin de pouvoir y soutenir ses conclusions.

Remarquons qu'en france, la répression des délits forestiers, spécialement des délits commis par les délinquants insolvables est insuffisante et il résulte de celte situation fâcheuse que les propriétaires de bois n'ont en général qu’à supporter les délits en silence; leurs gardes

(1) Code forestier, art. 188: "Les délits el contraventions commis dans les bois non soumis au régime forestier, sont recherchés et constatés, tant par les gardes des bois et foréts des particuliers, que par les gardes champétres des communes, les gendarmes, et en général par tous les officiers de police judiciaire chargés de rechercher et de constater les délits ruraux; les procès-verbaux feront foi jusqu'à preuve contraire. Ces procès-verbaux, à l'exception do ceux dressés par les gardes particuliers, sont enregistrés en débet. "

(2) Code forestier, art. 117: «Les propriétaires qui voudront avoir pour la conservation de leurs bois des gardes particuliers, devront les faire agréer par le sous-préfet de l'arrondissement; sauf le recours au préfet en cas de refus. Ces gardes ne pourront exercer leurs fonctions qu'après avoir prèté serment devant le tribunal de promière instance. ") 
ne disposent d'aucun moyen efficace pour arriver à la répression.

Avec M. Broilliard nous appelons de tous nos vœux l'époque où le service des gardes champètres deviendra réel et bon.

Dans la pratique, la présence d'un garde dans une propriété boisée privée, agit surtout à titre préservatif.

b. Attributions degérance. - Les attributions du garde en fait de gérance proprement ditesont multiples ; d'abord il sert d'intermédiaire entre le propriétaire et les personnes qui ont affaire en forêt : marchand de bois et leurs ouvriers pendant la durée des exploitations, concessionnaires de menus produits, entrepreneurs de travaux culturaux, de travaux d'entretien, etc.; le garde a le rôle d'un contremaître ou d'un représentant du propriétaire qui dirige et surveille.

ll surveille spécialement les exploitations, le travail des ouvriers dans les coupes; il provoque le renvoi des chantier's, par l'entrepreneur ou l'adjudicaire, des mauvais ouvriers, etc.

En outre il participe aux opérations faites en forèt (arpentages, martelages ou balivages, récolements, dénombrements, etc.); il prépare le travail, recueille et fournit les renseignements sur les exploitations, etc.

Dans une boune gestion, il doit aussi faire fonction d'ouvrier, pour effectuer des travaux d'entretien (nettoyage de lignes d'aménagement, ete.) et un certain nombre d'opérations culturales qui ne sont jamais aussi bien failes par un ouvrier incompétent (dégagements de semis, plantations et repeuplements dans les vides et clairières, etc.). 'Tous ces travaux, dont il comprend le but, lui conviennent mieux qu'à un ouvrier quelconque; ils le portent à s'intéresser davantage à la forèl, et cela sans l'empècher d'exercer une bonne surveillance.

II faut qu'un garde forestier n'ait pas à s'occuper en dehor's de sa forct, et pour cela il doit stre valide et bien 
rétribué ; la rétribution qu'on lui donne doit être fixe; en général on traite à forfait, et le prix est proportionnel au nombre d'hectares qu'il a à surveiller.

La plupart desgardes, d'après M. Broilliard, ne sont pas convenablement rétribués; on paie 1 franc par hectare, on donne au préposé un peu de bois, quelquefois de l'herbe pour une ou deux vaches; or ce n'est pas 1 franc, mais 2 francs par hectare que raut la présence annuelle d'un garde dans de bonnes forèts productives, et c'est ce chiffre qui devrait de nos jours, ètre la base ordinaire de la rétribution des gardes forestiers.

Quant à l'étendue qu'on leur confiera à surveiller, elle doit être telle que le garde gagne suffisamment pour vivre en famille; ce minimum ne descend guère audessous de 1000 francs. Il convient donc que les propriétaires de petits bois s'entendent pour avoir un garde commun, ou qu'ils demandent l'assistance du garde forestier communal le plus voisin, ce qui est souvent la meilleure combinaison (1).

Ajoutons enfin que dans une gérance bien comprise, le garde doit avoir son chauffage, le droit de faire pâturer sur les chemins de la forêt un peu de bétail, et quelques petits suppléments à l'occasion, soil à titre de prime pour procès-verbaux, soit à titre d'indemnité pour travaux d'amélioration. Ces suppléments peuvent lui être payés d'après le nombre d'heures bien employées passées au travail, mais à un taux assez faible; dans l'administration des forèts de l'État, ce taux est de la moitié du salaire des ouvriers ordinaires (2).

(1) La demaude doit en ètre faite au Conservateur des Eaux et Forèts à qui l'on transmet en mème temps la commission à donner à ce garde. Une fois autorisé par l'administration forestière, le garde doit ètre agré j par le souspréfet, et ne peut exercer ses fonctions qu'après avoir prèté serment devant le tribunal de première instance (Code forestier, art. 117).

( $($ ) Nous conseillons beaucoup aux gardes forestiers de faire de l'apiculture. Avec dix ruches qu'un préposé peut très facilement surveiller et diriger sans peine, on peut obtenir dans beaucoup de régions apico'es un revenu de 150 à 200 francs. Pour un homme chargé de famille, ce gain supplémentairc 


\section{3. - Agents naturels de production.}

L'air, la lumière, l'humidité, la chaleur, les éléments minéraux du sol, etc. sont les véritables artisans de la production forestière. Comme ils sont toujours abondants et gratuits, l'exploitant n'a jamais à s'en préoccuper, autrement que pour diriger leur action au point de vue cultural; les trois premières parties de notre ouvrage sont relatives à ce sujet.

\section{II. - PRODUIT BRUT. - PRODUIT NET.}

L'ensemble des produits vendus ou utilisés par le propriétaire, exprimé en argent, et rapporté à l'hectare, constitue le produit brut de l'exploitation forestière. Si de ce produit brut on déduit es frais de production, on a le produit net.

\section{1. - Produit brut.}

L'importance du produit brut d'une forêt normale est liée au volume de la récolte et à ses prix.

a. Volume de la récolte. - Le volume de la récolte qu'on peut demander aux forèts sans les appauvrir varie suivant les essences, la station (climat, nature du terrain, humidité, exposition, etc.), le mode de traitement cultural et l'âge d'exploitation.

Ces différents facteur's peuvent faire varier le volume de la récolte moyenne, obtenue par hectare et par an, dans des proportions très fortes; là, un taillis simple, situé en maurais sol, et exploité à des courtes rérolutions n'est susceptible de donner qu'une faible production; par exemple un mètre cube de matière ligneuse

n'est pas a dédaigner. (Voir : "Apiculture en maison forestière", par A. Fron, Bull. de la Soc. d'Igriculture de l'urrondissement de Charolles. 1898, Jibrairie Guillemin (Charolles) et Revie des Eaux et Foréts, 1898. 
par hectare et par an ; ailleurs, la production sera beaucoup plus forte; un exemple de fort rendement est celui donné par les massifs de Bercé dans la Sarthe; cette forêt en futaie de chène pur aménagée à la révolution de deux cent seize ans renferme à l'hectare dans sa parcelle $\mathrm{A}^{3}$ un volume de 975 mètres cubes; le rendement annuel moyen depuis la naissance a été de $6^{\mathrm{mc}}, 75 \mathrm{et}$ il est fixé aujourd'hui à $6^{\mathrm{mc}}, 21$ (1).

Dans le Jura certaines futaies résineuses peuvent donner jusqu'à 8 mètres cubes et plus par hectare et par an.

Les écarts de production de forèt à forèt sont donc très considérables; toutefois, dans une mème station et avec les mèmes essences, la production en volume de matière ligneuse est à peu près constante, quel que soit l'âge d'exploitation.

b. Prix de l'unité de volume. - Les prix des diverses unités de volume de matière ligneuse dépendent de leur degré d'utilité et de leur rareté; ils varient suivant les pays et les régions d'après l'offre et la demande, et dans la mème région ils varient arec les années.

Toutes choses égales d'ailleurs, on peut dire qu'en général, le prix de l'unité de volume grume, augmente avec l'àge d'exploitation, tout au moins dans certaines limites, et sauf circonstances locales. Pour un arbre considéré isolément, le prix à l'unité de volume de sa matière ligneuse croitra avec son diamètre; pour un peuplement, le prix à l'unité de volume des produits en matière de ce peuplement croitra avec le volume total, et surtout arec la proportion en gros bois ou bois d'ouvre que ce volume contient.

Toutefois, des utilisations spéciales modifient très souvent les prix de certaines catégories de produits, et

(1) Dans la forêt de Bercé, les fùts atteignent 20 ou 30 mètres, et les diamètres oscillent entre $0^{\mathrm{m}}, 60$ et $1^{\mathrm{m}}, 20$. Il y a de plus un épais sous-étage de hêtre.

A. Fros. - Sylviculture. 


\section{9' NOTIONS GÉNÉRALES D'ÉCONOMIE FORESTIĖLE.}

rendent alors cette loi inexacte, de même que des conditions défavorables de vidange, ou une insuffisance de moyens d'utilisation, peuvent y apporter de nouvelles atténuations. Dans les environs d'une mine par exemple, des bois taillis passeront brusquement d'une valeur correspondant à leur utilisation comme chauffage, à une valeur toute spéciale, souvent très supérieure si on les laisse vieillir quelques années de plus et acquérir les dimensions requises pour le boisage des galeries; au delà de ces dimensions, ils perdront cette majoration accidentelle. Il en sera de mème pour des plantations résineuses; à trente-cinq ans, si la dimension la plus favorable pour cette utilisation spéciale (étais de mine) correspond à cet âge, les bois se vendront un prix correspondant à 15 francs le mètre cube ; au delà de cet âge, les tiges peuvent ne plus ètre aussi recherchées et par suite se vendre moins avantageusement.

Dans le Jura, où le sapin est débilé en planches de sciage de dimensions déterminées par le commerce local, le sapin passera presque subitement de la valeur de 15 francs au mètre cube par exemple, à celle de 30 francs dès qu'il aura atteint les dimensions requises.

Enfin, dans certaines localités, l'arbre perd de son prix, quand sa dimension ne permet pas de le sortir de la forêt, ou encore quand les modes de déhit en usage dans la région ne sont pas susceptibles de se prèter à ces dimensions; c'est ainsi que dans les Pyrénées, des hètres magnifiques ont été abandonnés sur le terrain, faute de pouvoir les utiliser; de même dans le Jura, il n'y a pas encore bien longtemps, le sapin de 70 centimètres de diamètre se vendait moins cher à l'unité de volume, que celui de 60 centimètres, en raison du mauvais élat des scieries.

En somme, le prix de l'unité de volume de matière ligneuse est trés variable ; il appartient au propriétaire de connaitre dans la région qu'il habite les utilisations du bois aux divers ages, el de voir l'application qu'il 
peut en faire en ce qui concerne le fonctionnement économique de son domaine boisé.

Ces deux facteurs, volume de la récolle, et prix de l'unilé de volume, en se multipliant, donnent des résultats très variables pour la production moyenne en argent d'une forèt par hectare et par an. Dans les forèts domaniales françaises, cette production va depuis 3 francs en Corse, jusqu'à 8 ă francs dans la conservation d'Amiens, et exceptionnellement elle monte jusqu'à 200 et 300 francs ; dans la forèt domaniale de Bercé (Sarthe) la production moyenne était estimée en 1898 à 280 francs par hectare et par an. La statistique forestière dressée en 1878 constate que la production moyenne des forêts de l'État est de $40 \mathrm{fr} .25$ par hectare et par an ; ce chiffre n'est qu'une moyenne prise arec les forêts les plus diverses; la production dépasse notablement cette moyenne dans les conservations du nord et de l'est (Amiens, 89 francs; Epinal, 71 francs; Nancy, $; 8$ francs); par contre elle s'abaisse au-dessous de cette moyenne dans le midi; elle tombe au-dessous de 5 francs par hectare et par an dans six conservations formant 16 départements (Toulouse, Pau, Bordeaux, Ajaccio, Chambéry, Nice); cet abaissement tient non seulement à ce que les exploitations sont réglées à des âges plus faibles que dans le nord, mais en outre à ce que les forêts livrées au pâturage, sont en mauvais état, ou bien à ce qu'elles sont situées dans des régions dépourvues de moyens de vidange.

En résumé, on peut dire qu'en général, et sauf des circonstances locales, dues à la difficulté des transports et des débouchés, et surtout à une demande avantageuse de produits déterminés: plus l'âge d'exploitation est élevé, plus le revenu brut annuel donné par les foréts par hectare et par an est considéruble; autrement dit en culture forestière, l'exploitation intensive, c'est-à-dire celle qui correspond au revenu brut le plus élevé, comporte des âges d'exploitation élevés. 
Remarquons que dans le produit brut d'un domaine boisé peuvent entrer en ligne de compte un certain nombre de revenus annuels, tels que le fermage de la chasse, certaines concessions de menus produits, etc., ou bien des produits périodiques et même accidentels (extraction de pierres, bois chablis, bois de délit, etc.).

\section{2. - Produit net.}

Pour passer de ce premier résultat au produit net, il faut dẻcompter toutes les dépenses:

a. Dépenses d'outillage et d'amortissement. - Telles sont les dépenses que nécessite la mise en valeur du domaine, comme la détermination des limites et leur fixation sur le terrain (frais de délimitation et de bornage), la confection du plan du domaine et de l'aménagement, les travaux nécessaires pour ouvrir et asseoir sur le terrain les lignes d'aménagement, la création ou l'amélioration des voies de vidange, l'établissement de rigoles, de fossés, etc. Ces dépenses de premier établissement sont d'ailleurs communes à toutes les surfaces rurales; on pourra se proposer, comme en agriculture, de les amortir en un temps donné par le prélèvement d'annuités fait chaque année sur la récolte, et cette catégorie de dépenses rentre alors dans les dépenses annuelles et temporaires (1).

b. Dépenses d'entretien. - Ce sont les frais d'administration, de gérance et de surveillance; cette catégorie de dépenses esi représentée par un salaire annuel et rentre dans les dépenses annuelles permanentes.

c. Dépenses pour disposer de la récolte. - Ce sont, tantot les salaires des ouvriers, ou le prix déterminé à l'avance par un contrat (exploitation directe), tantot les

(1) Si ces dépenses sont effectuées depuis un temps ussez long, on pourvia les considérer comme amorties, et leur enpital représentatif peut s'incorporer alors, suivant les cas, à titre de plus-value, dans la valeur du fonds. 
frais de vente, de timbre et d'enregistrement des actes, de publicité, etc.

Cette catégorie de dépenses est défalquée immédiatement du produit en argent des récoltes correspondantes.

d. Impot foncier. - Tout immeuble est frappé en France d'un impôt spécial, l'impôt foncier.

Pour se rendre compte en détail de l'impôt qui frappe une propriété non bâtie (bois), le propriétaire ne peut y parvenir qu'en se procurant l'extrait du folio de la malrice cadustrale où figurent les parcelles constitutives de sa propriété. Cet extrait est délivré par le Directeur des contributions direcles; nous reproduisons un modèle d'extrait sur lequel figurent deux parcelles de la commune de Jouy qui nous serviront d'exemple.

\begin{tabular}{|c|c|c|c|c|c|c|}
\hline 总 & $\begin{array}{c}\text { No } \\
\text { du } \\
\text { plan. }\end{array}$ & $\begin{array}{c}\text { CANTON } \\
\text { Triage } \\
\text { ou lieu dit. }\end{array}$ & 密 & $\begin{array}{l}\text { CONTE- } \\
\text { NANCE. }\end{array}$ & 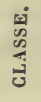 & $\begin{array}{l}\text { REVENU } \\
\text { cadastral. }\end{array}$ \\
\hline A. & 332 & La filandrerie. & Bois.. & 1 h. $.50^{\text {a }}$. & 1 & $12 \mathrm{fr}$. \\
\hline B. & 570 & Bois blanes... & Bois.. & $1^{\mathrm{h}} .50^{\mathrm{a}}$. & 2 & $7 \mathrm{fr}, 50$ \\
\hline
\end{tabular}

La contribution foncière est répartie par égalité proportionnelle sur toutes les propriétés foncières; ce qui veut dire que dans la même commune, chaque nature de culture de la méme classe comporte par hectare le même revenu cadastral.

Ainsi, d'après l'extrait précédent, toutes les parcelles cultivées en bois dans la commune de Jouy et qui au moment du cadastre ont été classées en bois de première et de deuxième classe, paient l'impôt foncier sur un revenu cadastral de 8 francs l'hectare pour la première classe, et de 5 francs l'hectare pour la deuxième classe.

Ces prix de 8 francs et de 5 francs que nous obtenons en divisant le revenu cadastral porté en regard des par- 
celles sur l'extrait par la contenance de ces parcelles sont ceux qui ont été fixés par le tarif des évaluations arrêté par les classificateurs lors de la confection du cadastre de la commune. Ce tarif des évaluations figure sur la feuille de tète du premier volume de la matrice carlastrale de la commune. Mais comme les propriétaires n'ont pas toujours la facilité de prendre communication de ce document, ils peuvent rétablir ce tarif comme il est dit plus haut, en divisant le revenu cadastral de chaque parcelle de la mème classe par la contenance de ces mèmes parcelles.

Dans les deux parcelles qui figurent sur l'extrait que nous avons reproduit, le revenu net de ces deux classes n'apparaît pas, par le motif que par revenu net des propriétés non bâties (bois), on entend ce qui reste au propriétaire par an, déduction faite sur le revenu brut des frais de garde, d'entretien, etc., etc., et que le revenu net imposable est le revenu net moyen, c'est-à-dire calculé sur un nombre d'années déterminé.

C'est ce revenu net imposable qui, au moment de la confection du cadastre de la commune, a servi à fixer le revenu cadastral, c'est-à-dire le chiffre résultant de l'application du tarif des évaluations au classement.

Le revenu cadastral, qu'on appelle aussi revenu matriciel, peut être le revenu net imposable lui-mème; mais le plus souvent il représente une fraction, un tant pour cent du revenu net imposable, et ce tant pour cent a été délerminé au moment de l'expertise cadastrale (1).

(1) La proportion d'atténuation qu'on a fait subir au revenu net pour le convertir en revénu cadastral, varie de commune à commune, de telle sorte que le revenu cadastral porté sur les matrices, n'est qu'un chiffre fictif qui sert de base à la répartition individuelle dans l'intérieur de la comnune.

Pour les bois, les classificateurs ont en général évalué le revenu cadastral aux deux tiers ou à la moitié du revenu inıposable réel.

En 1851 et en 1879 , une nouvelle évaluation des revenus territoriaux a été faite, afin de rechercher pour toutes les natures de cultures les proportions d'atténuation à ces éporques; ces chillres ont permis de modifier le contingent foncier de communes surtaxées ou imposées trop faiblement. 
Ces définitions posées, nous pourons calculer l'imposition que doit payer le bois de 3 hectares situé sur la commune de Jouy, et formé des deux parcelles $n^{\text {os }} 332$ $\mathrm{S}^{\circ \mathrm{n}} \mathrm{A}$ et $570 \mathrm{~S}^{\circ \mathrm{o}} \mathrm{B}$ :

$1^{\circ}$ Le revenu cadastral de chacune des deux parcelles s'obtient en multipliant la contenance de chacune d'elles par le prix du tarif des évaluations, soit 8 francs pour la première classe (bois) et 5 francs pour la deuxième classe (bois); nous avons: $1 \mathrm{~h} .50 \times 8=12$ francs et $1 \mathrm{~h} .50 \times 5$ $=7 \mathrm{fr} .50$.

$2^{\circ}$ Le montant de l'impôt s'obtient en multipliantle revenu cadastral de chaque parcelle parle centime-le-franc (1) de la contribution foncière non bâtie, chiffre qui est inscrit sur la feuille d'avertissement de chaque année :

Supposons qu'en 1903 le centime-le-franc soit dans la commune de Jouy de 0.4025783 le calcul donne

l'our la parcelle $n^{\circ} 332$ Son A un impôt de $12 \times 0,402578=4^{\mathrm{fr}, 83}$ no 570 Son $B-7,50 \times 0,402578=3^{\text {fr }, 02}$ Le montant total de l'impôt à payer est de... $\overline{7_{\mathrm{i}}^{\mathrm{fr}}, 85}$

Cette catégorie de dépenses rentre dans les dépenses annuelles permanentes.

Les bois étant fortement imposés dans certaines communes par rapport aux autres biens fonciers, les proprié-

(1) Le centime-le-franc, calculé chaque année par la Direction des contributions directes, varie suivant les impositions de la commune. Dans le centimele-franc, le principal de l'impôt est ce qui revient entièrement à l'Etat; mais des centimes additionnels sont ajoutés au principal, soit pour accroltre les recettes du trésor, soit pour subvenir aux dépenses locales des départements et des communes, soit pour couvrir les non-valeurs et les frais de perception; on distingue les centimes généraux, départementaux et communaux.

En 1901, 1 hectare de bois en France, comme moyenne générale, a payé environ comme impòt :

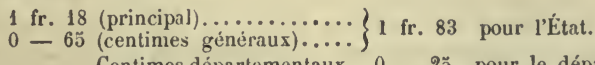
Centimes départementaux. $0-25$ pour le département.
Centimes conmunaux.... $0-28$ pour la commune.
Total (impòt moyen de 1 h. de bois). $\overline{2 \text { fr. } 36}$ 
taires ne peuvent que désirer la révision du tarif des évaluations (1).

e. Assurance. - Très peu de compagnies d'assurances consentent à assurer les forèts contre l'incendie; aucune ne les assure dans les régions dangereuses, notamment dans le midi. Les compagnies distinguent ordinairement et assurent séparément plusieurs sortes de risques; ceux du taillis, de l'ensouchement, de la futaie (gros bois), et du trouble d'aménagement; les primes d'assurance, fortes pour les jeunes bois, sont en général de plus en plus faibles pour les vieux bois (Voir p. 515).

Quoi qu'il en soit, l'assurance du domaine boisé contre l'incendie, si elle est possible, se traduit par le paiement d'une prime totale annuelle, plus cu moins forte suivant les cas; c'est une charge annuelle; elle rentre dans les dépenses annuelles permanentes.

Variations du produit net. - En résume, pour une forèt donnée, la plus grande partie de ces dépenses ne varie guère avec la valeur superficielle et l'âge de la récolte, ou tout au moins varie dans des limiles assez restreintes; il en résulte que le produit net suit une marche croissante avec le produit brut, et par suite qu'il augmente avec l'âge d'exploitation, sauf exceptions locales dues à la difficulté des transports et des débouchés et surtout à une demande avantageuse de produits déterminés.

Dès lors, si le propriétaire recherche le plus grand produit net, il est conduit, dans la majorité des cas, à reculer jusqu'à un âge avancé l'exploitation de ses peuplements.

En fait, ce n'est pas ce qui se passe, et l'intérèt du propriétaire forestier est d'exploiter ses bois à des âges

(1) La loi du 21 juillet 159:, relative aux contributions directes et aux taxes y assimilées de l'exercice 1893 (Journal officiel, 22 juillet 1894), a prescrit dans son article 4 la transformation de la contribution foncière des propriétés nou baties en un impót sur le revenu net réel, qui serait indiqué sur les matrices cadastrales. Le travail nécessaire pour refaire toutes les évaluations ne paralt pas terminé. 
moins avancés ; cette contradiction apparente, tient à la constitution toute spéciale du capital engagé dans l'exploitation forestière et à son fonctionnement financier; la considération du taux de placement va préciser la question.

\section{III. - TAUX DE PLACEMENT DU CAPITAL ENGAGÉ DANS UNE EXPLOITATION FORESTIERE.}

Par taux de placement des fonds engagés dans une exploitation forestière, nous entendons la relation qui existe entre le revenu que donne le domaine boisé, et le capital d'exploitation.

Nous avons à distinguer deux cas :

Premier cas. - Forêts à exploitation périodique. - Le caractère de ce type est qu'on ne touche un revenu $\mathrm{R}$ que toutes les $n$ années; la relation qui existe entre le capital d'exploitation et le revenu en fonction du taux de placement est exprimée par la formule:

$$
\mathrm{C}(1+t)^{n}=\mathrm{C}+\mathrm{R}
$$

dans laquelle le premier membre représente le capital initial C ayant fonctionné à intérèts composés au taux $t$ de l'exploitation pendant $n$ années; et le deuxième membre représente ce capital initial augmenté du revenu $\mathrm{R}$, c'est-à-dire de la récolte touchée au bout de $n$ années.

Remarquons que la forèt à exploitation périodique peut fonctionner d'une façon plus compliquée ; elle peut donner diverses sortes de produits intermédiaires ou accessoires, avant la récolte principale de l'àge $n$. Dans ce cas, la relation qui existe entre le capital, le revenu et le taux de placement est moins simple à exprimer; il n'entre pas dans le cadre de notre ouvrage de nous y arrèter.

Deuxième cas. - Forêts aménagées. - Le caractère 
502 NOTIONS GÉNÉRALES D'ÉCONOMIE FORESTIÈRE.

de ce type est qu'on touche un revenu annuel (1) et sensiblement constant, tout en conservant la forêt dans le même état.

La relation qui existe entre le capital d'exploitation, le revenu et le taux de placement est donnée.par l'expression :

$$
\overline{\mathrm{C}}=t
$$

dans laquelle $\mathrm{R}$ est le reveu annuel, $\mathrm{C}$ le capital d'exploitation, et $t$ le taux de placement auquel fonctionne ce capital.

Remarquons qu'il est rare de rencontrer des bois régulièrement aménagés; en général la forèt aménagée, prise telle qu'elle est, n'est pas normalement constituée ; il y a des lacunes et des surabondances, soit dans les âges, soit dans les contenances, et l'analyse économique du fonctionnement de la forèt est alor's beaucoup moins símple.

Variations du taux de placement. - Nous n'entrer'ons pas dans l'étude détaillée de la comparaison entre la marche ascendante de la valeur du capital engagé avec l'âge, et la marche ascendante du revenu avec l'àge. Nous nous contenterons d'énoncer, avec M. Puton, la conséquence suivante qui résulte de la constitution du capital forestier: pour un terrain de néme valeur et peuplé des mémes essences, le taux de placement augmente avec l'áge, passe par un maximum, el décroit ensuite, de telle sorte que l'abaissement du taux de placement est la note caractéristique des exploitations intensives.

Toutefois, ce principe n'est vrai que si des circonstances toutes spéciales ne viennent pas influer sur la

(1) Ce revenu représente le produit net annuel, ou est en relation directe avec lui, quand l'exploitation n'a pas pour résultat de faire des abus de jouis. pance (molilisation d'une partie du capital bois qui se traduit par une exploiintion trop forte) ou au contraire de faire des épargnes (réalisation seulenent fartielle de ce qui doit ètre exploité, ce qui se traduit par un enrichissement du matériel superficie). 
valeur de certaines catégories de marchandises et leur donner une plus-value importante qui peut bouleverser, tout au moins dans certaines limites d'âges, les conditions économiques du fonctionnement de la forèt.

D'une façon générale, si l'on fait abstraction de révolutions très courtes (oseraies, taillis de micocoulier, taillis à écorce et taillis spéciaux, qui sont plutôt des exploitations industrielles) (1), le taux de placement en forêt, sauf peut-être dans le cas des taillis simples, ne peut guère dépasser celui des autres placements faits dans les mèmes conditions de sécurité, soit 3 à : j p. 100. Il est souvent inférieur à ces chiffres.

Les exploitations très intensives, à longue révolution, fonctionnent à des taux de placement très faibles, 2 p. 100,1 p. 100 et mème moins encore, dans le cas par exemple où il s'agit d'une forèt à matériel surabondant et à vidange difficile.

Dans les circonstances actuelles, les forèts les mieux outillées, traitées en haute futaie, ne fonclionnent pas à un taux supérieur à 2 p. 100 .

On peut se rendre facilement compte de ce fait, dit M. Puton, en considérant une forêt aménagée; plus l'âge d'exploitation augmente, plus le revenu annuel s'accroît; mais le capital augmente aussi dans des proportions telles que la relation entre le revenu et le capital engagé, c'est-à-dire le taux, va toujours en fléchissant. Il en est de mème des forèts à exploitation périodique; seulement pour celles-ci, le temps nécessaire à la réalisation du produit, fait ici le mème office que le matériel bois des forêts aménagées; il abaisse le taux de placement de la mème manière.

La conséquence qui découle de ce fait est posée nettement par M. Huffel dans les termes suivants: Quel que. puisse devenir l’ prix des bois, il sera toujours rrai qu'à des

(1) Certains taillis simples fonctionnent à un taux supérieur à 10 p. 100. 


\section{NOTIONS GÉNÉRALES D'ÉCONOMIE FORESTIÈRE.}

âyes d'exploitation élevés correspond un taux de placement extrémement faille; un renchérissement des bois de fortes dimensions n'a qu'une action insignifiante pour augmenter ce taux, car' s'il fait augmenter le revenu, il fait aussi augmenter le capital engagé. Aussi les propriétaires particuliers ne sont pas et ne seront jamais producteurs cle gros arbres; assez d'autres emplois offriront toujours, a vec une sécurité égale, une bien meilleure rémunération à leur's capitaux; c'est ainsi que des capitaux fournissent encore, avec toute la sécurité possible, mème dans le moment de dépression générale des taux de placement que nous traversons, des revenus de 3 et mème 3,5 p. 100 à de grands capitalistes, tandis qu'une forèt aménagée en vue de produire de gros arbres, à des àges de cent cinquante ou deux cents ans, ne fournira que 0,5 à 1,5 p. 100, parfois moins encore du capital engagé.

Il y a là un fait qui domine toute l'étude du fonctionnement financier des exploitations forestières: la production des gros bois ne rémunère que très faiblement les capitaux qu'on y emploie. Cette vérité si simple, qui découle immédiatement de ce que la récolte en gros arbres met deux siècles à mûrir, a été trop sourent méconnue, bien qu'il semble qu'il suffise d'un moment de réflexion pour s'en convaincre.

Il résulte de ces considérations que le propriétaire particulier, préoccupé des conditions de placement de son argent et des nécessités de l'existence, demande loujours à son capital de fonctionner à un taux de placement aussi élevé que possible. Ce qu'il considère comme critère de perfection de son exploitation, c'est le taux de placement.

Le besoin de ce taux de placement avantageux le conduit, quelle que soil la situation, à une culture plus ou moins extensive (1).

(1) II ne faudrait pas que cetto expression exagère notre pensée ; à notre avis, il appartieul a chaque proprićtaire particuler d'envisager la situation dans 
Ajoutons toutefois, et cela est important, que l'âge d'exploitation le plus avantageux pour le propriétaire n'est pas l'âge le plus faible possible. Cette théorie conduirait rapidement, dans la majorité des cas, à la ruine du domaine boisé; en outre elle serait fausse, car le taux de placement avant de décroître, augmente d'abord arec l'âge; de plus, mème lorsqu'il a commencé à décroître, l'arbre peut très bien acquérir ultérieurement des dimensions telles que le placement avantageux d'un produit spécial obtenu à cet âge d'exploitation, relève subitement et pour quelque temps le taux de placement; c'est une question de production spéciale, à laquelle le propriétaire doit toujours songer. Enfin, et comme dernière considération, il faut se rappeler que la préoccupation du fonctionnement économique de l'exploitation ne doit pas, sauf en cas de sol de fertilité supérieure, faire oublier, les exigences de la culture forestière au point de vue de la fertilité des stations.

Utilité des forêts de l'État. - Si nous considérons que la production des gros bois est aussi indispensable dans l'état actuel de nos sociétés, que celle du sucre et des tissus de coton et si nous constatons, avec M. Mélard (1), que la production des bois d'œurre est en

le milieu économique où il se trouve, et de régler sur cette situation le degré d'intensité de sa culture; d'après M. Mélard, ce serait un tort de prétendre que la production des bois d'œuvre est aujourd'hui interdite aux propriétaires particuliers à cause du faible taux auquel fonctionnent les capitaux engagés dans les futaies. Aujourd'hui, a dit cet auteur au Congrès international de Sylviculture de 1900, l'intérèt des valeurs de tout repos ne dépasse plus 3 p. 100; il descendra peut-ètre encore ; et dès lors, il semble qu'un particulier peut avoir profit à élever sur les taillis des réserves qui assurent à son épargne un intérèt au moins égal, ou à produire dans les sapinières des bois de sciage qui lui donnent 2 à 3 p. 100 du capital engagé, abstraction faite de l'augmentation cependant bien certaine du prix des bois.

Le même auteur ajoute que l'augmentation de la valeur du hois d'œuvre doit conduire aussi à en éviter le gaspillage, à être plus soigneux dans la manière de diriger le débit, à n'utiliser comme bois de feu que ce qui est absolument impropre aux usages industriels.

(1) A. MéLARD, Insuffisance de la produetion des bois d'auvre dans le monde.

M. Mélard, après avoir démontré l'insuffisance de la production des bois

A. Frov. - Sylviculture. 


\section{NOTIONS GÉNÉRALES D'ÉCONOMIE FORESTIËR.}

décroissance dans le monde, il n'est pas dificile de comprendre le rôle des forèts de l'État. Des observations que nous venons de faire sur le fonctionnement économique de la propriété boisée particulière, il résulte que la méthode de gestion de cette propriété est désastreuse au point de vue de la fortune générale du pays, de la richesse nationale et de la production des gros bois; dès lors il appartient à l'État, être impérissable, plus soc:cieux de l'avenir parce qu'il est plus durable, de se charger de cette production; seul il peut accepter un taux de placement faible dans le but d'aroir des forèts à gros capital, susceptibles de donner en gros bois une production par hectare et par an de beaucoup supérieure à celle des autres forèts. Cette considération est la justification et la raison d'ètre des forêts possédées par l'État.

\section{IV. - INSTITUTIONS FORESTIÉRES.}

Les institutions forestières sont de deux genres: les unes doivent leur organisation à l'État; les autres ont été créées par l'initiative privée et vivent plus ou moins complètement de leurs propres ressources.

Ministère de l'agriculture. - Tous les services forestiers officiels dépendent du Ministère de l'agriculture organisé sur les mèmes bases que les autres ministères, c'est-à-dire ayant à sa tète un ministre qui le représente devant le parlement et que secondent des directeurs, chefs de bureaux, etc. Il existe auprès de ce ministère un conseil supérieur, et des commissions spéciales chargées d'étudier les lois et mesures administratives propres à favoriser les intérèts agricoles ef forestiers.

d'ceuvre dans le monde, expose qu'il est nécessaire de prendre des nuesures pour assurer la production de ces gros bois, et il ajoute : "La production forestière ne s'improvise pas; il faut un sic̀cle ou un siècle el demi pour obtenir des bois de sciage, et la disette des bois d'auvre se fera peut-etre sentir avant cinquaute ans. " 
Enseignement forestier. - L'enseignement professionnel forestier donné par l'État comprend, si nous en exceptons les établissements spéciaux ayant surtout pour but de recruter les fonctionnaires supérieurs du service forestier :

$1^{\circ}$ Le cour's d'enseignement supérieur de sylviculture et de culture pastorale, professé à l'Institut national agronomique à Paris, destiné à donner aux futurs ingénieurs agronomes les règles générales de sylviculture et de culture pastorale qui leur sont piécessaires, soit pour diriger dans un but pratique une exploitation forestière, soit pour enseigner dans les départements les meilleures règles de gestion d'un domaine boisé, soit ypour faire connaître et propager les moyens d'améliorer ou d'utiliser par le reboisement les friches, les mauraises pâtures et les terrains de qualité inférieure du domaine agricole. Accessoirement cet enseignement est destiné à préparer les futurs élèves de l'École nationale des Eaux et Forèts à suivre les cours supérieurs de Nancy.

$2^{\circ}$ Les cours d'enseignement secondaire de sylviculture, professés dans les Écoles nationales d'agriculture de Grignon (Seine-et-Oise), de Montpellier (Hérault) et de Rennes (Ille-et-Vilaine).

$3^{\circ}$ Les cour's professés à l'école pratique de sylviculture des Barres, annexée à l'Arboretum national des Barres, commune de Nogent-sur-Vernisson (Loiret); cette école est destinée à former des gardes particuliers, des régisseurs agricoles et forestiers, et subsidiairement des candidats à l'emploi de préposé forestier.

$4^{\circ}$ Les cour's d'enseignement primaire de sylviculture, professés dans certaines écoles pratiques d'agriculture disséminées dans les différentes régions de la France; l'école pratique de Saint-Pau (Lot-et-Garonne), dirigée par M. Capgrand-Mothes, dont le nom est si connu dans les pays de chêne-liège, se trouve au premier rang parmi les écoles pratiques qui ont compris que les forèts se 
raltachent intimement à l'agriculture, el qui ont inséré dans leurs programmes l'enseignement des sciences et industries forestières. De ces écoles peurent sortir des élèves diplômés qui fournissent des régisseurs, horticulteurs, chefs de laboratoire, etc., susceptibles d'ètre aussi bons forestiers qu'intelligents agriculteurs.

L'enseignement forestier est en outre donné assez fréquemment aujourd'hui aux élèves de certains lycées et collèges, ainsi qu'aux élères des écoles normales supérieures, des écoles normales primaires, el des éroles primaires élémentaires; enfin il est organisé depuis peu d'années dans quelques facultés. Les conférences faites dans chaque département par le professeur départemental d'agriculture, dans chaque arrondissement par les professeurs spéciaux, vulgarisent partout, où cela est utile, les applications pratiques de la science forestière.

L'administration des Eaux el Forêts, soucieuse de propager dans les campagnes les premières notions diart forestier, el les bienfaits qu'on est en droit de demander au reboisement, délivre gratuitement, aux instituteurs qui en font la demande, des graines et de jeunes plants l'arbres forestiers. Nous connaissons nombre d'instituteurs qui dans la mesure des moyens dont ils disposent ont constitué dans le jardin de l'école une pépinière el un petit champ de démonstration qui, tout modeste qu'il est, ne manque pas d'exercer une heureuse influence sur l'esprit des élèves; quelques-uns mème joignent à leurs conseils théoriques dexcellentes leçons pratiques en reboisant ávec leurs élèves peu à peu, et sous le prélexte d'une fìte de l'arbre, des friches incultes et improductives appartenant à la commune.

Quant à l'enseignement spécial, particulièrement destiné aux futurs agents des Eaux et Forêts, fonctionnaires chargés de la gestion des forêts de l'État el des lerrains soumis au régime forestier (bois des communes, 
des établissements publics, etc.), il est donné dans les deux établissements suivants:

$1^{6}$ L'école nationale des Eaux ct Forêts à Nancy (Meurthe-et-Moselle), dont les élèves se recrutent parmi les élèves sortant de l'Institut national agronomique et de l'École polytechnique; cetle école forestière admet également à ses cours des auditeurs libres français et étrangers.

$2{ }^{\circ}$ L'école secondaire des Barres, établie dans l'Arboretum National des Barres, commune de Nogent-surVernisson (Loiret), dont les élèves se recrutent parmi les préposés forestiers reconnus par voie de concours aptes à devenir agents des Eaux et Forèts.

Sociétés forestières. - Contrairement à ce qui se passe à l'étranger, le nombre des sociétés forestières est très restreint en France; touterois, depuis quelques années, un grand mourement se dessine dans notre pays en faveur de l'étude des questions forestières; à tous points de vue il est à encourager. Nous arons à signaler:

La Société nutionale dragriculture rle France, dont la troisième section a pris le titre de section de sylviculture.

La Société des agriculteur's ae $F_{1}$ 'ance qui a une section de sylviculture.

La Société for'estière de Franrhe-Comte et Belfort, fondée en 1890, et dont le siège est à Besançon; c’est une association amicale entre propriélaires de bois, forestiers, marchands de bois, industriels utilisant le bois, et tous ceux enfin qui s’intéressent aux forèts à quelque titre que ce soit. Cette association a pour but de contribuer: $1^{\circ}$ à l'arancement et à la propagation des connaissances se rapportant à l'économie forestière, ainsi qu'à l'exploitation et aux divers emplois des produits des forèts; $2^{\circ}$ à la conservation des richesses forestières existantes, à l'amélioration des forèts de peu de valeur, au reboisement des terres incultes, etc. Ses congrès 
annuels, suivis d'excursions en forêt, ont une vogue de plus en plus grande; des conférences sur les sujets les plus variés y sont faites par des amateurs compétents; des concours avec médailles, encouragrements de toutes sortes y sont organisés en fareur des reboisements, des perfeclionnements des industries forestières; enfin des questions intéressant l'économie forestière y sont mises à l'étude et ont déjà donné lieu à des mémoires remarquables publiés dans un bulletin trimestriel concurremment arec d'autres articles sur la gestion et l'exploitation des forêts (1) (Deroye). Pour faire suite à celle impulsion, et pour venir en aide aux nombreux préposés forestiers, gardes particuliers, propriélaires de bois, la Société, sur la proposition de son office de statistique et de recherches, a décidé de faire paraître un Agenda du forestier. Les citations de cette publication que nous avons faites au cours de notre ouvrage, suffisent à justifier l'éloge que nous en faisons; ce petit volume doit ètre dans la poche des propriétaires et employés vivant de la forêt, el autour de la forêt; c'est le vade-mecum de tout forestier.

La Société forestière française des amis des arbros;

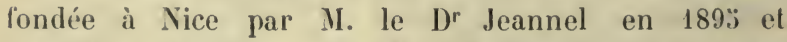
transférée à Paris par M. Demontzey; son siège social est actuellement, 2\%, quai Saint-ylichel (2); elle a pour but: $1^{\circ}$ d'inciter et seconder en france et dans les colonies françaises linitiative individuelle en vue de la plantation, de la protection, de l'amélioration el dis la propagation des arbres isolés ou en massif; $2^{\circ}$ d'aider par son concours, au groupement des honnes volontés et à la défense des intérèts des propriétaires.

Cette Société a déjà rendu d'éminents services; comme

(1) Pour faire partie de celte Sociéte, il suflit d'en for nuler In demande ('ll se faisunt présenter par un sociétaire; la cotssation annuelle est de á finnes.

(2) Membres souscripteurs, of francs par an; membres actifs. I francs par nu avec un engagement de planter au moins un arhre par an. 
la précédente, elle encourage par tous les moyens l'initiative individuelle, et nous renvoyons le lecteur au bulletin trimestriel fort instructif que publie cette association.

Nous arons à citer enfin des associations locales non moins dignes d'intérêt; telles sont : la Société agricole et forestière de Sologne; la Société horticole vigneronne et forestière de l'Aube; la Sociéti horticole, viticole et forestière de. Sens; la Société de protection des urbres et des forêts. Signalons ici la Section lorraine de la Société forestière française des amis des arbres, qui a été inaugurée en 1902 à Nancy, la Section Tarentaise et la Section d'Annecy qui, tout en étant rattachées à la Société mère de Paris, étendent la zone d'action de celte Société en groupant sur place les bonnes volontés individuelles.

Mentionnons à part les Socièlés scolaires forestières, dont la première a été fondée en 1899 par M. Nayet, instituteur à Avignon (Jura), a vec le concours de MI. Cochon, alors inspecteur des forêts et Delsiriès, inspecteur primaire à Saint-Claude. Leur nombre s'accroìt tous. les jours, et nous avons vu, au cours de notre carrière forestière trop de régions, où une initiative de ce genre serait susceptible de rendre d'éminents services, pour he pas souhaiter de les roir se généraliser dans tous les départements forestiers; nous empruntons sur leur formation en Franche-Comté, les lignes suivantes écrites par II. Émile Cardot (1): La plupart des communes de Franche-Comté possèdent des pâturages étendus où les habitants ont la faculté précieuse de conduire leur bétail pendant la saison d'été; malheureusement ces pâturages sont dégradés, appauvris peu à peu et ne fournissent plus au bétail qu'une alimentation tout à fail insuffisante. Ces pâturages, d'autre part, étaient autrefois partiellement boisés; de nombreux bouquets de taillis ou futaies procuraient du bois aux habitants et des ressources aux

(1) Émit. Cardot, Petit manuel à l'usage des Sociétés scolaires pastorales-forestises de Franche-Comte. 


\section{ä12 NOTIONS GÉNÉRALES D'ÉCONOMIE FORESTIÈRE.}

raisses communales. Aujourd'hui, ils ne renferment plus, pour la plupart, que des broussailles sans valeur.

Nos intelligentes populations franc-comtoises ont compris que l'on ne pouvait laisser plus longtemps dans un pareil état d'improductivité des surfaces considérables qui, comme les terres agricoles ou forestières du pays, reçoivent du soleil et de la pluie; et pourraient, élant remises en valeur et soignées, donner en abondance de lherbe et du bois.

Les instituteurs ont eu l'heureuse idée d'organiser dans nos villages de petites sociétés scolaires en vue de faire participer les en "ants à cette curre de restauration pastorale et forestière, et de graver dans leur's esprits celte idée que le bien comımunal doit être soigné, entretenu, añelioré dans l'in'érêt de tous.

Ces Sociétés se sont mises bravement à l'ourre, et par les résultats qu'elles commencent à donner on peut apprécier le rôle important qu'elles peuvent exercer dans l'avenir (1).

Aujourl'hui, dans le seul département du Jura, on ne compte pas moins de quarante-lrois sociétés scolaires, fondées sur le mème principe. Elles commencent à se répandre ígalement dans les départements du Doubs e de l'Ain. Les communes aussi, encouragées par les conseils généraux, les préfets, l'administration des Eaux et forèts, se mirent à la besogne; les plus pauvres volèrent des crédits. Résultat: 168.000 résineux plantés en 1901 dans les patturages communaux; ils donneront de l'abri au bétail, de la fraicheur aux gazons, en mème temps qu'ils auront pour effet de reconstituer le sol végé-

(1) Voir : Comples rendus au bulletin trimestriel de la Sociéte forestiére de Franche-Comté et Belfort.

Voir aussi au Bulletin $7^{\circ} 38$, anné 1902. de la Societé foresticre des amis des urbres, le rapport de . . E. Cardot sur le concours forestier entre les instituteurs, organisé par la société forestière franģaise des amis des arlues. 
tal sur les parties dénudées el de substituer aux broussailles sans valeur des bois de futaie (Cardot).

Mentionnons en outre, dans le département des Vosgres, une petite Socièté mutuelle forestière créée par M. Chipon, instituteur à Lissieux; deux Sociétés scolaires forestières du mème genre fondées dans le département de l'Ariège, dont le but, dit l'un des fondateurs de ces œuvres intéressantes M. Durrieu, est de faire comprendre en prèchant le reboisement dans des conférences ou des cours d'adultes la nécessité de faire un effort vigoureux pour empècher les inondations qui stérilisent le sol en le courrant d'une couche de cailloux et de graviers.

Citons enfin les Sociétés mutuelles scolaires de la Loire qui sur l'initiative de M. Audiffred, député de la Loire, se proposent d'acheter, soit au moyen de leurs propres ressources, soit à l'aide de dons et de subventions une certaine étendue de terrains improductifs pour les mettre en valeur par des travaux de reboisement.

Sociétés d'agriculture. - Comices agricoles. Syndicats. - 11 existe aujourd'hui en France un grand nombre de comices et de sociétés d'agriculture, dont le but principal est d'organiser des concours, arec les ressources fournies par les cotisations de leur's membres, et de décerner des récompenses aux agriculteurs les plus méritants.

Dans les régions forestières, ces sociétés et comices agricoles sont désignés pour encourager les améliorations forestières en décernant des récompenses aux sylviculteurs; sur leur initiative, nous voyons s'adjoindre à certains concours régionaux des expositions foresticres qui font ressortir par les échantillons et les travaux exposés l'importance et l'utilité des forèts de la région.

Des syndicats dépendant de ces sociétés ou de ces comices peuvent se créer, dans le but, soit de faire en commun et arec les garanties suffisantes les achats de graines et de plants, soit d'entreprendre des reboisements 
514 NOTIONS GÉNERALES D'ÉCONOMIE FORESTIÈRE.

importants à l'aide de pépinières créées el entrelenues par le syndicat.

Signalons à part le syndicat agricole et furestier de Campignol (Aude) fondé par M. Fabre, directeur de l'école publique de Gruissan; ce syndicat comprend plus de 2.30 heclares de lerres conquises sur les étangs par le colmalage; M. Fabre encourage les propriétaires à planter des arbres en bordure sur ces terrains assainis, et aujourd'hui déjà des milliers d'arbres assainissent le pays et en agrémentent l'aspect.

Crédit foncier. - Prêts hypothécaires sur biens forestiers. - Dans le langage économique, le mot crédit comprend toutes les opérations qui ont pour objel le prèt des capitaux, ou, si l'on veut, qui ont pour but de faire passer les capitaux en instruments de production de la main de celui qui les possède et ne les fait pas valoir, dans la main de celui qui ne les possède pas et qui veut les consacrer à la production. Les institutions qui servent ainsi à faire passer les capitaux d'une main dans l'autre sont des établissements de crédit (1).

La Soaieté clu Créclit foncier (2) dispense le crédit reposant sur des garanties matérielles, telles que l'affectation hypothécaire d'une propriété. Aux propriétaires d'inmeubles en général, le Crédit foncier prète en numẻraire, sur première hỵpothèque, jusqu'à concurrence de la moitié de la valeur des biens offerts en grarantie (3). Le plus souvent, les propriétaires d'immeubles boisés sont bien moins favorisés; l'estimation de leur propriété est faite très sévèrement par les inspecteur's du Crédit foncier; de plus le prèt qui leur est garanti par la Société n'estque du tier's de célte valeur estimative. Celte.

(1) Voy. le volume de l'Excrccopêde agucole: Économie agricole par Jouzier.

(2) Vuir A. Covunay, Crédit foncier de France. Prêts aux particuliers ; conditiuns et avantages.

(3) Ce prêt n'est que du tiers de la valeur, si les immeubles consisteut en vigues. 
sévérité s'explique par la nature même du gage offert et la durée très longue des prêts. Nous arons vu que le capital forestier, en raison de sa constitution toute spéciale, est toujours réalisable, au moins en partie; si le propriétaire exploite mal sa forèt, s'il ne suit pas les révolutions établies, s'il sacrifie une partie du capital superficie, par exemple des réserves dans un taillis composé, jour se faire de l'argent, ce qui arrive très souvent lorsque l'emprunteur est gèné, l'immeuble offert en garantie perd de suite une partie de sa valeur.

De ce fait résulte la conséquence suivante : les avantages que peuvent retirer les propriélaires de forèts des prèts du Crédit foncier, sont les mèmes que ceux dont profitent les autres propriétaires fonciers, mais la la somme qui leur est offerte est proportionnellement bien moins élévée; les propriétaires de biens forestiers sont donc obligés d'hypothéquer un gage, qui pour eux a une valeur trois et mème quatre fois plus grande que la somme qui leur est prètée.

\section{V. - ASSURANCES DES BOIS ET FORÊTS CONTRE L'INCENDIE.}

La question de l'assurance des forêts est aujourd'hui encore fort discutée par les assureurs, et par les propriétaires de forèts; les premiers la repoussent généralement, parce qu'on leur demande de garantir des risques qui peurent devenir à l'occasion fort importants; les seconds hésitent, parce qu'en prévision de sinistres graves, ils ont à payer des primes souvent fort lourdes.

Très peu de compagnies d'assurances consentent à assurer les forêts ; celles qui le font (1) n'assurent même pas les forêts dans toutes les régions de la France; elles se sont imposé des limites qu'elles ne dépassent pas, el

(1) Nous citzron; l'Assurance générale, la Providence qui consentent ì assurer les forèts dans certains cas. 


\section{¡i 6 NOTIONS GÉNÉRALES D’ÉCONOMIE FORESTIÈRE.}

qui sont laséez sur la sécheresse plus ou moins présumée les terrains, la durée de l'élé, elc. Ces limites sont restreintes au nord de la France, et les Compagnies ne paraissent pas descendre, en général, en dessous d'une ligne qui partant de Nantes, remontant la Loire et l'Indre jusqu'à Châteauroux, se dirige sur Chambéry en passant par Moulins, Màcon et Lyon. Elles font toutefois exception : $1^{\circ}$ pourcertaines parties du massif central dans lesquelles le terrain boisé, humide par nature, est moins susceptible d'ètre ravagé par un sinistre; $2^{\circ}$ pour les petites forèts ou bois, lor'sque ces terrains boisés dépendent d'unegrande propriétéassurée pour un gros risque ; loutefois l'assurance de la partie boisée ne doit itre que l'accessoire et la valeur du bois assuré ne doit pas dépasser 10 p. 100 du risque total assuré.

Contrairement à ce qui se passe en matière d'assurance ordinaire, lorsqu'il s'agil d'assurances de forèts, c'est l'assuré qui détermine lui-mème la valeur du risque à assurer. S'il assure une forèt d'une valeur de 10.000 ou de 100.000 francs, il évalue lui-mème l'importance de son risque et limite son assurance au chifre qu'il désigne. Cependant il ne doit pas oublier qu'il n'assure que le matériel sur pied; il peut assurer en oulre le trouble dans l'aménagement, ou l'ensouchement, mais il aura à payer en sus des primes spéciales correspondant à ces sortes de risques.

Ainsi, le lrouble dans l'aménagement est assuré par les Compagnies pour une valeur égale à la valeur estimée de la forèt; si une forèt évaluée :50.000 franes est assurée en mème lemps contre les risques d'incendie du matériel sur pied el contre le trouble apporté dams l'aménagement par un sinistre, l'assuré derra payer leux primes; la premiere caleulée sur $\$ 0.000$ frants pour l'assurance de la superficie, la seconde calculée aussi sur 50.000 fianes pour le trouble dans l'aménagement. 
Il faut remarquer toutefois que les évaluations de la forèt données par l'assuré, ne sont faites que pour établir une base à la perception des primes, car en cas de sinistre, le soin d'évaluer les pertes est confié à des experts nommés à l'amiable par les parties, si elles peuvent se mettre d'accord, ou sinon désignés par les tribunaux.

En matiìre d'assurance les Compagnies distinguent deux sortes de bois: les bois non résineux et les bois résineux.

Rois nın résineux (feuillus). - En ce qui concerne les bois non résineux, l'assurance est générale et s'applique à toute la forèt, sauf : $1^{0}$ aux sous-bois, landes, bruyères, brandes et ajoncs, qui sont exclus de l'assurance ; $2^{\circ}$ à l'ensouchement qui est exclu de l'assurance, et pour lequel l'assuré doit payer une prime spéciale s'il veut assurer en mème temps le repeuplement par rejets de souche.

Quant à la prime à payer, il y a lieu de distinguer selon que l'assurance s'applique à des taillis ou des futaies; pour les taillis âgés de moins de vingt-cinq ans et les taillis composés avec baliveaux, modernes ou anciens, la prime est généralement la mème, en moỵenne 0,73 p. 100 du capital assuré, étant entendu que la compagnie accorde une tolérance de un dixième de résineux disséminés. Pour les hautes futaies, à la condition qu'il n'y ait pas de jeunes hois an-dessous, la prime est seulement d'environ $0,40 \mathrm{p} .100$ du capital assuré; cependant cette prime diminue à mesure que le bois est plus âgé, car les chances d'incendie diminuent, ainsi d'ailleurs que l'importance des risques en cas de sinistre.

Bois résineux. - En ce qui concerne les bois résineux, l'assurance en général est refusée par les compagnies qui consentent rarement à passer un contrat dans ces conditions à cause du gros risque qu'elles courent; cependant, si elles y consentent, elles en excluent toujours, d'une part l'ensemencement, d'autre part les sous- 
bois, landes, bruyires, brandes et ajoncs. Pour ce genre te forèts, de mème que pour les futaies non résineuses, plus le bois esı âgé, plus la prime diminue.

La prime exigée est très variable; pour les bois résineux âgés de moins de dix ans elle atteint le chiffre très: élevé de 10 p. 100 du capital assuré; elle tombe à 1,:00 p. 100 pour les bois de cinquante ans et au-dessus ; entre ces limites elle varie de $\ddot{j}$ à $1,: 00 \%$. 100 , devenant d'aulant plus faible que les arbres sont plus âgés.

Évaluation du dommage en cas de sinistre. - Les polices d'assurances comprennent des clauses spéciales pour chaque genre d'aménagement; elles contiennent toutes le principe suivant : évaluer la valeur que les arbres devaient avoí au moment où aurait eu lieu la coupe normale; évaluer la valeur des arbres aussitôt après lr. sinistre, déduction faite toutefois du sauvetage; léduire celle seconde évaluation de la première; la difrérence représente la perte occasionnée par le sinistre. C'est en affet à l'époque de l'exploitation normale que l'on toil s. reporter pour déterminer le véritable dommage causé à l'assuré.

La compagnie ràgle inmédiatement le montant du sinistre; mais en agrissant ainsi elle paye un capital d'avance à l'assuré, et il est fort équitableque la compagnirfentc à se faire tenir comple par les assurés des intérèts composés de la somme à eux versée, entre le jour où la compagnie liquide l’indemnité et l'époque à laquelle le sinistré aurail effectivement touchéle prix do l'exploitation normale de sa foret. A cet efret, les compagnies ajoutent en général dans la police une clause aux termes de laquelle elles retiendront, lor's du paiement de l’indemnité, Im escomple de f p. 100 par an sur la somme versée, escomptequi sera ealculé pour autant d'années qu'il en existe entre la date du règlement du sinistre, et celle de l'àge normal d'exploilation.

En ce qui concerne l'ensouchement, le dommage causé 
aux souches par le passage du feu, si l'ensouchement est assuré, est en général évaluépar les compagnies à raison de deux plants par souche à planter dans les intervalles suirant l'usage.

Quant au trouble apporté par le sinistre dans l'aménagement, les compagnies suivent des règles qui varient arec les circonslances.

En résumé, les Compagnies d'assurances ne cherchent pas à assurer les forèts; lorsqu'on le leur demande, elles se tiennent sur une certaine réserve, se refusent à descendre au-dessous d'une ligne déterminée, ont enfin des tarifs spéciaux pour chaque groupement d'arlbres, tarifs. qui varient dans de grandes proportions, derenant d'autant plus élerés que les massifs sont plus exposés aux dangers dincendie, ou donnent une prise plus effective à l'action du feu. 


\section{DEUXIËME SECTION}

\section{Notions générales d'estimation des bois.}

Pour apprécier la valeur d'un arbre, d'une coupe ou d'un peuplement, autrement dit pour faire une estimation, il faut saroir déterminer le rendement en matière, c'est-à-dire en bois d'œurre, en bois de feu, en produits de toute nature que ces arbres ou peuplements sont susceptibles de donner.

L'estimation en argent consiste à appliquer à ces résultats les prix correspondants.

Nous nous proposons d'exarniner successivement l'etimation en matière ou les principes de cubage, et l'est:mation en argent.

\section{I. - estimation EN MATIĖRE; PRINCIPES DE CUBAGE.}

\section{1. - Cubage des bois abattus.}

Les bois abattus se décomposent généralement:

$1^{\circ}$ En bois d'ceurre fourni par le tronc et quelques branches principales;

$2{ }^{\circ}$ En bois de chaulfage et hois à charbon ou charbonnette, fournis par les parties de lit lige et des branches impropres au bois d'ouvre, et parle houppier;

$3^{\circ}$ En fagots et bourrées, composés de menus bois qui n'ont pas pu ètre compris, à cause de leurs faibles dimensions ou de leur forme, dans la charbonnette; 
$4^{\circ}$ En produits spéciaux, demandés par le commerce lans des conditions déterminées par une utilisation locale.

$\S 1$. Bois d'œuvre. - - Les gros bois, ou bois d'œuvre, se divisent en bois de service ou de construction et bois d'industrie ou de travail.

Les premiers renferment : les bois de charpente, les traverses, les poteaux télégraphiques, les étais et perches de mine, les perches à houblon; dans les seconds sont conıpris : les bois de sciage, les bois de fente (merrains et échalas).

Les bois d'œuvre se mesurent de bien des manières, suivant les usages, les habitudes ou les traditions des localités. Mais il y a lieu de distinguer les bois en grume et les bois équarris.

Cubage des bors en grume. - Le hois en grume s'entend des bois ronds, revètus de leur écorce. Ainsi considéré, le bois se prète à toute espèce de débit et d'utilisation.

Le cubage en mètres cubes grume devrait être le seul pratiqué, le procédé légal universel; il peut s'appliquer à toute espèce de marchandises; tout autre mode de cubage est affaire de conventions et d'habitude (1).

Le cubage en grume consiste à assimiler la tige considérée à un volume géométrique d'après les dimensions extérieures (longueur d'une part, et circonférence ou

(1) Nous appelons l'attention du propriétaire particulier sur les procédés de cubage en usage dans les différentes régions. Dans toute transaction, pour s'éviter des mécomptes, le mode de cubage doit ètre nettement déterminé ; les unilés de volume qu'emploie le commerce, mème lorsqu'elles sont exprimées en mètres cuhes, ne sont pas les mèmes (mètre cuhe grume, mètre cuhe au quart sans déduction, etc.); il est par suite nécessaire de les préciser, car le mème arbre, culé par le mème individu par des procédés exacts et admis dans le commerce, pourra donner un volume différent, bien qu'exprimé en mètres cub's, si la convention passée entre l'acheteur et le vendeur nª pas précisé la méthode de cubage; c'est ainsi qu'une mème personne, suivant qu'elle achète ou qu'elle vend, peut arriver à exprimer le volume d'un mème arbre par des chiffres différents; il lui suffit de choisir, suivant les cas, le procédé de cubage le plus avantageux. 


\section{¿22 NOTIONS GÉNÉRALES D'ESTIMATION DES BOIS.}

diamètre (l'autre part) et à déterminer par le calcul ce volume géométrique.

On appelle décroissance d'un arbre la loisuivant laquelle la grosseur de sa tige diminue de la base au sommet.

Si dans l'arbre, la décroissance était uniforme, la tige serait dlans ce cas exactement assimilable à un tronc de cône, se rapprochant plus ou moins du cylindre; mais en général la décroissance n'est pas uniforme sur toute la Jongueur. On admet cependant qu'elle l'est sur des longueur's partielles, de sorteque la tige peut ètre assimilée à une série de troncs de cône superposés l'un à l'autre. En réalité, il est plus exacl de dire qu'elle est un paraboloïde de révolution; et le volume exact de ce parabolö̈le est le volume réel du tronc.

Dans la pratique, assimilant le volume de la pièce à cuber à un cylindre, on en mesure la longueur, et la circonférence (ou le diamètre) au milieu; le volume ainsi obtenu dit volume cylindrique est donné par les deux formules suivantes :

a. En fonction de la circonférence au milieu C et de la liauteur du tronc $\mathbf{~ I}(1)$

$$
\mathrm{V}=0,0796 \mathrm{C}^{2} \mathrm{II} \text {. }
$$

b. En fonction du diamètre correspondant D (diamètre de la circonférence prise au milieu de la tronce) et de la hauteur

$$
\mathrm{V}=0,78: \mathrm{I}^{2} \mathrm{D}^{2} \mathrm{H} \text {. }
$$

Si la tige est irrégulière, on la partarre en billes ou tronces aussi régulières que possible, ef l'on cube parliellement chaque fraction, sauf à additionner les résullats partiels.

On appelle découpe l'opération qui a pour hut de diviser la lige entière endeux ou plusieurs piècestermanièreàen

(1) Formule simplifice de l'expression $V=\frac{1}{4 \pi}$ C2II. 
tirer le meilleur parti possible, sans compromettre les dimensions recherchées par le commerce pour les pièces de charpente; la découpe influe sur le cubage, et un exemple que nous empruntons à M. Frochot(1) est bon à citer pour faire saisir l'effet d'une découpe plus ou moins habile: la tige d'un chêne de 26 mètres de hauteur a une circonférence au milieu de $1^{\mathrm{m}}, 44$; le cubage en mètres cubes grume, donne, si l'on applique la formule précédente sans faire de découpe :

$$
\begin{gathered}
\mathrm{V}=0,796(1,44)^{2} \times 26 \\
=4 \mathrm{mc}, 290 .
\end{gathered}
$$

Mais si l'on remarque, comme on peut s'en rendre compte sur la figure 51 , que la partie AB par exemple a une qualité supérieure à la partie BD, eu égard à ses fortes dimensions, à sa forme régulière, à l'absence de noeuds, etc., et que par conséquent on peut lui appliquer un prix en rapport avec ces qualités, on est amené à faire une première

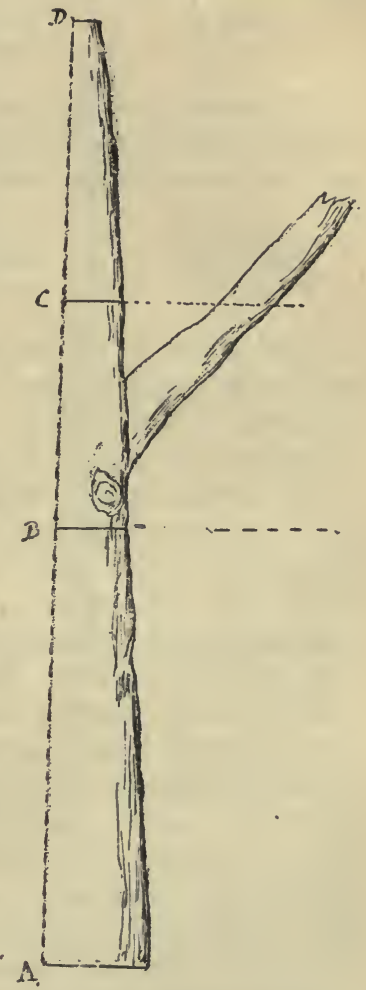

Fig 51.- Découpe d'un tronc d'arbre. découpe en $\mathrm{A}$. Si la tige est divisée en deux tronces A B et B D le cubage en mètres

(1) A. Frochot, Ciubage et estimation des bois. 
วั24 NOTIONS GÉNÉRALES D'ESTIMATION DES BOIS.

cubes grume donne si l'on applique la mème formule à chacune des tronces:

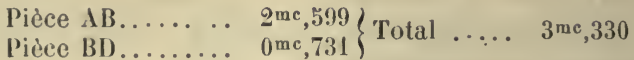

Ce résultat par trop défarorable, quant au volume, doit ètre modifié par une seconde découpe entre B et D, en C par exemple; par cette nouvelle combinaison on a :

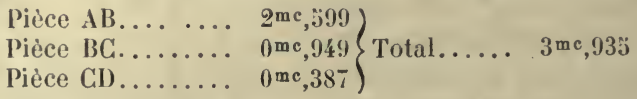

Les détails de ce dernier calcul font voir que le cube de la pic̀ce $B$ C est plus avantageux l'une manière absolue que celui de la pièce B D; c'est surtout à des anomalies te cette nature que les découpes doivent obvier.

Une découpe habile sait rendre le cubage aussi avantageux que possible, tout en permettant de classer les produits suivant la qualité spéciale que leur donnent leurs dimensions (charpente première qualité, charpente deuxième qualité, industrie, etc.), et que leur donnent aussi la forme régulière, l'absence de nocuds, etc. (1).

Mesure des hauteurs. - Les hauteurs ou longueurs des troncs d'arbres abattus se mesurent à la chaine métrique ou au ruban gradué. La plupart des marchands de hois préfèrent se servir d'un compas en bois à pointes en fer, ayant 1 mètre ou $1^{\mathrm{m}}, 30$ d'ourerture; ce compas est une simple règle graduée de 1 mètre de long, munie de leux pointes aux extrémités, pointes qui sont dirigées perpendiculairement à la direction de la riggle.

Mesure des diamétres. - Les diamètres se mesurent à l'aide ducompas forestier (fig. 32 ); ce compas se compo:e d'une rigle graduée, portant denx autres règles à angle

(1) Ce fait est encore h signaler au propriétaire particulier ; suivant la découpe adoptée, le volume des unités de mème qualité (charpente, industrie, etc.) peut varier; et si dans une transaction le cas n'est pas prévu, le juropriétaire peut se trouver lésé, faute d'avoir sjúcifié la méthode de cuhage. 
droit, celle de l'extrémité fixe, l'autre mobile sur la règle; on place horizontalement cet instrument de manière à embrasser entre les deux règles parallèles, le corps de l'arbre à la hauteur où on veut le me surer ; la règle mobile indique sur celle qui est graduée le diamètre cherché. Le zéro de la division est donc placé sur l'arète intérieure de la règle fixe, et la graduation en centimètres est établie à partir de ce point (1).

Mesure des circonférences. Les circonférences se mesurent à l'aide d'un ruban gradué qui se compose d'une boîte cylindrique dans laquelle s'enroule autour d'un axe un ruban en fil, rendu imperméable au moyen d'une préparation; sa longueur varie, mais généralement elle est de 10 mètres, ce qui lui a fait donner le nom de décamètre.

(1) Le compas est gradué sur une face de 2 en 2 centimêtres, et permet d'obtenir, pour un cubage approché, les diamètres de 2 en 2 centimètres.

S'il s'agit au contraire de mesurer rapidement un grand nombre d'arbres, dans une opération dont on inscrit les résultats sur un calepin, on doit en pratique, se contenter d'appeler les diamètres de 5̃ en 5 centimètres, ce qui réduit de beaucoup le nombre des colonnes du calepin; afin d'obtenir que les erreurs ainsi commises se compensent et tendent à s'atténuer, on doit appeler comme arbre ayant 20 centimètres de diamètre celui qui a depuis 17 centimètres et demi à 22 centimètres et demi, et ainsi de suite. On peut graduer en conséquence l'autre face du compas, de façon qu'à la lecture, le chiffre 20 soit découvert par la glissière mobile du compas, depuis l'arbre de $17^{\mathrm{cm}}, 5 \mathrm{~d}$ celui de $22^{\mathrm{cm}}, 5$, et de telle sorte qu'immédiatentent après, dès l'arbre de 23 centimètres de diamètre, ce soit le chiffre 2ă qui devienne apparent, et ainsi de suite. Cette disposition permet d'éviter un grand nombre d'erreurs de lecture, et supprime les hésitations en rendant la lecture très facile. 


\section{NOTIONS GÉNÉRALES D'ESTIMATION DES BOIS.}

Ces rubans sont soumis à un retrait parfois assez considérable, et peuvent donner des indications fausses; aussi est-il plus commode de se servir d'une corde bien tressée, peu susceptible d'allongement, armée à son extrémité d'une sorte de grande aiguille en fer, qui permet de la passer sous les troncs à terre; il sulfit alor's de reporter la longueur de la ficelle sur une règle graduée pour avoir la circonférence (1).

Tenue du calepin. - Dans le cas où les mesurages portent sur un certain nombre de troncs abattus (dénombrements), il y a lieu de préparer à l'avance un calepin à double entrée, l'une pour les diamètres (ou les circonférences), l'autre pour les hauteurs, avec distinction d'essences s'il y a lieu. Chaque arbre appelé est pointé à sa place sur le calepin de la manière générale que nous avons indiquée précédemment.

Cubage au quart sans déduction. - Ce procédé de cubage correspond à déterminer immédiatement, d'après la mesure directe de la circonférence du milieu (ou de la moyenne des circonférences des deux bouts), le volume que conservera la pièce de bois après un équarrissage grossier; c'est ce qu'on fait habituellement pour les sapins.

Pour obtenir le volume au quart sans déduction, il suffit de prendre le quart de la circonférence, de le multiplier par lui-même, et de multiplier le produit obtenu par la longueur de la pièce.

Ce cubage donne un volume représentant un peu plus des trois quarts et très approximativement 0 , 783 du volume en grume.

Ce nombre 0, 785 est le coefficient qui permet de passer du volume cylindrique au volume auquarl sans déduction.

Cubage au cinquième déduit. - Ce cubage correspond a déterminer immédiatement, d'après la mesure directe de la circonférence du milieu fou de la moyenne des

(1) Avec ce système, surveiller les erreurs ou les fraudes; les commis do twis savent faire glisser la corde en la rapportant sur le mètre gradué. 
circonférences des deux bouts), le volume que conservera la pièce après un équarrissage à vive arète et sans aubier ; le déchet est évidemment plus considérable dans ce dernier cas que dans le précédent.

Pour obtenir le volume au cinquieme déduit, il suffit de prendre le cinquieme de la circonférence, de le multiplier par lui-méme, et de multiplier le produit obtenu par la longueur de la pièce. - Ce cubage donne un volume représentant à peu près exactemement la moitié du volume en grume; le coefficient 0,5 permet de passer du volume cylindrique au volume au cinquième déduit.

Nous résumons dans le tableau suivant, emprunté à M. Frochot, les coefficients nécessaires : $1^{0}$ pour passer de l'un quelconque des cubages à un autre cubage; $2^{\circ}$ pour déduire le prix du mètre cube, correspondant à un cubage donné, du prix connu du mètre cube dans un autre système du cubage (1).

Tarifs pour le cubage des bois abattus. - Pour constituer un tarif de cubage soit en grume, soit au quart sans déduction ou au cinquième déduit, etc., pouvant servir d'une façon rapide, il suffit de calculer les rolumes successifs de billes d'un mètre courant ayant, soit les diamètres, soit les circonférences successives qui peuvent se présenter, et d'en dresser un tableau à deux colonnes; ces tarifs doivent être aussi réduits que possible, et très simples; on doit y supprimer toutes les décimales inutiles.

A l'aide de ces tarifs le calcul de cubage devient alors très simple; on applique son tarif à l'ensemble des arbres ayant le mème diamètre (ou la mème circonférence), en multipliant la somme de leurs hauteurs par le volume

(1) Il est évident que le mètre cube grume ne peut avoir la mème valeur que le mètre cube au quart sans déduction, au cinquième déduit, etc. Toutes ces unités se rapportent donc à des marchandises différentes; en matière de commerce des bois, donner un volume en mètres cubes n'est pas suffisant; il faut préciser de quelle marchandise il s'agit. 
528 NOTIONS GÉNÉRALES D'ESTIMATION DES BOIS.

\begin{tabular}{|c|c|c|c|c|c|c|}
\hline \multirow{4}{*}{ 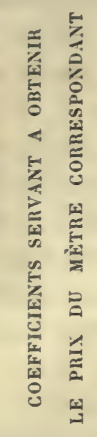 } & \multirow{4}{*}{ 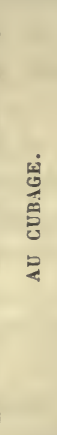 } & $\frac{\infty}{\grave{\Xi}}$ & $\underset{\sim}{\stackrel{20}{\circ}}$ & $\begin{array}{l}8 \\
-1 \\
-1\end{array}$ & $\begin{array}{l}\text { बे } \\
\text { : }\end{array}$ & $\stackrel{8}{8}$ \\
\hline & & 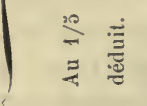 & $\begin{array}{l}20 \\
\mathscr{0} \\
\stackrel{8}{\circ} \\
\stackrel{-}{-}\end{array}$ & $\begin{array}{l}20 \\
9 i \\
0 \\
0 \\
0\end{array}$ & $\stackrel{8}{8}$ & 商 \\
\hline & & 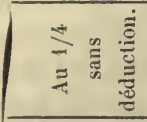 & 일 & $\begin{array}{l}8 \\
\stackrel{0}{0} \\
\stackrel{-}{-}\end{array}$ & @ँ & 苛 \\
\hline & & 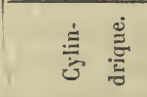 & $\stackrel{8}{8}$ & $\begin{array}{l}\overrightarrow{0} \\
0 \\
0 \\
0 \\
0\end{array}$ & $\begin{array}{l}\text { जิ } \\
\text { जิ } \\
0 \\
0 \\
0\end{array}$ & 势 \\
\hline \multirow{4}{*}{ 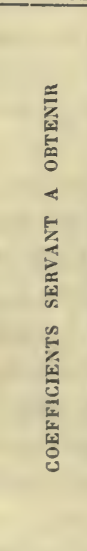 } & \multirow{4}{*}{ 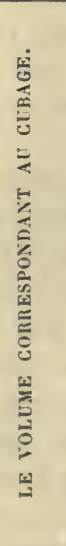 } & $\stackrel{0}{\stackrel{\vec{\Xi}}{\Xi}}$ & $\begin{array}{l}\frac{61}{4} \\
\frac{20}{30} \\
20 \\
0\end{array}$ & 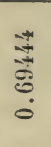 & $\begin{array}{l}\mathscr{0} \\
0 \\
\infty \\
0 \\
\dot{0}\end{array}$ & 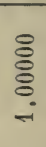 \\
\hline & & $\stackrel{20}{\stackrel{3}{\Xi}}$ & 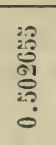 & $\begin{array}{l}8 \\
0 \\
0 \\
0 \\
0\end{array}$ & $\begin{array}{l}8 \\
8 \\
8 \\
\dot{0}\end{array}$ & $\frac{20}{\stackrel{0}{0}}$ \\
\hline & & 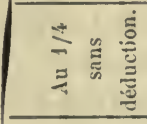 & $\begin{array}{l}0+1 \\
0 \\
0 \\
0 \\
0\end{array}$ & $\stackrel{8}{8}$ & 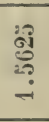 & : \\
\hline & & 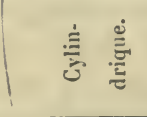 & 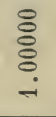 & 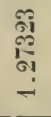 & $\begin{array}{l}0 \\
\stackrel{\infty}{\infty} \\
\stackrel{0}{0}\end{array}$ & $\begin{array}{l}\overrightarrow{20} \\
\hat{\overbrace{}} \\
\infty \\
0 \\
0\end{array}$ \\
\hline & $\begin{array}{l}0 \\
0\end{array}$ & 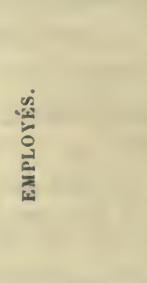 & 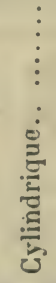 & 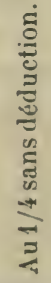 & 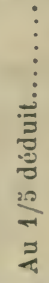 & 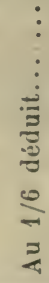 \\
\hline
\end{tabular}


donné au tarif pour la bille de 1 mètre de long ayant ce diamètre (ou celle circonférence).

Cubage des Lois équar'is. - On appelle équarrir une pièce de bois l'opération qui consiste à la transformer, à l'aide de la hache ou de la scie, en un parallélipipède rectangle ou en un tronc de pyramide droit.

- L'équarrissage peut ètre à vive arète sur franc bois, ou avec tolérance d'aubier.

On dit que la pièce équarrie présente des flaches quand les faces adjacentes ne se terminent pas chacune par une arète commune.

Les pièces ainsi obtenues présentent des formes géométriques connues, et il est facile de calculer les volumes en fonction des dimensions de ces pièces.

Règle à cubage de $\mathbf{M}$. de Montrichard (1). - La règle à cubage de $\mathbf{M}$. de Montrichard fournit un procédé de calcul rapide et essentiellement pratique pour obtenir les volumes; elle secompose, comme la règle à calcul de deux parties: d'une règle fixe, el d'une réglette mobile engagée à plat dans une coulisse pratiquée à l'intérieur de la règle, de façon à glisser parallèlement à celle-ci; elle porte trois échelles affectées, l'une aux circonférences (partie inférieure de la règle), l'autre aux hauteurs (partie supérieure de la réglette), la troisième aux volumes (partie supérieure de la règle); en outre vers le milieu de la réglette, et au dessous de l'échelle des hauteurs sont marquées des divisions qui constituent ce que nous appellerons l'échelle des repères, et qui servent à placer la réglette mobile dans la position voulue pour obtenir le volume cherché suivant le mode de cubage dont on a fait choix.

Si l'on veut cuber en grume, au quart sans déduction, au sixième ou au cinquième déduit des arbres abaltus, on choisit pour repère le trait au-dessus duquel est inscrit le mode de cubage adopté; ce trait amené au-dessus de

(I) D'après M. Frochot.

A. Fron. - Sylviculture. 
la division qui exprime la circonférence donnée, sur l'échelle inférieure de la règle, permet de placer la réglette mobile dans une position telle que la hauteur de l'arbre, lue sur l'échelle des hauteurs, coïncide avec la division qu'il faut lire sur l'échelle des cubes pour connaitre le volume cherché; ce volume est précisément le volume en grume, au quart sans déduction, au sixième ou au. cinquième déduit, selon le repère dont on s'est servi. II faut, bien entendu, dans cette opération, se donner la circonférence moyenne de l'arbre.

$\S 2$. Bois de feu. - Tous les bois impropres à la charpente et à l'industrie, ou ceux qu'on a intérèt à convertir en bois de chauffage sont utilisés comme combustible.

On distingue en général les bois de corde et la charbonnette; nous donnons ci-dessous d'après l'Agenda du furestier les catégories habituelles.

$1^{\circ}$ Bois de corde. - Petit rondin; de $0^{\mathrm{m}}, 06$ à $0^{\mathrm{m}}, 10$ de diamètre, soit $0^{\mathrm{m}}, 20$ à $0^{\mathrm{m}}, 30$ de tour. - Quartier; au-dessus de $0^{\mathrm{m}}, 13$ ou $0^{\mathrm{m}}, 13$ de diamètre.

Les longueurs habituelles des bûches sont $1^{\mathrm{m}}, 14$ à

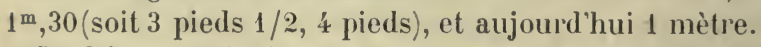

Parfois on trie à part le chène, le tremble, les bois tendres (blancs), les bois durs, le bois écorcé ou non écorcé, le bois de tige ou celui des branches ou ramiers; on distingue aussi, d'après les usages du commerce: le bois neuf, coupé depuis un an; le bois vieux, ayant plus d'un an de coupe, etc.

$2^{\circ}$ Criarbonnette. - Bois de toutes essences, de Ia grosseur de $0^{\mathrm{m}}, 06$ à $0^{\mathrm{m}}, 20$ de tour, débilés sur une longueur de $0^{\mathrm{m}}, 65$ à $0^{\mathrm{m}}, 80$ ( 2 pieds à 2 pieds $\left.1 / 2\right)$.

Le bois de chaufrage est empilé en las ou ròles portant souvent le nom de cordes; ces cordes offrent des dimensions très variables suivant les pays.

Empilage. - Les bûches sont empilées, de manière à ne laisser que le moins d'interstices possible entre elles, dans une membrure (fig. 53), dont les dimensions sont 
fixées d'arance, de façon que l'espace occupé par la pile, forme un parallélipipède droit dont les dimensions sont: longueur de bûche $\mathrm{L}$; hauteur de pile $\mathrm{H}$; longueur de la pile ou couche $\mathrm{C}$.

On n'empile jamais ensemble que les bois ayant même longueur de bûche; cette longueur de bûche dépend des régions et des emplois divers auxquels le bois est destiné ; c'est le seul facteur qu'on ne peut modifier. Partant de cette longueur de bûche, on calcule en centimètres la hauteur $\mathrm{H}$ à donner à la pile, de façon qu'en multipliant cette hauteur par la longueur de bûche, le produit soit

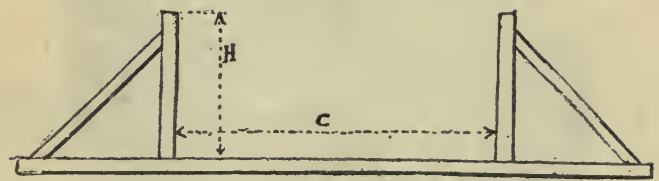

Fig. 53. - Membrure pour empilage.

exactement égal à un mètre carré, ( $\mathrm{L} \mathrm{H}=1 \mathrm{mq}$ et par par suite $\mathrm{H}=\frac{1}{\mathrm{~L}}$ ).

Ainsi construite, la pile représente sur une longueur de couche de un mètre, l'unité de volume qui est le stère (1) en matière de bois de chauffage, et le volume exprimé en stères et décistères se trouve représenté par la longueur de couche exprimée en mètres et décimètres (fig. ว̌́).

On peut aussi convenir qu'on donnera à la longueur de couche dans chaque pile uniformément soit 1 mètre, soit 2 ou 3 mètres, et que la hauteur de chaque pile sera cal-

(1) Le stère de bois de chauffage est la quantité de bùches empilées que peut contenir un espace mesurant 1 mètre cube. Le stère est une unité de volume du système métrique. Bien que le système métrique soit d'origine essentiellement frangraise, et que la plupart des nations l'aient adopté, le commerce de hois français continue à se servir d'autres mesures, corde, moule, etc., dont les volumes sont variables suivant les régions. Il est à désirer qu'on se décide en France à adopter d'une façon uniforme le système nétrique, dans toutes les circonstances où il n'y a pas de raisons sérieuses d'agir autrement. 
532 NOTIONS GÉNÉRALES D'EsTIMATION DES BOIS.

culée d'apròs la longueur des buches, de façon que le volume de la pile représente exactement le stère, le double slìre el le demi-dlécastère (2).

Dans la pratique, pour tenir compte du lassement qu se produit toujours el qui a pour effet de diminuer la hauteur du tas de 4 à "i p. 100, on doit donner à la hauteur du tas, au moment de lempilage, 3 à 4 centimètres de plus que la hauleur normale.

Le bois de chauffage s'entasse de manières bien diffé-

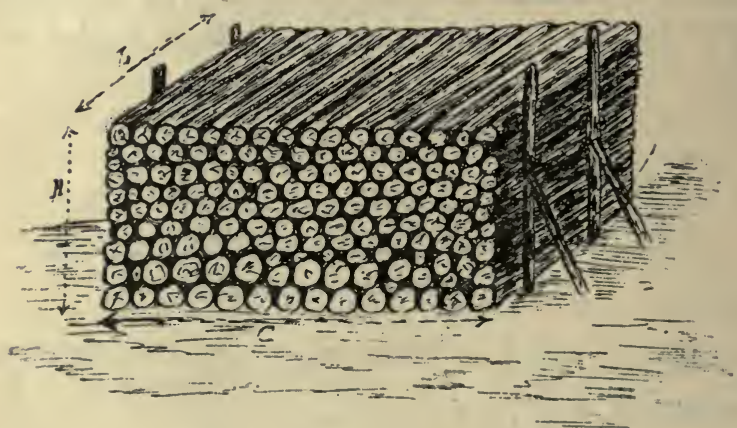

Fig. วั4. - Empilage des bois de feu.

rentes, suivant qüll est plus ou moins droit, qüil est en bûches plus ou moins longues, ou enfin quïl est noueux ou tordu; il en résulte que le volume réel de maliore ligneuse contenue dans un stere de bois est variable; on a rapporté ce volume au mètre cube et le tableau suivant que nous extrayons de l'A genda forestier donne les facteurs qui permettent de passer du volume exprimé en stires au volume réel de hois exprimé en mètres cubes at inversement, d'un volume réel exprimé en mètres cubrs, au volume en stires que donneront les mèmes bois apris

(2) Loi du 4 juillet 1837 et ordonnance du 16 juin 1839. - Renarquous 4ne le demi-ilécnstère n'est pas une mesure dn systinue métrique. 
empilage; ces facteurs varient avec les essences et arec la nature des bois empilés.

\begin{tabular}{|c|c|c|c|}
\hline ESSENCE. & NATLRE DES BOIS. & $\begin{array}{c}\text { Volume } \\
\text { en mètres cubes } \\
\text { du bois dans } \\
\text { le stère ou fac- } \\
\text { teur pour } \\
\text { passer du stère } \\
\text { au mètre cube. }\end{array}$ & $\begin{array}{c}\text { Facteur } \\
\text { pour passer } \\
\text { du mètre } \\
\text { cube au stère } \\
\text { ou facteur } \\
\text { d'empilage. }\end{array}$ \\
\hline $\begin{array}{c}\text { Sapin } \\
\text { Epicéa.. }\end{array}$ & $\begin{array}{l}\text { Bonne fente, écoree } \\
\text { unie (tige).......... } \\
\text { Fente difficile, écorce } \\
\text { raboteuse........... } \\
\text { Écorce très unic, fente } \\
\text { très bonne.......... } \\
\text { Ecorce raboteuse, fente } \\
\text { assez difficile........ } \\
\text { Rondin, écorce assez } \\
\text { unie............. } \\
\text { Rondin de cime, bran- } \\
\text { ches courbes....... } \\
\text { Écorce unie, fente fa- } \\
\text { cile................. } \\
\text { Ecorce raboteuse,fente } \\
\text { assez difficile........ } \\
\text { Cimeaux assez droits. } \\
\text { Branches courbes... }\end{array}$ & $\begin{array}{l}0.77 \\
0.63 \\
0.60 \\
0.88\end{array}$ & $\begin{array}{l}1.29 \\
1.34 \\
1.63 \\
1.72\end{array}$ \\
\hline
\end{tabular}

Un stère de bois ne renferme qu'un volume réel variant de $0^{\mathrm{mc}}, 46$ à $0^{\mathrm{mc}}, 77$ soit en moyenne $0^{\mathrm{me}}, 62 \%$ c'est-à-dire environ les six dixièmes.

En moyenne, le facteur d'empilaye varie de 1,3.j à 1,45 pour les bois droils : de 1,60 à 1,80 et quelquefois plus pour les bois tortus et noueux; il a élé constaté en outre que ce facteur dempilage augmente arec le nombre de búches susceptibles de constituer un slìre, et aussi en raison de la longueur des bûches; enfin qu il est plus fort pour les bois de quartier que pour les br is ronds. 


\section{NOTIONS GÉNÉRALES DESTIMATION DES BOIS.}

\$3. Fagots et bourrées (1). - Les dimensionsordinaires des fagots sont tris variables; leur longueur courante est le: $1^{\mathrm{m}}, 30 ; 1^{\mathrm{m}}, 33$ ou $1^{\mathrm{m}}, 40$; parfois de toutecelledeshrindilles; la longueur de tour est de $0^{\mathrm{m}}, 80,1 \mathrm{mithe}$ ou $1^{\mathrm{m}}, 33$.

Ces fagols sont retenus par un ou deux liens ou harts, et contiennent cing à huits brins dits parements ou jarrets de $0^{\mathrm{m}}, 06$ à $0^{\mathrm{m}}, 08$ de diamètre. Les dimensions des bourrées sont également variables, mais plus petites; leur longueur est de $0^{\mathrm{m}}, 80,1$ mètre ou $1^{\mathrm{m}}, 33$, la longueur de tour est de $0^{\mathrm{m}}, 50,0^{\mathrm{m}}, 80$ ou 1 mètre; elles ne renferment pas de parements, et ne sont retenues que par un lien.

Dans la pratique, on ne cube pas les fagots et les bourrées; on les estime au cent ou au mille, el les prix varienl suivant leurs dimensions el les régions.

Le volume en mètres cubes de la matière ligneuse réellement contenue dans les fagots a été détermininé par immersion dansl'eau ; il peut être intéressant à connaître. D’après M. Hüffel, le rendement de cent fagots de boisde moins de $0^{\mathrm{m}}, 20$ de tour serail exjrimé ainsi qüil suit:

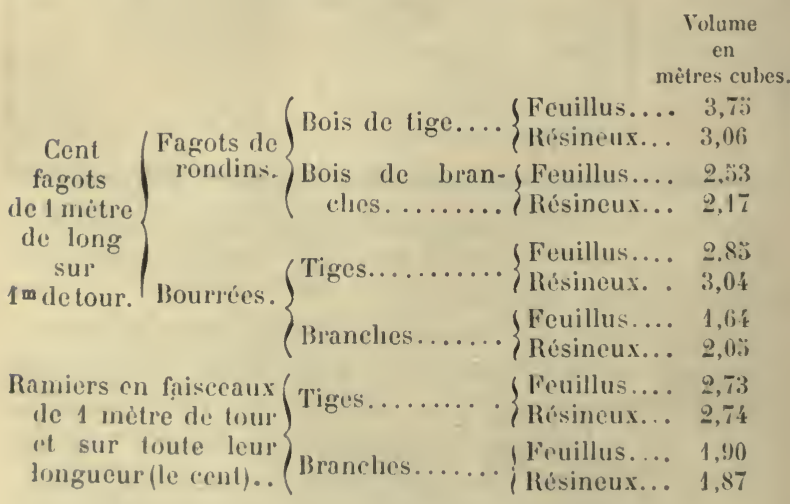

La Société forestièe de Franche-Comté al Belfort a

(1) I'nup's l'Ayenda du forestier. 
adopté comme base de ses expériences comparatives les facteurs moyens suivants pour des fagots et bourrées ayant $1^{\mathrm{m}}, 33$ de long et 1 mètre de tour:

3 mètres cubes pour 100 fagots pesant environ 2' kilos chacun ;

$1^{\mathrm{mc}},: 30$ pour 100 bourrées pesant environ 12 kilos chacun ;

Mesures de volume pour les bois de feu en France (1). Mesures de volume. Volume en stères.

Stère......................... 1,000

Double stère.....................

Décastère..................... 10,000

Corde des eaux et forcts 18 pieds de couche, 4 pieds de hauteur, 3 pieds $1 / 2$ de longueur). $\quad 3,839$ Corde de taillis (mêmes dimensions, sauf la longueur des bûches qui est de 2 pieds $1 / \mathcal{\imath})$. 2, 742 Corde de moule (mèmes mesures, longueur de bûches 4 pieds) $\ldots \ldots \ldots \ldots \ldots \ldots \ldots \ldots \ldots$ 4,387

Corde sur Eure................... \&,009

- sur l'Oise et l'Aisne............. . $\$ \mathbf{5 , 0 0 0}$

- sur la Marne et l'Oureq........... 4,008

- sur les ports de l'Yonne........... 4,007

- sur les ports de la Seine.......... 5,000

- sur le port de Montargis.......... \$ \$,003

Le Tonneau (Gironde)............. . 3,636

La Brasse (Gironde).............. $3,5,50$

\$. 4. Souches et rémanents. - La souche ou partie souterraine de l'arbre, donne des produits appréciables. Pour la plupart des essences, le volume de la partic souterraine est de 12 à $2 \%$ p. 100 (1/8 à 1/4) de la partie aérienne, lorsqu'on coupe rez terre.

Ces produits, ainsi que les copeaux d'abatage, les copeaux ou ételles d'équarrissage, menus débris ou rémanents d'exploitation, se rendent soit au stère, soit à la voiture, à la banne.

(1) D'après M. Frochot. 


\section{2. - Cubage des bois sur pied.}

Il y a lieu de distinguer le cubage des arbres de futaie et le cubage des bois taillis.

$\$ 1^{\mathrm{er}}$ Estimation en matière des arbres de futaie. Le cubage des arbres de futaie consisteà déterminer pour chaque arbre pris isolément: $1^{\circ}$ le volume de la tige; 20 le volume de la cime autrement dit du houppier.

$1^{\circ}$ Volume de la tige. - Les méthodes de cubage des arbres sur pied ont toutes pour objet principal la détermination des deux éléments du volume, hauteur de la lige el grosseur moyenne.

a. Nesure des huuteurs. - On apprécie la hauteur d'un arbre, ou plus exactement celle de la partie du trone propre à utiliser comme bois d'ceurre (1), soit à rue, soit à l'aide d'instruments spéciaux, dits dendromètres, planthelles, elc.

Mesure des hantenrs à vue d'œil. - Remarquons tout d'ahord que dans le calcul du volume, la hauteur' n'entre comme facteur qu'à la première puissance, et affecte beaucoup moins le résultat que le diamètre (ou la circonférence), qui y entre au carré; d'ailleurs il est inutile de chercherdans l'appréciation des hauteur's un degréd'exactitude que ne comporte pas la question.

Un xil exercé arrive facilement à apprécier les hauleurs à vue, arec une approximation d'un mètre au moins, sil a soin de contrôler de temps en temps son appréciation, soit à l'aide du dendromètre, soit à l'aide du pricédé suirant: dressant une perche de 2, 3 ou mieux f mètres le long de la lige à la hase de l'arhre, il se poste à une certaine distance de l'arbre, place devant ses yeux et rerticalement un crayon par exemple, qüil éloigne ou rapproche jusqu à ce que les rayons visuels passant

(1) Généralement partie du trone comprise entre te sol et le point où s'iusirent les premières branches principales. 
au-dessus et au-dessous du crayon aboutissent exactement aux deux extrémités de la perche; il élève ensuile le crayon dans la mème verticale, de façon à faire intercepter le long du tronc une hauteur égale, et ainsi de suite jusqu'au sommet. Le nombre des portées, mulliplié par la longueur de la perche appliquée contre l'arbre, donne la hauteur totale de la tige.

Pour diminuer les causes d'erreur, il y a lieu de s'éloigner d'autant plus de l'arbre que cet arbre est plus haut.

Ilesure des hauteurs au dendromètre, à la planchetle, etc. - II existedes instruments à l'aide desquels on peut mesurer exactement celle hauteur ; ces instruments appelés dendromètres , planchettes,etc.,

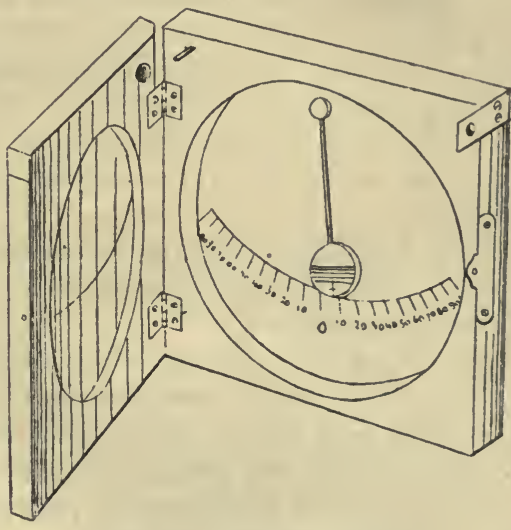

Fig. 55. - Clisimètre Goulier. sont très variés ; citons ceux de MIN. d'Arbois de Jubainville, Bayard, Bouvard, Regnault, etc. In des plus pratiques parmi ces dendromètres est le clisimètre Goulier, adapté à cet emploi par II. Bellieni, ingénieur opticien à Nancy (fig. 5\%).

b. Détermination d: la grosseur moyenne. - La seule mesure pratique dont on puisse disposer est celle qui est prise à la base de l'arbre; celle-ci, qui est toujours accessible, s'appelle grosseur à hautenr d'homme; elle se mesure à une hauteur au-dessus du sol variant entre $1^{\mathrm{m}}, 30$ ì $1^{\mathrm{m}}, \mathrm{50}$ et parfois 2 mètres, c'est-à-dire à un point assey. 
élevé pour que l'empâtement des racines ne s'y fasseplus sentir.

On se servira, suivant ses préférences, du diamètre ou de la circonférence (1).

Le diamètre à hauteur d'homme se mesure avec le compas forestier, comme nous l'avons vu précédemment; toutefois, dans des cubages précis, il est bon de mesurer deux diamètres en croix, l'un dans le sens de la plus forte épaisseur, l'autre dans celui de la plus faible, et de prendre la moyenne des deux lectures, spécialement quand la section de l'arbre est irrégulière.

La circonférence se mesure avec le décamc̀tre, avec une corde bien tressée qu'on reporte sur une règle graduée, ou encore avec le compas forestier gradué à cel effet.

En général, les diamètres s’estiment de z̈ en 5 centimètres el les circonférences de 20 en 20 ou mème de 23 en 2:j centimètres.

On conçoit aisément que si des tarifs spéciaux, élablis à l'aide de mesurages répétés, permettent de passer de la dimension de la tige à hauteur d'homme, à la dimension de cette tige en son milieu, le problème de cubage sera résolu; il en sera de mème si on peut passer directement de la donnée précédente au volume de l'arbre suivant sa hauteur. Dès lors on est conduit à élablir deux systèmes de tarifs :

a. - Tarif de decroissance des diamètres. - Leduc indique les facteurs suivants par lesquels il faut multiplier les diamètres à hauteur d'homme pour obtenir les diametres moyens:

Arbres de 3 à 5 mètres de hauteur. Multiplier le diamètre à hauteur d'homme par.............. 0,933 Arbres de 6 a 8 métres de hauteur. Multiplier le diamètre à hauteur d'homme par............. 0,90 -

(1) Le diametre est une dimension qui parte plus ì l'ail; nous le préféro a re titre. 
Arbres de 9 à 11 mètres de hauteur. Multiplier le diamètre à hauteur d'homme par.............. 0,880 Arbres de 12 à 1 '́ mètres de hauteur. Multiplier le diamètre à hauteur d'homme par.............. 0,833 Arbres de 15 à 17 mètres de hauteur. Multiplier le diamètre à hauteur d'homme par.............. 0,827 Arbres de 18 à 20 mètres de hauteur. Multiplier le diamètre à hauteur d'homme par.............. 0,800

Remarquons que les décroissances varient avec la hauteur des arbres; mais elles varient aussi suivant leur grosseur, et enfin d'essence à essence, de sorte que, dans une coupe renfermant des arbres de toutes grosseurs et hauteurs, adopter un facteur unique de décroissance n'est ni facile ni prudent.

b. - Turifs de cubage. - En France, on a l'habitude de se servir de tarifs spéciaux, donnant directement le volume pour les diverses catégories de grosseurs mesurées à hauteur d'homme; le mode d'usage de ces tarifs n'a pas besoin d'explications.

Le nombre de ces tarifs est incalculable; souvent chaque forèt a son tarif.

Nous renvoyons à ceux que donne l'Agenda duforestier publié sous le patronage de la Société forestière de FrancheComté et Belfort (1).

$2^{\circ}$ Cubage du houppier. - Le houppier est constitué par les branches, rameaux et ramilles qui forment la cime de l'arbre; les produits que donnent ce houppier ne consistent qu'en bois de chauffage ; on évalue leur volume, en comptant le nombre de stères que produisent ces branches façonnées comme bois de feu, ainsi que le nombre de fagots qui proviennent des produits impropres à l'empilage.

Dans la pratique, on a déterminé pour chaque classe d'arbres la valeur du houppier en fonction du volume du tronc, et on se contente dans une opération de cubage,

(1) Librairie P. Jacquin, Besançon (Doubs). 
a près avoir évalué le volume du trone, de calculer direclement celui du houppier à l'aide de ces coefficienls (1).

Pratique de l'opération du cubage; estimation en matière d'un lot d'arbres de futaie. - L'opération de* cubage d'un groupe d'arbres consiste à mesurer la grosseur de chaque arbre à hauteur d'homme, à en évaluer la hauteur en bois d'oeurre et à en déterminer le rolume.

Mais en pralique, lorsquion efleclue celle opération sur une coupe ou parcelle de forèt, on cherche à coorlonner l'opération; pour cela l'agent pointeur a préparéà l'a vance un carnet à deux entrées, lui permetlant de pointer les arbres, au fur et ànıesure de leur appellation,en les classant suivant leur diamètre d'une part, el leur hauteur de l'autre, et souvent aussi en les répartissant par classes d'essences.

L'opération finie, il faut relever les résultats; généralement, et dans un but de simplification, on donne comme hauteur aux arbres d'une mème catégorie, la moyenne les diverses hauteurs appelées pour ces arbres; et l'on n’a plus qu'un seul calcul à faire par catégorie de circonllérence ou de diamètre.

Les diverses calégories de grosseurs sont groupées ją classes, selon l'usage adopté dans la région. Entin on délerminedans une colonne spéciale le volume des houppiers en fonction du volume des tiges, et généralement l'appréciation du houppier se fait en bloc sur le volume total des tiges.

Dans certaines forèts particulières, il est d'usagre de numéroter chacun des arbres mis en vente et de prendre note des dimensions de clrapue arbre; les calepins de pointage doivent être lisposés en conséyuence $(2)$.

§. Estimation en matiere des taillis. - On esline le rendement en matière d'un taillis à vue d'oeil; c'est

(1) Voir Agenda du forestier, " tarifs divers ".

(2) Ia librairie Radenez à Montdidier (Somme), met en veute des calepins disposés pour ces opérations. 
ESTIMATION EN MATIERE.

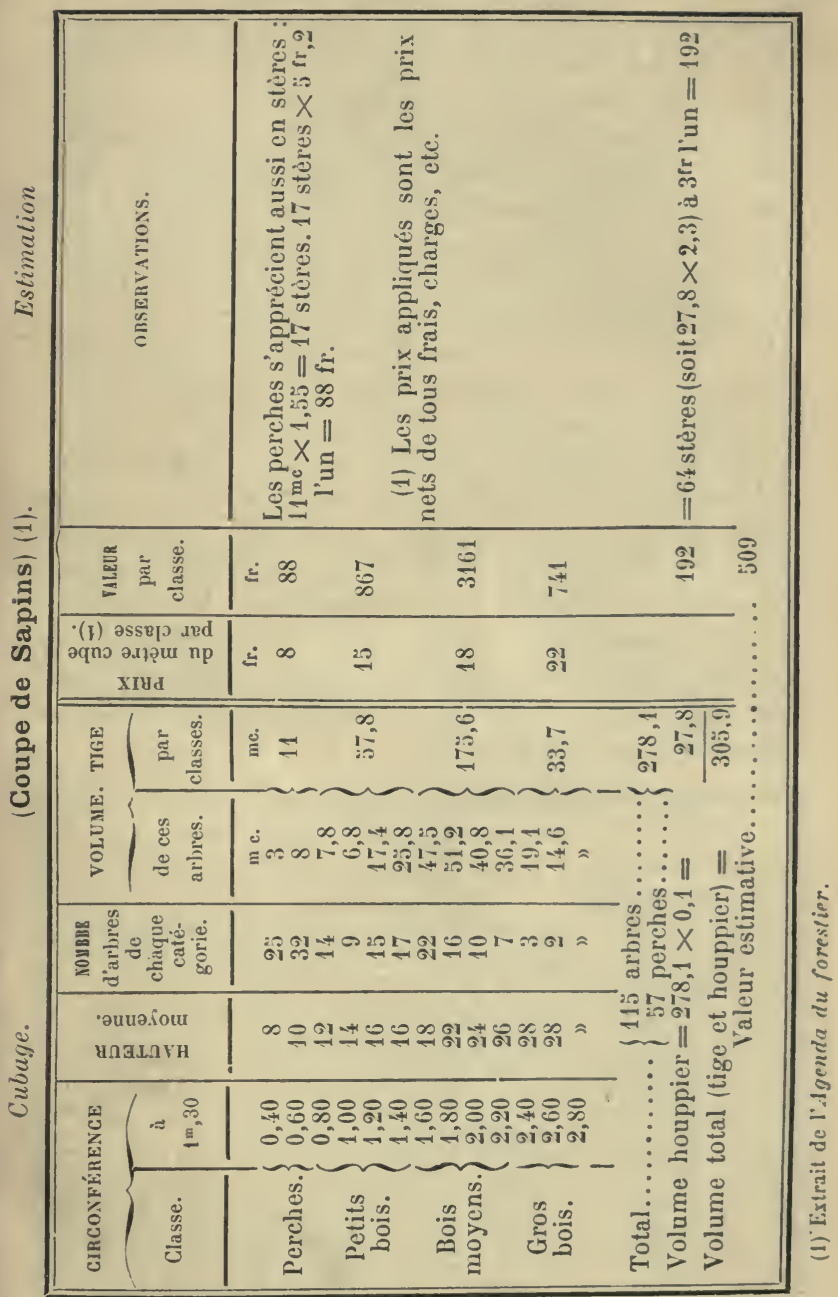

A. Frox. - Sylviculture. 


\section{NOTIONS GÉNÉRALES D'ESTIMATION DES BOIS.}

affaire d'habitude; un opérateur se forme aux estimations s'il se donne la peine de vérifier au cours de l'exploitation, la quantité de stères, de fagots ou de bourrées que fournit le peuplement qu'il a estimé sur pied; en comparant ces résultats à son estimation, il rectifie très facilement ses crreurs d'appréciation. Un bon préposé doit agir ainsi; il doit relever sur un calepin tous les résultats des exploitations qu'il surveille afin d'ètre à mème de fournir sur elles tous les renseignements nécessaires.

Un estimateur peu exercé peut s'aider en faisant des expériences sur des places d'essai ; à cet effet il choisit des emplacements, d'un are par exemple, dans les parties du peuplement présentant une composition moyenne (il peut faire des essais dans les niauvaises parties, dans les moyennes et dans les honnes, pour comparer les résultats) ; sur chaque place d'essai il compte les tiges, mesure leur grosseur à hauteur d'homme, apprécie leur hauteur, se rend compte de la consistance du taillis puis fait tout façonner devant lui en stères, fagots et bourrées, suivant l'usage courant. Quelques essais de ce genre forment rapidement un estimateur.

\begin{tabular}{|c|c|c|c|c|c|c|c|c|}
\hline \multirow{2}{*}{$\begin{array}{l}\text { CIRCoNF́́rexces } \\
\text { moyennes des tiges. }\end{array}$} & \multicolumn{8}{|c|}{$\begin{array}{l}\text { NOMBRE DE TIGES NéCESSAIRES POUn } \\
\text { faire un stère, la hauteur moyenne étant de }\end{array}$} \\
\hline & $4^{m}$ & $5^{m}$ & $6^{m}$ & $7^{m}$ & $8^{m}$ & $9_{\mathrm{in}}$ & $10^{\mathrm{m}}$ & $11 \mathrm{~m}$ \\
\hline $0^{\mathrm{m}}, 20$ & ร0 & 40 & 34 & 29 & $2: i$ & 23 & ” & ) \\
\hline $0 \mathrm{~m}, 21$ & 47 & 38 & 31 & 27 & 24 & 21 & ” & $"$ \\
\hline $0 \mathrm{~m}, 22$ & 43 & $3 i$ & 29 & 23 & 22 & 19 & $"$ & " \\
\hline $0^{\mathrm{m}}, 23$ & 40 & 32 & 27 & 23 & 20 & 18 & " & ” \\
\hline $0^{\mathrm{m}}, 24$ & 37 & 30 & 23 & 21 & 19 & 17 & " & $"$ \\
\hline $0^{\mathrm{m}}, 2 \mathrm{j}$ & $3 t$ & 28 & 23 & 20 & 17 & 16 & $"$ & 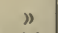 \\
\hline $0 \mathrm{~m}, 26$ & 32 & 26 & 22 & 19 & 16 & 15 & 13 & 11 \\
\hline $0 \mathrm{~m}, 27$ & 30 & $2 !$ & 20 & 17 & 13 & 14 & 12 & 11 \\
\hline $0 \mathrm{~m}, 28$ & 28 & 23 & 19 & 16 & 14 & 13 & 12 & 10 \\
\hline $0 m, 29$ & 26 & 21 & 18 & 15 & 13 & 12 & 11 & 10 \\
\hline $0 \mathrm{~m}, 30$ & פ̇ं & 20 & 17 & 14 & 13 & 11 & 10 & 9 \\
\hline
\end{tabular}


M. Frochot donne dans le tableau précédent des chiffres d'expérience à l'aide desquels on peut s'aider pour lívaluation d'un matériel sur pied; pour utiliser ces données il suffira de rendre compte aussi approximativement que possible du nombre de tiges, de leur grosseur, et de leur hauteur moyenne par hectare dans chaque catégorie.

Quant aux fagots, on peut en déterminer le nombre d’après la quantilé de stères el admeltre qüil y en aura de 8 à 1. par stère, suivant que le taillis sera plus ou moins jeune et plus ou moins fourré.

Pratique de l'estimation en matière des taillis. - On se sert des virées ou bandes de peuplement de 13 à 20 mètres de largeur faites pour le balivage; autant que possible ces virées sont dirigées dans le sens de la plus grande longueur de la coupe; on cherche à leur donner une forme régulière, et des contenances sensiblement. égales.

L'estimateur les parcourt une à une dans tous les sens, examine attentivement le taillis, sa composition, les essences qui y dominent, la grosseur et la hauteur des bois, la quantité de tiges qu'on peut compter en moyenne sur un are, l'élat de la végétation, la quantité de cépées, etc. ; après avoir ainsi étudié cette virée, il inscrit sur le carnet les résultals de son examen, et il estime le rendement à l'hectare du taillis de cette virée. Il opère de mème pour les autres virées; pour obtenir le protuil total de la coupe, il totalise les produits partiels de chaque virée.

Dans la pratique, et spécialement quand le taillis est assez homogìne dans toutes ses parties, il se contente de comparer les rendements à l'hectare qu il a adoptés pour chaque virée arec les contenances de ces virées et il délermine pour l'ensemble du taillis un rendement moyen à l'hectare; ce chiffre, multiplié par la contenance totale de la coupe, donne l'estimation en matière de cette coupe.

I'roduits spéciaux. - Lorsque le peuplement est sus- 
5'ł ' NOTIONS GÉNÉRALES D'ESTIMATION DES BOIS.

ceptible de donner des produits spéciaux, dont on doit tenir compte dans l'estimation (bois d'échalas) elc., on rapporte les produits spéciaux au stère, ou bien on se forme à l'estimation directe par des expériences et des places d'essai.

Production en écor'ce. - La production en écorce des taillis s'estime à vue, par comparaison avec les rendements des coupes précédentes; d'après M. Bouvard, pour obtenir 1000 kilogrammes d'écorce sèche, il faut écorcer en moyenne, dans les régions du nord :

21 stères de taillis de 12 ans, soit un rendement en poids de... $47 \mathrm{~kg}, 6$ par stère.

19 stères de taillis de 20 ans.

soit un rendement en poids de... $\ddot{2} 2^{\mathrm{kg}}, 6$ par stère.

18 stères de taillis de 25 ans,

soit un rendement en poids de... $\check{3} \ddot{\mathrm{kg}}, \ddot{\mathrm{k}}$ par stère.

17 stères de taillis de. 30 ans,

soit un rendement en poids de.... $58 \mathrm{~kg}, 8$ par stère.

Dans le midi, le rendement serait plus éleré, notamment en ce qui concerne les taillis de chène tauzin, où l'on peut extraire jusqu'à 120 à 12 kilogrammes d'écorce par stère, le poids de ce stère étant d'environ 500 kilogrammes.

J)ans l'estimation en matière d'un taillis, on apprécie le rendement d'écorce à l'hectare au poids; les ventes d'écorce s'effectuent soit par 1000 kilogrammes d'écorce. siche, soit par 100 boltes de 20 kilogrammes.

Dans l'estimation des taillis à écorce il y a lieu de tenir comple que le prélèvement de l'écorce réduit le rendement en stères du taillis calculé pour des bois non écorcés, le 8 à 12 \%. 100 environ.

\section{II. - ESTIMATION EN ARGENT.}

Lestimation en argent consiste à appliquer à chaque catégorie de marchandises un prix déterminé. 
$1^{\circ}$ Bois abattus et façonnés. - Les mercuriales donnent généralement les prix des diverses unités de marchandises rendues en gare, ou dans deslieux de dépòt hors forèt; or il est en général plus commode pour le propriétaire de livrer les produits en forèt, afin de ne pas avoir à se préoccuper de faire effectuer les transports.

Il y a là une distinction pour le choix du prix de chaque unité dont il faut tenir compte. Si le transport est difficile, le prix à appliquer en forèt sur le parterre de la coupe doit être inférieur à celui des mercuriales; la différence représente les frais de transport.

Les prix du commerce courant s’entendent généralement, le produit étant lirré (ou supposé livré) façonné sur le parterre de la coupe. Nous appellerons ce prix de l'unité façonnée sur le parterre de la coupe, le prix normal.

Pour estimer en argent une coupe vendue dans ces conditions, on applique à chacune des catégories de produits que comporte le dénombrement fait après façonnage, le prix normal correspondant.

$2^{\circ}$ Bois sur pied. - Il est d'usage lorsqu'on estime des bois sur pied de leur appliquer les prix normaux. On obtient ainsi l'estimation du matériel supposé façonné sur le parterre de la coupe.

Il reste, après ces calculs, à en défalquer tous les frais, de quelque nature qu'ils soient, qui sont nécessaires pour faire passer les bois de l'étal sur pied à l’élat façonné sur le parterre de la coupe. Cette différence représente la valeur nette de la coupe sur pied, c'est-à-dire le prix qu'on pourra en offrir pour s’en rendre acquéreur ou qui pourra servir de mise à prix si l'on est vendeur (1).

Le devis détaillé de tous ces éléments peut sétablir ainsi quil suit :

(1) Il ne faut pas oublier que l'intermédiaire retiendra un lant p. 100 qui représente le bénéfice de son travail, et qu'il y a lieu d'en tenir compte. 
$\$ 46$ NOTIONS GÉNÉRALES D'ESTIMATION DES BOIS.

10 Estimation du matériel brul sur le varterre de la coupe. fr. c.

fr. c.

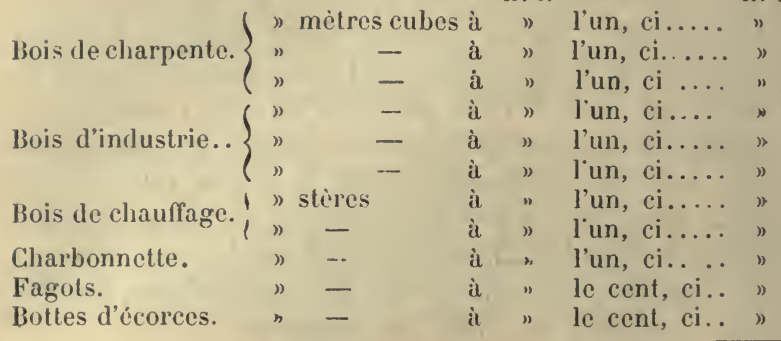

Total de l'estimation brute........... "

20 A déduire.

10 Bénéfice de l'exploitant à raison de » p. 100 de l'estimation ci-dessus................ "

2o Frais de surveillance de l'exploitation, frais de déplacements, etc................ »

$3^{\circ}$ Frais d'exploitation proprement dite :

Ébranchement des arbres à " fr. "lü, ci..... "

Abattage des arbres de futaie à " fr. " l'un, ci.. "

Abattage et façon du bois de chauffage, à » fr. »

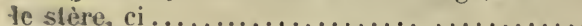

Abattage et fason du bois à charbon à " fr. " ci..

Façon des fagols a $\gg$ fr. le cent, ci......... "

- des écorces à $)$ fr. "les cent boltes, ci...

$4^{\circ}$ Travaux divers à la charge de l'exploitant, amćlioration et entretien de chemins, etc., ci. "

Reste................

5० 3 p. 100 de ce reste à déduire pour frais d'enregistrement et d'adjudication, ci.

Reste net.............

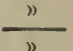




\section{TROISIEME SEGTION}

\section{Valeur de la propriété boisée.}

\section{I. - VALEUR COMMERCIALE DES FORÊTS OU VALEUR DE REALISATION.}

La valeur commerciale des forèts, ou valeur de réalisation est déterminée par celle des éléments qui composent le domaine boisé, dont les deux principaux sont le sol et les bois sur pied, et par la valeur commerciale de ces éléments au cours du jour et de la localité.

Ce procédé d'estimation, dit des marchands de bois, est appliqué par tous les marchands de bois et les spéculateurs de biens fonciers; il consiste, dit M. Puton, à compter, mesurer et cuber toutes les tiges, à en distribuer le volume par essences en marchandises les plus usuelles, à leur appliquer les prix nets du moment et de la localité et à estimer le sol par l'emploi dont il est susceptible à raison du prix des lerres d'égale qualité dans les environs.

Cette évaluation directe présente évidemment des difficultés; il faut compter et apprécier tous les arbres de la forèt, les estimer en matière puis en argent, estimer les taillis, etc.; et lintéressé est arrèté par la crainte de procéder à des dénombrements considérables et coûteux. Cependant lopéralion est nécessaire.

On ne songerait pas à vendre ni à acquérir une coupe à exploiter, si petite qu'elle soit, sans en aroir soigneusement estimé tous les produits; et on voudrait vendre ou acheter une forèt tout entière sans s'embarrasser de 
ce souci! Aussi voit-on souvent une opération de ce genre donner bien des mécomptes, les affiches relatives aux ventes de forèts n'en font connaître généralement que la situation et la contenance; parfois elles indiquent en outre le revenu des dernières années, ou bien on s'en informe chez le vendeur; puis, comme il est évident que le revenu perçu jusqualors peut ne pas ètre conforme à la production de la forèt, on fait soi-mème ou on fait opérer une reconnaissance de la forèt pour voir si, $\dot{a}$ l'aspect, elle semble être toujours en bon élat. Mais ce n'est hien sourent que d'une reconnaissance sommaire dont il s'agit, et on ne procède pas à l'estimation absolue; c'est sur cette base insuffisante que se discule le prix.

Profitant de cet état de choses, d'habiles acquéreurs de hiens boisés, ont pu acheter ainsi un domaine, réaliser en bloc, en quelques années, tout le matériel sur pied, et rentrer immédiatemeut par celle simple opération dans le montant de leurs déboursés, faire mème des bénéfices dans celle opération et se trouver, par-dessus le marché, propriétaires du terrain en surplus; à moins toutefois que l'acheteur, aussi inexpérimenté que le vendeur, n`ait, lui aussi, lenté celle opération hasardeuse sans renseignements suffisants, et ne se trouve, après exploilation, en déficit sur ses prévisions el propriélaire d'un sol maladroitement ruiné et presque improductif.

Pour agrir avec sécurité dans une évaluation de forèl, faite dans le but d'apprécier celle valeur vis-à-vis de lout acquéreur possible, la première estimation à faire est l'estimation commerciale, estimation brute ou absolue, basée non sur le parti qu'on en tire actuellement, ni sur celui qu'on pourrail en tirer uniquement comme forit, mais sur le meilleur parti qu'un acquéreur éventuel quelconque peut en lirer soil comme bois, soit autreinent (1).

(1) Bien souvent, on n'agit pas autrement dans l'évaluation d'un immeuble rural, quel que soit to capital qu'ait pu engager le propriétaire dans cet 
Application de la méthode. - a. Division en parcelles. - Pour faciliter l'opération matérielle, si la forèt a une certaine étendue, elle est divisée en parcelles. Pour cela, il faut d'abord se procurer un plan du domaine boisé : un calque du cadastre, relevé à la mairie du lieu de la situation desbois, peut suffire à la rigueur; ce plan, dont nous conseillons alors de ne se servir qu'à titre de renseignement, servira à établir la contenance approximative du domaine boisé, en totalisant la surface des parcelles cadastrales dont il se compose ; il donnera une première indication sur les limites ou les propriétés riveraines.

Prenant comme point de départ un repère fixe, facile à déterminer sur le terrain, on divisera la forèt en un certain nombre de parties dans chacune desquelles le terrain sera de mème nature et de mème qualité. Cette première subdivision parait sulfisante pour une simple estimation; mais, dans le but de faciliter des opérations ultérieures daliénation partielle, de partage, de morcellement ou toute autre opération de ce genre, et aussi de rendre plus rapide le comptage et l'estimation du matériel, on subdivisera chacune de ces parties en parcelles renfermant des peuplements de mème nature, c'est-à-dire provenant d'une même exploitation et ayant ainsi approximativement les mèmes dimensions (ou les mèmes âges).

Chacune de ces parcelles sera délimitée sur le terrain par un filet spécial ; elle ne devra pas avoir une trop trande étendue ; les sinuosités du périmètre en seront aussi simplifiées que possible; leur contenance en sera soigneu-

immeuble. Que de construclions ou de dépenses faites avec luxe sur une propriété rurale, et représentant un capital considérable incorporé à l'inmeuble, ne peuvent donner au domaine, en cas de vente, qu'une majoration de valeur insignifiante, parce qu'elles ne représentent pas pour l'acquéreur possible une utilisation directe et par suite une valeur corrspondant à ce capital engagé. 
sement arpentée et leur figure sera exactement reportée sur le plan d'ensemble (1).

b. Comptages. - Estimation de claque parcelle. Cliaque parcelle est alors estimée isolément de la manière suivante :

Toutes les tiges qui n'ont pas une trop faible dimension pour être vendables doivent ètre comptées; le travail s'effectue par virées; les tiges sont griffées au fur et à mesure du passage des gardes, et l'opérateur note sur un calepin préparé à cet effet la hauteur et la dimension (diamìtre ou circonférence) de chaque tige, en les répartissant par essences et en les groupant par catégories de diamètre d'une part et de hauteur de lautre.

Le taillis est estimé en stères, fagots el bourrées, par places d'essai quon fail faconner sur place ou à rue.

c. Opération au bureau. - Cubage et estimation des produits. - L'agent estimateur effectue les cubages, distribue les volumes jar essences en marchandisesles plus usuelles, et procède à l'estimation en argent, comme il a été dit pour les bois sur pied.

Il y a soin de faire subir à celle estimation les déductions nécessaires, tient compte du légilime profit de l'exploilant, de la durée probable de sa réalisalion et des pertes d'intérèt qui en sont la conséquence, enfin de la bonifiration quon fait en toules circonstances à l'acheleur en gros, bonification toujours plus forte que celle consentieà l'acheteur en détail.

Le résultat obtenu est la valeur nelte du matériel sur pied vendable.

d. Tiges mon vendables. - La jeunesse, les recrus et les semis n'ayant pas encore alteint une dimension

(1) Si la forèt est aménagée, la division en parcelles est toute faite; à moins qu'il $n^{\prime} y$ ait encore lieu de subdiviser ces parties en raison de la qualité el de la nature du sol. 
suffisante pour ètre vendables, sont négligés et il y a lieu de ne leur attribuer aucune valeur (1).

e. Sol. - Quant au sol, il est estimé d'après les ventes de terres de même qualité dans les environs, défalcation faite, s'il y a lieu, de certains frais de mise en état.

La somme de ces deux valeurs, valeur du sol, valeur de la superficie telle que nous l'avons déterminée, donne l'estimation absolue ou valeur commerciale du domaine boisé.

\section{II. - VALEURS RELATIVES DES FORÊTS.}

Dans la détermination de la valeur commerciale, nous n'avons pas tenu compte des éléments non rendables, et par suite sans valeur commerciale courante, renfermés soit dans le sol (état spécial du sol forestier, ensouchement, semis), soit dans la superficie (bois jeunes en croissance, jeunes plants, rejets, drageons, etc., qu'on ne peut ni exploiter ni façonner). Nous avons tenu compte des produits moins jeunes en les façonnant immédiatement, leur donnant ainsi une valeur immédiate de commerce et négligeant leur valeur d'avenir.

Tout cela représente, pour le propriétaire ou l’acquéreur qui veut demander au domaine boisé de continuer à fonctionner comme forèt, une valeur indiscutable, une somme de travail ou d’épargne accumulée sur la propriété, une faculté de production toute spéciale, susceptible de modifier pour lui, et pour lui seul, en raison mème du

(1) L'estimation des bois en croissance, réduite à cette limite des tiges non vendables devient, d'après M. Puton, une quantité en quelque sorte négligeable, dans l'evaluation commerciale d'un immeuble boisé; la négliger, c'est rester dans les tolérances de toute estimation, c'est-à-dire dans les limites de l'aléa qui accompagne tout achat ou toute vente.

Tel n'est pas l'avis d'autres auteur's forestiers faisant autorité comme II. Puton en matière d'estimation forestière. Les divergences d'opinions de's maftres en sylviculture prouvent sinılement, qu'en matière d'estimation forestière, il n'est pas de règle parfaite ni absolue. Dans bien des cas, tout dépend des circonstances et du point de rue auquel on se place. 
but quil se propose, l'estimation de la forèt en fonds et superficie.

Dans ce cas particulier, le domaine boise a de nouvelles valeurs, valeur's relatives à son degré d'utilisation.

Si le taux d'accroissement de valeur des peuplements avec l'âge était uniforme; si, d'autre part, la récolte du produit bois ne pouvail se faire qu'à un âge donné où le produit serait mûr (comme en agriculture), n'étant pas utilisable avant cet âge, devenant inutilisable après cet àge de maturité, le calcul de la valeur relative d'une forèl ne serait pas susceptible de plusieurs solutions.

Mais, en réalité, il n'en est pas ainsi, et le problème de l'estimation relative des forèts devient très complexe.

Il peut consister à chercher quelle est la valeur de la forế, si on suppose qu'elle est destinée à rester à l'état de forêt; qu'on ne modifiera ni le régime, ni le mode de traitement; enfin qu'on maintiendra le terme d'exploitabilité fixé par les anciennes exploitations.

La forèt a, pour celui qui pose ces conditioñs, une valeur toute spéciale que nous pouvons appeler valeur d'avenir, car elle est relative aux produits que donnera la forèt dans l'arenir, en fonctionnant sur des données connues.

II peut cousister aussi à chercher quelle est la valeur de la forêt, si on suppose qu'elle est destinée $\dot{a}$ rester a l'état de forét; quion se propose, s'il y a lieu, de modifier le régime ou simplement le mode de traitement; enfin qu'on se propose d'exploiter à l'àge le plus acantugeux.

La forêt a, pour celui qui se pose ces conditions, une nouvelle valeur que nous pourons appeler valeur de lacement, car elle est relative ici au but cherché qui est un placement de fonds.

Méthode de M. Puton. - Dans celle recherche de la valeur relative d'une forêt, M. Puton se place au point 
de vue suivant: combien vaut une propriété boisée, pour un capitaliste qui veut faire emploi de ses fonds à un taux déterminé, en exploitant cette forèt à un âge également déterminé. Il ajoute que ce capilaliste sait que s'il réussit à acheter cette forèt au prix C, en l'exploitant à l'àge $\mathrm{A}$, son argent fonctionnera au taux qu'il s'est fixé (1); que, s'il peut exploiter à un âge inférieur à $\mathrm{A}$, son capital sera placé à un taux différent.

Pour résoudre la question, il établit les formules générales suivantes :

$1^{\circ}$ Pour les exploitations périodiques:

$$
\frac{\mathrm{X}=\mathrm{R}(1+t)^{m}}{(1+t)^{n}-1}
$$

relation dans laquelle $X$ est la valeur cherchée de la forêt à l'époque $m$; R, le revenu périodique donné par, la forèt toutes les $n$ années, âge quil se donne; $t$, le taux de placement qu'il demande à son placement.

20 Pour les forêts aménagées:

$$
\mathrm{X}=\mathrm{R} \times \frac{1}{t}^{-}
$$

relation dans laquelle $\mathrm{X}$ est la valeur cherchée de la forêt, $\mathrm{R}$ le revenu net de la forèt si elle est normalement constituée, ou, dans le cas contraire, le revenu d'une forèt équivalente mais normalement constituéc dont il détermine la constitution, et $t$ le taux de placement.

Toute lapplication de la méthode consiste dans l'appréciation la plus exacte possible dans chaque cas particulier du revenu $R$, base de son estimation relaive.

(1) Ce taux doit être évidemment dans les limites qui peuvent convenir a un placement en forèt; en général, il doil avoir comme maxinum \& it 5 p. 100. 
La détermination de ce revenu $\mathrm{R}$, simple dans certains cas, est souvent très délicate dans la pratique.

Méthode de M. Broilliard. - M. Broilliard présente autrement la question: il suppose connu le taux de placement (taux des placements en forêts similaires dans la localité); il suppose connu le rendement en matière de la forèt aux différents âges (raleurs déduites des faits constatés, soit dans la forèt même, soit dans d'autres forèts semblables). De ces deux faits constatés, il déduit l'àge d'exploitation le plus avantageux. Enfin, à l'aide des calculs dintérèts composés, il détermine la valeur de la forèt, en la supposant exploitée à cet âge le plus avantageux (1).

Toutefois cette méthode comporte des restrictions, ef nous devons mentionner les différents cas qu'envisage cet auteur :

$1^{\circ}$ Pour le taillis simple ou la futaie régulière. - II admet qu'on détermine tout d'abord le taux de placements en forèts similaires dans la localité, et la base de sa méthode est d'estimer chacune des coupes séparément. II calcule l'âge auquel il serait le plus avantageux d'exploiter; pour cela, il détermine la valeur à l'hectare des coupes aux différents âges, en déduisant ces valeurs des faits constatés soit dans la forèt mème, soit dans d'autres forèts semblables d'après le façonnage de: produits.

Par un simple calculd'intérèts composés, connaissant le taux, il cherche quel estle revenu le plus avantageux (2); celui-ci lui montre l'âge de la coupe exploitable, soit $n$, par suite la récolte $\mathrm{A}$ que donnera celte coupe.

Sol. - Il estime le sol, comme capital susceptible de

(1) Pour l'application de cette méthode, nous conseillons vivement de se reporter à la nouvelle édition du "Traitement des bois en France ", do M. Broilliard. Ia notoriété de l'auteur suflit à justifier ce conseil.

(2) Le revenu le plus avantageux est celui qui se rapporte au capital correspondant le plus élevé, capital susceptible de douner le reveu aunuel le: plus élevé. 
donner toutes les $n$ années, au taux $t$ connu, une récolteA. Soil C ce capital; il est donné par la formule

$$
\mathrm{C}=\mathrm{A} \times \frac{1}{(1+t)^{n}-1}
$$

dans laquelle le facteur

$$
\frac{1}{(1+t)^{n}-1}
$$

peut se calculer très rapidement à l'aide du tarif III de Cotta (1).

Il estime au mème taux $t$ le capital correspondant aux frais annuels $a$; ce capital $c$ est donné par la formule.

$$
c=a \frac{100}{t}
$$

La différence de ces deux valeurs $C-c$ lui donne la valeur nette du sol.

Superficie. - La superficie, à un âge quelconque donné, est considérée comme la valeur acquise par. l'accumulation à intérèts composés du capital C.

Si la coupe a lieu à $m$ ans, le capital a été engagé pendant $m$ années, et la superficie est donnée par la formule :

$$
\mathrm{S}=\mathrm{C}\left[(1+t)^{m}-1\right]
$$

dans laquelle le facteur $(1+t)^{\mathrm{m}}$ se calcule très rapidement à l'aide du tarif I de Cotta (1).

La valeur à l'hectare en fonds et superficie est donnée par le total $(\mathrm{C}-c)+\mathrm{S}$, c'est-à-dire par la somme de la valeur nette du sol et de la superficie.

Pour obtenir la valeur de la coupe, il suffit de multiplier par la contenance de cette coupe.

(1) Ce tarif est dans l'Agenda du forestier. 
Enfin, toutes les coupes s'estiment de mème et la valeur de la forèt est la somme des résultats oblenus.

Remarquons que, par un calcul de mème ordre, il serait facile de tenir compte des produits accessoires à récoller avant l'exploitation principale, ainsi que des dépenses à faire, soit à un moment donné, soit périodiquement.

$2^{\circ}$ Pour le taillis composé. - M. Broilliard admet qu’on détermine le taux de placement en forèts similaires dans la localité. Comme dans le cas précédent, il cherche à estimer chacune des coupes séparément, et le point de départ de sa méthode est que la valeur en fonds et superficie doit se déduire des revenus successivement réalisables, en ayant soin de bien connaître les époques auxquelles on réalisera ces revenus, et de les escompter à l'époque où se fait l'estimation.

Il admet, bien entendu, qu'il connaît, d’après l'état de la forèt, le rendement en argent de chaque coupe, à l'expiration des périodes successives où elles seront exploitables.

Notons enfin que M. Broilliard indique comme procédé usuel le procédé empirique suivant:

Pour estimer les taillis composis, on se borne souvent $a$ calculer la valeur venale de tous les arbresfaits, modernes et anciens, et à y ajouter, pour les sous-bois et les baliceaux de l'äge d'une part, pour le sol d'autre part, des valeur's deduites du revenu de la forêt traitée en taillis simple avec baliveaux de l'âge seulement.

Pour un hectare de taillis composé portant un sousbois âgé de qualorze ans qui sera exploitable à trente ans, le taux de placements en forì étant dailleurs 3 p. 100 dans la localité, M. Broilliard donne l'estimation suivante : 
Arbres constitués (de $0 \mathrm{~m}, 20$ de diamètre et plus). Sol capable de donner tous les 30 ans $700 \mathrm{fr}$., savoir $600 \mathrm{fr}$, de sous-bois et $100 \mathrm{fr}$. de petits modernes :

Valeur brute $700 \frac{1}{(1+0,03)^{20}-1} \ldots \ldots \ldots \ldots .490,42$

Frais capitalisés à déduire........... 200,00

Recru de 14 ans et baliveaux.

Valeur nette........ $\overline{290,42} 290,42$ $490,40\left[(1+0,03)^{14}-1\right]$ c'est-ì-dire intérêts du capital sol et frais pendant 14 ans..........

Valeur à l'hectare....... . $\frac{206,00}{1063,00}$

La valeur ainsi obtenue par ce procédé simplifié donne un chiffre inférieur à la valeur réelle.

$3^{\circ}$ Sapinière jardinée. - Dans ce cas, la méthode de M. Broilliard est la méthode commerciale. Une chose est certaine, dit-il, c'est la valeur actuelle des bois sur pied, grénéralement grande dans ces forèts. On peut la calculer en comptant et mesurant de 5 en 5 centimètres, par exemple, les diamètres de tous les arbres, et en appliquant à chaque calégorie de grosseur les prix du commerce. Si l'on ajoute à cette valeur des arbres ct perches, la valeur relativement faible du sol, simplement estimée par comparaison avec les terrains similaires du voisinage (1), on obtient une base destimation convenable.

Mais la forèt vaut, suivant les cas, plus ou moins que cette somme; si le matériel sur pied est très considérable et en grande partie exploitable, la forèt vaut moins que ne l'indique le chiffre de l'estimation en valeurs actuelles pour un capitatiste qui reut la conserver à lélat de forìt; si elle est à vendre, ce sont les marchands de bois qui l'achèteront.

(1) Soit, par exemple, 200 francs, et s'il y a lieu de tenir compte des semis qu'il porte, on peut y ajouter la valeur d'un reboisement effectué depuis quelques années, soit encore 200 francs. Le sol avec les semis vaut alors environ 400 francs l'hectare. 
Si, au contraire, les bois sont jeunes et en bon état de végétation, si ce sont principalement des perches par exemple, c'est le capitaliste, qui a l'intention de fairt un placement d'argent en forèt, qui sera l'acquéreurprobable, car la forèt vaut pour lui plus que ne lintlique le prix actuel du matériel sur pied.

Méthode de M. Galmiche. - Enfin M. Galmiche, s'occupant plus spécialement du taillis composé, envisage autrement la question.

II écarte d'abord toutes les consilérations de conve. nance personnelle qui pèsent d'un grand poids dans la plupart des marchés relatifs aux forèts, mais ne peurent ètre évalués que par l'acheteur lui-mème.

Recherchant la valeur du massif boisé, ergo omnes emplores, il écarte en outre :

$1^{\circ}$ la détermination de la valeur dú sol par comparaison arec celle des sols roisins lirrés à d'autres cultures, ou par les prix attribués au fonds dans les transactions dont les forèts voisines ont pu ètre l'objet:

$2^{\circ}$ l'admission d'un taux déterminé soit par les achats effectués dans la région, soit par les projets de l'acheteur.

A son point de vue, le matériel sur pied vaul, commer celui d'une coupe, le prix auquel il peut ètre vendu; les bois trop jeunes pour ètre commerçables, ont une raleur qu'il déduit du tracé d'une courbe allant de la valeur 0 à 0 ou aux valeur's réalisables à divers âges.

Quant au sol pourvu d'un ensouchement ou de jeunes semis, il admet que sa valeur fonctionne au mème taux (c'est le seul postulatum de sa méthode) que le matériel bois considéré à partir dn moment où il a acquis une valenr commerciale, constituant à compter de celte date le capital, fonds et superficie, qui produit le revenu. 


\section{PRINCIPAUX OUVRAGES CONSULTÉS}

Bannal. (J.-A.) et Sagnier (H.), Dictionnaire dagriculture, Paris, 1886. Hachette et Cie.

Bagxḱris, Manuel de sylviculture, Paris, 1873. Berger-Levrault et Cie.

Bossier (G.), Les plantes des champs et des bois, Paris, 1887. J.-B. Baillière et fils.

Boppe et Jolyet, Les foréts, Paris, 1901, J.-B, Baillière et fils.

Boper, Traite de sylviculture, Paris, 1889. Berger-Levrault et Cie.

Brolliand, Traitement des bois en France, nouvelle édition, Paris, 1894. Berger-Levrault et Cie.

Bocqeet de la Grye, Guide du forestier. - Éléments de sylvicullure, 9o édition, Paris, 1892. J. Rothschild.

Candot (E.), Petit manuel à l'usage des sociétés scolaires pastoralesforestières de Franche-Comté, Besançon, 1902. Paul Jacquin.

- Rapport sur le concours forestier entre les instituteurs organisé par la Socièté forestière des Amis des arbres (Bull. de la Soc. forest. des Amis des arbres, $\left.\mathrm{n}^{\circ} 38,1902\right)$.

Deмoxtzex, Traité pratique du reboisement et du gazonnement des montagnes, Paris, 1882. J. Rothschild.

Fооснот, Cubage et estimation des bois, 3e édit., Paris. J. Hetzel et Cie.

Galmiche (E.), Notes pour l'achat et la vente des foreits, Besangon, 1897. Paul Jacquin.

Gayer (Dr Karu) (de Munich), Traité de sylviculture. Traduit sur la 3 e édit. allemande par E. Visart de Bocarné, Bruges, 1901. G. Stock, Paris. Berger-Levrault.

Hexry (E.), Poids et composition de la couverture morte ( $C$. Rendus de l'Ac. des $S c ., 1896)$.

Huffer. (G.), Les arbres et les peuplements forestiers, Paris, 1893. BergerLevrault et Cie.

Lorextz et Tassx, Culture des bois, $6^{\circ}$ édition, Paris. 0. Doin.

МАтнıе, Flore forestière, $4^{\circ}$ édition, revue par P. Fliche, Paris, $189 \%$. J.-B. Baillière et fils.

Матнie, Le reboisement et le gazonnement des Alpes, Paris, 1875. Imprimerie Nationale.

MАтнex, Étude sommaire des taillis-sous-futaie dans le bassin de la Saone (Bull. de la Soc. forest. de Franche-Comté et Belfort, septembre 1898). Besangon, 1898. P. Jacquin.

Ińlafd (A.). Insuffisance de la production des loois d'ouvre dans le monde (Cong. intern. forest. de 1900).

Muel. (E.), Notions de sylviculture, Paris, 1884. Ducher et Cie.

Purox, Traité d'économie forestière, Paris, 1888. Marchal et Billard.

PARdé, Les principaux végétaux ligueux exotiques au point de vue forestier (Cong. intern. de sylviculture de 1900).

- Etude sur les essences forestières exotiques dont la naturalisation semble possible en France (Bull. de la Soc. forest. frang. des Amis des arbres, 1901).

Vull.Rmix (P.), Les Mycorrhizes (Rev. gén. des Sc., 1890).

Atlas de stat. forest. de l'Admin. des Eaux et Foréts; Bulletin de la Soc. forest. de Franche-Comte et Belfort; Bull. de la Soc. forest. des Amis les arbres; C. R. détaillè du Cong. intern. de sylviculture, tenu à Paris du 4 au 7 juin 1900, sous la présid. de M. Daul,rée, conseiller d'État, directeur des Eaux et Foréts; Revue des Eaux et Forêts; Agenda du forestier, Besançon. P. Jacquin. 



\section{TABLE DES MATIÉRES}

Ixrroduction, par M. le Dr P. Regnard, directeur de l'Institut national

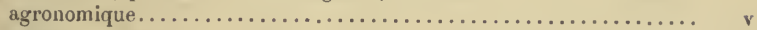

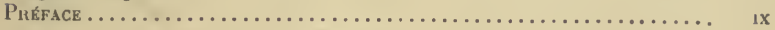

\section{PREMIÈRE PAR'TIE}

\section{La forêt en général et ses éléments constitutifs.}

I. Vie de l'arbre en général.....................

Gèneralités

I. Germination

II. Enracinement; yIscorrhizes.

III. Tige feuillée.-Ramification.

IV. Croissance en hatteur. Longévité. - Dimensions....

V. Floraison. - Fructification. .

VI. Croissance en diamètre. Bois....................

VII. Bourgeons .............

VIII. Rejets de souche. - Jrageons

IX. Composition et alimentation de l'arbre................

II. Forêt et peuplements.

Généralités...

I. Formation en massif........

II. Influence de l'état de massif sur les individus...........

III. Influence de l'état de massif sur la fertilité de la station...

IV. Accroissement total des peuplements.
V. Phases successives de la vie des peuplements............

VI. Conclusions générales ; enlèvement des feuilles mortes; écobuage................

Influence des forèts sur le régime gẻnéral des eaux...........

III. Principales essences forestières............ 70

Géneralités.............. 70

I. Chène rouvre et cliène pédonculé................. 71

II. Hetre............. 75

11I. Charme............. 77

IV. Sapin pectiné............ 80

V. Pin sylvestre........... 82

VI. Chène yeuse ou chène vert. 8 j

VII. Pin maritine.......... 87

41 VIII. Épicéa commun........ 88

42 IX. Mélèze............... 91

X. Pin d'Alep............. 93

44 XI. Chêne tauzin............ 94.

XII. Chène occideutal......... 95

51 XIII. Chène-liège........... 96

Tableau des essences secondaires

59 ou disséninées........... 98

1

5

7

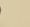

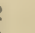


Tableau général pour reconnaitre les arbres, arbustes ou arbrisseaux..................

IV. Diverses formes de peuplements............

Généralités.............. 108

I. Futaie $\ldots \ldots \ldots \ldots \ldots \ldots \ldots$

1. Peuplements réguliers......

2. Peuplements irréguliers.....
3. Réserve sur coupe définitive. - Sous-étage............ 128

II. Taillis............. 132

III. Taillis composé......... 140

V. État de la forêt...... . 150

Gënéralités............. 10

I. Comparaison entre les diffé-

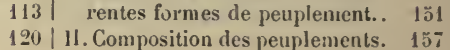

\section{DEUXIENIE PARTIE}

\section{Pratique sylvicole.}

I. Repeuplement........ 166

I. Repeuplement artificiel..... 166

Généralitès (essences indigènes, essences exotiques)..........

1. Boisement par semis direct..

2. Boisement par plantations...

3. Repeuplement par boutures et par marcottes...............

II. Repeuplement par semis naturel..................

1. Régénération par co:pes successives................

Ð. Régénération par coupe unique.

III. Repeuplement par rejels de souche et drageons..........

IV. Combinaison des différentes méthorles de repeuplement...

II. Opérations culturales. . 237
I. Action de l'homme sur le sol.. II. Action de l'homme sur les peuplements............. 264 . Répartition des jeunes sujets.... 26ä Dégagements ............. 257 Éclairícies. ............. 272

III. Mesures de gestlon.... 278

1. Plan du domaine, Limites... 278 212 II. Ordre des exploitations. Assiette des coupes.......... 11I. Notions succinctes d'aménagement.............. IV. Opérations relatives aux

V. Notions génćrales sur les ventes des coupes......... 323

249 V1. Travaux forestiers....... 331

\section{TROLSIEYL PARTIE}

\section{Principaux massifs forestiers.}

I. Etude speciale des taillis simples................

I. Généralités.............

II. Applications du taillis simple.

II. Étude spéciale des talllis composés.

I. Généralités............. 363

II. Applications du taillis composé. 386

III. Restauration des taillis conl- posés en maurais état...... III. Étude spéciale des futales.............. 399

Peuplement purs.......... $\$ 00$

I. Essences feuillues......... $\$ 00$

II. Essences résineuses....... \$16

Peuplements mélonges..... . \$7

I. Citudo succincte des divers mílanges............... 418 


\section{TABLE DES MATIÈRES.}

1. Mélange des essences d'ombre entre elles...............

2. Mélange des essences d'ombre avec les essences de lumière.

3. Mélanges des essences de 448 lumière entre elles........ 40.4 Des prés-bois............. 456

452 Éducation des arbres isolés.... 4 4

\section{QUATRIÈVE PARTIE}

\section{Le domaine boisé et ses éléments constitutifs.}

I. Notions générales d'économie forestière........ 430

I. Agents de la production forestière..................

1. Capital dexploitation.........

2. Travail. - Mode d'exploitation du sol................

3. Igents naturels de production.................. 492

II. Produit brut. - Produit net. 492

1. Produit brut............. 492

2. Produit net............. 496

III. Taux de placement du capital engagé dans une exploitation forestii re.............. 501

IV. Institutions forestières..... 506

V. Assurances des bois et forèts contre l'incendie......... ธิ1ว

II. Notions générales d'estimation des bois....... 520

I. Estimation en matière; principes de cubage.......... $\check{2} 20$

1. (ubage des bois aba.tus.... 520

2. Cubage des bois sur pied.... $\check{3} 36$

II. Estination en argent....... 5.44

III. Valeur de la propriété boisée................. 547

I. Valeur commerciale des forèts ou valeur de réalisation..... 547

II. Valeurs relatives des forèts.. วัว1

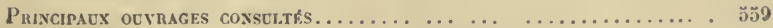




\section{LES FORETS \\ Traité pratique de sylviculture}

Par L. Boppe, directeur honoraire de l'École nationale des Faux et Forêts de Nancy, membre du Conseil supérieur de l'A griculture et Ant Jolyet, chargé de cours à l'Ecole nationale des Eaux et Forêts de Nancy. 1 vol. in-8 de 488 pages, arec 95̃ photogravures.............................. 8 fr.

MM. Boppe et JoLyet dans leur Traité de sylviculture considèrent d'abord l'arbre au strict point de vue forestier; puis ils étudient l'espéce, qui s'affirme par son tempérament, et la suivent dans ses rapports avec les phénomènes météoriques et avec le sol : à celte occasion, ils passent en revue les diflérentes essences qui peuplent nos plaines et nos montagnes, en donnant l'aire d'habitation de chacune, avec les lois qui président à cette distribution.

Ensuite, ils examinent comment ces essences se comportent quand elles sont à l'état isolé, ou réunies en massifs pour former les peuplements, dont l'ensemble constitue la foret. Celleci, influencée par le sol et le climat, change d'aspect dans chaque station et ils en montrent les principaux types.

Après avoir établi les exigences de la forèt spontanée dans chaque station, ils disent par quel genre de culture la forci aménagée, c'est-à-dire économiquement constituée, doit ètrc régénérée et améliorée, en vue de diriger la fabrication de la matière bois vers telle ou telle qualité de marchandise : ils abordent, alors, l'étude détaillée des régimes et des modes de traitement en usage avec leur application en toutes circonstances.

Après ces chapitres consacrés à la forèt en étal de rendement, ils examinent :

$1^{\circ}$ La protection de la forêt contre les dommages qu'elle peut subir ;

$2^{\circ}$ Le boisement des terrains nus, partout où l'exploitation rationnelle du sol le commande.

M.M. Bople el JoLvet ont donné à leur volume un sens prafique qui le met à la portée de tous les représentants de la richesse forestière en France, aussi bien des propriétaires farticuliers que des fonctionnaires de l'Etat. Ils ont éclairé le texte par despaysages forestiers, photographicis d'apre's nature el empruntés à l'album des Elives de l'Eeole Nationale des Eaux et Forèts. 




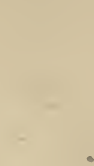

, 10 



\section{I.RRARY}

SD

Fron, Albert

391

Sylviculture

F65

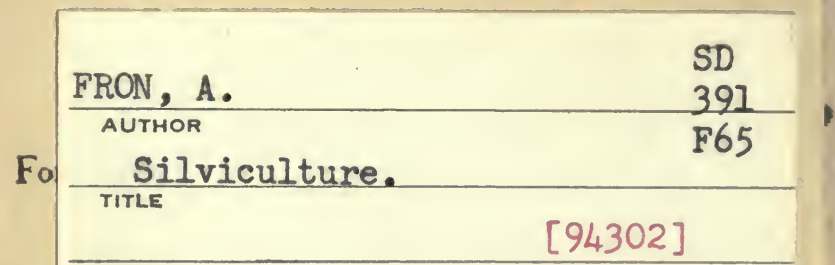

\begin{tabular}{l|l|}
\hline DATE & ISSUED TO \\
\hline
\end{tabular}

\section{[94302]}

\section{LIBRARY}

FACULTY OF FORESTRY

UNIVERSITY OF TORONTO 
